\title{
Label-free and Multi-parametric Monitoring of Cell-based Assays with Substrate-embedded Sensors
}

\section{DISSERTATION}

zur Erlangung des Doktorgrades der

Naturwissenschaften (Dr. rer. nat.)

der Fakultät für Chemie und Pharmazie

der Universität Regensburg

vorgelegt von

Maximilian Oberleitner

aus Obing (Traunstein)

2015

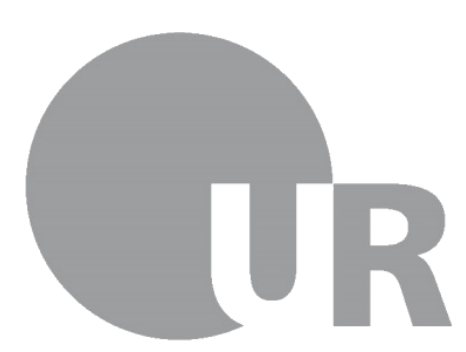

\section{Universität Regensburg}



Prüfungsgesuch eingereicht am:

Promotionskolloquium:

Diese Arbeit wurde angeleitet von:

Prüfungsausschuss:
18.12.2015

04.03 .2016

Prof. Dr. Joachim Wegener

Erstgutachter: Prof. Dr. Joachim Wegener

Zweitgutachter: PD. Dr. Rainer Müller

Drittprüfer: Prof. Dr. Antje J. Bäumner

Vorsitzender: Prof. Dr. Oliver Tepner 



\section{Contents}

Chapter 1 Introduction

1.1 Label-free Biosensors for Cell-based Assays

1.1.1 Electrochemical Transducers for Label-free CBB 4

1.1.2 Optical Transducers for Label-free CBB 7

1.1.3 Piezolectric Transducers for Label-free CBB 11

1.2 Multi-Parametric Label-free Cell-based Biosensing 12

\begin{tabular}{lll} 
Chapter 2 & Objectives & 15 \\
\hline
\end{tabular}

$\begin{array}{lll}\text { Chapter } 3 \text { Methods \& Instrumentation } & 19\end{array}$

3.1 Cell Culture 20

3.1.1 Cell Culture Conditions 20

3.1.2 Sub-cultivation of Cells $\quad 21$

3.1.3 Cell Storage 21

3.1.4 Cell Preparation for Experiments $\quad 22$

3.2 Impedance Spectroscopy 22

3.2.1 The Complex Impedance 23

3.2.2 Impedance Elements in Equivalent Circuits $\quad 25$

3.2.3 Equivalent Circuit Models 28

3.3 The Quartz Crystal Microbalance (OCM) 31

3.3.1 Basic Principles of the OCM Technique 32

3.3.2 Electro-acoustic Modeling and Impedance Analysis of TSM Resonators 37

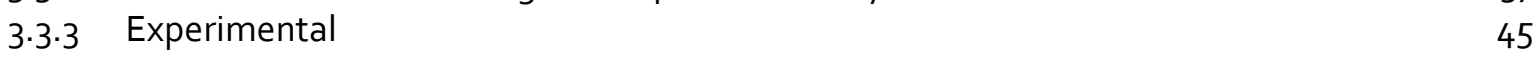

3.4 Electric Cell-Substrate Impedance Sensing (ECIS) 50

3.4.1 Basic Principles of the ECIS Technique 50

3.4.2 Impedance Properties of (Cell Covered) ECIS Electrodes $\quad 52$

3.4.3 Modeling the Impedance of the Cell-Electrode Interface 55

3.4.4 ECIS-QCM Combination in One Experimental Setup 56

3.4.5 Electroporation on ECIS-OCM Sensor (ELPO-ECIS-OCM) 59

3.5 Optical Temperature and Oxygen Imaging 61

3.5.1 Basic Principles of Luminescence and Luminescence Quenching 62

3.5.2 Fluorescence Lifetime Imaging (FLIM) using Rapid Lifetime Determination (RLD) 65

3.5.3 Experimental of FLIM-RLD 66

$\begin{array}{lll}3.6 & \text { Light Microscopic Techniques } & 73\end{array}$

3.6.1 Phase-Contrast Microscopy 73

$\begin{array}{ll}3.6 .2 & \text { Stereomicroscopy } \\ 3.6 .3 & 75\end{array}$

$\begin{array}{lll}3.6 .3 & \text { Fluorescence Microscopy } & 75\end{array}$

3.6.4 Confocal Laser Scanning Microscopy (CLSM)

3.6.5 Cytochemical Fluorescence Staining 78 
4.1 Layout and Oscillation Properties

4.1.1 2-Electrode OCM Layout

4.1.2 Oscillation in Air

4.1.3 Oscillation under Liquid Loading

4.1.4 Resonator Synchronization

4.1.5 Resonance Decoupling via Selective Rigid Coating 90

4.2 Decoupled 2ElOs: Impact of Film Thickness 93

4.2.1 Degree of Decoupling as a Function of Film Thickness 93

4.2.2 Film Thickness Determination 95

$\begin{array}{lll}4.3 & \text { Discussion } & 97\end{array}$

4.3.1 2ElQ Sensor Layout and Oscillation Properties $\quad 97$

$\begin{array}{lr}\text { 4.3.2 Decoupling of 2EIQ Resonators } & 102\end{array}$

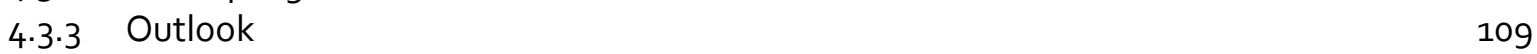

Chapter 5 OCM-ECIS: Combined Viscoelastic and Dielectric Sensing of Cells

5.1 OCM-ECIS Sensor Layout and Characteristics 113

5.1.1 Substrate-Integration of OCM-ECIS 113

5.1.2 Standard OCM-ECIS Sensor Layout $\quad 115$

5.1.3 Surface Characterization $\quad 115$

5.1.4 OCM-mode: Piezoelectric Characterization of the Sensor $\quad 117$

5.1.5 ECIS-mode: Electrochemical Characterization of the Sensor 120

5.1.6 OCM-ECIS Sensors: Systematic Variation of WE Sizes and Layouts 123

$\begin{array}{lll}5.2 & \text { OCM-ECIS Biosensor for Cell Attachment and Spreading } & 129\end{array}$

5.2.1 Sensor Preincubation with Culture Medium 129

5.2.2 MDCK-II Cell Attachment and Spreading 131

5.2.3 NRK Cell Attachment and Spreading 135

5.2.4 Cell Attachment and Spreading in Absence of $\mathrm{Ca}^{2+}$ and $\mathrm{Mg}^{2+} \quad 139$

5.3 OCM-ECIS Whole-Cell Biosensor 143

5.3.1 Degradation of the Actin Cytoskeleton 144

5.3.2 Cell Stiffening using Chemical Fixatives $\quad 149$

5.3.3 Stimulating Intracellular Signaling Cascades $\quad 154$

5.3.4 Electric Manipulation and Double-mode Impedance Analysis (ELPO-ECIS-OCM) $\quad 159$

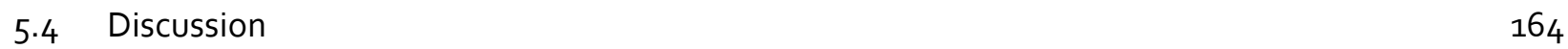

5.4.1 Electrode Layout $\quad 164$

5.4.2 OCM-mode: Piezoelectric Characterization of the Sensor 166

5.4.3 ECIS-mode: Electrochemical Characterization of the Sensor $\quad 171$

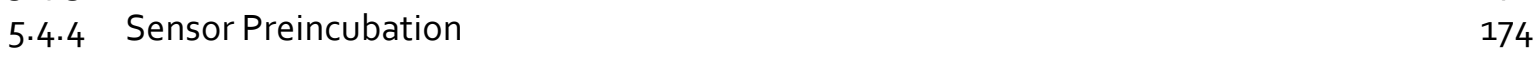

5.4.5 Cell Attachment and Spreading 175

5.4.6 Cell Attachment and Spreading in Absence of $\mathrm{Ca}^{2+}$ and $\mathrm{Mg}^{2+} \quad 183$

$\begin{array}{ll}\text { 5.4.7 Degradation of the Actin Cytoskeleton } & 189\end{array}$

5.4.8 Cell Stiffening using Chemical Fixatives $\quad 195$

5.4.9 Stimulating Intracellular Signaling Cascades 203

5.4.10 Electric Manipulation and Double-mode Impedance Analysis (ELPO-ECIS-OCM) 208

$\begin{array}{ll}5.4 .11 & \text { Summary } \\ 5.4 .12 & 216\end{array}$

$\begin{array}{ll}5.4 .12 \text { Outlook } & 218\end{array}$ 
Chapter 6 OCM-OCS: Optochemical Sensing of Temperature and $\mathrm{pO}_{2}$ in the Cell Surface Junction

6.1 Imaging the Frictional Heat Induced by High-Amplitude Quartz Oscillations

6.1.1 Quartz Coating and Characterization

6.1.2 Sensor Calibration $\quad 225$

6.1.3 Imaging the Temperature of High-Amplitude OCM in Air 226

6.1.4 Imaging the Temperature of High-Amplitude QCM in Buffer 230

6.2 Imaging the Oxygen Consumption Rate (OCR) beneath Adherent Cells 232

6.2.1 Cytocompatibility Testing of the Sensor Film 233

6.2.2 Sensor Calibration $\quad 235$

6.2.3 Imaging the OCR of Cells 239

6.3 Discussion $\quad 242$

6.3.1 Imaging the Frictional Heat Induced by High-Amplitude Quartz Oscillations $\quad 242$

6.3.2 Imaging the Oxygen Consumption Rate (OCR) beneath Adherent Cells 246

$\begin{array}{lll}6.3 .3 & \text { Outlook } & 251\end{array}$

\begin{tabular}{lll} 
Chapter 7 & Summary/ Zusammenfassung & 253 \\
\hline
\end{tabular}

$\begin{array}{llr}7.1 & \text { Summary } & 254\end{array}$

$\begin{array}{lll}7.2 & \text { Zusammenfassung } & 257\end{array}$

$\begin{array}{ll}\text { Appendix } & 261\end{array}$

$\begin{array}{lll}\text { A } & \text { Supplementary Information (SI) } & 262\end{array}$

B Abbreviations, Acronyms, and Symbols $\quad 281$

C List of Special (Bio)Chemical Reagents 284

\begin{tabular}{lr} 
References & 285 \\
\hline
\end{tabular}

$\begin{array}{ll}\text { Acknowledgement } & 313\end{array}$

$\begin{array}{lr}\text { CV } & 315\end{array}$

$\begin{array}{ll}\text { Eidesstattliche Erklärung } & 317\end{array}$ 



\section{Chapter 1 Introduction}

1.1 Label-free Biosensors for Cell-based Assays 2

1.2 Multi-Parametric Label-free Cell-based Biosensing 12 


\subsection{Label-free Biosensors for Cell-based Assays}

Following IUPAC, a biosensor is defined as

a self-contained integrated device, which is capable of providing specific quantitative or semi-quantitative analytical information using a biological recognition element (biochemical receptor) which is retained in direct spatial contact with a physicochemical transduction element. ${ }^{[1]}$

The biological recognition unit in biosensors can be represented by enzymes, antibodies/ antigens, nucleic acids, DNA strands, cell organelles or particles, micro-organisms, whole eukaryotic cells, or tissues. The setup of a cell-based biosensor device is illustrated on the right hand side in Fig. 1-1. It is composed of two major components: (1) an immobilized biological receptor unit (cells), which sensitively and selectively reports on the presence of an analyte or stimulus in a concentration-dependent manner by the change of a physicochemical quantity and (2) a transducer unit in close proximity to the biological recognition unit, which translates the physicochemical change of the recognition event into an electrical signal. Further components are the electronics for the detection of this signal and a control and signal output unit for controlling the transducer readout and further conversion, data processing, analysis, storage, and visualization of the signals measured.

With respect to cellular biosensing, one has to distinguish between biosensors that are based on adherent/ immobilized whole cells as the biological recognition unit for monitoring the cellular response to an externally applied analyte/ stimulus, and biosensors that utilize immobilized functional proteins or other structures on the sensor surface for the specific detection of cells as the analyte in a sample. In this thesis the term cell-based biosensor (CBBs) is used only for the former. Moreover, cell-based assays (CBAs) are classified in label-based and label-free approaches. Whereas the former require additional reporting compounds for detecting cellular responses, provided by radio-, enzyme-, fluorescence- or chromophoric labeling or overexpression of reporter proteins, the latter does not. Label-free CBAs exhibit several advantages over conventional label-based cellular assays. First, label-free approaches redundantize a costly and time consuming labeling step or genetic engineering. Moreover, there is no additional component introduced or generated in situ, which potentially interferes with the native cellular system under study. Label-based assays are typically endpoint approaches where the experiment is terminated at a predefined point and where cells are commonly sacrificed for analysis. By contrast, label-free sensor technologies for living cells themselves are characterized by their non-invasiveness of measurements. This allows an unrestricted and continuous monitoring and analysis of CBAs over any desired period of time. However, label-based methods are advantageous in other fields, like for intstance for identifying and analyzing of specific biomolecular interactions or in the throughput of CBAs. Therefore, label-free and label-based CBAs are considered rather as complementing than as competing approaches.

Label-free CBB technologies gained an increasing interest over the past two decades, as they are considered as a promising tool in various research fields, such as drug discovery, toxin and pathogen testing, clinical diagnostics, environmental monitoring, and biosecurity, just to name a few. ${ }^{[2-6]}$ Sensors based on cell cultures act as a bridging technology between the classical sensing of biomolecular recognition/ interaction and animal tests. Though cell-based biosensors cannot mimic the total response of a whole organism towards a stimulus, they provide a native biological environment on a small scale (the cellular level) that mirrors the real physiological situation much better than isolated biomolecules. 
Cells represent the smallest self-sustaining living entity. In in vitro cell cultures the basic biological processes at the cellular level (like spreading, proliferation, barrier formation, migration, gene expression, signaling, apoptosis, etc.) are similar to the processes in vivo. Hence, they are in many cases a representative functional model system for the whole body of an organism. In contrast to biomolecular sensors, in cell-based assays it is not the molecular interaction event of an analyte with a target structure that is detected, but it is rather the detection of the integrated physiological effect caused by an analyte or stimulus with all its complexity as a whole.

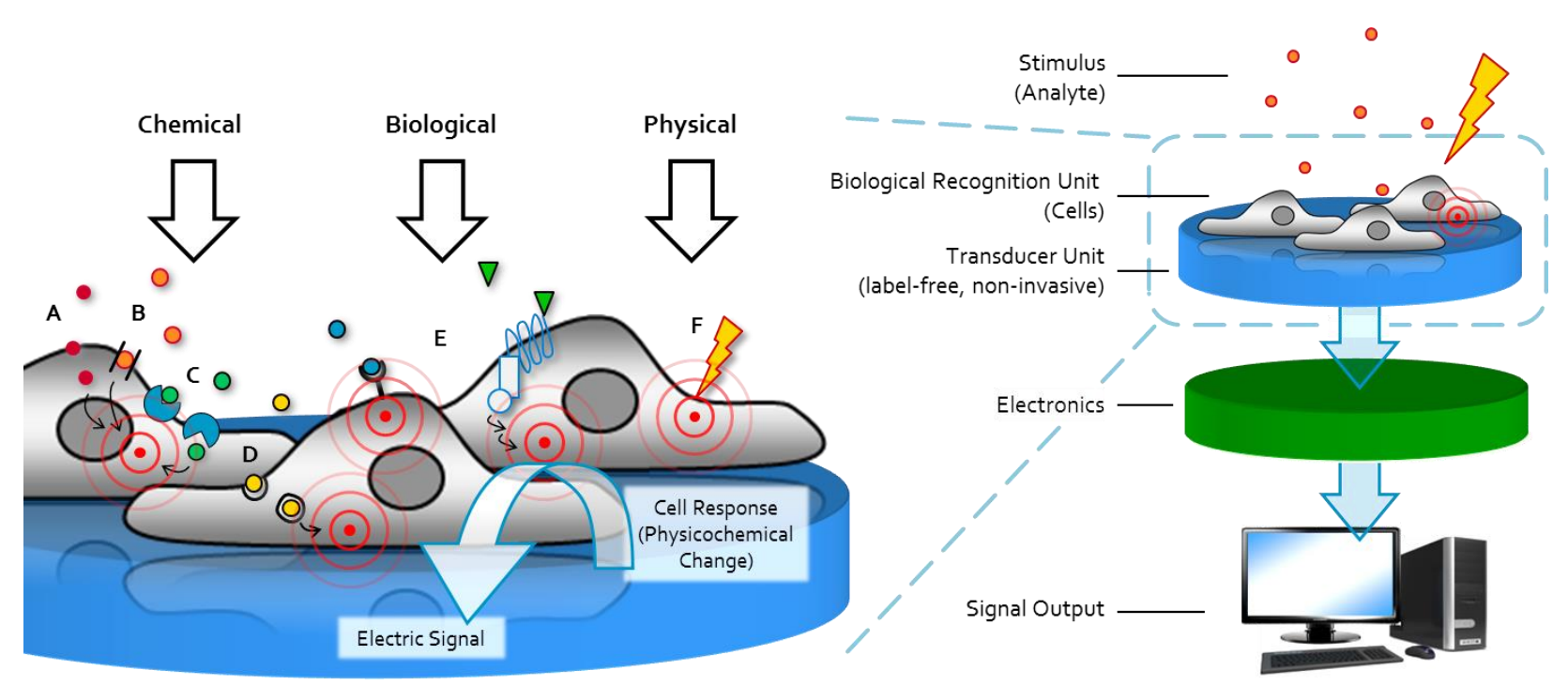

Fig. 1-1 On the right: setup of a label-free cell-based biosensor (CBB). On the left: ways how chemical, biological, and physical stimuli may interact with the cellular recognition unit to cause a cellular response (a change in physicochemical quantity) that is converted into a measurable electric signal by the transducer unit. In parts adapted from ${ }^{[7]}$.

There are many ways how an externally applied stimulus can be recognized by a cell layer. This is schematically shown on the left hand side in Fig. 1-1. In cases of a chemical (e.g. a metal nanoparticle) or a biochemical (e.g. a toxin) stimulus the respective substance can be recognized by the cells due to its uptake, via free diffusion across the cell membrane, channel-/ carrier-mediated transport, or endocytosis (Fig. 1-1 A-D), or due to its (specific or nonspecific) binding to cell surface receptors (Fig. 1-1E). A physical stimulus (e.g. heat) interacts as a whole with the total cellular system (Fig. 1-1 F). Any event of stimulation or cell-analyte interaction could cause the activation of intracellular processes and signal cascades that in turn could be finally expressed in any kind of structural, functional, and/ or metabolic cellular response. Thus, the physicochemical change might be of multidimensional and complex nature. For instance, a stimulus could cause a change in the cellular metabolism (affecting the equilibrium concentrations of e.g. glucose, dissolved oxygen, $\mathrm{HCO}_{3}{ }^{-}, \mathrm{H}^{+}, \mathrm{Na}^{+}, \mathrm{Ca}^{2+}, \mathrm{Cl}^{-}$, lactate, etc.), changes in the membrane potential, the barrier resistance and cell morphology (both affecting the dielectric properties of the cell layer), the distribution of mass, and in the micromechanics of the cells.

These cellular responses and the accompanying changes of physicochemical parameters are what is to be detected and transferred into a measurable electrical signal by the transducer units of the label-free biosensor devices (Fig. 1-1). There are various types of transducers, distinguishable by the underlying physicochemical phenomenon: electrochemical (including potentiometric, amperometric, and impedimetric), optical, thermometric, piezoelectric, and magnetic transduction. ${ }^{[8]}$ Tab. $1-1$ gives an 
overview on the most common transducer principles and sensor techniques hitherto presented for the label-free and non-invasive monitoring and analysis of cell-based assays. However, this list makes no claim of completeness, since there are several further methodical and metrological developments and enhancements of the various sensor platforms. A complete survey is beyond the scope of this introduction on label-free CBBs. For instance, there are various microscopy-based techniques that make use of a metrologically modified form of the various transducer principles. These approaches are not listed here. It has to be noted that the luminescence-based readout principles mentioned are confined to experimental setups in which the luminophore is immobilized in a sensor matrix. This means that the cells are not exposed to the luminescent reporter compound and the approache therefore is justifiable referred to as label-free. The basic operation principles of selected CBB techniques which are most relevant to the scope of this thesis (printed in bold type in Tab. 1-1) and exemplary applications in labelfree CBAs are considered in more detail below.

Tab. 1-1 Most common transducer principles and sensor techniques used in label-free cellbased biosensing (CBB). ${ }^{[3,4,6,8-22]}$ Techniques printed in bold type are described in more detail in subsections 1.1.1-1.1.3.

\begin{tabular}{|c|c|c|}
\hline Transducer Principle & Readout Principle/ Quantity & Sensor Technique \\
\hline \multirow[t]{6}{*}{ Electrochemical } & Potentiometry & LAPS/ MLAPS \\
\hline & & ISFET/ EnFET \\
\hline & Amperometry & ME/ Clark-type ME \\
\hline & Impedance & ECIS \\
\hline & Resistance & \\
\hline & Capacitance & \\
\hline \multirow[t]{9}{*}{ Optical } & Luminescence (OCS) & Intensity/ Anisotropy/ Lifetime \\
\hline & Scattering & Raman/ SERS \\
\hline & Refractive Index & SPR (Prism-/ Waveguide-/ Grating-Coupling) \\
\hline & & RWG \\
\hline & & Interferometry/ RIfS/ RICM \\
\hline & & Waveguide \\
\hline & & Optical Ring Resonator \\
\hline & & Photonic Crystal \\
\hline & & Resonant Mirror \\
\hline \multirow[t]{2}{*}{ Piezoelectric } & Shear Acoustic Wave & QCM \\
\hline & & SAW \\
\hline
\end{tabular}

LAPS: Light-Addressable Potentiometric Sensor, MLAPS: Multi LAPS, ISFET: Ion-Selective Field-Effect Transistor, EnFET: Enzyme Field-Effect Transistor, ME: Microelectrodes, ECIS: Electric Cell-Substrate Impedance Sensing, OCS: Optical Chemical Sensing, SERS: Surface-Enhanced Raman Spectroscopy, SPR: Surface Plasmon Resonance, RWG: Resonant Waveguide Grating, RIfS: Reflectometric Interference Spectroscopy, RICM: Reflection Interference Contrast Microscopy, QCM: Quartz Crystal Microbalance, SAW: Surface Acoustic Wave.

\subsubsection{Electrochemical Transducers for Label-free CBB}

Electrochemical signal transduction can be divided into various types, either depending on its mode of readout, i.e. potentiometric, amperometric, and impedimetric, or depending on the content of 
information in CBB applications, i.e. cell metabolism or cell morphology. Electrochemical sensors for the analysis of cell metabolites are described on the basis of ion-selective field effect transistors (ISFET), light-addressable potentiometric sensors (LAPS), and amperometric microelectrodes. Information on cell morphology, cytotoxicity, and cellular motility is monitored by impedance analysis of adherently grown cell monolayers, also referred to as electric cell-substrate impedance sensing (ECIS).

\section{ISFET}

Ion-selective field effect transistors (ISFETs) are ion sensors based on field effect devices (FEDs), where the metal gate of typical metal-insulator-semiconductor/ metal-metal oxide-semiconductor field-effect transistors (MISFETs/ MOSFETs) is replaced by a reference electrode and an electrolyte solution above the gate insulator. Ion-sensitivity in ISFETs is introduced by an additional ion-sensitive layer between electrolyte and insulator. ${ }^{[8]} \mathrm{A}$ schematic layout of an ISFET is shown in Fig. 1-2 A. The current $I_{D}$ between source and drain at a constant gate potential $U_{G}$ is determined by the surface potential at the interface between the ion-sensitive layer and the electrolyte. Changes in the ion concentration in the solution influence this surface potential, resulting in a change of the source-drain current. At the operation principle of constant drain current, suitable electronics then automatically adapt the gate voltage to restore the initial operational drain current and to compensate for the surface potential changes due to the changing ion (analyte) concentration, respectively. The change of the gate voltage thereby directly correlates with the analyte concentration. ${ }^{[8]}$

In $\mathrm{H}^{+} / \mathrm{pH}$-sensitive ISFETs ( $\mathrm{pH}$-ISFET) the surface of the gate insulator $\left(\mathrm{Si}_{3} \mathrm{~N}_{41} \mathrm{Al}_{2} \mathrm{O}_{31}, \mathrm{Ta}_{2} \mathrm{O}_{5}\right)$ directly acts as the proton-sensitive layer. The ISFET devices are also capable of quantitatively sensing other ionic species but $\mathrm{H}^{+}$by the introduction of additional ion-sensitive layers. These are either ionophore-based polymeric membranes, molecularly imprinted polymer membranes, or solid ion-responsive membranes. ${ }^{[8]}$ ISFETs for the sensitive and selective determination of the physiologically relevant species $\mathrm{Na}^{+}, \mathrm{K}^{+}, \mathrm{Ca}^{2+}$, and $\mathrm{Cl}^{-[23-29]}$ in cell-based assays have been developed. Besides these, ISFETs for metal-ions like $\mathrm{Ag}^{+}, \mathrm{Cd}^{2+}, \mathrm{Cu}^{2+}, \mathrm{Hg}^{2+}$, and $\mathrm{Pb}^{2+}{ }^{[8]}$ have also been reported. By coating the ion-selective layer of an ISFET with an enzyme layer that produces or consumes in an enzymatic reaction the ion to which the layer below is sensitive, one obtains a so-called enzymatic ISFET (EnFET). This allows the indirect determination of other reactants and products of the enzymatic reaction. EnFETs based on $\mathrm{H}^{+} /$ $\mathrm{OH}^{-}$detection (enzymes immobilized on a pH-ISFET) were utilized for the biosensing of various analytes, such as glutamate ${ }^{[30]}$, glucose ${ }^{\left[3^{1-34]}\right.}$, urea ${ }^{[35-40]}$, lactate ${ }^{[41]}$, penicillin ${ }^{[42,43]}$, and glykoalkaloids ${ }^{[44]}$. ISFETs are mostly used as pH-ISFETs in CBBs for cell microenvironment monitoring and sensing of the extracellular acidification rate $(E C A R) .{ }^{[10]}$ As the ECAR is correlated with the physiological state and serves as an indicator for the global metabolism of cells ${ }^{[45]}$, $\mathrm{pH}$-ISFETs have been widely used in cellbased screenings of anticancer drugs efficacy. ${ }^{[17]}$ Recently, a chip-based sensor approach has been realized for the simultaneous monitoring of both the ECAR and the oxygen consumption rate (OCR) (cellular respiration) with a single ISFET. ${ }^{[45]}$ Further multi-parametric CBBs were developed on that basis ${ }^{[45-65]}$, which will be addressed in 1.2 Multi-Parametric Label-free Cell-based Biosensing.

\section{LAPS}

Light-addressable potentiometric sensors (LAPSs) are multilayer field-effect devices similar to ISFETs ${ }^{[66]}$. A LAPS consists of a doped semiconductor covered with an insulator layer and a chemosensitive layer. The device is immersed in an electrolyte solution. The semiconductor is connected with a reference electrode in contact with the electrolyte (Fig. 1-2 B; reviewed in ${ }^{[1,17,67-70]}$ with respect to biological and cell-based applications). The insulator material of LAPSs is typically made up either of a single $\mathrm{SiO}_{2}$ layer 
or a double layer of $\mathrm{SiO}_{2}$ and $\mathrm{Si}_{3} \mathrm{~N}_{41} \mathrm{Al}_{2} \mathrm{O}_{3}$, or $\mathrm{Ta}_{2} \mathrm{O}_{5}$ on top, whose surface charge is sensitive to $\mathrm{H}^{+}$-ions in the solution. If a bias voltage $U_{B}$ with respect to the reference electrode is applied to the LAPS, a depletion layer is established at the insulator-silicon interface. By irradiating the device with modulated LED light pulses of appropriate (IR) wavelength, electron-hole pairs are generated in the silicon. This causes an AC photocurrent as a function of the DC voltage in the LAPS. The effective potential, thereby, is the sum of the applied bias potential in series to the surface potential at the insulator-electrolyte interface ${ }^{[8]}$ Hence, any change in the $\mathrm{H}^{+}$concentration in the solution causes a change in the surface charge, affecting the interface potential and consequently the photocurrent. As LAPSs are usually operated in constant-photocurrent mode, the required change of the bias voltage for compensating photocurrent alterations (using a feedback-control circuit) is the readout quantity and corresponds to $\mathrm{pH}$ changes ${ }^{[10]}$ For instance, a decrease in the $\mathrm{pH}$ due to an increased ECAR causes the protonation of silanol groups at the insulator-electrolyte interface. This means a decrease of the interfacial charge and a reduction of the surface potential, which goes along with a smaller depletion of the semiconductor and a decrease in the photocurrent. Like in ISFETs, sensitivity to other analyte ions but $\mathrm{H}^{+}$can be introduced by a chemoselective layer on the insulator (Fig. 1-2 B). ${ }^{[28]}$

The possibility of only irradiating locally defined regions of the semiconductor material enables to readout various areas of the cell-covered sensor independently. On the one hand, this can be utilized for multi-analyte sensing (multiplexing), also termed multi-LAPS (MLAPS), by implementing differently ionsensitive membranes distributed over the insulator surface. These are monitored either sequentially by LEDs with similar modulation frequency or simultaneously by several light sources with different frequency modulations. ${ }^{[71-77]}$ MLAPS applications have already been presented for the parallel monitoring of the ECAR, glucose uptake, oxygen uptake, and lactate production ${ }^{[78,79]}$ as well as for the simultaneous sensing of ions like $\mathrm{H}^{+}, \mathrm{Li}^{+}, \mathrm{Na}^{+}, \mathrm{K}^{+}, \mathrm{Ca}^{2+}$, and $\mathrm{Cl}^{-[27,72,73,77]}$. On the other hand, the locally defined LAPS readout can be applied for imaging the spatial distribution of a single analyte by either scanning the light source across the device or using an array of light sources. ${ }^{[80-82]}$ This has already been presented for the 2-D mapping of local pH values. ${ }^{[71,75,76,81,83]}$ ECAR and cell metabolism sensing by LAPS has been widely used in various research fields, as for instance in receptor-based studies, biodefense toxin discrimination, drug efficacy and cancer studies, or environmental toxin monitoring (see ${ }^{[10,67,69,70,79]}$ and references there). LAPSs in combination with cell-based assays for sensing extracellular acidification are often also referred to as cytosensor or microphysiometer as the trade name of the only commercialized LAPS system (Molecular Devices LLC; Sunnyvale, CA, USA). ${ }^{[67]}$

\section{Amperometric}

Besides the potentiometric field-effect-based transduction techniques ISFETs and (M)LAPS, amperometric transducers for chemosensing (Fig. 1-2 C) are described as well. The most commonly used sensor of this type is the amperometric oxygen sensor for the determination of the biological oxygen demand (BOD). ${ }^{[8]}$ The first amperometric sensor was presented by Clark in 1956 for monitoring and controlling of blood and tissue oxygen tensions ${ }^{\left[{ }^{[8]}\right]}$ In Clark-type amperometric oxygen sensors, the analyte solution is separated from an electrochemical cell by an oxygen-permeable Teflon membrane. The cell consists of a working and a counter electrode immersed in a $\mathrm{KCl}$ solution. Oxygen molecules that diffused across this membrane are reduced at a constant potential at the noble metal ( $\mathrm{Au}, \mathrm{Pt})$ working electrode (cathode) and cause an increase in the current measured. Hence, the reduction current as the readout quantity is a direct measure of the concentration of dissolved oxygen (DO) in the sample solution.

Clark-type microelectrodes (MEs) and planar amperometric $\mathrm{pO}_{2}$ sensor structures (a three electrode system controlled by a potentiostatic electronic circuit) were developed and integrated into miniaturized 
chip-based systems for the label-free and continuous monitoring of DO in cell-based assays, i.e. the oxygen consumption rate (OCR) of cells. ${ }^{[47,48,50,53,54,57,60-62]}$ The sensor chips with integrated amperometric oxygen sensors are typically build up as multi-parametric sensors and contain further physicochemical transducer structures like e.g. for cell morphology testing and ECAR monitoring. Besides the direct measurement of $\mathrm{DO}$, the amperometric sensors are also capable of sensing indirectly any species that is consumed or produced in an oxidase-based enzymatic reaction (amperometric enzyme sensor) via the oxygen or hydrogen peroxide concentration. For this purpose, the amperometric $\mathrm{pO}_{2}$ sensor is coated with a layer in which the respective enzyme is immobilized and which enables free diffusion of all reactants between the bulk solution and the sensor surface. This approach was first presented by Clark and Lyons as an amperometric sensor for glucose ${ }^{\left[{ }^{85]}\right.}$ and has also been applied as transduction method in cell-based assays for the label-free determination and monitoring of glucose and lactose ${ }^{[78,79]}$. A list of physiologically relevant analytes that have been successfully monitored by amperometric enzyme sensors is given in ${ }^{[86]}$.

\section{ECIS}

Electrical impedance-based sensing of adherent cells, also termed as electric cell-substrate impedance sensing (ECIS), utilizes the property of cells to impede AC current flow when they are in close contact to the substrate, i.e. a small gold-film working electrode (Fig. 1-2 D). This technique was presented first by Giaever and Keese in $1984^{[87]}$ as "morphological biosensor for mammalian cells" [88]. The principle of ECIS, the method of impedance spectroscopy, and the analysis, physiological meaning, and modeling of impedance data are extensively addressed in the methods section 3.4 Electric Cell-Substrate Impedance Sensing (ECIS). Briefly summarized, ECIS enables the time-resolved, label-free, and non-invasive monitoring of cell-substrate interactions like cell attachment and spreading, proliferation, as well as cell detachment, and is suitable for long-term measurements of cell-based assays. ${ }^{[89,90]}$ Additionally, changes in cell-cell interactions and cell morphology that open or close AC current pathways through the cell layer, such as for instance barrier formation and opening, can be sensitively measured in a change of the electrical impedance. ${ }^{[91-95]}$

Such cell morphological alterations in a cell layer can be caused by various stimuli and, thus, make impedance monitoring a versatile tool for studies of e.g. cell vitality (analysis of impedance fluctuation) [96-98], cell migration and wound healing [99-103], cell invasion [100,104-107], barrier function [91,93,108], cytotoxicity ${ }^{[109-116]}$, and drug efficacy ${ }^{[4,100,117]}$. The ECIS system has also been applied in actuation mode, by exposing cell layers to AC voltage pulses of high amplitude. This has been used either to electrically generate a defined wound in a cell layer or to electroporate (ELPO) cells on the working electrode, depending on the duration and amplitude of the voltage pulse, with subsequent non-invasive impedance monitoring and analysis of the electrically manipulated cell layer..$^{[7,118-120]}$ The principle of ELPO is addressed in more detail in subsection 3.4.5. Besides the use of one or more small circular working electrodes as the impedance-sensitive spot(s) vis-à-vis to a large counter electrode, impedance-based CBBs are also presented utilizing interdigitated electrode (IDE) structures. ${ }^{[51,60,61,112,121-123]}$

\subsubsection{Optical Transducers for Label-free CBB}

Optical biosensors in general can be categorized into the various properties of electromagnetic radiation that are used for readout: the wavelength of excitation/ emitted light, the intensity of absorbed light (UV-/ VIS-/ IR-absorbance), the intensity of reflected/ scattered light (reflectometry, interferometry, 
Raman), the intensity of emitted light (fluorescence, phosphorescence), and the luminescence lifetime and anisotropy.

Even though transmission light microscopic techiques for analyzing cells are not in the scope of this overview, they are important non-invasive tools for the visual evaluation of the cell morphology and thus should be briefly mentioned here. Aside from the already established optical contrast-enhancing techniques like phase contrast and digital interference contrast microscopy, digital holographic microscopy (DHM) is an emerging and promising non-invasive and label-free interferometric method. It provides 3-D images of transparent samples like cells with a high resolution along the optical axis (in zdirection). Additionally, it also provides a quantitative measurement of the optical path length, which is related to the optical density of the cell. Thus, DHM is well-suited for the image-based label-free screening and quantitative analysis of various events of cell morphological alterations. ${ }^{[124-126]}$

Up to now, almost any optical CBB with the purpose of label-free transduction makes use of refractive index (RI) changes that occur at the sensor surface due to cell-substrate interactions and surface binding. There a various RI-based biosensor techniques and setups (Tab. 1-1), distinguishable either by the optical components and the physical principles used for coupling light into the sensor/liquid interface or by the way how RI changes affect the properties of the light that is reflected and analyzed. RI sensors applied in cell-based assays utilize interferometry, ring resonators, waveguides, resonant mirrors, photonic crystals, surface plasmon resonance (SPR), and resonant waveguide grating (RWG). ${ }^{[9,127-130]}$ Amongst

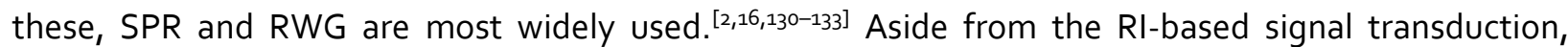
luminescence-based optical chemical sensing (OCS) is also used in label-free CBAs. The immobilization of the luminescent probe in a planar sensor matrix thereby implements the label-free approach in the experimental setup. The cells are hereby exposed neither intracellularly nor extracellularly to the probe and therefore this method is distinguished from conventional label-based biochemical assays using dissolved/ suspended probes.

\section{SPR}

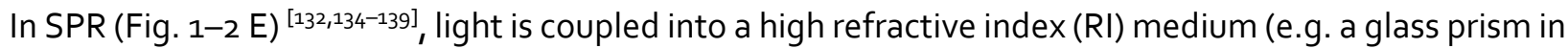
Kretschmann configuration ${ }^{[132,135]}, n_{1}$ ) in a certain angle $\theta_{i}$ of incidence so that total internal reflection (TIR) occurs at the interface with a low RI medium (e.g. water, $n_{2} ; n_{2}<n_{1}$ ). The critical angle above which TIR occurs depends on the wavelength of the incident light. If the requirements for TIR are met, a surfacebound electromagnetic (evanescent) wave is generated at the prism/liquid interface, which decays exponentially into the optical thinner medium, i.e. the liquid. The p-polarized component of the evanescent wave can excite free surface electrons in a thin noble metal layer ( $\mathrm{Au}, \mathrm{Ag}$ ) coated at the prism/ liquid interface. This generates so-called surface plasmons, i.e. longitudinal electron density oscillations (Fig. 1-2 E). Besides this method of prism coupling, surface plasmons can also be excited by means of waveguide coupling or grating coupling. ${ }^{[127,132,140]}$ There is a specific resonance condition at which a maximal fraction of light energy is coupled into surface plasmons. It depends on the wavelength and angle of the incident light as well as on the refractive indices of the prism (waveguide/ grating structure), the metal film and the neighboring medium. Resonance appears as minimum in the intensity of the reflected light (reflectance). Hence, if all parameters of the system are kept constant and the device is illuminated with monochromatic light at various angles (angle scan), a RI change of the liquid close to the metal layer can be measured by a shift of the angle of minimal reflectance. Alternatively, a frequency sweep of the excitation light at a fixed angle of incidence (frequency scan) provides spectroscopic data of the reflected light intensity and information on RI changes at the biosensor surface by means of resonance frequency changes. 
A

ISFET

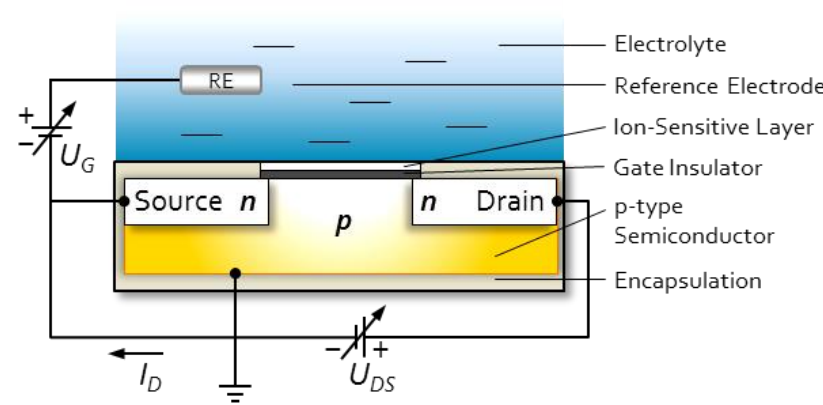

C

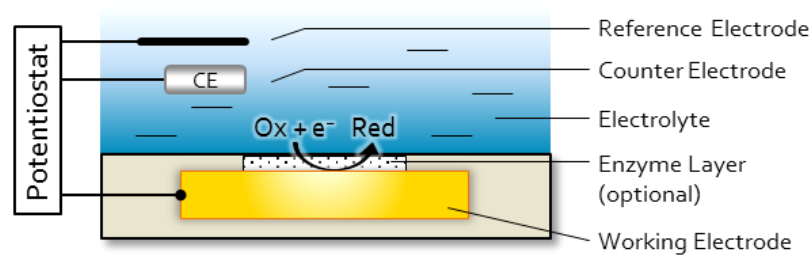

E

SPR

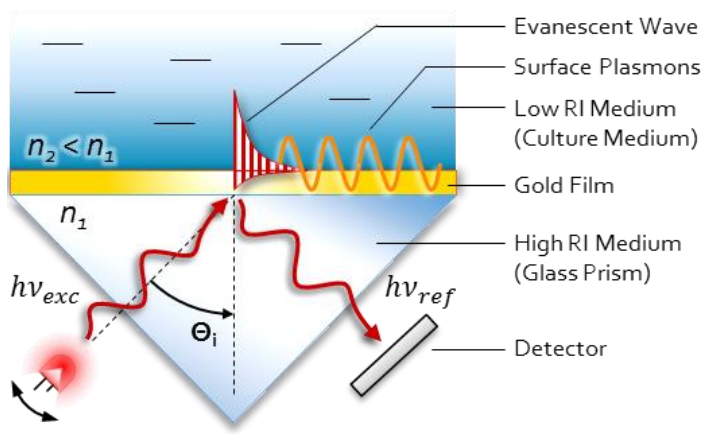

G

OCS

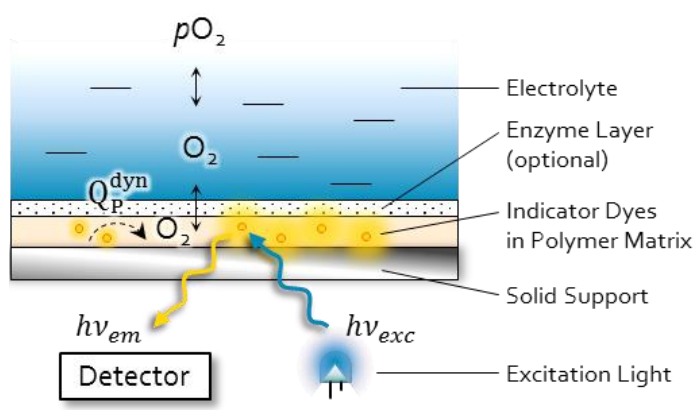

B

LAPS

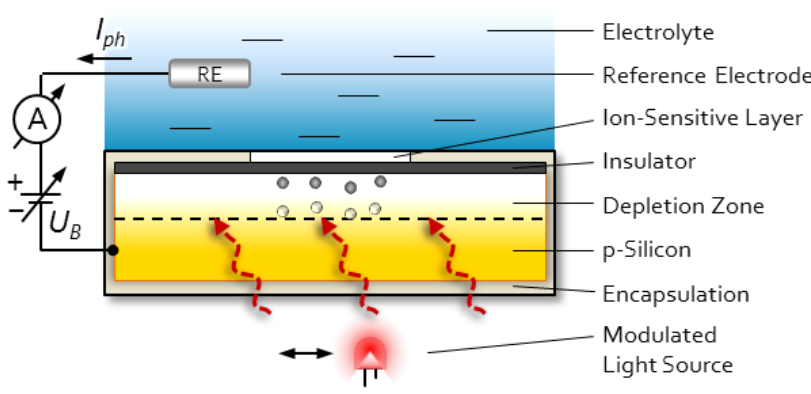

D

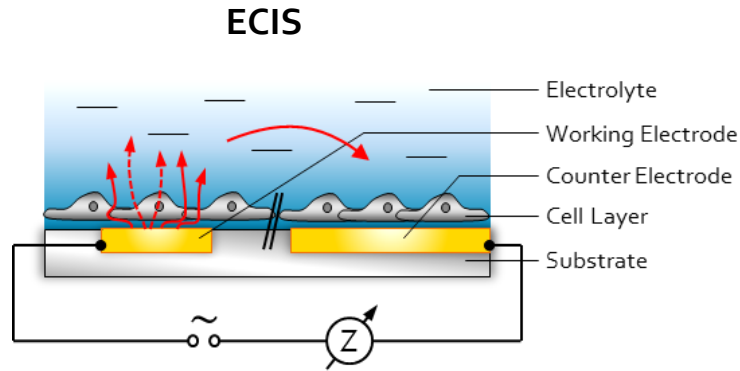

F

RWG

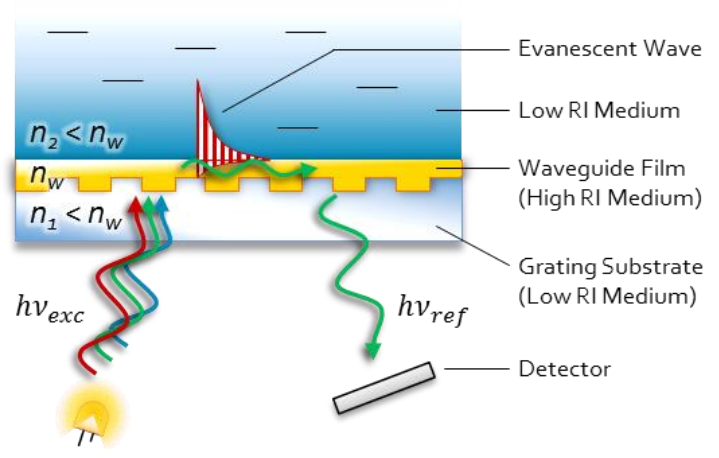

H

\section{QCM}

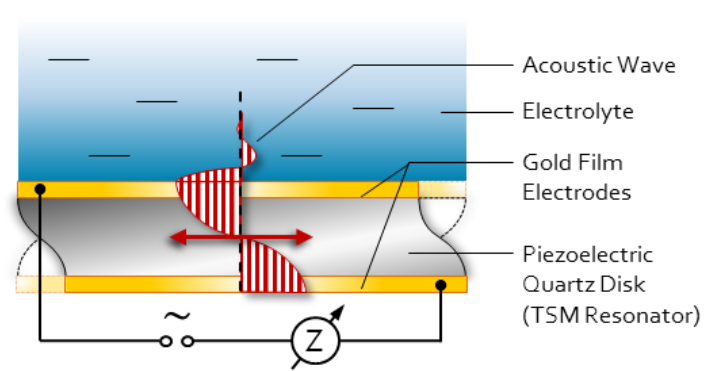

Fig. 1-2 Label-free transducer technologies applied for cell-based biosensing: (A) ISFET, (B) LAPS, (C) Amperometric (enzyme) sensor, (D) ECIS, (E) SPR in Kretschmann configuration, (F) RWG, (G) luminescence-based OCS, and (H) OCM; drawings not to scale. 
The SPR technique was initially developed for the analysis of biomolecular interactions. Its success in this field is apparent in a variety of commercial devices. Therefore, the SPR is often also termed affinity-based biosensor ${ }^{[139]}$ However, in recent years the SPR has been discovered as a valuable means to investigate not only molecular but also cell-substrate interactions and structural changes in the basal region of adherent eukaryotic cells, also referred to as dynamic mass redistribution (DMR) ${ }^{[141,142]}$. The SPR/ DMR sensors have been used for label-free and time-resolved investigations of structural and morphological processes during cell adhesion and proliferation ${ }^{[143-145]}$ and due to extra- or intracellular stimuli $[127,133,138,143,144,146-150]$. Aside from the spectroscopic approach, SPR has also been applied in a microscopic manner (SPR microscopy or SPR imaging) for imaging of cell-substrate contacts of living cells. ${ }^{[151-155]}$

\section{RWG}

In contrast to the prism-coupling of incident light in the SPR technique, grating-coupling is used in the RWG technique for generating an evanescent field and surface plasmons, respectively. ${ }^{[2,142,156-160]}$ In RWG (Fig. 1-2 F), an optical waveguide film of high refractive index and with a periodic metal diffraction layer (subwavelength structured) is illuminated with polychromatic p-polarized light. By the diffraction grating, light at a narrow band of wavelengths is selectively coupled into and propagates along the waveguide. ${ }^{[161]}$ This is accompanied by the generation of an evanescent wave at the interface of waveguide and low RI medium (the liquid and waveguide substrate). The resonant wavelength, i.e. the wavelength that is most effectively coupled into the waveguide, thereby depends on the refractive index at or close to the waveguide (sensor) surface. Thus, local changes in the RI due to DMR at the cellsubstrate interface ${ }^{[141,142]}$ cause a shift in the resonant frequency, which can be sensitively measured by the spectroscopic analysis of the reflected or transmitted light. ${ }^{[12,142,157,161-163]}$

RWG sensors have been successfully applied in a multitude of cell-based assays. Studies provided timeresolved data of cell-substrate and cell-cell interactions during cell adhesion and spreading on the RWG surface. ${ }^{[142,145,157,162,164]}$ The responses of cells in the form of spatial and temporal relocation of cellular structures (mass) in receptor and signaling studies ${ }^{[141,142,159,165-168]}$ and changes in the RI signature of adherently grown cells upon treatment with various membrane- and cytoskeleton-affecting drugs and compounds ${ }^{[142,167]}$ could be sensitively monitored by means of RWG as well.

\section{Luminescence-based OCS}

A non-electrochemical transducer principle for the screening of metabolic species in cell-based assays is based on the chemosensitive change of optical quantities. Such optical chemical sensors (OCS; reviewed in ${ }^{[169-173]}$ ) are often also termed as optodes as the optical analogue to the electrodes. They are categorized into the direct measurement of an analyte, by the change of an intrinsic optical property, and the indirect determination via the optical properties of an indicator molecule (probe). The latter case is the mostly used approach for screening metabolic or physiologically relevant species in cell-based assays, since such small, low molecular weight molecules typically possess no optical properties usable for their direct determination, such as for example absorbance or fluorescence in the visible spectral range. To comply with the approach of label-free CBAs, the probe has to be immobilized in a planar sensor matrix which prevents direct contact and interaction with cells (Fig. 1-2 G). Due to the high sensitivity of fluorescence-based analytical techniques, fluorescent probes are mainly used. The basic requirement for this method is a change in the fluorescence properties of the indicator molecule (intensity, anisotropy, lifetime) in a concentration-dependent manner specific for the analyte of interest. ${ }^{[174]}$ This could arise from either a physical interaction or a reversible chemical reaction of analyte and probe molecules, causing a quenching or the generation of luminescence. For instance, oxygen as 
one of the most relevant metabolic species in biological systems is an effective quencher of phosphorescence, making the intensity and lifetime of the indicator's phosphorescence valuable quantities for the sensitive monitoring of oxygen concentrations. ${ }^{[175]}$ For detailed information on oxygen sensing via luminescence quenching and the readout principle of fluorescence lifetime imaging the interested reader is referred to the methods-section 3.5 Optical Temperature and Oxygen Imaging. The range of accessible analytes is further increased tremendously by means of enzymatic reactions in which the analyte is consumed and compounds that affect the luminescence properties of a probe are either consumed as well or produced. The most popular biosensor of this category is the optical glucose sensor, where D-glucose is enzymatically consumed in a thin layer of enriched glucose oxidase (GOx) between the sample solution and the indicator layer (Fig. 1-2 G). Dissolved oxygen is consumed in this reaction while hydrogen peroxide and protons are produced, all of which are available for analytical detection with the respective chemosensitive indicator layers. ${ }^{[170]}$

Different configurations of OCS have been described in the introductory section of Chapter 6. An overview for various analytes that are of interest in cell-based assays and for which chemosensitive luminescent probes have been presented in the literature is also given here. Amongst these, probes for oxygen $\left(\mathrm{pO}_{2}\right)$ and $\mathrm{pH}$ are most commonly used in $\mathrm{CBBs}$, providing information on cell growth and cellular metabolism. Regarding optical oxygen sensors used for the sensing and imaging of the OCR of cells, a brief overview of various formats and applications is given in subsection 6.2 Imaging the Oxygen Consumption Rate (OCR) beneath Adherent Cells. Fluorescence-based OCSs have been presented in various formats with respect to miniaturization, parallelization, and multiplexing ${ }^{[58,173,176-195]}$ as well combined with other sensor principles for the purposes of multi-parametric whole-cell biosensing [46651,55,59] (cf. section 1.2).

\subsubsection{Piezolectric Transducers for Label-free CBB}

\section{QCM}

A third type of label-free CBBs is based on the inverse piezoelectric effect and the phenomenon of electromechanical coupling. ${ }^{[20,196-198]}$ The effect of piezoelectricity and how it is utilized for the electromechanical (acoustic) transduction in shear acoustic wave sensors is described in detail in the methods section 3.3 The Quartz Crystal Microbalance (OCM). One can distinguish between various shear acoustic wave devices depending on the dipole orientation in the material with respect to the electrodes and, thus, how the acoustic wave travels through the material: flexural-plate-wave (FPW), surface acoustic wave (SAW), and bulk acoustic wave (BAW). ${ }^{[20,196,197,199-202]}$

The acoustic wave sensor technique that is used by far most widely in (cell-based) biosensing applications is the quartz crystal microbalance (OCM). The OCM is a BAW device and is also referred to as thickness shear mode (TSM) resonator. ${ }^{[11,21,196,203,204]}$ A schematic cross section of a OCM sensor is illustrated in Fig. 1-2 $\mathrm{H}$. The core component of OCM acoustic sensors is an AT-cut quartz crystal. When an AC potential is applied via electrodes at the surface of such a material, the molecules between the electrodes are excited to perform a shear displacement. This mechanical oscillation travels in the form of an acoustic wave through the piezoelectric material and decays in exponential fashion into the surrounding media at its surfaces. Any mechanical alteration occurring within the decay length of the acoustic wave in the adjacent medium can be sensitively recorded by a change in the oscillation properties of the crystal. Besides measuring the adsorption of rigid masses on the microbalance surface via a decrease in its 
resonance frequency, the QCM is also capable of detecting changes in the viscoelastic properties of the quartz crystal loading by the damping of the oscillation amplitude. ${ }^{[205-207]}$

The OCM technique has been applied in various biomolecular interaction studies (including virus detection, protein adsorption, immunoassays, membrane formation and interaction, and oligonucleotide hybridization), studies aiming on bacteria detection and quantification, and in the recent past also in biosensing studies based on eukaryotic cells (CBB). ${ }^{[22,199,202,208-214]}$ Label-free and timeresolved acoustic CBB by means of OCM provided meaningful data of the micromechanical cellsubstrate interactions during the processes of cell attachment and spreading in a multitude of studies. ${ }^{[22,90,225-242]}$ Moreover, the viscoelastic properties of living adherent cells (predominantly determined by the cytoskeleton) have been studied under the influence of diverse chemical, biological and physical stimuli by OCM sensing as well. OCM studies of CBAs described in literature cover various research fields, such as toxicity testing of chemical compounds and environmental pollutants ${ }^{[216,243-251]}$, drug discovery $[4,19,22,206,216,222,225,234,242,243,248,251-255]$, cytocompatibility testing of growth substrate materials and coatings $[11,22,90,216-218,221,224,228,230,232,234,236,256-259]$, cell receptor and signaling investigation [220,260-262], cell motility and vitality monitoring in terms of cytotoxic efficacy ${ }^{[251,263]}$, and detection and quantification of the metastasis potential of cancer cells ${ }^{[264]}$ and of the beat rate of cardiomyocites ${ }^{[255]}$.

\subsection{Multi-Parametric Label-free Cell-based Biosensing}

Efforts to improve label-free sensor techniques for non-invasive monitoring of CBAs initially aimed at the miniaturization and parallelization of devices for high throughput screening (HTS) capabilities, as required in drug screening for instance. However, the technical possibilities of combining independent monitoring techniques and the analytical importance of high content screening (HCS) in cell-based studies were increasingly recognized, too. Cell-substrate and cell-cell interactions as well as cellular recognition of externally applied stimuli can result in multidimensional changes of cellular properties, such as for example the mechanics, morphology, and metabolism of cells. The readout signal of conventional CBBs however mirrors the complex processes that take place in the cellular system under study in condensed form and mostly only by one parameter. It is evident that this represents only a small fraction of the entire state and of the total response of cells. In order to gain a more detailed and a more comprehensive picture of the cellular state and of processes occurring in cell-based assays, it is of utmost relevance to improve the biosensing systems with respect to multiplexing and HCS on the basis of hyphenated techniques. ${ }^{[129]} A$ thus increased number of readout parameters provides the basis for a more a comprehensive and detailed description and evaluation of the cellular state and also allows a better understanding of metabolic and morphological cellular reactions due to various stimuli. This knowledge enables to intervene more specifically into the cellular system. This is not only of interest in terms of drug discovery but is also of great relevance in the fields of medical diagnostics, environmental monitoring, in early detection of biological and chemical warfare agents, and in basic research in life sciences in general. ${ }^{[2-6]}$

To date various approaches allowing for multi-parametric cell-based biosensing have been reported in literature that will pave the way for label-free HCS of cellular assays. In the following brief overview only hyphenated sensor approaches are considered with at least two independent transducer technologies combined in one biosensor platform. Approaches where the sensor spots of one transduction principle 
were multiplied and equipped with different selectivities or sensitivities only, such as for example MLAPS ${ }^{[27]}$, multi-ISFETs ${ }^{[265,266]}$, or multiple OCS ${ }^{[58,65,183]}$, are not taken into account here.

Janshoff et al. presented in 1996 a double-mode impedance sensor for the analysis of cell layers ${ }^{[219]}$. The core component of this dual sensor is a piezoelectric $5 \mathrm{MHz}$ quartz disk that is capable of performing shear oscillations. In this mode of operation ( $\mathrm{OCM}-/$ quartz-mode) the sensor enables monitoring the viscoelasticity of cell monolayers cultured on its surface by impedance analysis. Additionally, the dielectrical properties of the cell layer are monitored by impedance spectroscopy in ECIS-analogue manner (ECIS-/ cell-mode), with the help of a platinum wire electrode dipping into the culture medium from the top. This provides information about the degree of cell-coverage on the sensor and the formation of an intercellular barrier, which is complementary to the quartz mode-derived information about the mechanical cell-substrate interactions. Aside from studies of cell attachment and spreading, the system has been successfully applied in various types of cell-based assays adressing cytoskeleton perturbation, (bio)chemical stimulation, and cytocompatibility testing. ${ }^{[22,197,206,236,267,268]}$

In 1999, Baumann et al. ${ }^{[64]}$ developed a so-called "Cell Monitoring System" (CMS $\left.{ }^{\circledR}\right)$, which enables the simultaneous and label-free monitoring of multiple relevant cell layer parameters by the use of various microsensors arranged next to each other on a single surface. The multi-sensor system is build up of $\mathrm{pH}$ sensitive ISFETs, IDEs, and a temperature-sensitive transducer spot. The $\mathrm{pH}$-ISFET and the IDEs serve as sensors for the ECAR and the impedance-based morphological characterization of a studied cell layer, respectively. Various different $\mathrm{CMS}^{\circledR}{ }^{\circledR}$-chip versions were proposed to meet the requirements of different fields of applications. The system was further developed in the early 2000 s. The sensor chips were equipped with additional electrode structures capable of recording changes in dissolved oxygen and thus the OCR of cells by means of modified ISFETs ${ }^{[45]}$ and miniaturized Clark-type electrodes ${ }^{[60]}$. In this way, microsensor chips were developed for monitoring both, metabolic responses $\left(\mathrm{pH}\right.$ and $\left.\mathrm{pO}_{2}\right)$ and morphological alterations (impedance) of living cells to drug compounds. Later on, a three-electrode arrangement was integrated on the sensor chips that was operated with a potentiostatic circuit for amperometric oxygen measurements. ${ }^{\left[{ }_{3}, 62\right]}$ The microsensor systems were realized on glass chips, comprising an optical access for microscopic evaluations, as well as on silicon sensor chips with multiple ISFET readout spots. ${ }^{[48,53,54,62]}$ Based on these, a novel multi-parametric biosensor device called "Intelligent Mobile Lab" (IMOLA) was introduced by Wiest et al. ${ }^{[47]}$ in 2006 . The sensor chips of this system are fabricated either with silicon technology or thin film technology (ceramic chips). In the latter case, ISFETs for accessing $\mathrm{pH}$ changes are replaced by metal oxide sensors. The IMOLA system was commercialized by cellasys $\mathrm{GmbH}$ (Munich, Germany) in $2007^{[269]}$. A very similar chip system called "Bionas Discovery ${ }^{\mathrm{TM}} 2500$ System" was commercialized by Bionas GmbH (Rostock, Germany), capable of independent $\mathrm{pH} / \mathrm{pO}_{2} /$ impedance measurements ${ }^{[270]}$. Both systems have been applied in a multitude

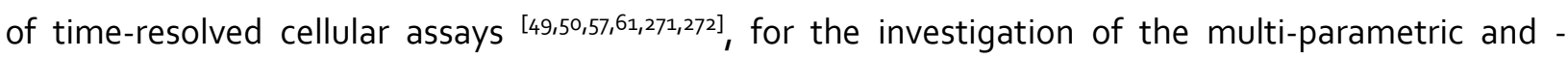
dimensional cellular responses (like in metabolic activity, cellular respiration, and morphology) towards various potential cytotoxic substances and drugs.

In another multiple CBB approach, electrochemical (ECIS) and chemosensitive optical (OCS) transduction were integrated next to each other on one sensor surface. ${ }^{[46,55,273]}$ The sensors are integrated on the bottom of 24-well-plates and comprise an IDE structure for impedimetric readout of cell

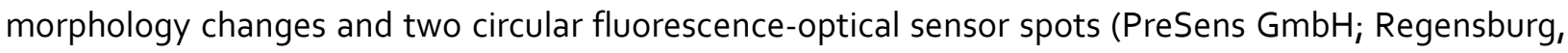
Germany) that enable the independent optical readout of $\mathrm{pH}(\mathrm{ECAR})$ and $\mathrm{pO}_{2}(\mathrm{OCR})$ in each well of the plate. The system under study can additionally be observed and analyzed (fluorescence-) microscopically, due to the transparency of the glass-based sensor chips. The parallelized multiparametric sensor system has been developed in order to increase aside from the information content 
also the screening throughput of cell-based assays. For this purpose, not only sensorchip-equipped multiwell-plates were presented but also an automated integrated analytical screening platform, including a $x-y$ movable microscope for automated imaging, a high-end CCD camera, a light source for fluorescence microscopy, and an integration control unit. The integration of further components for further automatation, a climate control and a fluidic system was proposed, too, and has already been considered in the development concept of the integrated screening system. The application of the system has been demonstrated in the time-resolved multi-parametric screening of cell attachment and spreading and of chemical and biological cell layer stimulations. ${ }^{[51,55,59]}$

Recently, the combination of the optical and electrochemical transducer principles, SPR and ECIS, in one sensor platform has been presented by Michaelis, Wegener and Robelek (2013). ${ }^{[274]}$ They integrated on gold film-coated SPR sensor chips an ECIS-analogue electrode layout by means of photolithographic structuring. This provides electrodes for impedance analysis on the one hand and two gold-covered areas of the substrate for SPR monitoring in cell-based biosensing applications on the other hand. The layout enables SPR sensing of two independent channels, where one is usually used as measurement channel and the other as control channel. SPR-ECIS monitoring of cells is capable of providing simultaneously and non-invasively information for the multi-parametric and time-resolved characterization of cell layers, due to the independency and the label-free nature of both sensor techniques. The content of information extractable from SPR-ECIS readings covers changes in the DMR and the morphology of cells, as well as alterations in the cell layers' integrity and barrier properties. The performance and the complementarity of information obtained from simultaneous SPR sensing and ECIS have been demonstrated by Michaelis et al. in several proof-of-concept studies, including investigations of cell attachment, spreading and differentiation on the dual sensor surface as well as the dual monitoring and analysis of confluent cell layers upon their exposure to an actin cytoskeleton disrupting drug.

Even though this has not yet been applied as CBB, the recently reported combination of OCM and SPR by Kim et al. (2010) ${ }^{[275]}$ is also briefly mentioned here, due to its specific relevance in the scope of this thesis and as it represents another jigsaw piece in the possible combinations of label-free biosensor techniques. The novel dual sensor was constructed on the basis of a typical AT-cut quartz crystal, with indium tin oxide (ITO) as transparent electrode material on the bottom side and with a gold film over a thin adhesion promoting titanium layer on the top side of the disk. The microbalance was mounted onto a glass prism via an index matching oil to meet the requirements for a SPR setup in Kretschmann configuration ${ }^{[132,135]}$. The proposed SPR-OCM setup, however, needs to be substantially improved, particularly in view of cell-based biosensing applications, since the SPR sensitivity of this setup is affected by disturbing reflections from the additional ITO and titanium layers and since the OCM oscillation exhibited a considerably increased motional resistance as a consequence of the damping oil film.

Finally, it should be noted that the non-invasive nature of all label-free CBB approaches and combinations described above offer the possibility to be extended with invasive end-point investigations and post-experimental label-based analytical methods, such as e.g. by AFM or fluorescence staining and microscopy. The cooperation of time-resolved non-invasive monitoring and of invasive techniques for a more in-depth analysis of cells enables the collection of a wealth of information, which allows a detailed profiling of cells. Thus, it is a promising key for attaining deeper insights into cellular states and a better understanding of the behavior of cells in its entirety. 
Chapter 2 Objectives 
Biosensors based on whole cells as the biological recognition unit (CBBs) have gained an increasing interest in the past years by research institutes focusing on the development of new technologies that can serve as basis for the label-free and non-invasive screening of living cells. Due to steady technical improvements in terms of throughput and handling, progresses in data analysis and interpretation, and the development of numerous cell-based assays, there is also an increasing interest in CBBs on the part of the pharmaceutical, food and biosecurity industry. ${ }^{[2-5,19,276]}$ In CBBs, cell lines can serve as a renewable biological recognition element for biomedical assays, such as pathogen and toxin detection and drug discovery. ${ }^{\left[{ }^{10]}\right]}$ Cells cultured In vitro can mimic on a small scale the functional effect of any substance or stimulus on tissue, organs or the whole organism and human body, respectively. Thus, biosensing of cells provides meaningful high-content information in a physiological context, which is not accessible by interaction studies of isolated biomolecules or by label-based and therefore invasive cell-based assays. In terms of drug discovery, for instance, the application of CBBs allows a pre-selection of candidate test substances and concentrations in preclinical screening studies to minimize the number of animal tests, which is desirable for both ethical and cost reasons.

Fig. 2-1 gives an overview of the technical state of the art of the three main CBBs used for monitoring structural changes of cells, QCM, ECIS, and SPR, with respect to available modes of operation and sensor combinations (indicated with a connecting line) at the beginning of this thesis. Sensing modes are indicated with blue arrows directed away from the sensor principle and actuating modes are highlighted in red with arrows pointing inwards to the cells. The stimulation of cells and their microscopic investigation, mostly used in addition to $\mathrm{CBB}$, are also illustrated for the sake of completeness. Fig. 2-2 shows the same overview, however, expanded with the contributions of this thesis (highlighted with blue borders). Methods and applications not used or not presented in this thesis are shaded in grey.

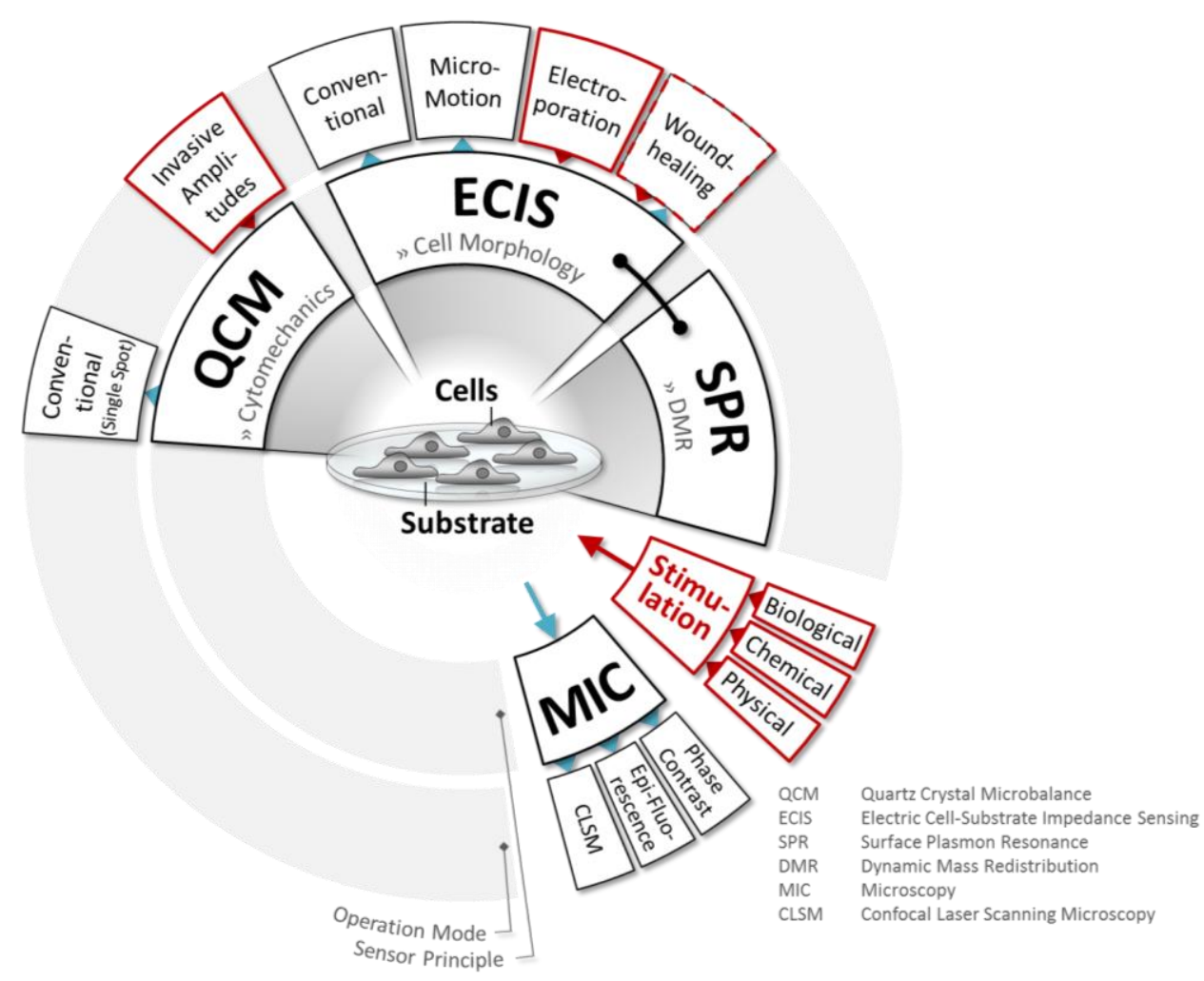

Fig. 2-1 State of the art of (combined) CBBs for monitoring structural changes of cells at the beginning of this thesis. 
It was the aim of this thesis to improve and develop real-time sensor devices that can be used to monitor assays with living cells label-free and non-invasively on the basis of the quartz crystal microbalance (OCM) technology. One focus thereby was set on the development of a multi-spot QCM sensor (MOCM), which should be designed, characterized, and optimized. The MOCM should be finally applied for investigating the mechanical properties of mammalian cells and their changes by analysis of the QCM impedance of cell-covered quartz resonators. Thus, the OCM should be established as a method that enables the continuous monitoring of cytomechanics under physiological conditions, with a high time resolution, manageable technical expenses, and a moderate throughput in cell-based screenings. This should be evaluated in various studies with compounds that affect the micromechanics of cells.

Another project aimed on the integration of the ECIS principle into the surface of the MOCM disks developed, in order to provide a novel hyphenated (OCM-ECIS) sensor combining piezoelectric and electrochemical transduction in one experimental setup. This should enable the label-free readout of cell morphology changes as well as cell-cell and cell-substrate interactions by means of ECIS in parallel to the viscoelastic profiling of cells by OCM recordings. The dual sensor platform should be developed using thin film technology and standard photolithography. An optimal electrode layout of the OCM-ECIS sensors should be evaluated, by means of microscopic investigations and analysis of the impedance characteristics of both QCM- and ECIS-mode readouts. Once a system suitable for cell-based biosensing applications is identified, the sensing performances of the individual transducer principles of the dual sensor should be evaluated on the basis of various cell adhesion and cell stimulation assays and, as a key issue, the added value of the hyphenated approach should be identified and discussed with respect to the separate sensor setups and readings.

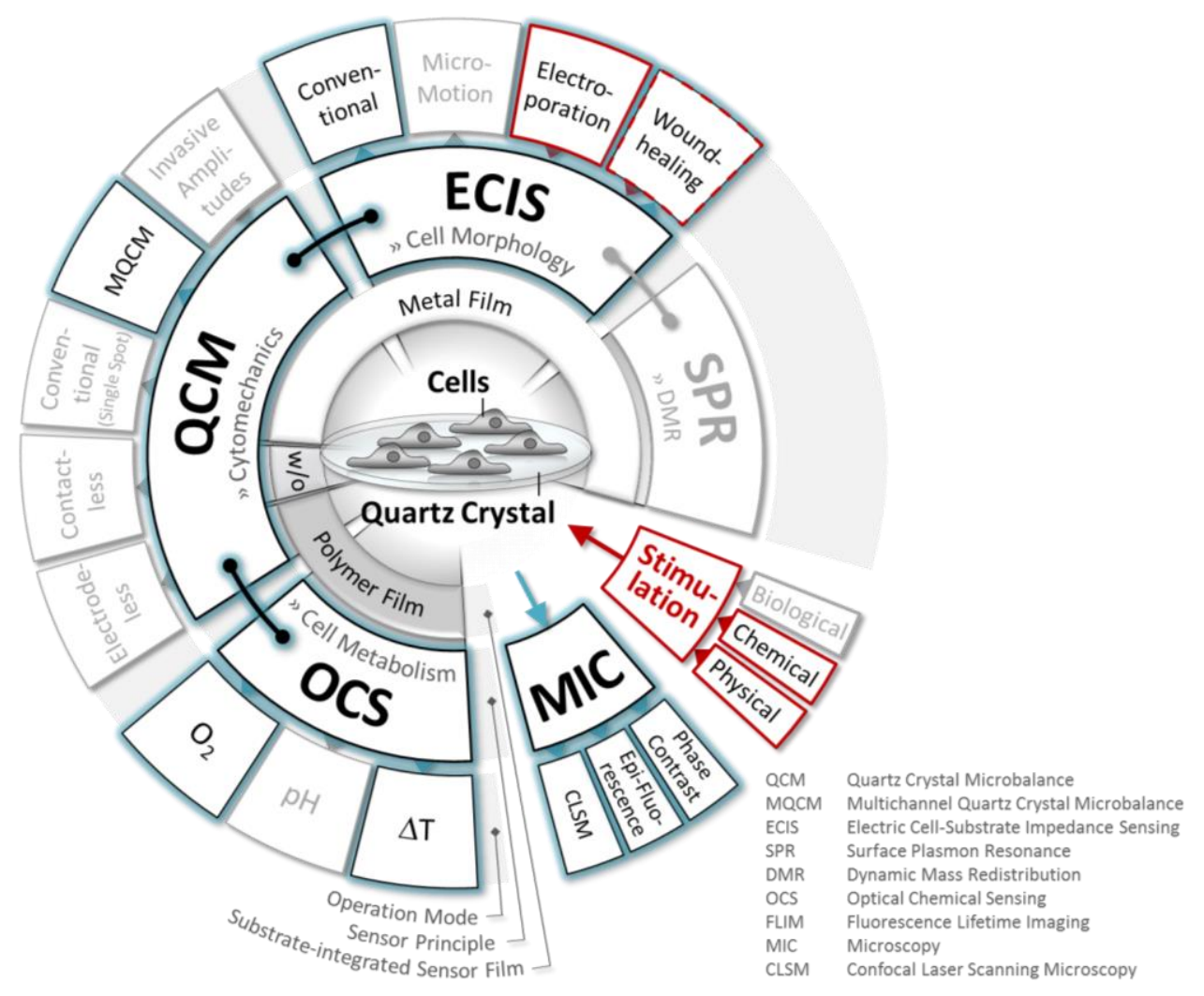

Fig. 2-2 State of the art of (combined) CBBs for monitoring structural and metabolic changes of cells at the end of this thesis. 
In a third project the QCM should be combined with luminescence-based OCS in order to provide another class of sensor combinations (OCM-OCS) for the purposes of multi-parametric CBB. A first OCM-OCS combination aimed on the experimental determination of temperature increases on the OCM surface, which are expected if the resonator is operated at elevated driving voltages and oscillation amplitudes, respectively. Two-dimensional OCS thereby should be implemented by means of a temperaturesensitive paint (TSP) and fluorescence lifetime imaging (FLIM). In another approach, an oxygen-sensitive sensor film should be developed which at the same time should serve as the growth substrate for mammalian cells. The cytocompatibility of the pressure-sensitive paint (PSP) used for this purpose should be investigated by means of QCM measurements and a PSP-coated substrate compatible for cell layer establishment should be realized. Finally, proof-of-concept studies should demonstrate the capability of the chemosensitive transducer for label-free and time-resolved sensing and imaging the oxygen consumption rate (OCR) of a cell layer upon stimulation. This should provide the basis for the substrate-embedded screening of further analytes, such as for instance $\mathrm{H}^{+}$or glucose, in cell-based assays by means of OCS. 


\section{Chapter 3}

\section{Methods \& Instrumentation}

3.2 Impedance Spectroscopy

3.3 The Quartz Crystal Microbalance (OCM)

3.4 Electric Cell-Substrate Impedance Sensing (ECIS)

3.5 Optical Temperature and Oxygen Imaging

3.6 Light Microscopic Techniques 


\subsection{Cell Culture}

For cell studies in this work the adherent epithelial cell line MDCK-II (Madin Darby Canine Kidney, strain II), and the adherent epithelial-like cell line NRK (Normal Rat Kidney, strain 52E) were used. Both cell lines were obtained from the Leibniz Institute DSMZ - German Collection of Microorganisms and Cell Cultures (Braunschweig, Germany). They grow on cell culture substrates as monolayers of cobblestonelike morphology.

\subsubsection{Cell Culture Conditions}

The cell lines were routinely incubated in tissue culture medium in $25 \mathrm{~cm}^{2}$ or $12.5 \mathrm{~cm}^{2}$ cell culture flasks, which were placed in a humidified incubator with a temperature of $37^{\circ} \mathrm{C}$ and an atmosphere that was aerated with $5 \% \mathrm{CO}_{2}$. A medium volume of $4 \mathrm{~mL}$ for $25 \mathrm{~cm}^{2}$ flasks ( $2 \mathrm{~mL}$ for $12.5 \mathrm{~cm}^{2}$ flasks) was adequate for completely covering the adherent cells on the bottom of the flask, providing nutrient supply for a few days. Cells were fed every two to three days or the day before an experiment, by exchanging the degraded culture medium for nutrient-rich medium from the stock solution. MDCK-II and NRK cells require different culture medium compositions and concentrations of nutrients. The respective medium compositions of basic medium and additives for both cell lines are listed below in Tab. 3-1.

Tab. 3-1 Composition for MDCK-II and NRK tissue culture medium. The basic medium contained different amounts of $\mathrm{NaHCO}_{3}$ and D-glucose, and were supplemented with fetal calf serum, penicillin/ streptomycin, and L-glutamin.

\begin{tabular}{lcc}
\hline & MDCK-Il medium & NRK medium \\
\hline Basic medium & $\begin{array}{c}\text { Minimum Essential Medium Eagle } \\
(\text { MEM) }\end{array}$ & $\begin{array}{c}\text { Dulbecco's Modified Eagle's Medium } \\
(\text { DMEM) }\end{array}$ \\
NaHCO $_{3}$ & $2.2 \mathrm{~g} / \mathrm{L}$ & $3.7 \mathrm{~g} / \mathrm{L}$ \\
D-glucose & $1 \mathrm{~g} / \mathrm{L}$ & $4.5 \mathrm{~g} / \mathrm{L}$ \\
+ Fetal calf serum (FCS) & $5 \%(\mathrm{v} / \mathrm{v})$ & $5 \%(\mathrm{v} / \mathrm{v})$ \\
+ Penicillin & 1 & $100 \mu \mathrm{g} / \mathrm{mL}$ \\
+ Streptomycin & $100 \mu \mathrm{g} / \mathrm{mL}$ & $100 \mu \mathrm{g} / \mathrm{mL}$ \\
+ L-glutamin $^{1}$ & $100 \mu \mathrm{g} / \mathrm{mL}$ & $2 \mathrm{mM}$ \\
\hline
\end{tabular}

${ }^{1}$ Sigma-Aldrich; St. Louis, MO, USA

Cell culture work was performed under sterile conditions in a laminar flow "HERAsafe KS/KSP class II biological safety cabinet" (Thermo Fisher Scientific; Waltham, MA, USA). Aseptic conditions in the laminar flow were ensured by spray- and wipe-disinfection of the bench, and all materials and equipment brought into the laminar flow, with $70 \%(\mathrm{v} / \mathrm{v})$ ethanol in deionized water. Sterility of solutions was achieved by either autoclaving at $120^{\circ} \mathrm{C}$ for $20 \mathrm{~min}$ (Systec DX-45; Wettenberg, Germany) or by sterile filtration (pore diameter: $0.2 \mu \mathrm{m}$ ), unless it was delivered already sterile by the manufacturer. Cell culture equipment was autoclaved likewise before usage, except the manufacturer delivered it in a sterile package. Cell culture waste, i.e. solutions and materials that were in contact with cells, was autoclaved before disposal. 


\subsubsection{Sub-cultivation of Cells}

MDCK-II and NRK cells were cultured on the bottom of culture flasks, where they proliferate until a confluent monolayer is reached. When the cells reach a confluent state, contact inhibition within the cell layer occurs, which means that the cells slow down and finally stop proliferation. For maintaining an active cell cycle and a continued proliferation rate, the cells were sub-cultured on the verge of reaching contact inhibition, by seeding a fraction of the cells in new flasks. The seeding densities were adjusted for a sub-cultivation of cells once a week.

Sub-cultivation was performed according to a standard cell culture protocol. The divalent cations, $\mathrm{Ca}^{2+}$ and $\mathrm{Mg}^{2+}$, are essential for the formation of cell-cell and cell-substrate contacts. They are, among other factors, responsible for the adherence of the cells to the extracellular matrix deposited on the culture substrates. In order to detach the cells from such a surface, these contacts were weakened by elutriation of the divalent cations. Firstly, the supernatant medium was removed, and the cell layer was washed twice with phosphate buffered saline ( $\mathrm{PBS}^{--}$, without $\mathrm{Ca}^{2+}$ and $\mathrm{Mg}^{2+}$ ). The depletion of cations was maximized by subsequently incubating the cell layer with a solution of $1 \mathrm{mM}$ EDTA (ethylenediaminetetraacetic acid) in $\mathrm{PBS}^{--}$for $5-10 \mathrm{~min}$ at $37^{\circ} \mathrm{C}$. The chelating agent possesses a very high binding affinity towards divalent cations. Rounding up of the cells indicated the loss of cell-cell and cell-substrate contacts, and consequently a sufficient period of EDTA incubation. Afterwards, the cell layer was incubated with a solution of $0.25 \%$ (w/v) Trypsin/ $1 \mathrm{mM} \mathrm{EDTA}$ in PBS-- (for $5-10$ min at $37^{\circ} \mathrm{C}$ ), for a complete detachment of the cells from the flask surface. The trypsinization process was stopped by adding a 10-fold excess volume of the respective culture medium to the flask. Then the suspended cells were centrifuged at $110 \mathrm{~g}$. After removal of the supernatant, the spun down cell pellet was re-suspended in fresh culture medium and seeded into a new cell culture flask in a certain dilution.

\subsubsection{Cell Storage}

The cell lines were deep-frozen in a cryogenic tank in order to store the cell line for a longer period. For this, a confluent cell layer on a flask was treated the same way as for sub-cultivation. After centrifugation, the cell pellet was re-suspended in a special freezing-medium, consisting of normal calf serum and $10 \%$ ( $\mathrm{v} / \mathrm{v})$ DMSO (dimethylsulfoxide). DMSO is a non-crystalizing alternative to a pure water-based medium. The latter would form ice crystals when freezing, which could damage the cell membrane and finally lead to cell death. The re-suspended cell suspensions were filled in cryovials and, in a first cooling step, slowly cooled down to $-80^{\circ} \mathrm{C}$ for $24 \mathrm{~h}$. A slow and constant cooling rate of $1^{\circ} \mathrm{C} / \mathrm{min}$ was achieved by placing the vials into a freezing container filled with isopropyl alcohol. Afterwards, the vials were placed in a rack and were immersed in liquid nitrogen in a cryogenic tank $\left(-196^{\circ} \mathrm{C}\right)$, for long-time storage of the cells.

Thawing of the cryogenically conserved cells and bringing them back into culture had to be performed gently. In a first step, the cryovial with the deep-frozen cell suspension was transferred from the cryogenic tank into a freezer, where it was stored for about $1 \mathrm{~h}$ at $-20^{\circ} \mathrm{C}$. Then, the vial was warmed to $37^{\circ} \mathrm{C}$ in a water bath. Since the high concentration of DMSO in the thawed cryoprotective solution is cytotoxic, it has to be diluted and removed from the cell suspension, respectively. Firstly, the cell suspension was transferred into a centrifugation tube, and $10 \mathrm{~mL}$ of pre-warmed culture medium were added drop-wise. A careful but quick addition was necessary in order to minimize the osmotic stress for the cells. Afterwards, the cell suspension was centrifuged at $110 \mathrm{~g}$, the DMSO containing medium was removed, and the cell pellet was re-suspended in fresh culture medium. The cells were then cultivated by seeding into flasks with a sub-confluent cell density. Subsequent treatment of the cells was carried out 
in accordance with the sub-cultivation procedure, mentioned above. Thawed cells were not used for experiments before at least one sub-cultivation.

\subsubsection{Cell Preparation for Experiments}

By default, cells were fed with fresh culture medium the day before they were seeded on a substrate for an experiment. This was necessary to remove dead cells and to maintain a consistent vitality and metabolic activity of the cell layer for all cell experiments. The cells were seeded on the respective substrate for an experiment after removing them from the culture flask, according to the standard protocol of sub-cultivation. In order to ensure aseptic conditions for cell experiments, the growth substrates were sterilized by treatment with argon plasma for $1 \mathrm{~min}$, unless the manufacturer delivered the substrate sterile. For purposes of comparability of experiments, the cells were seeded on the substrates with a defined cell number per unit area. Therefore, the number of cells after re-suspension of the cell pellet was counted (using a Bürker Hemacytometer from Marienfeld Superior, LaudaKönigshofen, Germany), and the cell suspension was appropriately diluted with culture medium, before seeding. In cases where control of the cell density was important, a cell number of $450000 \mathrm{cells} / \mathrm{cm}^{2}$ was defined for both MDCK-II and NRK cells. At this seeding density the cells were seeded to confluence, meaning that immediately after cell attachment and spreading the whole sensor surface is completely covered with cells. ${ }^{[215]}$ This means that the cells form a confluent monolayer after cell adhesion and spreading, without the need for proliferation. The cell layer possessed fully established cell-cell and cellsubstrate contacts about $12 \mathrm{~h}$ after seeding. Cell experiments on a confluent monolayer were usually performed $24 \mathrm{~h}$ after seeding to confluence. Tab. 3-2 gives an overview of different kinds of growth substrates, and the respective seeding volumes and concentrations of the cell suspensions for achieving a seeding density of 450000 cells $/ \mathrm{cm}^{2}$.

Tab. 3-2 Volumes and concentrations of the cell suspensions (MDCK-II and NRK) for maintaining a consistent cell number of 450000 cells $/ \mathrm{cm}^{2}$ on different kinds of substrates with different growth areas.

\begin{tabular}{lcccc}
\hline Growth substrate & $\begin{array}{c}\text { Growth area/ } \\
\mathrm{cm}^{2}\end{array}$ & $\begin{array}{c}\text { Cell number } \\
\left.\text { (for } 450000 \text { cells/ } \mathrm{cm}^{2}\right)\end{array}$ & $\begin{array}{c}\text { Volume/ } \\
\mathrm{mL}\end{array}$ & $\begin{array}{c}\text { Cell concentration/ } \\
\text { cells/ } \mathrm{mL}\end{array}$ \\
\hline OCM/ OCM-ECIS disks & 1.13 & 508500 & 1.5 & 339000 \\
8-well ECIS array & 0.75 & 337500 & 0.4 & 843750 \\
Petridish (small) & 9.04 & 4068000 & 3 & 1356000 \\
12-well culture plate & 3.66 & 1647000 & 3 & 549000 \\
\hline
\end{tabular}

\subsection{Impedance Spectroscopy}

Impedance spectroscopy (IS) is a measurement principle that delivers information on the electrical impedance (complex electrical resistance) of a system under test as a function of frequency. By definition, the electrical resistance is the hindrance of electrical current flow in a system, when a certain voltage is applied. Both transducer devices applied in this study, quartz crystal microbalance (OCM; section 3.3), and Electric Cell-Substrate Impedance Sensing (ECIS; section 3.4), are based on measuring 
the impedance of the system as a function of frequency. In $\mathrm{OCM}$, one obtains with the electrical impedance a quantity that is directly proportional to the acoustic impedance of the quartz oscillation (via electromechanical coupling), whereas ECIS provides the passive, electrical impedance of cells covering the electrodes. The following sections firstly focus on the mathematical description of the complex impedance. Afterwards, the impedance properties, and the graphical representation of the three basic circuit elements, resistance, capacitance, and inductance, are discussed. Finally, impedance properties of simple equivalent circuit models are considered.

\subsubsection{The Complex Impedance}

When applying a DC voltage (current) to an electrically closed system, the relation between resistance $R$, voltage $U$ and electrical current $I$ is given by the Ohm's law:

$$
R=U / I
$$

However, in IS the input signal is not a time-invariant DC but a sinusoidal AC voltage (current), which is varied in a wide frequency range. In IS small amplitudes of the input signal are used. Thus, IS can be used for non-invasive and non-destructive examinations of (biological) samples. The AC voltage $u(t)$ is a function of time and frequency (which is indicated by small symbols) and can be mathematically expressed in the trigonometric form:

$$
u(t)=\hat{u} \cdot \sin (\omega t)
$$

Hence, the voltage $u(t)$ at a certain time point is characterized by an amplitude $\hat{u}$ and a phase (Fig. 3-1), which is expressed by the variable angle $\omega t$ and where $\omega=2 \pi f$ is the angular frequency and $f$ the AC frequency of the excitation signal.

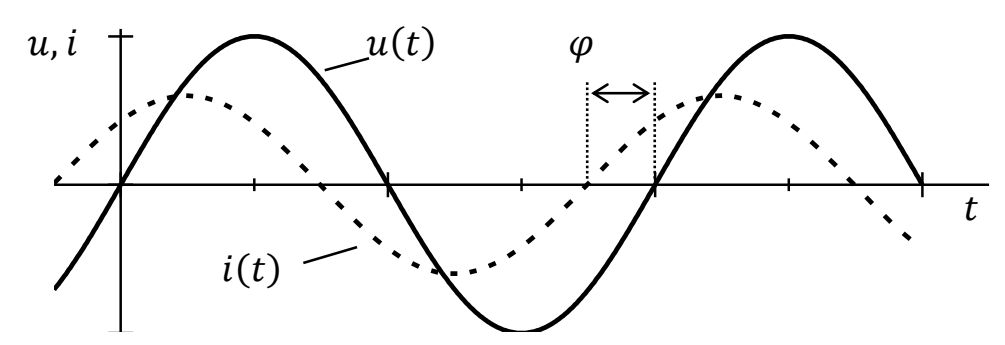

Fig. 3-1 Sinusoidal voltage $u(t)$ and corresponding current $i(t)$ for an arbitrary system under test. The voltage (current) is characterized by amplitude $\hat{u}(\hat{l})$ and phase $\varphi_{u}\left(\varphi_{i}\right)$. When there is only a pure Ohm's resistor in the electric circuit, $u(t)$ and $i(t)$ have the same phase $\left(\varphi=\varphi_{u}-\right.$ $\left.\varphi_{i}=0\right)$. The frequency dependent devices capacitor and inductor, however, cause phase shifts of the current with respect to the voltage of $\varphi=-\pi / 2$ and $\varphi=+\pi / 2$, respectively.

The voltage can also be expressed as a complex quantity (indicated by underlined symbols):

$$
\begin{aligned}
\underline{u}(\omega t) & =\hat{u} \cdot(\cos (\omega t)+\mathrm{j} \cdot \sin (\omega t)) \\
& =\hat{u} \cdot e^{\mathrm{j}(\omega t)}
\end{aligned}
$$


where the time and frequency dependent voltage is expressed as combination of a real and an imaginary part (with the imaginary unit j). The resulting current has a sinusoidal form of the same frequency as the exciting voltage (Fig. $3-1$ ) and can be mathematically expressed in the form:

$$
i(t)=\hat{\imath} \cdot \sin (\omega t)
$$

or as complex current:

$$
\begin{aligned}
\underline{i}(\omega t) & =\hat{\imath} \cdot(\cos (\omega t)+\mathrm{j} \cdot \sin (\omega t)) \\
& =\hat{\imath} \cdot e^{\mathrm{j}(\omega t)}
\end{aligned}
$$

The complex impedance $\underline{Z}$ of an AC circuit can be calculated, according to Ohm's law for DC circuits, by the ratio of the complex voltage, Eq. (3), and the complex current, Eq. (5):

$$
\begin{aligned}
\underline{Z} & =\frac{\underline{u}(\omega t)}{\underline{i}(\omega t)}=\frac{\hat{u}}{\hat{\imath}} \cdot e^{\mathrm{j}\left(\varphi_{u}-\varphi_{i}\right)} \\
& =|Z| \cdot e^{\mathrm{j} \varphi} \\
& =|Z| \cdot(\cos \varphi+\mathrm{j} \cdot \sin \varphi)
\end{aligned}
$$

in which the complex impedance $\underline{Z}$ is fully characterized by its magnitude $|Z|$ and phase $\varphi$, which represents the phase shift between voltage and current $\left(\varphi=\varphi_{u}-\varphi_{i}\right)$. In so-called Bode-plots, $|Z|$ and $\varphi$ are displayed as functions of frequency. As a complex quantity, the impedance can also be described by the sum of its real part $\operatorname{Re}(\underline{Z})$ and imaginary part $\operatorname{Im}(\underline{Z})$ :

$$
\begin{aligned}
\underline{Z} & =\operatorname{Re}(\underline{Z})+\mathrm{j} \operatorname{lm}(\underline{Z}) \\
& =|Z| \cos \varphi+\mathrm{j}|Z| \sin \varphi \\
& =R+\mathrm{j} X
\end{aligned}
$$

All frequency independent resistivities in the electrical circuit are merged to the real part $\operatorname{Re}(\underline{Z})$ of the impedance. As the real part behaves as an Ohm's resistor it is called Ohm's resistance, effective resistance or just resistance $R$. The real part mirrors energy losses within the system. The imaginary part of the impedance is expressed by the reactance $X$ and contains capacitive and inductive components of the electric circuit. They influence the permeability of the current in a frequency dependent manner, and mirror the storage of energy within the system.

The complex impedance can be displayed (besides the Bode-plots $|Z|(f)$ and $\varphi(f)$ ) also in so-called Nyquist diagrams. Here, the impedance is plotted as a vector in the Gaussian plane (complex plane), in which the imaginary part is plotted as a function of the real part (Fig. 3-2). The vector length corresponds to the magnitude $|Z|$ and the angle with the (real) $x$-axis of the vector is the phase $\varphi$ of the impedance. Thus, real part and imaginary part can be converted to magnitude and phase by:

$$
\begin{gathered}
|Z|=\sqrt{\operatorname{Re}(\underline{Z})^{2}+\operatorname{Im}(\underline{Z})^{2}}=\sqrt{R^{2}+X^{2}} \\
\varphi=\arctan \left(\frac{\operatorname{Im}(\underline{Z})}{\operatorname{Re}(\underline{Z})}\right)=\arctan \left(\frac{X}{R}\right)
\end{gathered}
$$




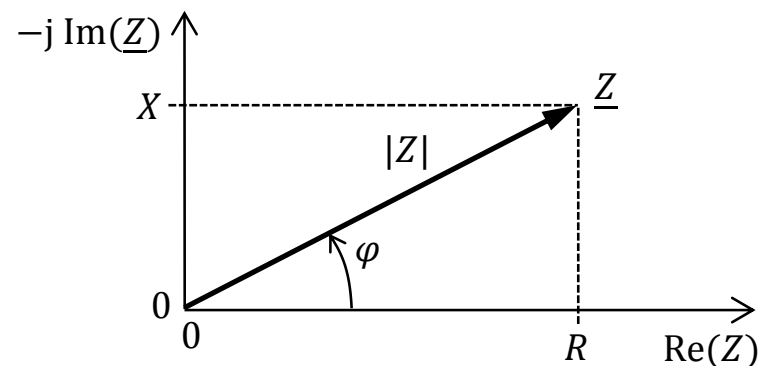

Fig. 3-2 Geometric representation of the impedance $\underline{Z}$ in the complex plane with the $x$-axis as real part $\operatorname{Re}(\underline{Z})$ and the $y$-axis as imaginary part $\operatorname{Im}(\underline{Z})$. The impedance can be fully described either by the Cartesian coordinates, resistance $R$ and reactance $X$, or in polar coordinates by the vector length impedance magnitude $|Z|$ and the angle $\varphi$ representing the phase shift. Both ways of representation can be converted into each other by equations (7), (8), and (9).

\subsubsection{Impedance Elements in Equivalent Circuits}

The current flow in an AC circuit and thus the complex impedance is determined by the three basic passive circuit elements: Ohm's resistors, capacitors and inductors. In the following discussion, an ideal electric behavior of these devices is assumed for all frequencies, meaning that e.g. a capacitor shows only capacitive behavior and has no additional resistive property, which a real capacitor would exhibit. A closer look on the impedance properties of the individual circuit elements and their characteristics in impedance plots helps for understanding and interpreting much more complex electrical systems with combined elements (cf. 3.2.3 Equivalent Circuit Models). ${ }^{[277-279]}$

\section{Resistor}

An electrical circuit consisting of a pure, ideal (Ohm's) resistor is free of any capacitive or inductive influences. Thus, the impedance is resistive and has no imaginary component at all AC frequencies. Applied to equations (6) and (7), this leads to the impedance $\underline{Z}_{R}$ of an ideal resistor:

$$
\underline{Z}_{R}=\operatorname{Re}(\underline{Z})+\mathrm{j} 0=\frac{\hat{u}}{\hat{\imath}}=R
$$

with the corresponding phase shift angle $\varphi$ :

$$
\underline{Z}_{R}=R \cdot e^{\mathrm{j} \varphi}=R \rightarrow \varphi=\varphi_{u}-\varphi_{i}=0
$$

Equation (10) reflects the Ohm's law for DC circuits (Eq. (1)) transferred to AC circuits. The complex impedance $\underline{Z}_{R}$ is given by the resistance $R$, and is calculated by the ratio of voltage and current magnitude. Since an ideal resistor has only a real contribution, there is no reactance part and consequently voltage and current are not phase shifted. Typical Nyquist and Bode diagrams in Tab. 3$3 \mathrm{~A}$ illustrate the frequency independent impedance contributions of an ideal resistor in an electrical circuit.

\section{Capacitor}

The capacitor is another passive element in an AC circuit. In contrast to the ohm's law for resistors, in which the current is directly proportional to the applied voltage ((1) and (10)), for capacitors the current is proportional to the voltage change over time: 


$$
\underline{i}=C \cdot \frac{d \underline{u}}{d t}=C \cdot \underline{u} \cdot \mathrm{j} \omega
$$

After conversion we obtain for the impedance $\underline{Z}_{C}$ of a capacitor:

$$
\underline{Z}_{C}=\frac{u}{\underline{i}}=\frac{1}{\mathrm{j} \omega C}=-\mathrm{j} \cdot \frac{1}{\omega C}
$$

The phase $\varphi$ of the capacitive current is $90^{\circ}$ ahead of the voltage:

$$
\varphi=\varphi_{u}-\varphi_{i}=-\pi / 2
$$

Ideal capacitors possess only an imaginary component, without any resistance contribution to the impedance. In the complex plane, the impedance vector of an ideal capacitor is a straight line along the negative reactance-axis. $\underline{Z}_{C}$ decreases with increasing frequency and capacitance values. Typical Nyquist and Bode plots are shown in Tab. 3-3 B.

\section{Inductor}

The third basic, passive element in AC circuits is an inductor. It plays a minor role in equivalent circuits for describing the electrical properties of biological systems. However, it is needed to describe the piezoelectric properties of an oscillating system with electric equivalents (cf. 3.3 The Quartz Crystal Microbalance $(O C M)$ ). An ideal inductor shows, like an ideal capacitor, only reactive (imaginary) impedance contributions. For inductors the driving voltage is proportional to the current change with time:

$$
\underline{u}=L \cdot \frac{d \underline{i}}{d t}=L \cdot \underline{i} \cdot \mathrm{j} \omega
$$

After conversion, the inductive impedance $\underline{Z}_{L}$ is given by:

$$
\underline{Z}_{L}=\frac{u}{\underline{i}}=\mathrm{j} \cdot \omega L
$$

The phase $\varphi$ of the inductive current is lagging behind $90^{\circ}$ with respect to the voltage:

$$
\varphi=\varphi_{u}-\varphi_{i}=+\pi / 2
$$

The impedance of ideal inductors is represented as a straight line along the positive reactance-axis in the Gaussian plane (Tab. 3-3 C). The inductive impedance is directly proportional to the frequency and increases likewise.

\section{Constant Phase Element (CPE)}

In contrast to the previously mentioned equivalent circuit elements (resistor, capacitor, and inductor) the CPE is a non-ideal and empiric circuit element. Non-ideal circuit elements are often required for modeling the impedance characteristics of real systems. Such non-ideal behavior may be due to dielectric inhomogeneity or roughness of the electrodes. ${ }^{[278]}$ The CPE aims to describe the electrical properties of double layers that show non-ideal capacitive behavior. It is used to adequately describe the interface impedance of electrodes in contact with an electrolyte. The CPE contributes to the impedance by: 
Tab. 3-3 Impedance properties are shown for the basic AC equivalent circuit elements, (A) resistor, (B) capacitor, and (C) inductor. Typical impedance spectra (Bode diagrams) and Nyquist plots are shown for equivalent circuits with either $(\mathrm{A})$ one resistor $(R=10 \mathrm{kOhm})$, or $(\mathrm{B})$ one capacitor $(C=100 \mathrm{nF})$, or $(C)$ one inductor $(L=0.05 \mathrm{H})$ as single circuit element. In Nyquist diagrams the increasing frequency $\omega$ is indicated by an arrow. Frequency range: $10^{\circ}-10^{6} \mathrm{~Hz}$.

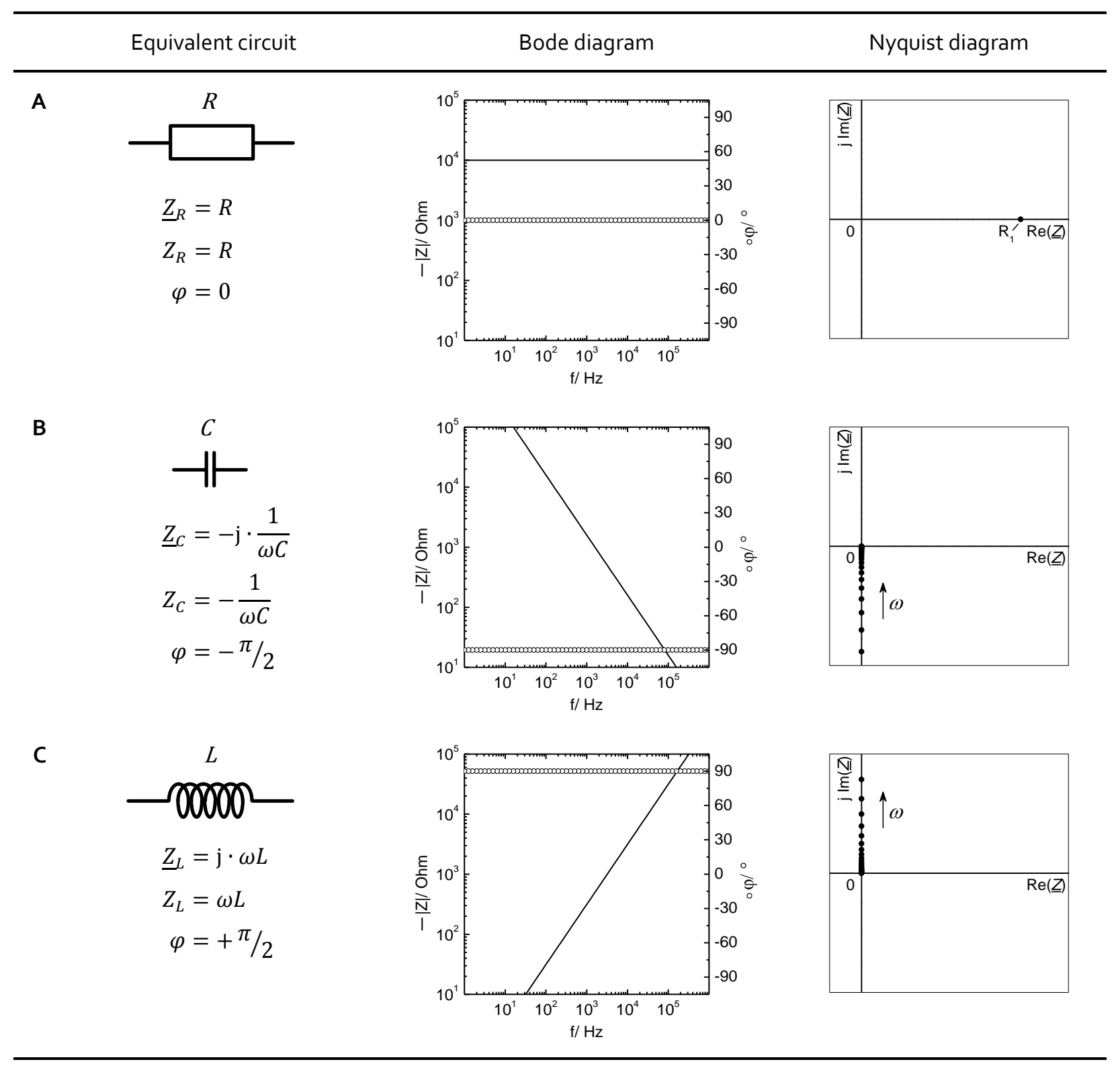

$$
\underline{Z_{C P E}}=\frac{1}{A \cdot(\mathrm{j} \omega)^{n}}, \quad \text { with } n=[0,1]
$$

where $A$ and $n$ are frequency independent parameters. The phase angle of the CPE is independent of the frequency, as the name, constant phase element, already indicates. The phase shift angle between AC voltage and current for a CPE is given by:

$$
\varphi=-n \cdot \pi / 2
$$

Two extreme cases can be considered for the electrical property of the CPE. In the first extreme case of $n=0$ the CPE describes an ideal resistor (with $1 / A=R$ ), and in the second extreme case of $n=1$ the CPE 
represents an ideal capacitor (with $A=C$ ). For any value of $n=] \mathrm{o}, 1[$ the CPE exhibits mixed electrical properties of a resistor and a capacitor with dominating capacitance for $n>0.5$ and dominating resistance for $n<0.5 .^{[278,279]}$

\subsubsection{Equivalent Circuit Models}

The basic idea of IS studies on biological systems is to get electric information that can be translated, with an appropriate model, into specific physical or chemical properties of the system of interest. In order to gain this information, the system has to be described by an electrical equivalent circuit. At first, such an equivalent circuit should take into account any impedance contribution within the system under test as an electric element in the equivalent circuit. Once an adequate equivalent circuit is created, the theoretical impedance of the circuit (integrating the impedance contributions of all individual impedance elements) can be expressed mathematically with a transfer function. The experimental impedance data is then fitted with the transfer function in order to obtain the parameters of all single circuit elements. The electrical properties of such an element are then correlated with specific physical properties of a structural component of the observed system. ${ }^{[279]}$

Biological systems are often very inhomogeneous and complex in their structure and composition. This is especially the case when one wants to describe the electrical or mechanical properties of whole cells in detail. Hence, it can be very difficult to design an accurate electrical model that characterizes the electrical or piezoelectrical properties of a sample in all details. Therefore, individual simplified equivalent circuits have to be designed that are adapted to the information one wants to extract. For instance, a very simple electrical model for the complex electrode-electrolyte interface is the combination of a resistor and a capacitor in series, whereas the impedance of a (not less complex) cell membrane can be modelled by the same two circuit elements, however, connected in parallel. ${ }^{[280]}$ The impact of a few basic, simple combinations of circuit elements (one (two) resistor(s) connected with a capacitor in various ways) on the impedance spectra and on the resistance and reactance values of the entire electrical circuit are described below.

\section{R-C Circuit}

An equivalent circuit with a serial combination of an ideal resistor and an ideal capacitor can be easily divided into resistance and reactance contributions. All resistance contributions originate from the resistor, whereas all reactive behavior is linked to the capacitor. The impedance of a resistor in series with a capacitor is given by $\underline{Z}_{R_{1}-C}=R_{1}-\mathrm{j} \cdot 1 / \omega C$. The impedance and phase spectra of a typical $R-C$ circuit is shown in Tab. $3-4 \mathrm{~A}$. The impedance is predominated by the capacitance in the low-frequency region, which is reflected in a negative slope and a phase angle of $-90^{\circ}$, respectively in the Bode plot. Whereas at high frequencies $(>1 \mathrm{kHz})$ the resistance dominates the impedance signal, with a frequency independent magnitude, parallel to the $x$-axis, and a phase shift of $0^{\circ}$. In the corresponding Nyquist diagram in Tab. 3-4 A the impedance of the $R-C$ circuit is displayed in the complex plane, by frequency independent real part $\left(R_{1}\right)$ and a frequency dependent imaginary (reactance) part $(-\mathrm{j} \cdot 1 / \omega C)$. The impedance locus in this case is a straight line parallel to the imaginary axis. The distance of this line from the origin and the $y$-axis, respectively, represents the value of the resistance $R_{1}$. The influence of the reactance (capacitance) on the impedance decreases with increasing frequency $\omega$, and thus contributes less to the entire impedance signal (indicated with an arrow in the Nyquist diagram). 
Tab. 3-4 Equivalent circuits and typical impedance spectra are displayed for the combination of a resistor and a capacitor in series $(A)$ and in parallel (B), as well as for a parallel circuit of a capacitor and a resistor in series with a second resistor (C). For each circuit typical Bode and Nyquist plots are shown. In Nyquist diagrams the increasing frequency $\omega$ is indicated with an arrow. Note: the imaginary axis is plotted negatively. Frequency range: $10^{\circ}-10^{6} \mathrm{~Hz}$; $R_{1}=10 \mathrm{kOhm}, R_{2}=100 \mathrm{Ohm}, C=100 \mathrm{nF}$.

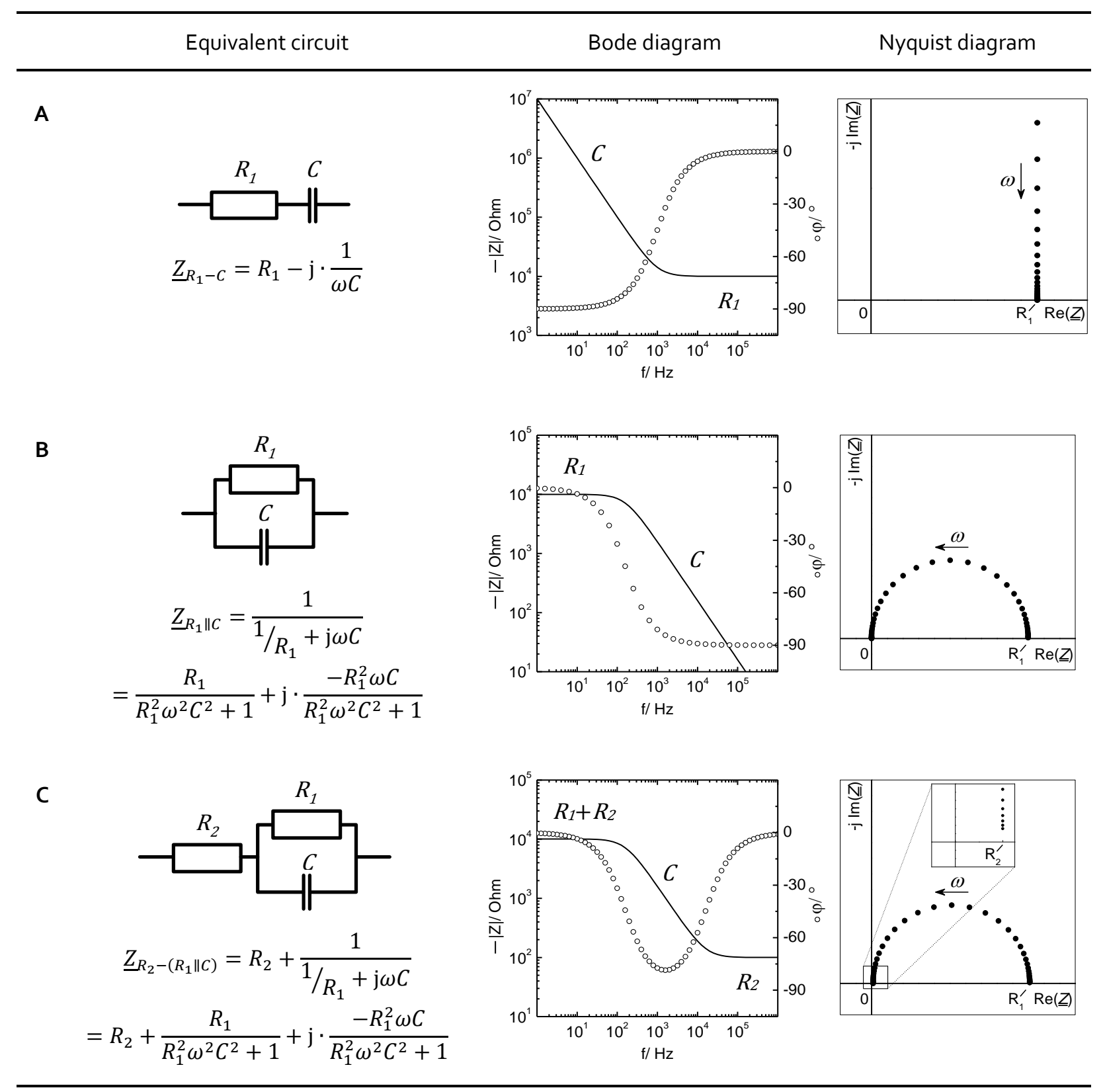

\section{RIIC Circuit}

The second possible combination of a resistor and a capacitor is the parallel connection of both (Tab. 3$4 \mathrm{~B}$ ). The impedance of a $R \| C$-circuit (\| stands for the parallel connection of the circuit elements) shows a completely different frequency dependence compared to the $R-C$ series circuit. The mathematical expression for the impedance $\underline{Z}_{R_{1} \| C}$ is given by $\underline{Z}_{R_{1} \| C}=\left(1 / R_{1}+\mathrm{j} \omega C\right)^{-1}$. Typical Bode and Nyquist diagrams are shown in Tab. 3-4 B. In $R \| C$ circuits the resistance dominates the impedance at lower frequency values, whereas the capacitance does at higher frequencies. In the respective Nyquist plot in Tab. $3-4 \mathrm{~B}$ it can be seen that the impedance of a $R \| C$ circuit has a semi-circle locus in the complex plane. 


\section{R-(R\|C) Circuit}

For the description of complexer systems, equivalent circuits with more than two electrical circuit elements are usually necessary. For instance, using the previous examples again, the description of a cell membrane in close contact to an electrode in an electrolytic solution requires the combination of the previously separately considered systems and equivalent circuits, respectively.

One possible circuit with three elements is the parallel combination of a resistor $R_{1}$ and a capacitor $C$, which is connected in series with a second resistor $R_{2}$ (Tab. 3-4 C). With respect to the aforementioned exemplary test systems, this circuit could be used as a simple model for describing the electrical situation of a cell membrane in contact with an electrolyte. The impedance of a $R_{2}-\left(R_{1} \| C\right)$ circuit is given by $\underline{Z}_{R_{2}-\left(R_{1} \| C\right)}=R_{2}+\left(1 / R_{1}+\mathrm{j} \omega C\right)^{-1}$. In contrast to the $R-C$ or $R \| C$ circuit, the impedance in an $R_{2}-\left(R_{1} \| C\right)$ circuit converges at low as well as at elevated frequency levels, according to:

$$
\begin{aligned}
& \omega \rightarrow \mathrm{0}: \quad \underline{Z} \rightarrow R_{1}+R_{2} \\
& \omega \rightarrow \infty: \quad \underline{Z} \rightarrow R_{2} \\
& \varphi \rightarrow 0^{\circ} \\
& \varphi \rightarrow 0^{\circ}
\end{aligned}
$$

This is illustrated in the Bode diagram of a test system in Tab. $3-4 \mathrm{C}$. The impedance magnitude at the low frequency region is determined by $R_{1}+R_{2}$, whereas it is dominated by $R_{2}$ at the upper end of the frequency window. The Nyquist representation of the $R_{2}-\left(R_{1} \| C\right)$ circuit impedance is similar to the Nyquist plot of the RIIC circuit in Tab. 3-4 B. However, there is an additional fixed real (resistance) contribution, originating from the second resistor $\left(R_{2}\right)$ in series to the parallel $R_{1} \| C$ circuit. This causes the impedance not to become purely imaginary at any frequency.

In Bode plots, either the impedance magnitude or the phase shift is plotted as a function of frequency in a two dimensional diagram. The presentation of only one parameter actually under-represents the impedance data, since magnitude and phase are complementary information and only the combination of both can fully describe the complex impedance of the system. Instead of presenting two individual plots, $|Z|(f)$ and $\varphi(f)$, or two-dimensional diagrams with two y-axes, the entire impedance information can also be merged in a 3-D Bode plot, with perpendicular axes of frequency ( $x$-axis), impedance magnitude ( $y$-axis), and impedance phase (z-axis). This provides an impedance spectrum in the threedimensional space that comprises any impedance information. A projection of the $3-D$ curve to the $|Z|$ $f(\varphi-f)$ plane provides the corresponding, two-dimensional representation of $|Z|(f)(\varphi(f))$, equal to the individual two-dimensional plot. Additionally, one gets in the third projection plane the frequency independent correlation between impedance magnitude $|Z|$ and phase $\varphi$. For instance, the impedance properties of the $R_{2}-\left(R_{1} \| C\right)$ circuit in Tab. 3-4 C are illustrated as a 3-D Bode plot in Fig. 3-3 A. The 2-D Bode plots, $|Z|(f)$ and $\varphi(f)$, equal to those in Tab. $3-4 C$, are represented in the respective projection planes. As additional information, the third projection plane provides the dependence of $|Z|$ and $\varphi$.

A Nyquist plot shows the imaginary part as a function of the real part with orthogonal $x$ - and $y$-axes. However, the frequency dependence of both, real and the imaginary part, is not directly visible in such diagrams. In order to make this frequency information accessible in a Nyquist plot, a third (frequency) axes can be plotted perpendicular to the complex plane of imaginary and real part. ${ }^{[277]}$ For resolution purposes, the frequency is plotted on a logarithmic scale. This results in a xyz or three-dimensional plot, which uncovers the full frequency dependence of real and imaginary parts. Moreover, it visualizes the frequency independent relationship between both quantities. Fig. 3-3 B displays a 3-D Nyquist diagram of the $R_{2}-\left(R_{1} \| C\right)$ circuit shown in Tab. $3-4 \mathrm{C}$, in the frequency range from $10^{\circ}-10^{6} \mathrm{~Hz}$. The impedance of 
the circuit is illustrated in 3 -D perspective as a function of excitation frequency ( $x$-axis), resistance ( $y$ axis), and reactance (z-axis). The projection of the 3 -D curve in Fig. 3-3 $B$ in the complex $\operatorname{Re}(\underline{Z})-\operatorname{Im}(\underline{Z})$ plane provides the same semi-circle as shown in the 2-D real-imaginary plot (Nyquist diagram) in Tab. 3${ }_{4} \mathrm{C}$. Moreover, the frequency dependence of either the imaginary part or the real part is accessible. It can be obtained by the projection of the $3-D$ curve in the $\operatorname{Im}(\underline{Z})-f$ plane and $\operatorname{Re}(\underline{Z})-f$ plane, respectively.

A

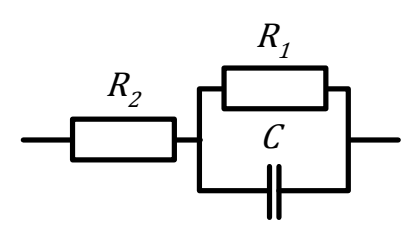

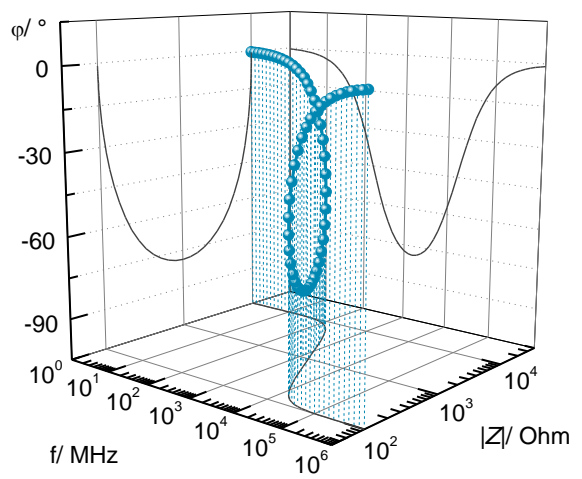

B

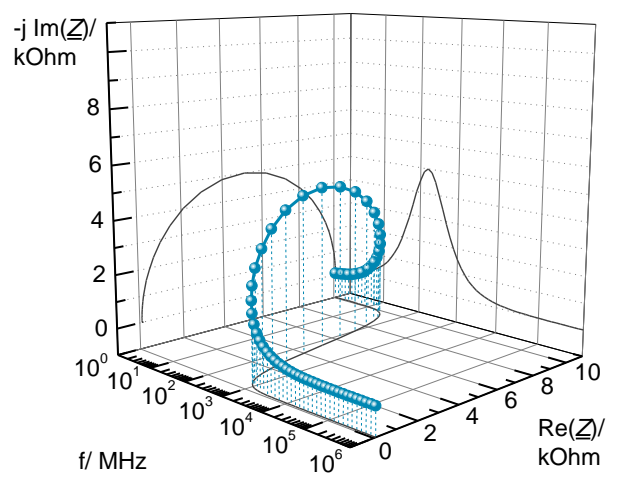

Fig. 3-3 (A) 3-D Bode plot and (B) 3-D Nyquist plot of the complex impedance of a $R_{2}-\left(R_{1} \| C\right)$ circuit. The complex impedance is plotted as a function of frequency $(f)$, impedance magnitude $(|Z|)$, and phase shift $(\varphi)$ in $A$ and as a function of real part $(\operatorname{Re}(\underline{Z}))$, negative imaginary part $(-\mathrm{j} \operatorname{Im}(\underline{Z}))$, and frequency $(f)$ in B. All axes are perpendicular to each other. Frequency range: $10^{\circ}-10^{6} \mathrm{~Hz} ; R_{1}=10 \mathrm{kOhm}, R_{2}=100 \mathrm{Ohm}, \mathrm{C}=100 \mathrm{nF}$.

\subsection{The Quartz Crystal Microbalance (OCM)}

The Quartz Crystal Microbalance (OCM) is a mass sensitive device which makes use of propagating acoustic waves in a solid material. Acoustic waves are low energy waves and occur between $10^{-2}$ and $10^{9} \mathrm{~Hz}$ in the electromagnetic spectra, adjacent to the microwaves in the upper frequency range (see Fig. 3-4). Acoustic waves can have different origins. However, their utilization for sensing applications requires a frequency-stable and continuous acoustic wave source, and moreover a sensitive detection device that is able to measure changes in the properties of acoustic waves with high accuracy. These requirements can be fulfilled by the use of piezoelectric materials as transducer unit in a sensor setup.

Materials that exhibit piezoelectric properties can be forced to oscillation by applying an external electrical AC voltage. The oscillation goes along with acoustic waves that propagate through (or on the surface of) the material, and decay in the surrounding medium in contact tothe material. The propagation (vibration mode) of acoustic waves in piezoelectric materials can be very diverse, as it depends on several properties of the resonating matter and its boundaries: the kind of the oscillating material, the thickness of the sensor plate, the lattice orientation, and also the orientation and geometry of the electrodes (which are needed for applying the oscillation voltage). Hence, a lot of different acoustic wave based sensor technologies were developed. Dependent on the kind of elastic wave, they can be roughly categorized into the three types: bulk-acoustic-wave (BAW) sensors ${ }^{1}$, surface-acoustic-wave

\footnotetext{
${ }^{1}$ bulk waves occur in a boundary-free medium
} 
(SAW) resonators ${ }^{2}$, and plate-acoustic-wave resonators ${ }^{3}$, like flexural-plate-wave (FPW) or shearhorizontal-acoustic-plate-mode (SH-APM) resonators. ${ }^{[196]}$ This big variety is also reflected by the large frequency range along which acoustic resonators are used for biosensing applications. The working range covers six orders of magnitude, from $1 \mathrm{kHz}$ to $1 \mathrm{GHz}$ (Fig. 3-4). ${ }^{[281]}$

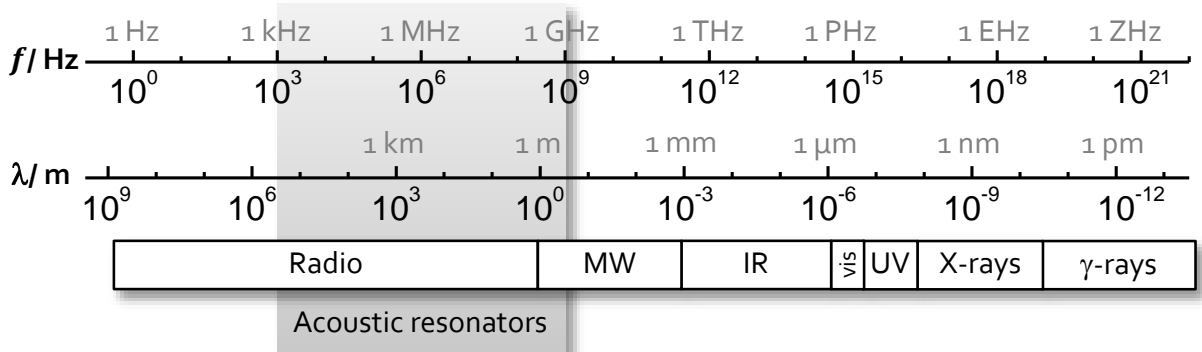

Fig. 3-4 The frequency range of acoustic resonators in the electromagnetic spectra. The region of radio waves covers about ten orders of magnitude. The relevant region for biosensing by means of acoustic waves range from $1 \mathrm{kHz}$ to $1 \mathrm{GHz}$. $f=$ frequency, $\lambda=$ wavelength, $\mathrm{MW}=$ microwave, $\mathrm{IR}=$ infrared, vis = visible light, $\mathrm{UV}=$ ultraviolet radiation.${ }^{[196,281]}$

The most important and most commonly used piezoelectric material is $\alpha$-quartz, as it exhibits very high temperature stability. Other materials that show piezoelectricity are lithium niobate, lithium tantalate, zinc oxide or aluminum nitride. ${ }^{[197,200,201]}$ For OCM applications, thin and round AT-cut disks from an $\alpha$ quartz crystal are used. They exhibit a very high frequency stability, with a temperature coefficient around zero between $0^{\circ} \mathrm{C}$ and $50^{\circ} \mathrm{C}$ (cf. Supplementary Information SI 1, p. 262). ${ }^{[282]}$ AT-cut terms the cutting angle of $35.25^{\circ}$ relative to the z-axis (optical axis) of the crystal. Theses $\mathrm{QCM}$ resonators generate bulk acoustic waves (BAW), and are referred to as thickness shear mode (TSM) resonators. TSM devices are characterized by transverse (shear) waves in the piezoelectric material that propagate perpendicular to the plate surfaces. The vibration in the solid continues at the solid surfaces and decays in the surrounding medium. Mechanical changes at the sensor interphase influence the oscillation of the quartz and thus can be detected by recording and analyzing the parameters of the resonant oscillation.

The following sections will focus on the basic principle of piezoelectricity in AT-cut quartz disks, its primary application for micro-weighing, and on how impedance spectroscopy in combination with electromechanical equivalent circuits could extend the OCM into a biosensor for cytomechanics.

\subsubsection{Basic Principles of the QCM Technique}

\section{Piezoelectricity of TSM Resonators}

As previously mentioned, the piezoelectricity of AT-cut quartz disks is the basic principle of OCM measurements. The piezoelectric effect was first discovered 1880 by the brothers Jacques and Pierre Curie. They describe the effect of generating electrical charges on the surface of a crystal by unidirectional, mechanical deformation. The basic requirement of a material or crystal for exhibiting

\footnotetext{
${ }^{2}$ surface waves occur along a single plane boundary (= semi-infinite solid)

${ }_{3}^{3}$ plate waves occur between two parallel plane boundaries
} 
piezoelectric properties is the absence of an inversion center. Within the crystal classes, 21 out of the 32 point groups are non-centrosymmetric, and 20 of them show piezoelectric behavior. ${ }^{[283]}$

A

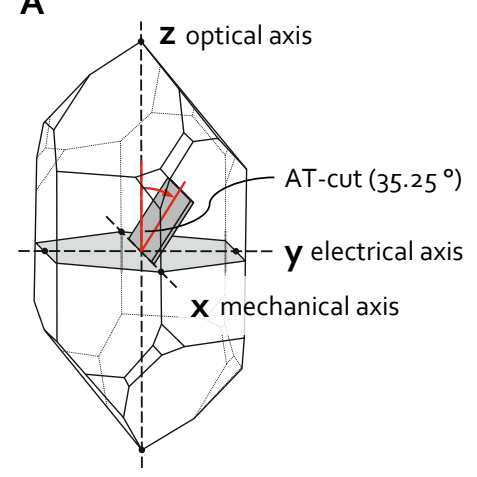

B

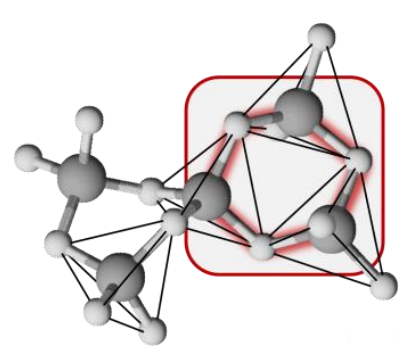

C

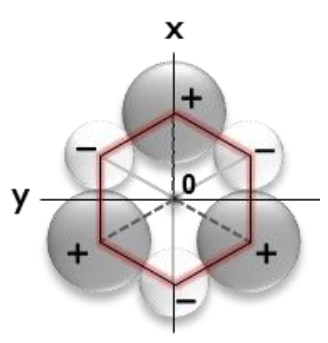

$z^{\prime} y$
D

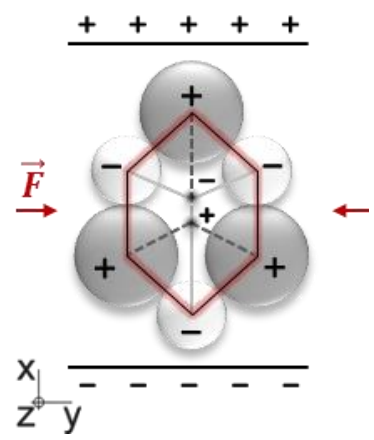

Fig. 3-5 (A) Crystal structure of an $\alpha$-quartz with indicated AT-cut plane $\left(35.25^{\circ}\right.$ with respect to the optical axis). (B) Oxygen linked $\mathrm{SiO}_{4}$ tetrahedrons form the crystal structure of the quartz; a hexagonal structural unit - composed of three silicon and three oxygen atoms from three linked tetrahedrons - is highlighted. (C) Simplified scheme of the distribution of charges within a hexagonal structural unit of quartz; positive and negative charges coincide in the center resulting in a neutral quartz surface. (D) Transversal piezoelectric effect: a mechanical stress along the $y$ axis causes transversal electric displacement (along the $x$-axis) and the formation of charges on the quartz surface. Illustrations were redrawn, according to ${ }^{[284,285]}$.

The piezoelectric effect can be explained by the orientation of charged atoms within the crystal lattice. Fig. 3-5 A shows a drawing of a quartz single crystal with hexagonal structure. Three different kinds of axes characterize the symmetry of the crystal: one z-axis (optical axis), which is vertical to the hexagonal plane, three $y$-axes (electrical axes) running through the edges of the hexagon, and three x-axes (mechanical axes) running through the hexagon vertices. The three $x$-axes are polar axes, whereas the $y$ axes and the z-axis are non-polar. This becomes evident when zooming into the atomic structure of the $\mathrm{SiO}_{2}$ lattice. Fig. 3-5 B shows a simplified structure cut-out of the $\alpha$-quartz crystal. Each silicon atom is connected with four oxygen atoms, whereas the oxygen atoms form the vertices of a tetrahedron (indicated with black lines) with a silicon atom in the center. Each oxygen atom again is part of a neighboring triangular pyramid. Three connected $\mathrm{SiO}_{4}$ tetrahedrons form a hexagonal structure consisting of three silicon and three oxygen atoms (highlighted in Fig. 3-5 B). This hexagon is a recurrent structure element in the quartz and is suitable for displaying the distribution of charges within the crystal. A simplified two-dimensional hexagon of silicon and oxygen in natural orientation is illustrated in Fig. 3${ }_{5} \mathrm{C}$. The center of positive $\left(\mathrm{Si}^{4+}\right)$ and negative $\left(\mathrm{O}^{2-}\right)$ charges coincide in the spatial center of the hexagon, which leads to a crystal with zero net charge.

When applying a mechanical stress in $y$-axis direction of the atomic structure, this causes a transversal displacement of the centers of (positive and negative) charges in $x$-axis direction (Fig. 3-6 D). Thus, the hexagonal structure, and consequently the whole crystal are electrically polarized with resulting opposing charges on the crystal surface. This effect is called transversal piezoelectric effect. In contrast, a mechanical stress applied in $\mathrm{x}$-axis direction causes a unidirectional displacement of atoms and charges in $x$-axis, which is referred to as longitudinal piezoelectric effect (SI 2, p. 262). Thus, a piezoelectric material can be termed as an electromechanical coupler, as it is capable of transforming mechanical energy into electrical, and vice versa. 
In OCM technology, the so-called converse (transversal) piezoelectric effect is applied. By plating two circular gold electrodes in the center of either side of the AT-cut quartz disk, the resonator can be electrically addressed (Fig. 3-6 A). Applying a constant electrical field on the quartz surfaces causes a transversal displacement of charges that induces a mechanical strain within the crystal (Fig. 3-6 B). If one applies an alternating potential difference, the quartz disk is forced to an alternating mechanical deformation (shear oscillation) that is perpendicular to the electric field. This strain can be described by two acoustic waves that travel - vertical to the surfaces - through the material in opposite directions (Fig. 3-6 C). At the electrode interface, the acoustic wave propagates and decays in the adjacent medium. The oscillation amplitude distribution over the quartz surface has a Gaussian shape. The amplitude possesses its maximum in the center of the electrode $(r=0)$ and vanishes almost to zero at the edges of the electrode $\left(r=r_{e l}\right)$ (Fig. 3-6 C). Hence, the center of the quartz is the most sensitive region of the surface for changes of the oscillation, and thus in OCM readings. At standard driving voltages of $0.15 \mathrm{~V}$ for $5 \mathrm{MHz}$ AT-cut quartz resonators, the maximal shear amplitude is $<1 \mathrm{~nm}$ in the center of the quartz surface. Thus, considering the average cell diameter of roughly $10 \mu \mathrm{m}$, the QCM is an essentially non-invasive sensing technique for investigating whole cells.

A

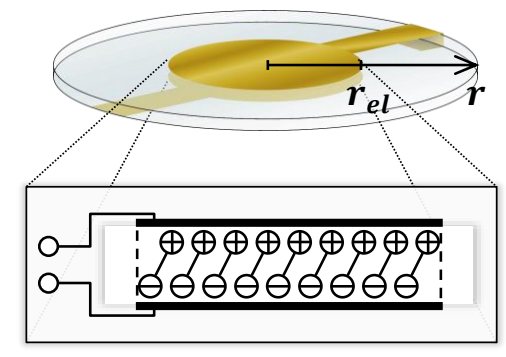

B

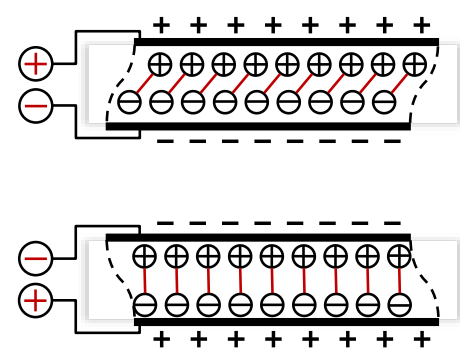

C

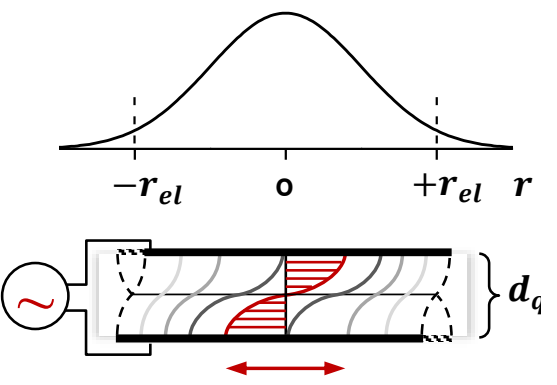

Fig. 3-6 (A) Drawing of a typical AT-cut quartz disk with evaporated, disk-shaped gold film electrodes on either side; the dipoles are in the natural orientation, without electrical charging of the quartz surface. (B) Converse transversal piezoelectric effect: a static external field causes a perpendicular displacement of charges, and thus a mechanical strain. (C) Applying an alternating voltage on the quartz surfaces causes a mechanical shear oscillation that travels, perpendicular to the surface, as an acoustic wave through the quartz; the oscillation amplitude has its maximum in the center of the electrodes with radius $r_{e l}$. Graphics were redrawn, according to ${ }^{[286]}$.

\section{TSM Resonators as Microbalances - Sauerbrey Relationship and Limitations}

Each piezoelectric element has characteristic eigenfrequencies, at which the opposing acoustic waves show positive interference and exhibit a standing acoustic wave within the crystal. This resonance condition is fulfilled, when an integer multiple $n$ of the wavelength $\lambda$ of the acoustic wave is twice the thickness $d_{q}$ of the quartz disk (Eq. (20)). At those resonance conditions the shear vibration shows minimal oscillation impedance.

$$
n \lambda=2 d_{q}, \quad \text { with } n=1,3,5, \ldots
$$

In this case, the disk thickness defines the wavelength of the fundamental $(n=1)$ and the harmonic $(n=$ $3,5,7 \ldots$ resonances. The factor $n$ must be an odd number, because only then the boundary conditions are met for opposing displacement on the quartz surfaces. The fundamental resonance condition $(\lambda / 2=$ 
$d_{q}$ ) is shown in Fig. 3-6C, where half of the wavelength corresponds to the thickness of the disk. The fundamental resonance frequency $f_{0}$ is related to the quartz thickness by the speed of sound and can be calculated according to:

$$
f_{0}=\frac{v_{q}}{\lambda}=\frac{v_{q}}{2 d_{q}}=\frac{\sqrt{\overline{c_{66}} / \varphi_{q}}}{2 d_{q}}=\frac{K_{R}}{d_{q}}
$$

with $v_{q}$ is the speed of sound $\left(3322 \mathrm{~m} \cdot \mathrm{s}^{-1}\right)$ and $\overline{c_{66}}$ is the "piezoelectrically stiffened" shear modulus $\left(2.947 \cdot 10^{11} \mathrm{~g} \cdot \mathrm{cm}^{-1} \cdot \mathrm{s}^{-2}\right)$ in an AT-cut quartz crystal, and $\varphi_{q}$ is the density of quartz $\left(2.648 \mathrm{~g} \cdot \mathrm{cm}^{-3}\right) . K_{R}$ is a frequency constant (the electromechanical coupling constant) of AT-cut quartzes and is defined as the half of the propagation velocity of the acoustic wave in a TSM quartz resonators, and is $1665 \mathrm{~m} \cdot \mathrm{s}^{-1} \cdot[204,287-$ ${ }^{289]}$ The quartz disks used in this work have a fundamental mode of $5 \mathrm{MHz}$, which corresponds to a plate thickness of $330 \mu \mathrm{m}$.

If the quartz thickness is increased by an additional layer of mass that features quartz-like behavior (similar $K_{R}$ value), the resonance frequency is decreased according to equation (21) (cf. $S / 3$, p. 262). Saverbrey described the mathematical relationship between the additional mass $\Delta m$ of an adsorbed layer and the resulting resonance frequency shift $\Delta f / f_{0}$ of the resonator:

$$
\frac{\Delta f}{f_{0}}=-\frac{\Delta d}{d_{q}}=-\frac{\Delta m}{d_{q} \cdot A \cdot \varphi_{q}}
$$

Expressing $d_{q}$ Eq. (22) by the relation in Eq. (21), and solving for the resonance frequency shift $\Delta f$, one then obtains the Saverbrey relation:

$$
\Delta f=-\frac{2 f_{0}^{2}}{\sqrt{\overline{\bar{c}_{66}} \cdot \varphi_{q}} \cdot A} \cdot \Delta m=-S_{f} \cdot \frac{\Delta m}{A}
$$

with $A$ as the vibrating area on the quartz surface (i.e. approx. the electrode area), and $S_{f}$ as the integral mass sensitivity and Sauerbrey constant, respectively. ${ }^{[282]}$ For $5 \mathrm{MHz}$ AT-cut quartz disks, a theoretical integral mass sensitivity of $S_{f}=0.0057 \mathrm{~Hz} \mathrm{~cm}^{2} \mathrm{ng}^{-1}$ was calculated. According to equation (23), the integral mass sensitivity $S_{f}$ is a function of the square of the fundamental resonance frequency. Hence, the mass sensitivity of the $\mathrm{OCM}$ can be increased by using quartzes with a higher fundamental resonance frequency. According to equation (21), an increase in $f_{0}$, however, goes along with a decrease in the quartz thickness. This loss in mechanical stability limits the applicability of resonators with very high fundamental frequencies. $5 \mathrm{MHz}$ quartzes, which were used in this thesis, are a good compromise with respect to sensitivity and mechanical stability.

In principle, Sauerbrey's relationship (Eq. (23)), is only valid for the adsorption of a quartz-like layer. Thin and rigid layers adsorbed on the quartz surface influence the eigenfrequency of the quartz only by their inertial mass, but not by their elastical properties. Thus, the deposition of any material influences the eigenfrequency - within the limits of the boundary conditions - equal to an additional layer of quartz of the same thickness. By this, it is possible to use the QCM as microbalance for the adsorption of small masses. The validity range for the Sauerbrey relation is limited to depositions of rigid, thin, and homogeneous layers in dry air, which have approximately the same density as quartz, and exhibit neither viscous nor elastic properties. 
Any deposited layer that is not ideally rigid shows deformation and a non-synchronous oscillation with the quartz. Deformation effects within the deposited material lead to loss of energy. Thus, viscoelastic loadings have an impeding impact on the quartz oscillation. Additional to the pure deposition of mass and the resulting frequency shift, there is a superimposed damping effect on the oscillation amplitude. This effect cannot be described by the Sauerbrey relation anymore. In order to derive physical information of the load material by OCM frequency readings, a lot of models have been developed in the past three decades. These models are often modified or expanded Sauerbrey based models. They connect the resonance frequency change of TSM resonators with the altered specific physical properties of the resonator itself and/ or the mechanical properties of the load material. Kanazawa and Gordon, for example, developed a model in 1985 that describes the effect of Newtonian liquids in contact with one face of a resonator on resonance frequency shifts. Their model combined a (lossless) oscillating quartz crystal with the damped propagation of the shear wave in a purely viscous liquid (a scheme is shown in $S / 4$, p. 263). The frequency shift is a function of the viscosity $\eta_{l}$ and the density $\rho_{l}$ of the liquid loading, according to:

$$
\Delta f=-\frac{f_{0}^{3 / 2}}{\sqrt{\pi \cdot \overline{c_{66}} \cdot \varphi_{q}}} \cdot \sqrt{\eta_{l} \cdot \rho_{l}}
$$

This model also describes the exponential decay of the mechanical displacement amplitude in the liquid. The damping of the acoustic wave in the viscous liquid can be described by the decay length of the shear wave, which is the reciprocal of the propagation constant. According to this model, the decay length $\delta$ is a function of liquid viscosity and density, and is given by:

$$
\delta=\sqrt{\frac{\eta_{l}}{\pi \cdot f \cdot \rho_{l}}}
$$

Applying this model to a $5 \mathrm{MHz}$ piezoelectric quartz resonator in water at $20^{\circ} \mathrm{C}$, the decay length of the acoustic wave into the liquid is calculated to about $0.25 \mu \mathrm{m} \cdot{ }^{[290,291]} \mathrm{A}$ historical overview of empirical and physical models for different loading conditions is given by Thompson. ${ }^{[204]}$

Although there are many different models explaining resonance frequency shifts in response to specific load materials, they mostly focus on only one or few parameters of the observed system. The KanazawaGordon model, for example, gives a relationship between resonance frequency change and the densityviscosity product of a liquid loading. However, any adsorption of mass would also come along with a decrease in $f$ (Eq. (23)), meaning that effects of both, mass loading and liquid properties on the resonance frequency cannot be distinguished. Thus, measurements of the frequency shift alone deliver too little information for describing more complex systems, such as found in real biosensor applications.

Another approach for obtaining more detailed information from OCM readings is based on reading not only the resonance frequency, but recording the whole oscillation spectra (close to the resonance frequency) of the quartz disk. Impedance spectroscopy of the oscillation provides more information about the observed system. This information is obtained as electrical impedance, which is connected with the acoustic (mechanical) impedance of the resonating system, due to the electro-mechanical coupling within the piezoelectric material. Hence, by developing appropriate electro-mechanical models, impedance spectroscopy is the method of choice to extract mechanical parameters of complex load materials. ${ }^{[206,292]}$ 
The following section will at first address the basic relationships between acoustic and electric properties of piezoelectric resonators. Subsequently, an appropriate electrical equivalent circuit for the OCM will be discussed. Finally, the impact of different loading conditions on the electrical parameters of the model will be discussed.

\subsubsection{Electro-acoustic Modeling and Impedance Analysis of TSM Resonators}

TSM oscillators are piezoelectric devices that are used as electro-mechanical transducers in OCM sensors. In such electro-mechanical devices mechanical energy is converted into electrical energy (direct piezoelectric effect) or vice versa (converse piezoelectric effect). Once an appropriate mechanical model for the resonator is developed, each mechanical element in the model can be expressed by an electrical analogue and consequently the mechanical oscillation by an electrical equivalent circuit. The model of the resonator is supplemented by any mechanical contribution originating from a particular loading on the resonator. This results in a more or less complex electrical equivalent for the system of interest. Measuring the whole oscillation impedance of the resonator around the resonance frequency (impedance spectroscopy) and subsequent fitting the impedance spectrum with an appropriate model (impedance analysis), all electrical contributions, and therefore the associated mechanical properties of the loading are accessible.

\section{Electro-mechanical Model for an Unperturbed Resonator}

A simple mechanical model for the resonance of a $\mathrm{QCM}$ is a damped oscillatory motion (Fig. 3-7 A). It consists of a mass $m$ in combination with a dash-pot (with a friction coefficient $r$ ), in parallel with a spring (with $k$ as Hooke's constant). The mass is displaced by an external force $F$ and brought to oscillation with a certain kinetic energy. This is related to the displacement of particles in the OCM, by applying an electrical field on the AT-cut quartz crystal. Storage of energy in the system during oscillation is represented by the spring, and the damping resistor represents energy loss. With respect to a quartz disk, oscillation energy is stored due to the elastic properties of the quartz crystal lattice. Energy dissipation appears in OCM resonators due to internal friction, losses in the electrodes, damping in the mounting, and acoustic losses at the surface between the crystal and the surrounding medium.

A

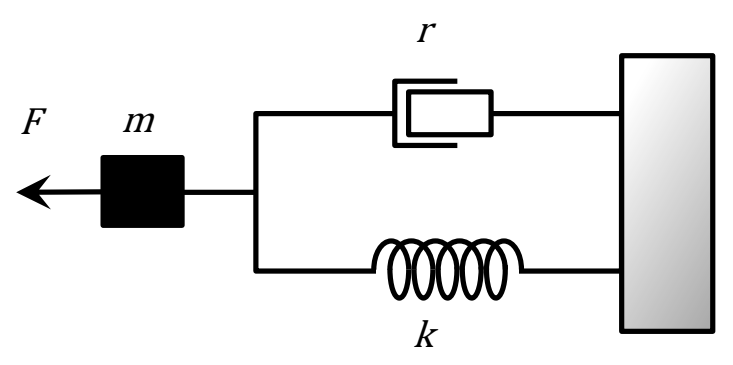

B

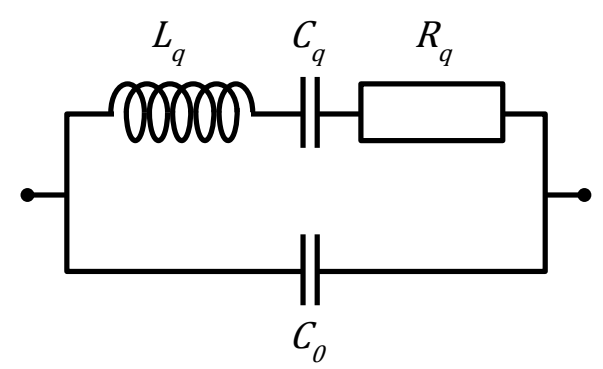

Fig. 3-7 (A) Mechanical model of a quartz oscillation, and (B) the electrical BVD equivalent circuit of the quartz oscillation, in which every circuit element represents a mechanical analogue. ${ }^{[293]}$ 
For the model of a mechanically oscillating system, consisting of a mass, a dash-pot, and a spring (Fig. 3$7 \mathrm{~A}$ ), the equation of motion is given by the balance of forces:

$$
F=m \cdot \frac{d^{2} x}{d t^{2}}+r \cdot \frac{d x}{d t}+k \cdot x
$$

where $F$ is the applied force, and $x$ is the resulting particle displacement. This mechanical model can be represented by an electrical equivalent circuit, consisting of an inductor $L_{q}$, a capacitor $C_{q}$, and a resistor $R_{q}$ in series (series branch in Fig. 3-7 B). The equation for the electrical oscillation of the LCR tank circuit is given by:

$$
U=L_{q} \cdot \frac{d^{2} q}{d t^{2}}+R_{q} \cdot \frac{d q}{d t}+\frac{1}{C_{q}} \cdot q
$$

where $U$ is the driving voltage and $q$ the amount of charge.

By comparing Eq. (26) and Eq. (27), one can easily see the isomorphic structure of the mechanical and the electrical model. The applied AC voltage $U$ and the generation of charges $q$ in the series branch is equivalent to the external force $F$ and the resulting displacement $x$ in the mechanical model. In the electrical circuit, the inductance $L_{q}$ represents the oscillating mass $m$ (inertia) in the mechanical model. In addition, the capacitance $C_{q}$ is (inversely) related to the spring constant $k$ and thus stands for mechanical elasticity. The resistance $R_{q}$ is linked with the friction factor $r$ and represents mechanical losses of the oscillation. The mechanical and the electrical oscillation equations are connected via the electro-mechanical coupling coefficient $\kappa(F=\kappa U) .{ }^{[294]}$ Thus, the mechanical properties of the motion are accessible by analyzing the electrical parameters of the equivalent network. ${ }^{[293,294]} A$ complete electrical equivalent circuit for a quartz resonator is obtained by complementing the LCR tank circuit with a capacitance $C_{0}$ in parallel (Fig. 3-7 B). It takes into account the sum of the static capacitance $C_{S}$ of the dielectric quartz plate between the two surface electrodes, and the parasitic stray capacitance $C_{P}$. Parasitic capacitances originate from the experimental setup and include wiring, crystal mounting and contacting contributions. The entire network (Fig. 3-7 B), of the LCR series resonance circuit (motional branch) and the parallel capacitance (dielectric branch), is also termed Butterworth-van-Dyke (BVD) equivalent circuit. ${ }^{[295]}$ In the BVD circuit, the parallel capacitance dominates the electrical circuit far from the resonance frequency. However, in the resonance frequency range the LCR series branch, i.e. the piezoelectric properties of the quartz, dominates the oscillation impedance.

The equations for calculating the values of the particular electrical parameters can be obtained by solving the wave equation of motion, using appropriate boundary conditions, and calculating the potential function at the quartz surface. The resulting mathematical expressions for the BVD electrical circuit elements $L_{q}, C_{q}, R_{q}$, and $C_{0}$ of an AT-cut quartz at the fundamental resonance are:

$$
\begin{aligned}
& L_{q}=\frac{d_{q}^{3} \rho_{q}}{8 A e_{26}^{2}} \\
& C_{q}=\frac{8 A e_{26}^{2}}{d_{q} \pi^{2} \overline{c_{66}}}
\end{aligned}
$$




$$
\begin{aligned}
& R_{q}=\frac{d_{q}^{3} r}{8 A e_{26}^{2}} \\
& C_{0}=\frac{\varepsilon_{22} A}{d_{q}}
\end{aligned}
$$

where $d_{q}$ denotes the quartz thickness, $\rho_{q}$ the density of the quartz, $A$ the electrode area, $e_{26}$ the piezoelectric stress constant, $\overline{c_{66}}$ the "piezoelectrically stiffened" shear modulus, $r$ the damping constant ( $d_{q}^{2} r=\eta_{q} \pi^{2}$ with $\eta_{q}$ as effective quartz viscosity), and $\varepsilon_{22}$ the dielectric constant of the quartz material. ${ }^{[197,293,295,296]}$

\section{Impedance Analysis of the BVD Model}

The BVD circuit parameters are obtained by carrying out impedance spectroscopy measurements of the shear oscillation and subsequent fitting the model to the raw data. A general introduction in impedance analysis is given in section 3.2 Impedance Spectroscopy (p. 22 ff.). There, the contribution of individual electrical circuit elements to the complex impedance is shown, and how this is reflected in the respective Bode and Nyquist plots. Moreover, the impedance characteristics of some exemplary electrical circuits are discussed in subsection 3.2.3 (p. 28).

In impedance spectroscopy a sinusoidal driving voltage of small amplitude is applied to the system of interest and the resulting current through the system is measured. This is done in frequency dependent manner, which is for quartz resonators a narrow range around the fundamental resonance frequency. The output signals of the measurements are the magnitude of the complex impedance $|Z|$ (the ratio of voltage and current amplitudes), and the phase angle shift $\varphi$ between voltage and current. Regarding the BVD circuit, the impedance of the resonator consists of the impedance of the motional branch $\underline{Z}_{m}$ (with $L_{q}, C_{q}$ and $R_{q}$ in series) and the parallel impedance $\underline{Z}_{C_{0}}$, i.e. the stray capacitance $C_{0}$ (cf. Fig. 3-7 B). For parallel arrangements of circuit elements, as in the BVD model, it is convenient to use the inverse of the complex impedance, which is the complex admittance $\underline{Y}$. Accounting for the Kirchhoff's law for electrical circuits, the complex admittance $\underline{Y}$ of the BVD model is expressed as ${ }^{[204,294]}$ :

$$
\underline{Y}=\frac{1}{\underline{Z}}=\frac{1}{\underline{Z}_{m}}+\frac{1}{\underline{Z}_{C_{0}}}=\frac{1}{R_{q}+\mathrm{j} \omega L_{q}+\frac{1}{\mathrm{j} \omega C_{q}}}+\mathrm{j} \omega C_{0}
$$

As the admittance is a complex number of the form $\underline{Y}=G+\mathrm{j} B$, equation (32) can be separated into its real part (conductance $G$ ) and imaginary part (susceptance $B$ ), with the expressions ${ }^{[204,294] \text { : }}$

$$
\begin{aligned}
& G=\frac{R_{q}}{R_{q}^{2}+\left(\omega L_{q}-\frac{1}{\omega C_{q}}\right)^{2}} \\
& B=\frac{-\left(\omega L_{q}-\frac{1}{\omega C_{q}}\right)}{R_{q}^{2}+\left(\omega L_{q}-\frac{1}{\omega C_{q}}\right)^{2}}+\omega C_{0}
\end{aligned}
$$

Inversion of equation (32) delivers the complex impedance $\underline{Z}$ of the BVD circuit as 


$$
\underline{Z}=\frac{1}{\underline{Y}}=\frac{R_{q}+j \omega L_{q}+\frac{1}{j \omega C_{q}}}{1-\omega^{2} L_{q} C_{0}+j \omega R_{q} C_{0}+\frac{C_{0}}{C_{q}}}
$$

The respective complex impedance real part (resistance $R$ ) and imaginary part (reactance $X$ ) are connected with conductance $G$ (Eq. (33)) and susceptance $B$ (Eq. (34)) by the following relations:

$$
\begin{aligned}
& R=\frac{G}{G^{2}+B^{2}} \\
& X=\frac{-B}{G^{2}+B^{2}}
\end{aligned}
$$

Besides complex numbers, the complex impedance can also be fully described by its magnitude $|Z|$ and phase $\varphi$, according to Eqs. (8) $\left(|Z|=\sqrt{R^{2}+X^{2}}\right)$ and (9) $(\varphi=\arctan (X / R))$.

The impedance of the oscillation near resonance is commonly plotted as a Bode plot, by the impedance magnitude as a function of frequency. Often, the respective phase angle is also displayed, as it complements the impedance properties of the oscillation. A characteristic Bode diagram of an unperturbed resonator with a fundamental frequency of $5 \mathrm{MHz}$ is shown in Fig. 3-8 A. Damping of the motional oscillation can be considered negligible, with an energy loss of $R_{q}=10 \mathrm{Ohm}$. This is due to the high quality factor of the quartz. Two characteristic resonance frequencies can be defined in this case, i.e. the resonant $\left(f_{R}\right)$ and antiresonant $\left(f_{A}\right)$ frequency. There, the imaginary part of the impedance and the phase are zero. At the lower resonant frequency $f_{R}$ the motional branch exhibits resonance, i.e. the inductance $L_{q}$ and the capacitance $C_{q}$ are resonant. In this case, the impedance magnitude shows a minimum (solid line in Fig. 3-8 A). For frequencies $f>f_{R}$, the motional branch possesses inductive properties, which is reflected by a phase shift to $+90^{\circ}$. This net inductance is in resonance with the parallel capacitance $C_{0}$ at the antiresonant frequency $f_{A}$, where the total reactance of the BVD-circuit is zero and where the impedance magnitude has its maximum. Below $f_{R}$ and above $f_{A}$ the parallel capacitance $C_{0}$ is dominating the entire BVD impedance, which can be seen by a phase shift (dashed line in Fig. 3-8 A) of $-90^{\circ}$.

However, if damping effects are taken into account (e.g. $R_{q}=10^{3} \mathrm{Ohm}$ ), four different resonant frequencies can be specified (Fig. 3-8 C). With increasing damping the maximal phase $\varphi_{\max }$ decreases, and the phase spectrum is broadened, too. Thus, the frequency of minimal impedance differs from the frequency of zero phase at series resonance. And also the frequency of maximal impedance differs from the frequency of zero phase at parallel resonance. Hence, the resonant frequency $f_{R}$ divides into the frequency of minimal impedance $f_{|Z|_{\text {min }}}$ and the series resonant frequency $f_{S}$, at which the reactance, i.e. the impedance phase, of the motional branch in the BVD circuit is zero. The antiresonant frequency $f_{A}$ separates likewise into the frequency of maximal impedance $f_{|Z|_{\text {max }}}$ and the parallel resonant frequency $f_{p}$ at the high frequency region (Fig. 3-8C). The frequencies $f_{|Z|_{\min }}$ and $f_{s}$, as well as $f_{|Z|_{\text {max }}}$ and $f_{p}$, diverge with increasing damping values of $R_{q}$, as indicated in Fig. 3-8 B.

The presentation of the complex impedance of a BVD circuit in the Gaussian (complex) plane shows a circular shape (Fig. 3-8 D). The frequency with the shortest impedance vector represents $f_{|Z|_{\text {min }}}$, whereas

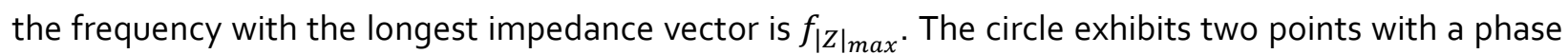
angle of zero (where the circle crosses the real axis), the respective frequencies are $f_{s}$ at lower frequencies and $f_{p}$ at elevated frequency values. Tab. 3-5 lists the mathematical expressions for 
calculating the characteristic parameters of a quartz resonator by the BVD equivalent circuit elements. The effect on the oscillation impedance curve shape (Fig. 3-8 A) by variations in the BVD circuit parameters $L_{q}, C_{q}, R_{q}$, and $C_{0}$ was described and discinto ussed by Yang and Thompson. ${ }^{[296]}$
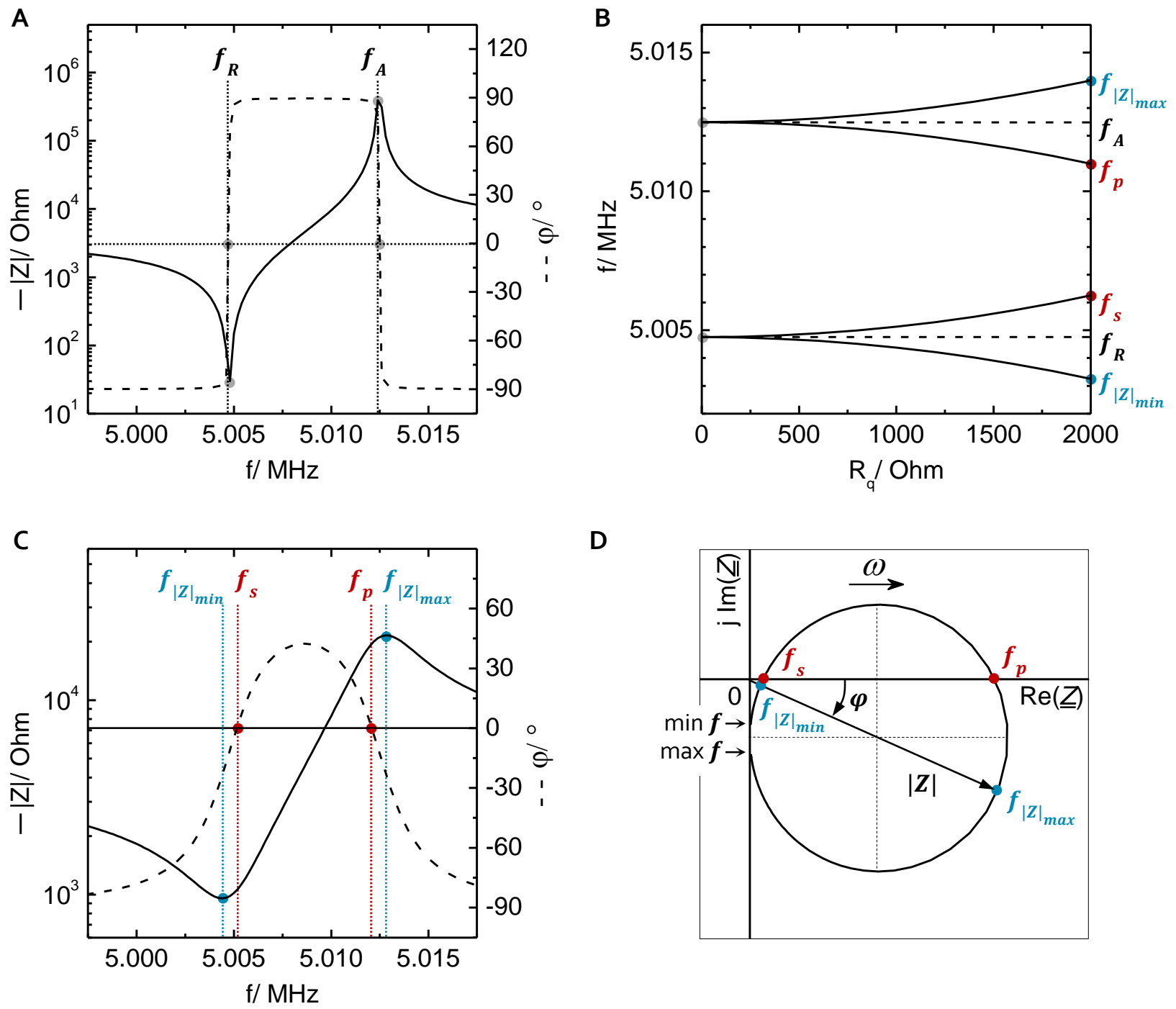

Fig. 3-8 (A) Bode diagram of a BVD equivalent circuit for a $5 \mathrm{MHz}$ quartz resonator with negligible energy losses of the oscillation $\left(R_{q} \rightarrow 0\right)$, with the impedance magnitude $|Z|$ (solid line) and the phase shift $\varphi$ (dashed line) as a function of frequency $f$ near resonance. At the resonant $\left(f_{R}\right)$ and the antiresonant $\left(f_{A}\right)$ frequency there is a zero-crossing of $\varphi$, and $|Z|$ is minimal (@ $f_{R}$ ) or maximal (@ $\left.f_{A}\right)$, respectively. (B) Increasing damping $\left(R_{q}\right)$ of the oscillation causes separation of the resonant frequency $f_{R}$ (antiresonant frequency $f_{A}$ ) into frequency of minimal impedance $f_{|Z|_{\min }}$ (frequency of maximal impedance $f_{|Z|_{\max }}$ ) and series resonant frequency $f_{s}$ (parallel resonant frequency $f_{p}$ ). (C) Bode plot and (D) Nyquist plot of BVD equivalent circuit for a damped $5 \mathrm{MHz}$ quartz resonator $\left(R_{q}>0\right)$. According to $\mathrm{A}, f_{R}$ is separated into $f_{|Z|_{\min }}$ and $f_{S}$ and $f_{A}$ into $f_{|Z|_{\max }}$ and $f_{p}$, respectively, with increased values of $R_{q}$. The impedance has a circular shape in the complex plane (Nyquist plot). The characteristic frequencies are indicated with arrows. $R_{q}=10 \mathrm{Ohm}(\mathrm{A}), R_{q}=1 \mathrm{kOhm}(\mathrm{C}), L_{q}=46.7 \cdot 10^{-3} \mathrm{H}, C_{q}=2.1655 \cdot 10^{-14} \mathrm{~F}, C_{0}=7 \cdot 10^{-12} \mathrm{~F}$.

The impedance spectra of an undamped and a damped BVD oscillator, as they are shown in Fig. 3-8, are illustrated in 3-D representations in $S / 5$ (p. 263), by 3-D Bode plots ( $Z \mid$ vs. $\varphi$ vs. $f$ ) and 3-D Nyquist plots $(\operatorname{Re}(\underline{Z})$ vs. j $\cdot \operatorname{Im}(\underline{Z})$ vs $f)$. 
Tab. 3-5 Impedance parameters for an AT-cut quartz resonator expressed by the electrical elements of the BVD equivalent circuit. ${ }^{[197,296]}$

\begin{tabular}{cllll}
\hline Parameter & Expression & Parameter & Expression \\
\hline $\boldsymbol{f}_{\boldsymbol{R}}$ & $\frac{1}{2 \pi} \sqrt{\frac{1}{L_{q} C_{q}}}$ & (38) & $\boldsymbol{f}_{\boldsymbol{A}}$ & $\frac{1}{2 \pi} \sqrt{\frac{1}{L_{q} C_{q}}+\frac{1}{L_{q} C_{0}}}$ \\
$\boldsymbol{f}_{|\boldsymbol{Z}|_{\text {min }}}$ & $\frac{1}{2 \pi} \sqrt{\frac{1}{L_{q} C_{q}}\left(1-\frac{C_{0} R_{q}^{2}}{2 L_{q}}\right)}$ & $(40)$ & $\boldsymbol{f}_{|\boldsymbol{Z}|_{\max }}$ & $\frac{1}{2 \pi} \sqrt{\frac{1}{L_{q} C_{q}}}\left(1+\frac{C_{0} R_{q}^{2}}{2 L_{q}}+\frac{C_{q}}{2 C_{0}}\right)$
\end{tabular}

\section{Impact of Different Loadings on the Resonator Oscillation}

As shown in the previous section, the mechanical oscillation of an unperturbed quartz resonator can be described by an equivalent electrical oscillation, due to electro-mechanical coupling of piezoelectric materials. The BVD circuit is an appropriate electrical equivalent circuit for a quartz resonator that resonates unperturbed in air. The correlations between the BVD circuit parameters and the mechanical properties of an AT-cut quartz disk are given in equations (28) - (31). However, any rigid, viscous, or viscoelastic loading (or any combination) has an impact on the oscillation properties of the quartz. This is reflected in changes of the characteristic impedance parameters of the oscillation. The perturbation by the surface loading can be treated as an additional motional impedance contribution $\underline{Z}_{m}^{1}$ in series to the motional impedance $\underline{Z}_{m}^{0}$ of the blank unperturbed resonator. ${ }^{[197,297]}$ In the BVD circuit, this is considered by an additional impedance element in series to the motional branch, as it is illustrated in Fig. 3-9.

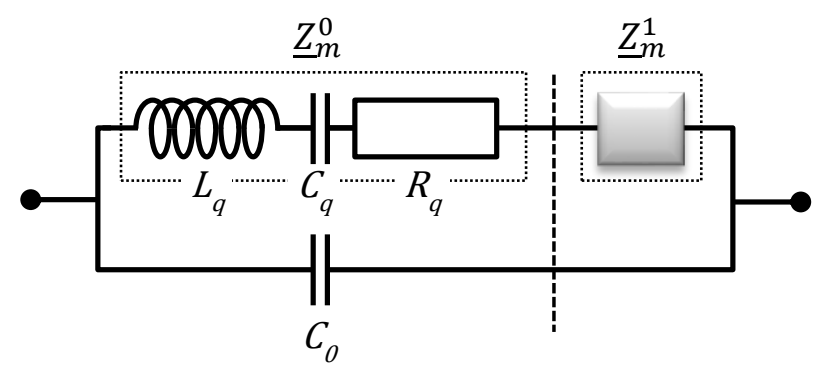

Fig. 3-9 A perturbation of a resonator by a surface loading can be considered in the BVD equivalent circuit by an additional motional impedance $\underline{Z}_{m}^{1}$, in series to the motional branch impedance of an unperturbed resonator, $\underline{Z}_{m}^{0}$.

Thus, the motional impedance $\underline{Z}_{m}$ of a perturbed quartz oscillation is given by: 


$$
\underline{Z}_{m}=\underline{Z}_{m}^{0}+\underline{Z}_{m}^{1}
$$

With Eq. (46), Eq. (32) then can be extended to:

$$
\underline{Y}=\frac{1}{\underline{Z}}=\frac{1}{\underline{Z}_{m}^{0}+\underline{Z}_{m}^{1}}+\frac{1}{\underline{Z}_{C_{0}}}
$$

By determining the circuit parameters of an unperturbed quartz prior to a loading, it is possible to calculate the impedance that arises from the loading. With an equivalent electrical model for the loading, the BVD circuit (for an unloaded resonator) can be extended by specific, corresponding impedance contributions. In this case, it is possible to calculate specific mechanical properties (and their changes) of the quartz loading, by impedance analysis of an OCM measurement. According to Bandey (1999) [297], the additional motional impedance of a perturbed resonator can be approximated by:

$$
\underline{Z}_{m}^{1} \approx \frac{n \pi}{4 K^{2} \omega_{S} C_{0}}\left(\underline{\underline{Z}}_{q}\right)=\psi\left(\frac{\underline{Z}_{S}}{\underline{Z}_{q}}\right)=R_{L}+\mathrm{j} X_{L}
$$

where $K$ is the electromechanical coupling factor for quartz, $\omega_{s}$ is the angular series resonant frequency $\left(\omega_{s}=2 \pi f_{s}\right), \underline{Z}_{s}$ is the surface mechanical impedance, and $\underline{Z}_{q}$ is the quartz characteristic impedance $\left(\underline{Z}_{q}=\sqrt{\rho_{q} \mu_{q}}\right.$, where $\rho_{q}$ is the quartz density and $\mu_{q}$ is the quartz shear stiffness). $R_{L}$ and $X_{L}$ represent the real and imaginary parts of the motional impedance of the surface load. Expressions of $Z_{S}, R_{L}$, and $X_{L}$ for three basic loading conditions (ideal mass layer, Newtonian liquid (semi-infinite), and semi-infinite viscoelastic layer) are summarized in Tab. $3-6 .^{[197,297]}$

\section{Rigid Mass}

An ideal mass layer (rigid and sufficiently thin) as surface loading on the resonator fulfills the Sauerbrey requirements. The additional mass layer synchronously oscillates with the resonator. Hence, an ideal mass loading causes no energy losses of the oscillation $\left(R_{L}=0\right)$, and any oscillation energy is stored as kinetic energy of the moving mass. This is reflected in the motional impedance of the loading being purely imaginary. The load impedance $\underline{Z}_{S}$ of a rigid mass is a function of frequency and density $\rho_{s}$ of the solid layer, and thus is purely inductive $\left(\underline{Z}_{m}^{1}=\mathrm{j} X_{L}=\mathrm{j} \omega L_{L} ;\right.$ Tab. $\left.3-6 \mathrm{~A}\right)$. Consequently, $\rho_{s}$ influences the series and parallel resonant frequencies, whereas it does not contribute to the magnitude of impedance minimum and maximum (cf. Tab. 3-5). This is in agreement with the Sauerbrey equation (Eq. (23)) that relates a deposition of mass with a negative frequency shift.

\section{Newtonian Liquid}

In the case of a liquid in contact with one resonator surface, both energy dissipation $\left(R_{L}\right)$ and energy storage $\left(L_{L}\right)$ of the oscillation can be observed. The liquid viscosity and density cause damping of the acoustic wave propagation. In cases of a Newtonian liquid of semi-infinite thickness (film thickness >> decay length of the acoustic wave), the motional impedance of the loading can be expressed by equal reactance and resistance parts (Tab. 3-6 B). The surface mechanical impedance $\underline{Z}_{s}$ is a function of the density-viscosity product of the liquid. Hence, any characteristic impedance parameter $\left(f_{|Z|_{\text {min }}}|Z|_{\text {min }}\right.$ $f_{s,} f_{|Z|_{\text {max }}}|Z|_{\text {max }}, f_{p}$, and $\varphi_{\text {max }}$ ) is altered, with respect to the unperturbed quartz (cf. Tab. 3-5). 


\section{Semi-infinite Viscoelastic Body}

The impedance contribution of a viscoelastic surface load (of semi-infinite dimensions) to the entire oscillation impedance can be described by its complex shear modulus $\underline{G}$ (Tab. $3-6$ C). ${ }^{[197,297]}$ For instance, viscoelastic damping occurs, when cells are seeded on the quartz resonator. A cell layer exhibits viscous properties, which are mainly due to the cytoplasm and the viscosity of the interfacial layer between cells and quartz surface. On the other hand, the lipid membranes and the cytoskeleton network add elastic properties to the cells. Aside from the complex mechanical properties of individual cells, consisting of the mechanical contributions of all cellular components, also mechanical attributes arising from next higher organizational structures of individual cells, i.e. 2-D monolayers or 3-D clusters, contribute to the total mechanical characteristics of cells. Hence, there is not yet a suitable mechanical model available for cells. ${ }^{[297,298]}$ However, the viscoelastic properties of a cell layer, and their changes, can be measured sensitively by means of impedance readings of the quartz oscillation and subsequent impedance analysis. This delivers information about the mechanical interaction of cells with the growth substrate during the processes of cell attachment and spreading, as well as information about subsequent cytomechanical alterations. Hence, the OCM can be utilized as a whole-cell biosensor for detecting mechanical cell responses towards specific physical, chemical, or biochemical external and internal stimuli.

Tab. 3-6 Three basic loading conditions on a TSM resonator: (A) Rigid mass, (B) Newtonian liquid, and (C) Semi-infinite viscoelastic layer and their respective BVD equivalent circuit extensions. Expressions for the surface mechanical impedance $\underline{Z}_{s}$, the resistance $\left(R_{L}\right)$, and reactance $\left(X_{L}\right)$ of the complex motional impedance of the load $\left(\underline{Z}_{m}^{1}\right)$ are listed. Thereby, $\rho_{S}$ is the density of the solid layer, $\rho_{l}$ is the density and $\eta_{l}$ the viscosity of the liquid loading, $\rho_{v}$ is the density of the viscoelastic film, and $\underline{G}$ is the complex shear modulus of the viscoelastic layer $(\underline{G}=$ $G^{\prime}+\mathrm{j} G^{\prime \prime}$ and $|G|=\sqrt{\left(G^{\prime}\right)^{2}+\left(G^{\prime \prime}\right)^{2}}$, where $G^{\prime}$ is the storage modulus and $G^{\prime \prime}$ is the loss modulus). ${ }^{[197,297]}$

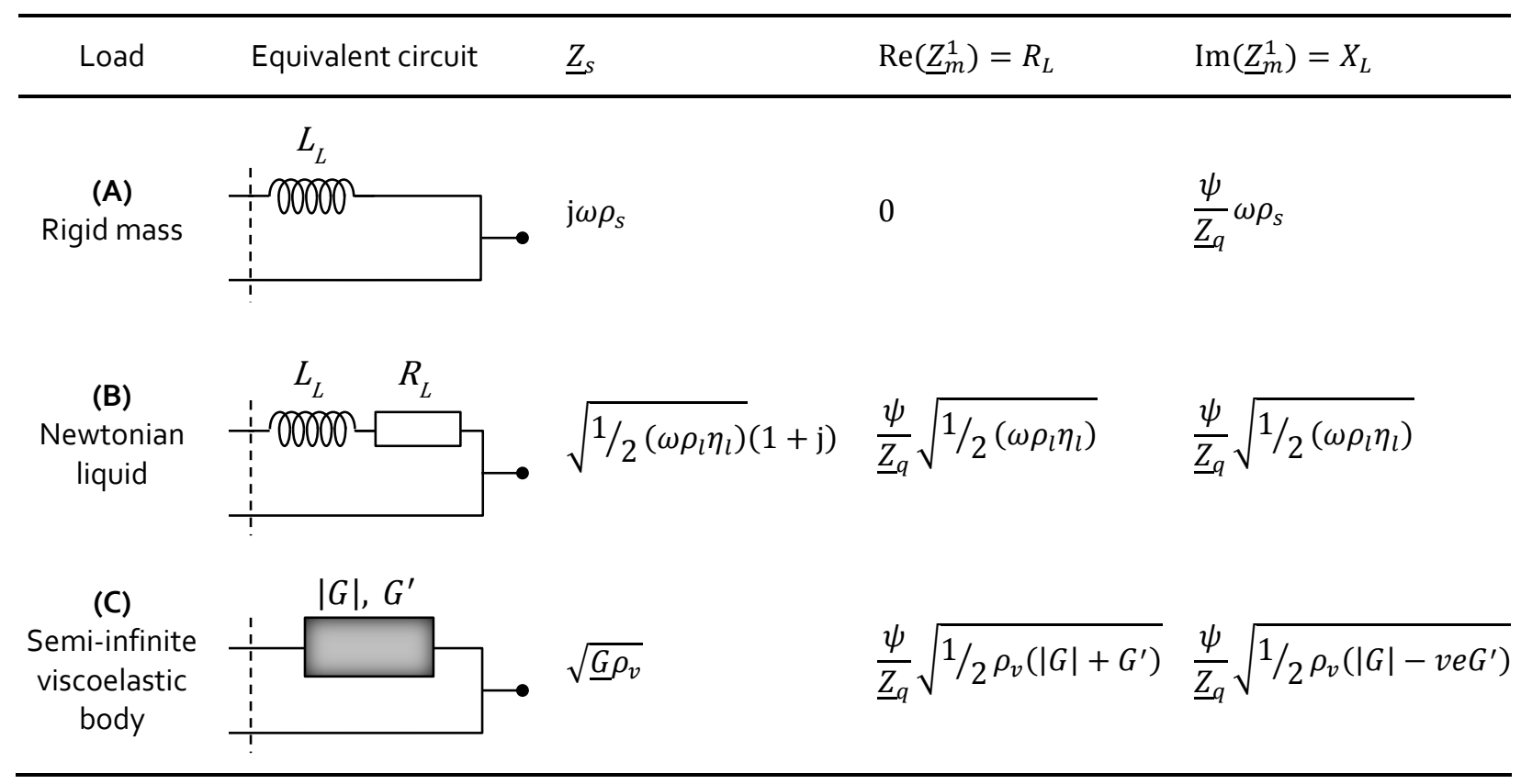




\subsubsection{Experimental}

\section{Quartz Resonator Layout}

In this work, quartz resonators with different electrode layouts were used. The classical 1-Electrode quartz (1EIO) layout with a circular gold film electrode evaporated on both sides of the quartz disk is shown in Fig. 3-10 A. On the basis of this classical layout, a novel electrode layout with two circular gold film electrodes on both quartz surfaces ( $2 \mathrm{EIO}$ ) has been developed in this work (Fig. 3-10 B). The $2 \mathrm{EIO}$ layout is used for $\mathrm{OCM}$ readings with two sensor spots and, after further modifications, for combined QCM-ECIS measurements. The sensor layout and the implementation of OCM-ECIS measurements with the $2 \mathrm{ElOs}$ is described below in section 3.4.4 (p. 56 ff.). The $5 \mathrm{MHz}$ AT-cut quartz resonators were purchased from KVG Quartz Crystal Technology GmbH (Neckarbischofsheim, Germany). The disks had a diameter of $14 \mathrm{~mm}$ and were produced with either one circular gold film electrode $(\varnothing=6 \mathrm{~mm} ; \mathrm{Fig}$. 3$10 \mathrm{~A})$ or with two electrodes $(\varnothing=3.5 \mathrm{~mm}$; Fig. 3-10 B) on either side, and with electrical supply lines from the disk edge to the electrodes in the center.

A

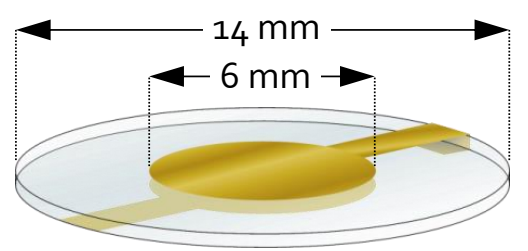

B

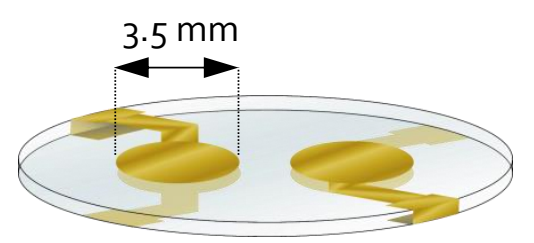

Fig. 3-10 Different electrode layouts were established on $5 \mathrm{MHz}$ AT-cut quartz disks $(d=330 \mu \mathrm{m})$. On the resonators either one $(\mathbf{A} ; 1 \mathrm{ElO})$ or two $(\mathbf{B} ; 2 \mathrm{ElQ})$ circular gold film electrode/s was/were evaporated on both quartz surfaces.

\section{Quartz Cleaning}

Prior to an experiment, the quartz disk was cleaned by a three-step standard cleaning protocol. Firstly, it was treated with a $5 \%$ (v/v) "elma clean 10" solution (Elma Hans Schmidbauer GmbH \& Co KG; Singen, Germany) in an ultrasonic bath at $70^{\circ} \mathrm{C}$ for 30 min. Afterwards, the resonator was washed $3-5$ times with deionized water and subsequently sonicated in deionized water at $70^{\circ} \mathrm{C}$ for $30 \mathrm{~min}$. This step was repeated once again, for completely removing residues of quartz loading and cleaning solution from the surfaces. Finally, the resonator was air dried over night, or dried in an incubator for $\sim 1 \mathrm{~h}$ at $50^{\circ} \mathrm{C}$. The cleaned quartzes were stored under dry and dust-free conditions until usage.

The gold film at the edge of the disk, needed for contacting the top electrode from the lower side of the resonator, was often damaged in the course of the cleaning process. This connection was renewed by connecting the electrical supply line on the top side and the contact pad on the lower side of the quartz disk with a drop of silver conductive paste (Busch; Viernheim, Germany). The conductive paste was either dried over night at RT or for $1 \mathrm{~h}$ at $50^{\circ} \mathrm{C}$. Thereby, the conductivity and consequently the oscillation excitation of the resonator could be preserved. 


\section{Spin Coating and Photolithography on Quartz Disks}

Structuring of the electrodes on a $2 \mathrm{EIO}$ sensor (Fig. 3-10 B) was necessary, in order to systematically load one surface electrode of a $2 \mathrm{EIO}$ sensor with a rigid mass layer and to realize and optimize ECIS-mode measurements on $2 \mathrm{ElO}$ substrates (cf. section 3.4.4, p. 56 ff.). This was achieved by spin a coating photopolymer (PhoP) on one side of the resonator and subsequently removing defined areas of photoresist by means of photolithography.

Coating was performed using a self-made spin coater (Dr. F. Höhn; Institute of Biochemistry, University of Münster, Germany). Prior to coating, the cleaned quartz substrates were sequentially washed with acetone and isopropyl alcohol, in order to remove organic impurities as well as dust from the surface. The substrates were subsequently heated to $120^{\circ} \mathrm{C}$ for $30 \mathrm{~min}$, to remove solvents residues and any surface water. Spin coating and photolithography of the photoresist were performed step-wise according to the schematic illustration in Fig. 3-11.

A
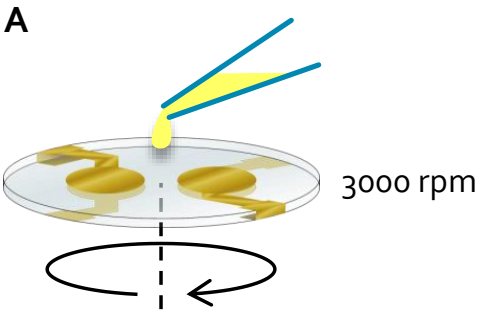

B

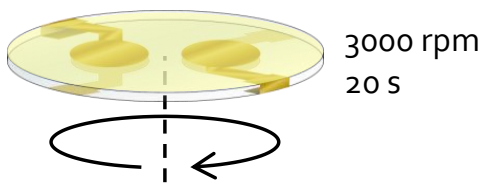

C

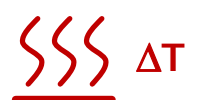

D

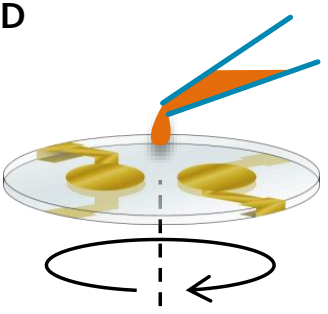

$\mathrm{E}$

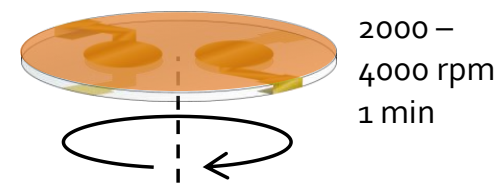

$F \quad \int S \int \Delta T$

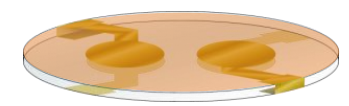

$2000-$ $4000 \mathrm{rpm}$
G
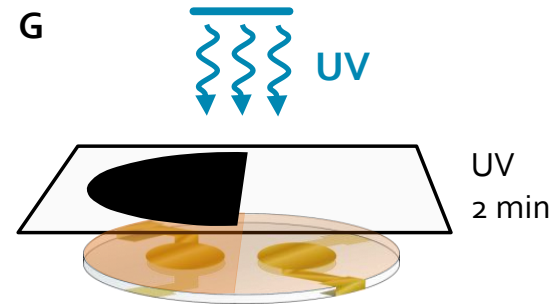

H

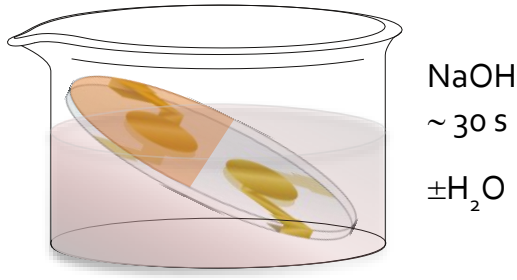

I

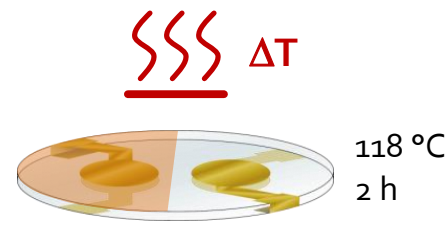

Fig. 3-11 Schematic illustration of the spin coating and photolithography processes on a $2 \mathrm{EIO}$ substrate. Firstly, the substrate was pre-coated with an adhesion promoter (A), (B), followed by its thermal activation (C). Then, a positive photoresist was spin coated at defined rotational speed (D), (E). After a short soft-baking period (F), the photoresist was illuminated with UV light through a specific mask (G). At irradiated areas the photoresist was destabilized, which was subsequently removed/ developed by a sodium hydroxide solution $(\mathrm{H})$. After rinsing with deionized water, the photoresist structure is stabilized in a final hard-bake (I).

In a first spin coating process, "TI Prime" (Microchemicals; Ulm, Germany) was applied, which served as adhesion promoter for the PhoP. The quartz disk was placed on the spin coating holder by adhesive plasticine. A drop of the promoter was carefully placed in the middle of the rotating substrate at ambient temperature, and at a spin velocity of $3000 \mathrm{rpm} .20 \mathrm{~s}$ of spin coating were sufficient for obtaining a uniformly thin layer of the adhesion promoter. The substrate was placed in the oven at $130^{\circ} \mathrm{C}$ for $10 \mathrm{~min}$, in order to activate the promoter. By this step, the primarily hydrophilic quartz surface was converted into a more hydrophobic surface, which then promoted a much more uniform spreading and adhesion of 
the spin coated hydrophobic PhoP. The Photoresist "AZ ${ }^{\circledR}$ ECl 3027" (Microchemicals; Ulm, Germany) was spin coated at a defined velocity and with a spinning duration of $1 \mathrm{~min}$. The applied rotation speed was adjusted to values between $2000 \mathrm{rpm}$ and $4000 \mathrm{rpm}$, depended on the required thickness of the film. Standard structuring of the $2 \mathrm{EIO}$ surfaces was performed at a spin velocity of $3000 \mathrm{rpm}$. After applying the photoresist, the substrate was placed in the oven for soft baking at $100^{\circ} \mathrm{C}$ for $30 \mathrm{~min}$. The coated substrate was then illuminated through a specific mask with UV-light for $2 \mathrm{~min}$. The photolithography masks were drawn with "CorelDRAW ${ }^{\circledR}$ Graphics Suite X6" software (Corel Corporation; Ottawa, Ontario, Canada) and ink-jet printed on a transparent slide in black at maximum saturation (masks are shown in

Fig. 3-12).

A

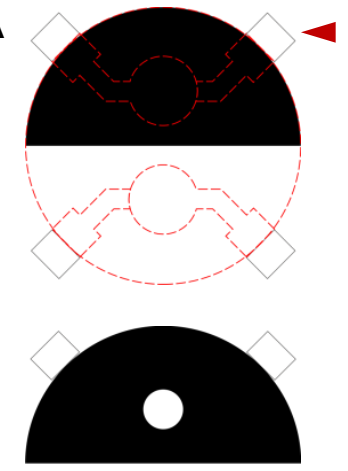

B
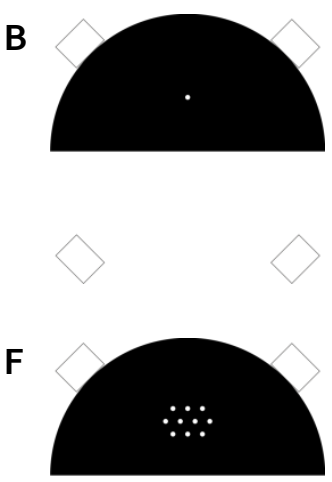

$\mathrm{C}$
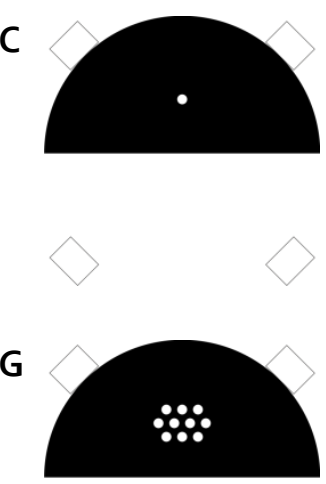

D

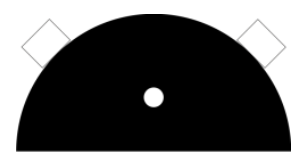

$\mathrm{H}$

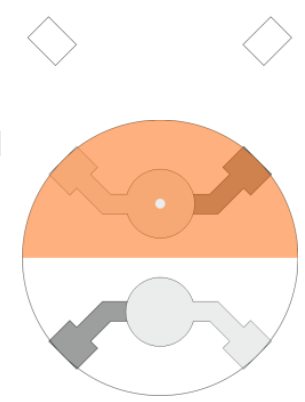

Fig. 3-12 Photolithography masks (scale of 2:1) for structuring 2ElOs with a positive photoresist. Illuminated areas of PhoP are destabilized; opaque areas represent the remaining PhoP after photolithography. (A) Mask for completely covering one surface electrode (half of the disk surface) with photopolymer. The dashed red line (not part of the mask) indicates the positioning of the mask on a $2 \mathrm{EIO}$. Rectangular structures at the borders of the mask (indicated with an arrow) helped for an exact and reproducible positioning. (B) - (G) Photolithography masks to establish different working electrode layouts on quartz resonators for ECIS mode measurements. Hole diameters were adjusted to (B) $250 \mu \mathrm{m}$, (C) $500 \mu \mathrm{m}$, (D) $1 \mathrm{~mm}$, (E) $2 \mathrm{~mm}$, (F) $10 \times 250 \mu \mathrm{m}$, and (G) $10 \times 500 \mu \mathrm{m}$. (H) Exemplary, final PhoP structure (orange) on a $2 \mathrm{ElO}$, after spin coating and subsequent photolithography by applying mask $C$.

As the applied photopolymer was a positive photoresist, the opaque masks represented the remaining photopolymer structure on the substrate after the photolithography process. The UV-illuminated photopolymer was destabilized and removed by rinsing the substrate with $\mathrm{NaOH}$ solution $(7 \mathrm{~g} / \mathrm{L}$ in deionized $\mathrm{H}_{2} \mathrm{O}$ ) for $20-30 \mathrm{~s}$. After that, the substrates with the final photopolymer structure were rinsed twice with deionized water, for completely removing the base with the dissolved photopolymer. In a final hard bake, the substrate was tempered at $118^{\circ} \mathrm{C}$ for $2 \mathrm{~h}$, in order to achieve maximal thermal, physical, and chemical stability of the coating.

Quartz disks with photopolymer structure were stored dry and protected from light and dust until usage. In the forerun of an experiment under liquid, like cell attachment studies in culture medium, each sensor with a coated photopolymer layer was immersed in serum-free medium (SFM) for at least $20 \mathrm{~h}$. This was done in order to uncouple equilibration effects from the cell signals in the course of an experiment. 


\section{Hardware - Measurement Chamber}

For QCM experiments, the quartz disk was mounted between two components of the home-made Teflon measurement chamber, as illustrated on the right hand side of Fig. 3-13. The lower component comprises two/ four gold-pated spring contacts to make contact to the impedance analyzer with the ${ }_{1} \mathrm{EIQ} / 2 \mathrm{ElQ}$ resonator from the lower side. An illustration of the modified chamber to contact $2 \mathrm{ElOs}$ for OCM-ECIS measurements is shown in Fig. 3-18 (p. 58). The second Teflon element was a cylindrical chamber that was mounted from the top on the resonator and which served as reservoir for OCM measurements under liquid. O-rings were sandwiched between the quartz disk and each Teflon component (Fig. 3-13). These served for a tensionless fixture of the resonator between the Teflon components on the one hand, and for sealing the reservoir on the other hand. Mounted in the measurement chamber, the quartz possessed an accessible cell growth area of $\sim 1.13 \mathrm{~cm}^{2}$.

In the course of OCM measurements under liquid, an additional Teflon stamp was used to seal the cylindrical chamber. This was used to minimize the evaporation of the liquid from the chamber, since continuous changes in liquid height cause periodical interference in the measured OCM signal by longitudinal waves that are reflected at the liquid-air interface. ${ }^{[299]}$ Moreover, the sealing with a stamp ensured sterility to a certain extent within the reservoir. However, the stamp had small holes in order to guarantee air exchange between the measurement chamber, i.e. the cell culture medium and the cells on the quartz, and the ambient atmosphere. The whole $\mathrm{OCM}$ measurement chamber was placed into an incubator with temperature $\left(37^{\circ} \mathrm{C}\right.$ ) and $\mathrm{CO}_{2}$ control ( $5 \%(\mathrm{v} / \mathrm{v})$ in air) (Revco, Thermo Fisher Scientific; Waltham, MA, USA) to meet cell culture conditions. Cables for contacting the quartz were led from inside the incubator through the rubber seal of the incubator door to the outside and were connected to a computer-controlled "1260A Impedance/ Gain-phase Analyzer" (Solartron Analytical; Farnborough, UK). The IA featured an operating frequency range from $10 \mu \mathrm{Hz}$ to $32 \mathrm{MHz}$, with a frequency resolution of $0.015 \mathrm{ppm}, 0.1 \%$ magnitude and 0.1 degree accuracy, and $0.001 \mathrm{~dB}$ and 0.01 degree resolution. ${ }^{[300]}$
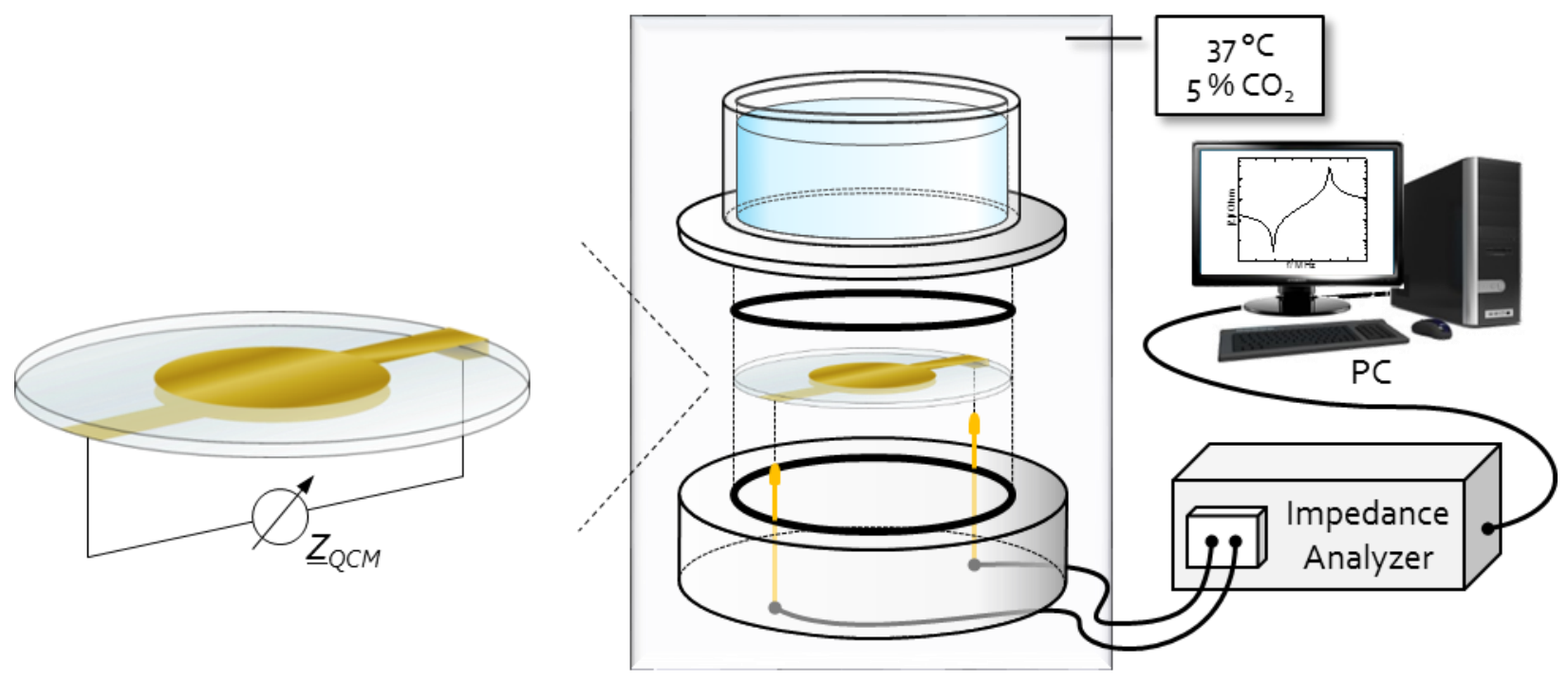

Fig. 3-13 Experimental setup for measuring the oscillation impedance $\underline{Z}_{O C M}$ of a conventional ${ }_{1} E I O$. The quartz disk is mounted in a home-made Teflon measurement chamber, for electrical contacting of the electrodes, and in order to have a liquid vessel on top of the resonator. The measurement chamber was placed in an incubator for cell experiments $\left(37^{\circ} \mathrm{C}, 5 \% \mathrm{CO}_{2}\right)$, with the wiring brought to the outside to connect it with the IA and the PC. 


\section{Software - OCM Measurements}

The impedance analyzer was controlled by a LabVIEW based software (National Instruments ${ }^{\mathrm{TM}}$; Austin, Texas, USA), programmed by J. Wegener (Institute of Analytical Chemistry, Chemo- and Biosensors, University of Regensburg, Germany). A sinusoidal voltage of $150 \mathrm{mV}$ was applied to the electrodes on the quartz surfaces and the resulting complex impedance (phase and magnitude) was measured by the IA. The data was read-out and stored by a personal computer. This was done for $150-200$ equidistant frequencies near the fundamental resonance. For the standard range of $4.97-5.04 \mathrm{MHz}$, this means a frequency resolution of $466-300 \mathrm{~Hz}$. Impedance spectroscopic scanning of the oscillation was performed in time-resolved manner. The scanned frequency range and the number of frequencies within this range were adjusted for each experiment to meet time- or frequency-resolution of the spectra as required.

\section{Experimental Implementation}

Before all QCM experiments, an impedance spectrum of the mounted quartz in air was recorded and the oscillation parameters of an unperturbed quartz disk were obtained. Moreover, it was used to check the quartz for contacting problems and for its unperturbed oscillation, prior to starting an experiment. The parameters of the lossless oscillation were also needed for modeling the oscillation impedance, i.e. calculating the contributions of the loading to the entire acoustic impedance.

Prior to cell experiments the whole OCM chamber was sterilized by plasma treatment. In this case, the Teflon measurement chamber, comprising the mounted quartz and the stamp for closing the chamber, was treated with argon plasma in a plasma cleaner (Harrick Plasma; Ithaca, New York, USA) for 1 min and at highest energy level. Immediately after plasma sterilization, the measurement chamber was sealed with the stamp and covered with aluminum foil, in order to avoid contamination. The cell suspension was added to the QCM chamber in the laminar flow under sterile conditions. Afterwards, the measurement chamber was placed in an incubator $\left(37^{\circ} \mathrm{C}, 5 \%(\mathrm{v} / \mathrm{v}) \mathrm{CO}_{2}\right)$, the electrodes were contacted to the IA, and the $\mathrm{OCM}$ recording was started. Experimental variations and the details of cellular experiments are described in the results sections.

\section{Data Processing}

The characteristic oscillation parameters $f_{|Z|_{\text {min }}}$ and $|Z|_{\text {min }}$ were extracted by fitting the impedance spectra with a spline function in the resonance region. $|Z|_{\min }$ was plotted as function of time since it represents the OCM key parameter for detecting and monitoring viscoelastic changes at the sensor surface. The time courses were presented normalized to a certain reference data point pf the experiment. On the one hand, this facilitated to average measurements with different initial values and, on the other hand, this also enabled the graphic comparison of time courses obtained from different experiments. Potential differences in the extent or/ and in the kinetics of $|Z|_{\min }$ changes were visualized in this way.

Experiments were usually repeated several times in order to generate data packages for the purpose of averaging. This served as basis to resolve and discriminate random signal fluctuations and distinct signal changes of the monitored cell population and, thus, to confidently identify significant and reproducible viscoelastic effects related to the cell layer. 


\section{Sensor Regeneration}

Quartz disks were typically reused several times for experiments. The quartz disks were regenerated by multiple cleaning steps in order to re-establish a pure sensor surfaces and, thus, to ensure consistently good oscillation properties. At first, any organic adsorption on the quartz disk was oxidized by washing it in a $3: 1$ mixture of conc. $\mathrm{H}_{2} \mathrm{SO}_{4}$ and $30 \%(\mathrm{v} / \mathrm{v}) \mathrm{H}_{2} \mathrm{O}_{2}$ for about $30 \mathrm{~min}$. After several washing steps with deionized water, the resonator was further cleaned before re-usage, according to the procedures described above in subsection Quartz Cleaning (p. 45).

\subsection{Electric Cell-Substrate Impedance Sensing $(\mathrm{ECIS})$}

Electric Cell-Substrate Impedance Sensing (ECIS) is a non-invasive and label-free measurement technique that was presented by Giaever and Kees in 1984. As "morphological biosensor for mammalian cells" ${ }^{[87]}$ this technique has been widely used in a variety of cell-based applications and has been further developed in the past 30 years. ${ }^{[88,89,301,302]}$ It has been successfully applied as very sensitive technique to monitor barrier functionality changes of cell layers towards different kinds of stimuli. ECIS is already a well-established means for the fast and sensitive testing of cytotoxic effects as well, which is due to its sensitivity for cell morphological changes. . $4,17,109-111,113-117,303-305]$ Moreover, the ECIS principle has proven to be a usefull tool for monitoring cellular motility, referred to as micromotion, by time-resolved readings of impedance fluctuations. ${ }^{[96,97,264,306]}$ Besides theses various applications of cellular sensing, the ECIS electrodes have also been utilized as actuators for the directed manipulation of cell layers by electrical fields. This can be basically categorized into the non-destructive and reversible short-term permeabilization and the irreversible damaging and wounding of the cells sitting on the ECIS working electrode(s), depending on the chosen values of the electric pulse parameters, frequency, duration, and magnitude. By applying the principle mentioned first, a short-term opening of the cell mambranes enabled the diffusion-driven transfer of molecules and xenobiotics that actually are cell-impermeant directly into the cytoplasm. The cell response towards the pulse itself or to the introduction of substances were then monitored by ECIS. ${ }^{[7,119,120]}$ Following the second principle, by applying voltage pulses of high amplitude and long duration (few seconds), the cell layer on the working electrode was successfully wounded. The thus generated, well-defined wound allowed for various wound healing assays that were monitored by ECIS. ${ }^{[7,99,303,307-313]}$ Further developments of ECIS aimed for combining it with other labelfree sensor techniques like SPR (surface plasmon resonance) or OCM. ${ }^{[11,22,236,267,274]}$ The following sections will address the ECIS principle and the impedance properties of cell-covered electrodes. Finally, the integration of ECIS-like measurements in the OCM setup provided simultaneous sensing of both viscoelastic and electrical properties of adherent cells in one experimental setup.

\subsubsection{Basic Principles of the ECIS Technique}

Like OCM, ECIS is an impedimetric measurement technique. A schematic drawing of the ECIS principle is shown in Fig. 3-14. Cells are seeded on two coplanar gold electrodes that serve as growth substrate for the cells. The buffer or cell culture medium on top of the cells conductively connects both electrodes. A weak and non-invasive AC voltage is applied between the electrodes, and the resulting AC current flow is measured. Thus, one obtains the complex impedance of the system. 


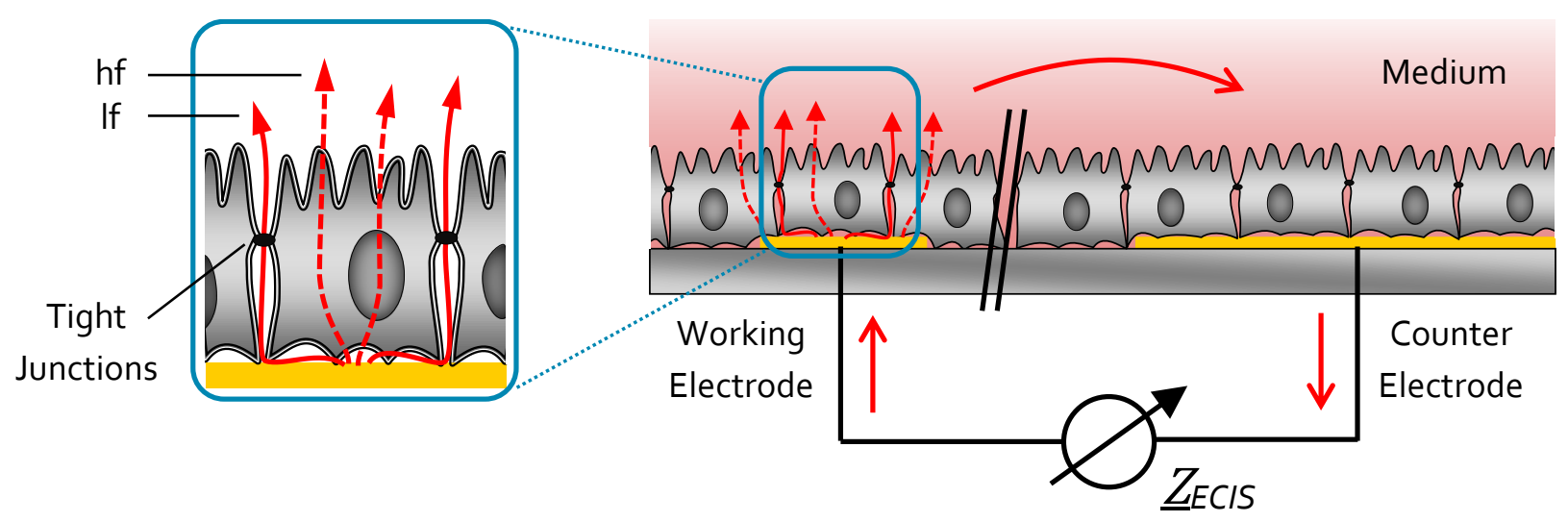

Fig. 3-14 Schematic drawing of the ECIS measurement principle. Two electrodes (a small working electrode, and a >100-fold bigger counter electrode) are used as culture substrate for cells. Cells impede current flow between the electrodes. High frequency (hf) current passes the cells on transcellular pathways, whereas low frequency (If) current flows on paracellular pathways beneath and between the cells and through tight junctions (zoomed-in detail in the drawing).

The impedance is measured spectroscopically in a wide frequency range (cf. 3.2 Impedance Spectroscopy, p. $22 \mathrm{ff}$.). In the cell-free situation, the entire impedance signal is determined by the interface impedance of the electrode at low frequencies and by the bulk resistance of the medium in the high frequency range. If cells cover the electrodes, the current cannot flow directly and faces extra impedance contributions, since cells behave like insulating particles. This is reflected in an increase of the system's impedance with the gradual coverage of the electrodes (Fig. 3-14). As the applied potential between the two coplanar electrodes is in the range of $10 \mathrm{mV}, \mathrm{ECIS}$ is non-invasive. By means of ECIS one can monitor the processes of cell adhesion, spreading and proliferation as they all affect the impedance of the system. Moreover, ECIS can also be used to study established confluent cell layers without the need to label the cells. Changes in the cell shape, membrane capacity, cell-cell as well as cell-substrate contacts give rise to changing current pathways and impedance, respectively. Additionally, cellular motility is expressed by the dynamic formation and release of cell-cell and cell-substrate contacts. This cellular micromotion is reflected in impedance fluctuations of the cell layer, which can be measured by performing rapid impedance monitoring at a single frequency.

The way current flows through the cell layer is determined by the frequency of the applied AC voltage (magnified scheme in Fig. 3-14). The impedance contribution and the frequency dependence of current pathways, however, are different for each cell type and has to be individually determined. At low frequencies the current is forced to flow on paracellular pathways through the cell layer. This means the current flows under the insulating cell layer and through the clefts between neighboring cells (indicated in Fig. 3-14 by arrows with a solid line). Thus, the low frequency impedance is dominated by impedance contributions arising from the small cleft between the electrode and the cells as well as from the electrical properties of the cell-cell contacts. In the high frequency region the current flows transcellularly through the cell and it has to cross the lower (basal) and the upper (apical) membrane of the (epithelial) cell layer (indicated in Fig. 3-14 by arrows with a dashed line). At elevated frequencies the lipid bilayer behaves electrically as a capacitor, which means that the entire transcellular impedance can be electrically described by a series combination of two capacitors.

The ECIS electrodes are of different size, in order to define a small working electrode (WE) and a ( 500-fold) bigger counter electrode (CE). By this, the overall impedance of the system is not dominated by the bulk resistance of the electrolyte between the electrodes and the counterelectrode itself. The 
bottleneck of current flow is now the interface impedance between the WE and the electrolyte. The latter is affected by the electrical properties of single cells or a confluent cell layer. The sensitivity of impedance measurements can be tuned by changing the size of the WE. Higher impedance sensitivities are achieved with a smaller WE size. However, with decreasing electrode area the electrical properties of only few cells of the whole cell layer are measured. This is a drawback, because the measured signal may not be representative for the whole cell layer. Hence, the electrode sizes have to be adjusted to the specific requirements of an ECIS experiment.

\subsubsection{Impedance Properties of (Cell Covered) ECIS Electrodes}

When immersing ECIS electrodes in an electrolyte and measuring the impedance of the cell-free system over a broad frequency range from $1 \mathrm{~Hz}$ to $1 \mathrm{MHz}$, one obtains characteristic curve shapes for the impedance magnitude $|Z|(f)$ and the phase shift angle $\varphi(f)$. Simulated impedance spectra for ECIS electrodes in contact with a physiological buffer are shown in Fig. 3-15 (--). Looking at the phase spectrum of the cell-free ECIS electrodes, it can be seen that its characteristics are similar to that of a simple RC series circuit, which has been discussed above (subsection 3.2.3, p. 28), and whose impedance spectrum is shown in Tab. $3-4 \mathrm{~A}$ (p. 29). In the high frequency range $\left(f>10^{4} \mathrm{~Hz}\right)$ the phase angle converges to zero. Hence, the systems impedance is solely resistive and is dominated by the bulk resistance of the electrolyte, $R_{\text {bulk }}$. This can also be seen in the impedance amplitude spectrum of the cell-free system (Fig. 3-15 A, ---), where the impedance magnitude exhibits a frequency independent value, i.e. only resistance contributions, at higher frequencies. In contrast, the lower frequency range $\left(f<10^{2} \mathrm{~Hz}\right)$ is almost exclusively represented by a capacitor, as the phase angle converges to a constant value close to $-90^{\circ}$ and the impedance magnitude exhibits the characteristic frequency dependence of a capacitor. This capacitive impedance originates from the electrical double layer properties of the WE. However, non-ideal capacitive behavior of the WE can be recognized in the phase shift angle, as the phase is not exactly $-90^{\circ}$ but is shifted to slightly higher values. This is due to roughness and inhomogeneity of the electrode surface, which give small but significant resistive contributions to the almost entirely capacitive impedance of the working electrode. Hence, the impedance of the electrode is better represented by a CPE, which considers these effects.

With cells covering the electrodes, the impedance situation is different. The impact of a confluent cell layer on the impedance of ECIS electrodes is simulated in Fig. 3-15 (-). The impedance is neither influenced in the very high nor in the very low frequency region by the cell layer. The spectra with cells superpose with the spectra of the cell-free electrodes for $f\left\langle 10^{1} \mathrm{~Hz}\right.$ and $f>10^{5} \mathrm{~Hz}$. However, impedance contributions due to the cells arise in the frequency region in between. In the phase spectra the cells induce a phase shift maximum and minimum. Coming from higher frequencies, there is an earlier decrease in the phase for cell-covered electrodes in comparison to cell-free electrodes. This is due to additional capacitive contributions to the system, which originate from the lower and the upper lipid bilayer of the cell membrane. At lower frequencies the phase rises to a maximum value and subsequently decreases and approximates to the value of the cell-free electrode. This phase recovery is caused by the increasing resistive properties of the cell layer in the lower frequency band. The resistive contribution is maximal at the frequency of the maximal phase shift. In the impedance magnitude, this cellular resistance contribution is reflected in an almost frequency independent plateau. This is related to current pathways beneath and around the insulating cell bodies, in particular from current flow through cell-cell contacts. The impedance curve shape of the cell-covered electrodes in the frequency range of 
$10^{1}-10^{5} \mathrm{~Hz}$ can be compared with the electrical properties of an $R C$ parallel circuit $(R \| C)$, which were previously discussed (cf. Tab. 3-4, p. 29).

It has to be mentioned that the simulated impedance characteristics of the cell-covered electrode in Fig. 3-15 are not only determined by a capacitance $C_{m}$ in parallel to a resistance $R_{b}$ (reflecting the capacitance of the cell membrane and the resistance of cell-cell contacts, respectively), but also by a third parameter, $\alpha$, which reflects the frequency dependent impedance contribution of the subcellular cleft (Fig. 3-17; for details, see subsection 3.4.3). The further parameters of the used transfer function are the membrane resistance, $R_{m}$, and the parameters $n_{\mathrm{CPE}}$ and $A_{\mathrm{CPE}}$ of the empirical constant-phase element (Eq. (18)).
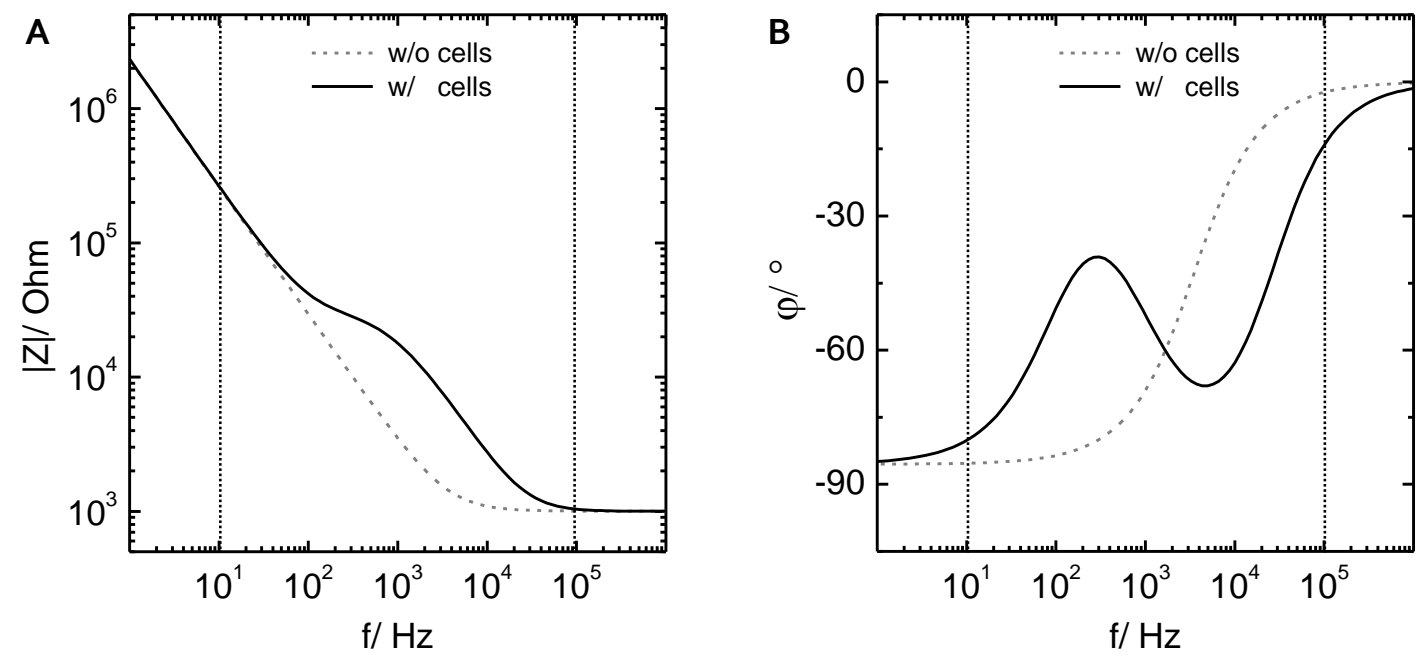

Fig. 3-15 Simulated impedance spectra (Bode plot) of the magnitude $|Z|(\mathbf{A})$ and the phase shift angle $\varphi$ (B) for ECIS electrodes in contact with an electrolyte. Spectra are shown for cell-free electrodes (w/o cells; ${ }^{---}$), and for electrodes covered with a confluent cell layer (w/ cells; - ). For simulation, the model proposed by Wegener and Hakvoort et al. (2000) ${ }^{[314]}$ was used, which is a slight modification of the original model of Giaever and Keese ${ }^{[96]}$ (cf. 3.4.3). Following parameter values were applied: $R_{b}=90 \Omega \cdot \mathrm{cm}^{2}, C_{m}=3 \mu \mathrm{F} \cdot \mathrm{cm}^{-2}, \alpha=16 \Omega^{1 / 2} \cdot \mathrm{cm}, R_{m}=2000 \Omega, R_{\text {bulk }}=1000 \Omega$, $n_{\mathrm{CPE}}=0.95$, and $A_{\mathrm{CPE}}=1.5 \cdot 10^{-5} \mathrm{~F} \cdot \mathrm{s}^{n-1} \cdot \mathrm{cm}^{-2}$, for an electrode area of $5 \cdot 10^{-3} \mathrm{~cm}^{2}$.

The spectra of the complex impedance shown in Fig. 3-15 can be split into real part and imaginary contributions, according to Eq. (7) (p. 24). The real part thereby represents an equivalent resistance and the imaginary part represents the equivalent capacitance of the system. Thus, the impedance spectra (in Fig. 3-16 A) can be broken up in the two individual spectra of the resistance (Fig. 3-16 A) and capacitance (Fig. 3-16 B).

The separation of the complex impedance into equivalent resistance (real part of $\underline{Z}$ ) and equivalent capacitance (imaginary part of $\underline{Z}$ ) allows for a more detailed and analytical view on the electrical properties of the cell layer. The resistance and capacitance spectra in Fig. 3-16 can be roughly separated into four frequency ranges, in which different impedance contributions dominate over the others. As already mentioned above, in the low frequency region $\left(f<10^{1} \mathrm{~Hz}\right.$, range I in Fig. $\left.3^{-16}\right)$ the impedance arises mainly from the double-layer capacitance (CPE) of the electrode-electrolyte interface, whereas the bulk resistance of the electrolyte dominates the impedance in the high frequency range $\left(f>10^{5} \mathrm{~Hz}\right.$, region IV). Two regions can be defined in between. The first is centered around $10^{2} \mathrm{~Hz}$ (region II), the second around $10^{4} \mathrm{~Hz}$ (region III), whereas the resistance is very sensitive to the cell layer in the former, 
the capacitance is in the latter. The low-frequency resistance is mainly provided by paracellular pathways of the current and thus can be used for following changes in the barrier resistance of cell-cell contacts as well as of the gap resistance between the basal cell membrane and the electrode surface (cf. Fig. 3-14). Independent of these properties the transcellular current pathways are reflected in the capacitance values at high frequencies. Hence, it is measure for the degree of membrane-coverage on the electrode and thus the hf capacitance can be applied to monitor the intactness of the cell membrane, cell attachment, and cell detachment, respectively.
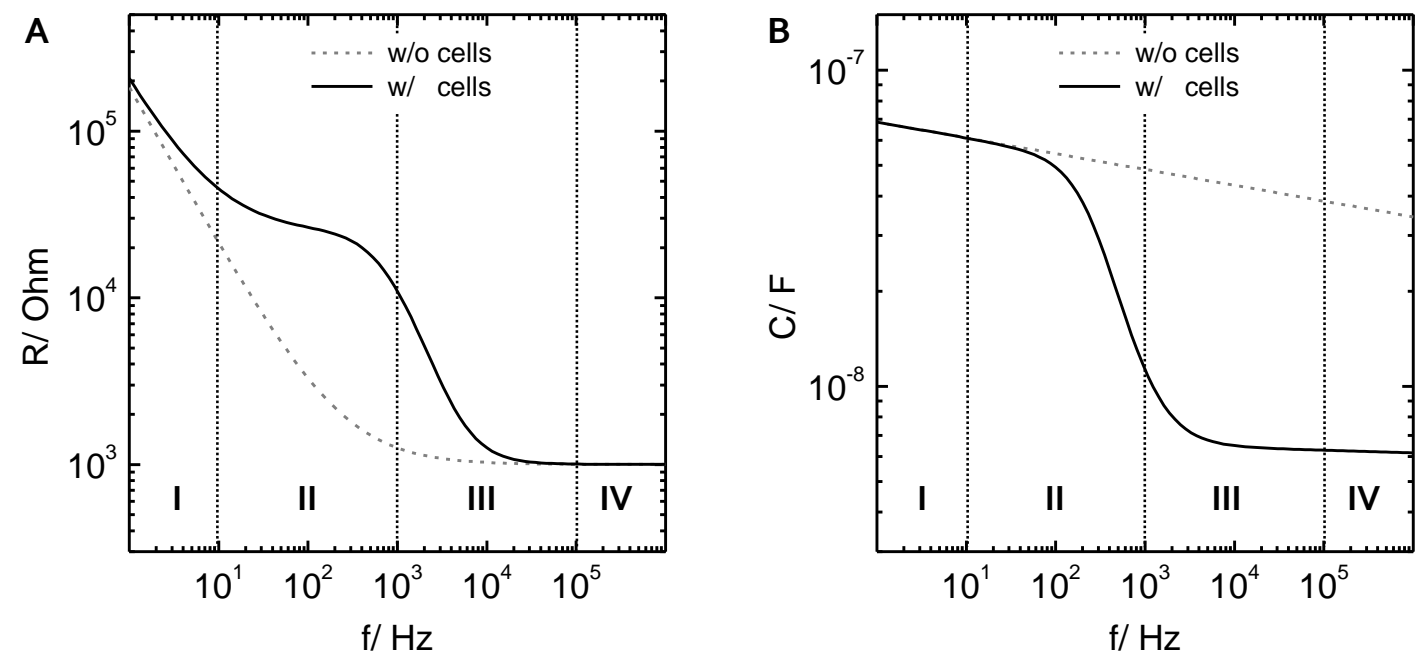

Fig. 3-16 Spectra of the equivalent resistance $(A)$ and the equivalent capacitance $(B)$ were extracted from the impedance spectra shown in Fig. 3-15. Thereby, $R$ represents the real part and $C$ ic calculated from the imaginary part of the complex impedance $\underline{Z}$. Spectra are shown for the cell-free (---) and the cell-covered (-) situation on ECIS electrodes. For simulation, the model proposed by Wegener and Hakvoort et al. (2000) ${ }^{[314]}$ was used, which is a slight modification of the original model of Giaever and Keese ${ }^{[96]}$ (cf. 3.4.3). Following parameter values were applied: $R_{b}=90 \Omega \cdot \mathrm{cm}^{2}, C_{m}=3 \mu \mathrm{F} \cdot \mathrm{cm}^{-2}, \alpha=16 \Omega^{1 / 2} \cdot \mathrm{cm}, R_{m}=2000 \Omega, R_{b u l k}=1000 \Omega$, $n_{\mathrm{CPE}}=0.95$, and $A_{\mathrm{CPE}}=1.5 \cdot 10^{-5} \mathrm{~F} \cdot \mathrm{s}^{n-1} \cdot \mathrm{cm}^{-2}$, for an electrode area of $5 \cdot 10^{-3} \mathrm{~cm}^{2}$.

By extracting the equivalent resistance and the equivalent capacitance from the ECIS readings, impedance spectroscopy provides a basis for sensing the morphological properties of a cell layer and allows a quantitative evaluation of the experimental data in terms of cell morphology. By monitoring the complex impedance in the relevant frequency regions, one can follow the general resistance and capacitance contributions of a cell layer in the course of an assay. This may be the capacitance drop in the course of cell adhesion and spreading, the increase in resistance upon formation of cell-cell contacts, or a capacitance increase due to rounding of cells and detachment from the surface when they die. The latter effect can be used for evaluating the cytotoxicity of certain substances or stimuli to the cell layer. If there is no need for a structural resolution of the toxic process, the complex impedance and the equivalent resistance and capacitance, respectively, is usually sufficient for a "yes or no" statement of cytotoxicity. The impedance raw data thus provides enough information for a general discussion of the system. This is sufficient in many assay formats.

However, if a detailed analysis of the biological origin of impedance contributions is of interest, modelling of the integral impedance data is necessary. A suitable electrical model for the impedance of a cellcovered electrode is addressed in the following section. 


\subsubsection{Modeling the Impedance of the Cell-Electrode Interface}

As mentioned above, impedance spectra provide the integral electrical properties of the entire system. Changes of the total impedance in a complex system therefore are difficult to interpret. More detailed information can be extracted from the recorded impedance spectra by subsequently fitting the impedance data with a transfer function of an appropriate electrical model.

Such an electrical equivalent model for a confluent cell layer on electrodes was introduced by Giaever and Keese in 1991. ${ }^{[96]}$ The model takes structural elements of a cell layer and properties of the cellelectrode interface into account. The similarity of the impedance spectrum of a cell-free electrode and the impedance of a RC series circuit was already discussed in the previous section. Thus, in a first approximation an R-C equivalent circuit adequately describes the interface of an electrode in contact with an electrolyte. However, the capacitance of the electrode is replaced by a CPE in the original model, according to the proposal of Wegener and Hakvoort et al. ${ }^{[314]}$, in order to take non-ideal behavior of the electrode into account (cf. p. 27). The CPE is connected in series with the bulk resistance $R_{\text {bulk }}$ of the electrolyte. In the case of a confluent cell monolayer on the electrode an additional impedance contribution $\underline{Z}_{C}$ due to the cell layer has to be considered. A schematic illustration of the model and the respective equivalent circuit is shown in Fig. 3-17. For this model, cells are approximated as uniform, cylindrical bodies of same size (radius $r_{C}$ ) and distance $(d)$ from the electrode surface. The impedance $\underline{Z}_{C}$ includes three characteristic parameters arising from the cell layer, which are the specific average membrane capacitance $C_{m}\left[\mu \mathrm{F} \cdot \mathrm{cm}^{-2}\right]$ of two membranes in series, the barrier resistance of the cell-cell junctions $R_{b}\left[\Omega \cdot \mathrm{cm}^{2}\right]$, and the parameter $\alpha\left[\Omega^{1 / 2} \cdot \mathrm{cm}\right]$ (Fig. 3-17). The latter describes the electrical properties of the cleft between the cells and the gold electrode. Hence, $\alpha$ is a function of the cellelectrode distance $d$, the average cell radius $r_{C}$, and the specific resistivity of the subcellular electrolyte $\rho_{\text {sub }}$, and is defined by:

$$
\alpha=r_{C} \cdot \sqrt{\frac{\rho_{s u b}}{d}}
$$

The total impedance of the cell-covered electrode $\underline{Z}_{\text {total }}$ is a function of the CPE impedance $\underline{Z}_{C P E}$ (cf. Eq. (18), p. 27), the membrane capacitance $C_{m}$ (expressed as cell membrane impedance $\underline{Z}_{m}$ ), the junctional or paracellular barrier resistance $R_{b}$, the parameter $\alpha$ (represented by the unitless term $r_{C} \gamma$, according to Eq. (51)), and of the bulk resistance $R_{\text {bulk }} \cdot \underline{Z}_{\text {total }}$ is given by:

$$
\underline{Z}_{\text {total }}=\left[\frac{1}{\underline{Z}_{C P E}}\left(\frac{\underline{Z}_{C P E}}{\underline{Z}_{C P E}+\underline{Z}_{m}}+\frac{\frac{\underline{Z}_{m}}{\underline{Z}_{C P E}+\underline{Z}_{m}}}{\frac{r_{C} \gamma}{2} \frac{I_{0}\left(r_{C} \gamma\right)}{I_{1}\left(r_{C} \gamma\right)}+R_{b}\left(\frac{1}{\underline{Z}_{C P E}}+\frac{1}{\underline{Z}_{m}}\right)}\right)\right]^{-1}+R_{\text {bulk }}
$$

where

$$
r_{C} \gamma=r_{C} \cdot \sqrt{\frac{\rho_{s u b}}{d}\left(\frac{1}{Z_{C P E}}+\frac{1}{Z_{m}}\right)}=\alpha \cdot \sqrt{\frac{1}{Z_{C P E}}+\frac{1}{Z_{m}}}
$$

and where $I_{0}$ and $I_{1}$ are modified Bessel functions of the first kind of order 0 and 1. 


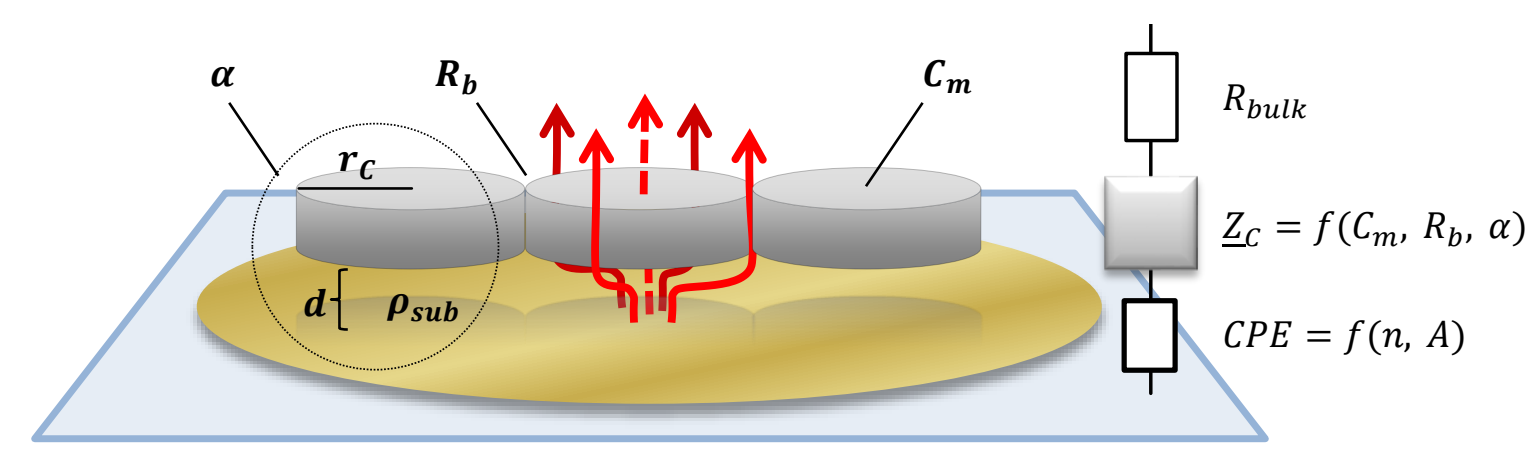

Fig. 3-17 Illustration of the impedance model for a cell-covered electrode according to Giaever and Keese ${ }^{[96]}$ and Wegener and Hakvoort et al. ${ }^{[314]}$. Cells are approximated as cylindrical disks, with an uniform height, an average radius $r_{C}$, and a mean distance $d$ to the electrode surface. The cell layer augments the impedance of the cell-free system (a CPE in series with the bulk resistance $R_{\text {bulk }}$ of the electrolyte) by an additional impedance $\underline{Z}_{C}$, which includes contributions of the cell membrane capacitance $C_{m}$, of the paracellular barrier resistance $R_{b}$, and of the parameter $\alpha$ that stands for the cleft impedance between the cells and the electrode.

The impedance measurement of the cell-free electrodes (immersed in the electrolyte) prior to seeding cells provides the values for $\underline{Z}_{C P E}$ as well as for $R_{\text {bulk }}$ by linear regression of the experimental data $\left(\log \left(|\underline{Z}|_{\text {total }}\right) v s \cdot \log (f)\right)$ in the low and high frequency range, respectively. ${ }^{\left[{ }^{14]}\right]}$ For impedance analysis of a cell-covered electrode, the only variables in the model are $C_{m}, R_{b}$, and $\alpha$. These characteristic cellrelated parameters are obtained by fitting the model in Eq. (50) to the experimental data by a numeric non-linear least-square optimization. By this, the time dependent changes of $C_{m}, R_{b}$, and $\alpha$ during an experiment are available from time-resolved recordings of impedance spectra. Stolwijk (2011) demonstrated by simulations the impact of the individual cell-layer specific parameters on the shapes of the impedance, resistance and capacitance spectrum of a cell-covered electrode..$^{[7]}$

\subsubsection{ECIS-OCM Combination in One Experimental Setup}

The ECIS results shown in the experimental part of this thesis were not obtained by using the conventional ECIS substrates and instrumentation. One key objective was to integrate an ECIS-like electrode arrangement on a quartz resonator in order to combine the two label-free measurement techniques of OCM and ECIS on one substrate. Double-mode impedance analysis of cells by combining OCM-mode measurements with ECIS-mode measurements was already presented earlier. ${ }^{[206,219,236,267,268]}$ For this, a conventional measurement chamber (1EIQ) was supplemented with an additional, vertical platinum (Pt) dipping electrode. ECIS-mode measurements were performed by immersing the $\mathrm{Pt}$ electrode into the physiological buffer above the quartz electrode. Thereby, the top electrode of the quartz resonator served as working electrode (WE) and the dipping electrode as counter electrode (CE). With this setup it is possible to measure both the viscoelastic properties of cells by OCM-mode readings as well as the morphological properties of the cell layer by ECIS-mode measurements on one OCM sensor. By this extension, the " $1^{\text {st }}$ generation $Q \mathrm{OCM}^{\text {" ( }}$ (simply a $\mathrm{QCM}$ sensor) was technologically upgraded to the " $2^{\text {nd }}$ generation $\mathrm{OCM}$ ", a combined ECIS-OCM sensor. However, the additional dipping electrode in " 2 nd generation $\mathrm{OCM}$ " leads to some disadvantages, like a more complicated experimental setup and the introduction of an additional entry point for contamination. Moreover, drawbacks arise from the kind and shape of the dipping electrode as well as from a defined geometrical arrangement of the CE above the WE. However, reproducible experimental conditions are prerequisites to compare impedance data of cells from different experiments. In order to overcome the disadvantages of the dipping electrode, a 
novel sensor layout was developed in this project. In this, a co-planar electrode arrangement was implemented on a piezoelectric quartz disk, similar to the situation on conventional ECIS substrates. By this, a well-defined CE was integrated on the sensor surface, which redundantizes the disadvantageous dipping electrode for ECIS. Hence, the " $2^{\text {nd }}$ generation $\mathrm{QCM}$ " was technologically upgraded to the " 3 rd generation $\mathrm{QCM}$ " in this project.

The development steps from the " $2^{\text {nd }}$ generation $\mathrm{QCM}$ " to the " $3^{\text {rd }}$ generation $\mathrm{OCM}$ " are described in the results sections. The capability of the novel " 3 rd generation $\mathrm{OCM}$ " sensor for double-mode measurements was investigated in a variety of different cellular assays. Thereby, the complementarity and gain of information provided by simultaneous ECIS- and QCM-mode measurements of the same cell population were demonstrated (cf. 5.2 QCM-ECIS Biosensor, p. 129 ff.).

\section{Quartz Resonator Layout for ECIS Integration}

For the ECIS-mode measurements that are shown in this work a newly sensor layout was developed. The basis of the sensor was a disk-shaped $5 \mathrm{MHz}$ AT-cut quartz resonator (KVG Quartz Crystal Technology

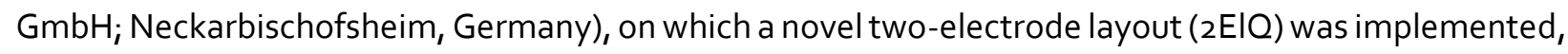
as illustrated in Fig. 3-10 B. Evaporation of the circular gold-film electrodes $(\varnothing=3.5 \mathrm{~mm})$ and the contact paths was carried out by KVG, based on a self-developed mask for the electrode layout. Having two coplanar electrodes on the surface of the quartz disk, ECIS measurements were performed without dipping a counter electrode into the electrolyte. Thus, additionally to OCM measurements ECISanalogue measurements are available with this setup.

In order to meet the experimental prerequisites for meaningful ECIS mode measurements the commercially provided gold film electrodes had to be further processed. Surface structuring of the sensor was achieved by spin coating and subsequent photolithography of an insulating photoresist. The experimental procedures for this, the applied photolithography masks (Fig. 3-12), as well as preliminary sensor cleaning steps are described above in subsection 3.3.3 (p. 45 ff.).
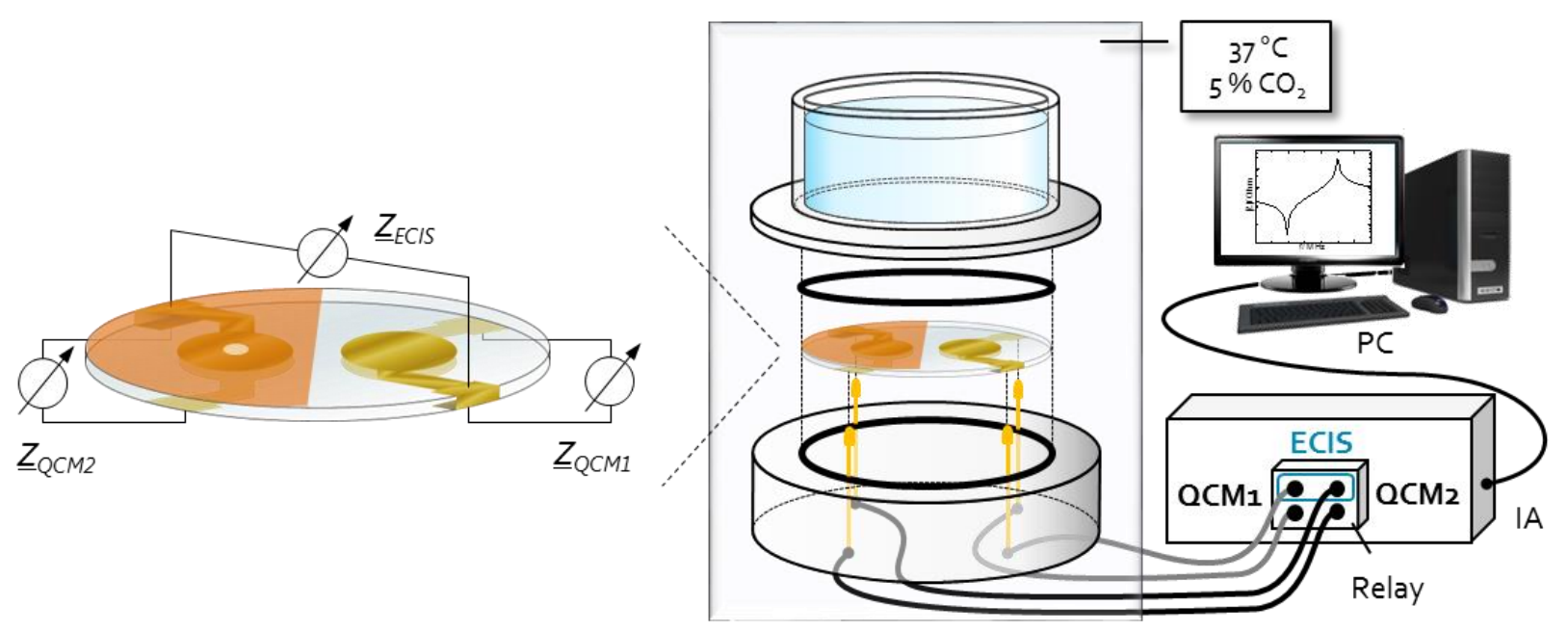

Fig. 3-18 Measurement chamber and experimental setup for a 2 ElQ sensor. The electrodes on the sensor were contacted from below by four contact pins and connected via a PC-controlled relay to the IA. This setup allowed reading the oscillation impedances of the two QCM sensor spots $\left(\underline{Z}_{O C M_{1}}\right.$ and $\underline{Z}_{O C M_{2}}$ ) as well as the electrical impedance between the two top surface electrodes $\left(\underline{Z}_{E C I S}\right)$. For cell experiments the whole measurement chamber was placed in an incubator $\left(37^{\circ} \mathrm{C}\right.$, $\left.5 \% \mathrm{CO}_{2}\right)$. 


\section{Hardware - Measurement Chamber}

The measurement chamber used for $2 \mathrm{ElOs}$ was similar to that for conventional $1 \mathrm{ElOs}$, which was previously described (p. 48). However, for $2 \mathrm{ElO}$ s the lower part of the chamber comprised four contact pins instead of two, and an additional relay was interconnected between the sensor and the impedance analyzer (Fig. 3-18). With this PC-controlled relay different pairs of electrodes of the 2 EIO were addressed. Hence, the impedance analyzer was switched between readings of the oscillation impedances ( $\underline{Z}_{O C M_{1}}$ and $\underline{Z}_{O C M_{2}}$ ) and the electric cell-substrate impedance ( $\left.\underline{Z}_{E C I S}\right)$ ((Fig. 3-18). More details of the experimental setup are mentioned in the OCM subsection Hardware - Measurement Chamber (p. 48).

\section{Software-ECIS-OCM Measurements}

The software used for combined ECIS-OCM measurements is an extended version of the LabVIEW based OCM software described previously (Software - OCM Measurements, p. 49). Switching the additional relay was integrated in the software for automated and sequential measurements of $\underline{Z}_{\mathrm{OCM}} \underline{Z}_{\mathrm{OCM} 2}$, and $\underline{Z}_{E C I S}$. In OCM-mode impedance was recorded successively for the two sensor spots, according to the procedure described in the experimental OCM section. For ECIS-mode scanning of the complex impedance a sinusoidal, non-invasive $A C$ excitation voltage of $10 \mathrm{mV}$ was applied between both upper surface electrodes of the quartz disk ((Fig. 3-18). The impedance of the system was measured in a frequency range from $1 \mathrm{~Hz}$ to $1 \mathrm{MHz}$ for 61 frequencies that were distributed equidistantly on a log-scale (10 data points per decade).

\section{Experimental Implementation of ECIS-OCM Measurements}

Combined ECIS-OCM measurements were performed similarly to OCM experiments described above (Experimental Implementation, p. 49). However, before seeding cells on the ECIS-OCM sensor the photopolymer had to be pre-incubated with liquid. It is known for photoresist-coated resonators that equilibration of the polymer in liquid has a non-negligible effect on the acoustic impedance. ${ }^{[236]}$ In order to avoid an overlap of swelling with the cell-related signal and to gain a constant baseline signal of the motional $(\mathrm{OCM})$ impedance the polymer on the $2 \mathrm{ElO}$ was immersed with liquid for $\sim 20 \mathrm{~h}$.

\section{Data Processing}

The recorded OCM data was analyzed according to the procedure described earlier (Data Processing, p. 49). A set of impedance spectra (magnitude and phase) was recorded by ECIS-mode measurements during an experiment. The data set was processed by LabVIEW based software (written by J. Wegener, University of Regensburg), which extracted the equivalent resistance and the equivalent capacitance value for each AC frequency (cf. 3.4.2 Impedance Properties of (Cell Covered) ECIS Electrodes). By dividing the impedance (resistance/ capacitance) spectra of a cell-covered electrode by the impedance (resistance/ capacitance) spectra of the corresponding cell-free electrode, the frequency at which the impedance (resistance/ capacitance) is most sensitive to the cell layer is obtained. At these frequencies the impedance, resistance, and capacitance were plotted as a function of time. The experimental time was usually normalized with respect to a characteristic point of the experiment, like e.g. the seeding of cells, the addition of a substance, or any other stimulation of the cells. The values of $|Z|, R$, or $C$ were also normalized with respect to their value of the data point that was defined as time zero. In this way, absolute signal changes in percent were plotted, which made the data more comparable to other experiments. The time courses of the resistance at low and of the capacitance at high frequencies were 
then used for obtaining information regarding alterations of the junctional resistance (barrier functionality) and changes of the membrane capacitances of the cells, respectively. Combined ECISOCM measurements were repeated several times and parameter changes in the course of a test series were average and statistically evaluated.

\section{Sensor Regeneration}

Before regeneration and cleaning of the resonators, the photopolymer on the $2 \mathrm{ElO} s$ had to be removed. This was achieved by rinsing the substrate in acetone for $\sim 1 \mathrm{~min}$. Afterwards, the quartz was cleaned in a piranha solution ( 3 parts ( $/ / v)$ of conc. $\mathrm{H}_{2} \mathrm{SO}_{4}$ and 1 part $(\mathrm{v} / \mathrm{v})$ of $30 \%(\mathrm{v} / \mathrm{v}) \mathrm{H}_{2} \mathrm{O}_{2}$ ) for $\sim 30$ min in order to remove organic residuals from the sensor surface. Then the quartz disk was rinsed several times with deionized water in order to get rid of the acid. Before re-using the quartz, it had to be cleaned and prepared again, according to the protocols in subsection Quartz Cleaning (p. 45).

\subsubsection{Electroporation on ECIS-OCM Sensor (ELPO-ECIS-QCM)}

The introduction of membrane impermeable substances into the cytoplasm of adherent cells by means of in situ electroporation has been previously described. ${ }^{\left[{ }^{13-318]}\right.}$ It has also been shown, how ECIS electrodes were not only used for sensing cells, but also for actuating and manipulating cells by external electric fields. ${ }^{[7,99,118-120]}$ In ECIS, the electrodes were used for electrically monitoring the cellular viability and morphology before and after applying a pore forming electroporation (ELPO) pulse to adherent cells (ELPO-ECIS).

\section{Electroporation Principle}

In general, electroporation describes the formation of pores in a membrane caused by an electric field. The strategy of introducing a cell membrane impermeable substance into the cytoplasm of a cell is schematically illustrated in Fig. 3-19. For ELPO, two electrodes need to be introduced into the system, which are on opposite sites of the membrane that is to be electroporated (Fig. 3-19 A). By applying an appropriate electric field, the membrane can be polarized and consequently destabilized to an extent to cause the collapse of the membrane potential. This breakdown of the membrane potential goes along with the formation of pores in the lipid bilayer (Fig. 3-19 B). In order to form pores in cell membranes the electric field has to fulfill cell membrane specific requirements. Depending on the frequency, the AC voltage magnitude, and the duration of the pulse, the electroporation is invasive and initiates cell death or it is non-invasive, which leaves the cells viable after the electric pulse. In the latter case the cell membrane is only transiently opened, the pores are closed as soon as the field is turned off, and the impermeable membrane is restored within a short period after the pulse (Fig. 3-19 C). Thus, it is possible to transfer membrane impermeable substances into the cytoplasm of cells, just by providing a sufficiently high extra-cellular concentration of the respective molecules and by opening the cell membranes for diffusion by applying an adjusted electroporation pulse.

By combining this electroporation technique with a sensor technique like ECIS, one can follow the cellular response towards the voltage pulse and the introduction of substances immediately, non-invasively, and in time resolved manner. However, only cells on the working electrode are affected. This is because the smaller working electrode dominates the entire impedance of the system and the voltage almost completely drops across it. A sinusoidal AC voltage of $40 \mathrm{kHz}$, amplitudes of $3-7 \mathrm{~V}$, and pulse durations between 200-500 ms were found to be adequate parameters for an efficient, non-invasive electroporation of a variety of adherent cells on ECIS substrates (working electrode area: $5 \cdot 10^{-4} \mathrm{~cm}^{2}$ ). ${ }^{[7]}$ 
The principle of ELPO was also introduced into the newly developed ECIS-OCM setup (cf. 3.4 .4$, p. 56 ff.). Thus, ELPO was combined with instantaneous real-time monitoring of the cell responses in both ECISand OCM-mode measurements (ELPO-ECIS-OCM). With this, cytomechanical cell responses upon the introduction of membrane-impermeable xenobiotics can be investigated in addition to the cell-shape and vitality monitoring by ECIS.

A

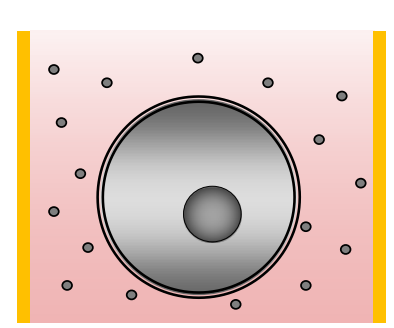

B

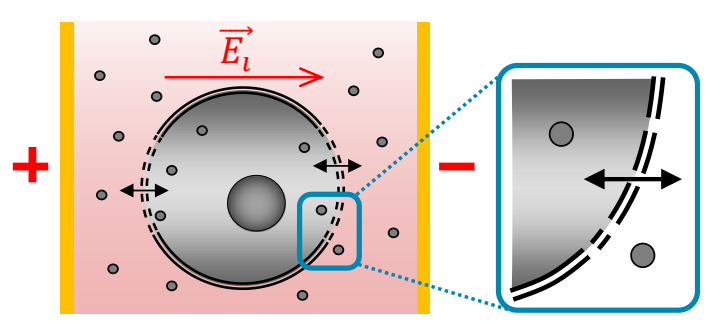

C

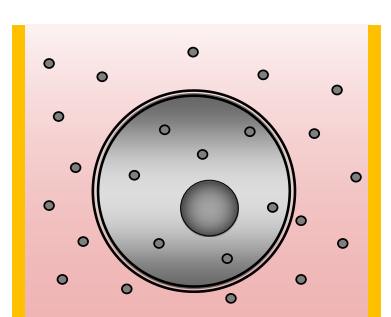

Fig. 3-19 Electroporation principle. (A) A cell with intact lipid bilayer membrane is placed between two electrodes (yellow bars) in an electrolyte that contains a membrane impermeable substance (grey spheres). (B) Applying a potential between the electrodes generates an electric field $\overrightarrow{E_{l}}$ in the solution. The cell membrane is polarized in this electric field, and pores are formed in the cell membrane at a critical threshold value of polarization. During electroporation until membrane regeneration extra-cellular molecules can diffuse through the pores into the cells, according to the gradient of concentration. (C) The cell membrane reseals again after (noninvasive) electroporation, leaving the membrane impermeable substance remaining in the cytoplasm and, thus, being introduced into the cell.

\section{Hardware for Electroporation}

For realizing electroporation on quartz disks, the hardware requirements were basically the same as for ECIS mode measurements on OCM resonators, regarding the sensor layout itself as well as the experimental setup (cf. 3.4.4). The $2 \mathrm{ElOs}$ comprised two coplanar electrodes on the top side of the sensor, similar to the ECIS substrates. The size of the working electrode was systematically optimized by preparing $2 \mathrm{EIO}$ resonators with working electrodes of the following diameters: $250,500,1000$, and $2000 \mu \mathrm{m}$ (cf. Spin Coating and Photolithography on Quartz Disks, p. 46 f.).

Electroporation was integrated into the running ECIS-OCM measurement. For this, the ECIS-OCM setup was supplemented by a "33120A $15 \mathrm{MHz}$ Function/ Arbitrary Waveform Generator" (Hewlett-Packard; Palo Alto, CA, USA) (Fig. 3-20). The frequency generator served as AC voltage source for applying a defined voltage magnitude of a certain frequency and for a defined period of time between the working and the counter electrode and, thus, to the adherently grown cells on the working electrode. For visualizing and controlling the electroporation pulse, an "oscilloscope HM 1005" (HAMEG; Frankfurt/ Main, Germany) was implemented between the frequency generator and the connecting wires of the two top electrodes of the $2 \mathrm{EIO}$ (Fig. 3-20).

\section{Experimental Implementation}

Electroporation pulses were applied in the course of an ECIS-OCM measurement, after cells had formed a confluent monolayer. Before electroporation, the processes of cell attachment, cell spreading, and the establishment of a confluent cell layer were monitored by OCM-mode and ECIS-mode readings. Moreover, the extracellular influence of a membrane-impermeable substance of interest was recorded 
in real-time by ECIS-OCM. Equilibration of signals was awaited, before applying the ELPO voltage pulse. Thus, effects of extra-cellular stimulation were distinguished from cell responses to ELPO and intracellular stimulation. To apply the electroporation pulse the ECIS-OCM recording was shortly paused and the upper surface electrodes of the $2 \mathrm{EIO}$ (ECIS electrodes) were connected to the frequency generator. Then, an AC voltage pulse of a certain magnitude, at a defined frequency, and for a specific period of time was manually applied to the cell layer on the working electrode. Afterwards, the cables were replugged between frequency generator and impedance analyzer and ECIS-OCM measurements were instantaneously continued. This experimental setup allowed to monitor non-invasively and in real-time both the morphological as well as the viscoelastic effects on the cell layer triggered by the electroporation pulse and the substance introduced into the cytoplasm.

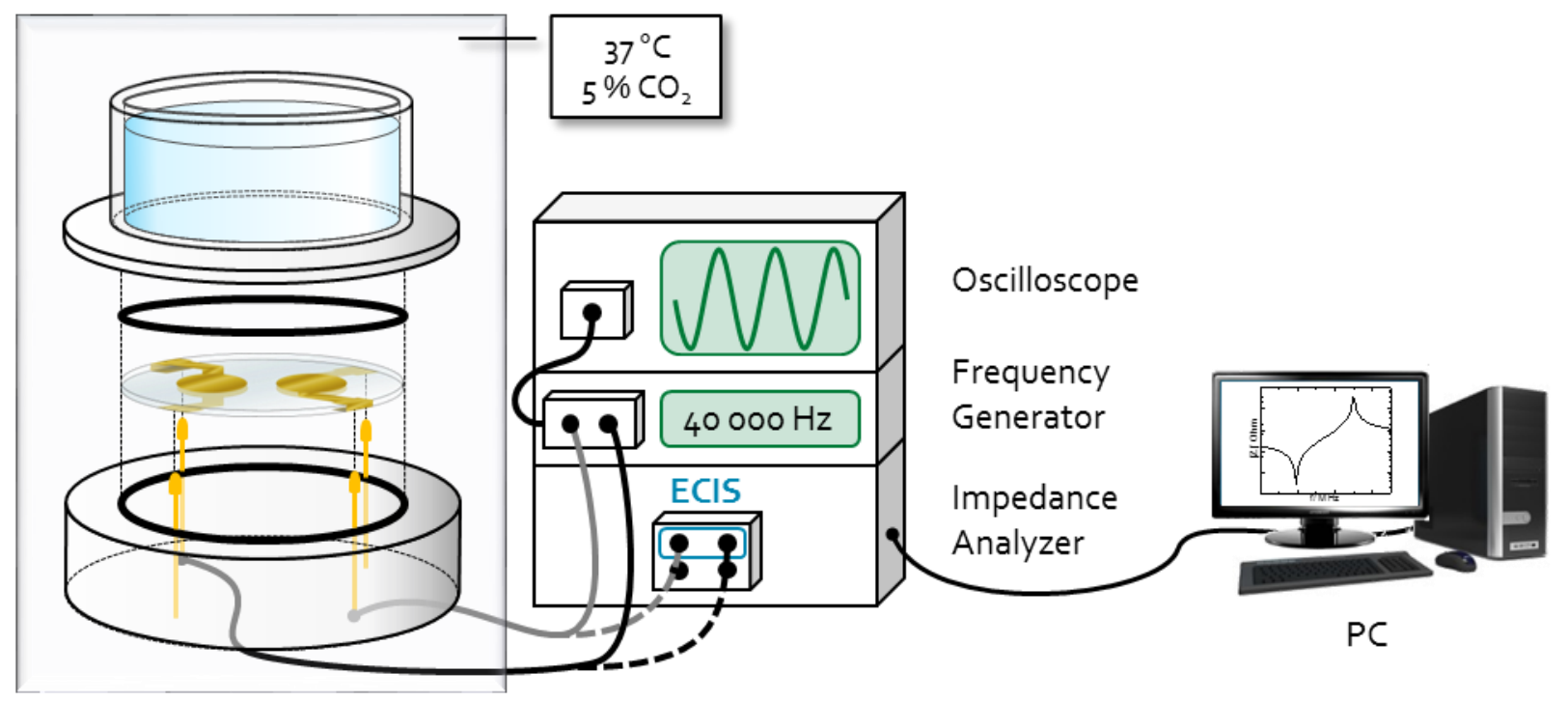

Fig. 3-20 Schematic illustration of ELPO integration in ECIS-OCM measurements (ELPO-ECISQCM). For simplification, the wires for QCM-mode measurements are left out, and only the connecting wires for the two upper surface electrodes (for ECIS-mode measurements) are shown. For applying an electroporation pulse during a running measurement, the ECIS-OCM recording is temporarily stopped and the upper electrodes are connected to the frequency generator. After manually applying a defined electric pulse, cables were replugged again, and ECIS-QCM measurements were instantaneously continued. Further details of the experimental setup were previously described (cf. 3.4.4, p. 56 ff.; Fig. 3-18).

\subsection{Optical Temperature and Oxygen Imaging}

Optical sensors are based on the detection of changing optical properties of a sensor probe, caused by its interaction with an analyte. The optical properties of a probe that are used for sensing are absorbance, emission (luminescence wavelength and intensity), anisotropy, reflectivity, or the luminescence lifetime of a luminophore. ${ }^{[174]} \mathrm{An}$ increase in temperature $(\mathrm{T})$ as well as elevation of the oxygen partial pressure $\left(p \mathrm{O}_{2}\right)$ cause a decrease of the luminescence intensity and lifetime, respectively. $\mathrm{T}$ and $p \mathrm{O}_{2}$ are both luminescence quenchers that promote radiation-less relaxation processes of excited fluorophore molecules. Imaging of the luminescence intensity (lifetime) of a sensor film provides a two-dimensional resolution of the luminescence properties on the photographed sample. One thus obtains the twodimensional temperature (oxygen) distribution on the sample surface by luminescence lifetime imaging 
of a temperature (oxygen) sensitive probe. However, the luminescence lifetime of most probes is sensitive to quenching by both $\mathrm{T}$ and $\mathrm{pO}_{2}$. Therefore, the quenching effect due to other influences has to be excluded or kept constant for a quantitative analysis of only one parameter. ${ }^{[176,177,319,320]}$

The following section addresses the basic principles of light absorption, emission, and luminescence quenching. Subsequent sections focus on FLIM as it was usedin this study, the experimental setup, the specific experimental implementation of $\mathrm{T}$ and $\mathrm{pO}_{2}$ imaging, and finally on the way of data processing and data presentation of measurements.

\subsubsection{Basic Principles of Luminescence and Luminescence Quenching}

The processes of light absorption, emission, and luminescence quenching can be described best by a Jablonski energy-level diagram ${ }^{[321]}$, as shown in Fig. 3-21. If a luminophore in its energetic ground state is illuminated with photons $\left(h v_{\text {exc }}\right.$ ) of certain energy, this energy is instantaneously absorbed $\left(10^{-15} \mathrm{~s}\right)$ by exciting electrons from the electronic (singlet) ground state $S_{0}$ to higher energy levels. If the energy of the photons is high enough, the electrons can be excited from $S_{0}$ to vibrational levels of the first or second excited singlet state $\left(S_{0} \rightarrow S_{1}, S_{0} \rightarrow S_{2}\right.$ ). Electrons in elevated vibrational levels (of $S_{1}$ or $S_{2}$ ) immediately fall back to the vibrational ground state of $S_{1}$ by internal conversion (IC) from $S_{2} \rightarrow S_{1}$, and subsequent nonradiative (vibrational) relaxation $\left(\sim 10^{-12} \mathrm{~s}\right)$. The IC process describes the lossless energy conversion of electrons from an excited electronic state to a vibrational level of a lower electronic state of the same multiplicity $\left(S_{2} \rightarrow S_{1}, S_{1} \rightarrow S_{0}\right) .^{[174]}$
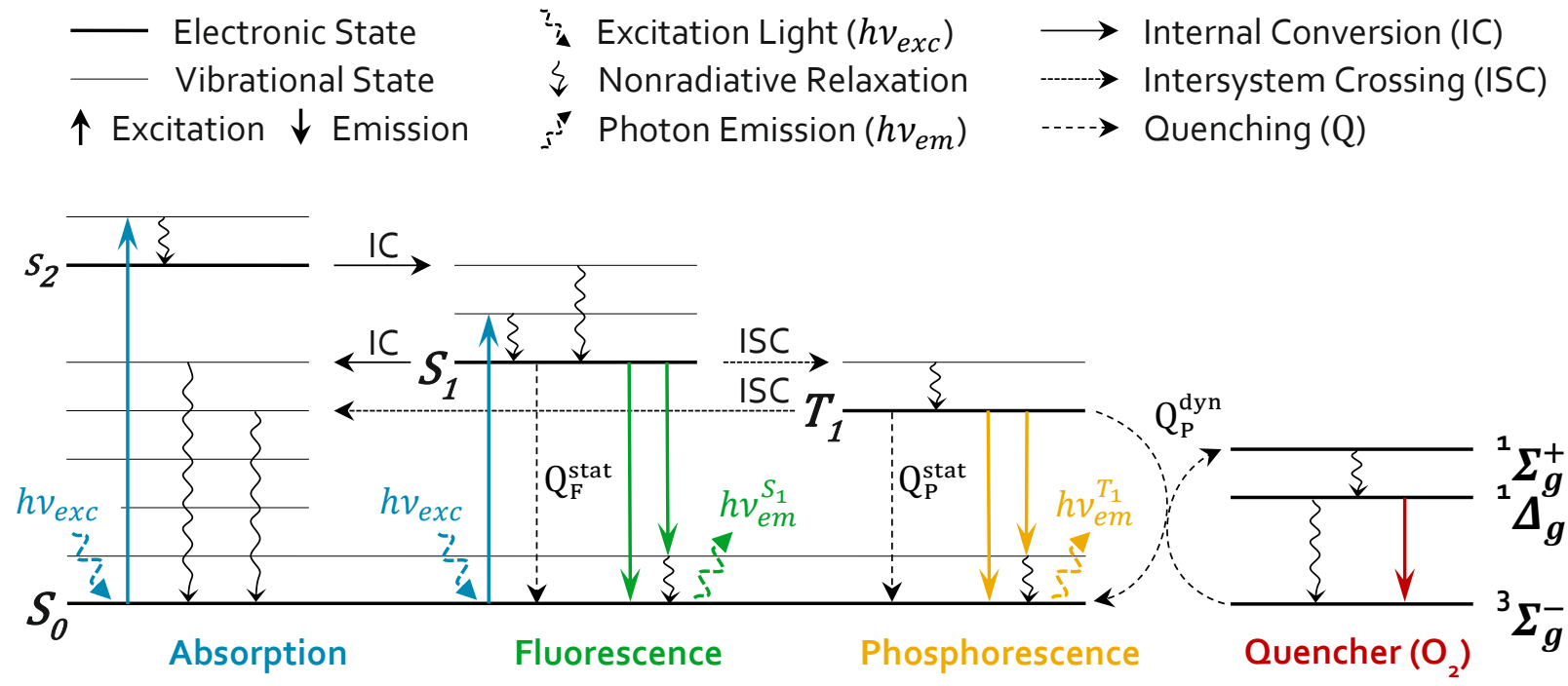

Fig. 3-21 Jablonski diagram; modified after ${ }^{[322,323]}$.

From $S_{1}$, the electrons can fall back to $S_{0}$ in a multitude of different ways of energy transfer. Like for the radiationless deactivation from $S_{2} \rightarrow S_{1}$, the $S_{1}$ energy can be further dissipated without radiation by IC ( $S_{1} \rightarrow S_{0}$; first-order rate constant $k_{I C}=10^{5}-10^{9} \mathrm{~s}^{-1}$ ) and subsequent instantaneous vibrational relaxation to the vibrational ground state. However, electrons can also fall back to $S_{0}$ by emitting the energy in the form of photons $\left(h v_{e m}^{S_{1}}\right)$, which is fluorescence emission. Fluorescence is a spin-allowed process and exhibits a rate constant of $k_{F}=10^{6}-10^{9} \mathrm{~s}^{-1}$. Beside the previously mentioned nonradiative 
relaxation via IC, fluorescence emission can be decreased by static or dynamic quenching processes $\left(Q_{\mathrm{F}}^{\mathrm{stat}}, \mathrm{Q}_{\mathrm{P}}^{\mathrm{dyn}}\right) \cdot{ }^{[174]}$

In a fourth transition process, $S_{1}$ is depopulated by intersystem crossing (ISC) of the electrons to the first excited triplet state $\left(S_{1} \rightarrow T_{1}\right)$. ISC to higher vibrational levels of $T_{1}$ is followed by an immediate radiationless relaxation to the fundamental vibrational state of $T_{1} . S_{1} \rightarrow T_{1}$ is actually a spin-forbidden transition (electrons have to change their spin orientation during ISC). However, ISC is partially allowed, due to effects of spin-orbit coupling, which mixes singlet and triplet states. Spin-orbit coupling is highly increased by the presence of heavy metal atoms like platinum within the probe, which results in an increased probability and transition rate of ISC $\left(k_{I S C}^{S_{1} \rightarrow T_{1}}=10^{4}-10^{12} \mathrm{~s}^{-1}\right)$. For the platinum porphyrine complex PtTFPP the triplet quantum yield (the fraction of absorbed photons that lead to a population of $T_{1}$ ) reaches values of $\sim 100 \%$. ${ }^{[322,323]}$

Once in the $T_{1}$ state, there are again a number of possible energy transfer processes for the electrons. First, an ISC back to the $S_{1}$ can take place under thermal activation $\left(T_{1} \rightarrow S_{1}\right)$, followed by de-excitation processes from $S_{1}$ to $S_{0}$. Thermal activation is necessary, as $T_{1}$ is a lower energy level than $S_{1}$. A second ISC can occur from the excited triplet to one of the vibrational levels of the singlet ground state $\left(T_{1} \rightarrow S_{0}\right.$; $\left.k_{I S C}^{T_{1} \rightarrow S_{0}}=10^{1}-10^{9} \mathrm{~s}^{-1}\right)$, followed by nonradiative relaxation. Similar to fluorescence, the electrons in $T_{1}$ can relax to the electric ground state $\left(T_{1} \rightarrow S_{0}\right)$ under emission of radiation $\left(h v_{e m}^{T_{1}}\right)$, too. However, phosphorescence is an intersystem radiative transition and, thus, is a spin-forbidden process. This causes a delayed emission of phosphorescence $\left(k_{P}=10^{-2}-10^{6} \mathrm{~s}^{-1}\right)$ in comparison to fluorescence $\left(k_{F}=10^{6}-\right.$

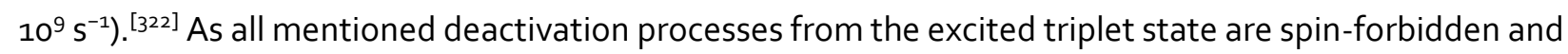
therefore possess small rate constants, $T_{1}$ exhibits a long lifetime that varies from $10^{-6} \mathrm{~s}$ to several seconds. Besides the previously mentioned ISCs, the quantum yield for phosphorescence depends on two further radiationless deactivation processes, which are static quenching $\left(Q_{P}^{\text {stat }}\right)$ and dynamic quenching $\left(Q_{P}^{\text {dyn }}\right)$. Both quenching effects are based on a molecular interaction between the fluorescent probe and the quenching molecule. In static quenching the luminophore molecule and the quencher molecule (Q) form a complex that exhibits no fluorescence properties. In dynamic quenching the energy of the excited fluorophore is transferred to the quencher molecule. By this, the luminophore is de-excited to its ground state $S_{0}$. Dynamic quenching is also termed collisional quenching, as the fluorophore and the quencher must be in close proximity to each other ( $20 \AA$ and less) for the energy transfer. The exact mechanism of energy transfer is not jet fully understood. ${ }^{[174,322,323]}$

\section{Oxygen Sensitivity of Luminescence}

Molecular oxygen is a well-known dynamic quencher of phosphorescence. An oxygen molecule naturally exhibits a triplet ground state $3 \Sigma_{\mathrm{g}}^{-}$(two unpaired electrons of parallel spin-orientation) and two excited singlet states, ${ }^{1} \Delta_{\mathrm{g}}$ and ${ }^{1} \Sigma_{\mathrm{g}}^{+}$, with energy difference of approximately $1.0 \mathrm{eV}$ between the ground state and the lowest singlet state (Fig. 3-21). Thus, oxygen in its triplet ground state can efficiently quench luminophores in their excited, long-living triplet state by triplet-triplet annihilation $\left(Q_{P}^{\mathrm{dyn}}\right)$. Thereby, the dye is deactivated from $T_{1}$ to the ground state and the excitation energy is absorbed by the oxygen molecule. The transferred energy excites the oxygen to the first or second singlet state. Singlet oxygen energy then dissipates by either phosphorescence with IR radiation $(\lambda>1240 \mathrm{~nm})$, or ISC with subsequent radiationless relaxation, or by energy transfer to molecules. The latter means chemical reactions of the highly reactive singlet oxygen with surrounding molecules. This singlet oxygen species 
is also the reason for phototoxicity in biological systems, when investigating oxygen quenching of a fluorescent dye.

The decrease of fluorescence of a fluorophore due to dynamic quenching can be described by the SternVolmer equation:

$$
\frac{I_{0}}{I}=\frac{\tau_{0}}{\tau}=1+K_{S V} \cdot[Q]=1+\kappa_{q} \tau_{q} \cdot[Q]
$$

where $I_{0}$ and $I$ are the luminescence intensities and $\tau_{0}$ and $\tau$ are the luminescence lifetimes in the absence and presence of the quencher, respectively, and $[Q]$ is the quencher concentration. $K_{S V}$ is the Stern-Volmer quenching constant and comprises the bimolecular quenching constant $\kappa_{q}$. Equation (52) is only valid for a homogeneous distribution of fluorophore molecules in a non-quenching matrix. If fluorophores are embedded in more than one matrix, the so-called multi-site model has to be used for describing fluorescence quenching:

$$
\frac{I_{0}}{I}=\frac{\tau_{0}}{\tau}=\sum_{i=1}^{n} \frac{f_{i}}{1+K_{S V}^{i} \cdot[Q]}
$$

with $i$ being the number of $n$ different matrices, $f_{i}$ is the fraction of fluorophore molecules in the respective matrix, and $K_{S V}^{i}$ is the according quenching constant.

In the case of oxygen being the quencher, the oxygen concentration $\left[\mathrm{O}_{2}\right]$ is proportional to the partial pressure of oxygen $\mathrm{pO}_{2}$ above the matrix, in which the luminophore is embedded, described by Henry's law. As the volume fraction of oxygen in air is constant $\left(20.95 \%(v / v)^{[324]}\right)$, the sensor can be used for air pressure sensing. Thus, sensor foils containing such oxygen quenchable probes are also referred to as pressure-sensitive paints (PSP). ${ }^{[322]}$

\section{Temperature Sensitivity of Luminescence}

Luminescence emission is also very sensitive to temperature. In contrast to collision quenching by oxygen, temperature does not provide an additional energy dissipation process that reduces the quantum yield for phosphorescence emission. The phosphorescence emission rate itself is weakly dependent on temperature as well as the triplet quantum yield $\left(\Phi_{T}\right)$ is almost temperature insensitive, as long as $\Phi_{T} \approx 1$. Temperature instead promotes non-radiative relaxation processes of the excited triplet state, which is the ISC of $T_{1} \rightarrow S_{0}\left(k_{I S C}^{T_{1} \rightarrow S_{0}}=10^{1}-10^{9} \mathrm{~s}^{-1}\right)$. The temperature dependence of the ISC rate constant $k_{I S C}^{T_{1} \rightarrow S_{0}}$ is given by the Arrhenius relationship: ${ }^{[325,326]}$

$$
k_{I S C}^{T_{1} \rightarrow S_{0}}(T)=A_{I S C} \cdot \exp \left(-\frac{\Delta E_{I S C}}{R T}\right) \cong k_{I S C}^{T_{1} \rightarrow S_{0}}\left(T_{0}\right) \cdot\left(1+\frac{\Delta E_{I S C}}{R T_{0}} \frac{\Delta T}{T_{0}}\right)
$$

where $\Delta E_{I S C}$ is the activation energy for ISC of $T_{1} \rightarrow S_{0}, T_{0}$ is a reference temperature, and $\Delta T=T-T_{0}$ is small relative to $T_{0} \cdot{ }^{[322]}$

With increasing temperature, the rate constant for ISC $T_{1} \rightarrow S_{0}$ is increased. This causes that more excited triplet states undergo radiationless de-excitation and the phosphorescence quantum yield is decreased. Consequently, a decrease in phosphorescence intensity and lifetime of the triplet state can be measured. According to the previously mentioned pressure-sensitive paints (PSPs), a sensor foil with embedded temperature sensitive probe is often called temperature-sensitive paint, or short TSP. 


\subsubsection{Fluorescence Lifetime Imaging (FLIM) using Rapid Lifetime Determination (RLD)}

There is a variety of possible measurement methods for the two-dimensional luminescence imaging of a luminophore in a matrix. Measuring the luminescence intensity is the most direct method. However, intensity measurements lead to some drawbacks, since the luminescence intensity is affected by a multitude of factors. Constant luminescence intensity requires a uniformly distributed dye within the matrix, an even illumination by the light source, a consistent light intensity, and constant geometrical parameters in the experimental setup. Moreover, luminescence intensity is strongly influenced by light scattering and photo-bleaching effects. With the help of ratiometric imaging methods and lifetimebased imaging techniques, these drawbacks can be overcome. Fluorescence lifetime imaging (FLIM) can be performed either in the frequency domain or in the time domain, whereby data from both methods can be transferred to each other by Fourier transformation. Time domain FLIM is the method of choice for metal-ligand complexes (MLCs) as oxygen and temperature sensitive dyes, since the MLCs exhibit luminescence lifetimes in the microsecond time scale. Using such long-living MLCs as probe has the additional advantage that background fluorescence (decay time < $200 \mathrm{~ns}$ ) can be suppressed by simply starting imaging after a delay time of $1 \mu$ s.

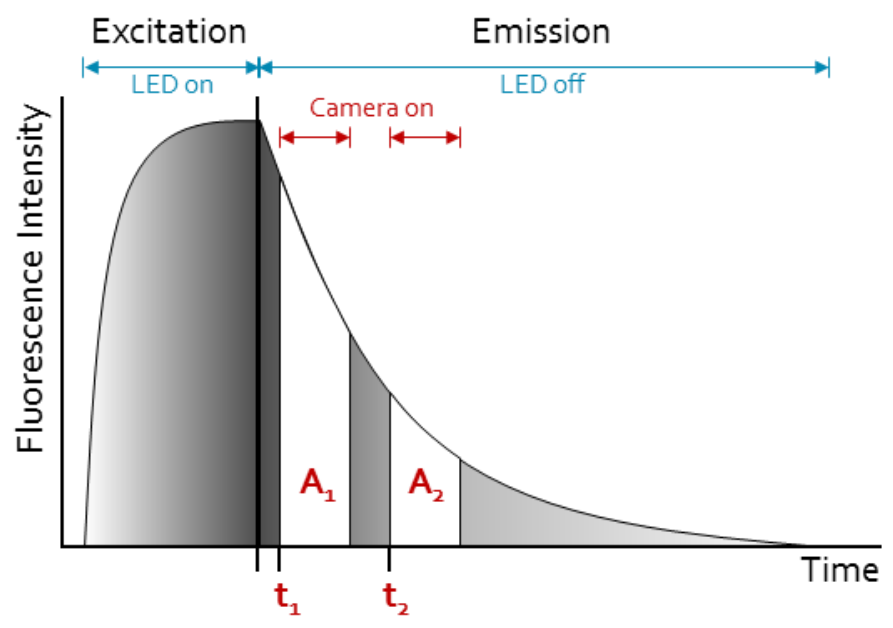

Fig. 3-22 Schematic illustration of fluorescence lifetime imaging with the rapid lifetime determination (RLD) principle. The fluorophore is excited by a LED light source, and two timegated intensity images $\left(A_{1}\right.$ and $\left.A_{2}\right)$ are recorded by the triggered CCD camera in the emission phase at time points $t_{1}$ and $t_{2}$, after the LED was switched off. The gate width for images $A_{1}$ and $A_{2}$ was set identically, and time point $t_{1}$ was set delayed, so that background fluorescence was completely decayed for imaging.

FLIM can be referenced intrinsically by time gated imaging in the decay phase of the MLC luminescence. Rapid lifetime determination (RLD) ${ }^{\left[{ }^{[27]}\right.}$ is one time gated method for acquiring self-referenced FLIM data. The principle of RLD is schematically shown in Fig. 3-22. The luminescent probe is excited by a rectangular light pulse of a light emitting diode (LED). During this excitation phase the luminescence increases until equilibrium between absorbed and emitted photons is reached. After turning the excitation light source off, background and MLC luminescence emission decay, according to their luminescence lifetimes. At defined time points, $t_{1}$ and $t_{2}$, after stopping excitation (and after background fluorescence has decayed), the shutter of the CCD camera is opened twice for identical time 
intervals. In this two time gates, $A_{1}$ and $A 2$, the CCD collects the emitted photons and integrates the luminescence intensity for each pixel of the sensor. Thus, by RLD one obtains two intensity pictures, $A_{1}$ and $A_{2}$, in the decay phase of the luminescent probe. With the ratio of the two intensity pictures, $A_{1} / A_{21}$ one can calculate the decay time $\tau$, according to:

$$
\tau=\frac{t_{2}-t_{1}}{\ln \frac{A_{1}}{A_{2}}}
$$

where $t_{1}$ and $t_{2}$ depict the time points of camera opening in the emission decay phase, and $A_{1}$ and $A_{2}$ represent the integrated intensities within the respective time gates.

It is noted that the absolute values for the decay time, calculated by Eq. (55), are only valid for monoexponential decays. Since the exact lifetime values were not of interest, and relative changes in lifetime were sufficient for imaging and quantifying the quenching effects by oxygen and temperature. ${ }^{[178,328]}$ As the luminescence decay time is independent of the intensity, the intensity image ratio in RLD eliminates variations in signal intensity. Hence, non-uniform matrix thickness, fluctuations in the excitation light intensity, reflections, background luminescence, or changing geometry between sample and camera causes no adverse effects in accuracy. Thus, the RLD method is a robust and powerful tool for FLIM applications. However, as RLD is a time-resolved imaging method, the imaging setup requires a camera with a fast shutter that is triggered synchronously with the excitation light source. Moreover, better signal-to-noise ratios can be achieved with cameras that exhibit a cooled CCD chip.

\subsubsection{Experimental of FLIM-RLD}

\section{TSP and PSP Sensor Preparation}

For temperature imaging, two different TSPs were prepared, containing either the temperaturesensitive probe Tris(dinaphthoylmethane)-bis(trioctylphosphineoxide)-europium(III) (Eu(dnm) $)_{3}(\text { topo })_{2}$; synthesized in our institute, according to Peng's procedure ${ }^{\left[{ }^{2} 2\right]}$ ]) or Tris(benzoylacetonato)-mono(phenanthroline)-europium(III) (Eu(benzac) $)_{3}($ phen); Sigma-Aldrich; St. Louis, MO, USA). As oxygensensitive dye, the platinum porphyrin complex 5,10,15,20-tetrakis-(2,3,4,5,6-pentafluorophenyl)porphyrin-platinum(II) (PtTFPP; obtained from Porphyrine Systems GbR; Appen, Germany) was used. The chemical structures of the dyes are shown in Fig. 3-23.

A

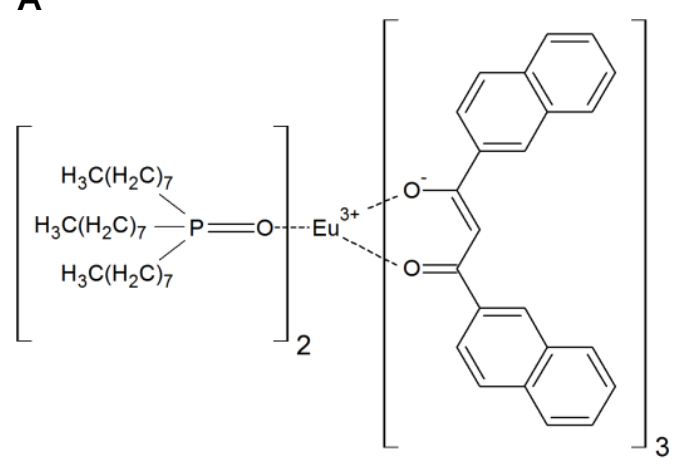

B

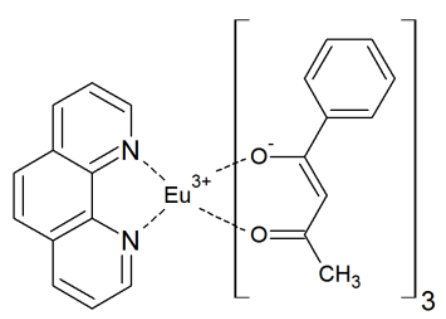

C

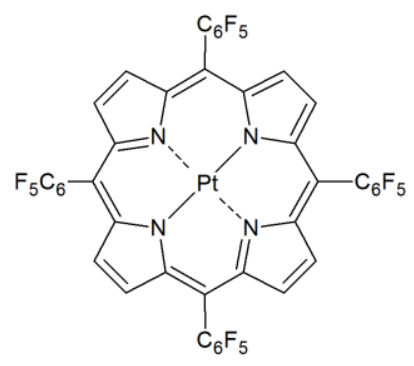

Fig. 3-23 Chemical structures of the temperature-sensitive probes (A) Eu(dnm) $)_{3}(\text { topo })_{2}$ and (B) Eu(benzac) $)_{3}($ phen), and of the used oxygen-sensitive probe (C) PtTFPP. 


\section{TSP Sensor Preparation}

The TSP sensor cocktail was prepared by mixing a polymer solution of $10 \%(\mathrm{w} / \mathrm{v})$ poly(vinylidenechloride/ acrylonitrile) (80:20) (Polysciences, Inc.; Warrington, PA, USA) in tetrahydrofuran (THF; SigmaAldrich; St. Louis, MO, USA) with $15 \%$ (w/w of polymer) of Eu(dnm) ${ }_{3}$ (topo) ${ }_{2}$ or Eu(benzac) ${ }_{3}$ (phen). This "cocktail" was sprayed from a distance of $\sim 15 \mathrm{~cm}$ onto the surface of a cleaned quartz resonator (1ElO) with a paint-spraying gun (Walther PILOT; Wuppertal, Germany). The quartz cleaning procedure is explained in subsection Quartz Cleaning (p. 45). The spray pressure was 2.5 bar, and the solvent was evaporated over night at room temperature. In this way, a TSP sensor film thickness of $\sim 0.7-2.5 \mu \mathrm{m}$ was obtained (determined by OCM measurements).

\section{PSP Sensor Preparation}

For the PSP sensor film, the oxygen-sensitive probe PtTFPP was embedded in a polystyrene (PS) matrix. In the sensor cocktail, the PtTFPP concentration was set to $3 \%$ (w/w of PS), and the PS concentration was either $3 \%(\mathrm{w} / \mathrm{v})$ or $5 \%(\mathrm{w} / \mathrm{v})$ in the solvent, which was either THF or tolvene (Merck; Darmstadt, Germany). The cocktail was protected from light and stored in a fridge until usage. A PSP sensor film was deposited on circular $(\varnothing=12 \mathrm{~mm}$ ) microscope cover glasses (VWR; Radnor, PA, USA) by either spray coating (cf. subsection TSP Sensor Preparation) or spin coating of the sensor cocktail. For spin coating a self-made spin coater composed of a hard disk drive was used (developed by Dr. F. Höhn; Institute of Biochemistry, University of Münster, Germany). Before coating, the glass substrates were cleaned by rinsing in acetone and isopropyl alcohol, in order to remove organic impurities from the surface. The solvents and surface water were subsequently removed by heating the substrates to $120^{\circ} \mathrm{C}$ for $30 \mathrm{~min}$. In order to guarantee a homogeneous solution of the cocktail for the coating process, the PSP was stirred and sonicated for at least $10 \mathrm{~min}$. Spin coating was performed at room temperature and at $1500-4000 \mathrm{rpm}$. A drop of the cocktail was placed in the center of the rotating substrate and spin coated for 1 min. After PSP coating, the substrate was dried at room temperature overnight. For some test series, the adhesion promoter "TI Prime" was spin coated prior to the PSP. "TI Prime" spin coating was performed at $3000 \mathrm{rpm}$ for $20 \mathrm{~s}$, followed by activation of the adhesion promoter in the oven $\left(130^{\circ} \mathrm{C}\right.$ for $10 \mathrm{~min})$. After this, the substrate was ready for PSP coating as described above.

\section{Hardware - Experimental Setup}

\section{Camera and Light Source}

Time-resolved luminescence measurements were performed with an "ImageX Time Gated Imaging system" (Photonic Research Systems; Newhaven, UK). It consisted of a cooled 12-bit b/w CCD camera, a trigger box, and the respective software for defining and controlling imaging parameters, as well as for image processing. The camera was combined with a pulsable "405-66-60" $405 \mathrm{~nm}$ LED from Roithner Lasertechnik (Vienna, Austria). The excitation light was filtered through a "BG12" excitation filter (Schott; Mainz, Germany). Emission filters were adapted to the luminescent dye and are mentioned below in the TSP- and PSP-specific setup section. The residual instrumentations for temperature imaging and oxygen imaging are also described in the respective sections.

\section{Setup for TSP Imaging}

A schematic drawing of the experimental setup for temperature imaging on quartz disks is shown in Fig. 3-24. The imaging instrumentation comprises the $405 \mathrm{~nm}$ excitation LED (E) with BG 12 excitation filter (F), a cooled CCD camera ( $G$ ) with a D610/6oM band-pass emission filter ( $H$ ) (Chroma; Rockingham, 
VT, USA), and a trigger box (A). The latter is connected with a PC and triggers the LED excitation with the CCD shutter, based on the settings in the imaging software.

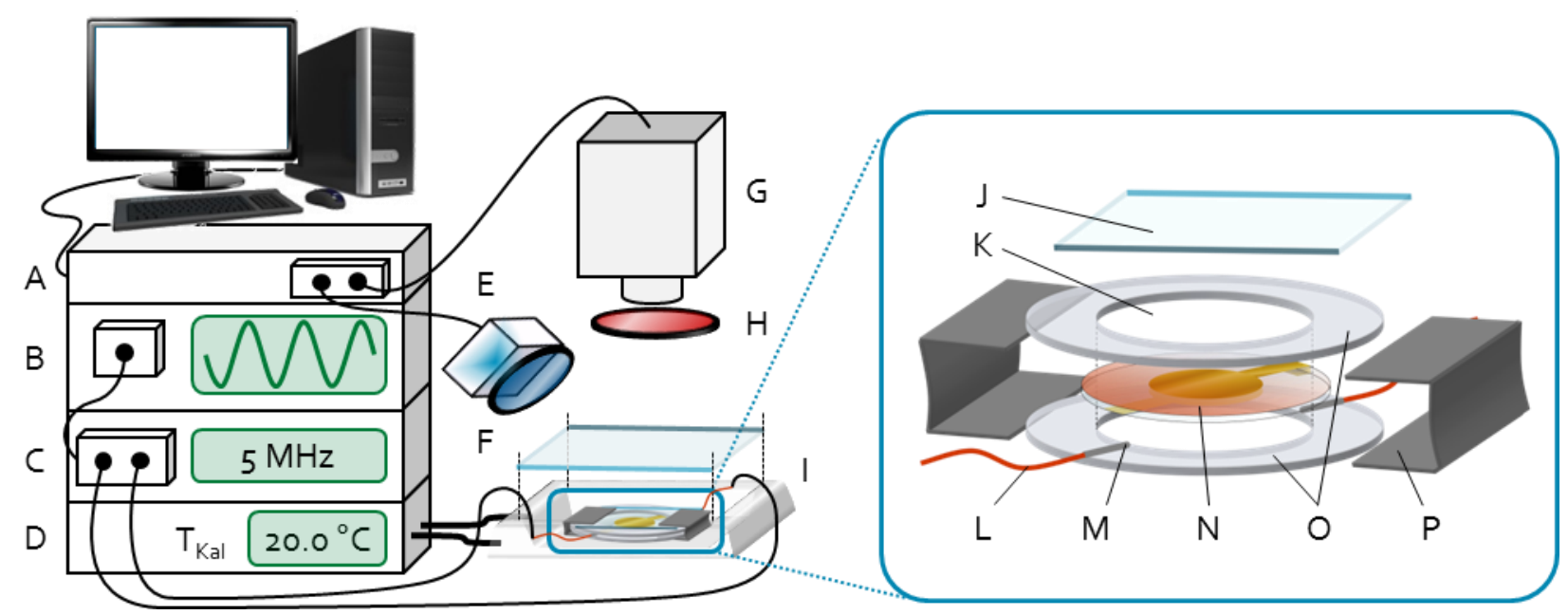

Fig. 3-24 Experimental setup for FLIM of a temperature-sensitive probe on an oscillating quartz resonator with (A) trigger box, (B) oscilloscope, (C) voltage supply, (D) temperature control, (E) LED, (F) BG 12 excitation filter, (G) cooled CCD camera, (H) D610/6oM emission filter, and (I) calibration chamber with optical window. In the miniaturized OCM chamber the TSP-coated quartz disk $(\mathrm{N})$ is sandwiched between two PDMS mats with circular holes $(\mathrm{O})$. A glass slide $(\mathrm{J})$ on the top PDMS mat forms a narrow cavity $(K)$ above the quartz. The whole QCM chamber is held together by two fixing clamps $(P)$, and the quartz electrodes were electrically addressed via conductive silver paint (M) and copper wires (L).

In order to be able to do temperature imaging and oscillation analysis of the quartz resonator together, a custom-made miniaturized OCM chamber was developed, in which the quartz disk was mounted and electrically contacted. Moreover, the miniaturized OCM chamber was small enough to be placed in a small temperature-controlled calibration chamber (I), which comprised an optical window that was transparent for excitation as well as for emission light. Temperature control (D) was necessary for calibration of the luminescence lifetime at defined temperature values. For experiments with the oscillating quartz, a constant ambient temperature was crucial for accurate FLIM of the TSP, too. The temperature of the calibration chamber was controlled by an "E100 + RE104" thermostat (Lauda; LaudaKönigshofen, Germany). A temperature sensor was additionally introduced inside the calibration chamber for temperature control. An exploded view drawing of the miniaturized QCM chamber is shown on the right hand side in Fig. 3-24. The OCM chamber consists of two PDMS mats (O), with a circular hole in the middle $(\varnothing=11 \mathrm{~mm})$, where the TSP-coated quartz disk $(N)$ was mounted in between. The PDMS mats were prepared with "Sylgard ${ }^{\circledR} 182$ silicone elastomer kit" (Dow Corning; Midland, MI, USA). Above the upper PDMS mat, a thin glass plate $(J)$ was integrated. This was necessary to form a sealed measurement chamber $(K)$ on the one hand, and to uniformly distribute the pressure of the fixing clamps $(P)$ over the entire quartz on the other hand. By this, a strain-less clamping of the quartz disk for unhindered oscillation was assured. Electrical connection of the quartz disk from the edge of the chamber was achieved by conductive silver paint paths on the lower PDMS mat (M). Copper wires (L) guided this connection to the outside of the calibration chamber, where they were plugged to the corresponding electronics (C), which was a "33120A 15 MHz Function/ Arbitrary Waveform Generator" (Hewlett-Packard; Palo Alto, CA, USA). With this, the quartz could be forced to oscillation, by applying a 
sinusoidal AC voltage of defined frequency and amplitude. Additionally, an "oscilloscope HM 1005" (HAMEG; Frankfurt/ Main, Germany) was switched in between, via a switchable resistance in series, for checking the current flow in the network and thus the resonance frequency of the quartz crystal $(B)$. The whole setup was placed in a dark room, as light pollution had to be avoided during the imaging. A picture of the TSP imaging setup is shown in SI 6 (p. 264)

\section{Setup for PSP Imaging}

The PSP imaging setup and the instrumentation needed for the oxygen calibration of the PSP are illustrated in Fig. 3-25. It comprises a personal computer with the "Imagex TGi" software for controlling the LED excitation light pulse $(H)$ and the shutter of the CCD camera $(D)$ via the trigger box $(A)$. For calibration of the PSP film, defined partial pressures of oxygen were streamed over the sensor, controlled by a gas mixing device $(B)$. In order to avoid temperature-quenching effects, the sensor was placed in a chamber (F) with temperature control (C). The LED excitation light was filtered through a BG12 filter (G), and fluorescence emission of the PtTFPP was filtered through a 630/75 band-pass filter (E). The vertical arrangement of the imaging components comprised some advantages. Using this arrangement, the camera could be brought as close as possible to the sensor surface without steric hindrance of the excitation light source, for the purpose of maximizing lateral resolution along the imaged surface. Illumination of the sensor from below was achieved by a hole in the temperature block. The close proximity of sensor and LED allowed a homogeneous and bright excitation. The imaging setup was further refined by mounting the CCD camera on the camera port of an "Axiotech vario" upright microscope (Carl Zeiss Microscopy GmbH; Jena, Germany) and using a 5-fold objective from Zeiss (EC Epiplan-NEOFLUAR, $5 \times / 0.13 \mathrm{HD}$, working distance $=14.5 \mathrm{~mm}$ ) for magnification. A picture of the PSP imaging setup with microscope is shown in $S I 7$ (p. 264). By this integration, the time-gated imaging setup was combined with the magnification properties of a microscope, resulting in an improved lateral resolution of the lifetime images.

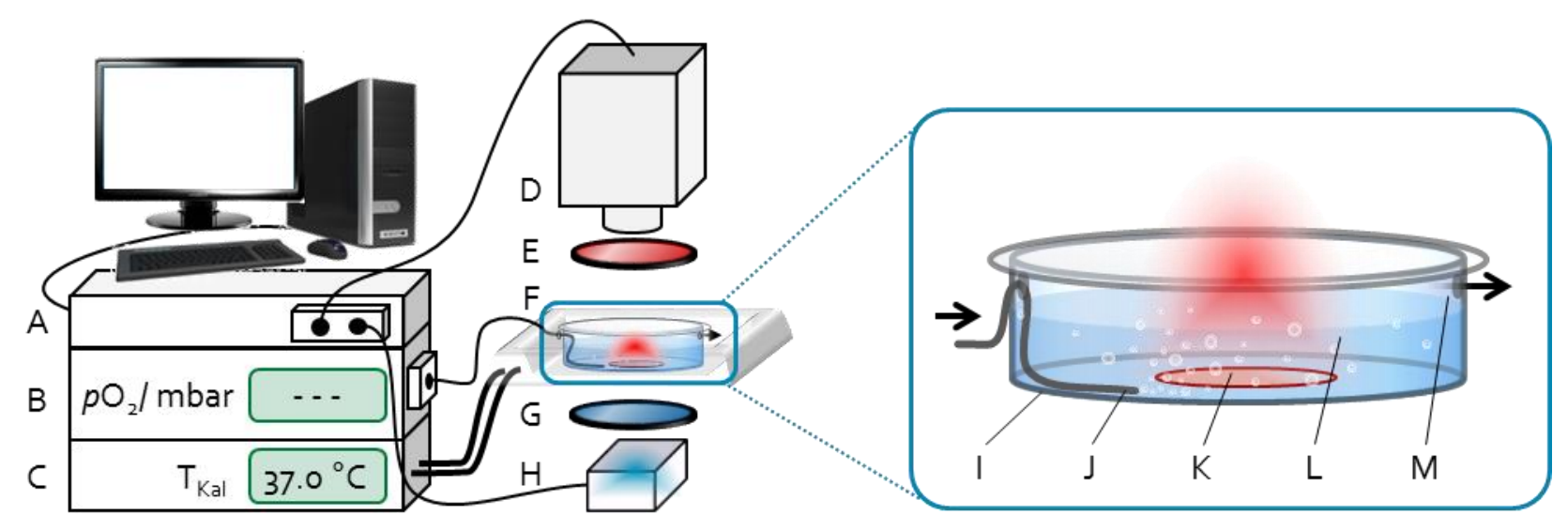

Fig. 3-25 Experimental setup for FLIM of an oxygen-sensitive probe. (A) Trigger box, (B) gas mixing device, $(C)$ temperature control, $(D)$ cooled CCD camera, $(E) 630 / 75$ emission filter, $(F)$ temperature calibration chamber, (G) BG 12 excitation filter, $(\mathrm{H})$ pulseable $405 \mathrm{~nm}$ LED. A small petri dish with sealed lid (I) served as measurement chamber for the PSP-coated glass substrate (K), immersed in a liquid (L). For calibration of the PSP with defined partial pressures of oxygen, the petri dish was equipped with a hose for gas inlet $(\mathrm{J})$, coming from the gas mixing device, and with an opposing small hole for gas outlet (M). 
Calibrations of PSP-coated glass disks were performed in a modified small petri dish (I) (enlarged in Fig. 3-25). The lid of the petri dish was sealed, in order to avoid air (oxygen) diffusion into the chamber. Two small holes were introduced into the chamber for having a defined gas inlet and outlet (M). The gas inlet was equipped with a thin hose $(J)$ that was plugged to the gas mixing device. Thus, defined partial pressures of oxygen $p \mathrm{O}_{2}$ could be flowed over the PSP-coated substrate (K), for calibration under liquid or air (L).

\section{Software}

For all imaging measurements the software "ImageX TGi v4.0" was used (Photonic Research Systems; Newhaven, UK). It allowed to individually control the exposure time and the time gating settings for up to 16 consecutive images of an image series. The time gating settings for the temperature- and oxygensensitive probes are listed in Tab. 3-7.

The settings were adjusted in a way that gate $A_{1}$ and gate $A_{2}$ were measured in consecutive images (cf. RLD in Fig. 3-22). Since the software allowed taking automatically 15 images in series, seven pairs of $A_{1}$ $A_{2}$ images and one blank image, where excitation light was not switched on and thus background fluorescence was measured, were taken in one series of images. First, three $A_{1}-A_{2}$ pairs were taken (images 1-6), followed by a blank image (image 7) and again four pairs of $A_{1}$ and $A_{1}$ images (images 8 -15). The images were processed with a software-integrated maths-script editor for image calculations. The software also provided an area-tool with which regions of interest (ROIs) were defined in the recorded images and which displayed the integrated intensity value for each ROI. Single images were exported as 16-bit TIFF files and further processed with the image editing software ImageJ [330,331] (developed by Wayne Rasband at the National Institutes of Health, USA).

Tab. 3-7 Time gating parameters for FLIM (RLD method) of the temperature sensitive-probes $\mathrm{Eu}(\mathrm{dnm})_{3}(\text { topo })_{2}$ and $\mathrm{Eu}(\text { benzac })_{3}$ (phen), and the oxygen-sensitive probe PtTFPP.

\begin{tabular}{lccc}
\hline & Eu(dnm) $)_{3}$ (topo $)_{2}$ & Eu(benzac) $)_{3}$ (phen) & PtTFPP \\
\hline Excitation pulse/ $\mu \mathrm{s}$ & 400 & 400 & 100 \\
Delay $\mathrm{A} 1\left(t_{1}\right) / \mu \mathrm{s}$ & 1 & 1 & 1 \\
Width $\mathrm{A} 1\left(\Delta t_{1}\right) / \mu \mathrm{s}$ & 80 & 120 & 40 \\
Delay $\mathrm{A} 2\left(t_{2}\right) / \mu \mathrm{s}$ & 41 & 241 & 21 \\
Width $\mathrm{A}_{2}\left(\Delta t_{2}\right) / \mu \mathrm{s}$ & 80 & 120 & 40 \\
Exposure time/ms & $1000-2100$ & 2100 & $1500-2000$ \\
Exposure frequency/ $\mathrm{Hz}$ & 1000 & 1000 & 5000 \\
\hline
\end{tabular}

\section{Experimental Implementation of $\mathrm{T}$ and $\mathrm{pO}_{2}$ Lifetime Imaging}

\section{Temperature Imaging on Oscillating Quartz Resonators}

Prior and after coating of a quartz disk with the TSP, the oscillation properties were determined by measuring an impedance spectrum close to the resonance frequency in the standard OCM chamber (cf. Fig. 3-13). By this, the oscillation properties of the quartz could be checked, and the resonance frequency could be determined. The resonance frequency was needed for exciting the quartz to resonant oscillation during temperature imaging experiments. Moreover, it was needed for the determination of 
the TSP thickness on the quartz, according to the Saverbrey relationship (Eq. (23)). QCM readings were performed, according to the experimental procedure described in subsection 3.3.3 (p. 45 ff.). The TSPcoated quartz disc was then mounted in the miniaturized OCM chamber for temperature imaging (Fig. 324). The impedance spectrum was recorded again with the impedance analyzer, in order to check the contacting and to verify the resonance of the quartz in the custom-made chamber, since the different mountings might have an influence on the oscillation. When temperature under liquid loading of the quartz resonator was to be investigated, the small cavity between the quartz and the glass slide (Fig. 3$24 \mathrm{~K}$ ) was filled with $550 \mu \mathrm{L}$ PBS buffer. The impedance spectrum of the quartz under liquid loading was then recorded, too. Afterwards, the OCM chamber was placed in the calibration chamber and connected with the frequency generator, according to Fig. 3-24. For temperature imaging of the oscillating quartz, a constant ambient temperature of $20^{\circ} \mathrm{C}$ or $37^{\circ} \mathrm{C}$ was adjusted in the calibration chamber. The equilibration duration was $\sim 20 \mathrm{~min}$ for measurements in air (at $20^{\circ} \mathrm{C}$ ), and $>45 \mathrm{~min}$ for measurements of the resonator in liquid (at $20^{\circ} \mathrm{C}$ and at $37^{\circ} \mathrm{C}$ ). The quartz was forced to oscillation at its resonance frequency and at increasing voltage amplitudes in the range of $0.15-10 \mathrm{~V}$. The quartz oscillated at least for $15 \mathrm{~min}$ at the specified voltage before imaging the luminescence lifetime of the TSP on the resonator. Additionally to that, the temperature was also imaged at $10 \mathrm{~V}$ at an "off resonance" driving frequency, which means at a frequency that was shifted by $-30 \mathrm{kHz}(-100 \mathrm{kHz})$ from the resonance frequency. The applied gate settings for image acquisition are listed in Tab. 3-7.

For calibration of a TSP, its fluorescence lifetime was imaged for a range of defined temperatures. This was $10-55^{\circ} \mathrm{C}$ for TSP imaging of the quartz oscillation in air (at $20^{\circ} \mathrm{C}$ ). For TSP imaging of the quartz oscillation under liquid loading, the temperatures for calibration were confined to $19.5-23.5^{\circ} \mathrm{C}$ (for imaging at $20^{\circ} \mathrm{C}$ ) and to $36.5-40.5^{\circ} \mathrm{C}$ (for imaging at $37^{\circ} \mathrm{C}$ ).

\section{Oxygen Imaging beneath Confluent Cell Layers}

After PSP-coating and drying, the cover glasses were fixed at the bottom of a petri dish using silicon glue. The substrates were sterilized in an argon plasma for $1 \mathrm{~min}$ and the polymer was allowed to swell in serum-free medium under sterile conditions in an incubator $\left(37^{\circ} \mathrm{C}, 5 \% \mathrm{CO}_{2}\right)$ for at least $20 \mathrm{~h}$. Cells were seeded in the petri dish $\left(\varnothing=9.04 \mathrm{~cm}^{2}\right)$ with the PSP-coated substrate at a seeding density of 450000 cells $/ \mathrm{cm}^{2}$. The day after seeding cells, cell coverage of the substrate was microscopically verified (Nikon Diaphot Inverted Tissue Culture Microscope; Tokyo, Japan) and documented (Nikon D500o DSLR camera; Tokyo, Japan). For oxygen imaging, cell culture medium was removed, and after washing twice, the petri dish was completely filled with $10 \mathrm{~mL}$ of $\mathrm{PBS}^{++}$, supplemented with $1 \mathrm{~g} / \mathrm{L}$ glucose $\left(\mathrm{PBS}^{++} / \mathrm{Glu}\right)$. The petri dish was subsequently sealed with the lid, which possessed a small hole for liquid exchange. The sensor chamber with the PSP- and cell-covered substrate was placed in a temperature-controlled chamber $\left(37^{\circ} \mathrm{C}\right)$ for FLIM. Injection of a test substance that was tested for its impact on cellular oxygen consumption was performed via a syringe with cannula, by replacing $5 \mathrm{~mL} \mathrm{PBS}{ }^{++} / G l u$ by $5 \mathrm{~mL}$ of a prewarmed test solution, double-concentrated in $\mathrm{PBS}^{++} / \mathrm{Glu}$. The time gate settings for image acquisition are listed in Tab. 3-7. Imaging experiments were usually followed by a microscopic inspection of the cell state.

Each PSP sensor was calibrated, in order to correlate the imaged luminescence lifetime information with a defined partial pressure of oxygen. For calibration, a gas mixture of defined oxygen content was applied to the calibration chamber via a small hose. This was done for the dry sensor by continuously flowing the gas through the chamber. In case of sensor calibration in liquid, the gas was either streamed through the headspace above the liquid or bubbled through the liquid. In both cases, gas was streamed through the chamber until an oxygen equilibration between air and liquid as well as liquid and sensor matrix was 
achieved. The total pressure of the gas flow was set to 1 bar, and the oxygen partial pressure was adjusted to $p \mathrm{O}_{2}=0-1000$ mbar, whereas the complementary gas was either argon ( $\mathrm{Ar}$ ) or nitrogen $\left(\mathrm{N}_{2}\right)$. In cases of sensor calibration after a cell experiment, the cells were previously removed from the sensor surface by rinsing twice with PBS $^{--}$, and subsequent EDTA/ trypsinization treatment.

\section{Data processing}

\section{FLIM of the TSP}

For each imaging time, seven $A_{1}$ images and seven $A_{2}$ images, as well as one blank image $A_{B}$ were obtained. Thereby, $A_{1}\left(A_{2}\right)$ represented the integrated intensity image of gate $\mathrm{A}_{1}\left(\mathrm{~A}_{2}\right)$ in the decay phase of the fluorophore (Fig. 3-22), and $A_{B}$ is an integrated intensity image without previous excitation (background fluorescence). $A_{1}, A_{2}$, and $A_{B}$ images of one image series were subsequently processed by using the software-integrated maths-script editor for image calculations. Following this procedure, images were firstly background corrected by subtracting $A_{B}$ from all $14 A_{1}$ and $A_{2}$ images (SI 8, Maths Script \#1, p. 265). Using the area-tool in the Imagex TGi measurement software, a region of interest (ROI) was defined that was the same for all images of a series. Within the ROI the integrated intensity value was extracted for every image. Based on these values, the ratio $A_{1} / A_{2}$ was calculated for each of the seven consecutively measured pairs of $A_{1}$ and $A_{2}$. Hence, seven $A_{1} / A_{2}$ ratios were obtained, which were subsequently averaged. Calibration data points were fitted either linearly (calibrations in liquid) or by an exponential fitting function (calibrations in air), dependent on the temperature range. Based on the obtained temperature- $A_{1} / A_{2}$ relationships, the temperature value was calculated for $A_{1} / A_{2}$ ratios of an imaging experiment. All experiments were repeated at least five times, in order to obtain sufficient high confidence levels.

\section{FLIM of the PSP}

Every single measurement point from FLIM of an oxygen-sensitive probe is calculated from 15 individually taken lifetime images. Intensity images of gate $A_{1}$ and gate $A_{2}$ were photographed consecutively three times. Afterwards, a blank image $A_{B}$ (FLIM w/o excitation) was taken, followed by another four times of FLIM of $A_{1}$ and $A_{2}$. The recorded images $A_{1}, A_{2}$, and $A_{B}$ were subsequently processed by using the Imagex TGi software-integrated math script for image calculations (SI 8, Maths Script \#2). Each image $A_{1}$ and $A_{2}$ was at first corrected by the blank image $A_{B}$, followed by averaging all seven corrected images of $A_{1}$ and $A_{2}$, respectively. The averaging was implemented in the math script by firstly multiplying each $A_{1}\left(A_{2}\right)$ image with $1 / 7$ and by subsequently summing up these seven $A_{1}\left(A_{2}\right)$ images. In the last step the ratio $A_{1} / A_{2}$ is calculated with the respective averaged images of $A_{1}$ and $A_{2}$. $A_{1} / A_{2}$ values obtained from calibration measurements were plotted as a function of oxygen pressure and data points were fitted by a suitable mathematical function that describes the relationship best (highest coefficient of determination, $R^{2}$ ), however does not follow a theoretical model. The obtained oxygen- $A_{1} / A_{2}$ correlation was used for converting $A_{1} / A_{2}$ ratios from cell experiments into respective oxygen concentrations.

It is important to note that the obtained oxygen concentrations and molar fractions are related to the gas composition in the headspace of the sensor. In order to obtain the actual oxygen concentrations in the liquid, Henry's law has to be applied. However, it has to be adapted due to the deviations from standard conditions, regarding temperature $\left(37^{\circ} \mathrm{C}\right.$ instead of $20^{\circ} \mathrm{C}$ ) and electrolyte concentration (PBS ${ }^{++}$ buffer instead of water). 


\subsection{Light Microscopic Techniques}

Different light microscopy techniques were used in this work, comprising stereo microscopy, phasecontrast microscopy, and the luminescence-based epi-fluorescence microscopy and confocal laser scanning microscopy (CLSM). Light microscopy was used for controlling the properties of experimental surface structures and for the validation and documentation of cell cultures under test. For instance, the degree of cell coverage on cell culture flasks or on sensor substrates was routinely checked. Moreover, staining of specific cellular structures combined with luminescence-based microscopy was utilized for supporting conclusions from ECIS- and QCM-measurements. An overview on the microscopes used in this work and the respective specifications is listed in Tab. 3-8.

Tab. 3-8 List of light microscopes that were used in this work.

\begin{tabular}{|c|c|c|c|}
\hline Microscope & Setup & Microscopic Method & Objectives* \\
\hline Nikon Diaphot & inverted & $\begin{array}{l}\text { Phase-Contrast } \\
\text { Epi-Fluorescence }\end{array}$ & $\begin{array}{l}\text { Plan 4x/ 0.13, PhL } \\
\text { Plan 10x/ 0.25, Ph1 } \\
\text { Plan 20x/ 0.40, Ph2 }\end{array}$ \\
\hline Nikon Eclipse goi & upright & $\begin{array}{l}\text { Phase-Contrast } \\
\text { Epi-Fluorescence } \\
\text { CLSM }\end{array}$ & $\begin{array}{l}\text { Plan 10x/ 0.25, Ph1, } \infty /-, \text { WD } 10.5 \\
\text { Plan 20x/0.40, Ph1, } \infty / 0.17, \text { WD } 1.2 \\
\text { LU Plan ELWD 50x/ 0.55 B, } \infty / 0, \text { EPI } \\
\text { NIR Apo 6ox/ 1.0W, DIC N2, } \infty / 0, \text { WD } 2.8\end{array}$ \\
\hline Nikon SMZ 1500 & upright & Stereo & Plan 1X \\
\hline
\end{tabular}

*type and magnification/ NA, immersion, contrast method, lens tube length/cover glass thickness, working distance

The following sections address the basic principles of the light microscopy techniques used here. Moreover, staining strategies of specific structural compounds of cells will be presented. The specific labeling of cellular structures with luminescent molecules allowed for their analysis by epi-fluorescence microscopy as well as by CLSM.

\subsubsection{Phase-Contrast Microscopy}

The principle of making thin, almost transparent, and unstained objects visible by phase-contrast microscopy was first described by Zernike, $1934 .{ }^{[332]}$ By nature, the human eye can only distinguish between structures that have a different color (variations in wave frequency) or which differ sufficiently in light intensity (variations in wave amplitude). However, very thin specimens like cells, of only $1-10 \mu m$ in height, absorb almost no visible light. Such specimens show almost no contrast in bright-, or in darkfield microscopy. This means that the amplitude of the background light cannot be distinguished from the slightly attenuated amplitude of light that has passed through the specimen. The contrast of the specimen $(C)$ is defined as the difference of background intensity $\left(I_{B}\right)$ and specimen intensity $\left(I_{S}\right)$, related to the overall background intensity:

$$
C=\frac{I_{S}-I_{B}}{I_{B}} \cdot 100[\%]
$$


Cells absorb not enough light, to reduce the amplitude of the diffracted light like an amplitude specimen, and to be visible without contrast enhancement. However, the speed of light that passes through a cell is retarded in comparison to the non-diffracted direct background light. Hence, the diffracted specimen light and the direct background light exhibit a phase shift after passing the object, which can be detected in the image plane. The phase shift $\delta$ is a function of the wavelength $\lambda$ of the incident light, of the thickness $t$ of the object, and of the refractive index difference between the specimen $\left(n_{2}\right)$ and the surrounding medium $\left(n_{1}\right)$. The correlation is given by:

$$
\delta=2 \pi \frac{\Delta}{\lambda}=2 \pi \frac{\left(n_{2}-n_{1}\right) \cdot t}{\lambda}
$$

where $n_{i} \cdot t$ is the optical path length (OPL) in a medium $i$, and $\Delta=\left(n_{2}-n_{1}\right) \cdot t$ is referred to as the optical path difference (OPD) between specimen and surrounding medium. The phase shift caused by a cell layer immersed in medium can be approximated to $\pi / 2\left(\Delta=\lambda / 4\right.$; with $t=5 \mu \mathrm{m}, n_{2}=1.36$, $n_{1}=1.335$, and $\lambda=500 \mathrm{~nm}$ (green light)).

Zernicke found a way to optically convert the phase shift of the light into an amplitude difference of the light in the image plane, resulting in an enhanced contrast of the image. For obtaining phase-contrast images two optically conjugated components have to be placed in the light beam of a brightfield microscope, a condenser annulus between light source and specimen, and a phase plate in the rear focal plane of the objective (Fig. 3-26). The condenser annulus causes a separation of diffracted and undeviated light emerging from the specimen in the objective rear focal plane. Thereby, the direct (undeviated) light is focused on the ring of the phase plate. By passing this ring, the direct light is both reduced in intensity (amplitude) and phase shifted, which is in contrast to the diffracted light that passes the phase plate unaltered. The phase of the surrounding light is either advanced (positive/ dark phasecontrast) or retarded (negative/ bright phase-contrast) by $\Delta=\lambda / 4$, when passing through the phase plate ring. When the direct and the diffracted light beam are reunited behind the phase plate, they exhibit then a net phase shift of either $180^{\circ}$ (destructive interference) or zero degrees (constructive interference) with each other. Focused in the image plane, the interferences of the reunited light beams generate a phase-contrast image of the specimen, which can be observed in the ocular. For instance, a destructive interference of diffracted and direct light let the details of the specimen appear dark against the brighter background (positive/ dark phase-contrast). ${ }^{[333-335]}$

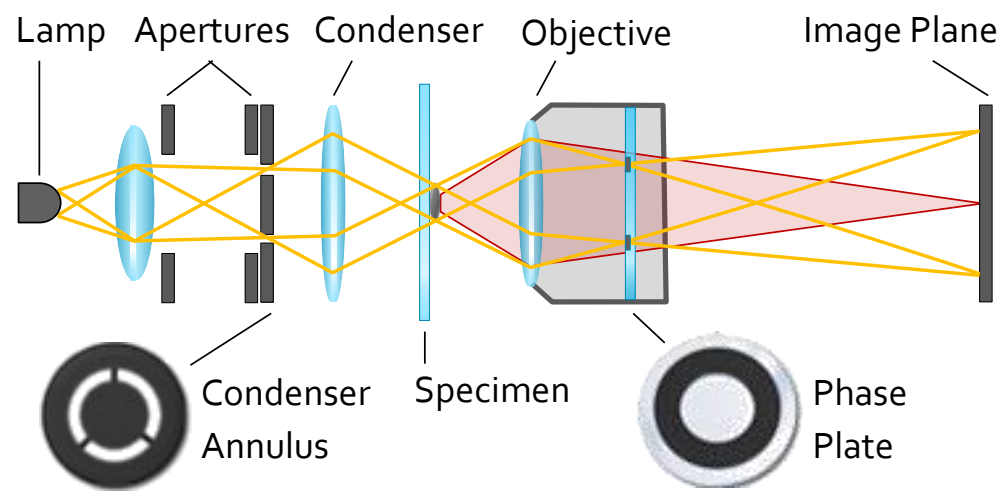

Fig. 3-26 Schematic illustration of the crucial components in a phase-contrast microscope together with the light path necessary to generate a phase-contrast image of a specimen in the image plane (modified after ${ }^{[333]}$ ). The undeviated light path is shown in yellow and light that is deflected when passing through the specimen is illustrated in red. 


\subsubsection{Stereomicroscopy}

Stereomicroscopes aim to generate a three-dimensional view of a given specimen. This makes it superior to other microscopic techniques in applications where a 3-D-observation and a view of depth are necessary for interpreting structures of a specimen. Moreover, stereomicroscopes have a magnification range up to $500 x$ (depending on zoom factor, eyepiece, and objective) in combination with large working distances of $2-20 \mathrm{~cm}$, which provides a comfortable working room between the specimen and the objective.

When observing a specimen through the eyepieces of a stereomicroscope, two separate light paths from the objective to the eyepieces provide the spatial impression and the perception of depth. The two pathways collect light from the specimen in slightly different angles $\left(10-20^{\circ}\right)$. Thus, the objective is observed through the left and the right eyepiece from slightly different perspectives. Due to the human ability of stereoscopic viewing, the brain is able to combine these two marginally different pictures of the object under observation to one three-dimensional picture with spatial depth.

In this work a "SMZ 1500" stereomicroscope (Nikon; Tokyo, Japan) with zoom factor range of $0.75-$ $11.25 \mathrm{X}$ was used for the survey of structures on sensor surfaces. A total magnification of $112.5 \mathrm{X}$ was achieved by the $1 x$ objective (Nikon Plan 1X), 10x eyepieces (Nikon C-W 10xB/22), and by using the maximum zoom factor of $11.25 x$. With the microscopes' specifications, depth of field values/ numerical apertures between $26 \mu \mathrm{m} / 0.131$ (zoom factor 11.25x) and $1.345 \mathrm{~mm} / 0.023$ (zoom factor 0.75x) were obtained. The stereomicroscope was equipped with a Nikon Coolpix 990 camera for digital imaging and documentation. ${ }^{[336,337]}$

\subsubsection{Fluorescence Microscopy}

Fluorescence microscopy is routinely used in bio-analytical sciences and has become an indispensable technique for studying the structural properties of cells. There are different strategies how fluorescence can be utilized for investigations of cells by means of fluorescence microscopy. One approach is based on the specific binding of probe molecules towards a target molecule that is located in a particular region or structural element of the cell. When labeling such specific binding molecules with a fluorophore a fluorescent probe can be directed to the subcellular structure of interest. The localization of the specific molecules and, consequently, of the subcellular structures they have bound to can be visualized and mapped by exciting the dye and imaging the fluorescence emission by means of fluorescence microscopy. This is mainly achieved by using fluorescence-labeled antibodies that specifically bind to the corresponding antigens in the cell (immunostaining). Another strategy aims on the in situ generation of fluorescent molecules in the cells. For instance, the absorption and emission spectra of a certain class of molecules is altered by intercalation in the DNA helix in a way that they show fluorescence emission in the visible range. Moreover, fluorophores can be generated within cells by employing enzymes. In an enzymatic reaction, a fluorescent dye can be formed from a non-fluorescent precursor molecule as substrate. This concept is applied in a variety of assays formats for visualizing enzyme activity in cells, which serves as reporter for cell viability.

The basic concepts of light absorption and fluorescence emission were previously described in section 3.5.1 (p. 62). Most fluorescence microscopes are used in reflected-light mode, with a collinear optical path for illuminating the object and for collecting fluorescence from the specimen. This is schematically illustrated for an upright epifluorescence microscope in Fig. 3-27, with excitation and luminescence detection of the object from the top side. Fluorescence microscopy requires a separation of excitation 
and emission light. This is achieved by an arrangement of optical filters, consisting of an excitation filter, a dichroitic mirror, and an emission filter (Fig. 3-27). The filter set has to be adapted to the spectral properties of the fluorophore that is to be detected. Excitation light from the light source is at first guided through an excitation filter. Thus, longer and redundant wavelengths for the excitation are cut off, in order to avoid stray light from the light source in the emission range of the dye. The dichroitic mirror (DM) is chosen to be reflective for the short-wavelength excitation light but transparent for the longwavelength fluorescence emission of the dye. Hence, excitation light is reflected at the DM by $90^{\circ}$ towards the object, whereas the induced fluorescence emission from the specimen straightly passes the DM. After passing an additional barrier (emission) filter, the emitted fluorescence is focused in the image plane. The 2-D fluorescence pattern can be observed by eyepieces and imaged with a digital camera.

The fluorescence microscope used in this study, a Nikon Eclipse goi, was equipped with three different filter sets, allowing for excitation of fluorophores in the UV, blue, and green range of the electromagnetic spectrum. The respective filter sets and the spectral properties of the excitation filters, the DMs, and the emission filters are listed in Tab. 3-9.

Tab. 3-9 List of filter sets that were used for fluorescence microscopy with collinear optical paths of excitation and emission light. With these filter sets fluorophores could be excited in the UV, blue, and green spectral range and the respective emission detected in the blue, green, and red channel. The channel color indicates the spectral range of the emission filter and denotes the color used for pseudo-color presentation of the fluorescence images.

\begin{tabular}{lcccc}
\hline Filter set & Excitation filter/nm & Dichroitic mirror/nm & Barrier filter/nm & Channel color \\
\hline Nikon UV-2E/C (DAPI) & $340-380$ & 400 & $435-485$ & blue \\
Nikon B-2E/C (FITC) & $465-495$ & 505 & $515-555$ & green \\
Nikon G-2E/C (TRITC)* & $540 / 25$ & 565 & $605 / 55$ & red \\
\hline
\end{tabular}

*the used barrier filter deviates from that in the original Nikon G-2E/C filter set, which contains a $620 / 60$ nm bandpass filter.

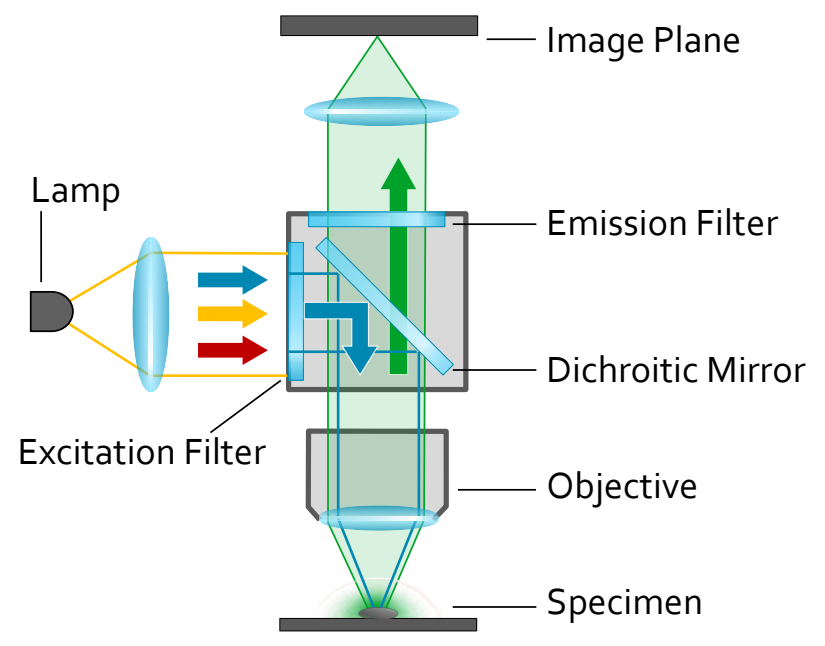

Fig. 3-27 Schematic illustration of the excitation and emission beam path through the filters of an epifluorescence microscope. The excitation filter cuts off long-wavelength light coming from the polychromatic light source (indicated by the blue, yellow, and red arrow). At the dichroitic mirror, the excitation light is reflected to the specimen (blue arrow). The induced fluorescence emission (green arrow) passes the DM, since it is transparent for long-wavelength light. The fluorescence light is finally guided through an emission filter and focused in the image plane. 


\subsubsection{Confocal Laser Scanning Microscopy (CLSM)}

The main characteristic of a confocal laser scanning microscope (CLSM) is the punctual scanning of fluorescence excitataion, which is in contrast to the wide-field imaging of fluorescence by an epifluorescence microscope. With CLSM, fluorescence is recorded from a defined and narrow focal plane, while stray light and background fluorescence from the out-of-focus plane is suppressed by pinhole apertures in the light path. Hence, CLSM images exhibit a better resolution compared to fluorescence images obtained by wide-field fluorescence microscopy. Collecting the fluorescence from defined optical planes in CLSM also allows for recording the fluorescence in optical sections, so-called z-stacks, from different focal planes of the specimen under investigation. After recording, the optical sections can be re-assembled to a three-dimensional fluorescence image of the object on the computer. However, CLSM images cannot be instantaneously observed in the eyepieces like in wide-field fluorescence microscopy. The fluorescence image in CLSM is acquired by scanning point by point and line by line the specimen with the laser beam and collecting the fluorescence photons by a photomultiplier tube (PMT). This onedimensional fluorescence information is then assembled into a two-dimensional fluorescence image with the CLSM software on the computer.

The components and the optical path in a basic confocal laser scanning microscope are schematically illustrated in Fig. 3-28. The discrimination of fluorescence light in the focal plane from fluorescence emission out-of-focus is achieved by two (confocal) pinhole apertures, being arranged in front of the laser light source and in front of the PMT. Emission light from the in-focus plane can pass the pinhole. Thus, it can be detected by the PMT. Fluorescence light coming from the out-of-focus plane is not focused on the pinhole by the optics in the microscope. Hence, the out-of-focus photons do not pass the pinhole aperture to be detected by the PMT and, consequently, do not contribute to the final fluorescence image. Thus, background fluorescence is almost completely separated, resulting in high-resolution images of fluorescently labeled structures of the cell.

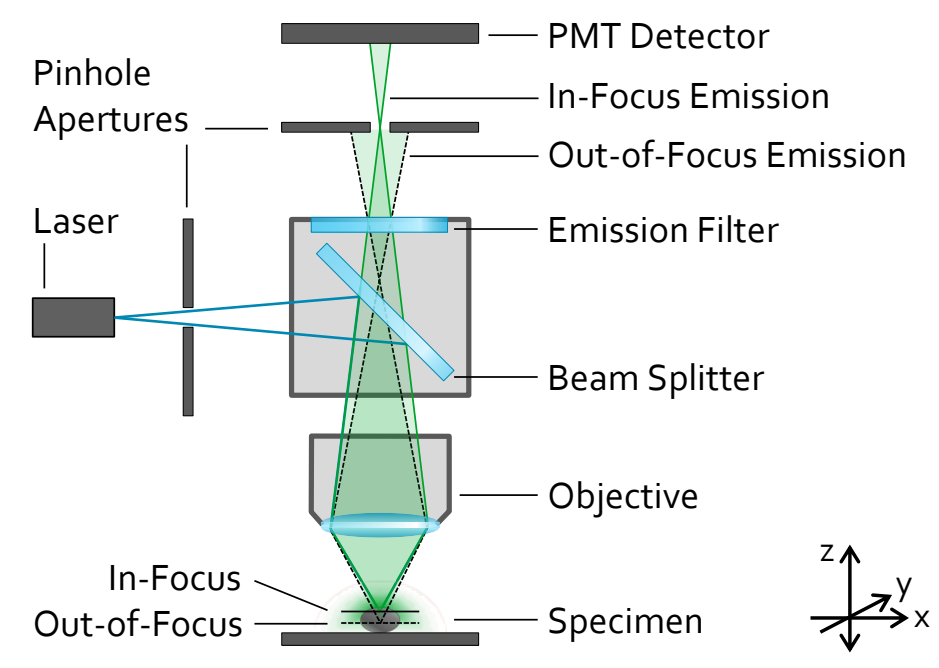

Fig. 3-28 Schematic drawing of the components, and the light path in a basic confocal laser scanning microscope (CLSM). Core components are the laser light source and the photomultiplier tube (PMT) detector in combination with two pinhole apertures. This allows for separating infocus and out-of-focus fluorescence emission from the specimen at the PMT. 
By increasing the pinhole diameter, the thickness of the focal plane from which fluorescence light is collected is increased. This causes an increase in the PMT current, higher fluorescence intensity, and consequently a better signal-to-noise ratio. However, an increasing pinhole diameter is accompanied by a loss in resolution and an increase in the depth of field. ${ }^{\left[33^{3-340]}\right.}$ The confocal microscope (Nikon Eclipse 9oi) was equipped with three different lasers, which allowed for excitation of fluorophores in the UV $(405 \mathrm{~nm})$, blue $(488 \mathrm{~nm})$, and green $(543 \mathrm{~nm})$ range of the electromagnetic spectrum. Fluorescence emission was discriminated by emission filters for blue (450/35), green (515/30), and red light (650 LP).

\subsubsection{Cytochemical Fluorescence Staining}

In order to investigate cell layers by fluorescence microscopy, cellular structures of interest had to be stained. Tab. 3-10 provides an overview on the cellular components, which were stained in this work, together with the probes used for staining and their spectral properties. The specified channel color in Tab. 3-10 indicates the spectral range in which the emission filter is transparent for light. In laser scanning microscopy fluorescence light is recorded by photon counting and the emission filter in the optical path solely provides spectral discrimination. Hence, the obtained fluorescence images are in fact greyscale images without wavelength (color) information. To cope with the wavelength of the collected photons, and for a better visualization, the grayscale images were pseudo-colorized on the computer with the respective channel color. In the following subsections, the experimental cytochemical staining protocols will be explained.

Tab. 3-10 Overview on cell structures and probes used for staining. For each dye the wavelengths of the absorption (Abs) and the emission (Em) maximum is listed. The channel color indicates the spectral range of the emission and denotes the colorization of greyscale images for pseudo-color presentation.

\begin{tabular}{llccc}
\hline Stained Cell Structure & Probe(s) & Abs/nm & Em/nm & Channel color \\
\hline Nuclei/ DNA & DAPI & 360 & 460 & blue \\
Cytoplasm (Live) & Calcein AM & 494 & 517 & green \\
Nuclei/ DNA (Dead) & Ethidium Homodimer-1 & 528 & 617 & red \\
Actin Filaments & Alexa Fluor ${ }^{\circledR} 488$ phalloidin & 495 & 518 & green \\
& TRITC phalloidin & 544 & 572 & red \\
Tight Junctions (ZO-1) & ZO-1 mouse monoclonal antibody & 556 & 573 & red \\
& + Alexa Fluor ${ }^{\circledR} 546$ rabbit anti-mouse IgG $(\mathrm{H}+\mathrm{L})$ & & & \\
\hline
\end{tabular}

\section{Nucleus Staining (DAPI)}

The nuclei of cells were visualized by the DNA-binding dye 4,6-diamidin-2-phenylindol (DAPI). The fluorescence emission intensity of DAPI is drastically increased ( $\sim 20$-fold) by its binding to AT clusters in the minor groove of a double-stranded DNA. DAPI can be excited by a mercury lamp or an UV laser, since it shows a broad absorption spectrum between $320-380 \mathrm{~nm}$, with an absorption maximum at $360 \mathrm{~nm} \cdot{ }^{[341,342]}$ Fluorescence occurs in the visible blue range with an emission maximum at $\lambda_{\mathrm{Em}}=460 \mathrm{~nm}$.

A DAPI stock solution (Sigma-Aldrich; St. Louis, MO, USA) of $100 \mathrm{ng} / \mathrm{mL}$ was prepared in deionized water for nuclei staining of adherently grown NRK or MDCK-II cells. The cells were fixed and permeabilized prior to staining, since DAPI exhibits only low membrane permeability. Fixation was 
performed at room temperature (RT) by incubating the cells for 10 min with $4 \%(\mathrm{w} / \mathrm{v})$ paraformaldehyde (PFA) in $\mathrm{PBS}^{++}$, followed by twofold washing with $\mathrm{PBS}^{++}$. Cells were subsequently permeabilized by incubation with $0.2 \%(\mathrm{v} / \mathrm{v})$ Triton-X-100 in $\mathrm{PBS}^{++}$for 10 min at RT. After two washing steps with $\mathrm{PBS}^{++}$, the cellular dsDNA was stained by incubating the cells with a 1:10 dilution (in $\mathrm{PBS}^{++}$) of the DAPI stock solution (10 ng/ $\mathrm{mL}$ ) for 2 min at RT. Unbound DAPI was removed by washing the cells three times with $\mathrm{PBS}^{++}$. DAPI was used as blue-emitting counterstain in combination with a green or/and a red emitting staining of other cellular structures.

\section{Actin Cytoskeleton Staining (phalloidin)}

Phalloidin is a toxin from the poisonous "death cap" mushroom (Amanita phalloides). It is known to bind specifically to filamentous actin ( $f$-actin), whereas it exhibits a much lower binding affinity to monomeric actin. The phallotoxin stabilizes $\mathrm{f}$-actin and inhibits the dynamic polymerization and depolymerization processes of actin filaments in the cells. This specific binding property of phalloidin is utilized for cellular imaging applications. For this, a fluorescent dye is covalently bound to phalloidin molecules. The actin cytoskeleton in the cells then can be visualized and imaged by exciting the phalloidin-linked dye and recording its emission with a fluorescence microscope. ${ }^{[343,344]}$ Two differently labeled phalloidins were used for $\mathrm{f}$-actin staining in cells. "Alexa Fluor ${ }^{\circledR} 488$ phalloidin" (Life Technologies; Carlsbad, CA, USA) served as green-emitting phalloidin derivative $\left(\lambda_{\mathrm{Exc}}=495 \mathrm{~nm} / \lambda_{\mathrm{Em}}=518 \mathrm{~nm}\right)$, whereas TRITC-phalloidin (Sigma-Aldrich; St. Louis, MO, USA) was applied as red-emitting f-actin marker $\left(\lambda_{\mathrm{Exc}}=544 \mathrm{~nm} /\right.$ $\left.\lambda_{\mathrm{Em}}=572 \mathrm{~nm}\right)$.

Tab. 3-11 Protocol to stain for $\mathrm{f}$-actin (TRITC-phalloidin) and DNA/ nuclei (DAPI).

\begin{tabular}{|c|c|c|c|c|c|c|}
\hline & Step & Rerun & Volume & Solution & Duration & Temp. \\
\hline 1 & Washing & $2 x$ & $500 \mu \mathrm{L}$ & $\mathrm{PBS}^{++}$ & & \\
\hline 2 & Fixation & & $500 \mu \mathrm{L}$ & PFA, $4 \%(w / v)$ in $\mathrm{PBS}^{++}$ & $10 \mathrm{~min}$ & RT \\
\hline 3 & Washing & $2 x$ & $500 \mu \mathrm{L}$ & $\mathrm{PBS}^{++}$ & & \\
\hline 4 & Permeabilization & & $500 \mu \mathrm{L}$ & Triton-X-100, $0.2 \%(v / v)$ in $\mathrm{PBS}^{++}$ & $10 \mathrm{~min}$ & RT \\
\hline 5 & Washing & $2 x$ & $500 \mu \mathrm{L}$ & $\mathrm{PBS}^{++}$ & & \\
\hline 6 & \multicolumn{6}{|c|}{ Transfer of cover glasses (12-well-plate $\rightarrow$ Parafilm) } \\
\hline 7 & Actin staining & & $200 \mu \mathrm{L}$ & TRITC-phalloidin, $3 \mu \mathrm{g} / \mathrm{mL}$ in $\mathrm{PBS}^{++}$ & $45 \min$ & RT \\
\hline 8 & Washing & $4 x$ & $200 \mu \mathrm{L}$ & $\mathrm{PBS}^{++}$ & & \\
\hline 9 & Nuclei staining & & $200 \mu \mathrm{L}$ & DAPI, 1:10 in $\mathrm{PBS}^{++}$ & $2 \min$ & RT \\
\hline 10 & Washing & $3 x$ & $200 \mu \mathrm{L}$ & $\mathrm{PBS}^{++}$ & & \\
\hline 11 & \multicolumn{6}{|c|}{ Transfer of cover glasses (Parafilm $\rightarrow$ petri dish $\left(\varnothing=9.04 \mathrm{~cm}^{2}\right)$ ) } \\
\hline 12 & Immersion & & $>2 \mathrm{~mL}$ & $\mathrm{PBS}^{++}$ & & \\
\hline 13 & Storage & & & & $1-2$ weeks & $6^{\circ} \mathrm{C}$ \\
\hline
\end{tabular}

Actin cytoskeleton labeling of either NRK or MDCK-II cells with TRITC-phalloidin was performed according to the staining protocol in Tab. 3-11. Cells were grown on small disc-shaped cover glasses in the wells of a 12-well tissue culture plate to investigate the stimulus of interest. Prior to staining, the stimulated cells were fixed and permeabilized, according to steps 1- 5 in Tab. 3-11. For staining the cells, the cover glasses at first were placed on a parafilm in a petri dish. Thereby, the hydrophobicity of the 
parafilm surface allowed to place small drops of staining solution on the cover glasses without spreading. Hence, volumes of the staining solutions could be minimized to $200 \mu \mathrm{L}$. Actin staining was performed by incubating the cells on each cover glass with $3 \mu \mathrm{g} / \mathrm{mL}$ of TRITC-phalloidin (in PBS $^{++}$) for 45 min at ambient temperature. Unbound phalloidin was removed by washing the cell layer four times with $\mathrm{PBS}^{++}$buffer. The protocol includes a blue-emitting counterstaining of the nuclei by DAPI, subsequent to the $f$-actin staining step. This was performed by incubating the cells with $200 \mu \mathrm{L}$ of a $1: 10$ dilution (in $\mathrm{PBS}^{++}$) of the DAPI stock solution $\left(c_{0}=10 \mathrm{ng} / \mathrm{mL}\right.$ ). After $\mathrm{f}$-actin and nuclei staining, the cover glasses were placed in small petri dishes, and immersed with buffer. Microscopic (Nikon Eclipse goi) observation was performed by means of a 6ox water-immersion objective. If protected from light and immersed under liquid, the stained samples could be stored in the fridge for $1-2$ weeks prior to the examination by microscopy.

F-actin staining of cells with the green-fluoresecent "Alexa Fluor ${ }^{\circledR} 488$ phalloidin" was applied in a threecolor staining together with DAPI (blue channel) and an orange-emitting (red channel) anti-ZO-1 staining (see Tight Junction Staining below and Tab. 3-12).

\section{Tight Junction Staining (ZO-1 Immunostaining)}

ZO-1 is a peripheral membrane protein that is, besides ZO-2, occluding, and the members of the claudin family, a major component of tight junctions (zonula occludens) in endothelia and epithelia of vertebrates. Tight junctions (TJ) seal the space between neighboring cells and serve as diffusion barrier along the paracellular pathways. Moreover, TJ are also responsible for the maintenance of epithelial cell polarity, since they prevent the lateral diffusion of membrane components between the apical and basal site of the cells. ${ }^{[345,346]}$ They are accessible to fluorescence microscopic examinations by a two-step immunostaining of the ZO-1 protein. Firstly, a "ZO-1 mouse monoclonal antibody" (Life Technologies; Carlsbad, CA, USA) was added to the cells. It is known to specifically bind to the ZO-1 protein in TJ. In a second incubation step, the fluorescence-labeled, secondary antibody "Alexa Fluor ${ }^{\circledR} 546$ rabbit antimouse IgG $(\mathrm{H}+\mathrm{L})^{\prime \prime}$ (Life Technologies; Carlsbad, CA, USA) was bound to the monoclonal anti-ZO-1 antibody. Thus, ZO- 1 can be visualized by the fluorescence of the "Alexa Fluor ${ }^{\circledR} 546^{6}$ " dye $\left(\lambda_{\mathrm{Exc}}=556 \mathrm{~nm} /\right.$ $\lambda_{E m}=573 \mathrm{~nm}$ ), which is detectable in the orange wavelength range.

The protocol for ZO-1 immunostaining is shown in Tab. 3-12. Staining for ZO-1 was applied in a triple staining together with staining for $\mathrm{f}$-actin, via "Alexa Fluor ${ }^{\circledR} 488$ phalloidin" $\left(\lambda_{E x c}=495 \mathrm{~nm} / \lambda_{\mathrm{Em}}=518 \mathrm{~nm}\right.$ ), and staining for DNA with DAPI $\left(\lambda_{E x c}=360 \mathrm{~nm} / \lambda_{E m}=460 \mathrm{~nm}\right)$. The fluorescence emissions of all three dyes was separated by the three optical filter sets (cf. Tab. 3-9), and the stained cellular structures were individually examined in the blue (DNA/ nuclei), green (f-actin), and red channel (ZO-1/ TJ). Cells were seeded in self-made cell culture wells for staining. These culture vessels were prepared by gluing the 8-well chamber of ECIS ${ }^{\circledR}$ arrays on the bottom of a tissue culture petri dish with silicone grease. By this, volumes for cell culture and the staining procedures could be minimized. After washing and fixing, the cells could be store for a few days under buffer in the fridge until resuming the staining protocol (steps 1-3 in Tab. 3-12). After the steps of permeabilization and blocking (steps $4-6$ in Tab. 3-12), the TJ of the cells were stained at first. The primary ZO-1 mouse monoclonal antibody was diluted 1:50 in PBS ${ }^{++}$ and was supplemented with $0.5 \%(\mathrm{w} / \mathrm{v})$ bovine serum albumin (BSA) before usage. The secondary, dyelabeled antibody was diluted 1:500 in $\mathrm{PBS}^{++}(\mathrm{w} / 0.5 \%(\mathrm{w} / \mathrm{v}) \mathrm{BSA})$ for incubation. It is noted that steps $6-11$ in the staining protocol (Tab. 3-12) were carried out at $37^{\circ} \mathrm{C}$. After TJ immunostaining, the actin cytoskeleton was stained (steps $11-14$ ), followed by nuclei staining (steps 15, 16). After the final washing step of the staining procedure, the wells were filled with $200 \mu \mathrm{LBS}^{++}$buffer and the 8-well chamber top was carefully removed from the bottom of the petri dish. Finally, the petri dish was filled with $\sim 20 \mathrm{~mL}$ $\mathrm{PBS}^{++}$buffer and the triple staining was examined using the $60 x$ water-immersion objective of the CLSM. 
Tab. 3-12 Protocol to triple stain for tight junctions (ZO-1 mouse monoclonal antibody + Alexa Fluor ${ }^{\circledR} 546$ rabbit anti-mouse lgG $(\mathrm{H}+\mathrm{L})$ ), Actin filaments (Alexa Fluor ${ }^{\circledR} 488$ phalloidin), and nuclei (DAPI).

\begin{tabular}{|c|c|c|c|c|c|c|}
\hline & Step & Rerun & Volume & Solution & Duration & Temp \\
\hline 1 & Washing & $2 x$ & $200 \mu \mathrm{L}$ & $\mathrm{PBS}^{++}$ & & \\
\hline 2 & Fixation & & $200 \mu \mathrm{L}$ & PFA, $4 \%(w / v)$ in PBS $^{++}$ & $10 \mathrm{~min}$ & $\mathrm{RT}$ \\
\hline \multirow[t]{2}{*}{3} & Washing & $2 x$ & $200 \mu \mathrm{L}$ & $\mathrm{PBS}^{++}$ & & \\
\hline & (Storage & & $400 \mu L$ & $P B S^{++}$ & 2 weeks & $\left.6^{\circ} \mathrm{C}\right)$ \\
\hline 4 & Permeabilization & & $200 \mu \mathrm{L}$ & Triton-X-100, $0.5 \%(\mathrm{v} / \mathrm{v})$ in $\mathrm{PBS}^{++}$ & $10 \mathrm{~min}$ & $\mathrm{RT}$ \\
\hline 5 & Washing & $2 x$ & $200 \mu \mathrm{L}$ & $\mathrm{PBS}^{++}$ & & \\
\hline 6 & Blocking & & $200 \mu \mathrm{L}$ & $\mathrm{BSA}_{1} 3 \%(\mathrm{w} / \mathrm{v})$ in $\mathrm{PBS}^{++}$ & $20 \mathrm{~min}$ & $37^{\circ} \mathrm{C}$ \\
\hline 7 & ZO-1 staining (1) & & $200 \mu \mathrm{L}$ & $\begin{array}{l}\text { ZO- } 1 \text { mouse monoclonal antibody, } \\
1: 50 \text { in } \mathrm{PBS}^{++} \mathrm{w} / 0.5 \%(\mathrm{w} / \mathrm{v}) \mathrm{BSA}\end{array}$ & $1 \mathrm{~h}$ & $37^{\circ} \mathrm{C}$ \\
\hline 8 & Washing & $3 x$ & $200 \mu \mathrm{L}$ & $\mathrm{PBS}^{++}$ & & \\
\hline 9 & ZO-1 staining (2) & & $200 \mu \mathrm{L}$ & $\begin{array}{l}\text { Alexa Fluor }{ }^{\circledR} 546 \text { rabbit anti-mouse } \lg G(H+L) \\
1: 500 \text { in } \text { PBS }^{++} w / 0.5 \%(w / v) \text { BSA }\end{array}$ & $40 \mathrm{~min}$ & $37^{\circ} \mathrm{C}$ \\
\hline 10 & Washing & $3 x$ & $200 \mu \mathrm{L}$ & $\mathrm{PBS}^{++}$ & & \\
\hline 11 & Actin staining & & $200 \mu \mathrm{L}$ & Alexa Fluor ${ }^{\circledR} 488$ phalloidin, 1:40 in $\mathrm{PBS}^{++}$ & $45 \mathrm{~min}$ & $37^{\circ} \mathrm{C}$ \\
\hline 12 & Washing & $3 x$ & $200 \mu \mathrm{L}$ & $\mathrm{PBS}^{++}$ & & \\
\hline 13 & Post-Fixation & & $200 \mu \mathrm{L}$ & PFA, $4 \%(w / v)$ in PBS $^{++}$ & $10 \mathrm{~min}$ & $\mathrm{RT}$ \\
\hline 14 & Washing & $3 x$ & $200 \mu \mathrm{L}$ & $\mathrm{PBS}^{++}$ & & \\
\hline 15 & Nuclei staining & & $200 \mu \mathrm{L}$ & DAPI, 1:10 in $\mathrm{PBS}^{++}$ & $2 \min$ & $\mathrm{RT}$ \\
\hline 16 & Washing & $3 x$ & $200 \mu \mathrm{L}$ & $\mathrm{PBS}^{++}$ & & \\
\hline 17 & Immersion & & $\sim 20 \mathrm{~mL}$ & $\mathrm{PBS}^{++}$ & & \\
\hline
\end{tabular}

\section{Cell Viability Staining (LIVE/DEAD $\left.{ }^{\circledR}\right)$}

The "LIVE/DEAD ${ }^{\circledR}$ viability/ cytotoxicity kit" for mammalian cells (Molecular Probes, Life Technologies; Carlsbad, CA, USA) was used for testing the viability of cells after an experimental stimulation. The live/dead assay is based on the complementary staining of live and dead cells with two fluorescent dyes of different color. ${ }^{[347,348]}$ Thereby, cells which are alive are visualized via their esterase activity. The enzyme converts the non-fluorescent and membrane-permeable calcein AM into the green-fluorescent calcein $\left(\lambda_{\mathrm{Exc}}=494 \mathrm{~nm} / \lambda_{\mathrm{Em}}=517 \mathrm{~nm}\right)$, which retains inside living cells. The second dye, ethidium homodimer-1 (Ethd-1), is not membrane-permeable due to its multiple positive charges. However, it can enter dead or dying cells with a destroyed and leaky plasma membrane. Ethd-1 binds to the DNA, supposed by an intercalation process, by which the fluorescence of the molecule undergoes a $>30$-fold enhancement $\left(\lambda_{E x c}=528 \mathrm{~nm} / \lambda_{E m}=617 \mathrm{~nm}\right)$. Thus, living and dead cells can be distinguished and examined by fluorescence microscopy in the green and the red emission channel, respectively.

Live/dead staining of cells was performed by carefully washing the respective cells with pre-warmed $\mathrm{PBS}^{++}$and subsequently adding the staining solution, containing $2 \mu \mathrm{M}$ calcein $\mathrm{AM}$ and $4 \mu \mathrm{M}$ Ethd -1 in $\mathrm{PBS}^{++}$. The cells were incubated for at least $30 \mathrm{~min}$ in the dark at $37^{\circ} \mathrm{C}$. After the staining process, the cells were washed once with pre-warmed $\mathrm{PBS}^{++}$and were then ready for fluorescence microscopic examination and documentation. 



\section{Chapter 4}

\section{MOCM: Multiple Cytomechanic}

\section{Sensing}

\subsection{Layout and Oscillation Properties}

4.2 Decoupled 2ElOs: Impact of Film Thickness

4.3 Discussion 
Quartz resonators have become a promising tool in bioanalytical sensing during the past decades. They allow for detecting oscillation changes (motional impedance and resonance frequency) arising from changes of the mechanical properties at the sensor interface, or more precisely within the decay length of the acoustic wave ( $250 \mathrm{~nm}$ in water). ${ }^{[349]}$ Such micromechanical changes are used to detect binding and detaching events of biomolecules to and from functionalized quartz substrates. The OCM was successfully applied in a multitude of biomolecular recognition and molecular interaction studies, like DNA/ RNA hybridization and immunosensing. ${ }^{[350]}$ Moreover, the OCM has become a versatile tool in cellular bioanalytics. Here, it is used as a label-free, substrate-integrated whole-cell biosensor to detect and monitor changes in the micromechanical properties of living, adherent cells. ${ }^{[22,206,209,215,219,231]}$

In order to make the OCM a more advanced sensing technology, sensor platforms with multiple OCM readout spots on a single quartz crystal surface have been designed. These OCM sensor arrays are also referred to as monolithic multichannel quartz crystal microbalances (MOCMs) and aim for multiselectivity (by different selective receptors/ ligands immobilized), multi-sensitivity, and multi-dynamic range mechanosensing (by sensor spots with different resonance frequencies) in a single measurement setup. The main field of MOCMs is their use as a piezoelectric nose (gas sensor) or tongue (chemical sensor) ${ }^{\left[35^{-355]}\right.}$ or as biosensor array ${ }^{[356-359]}$. Different recognition molecules, receptors and coatings on the different sensor spots of a MOCM allow for a label-free and time-resolved multi-component detection and analysis of a sample. Moreover, one sensor spot on a MOCM array can be applied as a sensor-integrated reference channel for compensation of environmental influences. ${ }^{\left[{ }^{360]}\right.}$ Besides different coatings for multi-component detection, a multiplication of sensor spots also increases the number of separate and independent readouts and provides information from different regions of an observed system. Hence, the number of measured parameters is multiplied, which results in a better statistical validation of the respective quantities.

In this study, a MOCM sensor with a double-electrode layout on a $5 \mathrm{MHz}$ AT-cut quartz crystal was developed, which is referred to as 2-Electrode Quartz ( $2 \mathrm{EIO}$ ) in the following. With this layout, the micromechanical interactions of adherent cells with the substrate surface were measured at two individual sensor spots. Moreover, the two neighboring, coplanar gold film electrodes on the top side of the quartz were used for the integration of ECIS-like measurements on the OCM substrate, without the need for an additional dipping electrode introduced from the top (Fig. 3-13, p. 48). This dual whole-cell biosensor allowed for multi-parametric, substrate-integrated, label-free, non-invasive, and real-time monitoring of adherent cells, thus providing mechanical (viscoelastic) and electrical information (electrode coverage, barrier resistance) from the same cell layer at the same time.

\subsection{Layout and Oscillation Properties}

\subsubsection{2-Electrode QCM Layout}

As basis for the 2-ElQ sensor layout, a blank, AT-cut $5 \mathrm{MHz}$ quartz crystal disk with a diameter of $14 \mathrm{~mm}$ was used. A thin film of chromium served as adhesion promoter for the deposition of gold structures onto the quartz surface. Two circular gold film electrodes were deposited on both sides, forming two resonator spots with congruent pairs of electrodes. The technical drawing for the electrode layout is shown in Fig. 4-1 A for the top side, and in Fig. 4-1 B for the bottom side of the quartz disk. Fig. 4-1 C illustrates a side view of the final $2 \mathrm{EIO}$ resonator. A diameter of $3.5 \mathrm{~mm}$ was chosen for each electrode, 
the distance between the coplanar electrodes was set to $2 \mathrm{~mm}$, and the minimal distance between the electrodes and the quartz edge was set to $3.5 \mathrm{~mm}$ (Fig. 4-1 A, B).

The size of the electrodes and their arrangement on the quartz disk were determined considering four main boundary conditions in order to achieve unperturbed oscillation properties of the two OCM spots. At first, a maximal electrode size has been selected, since the resonating area is inversely proportional to the quartz resistance $R_{q}$ (cf. Eq. (30)), which determines the intrinsic damping (mechanical loss) of the oscillator. According to the BVD equivalent circuit, a reduction of the resonator's area is reflected in higher $|Z|_{\min }$ and lower $|Z|_{\max }$ values (cf. Eqs. (44) and (45)), which means an increased dissipation factor and a lowered resonator sensitivity. Moreover, the bigger the electrode area the higher is its areato-circumference ratio. This minimizes the contribution of perturbing electrode edge effects to the total oscillation properties of the resonator. Third, perturbing influences of the disk edge should be avoided by a maximal distance between electrodes and edge. Finally, the inter-electrode distance on the quartz faces should be maximal in order to keep the interference of the neighboring sensor spots minimal.

Additional supply lines served for a contacting of the electrodes at the edge of the disk. The supply lines of upper and lower electrode were directed to opposite sides in order to avoid undefined and disturbing oscillation of the quartz material in between (Fig. 4-1). The two top side supply lines were conductively connected to contact pads on the bottom side via thin gold films on the periphery of the disk (red arrows in Fig. 4-1 B, C). Thus, all four electrodes could be individually contacted from the bottom side of the disk (arrows in Fig. $4^{-1}$ C). For recording the oscillation properties of the two sensor spots on 2ElOs, the selfmade measurement chamber for 1 ElOs was applied for quartz mounting, by upgrading it to four spring contacts (cf. Fig. 3-18, p. 57). The contact pads were broadened ( $2 \mathrm{~mm}$ in width) in comparison to the supply lines ( $1 \mathrm{~mm}$ in width) for the purpose of more tolerance in the arrangement of spring contacts and quartz disk.

\section{A: top view}

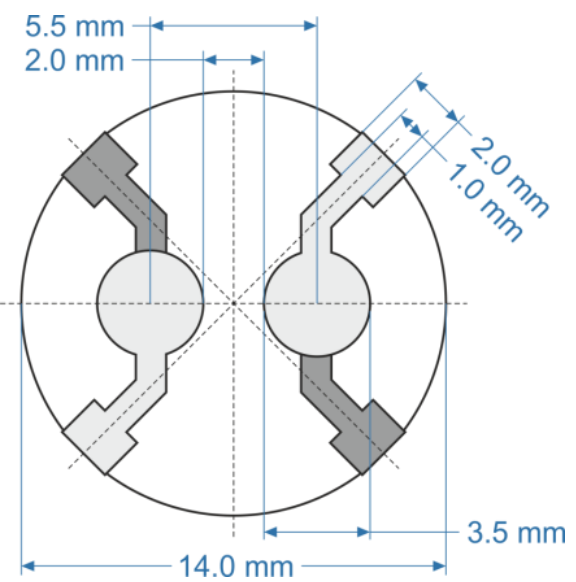

\section{B: bottom view}

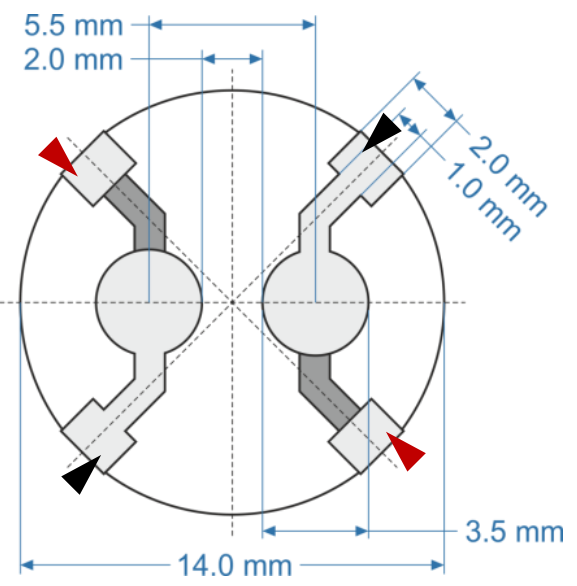

C: side view

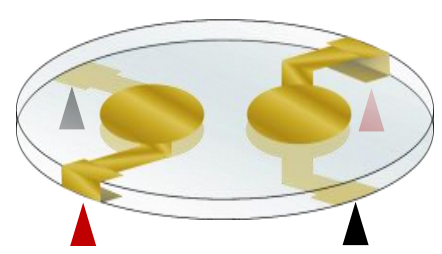

Fig. 4-1 Technical drawing of the $2 \mathrm{ElO}$ electrode layout on a $5 \mathrm{MHz}$ quartz crystal, in (A) top view and (B) bottom view. Dark grey areas indicate the electrode and supply line regions on the backside. Red arrows indicate the bridged contacting of the top side electrodes to the contact pads on the bottom side. Black arrows indicate the contact pads of the bottom side electrodes, which have no bridging contact to the top side. (C) All four electrodes could be contacted from the bottom side at the edges of the disk by electrical supply lines and contact pads. 


\subsubsection{Oscillation in Air}

The $2 \mathrm{ElO}$ was tested for the oscillation properties of the two individual sensor spots, $\mathrm{OCM}_{1}$ and $\mathrm{OCM}_{2}$ (Fig. 4-2 A). The quartz disk was mounted in the measurement chamber and the motional impedances of resonator one $\left(\underline{Z}_{O C M_{1}}\right)$ and resonator two $\left(\underline{Z}_{O \mathrm{CM}_{2}}\right)$ in air were consecutively recorded. Impedance spectra were recorded around resonance at 150 equidistant frequencies, in a frequency window from $4.97 \mathrm{MHz}$ to $5.04 \mathrm{MHz}$, which results in a frequency resolution of $470 \mathrm{~Hz}$ between two measurement points. While one resonator was excited to oscillation with a potential of $150 \mathrm{mV}$ for frequency scans the electrodes of the neighboring inactive resonator were on floating potential.

A

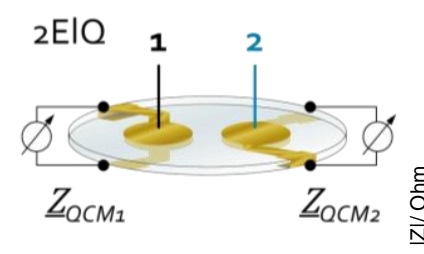

1EIQ

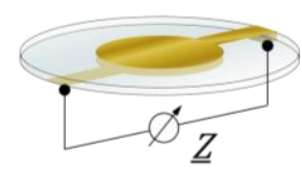

B

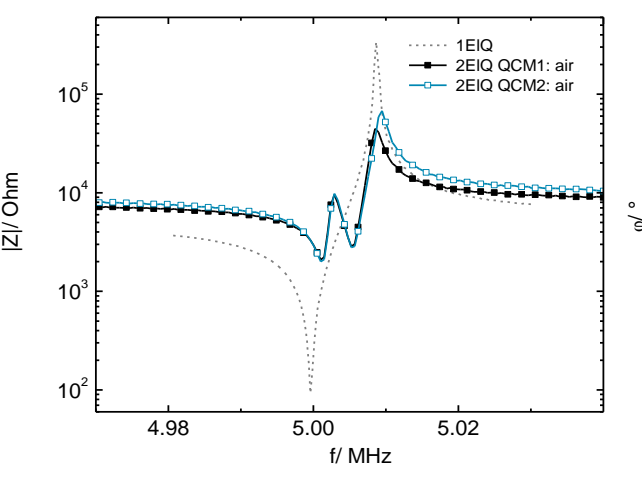

D
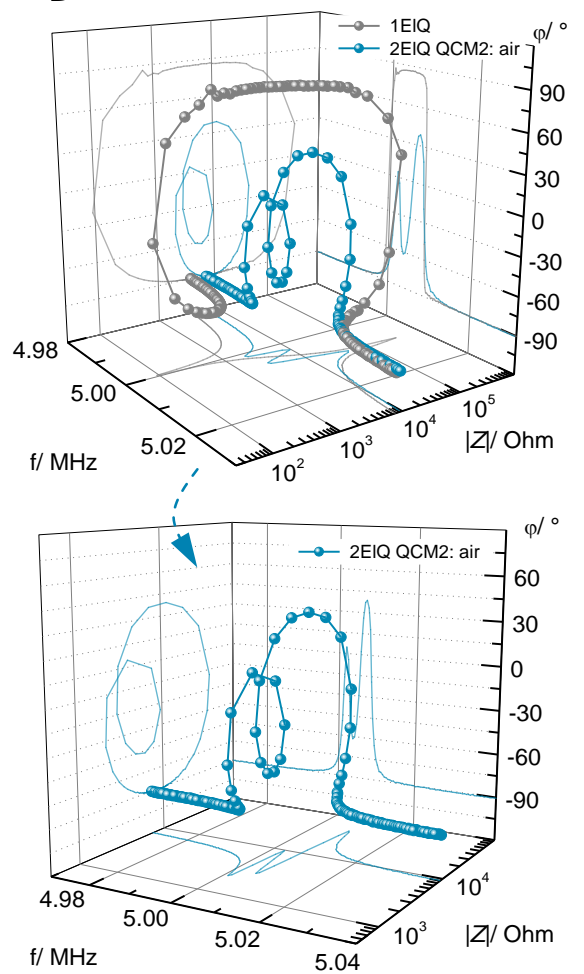

\section{C}

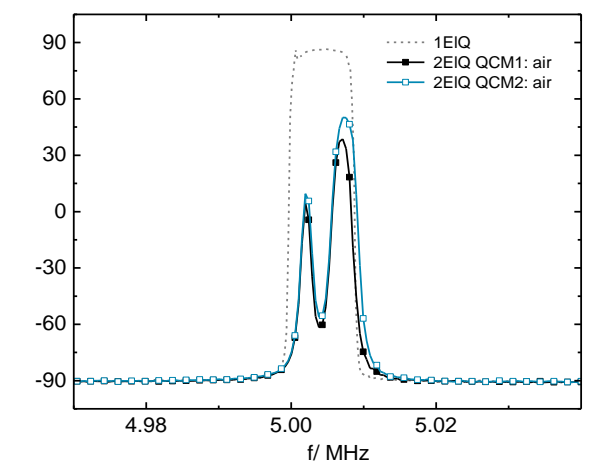

\section{E}
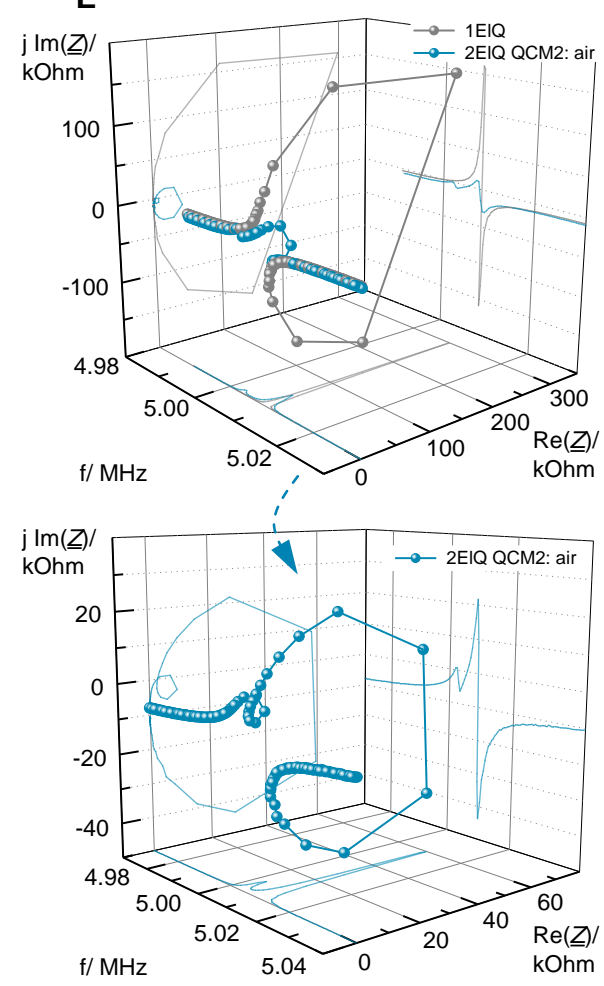

Fig. 4-2 Impedance data of $5 \mathrm{MHz} A T$-cut quartz disks with $2 \mathrm{EIO}$ and 1 ElQ layout performing shear oscillation in air (A). Oscillation properties are shown for both sequentially measured resonator spots of a $2 E_{1 Q}, \mathrm{QCM}_{1}(--)$ ) and $\mathrm{QCM} 2(-\square)$, as well for a 1 EIQ resonator $\left({ }^{---}\right)$. (B) Impedance magnitudes $|Z|$ and $(C)$ phase shifts $\varphi$ are shown as a function of AC frequency. Data points were reduced for sake of clarity. Corresponding (D) 3-D Bode plots ( $Z \mid$ vs. $\varphi$ vs. $f$ ) and (E) 3-D Nyquist plots $(\operatorname{Re}(\underline{Z})$ vs. $\operatorname{Im}(\underline{Z})$ vs. $f$ ) are shown for the $2 \mathrm{ElO}(\mathrm{QCM})$ and $1 \mathrm{EIQ}$. The $3-\mathrm{D}$ spectra of the $2 \mathrm{EIO}(\mathrm{OCM} 2)$ are in addition shown in separate diagrams for a more detailed view. 
The obtained complex impedances of both resonators are shown in Fig. 4-2 (OCM1: - , , OCM2: $\left.-{ }^{-}\right)$, split into impedance magnitude (B) and phase shift (C). Plots also comprise characteristic spectra of a classical ${ }_{1} \mathrm{EIO}$ resonator oscillating in air (---) for comparison. Fig. 4-2 D, E show alternative ways of spectra presentation by means of a three-dimensional Bode plot ( $Z \mid$ vs. $\varphi$ vs. $f$ ) as well as a three-dimensional Nyquist plot $(\operatorname{Re}(\underline{Z})$ vs. $\operatorname{Im}(\underline{Z})$ vs. $f)$. For sake of clarity, and since both resonators of the $2 \mathrm{ElO}$ exhibit similar spectra, only the oscillation of $\mathrm{OCM}_{2}$ of the $2 \mathrm{ElQ}$ is illustrated together with the $1 \mathrm{ElO}$ spectra in the respective $3-D$ plots. The $3-D$ spectra of the $2 \mathrm{EIO}(\mathrm{OCM} 2)$ are in addition shown in separate diagrams below for a more detailed view.

At parallel resonance frequencies $\left(f_{|Z|_{\text {max }}}\right.$ ), the two resonators exhibit impedance values of $|Z|_{\text {max }} \approx 7 \cdot 10^{4} \mathrm{Ohm}$, which is roughly ten times less in comparison to classical $1 \mathrm{ElOs}$. The maximal phase shift reaches values of $\varphi_{\max } \approx 45^{\circ}$, which is a distinct reduction in comparison to 1 ElOs $\left(\varphi_{\max } \approx 85-90^{\circ}\right)$. From impedance magnitude and phase spectra, as well as from the 3-D Bode and 3-D Nyquist plot, it is obvious that the oscillation properties of both resonators are disturbed at frequencies near serial resonance. The impedance magnitude does not exhibit a distinct minimum with expected values in the order of $|Z|_{\min } \approx 10^{3} \mathrm{Ohm}$. Instead, the curves possess a second, narrow maximum with $|Z| \approx 10^{4} \mathrm{Ohm}$. The serial resonance of each resonator is interfered by the presence of the not-energized second pair of electrodes, which are $2 \mathrm{~mm}$ apart. The interference results in $|Z|$ values, which are close to off-resonance values, where the parallel capacitance of the quartz dominates the signal. In the phase spectra, interferences from the passive resonator are reflected in a second, narrow phase peak to $\varphi \approx 0^{\circ}$ at frequencies below the series resonance (zero-phase crossing) of the active resonator. In both 3-D plots, the lateral oscillation coupling with the adjacent resonator spot appears as an additional small loop in front of the resonance loop of the active oscillator. In Fig. 4-2 D, the projections of the curve to the xyplane (bottom layer) and to the xz plane (right back plane) represent the 2-D Bode plots of impedance magnitude and phase shift, respectively, as illustrated in Fig. 4-2 A, B. The third projection to the yzplane (left back plane) illustrates the impedance magnitude as a function of phase shift. This is a rather non-common impedance representation but it nicely shows the deviation from an unperturbed oscillation due to oscillation coupling by an additional distinct loop in the curve profile. This as well applies for the 3-D Nyquist illustration of impedance (Fig. 4-2 E), where a second loop in the curve of the perturbed $2 \mathrm{ElO}$ oscillation can be observed (see magnified $2 \mathrm{ElO}$ spectrum) and which is not present in the curve shape of the $1 \mathrm{EIQ}$ spectrum.

\subsubsection{Oscillation under Liquid Loading}

Since the $|Z|_{\min }$ value should serve as an indicator for the viscoelastic properties of cells on the $2 \mathrm{EIQ}$, the mutual interference in the resonance region of the two resonators had to be eliminated. At first, the quartz was loaded with water to mimic the initial loading condition for cell experiments and to investigate whether the damping by the liquid (viscosity-density product; cf. Eqs. (24) and (25)) is already sufficient to decouple the resonators. The $2 \mathrm{ElO}$ was loaded with $1.5 \mathrm{~mL}$ deionized water and the motional impedance was recorded sequentially for each sensor spot. In Fig. 4-3, the obtained (B) impedance magnitude and $(C)$ phase shift are shown as a function of frequency. Fig. 4-3 D, E illustrate the $\mathrm{QCM}_{2}$ oscillation impedance as 3-D Bode and 3-D Nyquist plots (note: for comparison purposes the scales of axes in Fig. 4-3 D, E are chosen equal as in the respective diagrams in Fig. 4-2 D, E).

Impedance and phase spectra are similar for both resonator spots, $\mathrm{QCM}_{1}(--)$ and $\mathrm{OCM}_{2}\left(-{ }^{-}\right)$. The whole impedance spectra are damped in water with respect to oscillation in air (cf. Fig. 4-2). This is reflected in lower values of $|Z|_{\max }\left(\approx 3 \cdot 10^{4} \mathrm{Ohm}\right)$ and $\varphi_{\max }\left(\approx 15^{\circ}\right)$, and broader peaks of the resonance 
curves. In 3-D spectra (Fig. 4-3 D, E), damping due to loading the $2 \mathrm{ElO}$ with water is reflected in a decrease in the diameter of the resonance loop. Moreover, the whole spectra are slightly shifted to lower frequencies compared to the unperturbed quartz oscillation in air. Damping and frequency shift was due to the viscosity and density of water. In the BVD circuit, quartz loading with a Newtonian liquid causes an additional motional impedance $\left(\underline{Z}_{m}^{1}\right)$ in series to the motional branch impedance $\left(\underline{Z}_{m}^{0}\right)$ of an unperturbed resonator (cf. Eq. (48) and Tab. 3-6, p. 44), and contains dissipative (real part) as well as nondissipative (imaginary part) contributions. The damping of the oscillation also goes along with a damping of the interference peak in the serial resonance region of the 2-D Bode plots and with a reduction of the "coupling loop" in the respective 3-D plots.

Nevertheless, the mutual interference between neighboring sensor spots was not eliminated by simply loading the quartz with a Newtonian liquid like water or cell culture medium. The remaining, significant perturbation still precludes the extraction of $|Z|_{\text {min }}$ values from the spectra, which are required as indicator for time dependent alterations of the oscillation damping. In order to allow for this, the oscillations had to be uncoupled.

A

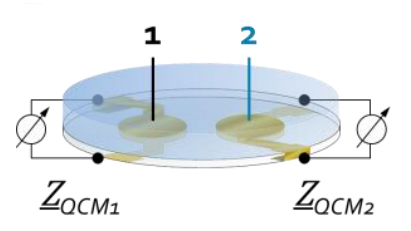

B

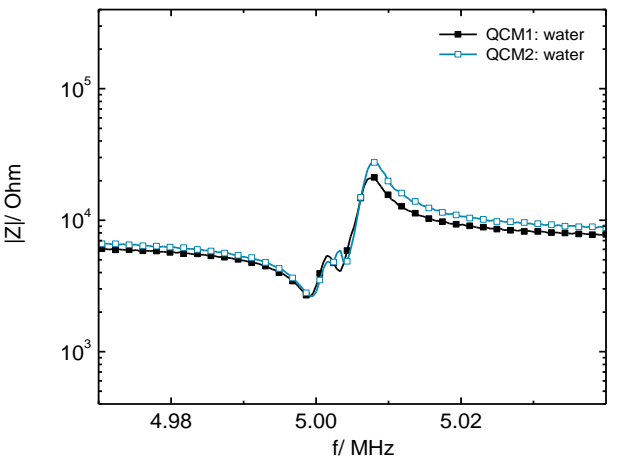

D

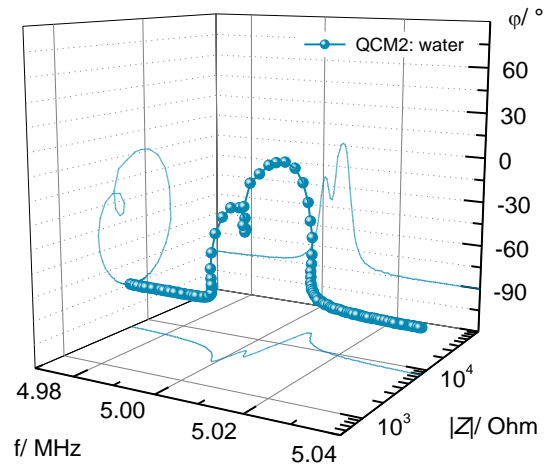

C

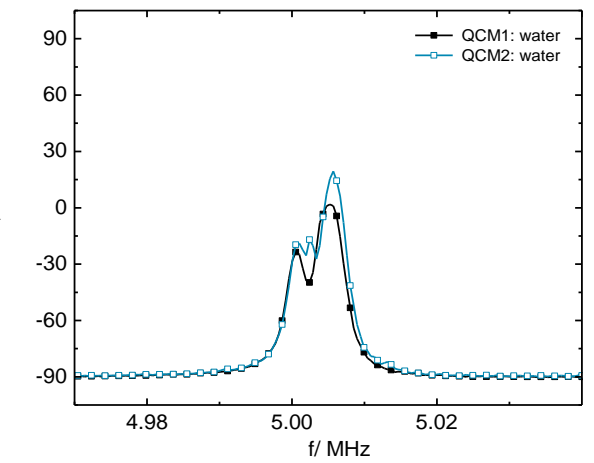

$E$

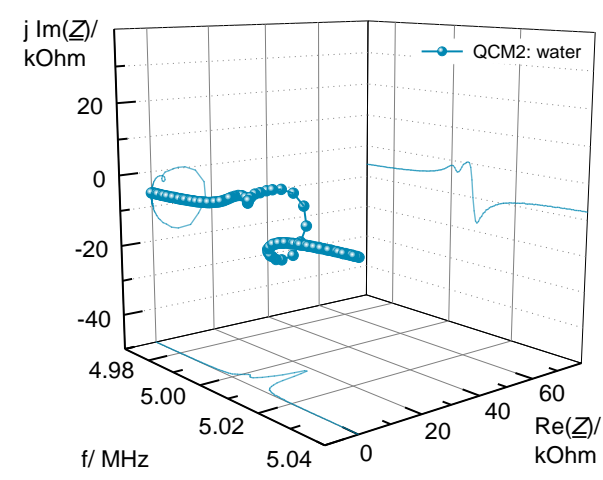

Fig. 4-3 Impedance data of a $5 \mathrm{MHz}$ AT-cut quartz disk with $2 \mathrm{ElO}$ layout that performs shear oscillation with one face immersed in water (A). Spectra are shown for the successively measured sensor spots, $\mathrm{OCM}_{1}(-)$ ) and $\mathrm{OCM}_{2}\left(-{ }^{-}\right)$. (B) Impedance magnitudes $|Z|$ and (C) phase shifts $\varphi$ are shown as a function of AC frequency. Data points were reduced for sake of clarity. A respective (D) 3-D Bode plot and (E) 3-D Nyquist plot are exemplarily shown for OCM2.

\subsubsection{Resonator Synchronization}

Based on the results presented in chapters 4.1.2 and 4.1.3 it is uncertain whether the disturbance near the serial resonance frequency of each sensor spot arises from the presence of the adjacent electrode or 
not. In order to exclude further parasitics, not the individual but the oscillation impedance $\left(\underline{Z}_{\mathrm{OCM}+2}\right)$ of the combined two resonators on a $2 \mathrm{ElO}$ were investigated. Therefore, the two electrodes on the top side as well as the two electrodes on the bottom side of the 2 ElO were short-circuited (Fig. 4-4 A). By applying an $\mathrm{AC}$ voltage, the two resonators oscillated simultaneously, resulting in an averaged resonance curve of the two separately oscillating sides. Impedance spectra near resonance were recorded for the oscillation in air, under liquid loading, and after attachment and spreading of adherent cells. The respective 2-D Bode plots are illustrated in Fig. 4-4 for impedance magnitude (B) and phase shift (C), as well as the 3-D Bode (D) and the 3-D Nyquist plots (E). $1.5 \mathrm{~mL}$ of cell culture medium were added to the measurement chamber for the measurement under liquid loading. The impedance spectrum for the resonator covered with a confluent layer of adherently grown MDCK-II cells ( $\mathrm{w} /$ cells) was recorded $24 \mathrm{~h}$ after seeding 450000 cells $/ \mathrm{cm}^{2}$ on the sensor surface.

A
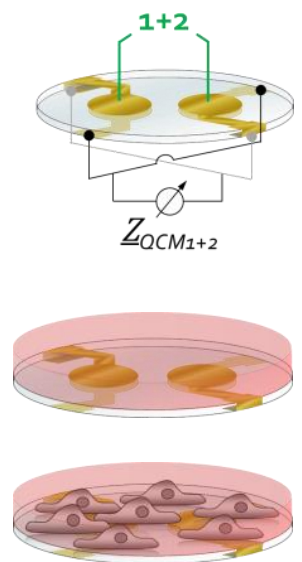

B

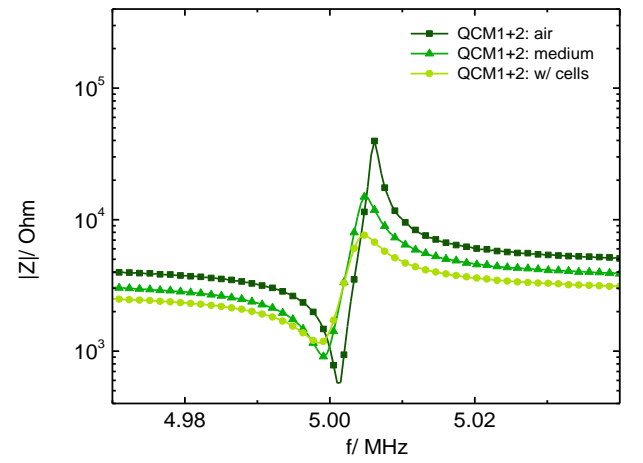

D

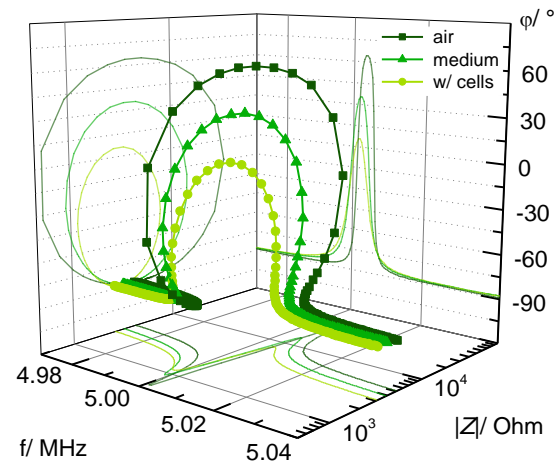

C

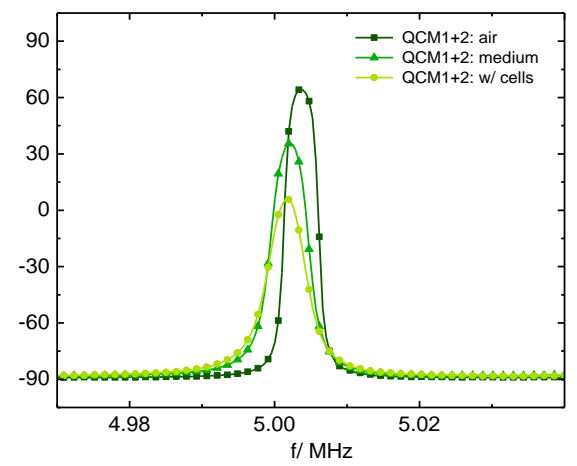

$E$

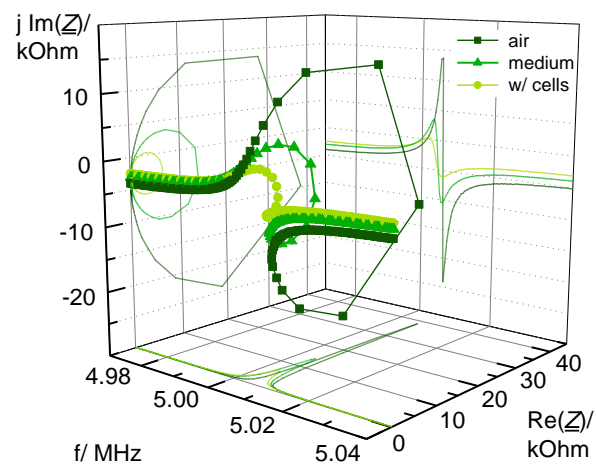

Fig. 4-4 The electrodes on both faces of a $2 \mathrm{ElO}$ disk were short-circuited (A). Impedance magnitude (B) and phase shift (C) are shown for the oscillation under different loading conditions. Oscillations were recorded in air $(-)$, under loading with medium $(-)$, and with a confluent cell layer on the surface $\left({ }^{-}-\right)$. Data points were reduced for sake of clarity. (D) 3-D Bode plots and (E) 3-D Nyquist plots for the respective oscillations.

Interference or disturbing effects near the minimal impedance are detectable neither in the impedance magnitude nor in the phase spectra of short-circuited $2 \mathrm{ElO}$ resonators, independent of the loading condition. The short-circuited spots exhibit a resonance curve similar to that of classical $1 \mathrm{EIO}$ measurements. In air $(\rightarrow)$ ), the double-resonator possesses characteristic impedance values of $|Z|_{\max } \approx 4 \cdot 10^{4} \mathrm{Ohm}$ and $|Z|_{\min } \approx 6 \cdot 10^{2} \mathrm{Ohm}$. The phase maximum $\varphi_{\max } \approx 65^{\circ}$ is reduced by $25^{\circ}$ with respect to the classical 1 EIO layout (cf. exemplary ${ }_{1} E I Q$ spectra in Fig. $4^{-2} \mathrm{~B}, \mathrm{C}$ ). This shift indicates an increase in energy dissipation caused by the new electrode layout. By loading the sensor surface with 
medium ( $\triangle$ ), the oscillation is further damped, resulting in values of $|Z|_{\max } \approx 1.5 \cdot 10^{4} \mathrm{Ohm}$, $|Z|_{\min } \approx 9 \cdot 10^{2} \mathrm{Ohm}$, and $\varphi_{\max } \approx 35^{\circ}$. After these measurements under liquid loading, the medium was exchanged by a suspension of MDCK-II cells. One day after seeding ( 450000 cells/ $\mathrm{cm}^{2}$ ), after cells have formed a confluent monolayer on the surface, the OCM impedance was recorded again $(--)$. The resulting spectra were further damped compared to measurements under liquid loading and in air. The maximal impedance and phase were reduced to $|Z|_{\max } \approx 0.75 \cdot 10^{4} \mathrm{Ohm}$ and $\varphi_{\max } \approx 6^{\circ}$, respectively, whereas the minimal impedance value increased to $|Z|_{\min } \approx 12 \cdot 10^{2} \mathrm{Ohm}$ upon loading the shortcircuited $2 \mathrm{EIO}$ resonators with a layer of viscoelastic cell bodies. In the 3-D spectra, Bode (Fig. 4-4 D) as well as Nyquist (Fig. 4-4 E) presentation, the gradual increase of damping from the initial oscillation in air to medium-loading and finally cell-loading is reflected in a decreased radius of the resonance loops. These representations further confirm unperturbed oscillations, due to the absence of "disturbing loops" in the curves.

Thus, the mutual interference of the resonators can be eliminated by simply short-circuiting them. However, this resembles the electrical situation for a classical $1 \mathrm{EIQ}$, but with the drawbacks of a smaller resonating area and the loss of two separate, individual measurement regions/ spots.

\subsubsection{Resonance Decoupling via Selective Rigid Coating}

The experiments shown above together with additional preliminary experiments, where the sensor spots were selectively damped with a highly viscous loading (data not shown), provided evidence that the perturbation in impedance signals on a $2 \mathrm{EIO}$ is due to the neighboring electrodes and that this coupling was reduced by loading one resonator with a damping material. According to the Saverbrey equation (Eq. (23)), deposition of a rigid mass on the resonator surface is directly proportional to a decrease in resonance frequency. Subsequent experiments aimed to systematically coat one resonator on the $2 \mathrm{EIO}$ with a thin layer of a rigid mass, while leaving the second resonator uncoated. Thereby, the resonance frequencies should be uncoupled to an extent that was sufficiently high for suppressing resonance interference between $\mathrm{OCM} 1$ and $\mathrm{OCM} 2$. As rigid material a photopolymer (PhoP) was used, which could be spin coated on the quartz disks and subsequently structured by photolithography. According to the spin coating protocol (cf. 3.3.3, p. 46), a film of PhoP was deposited onto the top surface of a 2 ElO disk at $3500 \mathrm{rpm}$. By means of photolithography, half of the PhoP was subsequently removed, resulting in a resonator with rigid loading on one half $(\mathrm{OCM})$ and an unloaded resonator $(\mathrm{OCM})$ on the other half of a 2 ElO top surface (Fig. 4-5 A).

After PhoP drying, the motional impedances of the structured $2 \mathrm{ElO}$ were recorded. Fig. $4-5$ shows the spectra of (B) impedance magnitude and (C) phase shift, for $\mathrm{OCM}_{1}$ and $\mathrm{OCM}_{2}$ measured successively $\left(-,-\square^{-}\right)$and for synchronized sensor spots (- - ). Fig. 4-5 D - G illustrate the oscillation impedances in 3-D-Bode (D, F) and 3-D-Nyquist diagrams $(E, G)$. Resulting characteristic impedance parameters $\left(f_{|Z|_{\text {min }}}\right.$ $|Z|_{\text {min }}, f_{s}, f_{p}, f_{|Z|_{\text {max }}},|Z|_{\text {max }}, \varphi_{\max }$ ) of the separately measured resonator units are summarized in Tab. 4-1. It is noticeable that the resonance of the coated $\mathrm{OCM}_{1}$ is shifted to lower frequencies by about $22.5 \mathrm{kHz}$ with respect to the resonance of the uncoated $\mathrm{OCM}$. However, $\mathrm{QCM} 1$ and $\mathrm{OCM} 2$ show similar profiles in the spectra of impedance magnitude and phase shift. This is reflected quantitatively in similar values of $|Z|_{\max }|Z|_{\text {min }}$, and $\varphi_{\max }$ for both resonators (Tab. 4-1). The similarities of OCM1 and QCM2 in oscillation parameters can be nicely seen in the 3-D impedance illustrations. The yz-projections of the 3-D Bode plot (Fig. 4-5 $\mathrm{D} ;|Z|-\varphi_{\max }$ plane) and the 3-D Nyquist plot (Fig. 4-5 $\mathrm{E}_{i} \operatorname{Re}(\underline{Z})-\operatorname{Im}(\underline{Z})$ plane) both visualize the similar resonance properties of $\mathrm{OCM}_{1}$ and $\mathrm{OCM}_{2}$, by almost perfectly congruent curves. The only difference of their resonance charcteristics is the mentioned offset along the frequency axis, as 
noticeable in the 3-D graphs and $x z$ - and xy-projections. No damping in the vibration of $Q C M_{1}$, due to the polymer film, can be observed. Hence, vibrational energy is stored and not dissipated by the PhoP coating, confirming the rigidity of the film. Moreover, $\mathrm{OCM}_{1}$ and $\mathrm{OCM}_{2}$ exhibit distinct impedance minima without any detectable perturbations. This is also reflected in the absence of any "couplingloops" in the 3-D impedance presentations of the two sensor units (Fig. 4-5 D, E) in contrast to the spectra of a not decoupled $2 \mathrm{EIO}(S / 9$, p. 265). Hence, near fundamental resonance, neither of the two sensor spots interfers with the other one anymore. With this approach, the two sensor spots were mechanically decoupled, while the vibrational energy and, thus, the impedance sensitivity of each sensor spot was preserved.

A
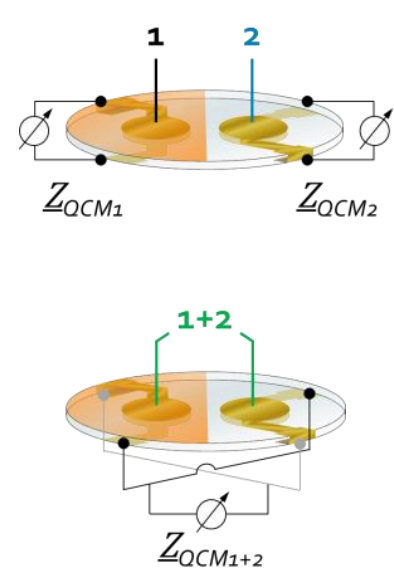

B

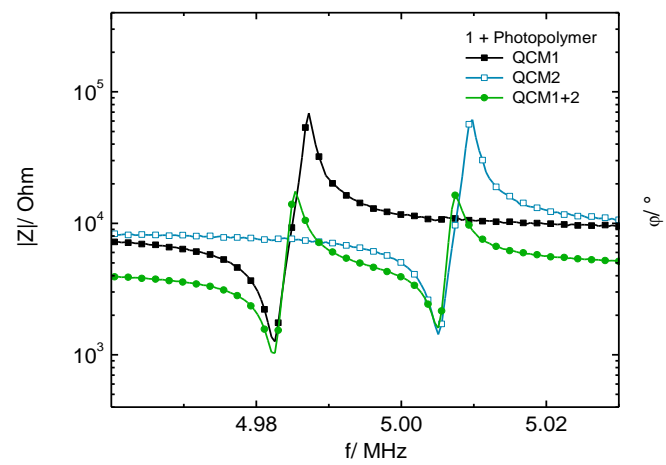

D

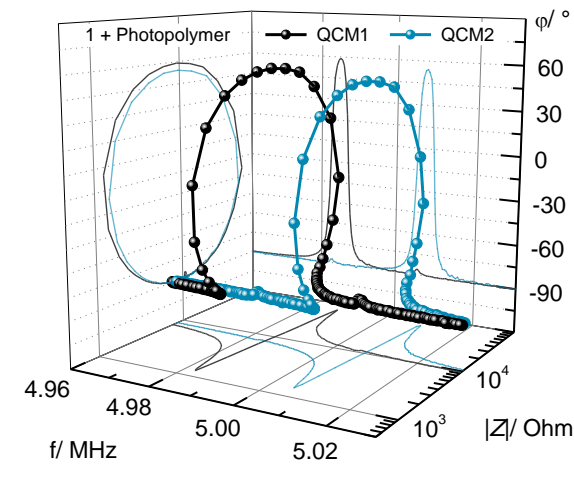

$\mathbf{F}$

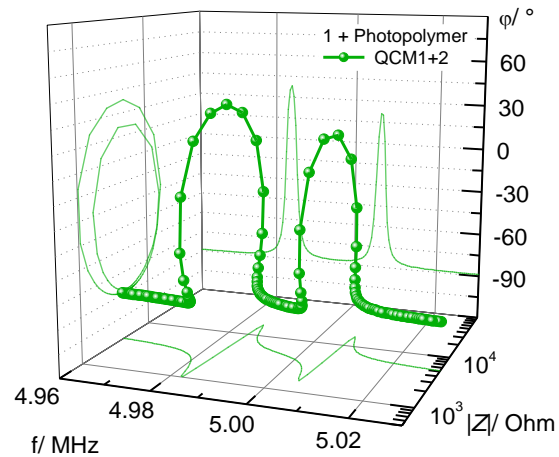

C

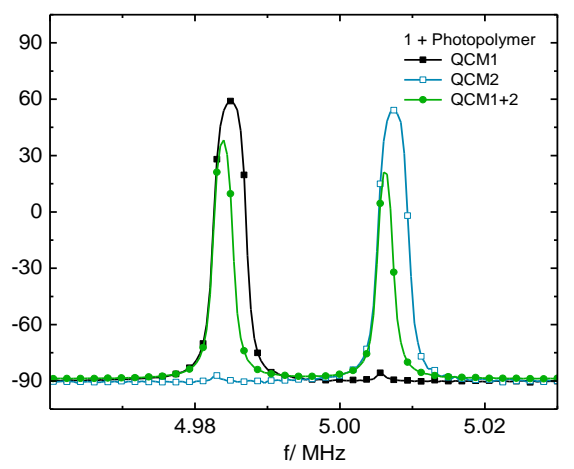

E

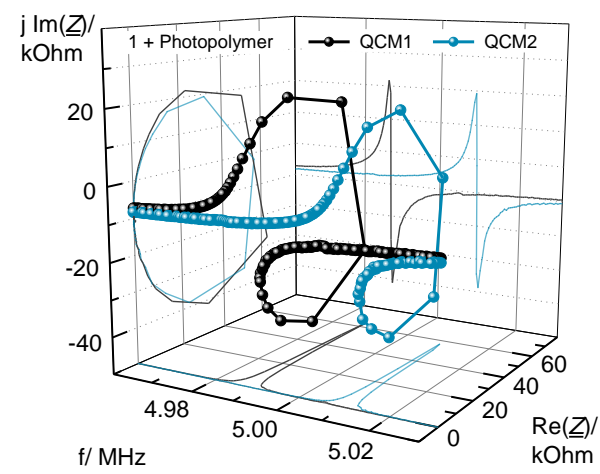

G

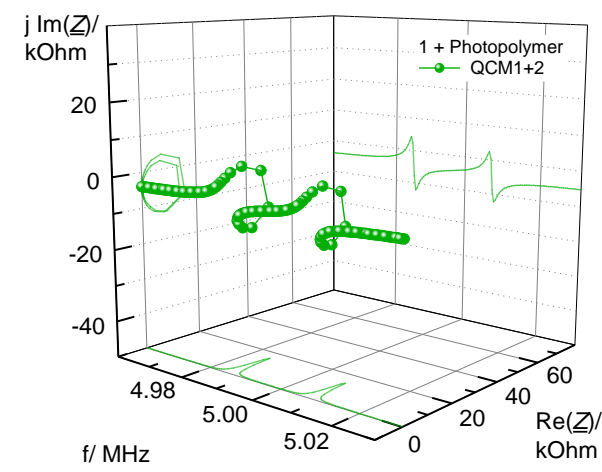

Fig. 4-5 Impedance data of $\mathrm{OCM}_{1}$ and $\mathrm{OCM}_{2}$ on a $2 \mathrm{ElO}$ in air, when one electrode on the top side was loaded with a rigid film of photopolymer (A). Spectra of impedance magnitude (B) and phase shift (C) were recorded for OCM1 $(-)$ ) and OCM2 $(-\square)$ in series, and for short-circuited $\mathrm{OCM}_{1+2}$ in parallel ( - ). Data points were reduced for sake of clarity. (D), (F) 3-D Bode plots and (E), (G) 3-D Nyquist plots of $\mathrm{OCM}_{1}$ (black), $\mathrm{OCM}_{2}$ (blue), and $\mathrm{OCM}_{1+2}$ (green). 
By short circuiting the decoupled $\mathrm{QCM} 1$ and $\mathrm{QCM} 2$ on a $2 \mathrm{EIO}$ (Fig. $4-5 \mathrm{~A}$ ), the combined oscillation impedances of both resonators, $\underline{Z}_{\mathrm{OCM} \mathrm{M}_{+2}}$, were recorded in parallel with a single frequency scan ( $-\bullet-$ in Fig. 4-5). The impedance spectra exhibit two consecutive resonances, which can be assigned to the $\mathrm{OCM}_{2}$ resonator in the higher frequency region and to $\mathrm{OCM}_{1}$ in the lower frequency region, since the resonance of the latter is shifted to lower values by the rigid loading. Significant differences can be identified between the impedance profiles of short-circuited resonators and impedance profiles of separately measured $\mathrm{OCM}_{1}$ and $\mathrm{OCM}$. The impedance parameters near parallel resonance as well as far from resonance were strongly affected by the synchronization of the two top electrodes and the two bottom electrodes on a $2 \mathrm{EIO}$. In the 3-D plots this is reflected by a compression of the loops of both sensor units along the impedance magnitude axis (Fig. 4-5 F vs. Fig. 4-5 D) and along the imaginary and real part axis (Fig. 4-5 G vs. Fig. 4-5 E). In the latter cases the resonance loops thereby are similarly reduced along both axis. Consequently, the circles in the Gaussian plane $(\operatorname{Re}(\underline{Z})-\operatorname{Im}(\underline{Z}))$ are reduced in diameter. In this context, it is striking that resistive and reactive contributions are minimized to an equal extent, resulting in a perfect circle in the complex plane.

Tab. 4-1 Typical oscillation properties of the two resonators, $\mathrm{QCM} 1$ and $\mathrm{QCM} 2$, on a $2 \mathrm{ElO}$ in air and $24 \mathrm{~h}$ after liquid loading with medium $(\mathrm{N}=1)$. $\mathrm{OCM}$ was coated with a film of photopolymer (PhoP). Values were estimated by spline-interpolation of data points in the corresponding frequency regions.

\begin{tabular}{|c|c|c|c|c|c|c|c|c|}
\hline Resonator & Load & $\begin{array}{c}f_{|Z|_{\min }} / \\
\mathrm{MHz}\end{array}$ & $\begin{array}{l}|Z|_{\min } \mid \\
\mathrm{kOhm}\end{array}$ & $\begin{array}{c}f_{s} / \\
\mathrm{MHz}\end{array}$ & $\begin{array}{c}f_{p} / \\
\mathrm{MHz}\end{array}$ & $\begin{array}{c}f_{|Z|_{\max }} l \\
\mathrm{MHz}\end{array}$ & $\begin{array}{c}|Z|_{\max } \mid \\
\mathrm{kOhm}\end{array}$ & $\varphi_{\max } 1^{\circ}$ \\
\hline \multirow[t]{2}{*}{$\mathrm{QCM}_{1}+\mathrm{PhoP}$} & air & 4.9825 & 1.2 & 4.9826 & 4.9870 & 4.9873 & 66.7 & 60 \\
\hline & medium & 4.9800 & 2.0 & $4 \cdot 9810$ & 4.9849 & 4.9859 & 23.9 & 26 \\
\hline \multirow[t]{2}{*}{$\mathrm{QCM}_{2}$} & air & 5.0051 & 1.5 & 5.0054 & 5.0094 & 5.0098 & 55.9 & 52 \\
\hline & medium & 5.0026 & 2.1 & 5.0039 & 5.0066 & 5.0080 & 17.0 & 16 \\
\hline
\end{tabular}

In subsequent experiments, the oscillation performance of the $2 \mathrm{EIO}$ resonators with partial PhoP coating were tested under liquid loading. $1.5 \mathrm{~mL}$ of cell culture medium were added to the quartz and the motional impedances of $\mathrm{OCM} 1$ and $\mathrm{OCM} 2$ were measured after $24 \mathrm{~h}$. This incubation period was required in order to enable equilibration in liquid (cf. Fig. 5-12 A, p. 130). Fig. 4-6 shows typical impedance spectra of PhoP coated $\mathrm{OCM}_{1}(-)$ and uncoated $\mathrm{OCM}_{2}\left(-\Delta^{-}\right)$under liquid loading. The oscillation profiles of the two resonators are damped to a similar degree upon liquid loading of the quartz, due to the viscosity of the medium. Moreover, the spectra of the two resonators are equally shifted by $\sim 2.5 \mathrm{kHz}$ to lower frequencies. Similar responses of $\mathrm{OCM}_{1}$ and $\mathrm{OCM}_{2}$ towards loading with medium were observed by similar changes of the characteristic impedance parameters $\left(f_{|Z|_{\text {min }},}|Z|_{\text {min }}, f_{s}, f_{p}, f_{|Z|_{\text {max }}},|Z|_{\text {max }}\right.$, $\varphi_{\max }$ ) of both resonators (Tab. 4-1). Hence, the PhoP coating on $\mathrm{QCM}_{1}$ does not influence the sensor response upon exposing the resonator surface to liquid.

These results prove that the polymer-modified $2 \mathrm{EIO}$ can be used as a MOCM in air as well as under cell culture conditions (= liquid loading). Seeding cells on the $2 \mathrm{EIO}$ surface should allow for sensing the cytomechanical properties at two separate and individual sensor spots. The twofold cytomechanic sensing is shown for a multitude of different assays in chapter 5.2 (p. $129 \mathrm{ff}$.), where the additional integration of ECIS-like measurements on the $2 \mathrm{EIO}$ substrates is demonstrated. 
A

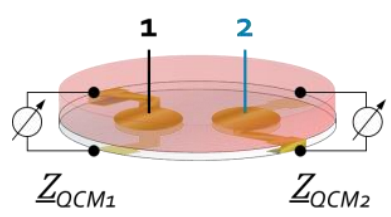

B

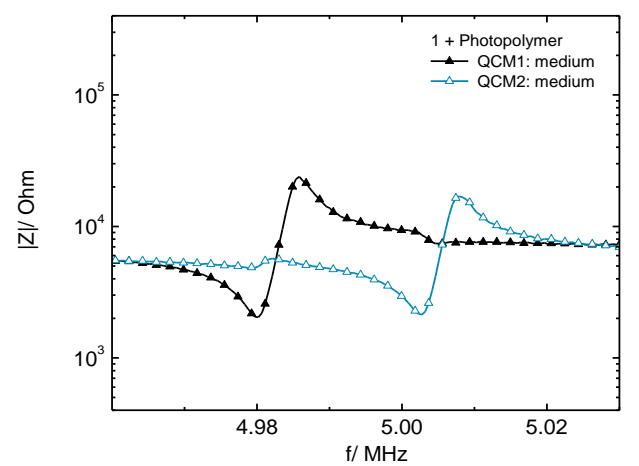

D

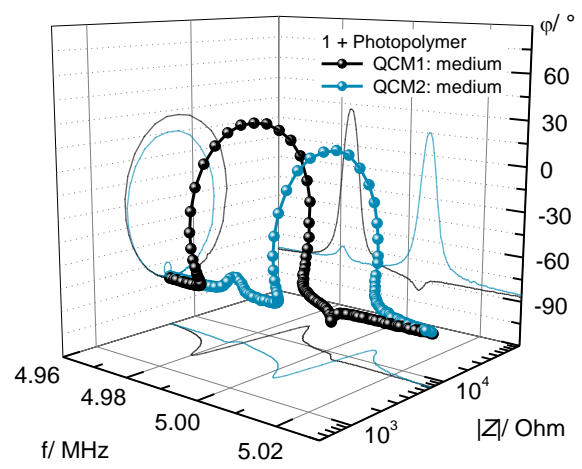

C

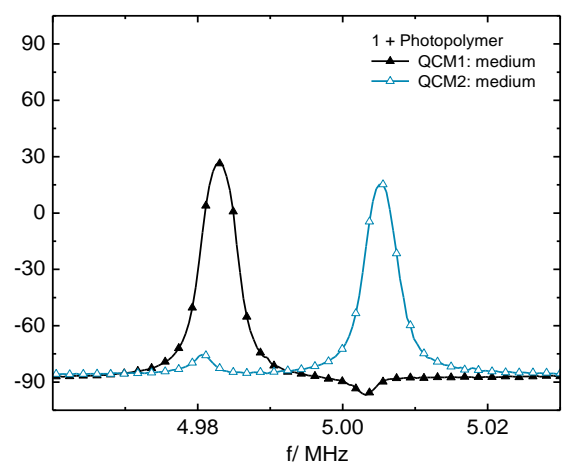

$E$

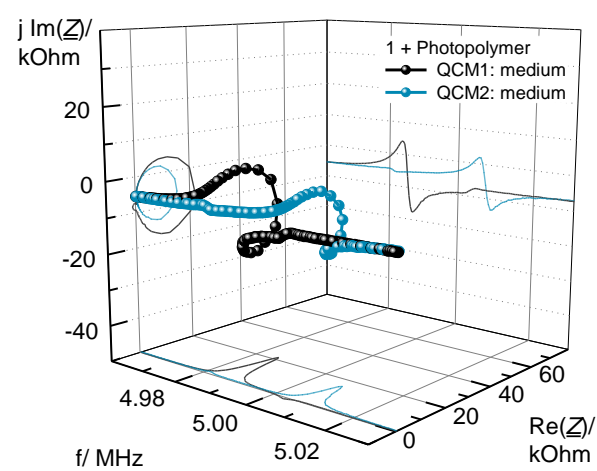

Fig. 4-6 Impedance data of $\mathrm{OCM}_{1}$ and $\mathrm{OCM} 2$ on a $2 \mathrm{EIO}$ under liquid loading, when one electrode on the top side was coated with a rigid film of PhoP (A). Spectra of (B) impedance magnitude and (C) phase shift were successively recorded for OCM1 $\left(-\boldsymbol{O}_{-}\right)$and $\mathrm{OCM} 2(-\square)$. Data points were reduced for sake of clarity. (D), (E) Corresponding 3-D Bode and 3-D Nyquist plots.

\subsection{Decoupled 2ElQs: Impact of Film Thickness}

It has been shown that the coupling between the two resonators, $\mathrm{OCM}_{1}$ and $\mathrm{QCM}$, on $2 \mathrm{ElO}$ s is avoided by loading one resonator with a thin layer of rigid photopolymer (PhoP; cf. Fig. 4-2 vs. Fig. 4-5). Due to the additional rigid mass on one resonator, the effective thickness of the (loss-less) oscillating material was increased. This increase resulted in a shift of the resonance frequency to lower values. The following section focusses on the correlation of the film thickness with the change of resonance parameters and the degree of decoupling. The height of deposited PhoP layer thereby was triggered via the spin coating velocity. These investigations also enabled to derive an empirical formula for the estimation of film thicknesses from the difference of resonance frequencies of both resonators on the $2 \mathrm{EIO}$.

\subsubsection{Degree of Decoupling as a Function of Film Thickness}

According to the spin coating and photolithography protocol (Fig. 3-11, p. 46), one electrode on $2 \mathrm{ElO}$ resonators was selectively coated with a thin layer of photoresist. In order to obtain different heights of the polymer film, coating was performed at different rotational velocities (between 2000 and $4000 \mathrm{rpm}$ ). Quartz discs were spin coated at the various velocities in two separate test series. The photopolymer used in both was the same, however the different batches had an age-related different flow viscosity (viscosity "old" > "new"). After spin coating, photolithography and drying of the $2 \mathrm{ElO}$, the resonators 
were mounted in $\mathrm{OCM}$ chambers and impedance spectra of the polymer-coated resonator $\left(\mathrm{OCM}_{1}\right)$ and the uncoated resonator ( $(\mathrm{OCM} 2)$ were recorded in air. Fig. 4-7 A shows typical impedance spectra of discs coated with "old" photopolymer. The frequencies of all spectra were normalized with respect to the resonance frequency of the corresponding uncoated resonator $\mathrm{OCM}$. Thus, the plot illustrates the resonance shift of the coated sensor spots relative to the uncoated ones. The reference spectrum of a $\mathrm{OCM} 2$ oscillation is shown as well (….). The same applies for the impedance representation of quartz discs coated with "new" photopolymer in Fig. 4-7 C. The results from both test series clearly show the spectral shift of the oscillation to lower frequencies with a decrease in the spin coating velocity, indicating an increase in the layer thickness. This shift is quantified by the frequency change of the minimal impedance $\Delta f_{|Z|_{\text {min }}}$. By the applied different velocities and polymer viscosities, spectral shifts in the order of $\Delta f_{|Z|_{\text {min }}} \approx-(15-30) \cdot 10^{3} \mathrm{~Hz}$ are obtained.

A

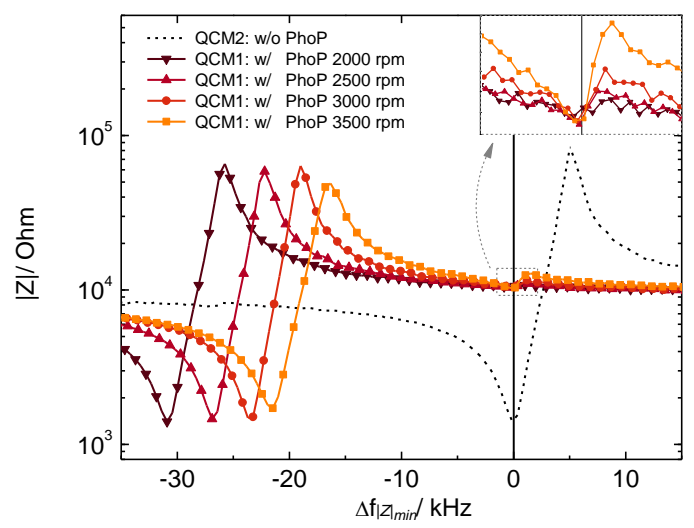

C

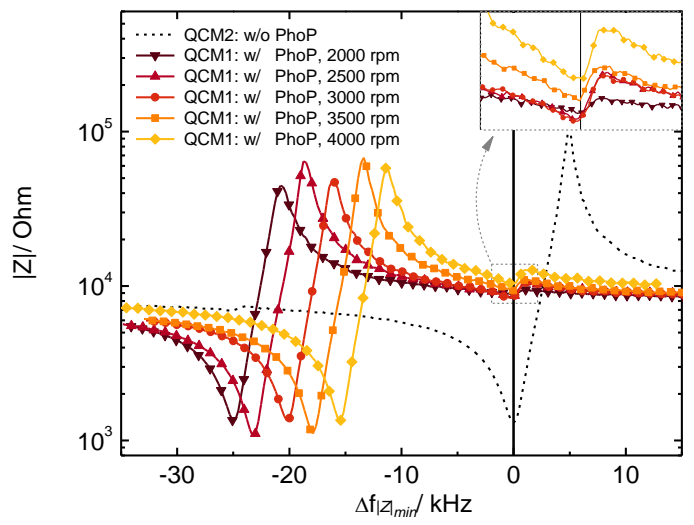

B

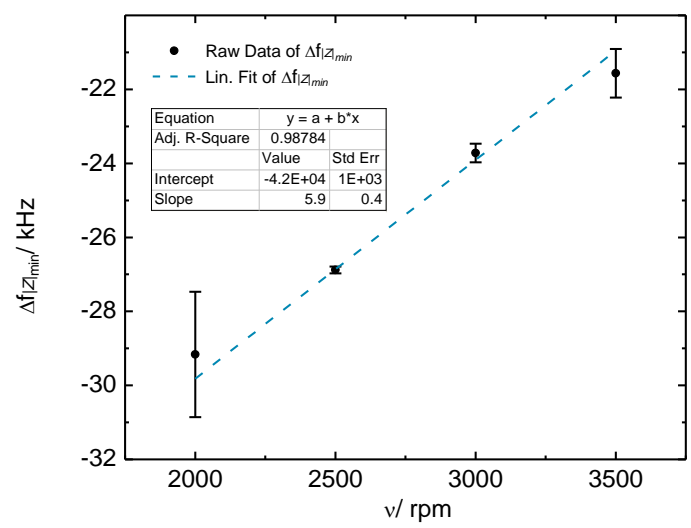

D

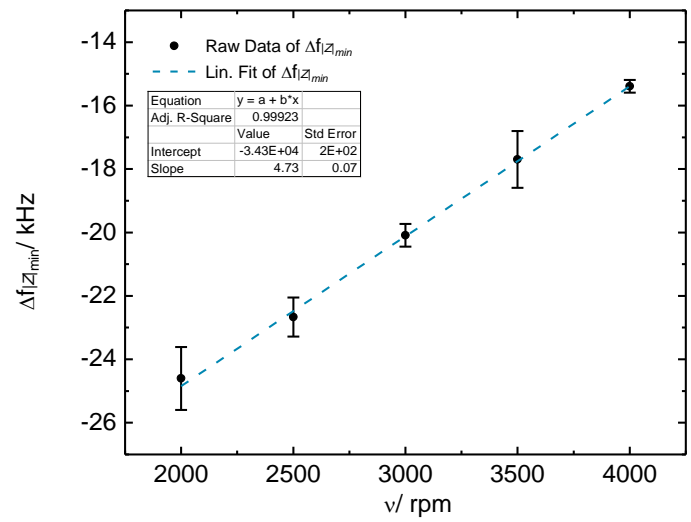

Fig. 4-7 (A), (C) Impedance spectra of OCM1 resonators on a 2ElQ, loaded with (A) "old" and (C) "new" photoresist at different spin coating velocities. Spectra were normalized with respect to the resonance frequency at $|Z|_{\min }$ of the corresponding uncoated resonator, $\mathrm{OCM} 2(\cdots \cdots \cdot)$. Data points were reduced for sake of clarity. Diagrams (B), (D) next to the spectra show the averaged spectral shifts of impedance minima, $\Delta f_{|Z|_{\text {min }}}$ due to polymer coating as a function of spin coating speed $v$ (mean $\pm \mathrm{SEM}, \mathrm{N} \geq 2$ ). Individual values and errors refer to Tab. $4^{-2}$ The dashed line (---) in each plot represents the outcome of a linear regression analysis of data points.

The deposited polymer layers in both test series prove to be sufficiently thick to decouple $\mathrm{QCM} 1$ and $\mathrm{OCM} 2$ resonances. This is evident in the impedance spectra by the disappeared interference near the fundamental resonances, which are observed for non-decoupled resonators (cf. Fig. 4-2). This even 
applies for the highest spin coating speed (4000 rpm) of the "new" photopolymer, which resulted in the smallest $\Delta f_{|Z|_{\text {min }}}$ value $(-15.4( \pm 0.2) \mathrm{kHz}$ (mean $\pm \mathrm{SEM} ; \mathrm{N}=5)$ ) and the thinnest deposited film of both test series ( $\rightarrow$ in Fig. $4-7$ C). However, one can recognize slight disturbances off resonance in the $\mathrm{QCM}_{1}$ impedance spectra, in the region of $\mathrm{OCM}_{2}$ resonance. These look like a side resonance due to interference with $\mathrm{QCM}$, which becomes apparent by a zoom-in to this region (inset graph in Fig. 4-7 A, C). It is also obvious, that this disturbance at $\Delta f_{|Z|_{\text {min }}}=0$ is significantly more pronounced with increased spin coating velocities, i.e. at decreased layer thicknesses and smaller shifts of the (main) resonance. What else can be stated is that, except for the frequency shift and the mentioned slight disturbances in the spectra, there is no significant difference in the overall shape of the impedance spectra between the $\mathrm{OCM} 1$ resonators with different polymer thicknesses, just as less as between the coated $\mathrm{OCM} 1$ and the uncoated $\mathrm{QCM}_{2}$ resonators. Hence, the dissipative oscillation properties of the $2 \mathrm{ElO}$ resonators are independent of the thickness of rigid loading within the investigated scope.

From normalized impedance spectra as shown in Fig. 4-7 A and C, the values of the shift of the resonance frequency relative to the uncoated resonator $\Delta f_{|Z|_{\text {min }}}$ were extracted and averaged for each spin velocity of each test series (individual mean values and errors refer to Tab. 4-2). Diagrams Fig. 4-7 B, D illustrstrate these $\Delta f_{|Z|_{\text {min }}}$ values as a function of spin coating velocity $v(\bullet)$. Corresponding results of a linear regression analysis are shown as well, by a dashed line (--). According to the linear relation in Fig. 4-7 B, a reduction of the shift by $\sim 6 \mathrm{kHz}$ per $1000 \mathrm{rpm}$ speed increase could be approximated for the "old" photoresist and conditions studied here. The linear regression was calculated considering standard errors of mean values by instrumental weighting. The big error bar at $2000 \mathrm{rpm}$ (Fig. 4-7 B) arises from only double determination of $\Delta f_{|Z|_{\text {min }}}$ values at this spin velocity. In the case of the "new" photoresist, a reduction of the frequency shift by $\sim 4.7 \mathrm{kHz}$ per $1000 \mathrm{rpm}$ speed increase was obtained, based on the slope of the linear regression. This reduction rate is significantly lower in comparison to the first test series with the "old" photoresist, which reflects the lower flow viscosity.

\subsubsection{Film Thickness Determination}

Additionally to the OCM impedance investigations of spin coated quartz disks, profilometry measurements were performed of similarly coated glass substrates in order to get information about the film thickness of the polymer coating. The height differences between the polymer film and the uncoated substrate were determined by means of white light vertical scanning interferometry by nanoAnalytics company (Münster, Germany), using a "NT3300 optical profiler" (Veeco Instruments Inc.; NY, USA). Three substrates were coated with "old" photoresist at different spin coating velocities (2000, 2500, 3000 , and $3500 \mathrm{rpm}$ ). By subsequent photolithography, half of the polymer was removed (Fig. 3-11, p. 46) and height profiles were determined at two different polymer edges for each substrate. Data from a typical optical profile measurement are illustrated in Fig. 4-8 A, obtained from a substrate that has been coated with photoresist at $3000 \mathrm{rpm}$. The image shows the field of view ( $1 \times 1 \mathrm{~mm})$ at the edge between substrate and polymer. In this particular measurement, a height difference of $h=3.477 \mu \mathrm{m}$ was determined between the planes of polymer and glass substrate. Fig. 4-8 B summarizes the profilometry measurements of all spin coated substrates. The thickness values were averaged for substrates with the same coating speed (values refer to Tab. 4-2) and plotted as a function of the rotational speed used for spin coating. Film thicknesses varied in the range of $1 \mu \mathrm{m}$, i.e. between $\sim 4.2 \mu \mathrm{m}$ for a velocity of $2000 \mathrm{rpm}$ and $\sim 3.2 \mu \mathrm{m}$ for $3500 \mathrm{rpm}$. In accordance to the data provided by the photoresist manufacturer ${ }^{[361]}$, an exponential correlation between layer thickness and coating speed can be assumed. This is represented by a dashed line in Fig. 4-8 B. 
A

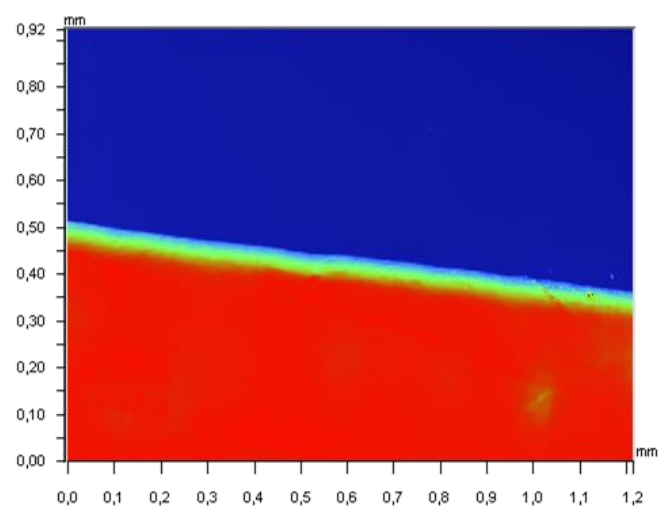

B

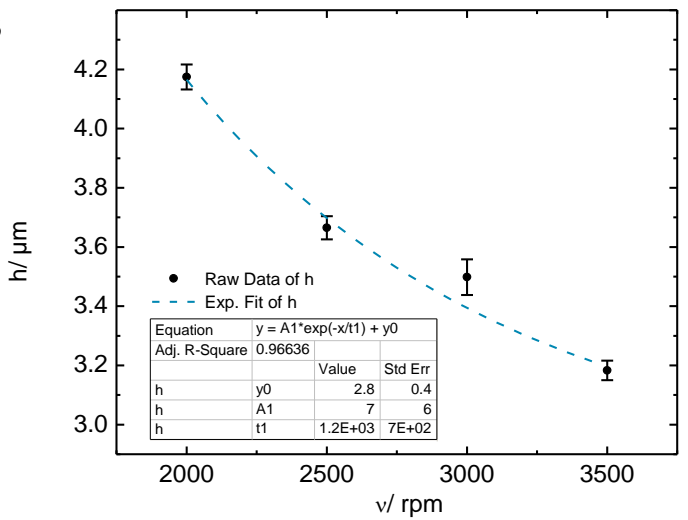

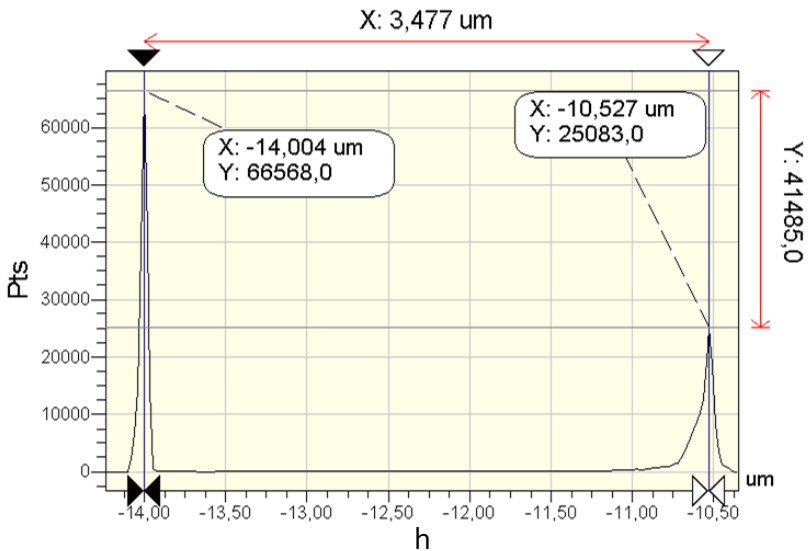

C

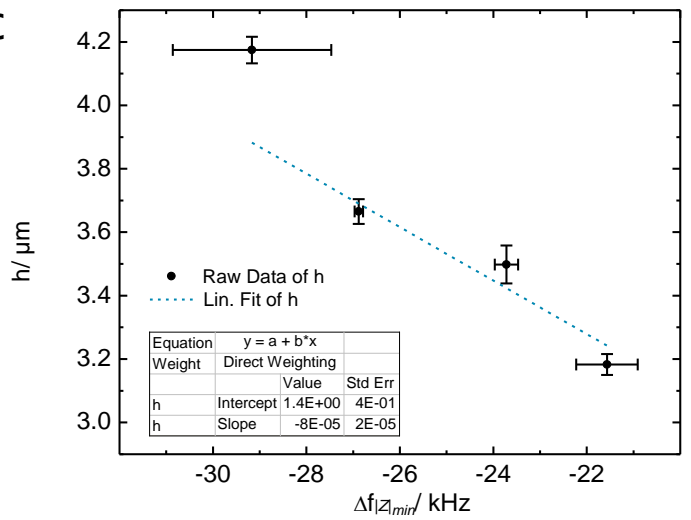

Fig. 4-8 (A) Result of white light vertical scanning interferometry of a glass substrate that was partially covered with PhoP (spin coating at $3000 \mathrm{rpm}$ and subsequent photolithography). (B) Representation of the measured height of PhoP as a function of spin coating velocity (mean $\pm \mathrm{SEM}, \mathrm{N}=6$ ). An exponential correlation between data points can be assumed (---). (C) For each coating speed, the polymer layer thickness $h$ and the resonance frequency shift $\Delta f_{|Z|_{\text {min }}}$ (cf. Fig. 4-7 B) were plotted against each other. Asssuming a linear relationship ( --- ), the corresponding equation is given by: $h=1.4( \pm 0.4) \mu \mathrm{m}-8( \pm 2) \cdot 10^{-5} \mu \mathrm{m} / \mathrm{Hz} \cdot \Delta f_{|z|_{\text {min }}}$.

Tab. 4-2 Resonance frequency shift $\Delta f_{|Z|_{\min }}$ of $2 \mathrm{ElO}$ resonators with one half of the sensor surface coated with PhoP layers of different thicknesses, via various spin coating velocities $v$ and subsequent photolithography (mean $\pm \mathrm{SEM}, \mathrm{N}=2-7$ ). Height values $h$ of layers with "old" PhoP were obtained from white light interferometry measurements (cf. Fig. 4-8 A). Applying the empiric calibration in Eq. (58) to the $\Delta f_{|Z|_{\text {min }}}$ values of resonators coated with "new" PhoP provides calculated values of the deposited polymer height, $h_{\text {calc }}$.

\begin{tabular}{ccccc}
\hline & \multicolumn{2}{c}{ "old" PhoP } & \multicolumn{2}{c}{ "new" PhoP } \\
$v / \mathrm{rpm}$ & $\Delta f_{|Z|_{\text {min }} / \mathrm{kHz}}$ & $h / \mu \mathrm{m}$ & $\Delta f_{|Z|_{\min } / \mathrm{kHz}}$ & $h_{\text {calc }} / \mu \mathrm{m}$ \\
\hline 2000 & $-29.2( \pm 1.7)$ & $4.17( \pm 0.04)$ & $-24.6( \pm 1.0)$ & $3.5( \pm 0.5)$ \\
2500 & $-26.9( \pm 0.1)$ & $3.67( \pm 0.04)$ & $-22.7( \pm 0.6)$ & $3.3( \pm 0.5)$ \\
3000 & $-23.7( \pm 0.3)$ & $3.50( \pm 0.06)$ & $-20.1( \pm 0.4)$ & $3.1( \pm 0.5)$ \\
3500 & $-21.6( \pm 0.7)$ & $3.18( \pm 0.03)$ & $-17.7( \pm 0.9)$ & $2.9( \pm 0.5)$ \\
4000 & - & - & $-15.4( \pm 0.2)$ & $2.7( \pm 0.5)$ \\
\hline
\end{tabular}


With the information about PhoP height and the corresponding resonance shift for different spin coating velocities, the first two parameters could be related to each other. In Fig. 4-8 C the measured PhoP height $h$ is represented as a function of the corresponding resonance frequency shift $\Delta f_{|Z|_{\text {min }}}$. Assuming a linear relationship between the data points, in consideration of standard errors (direct weighting) of $h$ (yEr) and $\Delta f_{|Z|_{\text {min }}}(\mathrm{xEr}), h$ is expressed as a function of $\Delta f_{|Z|_{\text {min }}}$ :

$$
h=1.4( \pm 0.4) \mu \mathrm{m}-8( \pm 2) \cdot 10^{-5} \mu \mathrm{m} / \mathrm{Hz} \cdot \Delta f_{|Z|_{\text {min }}}
$$

Hence, one could approximate for this type of photoresist that an increase of the layer thickness by $\sim 80 \mathrm{~nm}$ is correlated with a resonance frequency shift of $-1 \mathrm{kHz}$ of the coated with respect to the uncoated resonator.

Eq. (58) was applied to estimate the film thickness of spin coated "new" photoresist on 2ElOs by the determined values of $\Delta f_{|Z|_{\text {min }}}$ (cf. Fig. 4-7D and Tab. 4-2). This provides calculated heights $h_{\text {calc }}$ of the deposited PhoP layers in the range of $\sim 3.5 \mu \mathrm{m}$ for $2000 \mathrm{rpm}$ and $\sim 2.7 \mu \mathrm{m}$ for $4000 \mathrm{rpm}$ (values listed in Tab. 4-2). Standard errors of mean values were calculated according to the Gaussian error propagation. It is noted that these are mainly determined by the parameter uncertainties in Eq. (58) and less by the accuracy of $\Delta f_{|Z|_{\text {min }}}$ determinations. In comparison to the data of the "old" photoresist (cf. Fig. 4-8 B and Tab. 4-2), the deposited films with the new batch of photoresist were thinner at specific spin velocities.

\subsection{Discussion}

\section{4-3.1 2EIQ Sensor Layout and Oscillation Properties}

In this project, a new MOCM sensor was established that allowed for measuring mechanical changes at the quartz interface at two separate sensor spots. A novel electrode layout providing two electrodes on both faces of a $5 \mathrm{MHz}$ AT-cut quartz plate (Fig. 4-1) was designed. This novel dual QCM sensor is further referred to as 2-Electrode Quartz (2EIQ).

\section{Interference of 2EIQ Resonators}

The two resonators on a $2 \mathrm{ElO}, \mathrm{OCM}_{1}$ and $\mathrm{OCM}$, exhibited mutual disturbances in their oscillation characteristics in air (Fig. 4-2) as well as when one face of the disk was immersed in water (Fig. 4-3). Such lateral coupling effects between adjacent oscillation units of a MOCM sensor were already considered experimentally ${ }^{[353,355,360,362]}$ as well as in theoretical approaches ${ }^{[363,364]}$ in literature. Frequency interferences occur when two resonators are brought too close to each other, so that the decay lengths of their shear displacements overlap. This happens predominantly when the distance between resonators with similar electrode diameter and similar resonance frequencies is rather small. ${ }^{\left[{ }^{363}\right]}$ The litertaure studies, however, consider only the simultaneous oscillation of two adjacent resonators, which exhibit a parallel and an anti-parallel oscillation mode in cases of coupling. For a $5 \mathrm{MHz}$ AT-cut quartz with two detuned resonators (100 nm difference in electrode thickness) it was shown by simulation that an electrode distance of $1 \mathrm{~mm}$ (ratio distance-to-quartz thickness $d / d_{q}=2.96$ ) causes a cross-masssensitivity of $\sim 9 \%$ for the thinner resonator. This means that in cases of a homogeneous mass deposition on the quartz plate almost $10 \%$ of the detected frequency shift on the (thinner) resonator is due to mass deposition on the second resonator. This coupling factor was reduced to negligible values of $\sim 1 \%$ by increasing the electrode separation distance to $3 \mathrm{~mm}\left(d / d_{q}=8.88\right) .{ }^{\left[{ }^{664]}\right.}$ Mechanical inter- 
ferences between oscillating units imply that their shear displacements overlap. This means that the characteristic decay lengths of the oscillation amplitudes (of two simultaneously resonating units) have to cover at least half the distance between the electrodes. For a $5 \mathrm{MHz}$ AT-cut quartz crystals with similar double-electrode design, the oscillation region, i.e. the decay length of vibration beyond the electrode edges, was reported to be in the range of $\sim 1 \mathrm{~mm} \cdot{ }^{\left[{ }^{362]}\right.}$ The 2 ElOs used in the presented studies were designed with an electrode spacing of $2 \mathrm{~mm}$ (cf. Fig. 4-1) and the quartz oscillations of the two adjacent resonators were measured successively, so that a crosstalk between the oscillating unit and the inactive, not energized unit was not expected to occur. Nevertheless, interferences in oscillation were observed so that mechanical coupling obviously occurred across the inter-electrode, blank quartz material.

\section{Calculation of Shear Amplitudes of 2ElO Resonators}

According to a theoretical model by Martin et al. ${ }^{[365]}$, the shear amplitude of an AT-cut quartz resonator at a distance $r$ from the center $r_{0}$ of the quartz disk can be calculated. It is based on a Gaussian distribution of the shear amplitude of an oscillating quartz material sandwiched between two electrodes. The maximal amplitude is located in the center and decays radially to the edges of the electrode. However, motion at the edges of the electrode is not zero, since the oscillation amplitude decays across the electrode edges and beyond. The shear amplitude $x_{r}$ at a certain distance $r$ from the center of the electrode can be described according to the Gaussian function:

$$
x_{r}=x_{0} \cdot e^{\left(-w r^{2} / r_{e l}^{2}\right)}
$$

where $x_{0}$ is the maximal shear amplitude in the center $r_{0}$ of the electrode $(r=0), w$ is the width of the Gaussian function, and $r_{e l}$ is the radius of the electrode. For shear amplitudes of the quartz oscillation in air and in water, Gaussian distribution coefficients of $w^{\text {air }}=2.84$ and $w^{\text {water }}=2.03$ were found. ${ }^{[365]}$ Integration of Eq. (59) over the entire electrode surface provides the average shear amplitude $x_{a v}$ :

$$
\begin{aligned}
x_{a v} & =\int_{r_{0}}^{r_{e l}} x_{0} \cdot e^{\left(-w r^{2} / r_{e l}{ }^{2}\right)} 2 \pi r d r / \pi r_{e l}{ }^{2} \\
& =\frac{x_{0}}{w}\left(1-e^{-w}\right)
\end{aligned}
$$

According to ${ }^{[365]}, x_{a v}$ is also accessible from the electromechanical properties of the quartz plate and the applied amplitude of driving voltage $U_{0}$ by:

$$
x_{a v}=\frac{U_{0} d_{q}}{R_{t o t} e_{26} A \omega_{0}}
$$

In Eq. (61) $d_{q}$ is the quartz thickness, $R_{t o t}$ is the total motional resistance $\left(R_{t o t}=R_{q}+R_{L}\right.$, where $R_{q}$ and $R_{L}$ are the motional resistances of the quartz itself and of the loading, respectively), $e_{26}$ is the piezoelectric stress constant, $A$ is the electrode area, and $\omega_{0}$ is the angular resonance frequency of the quartz $\left(\omega_{0}=2 \pi f_{0}\right)$. Thus, $x_{a v}$ values dependent on specific resonator properties are obtained, which, inserted in Eq. (60), provide the maximal shear amplitude $x_{0}$ in the center of the electrode. Borovsky et al. used scanning tunneling microscopy to measure the lateral amplitude of vibration of an AT-cut quartz resonator ${ }^{[366]}$, revealing a relationship between $x_{0}$, the driving voltage $U_{0}$, and the quality factor $Q$ $\left(=X_{\text {tot }} / R_{\text {tot }}\right)$ of the resonator, according to: 


$$
x_{0}=1.4( \pm 0.1) \cdot 10^{-12}[\mathrm{~m} / \mathrm{V}] \cdot Q \cdot U_{0}
$$

Johannsmann and Heim ${ }^{[367]}$ have proven this equation for the amplitude of motion with a calculation based on a transmission line model. Using either the theoretically (Eqs. (60), (61)) or experimentally (Eq. (62)) obtained values of $x_{0}$, the shear amplitude $x_{r}$ at any distance $r$ from the electrode center can be calculated by applying Eq. (59). Based on this, Heitmann and Wegener ${ }^{[226]}$ calculated the lateral amplitude distribution of a classical $5 \mathrm{MHz}$ AT-cut quartz resonator, sandwiched between two circular gold-film electrodes of $6 \mathrm{~mm}$ diameter ( $1 \mathrm{ElO}$ ), under liquid loading and for various driving voltages. For a driving voltage of $0.15 \mathrm{~V}$ (which has been usually used in the $\mathrm{OCM}$ studies presented in this work), the calculations yield shear amplitudes of $x_{0}^{\text {theory }}=2.3 \mathrm{~nm}$ and $x_{0}^{\text {exp }}=0.5 \mathrm{~nm}$ in the center of the electrode. These calculations were now made for the 2 ElO resonators, too, for oscillation in air and under liquid (water) loading. For the sake of completeness and in order to have a $1 \mathrm{EIO}$ analogue for comparison with the calculated $2 \mathrm{EIO}$ values, calculations for $1 \mathrm{EIO}$ oscillations in air were additionally performed. The corresponding profiles of the shear amplitude $x_{r}$ as a function of distance $r$ from the electrode center are shown in Fig. 4-9 for $1 \mathrm{EIO}(\mathrm{A})$ and $2 \mathrm{EIO}(\mathrm{B})$ oscillations in air and when one quartz face is loaded with water. The parameter values that were used for the calculations $\left(R, A, R_{t o t}, X_{t o t}\right)$ and the calculated values of $Q, x_{a v}, x_{0}^{\text {theory }}$, and $x_{0}^{\text {exp }}$ for $1 \mathrm{EIO}$ and $2 \mathrm{EIO}$ oscillations in both air and water are listed in Tab. 4-3.

A
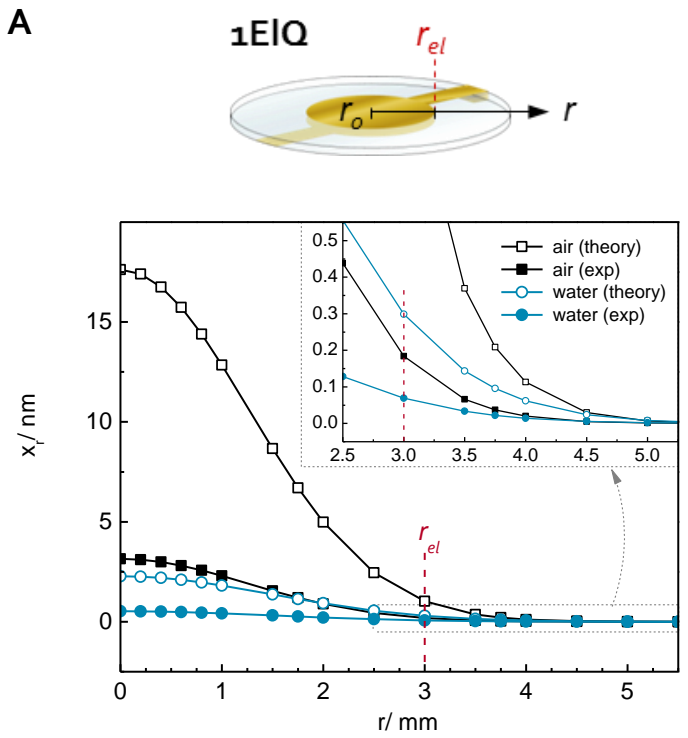

B
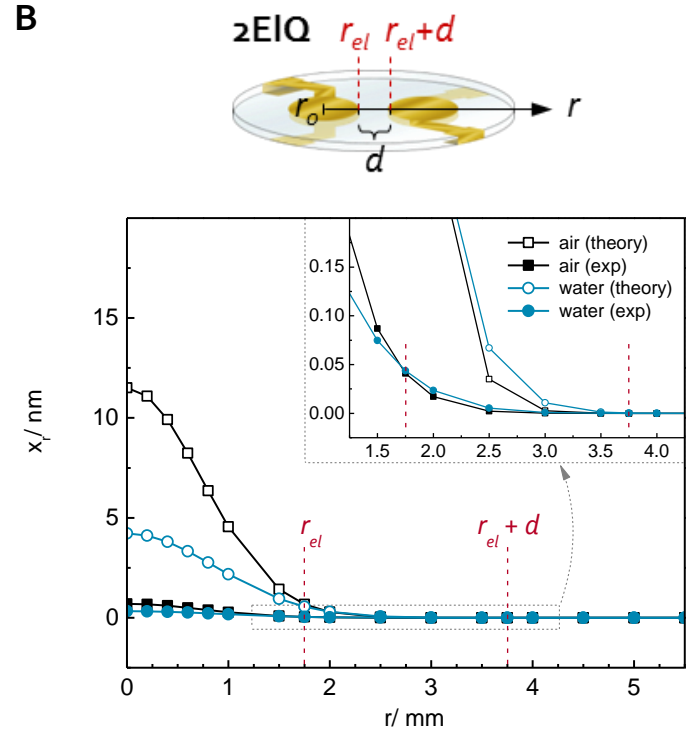

Fig. 4-9 Calculated shear amplitudes $x_{r}$ as a function of distance $r$ to the center of the electrode for a 1 EIO resonator (A) and one oscillating unit on a $2 \mathrm{EIO}$ (B) with $U_{0}=150 \mathrm{mV}$. Values were estimated with theoretically (theory) ${ }^{[365]}$ and experimentally (exp) ${ }^{[366]}$ based equations for oscillation at resonance in air and in water. $r_{e l}$ indicates the radius of the electrode and $d$ stands for the inter-electrode distance of a $2 \mathrm{EIO}$.

The graphs in Fig. 4-9 show maximal oscillation amplitudes at the electrode center $(r=0 \mathrm{~mm})$ and nonzero shear amplitudes at the electrode edges $\left(r=r_{e l}\right)$ for both types of resonators ( $1 \mathrm{EIQ}$ with $r_{e l}=3 \mathrm{~mm}$ and 2 EIO with $r_{e l}=1.75 \mathrm{~mm}$ ), in air as well as under liquid loading. The discrepancy between theoretically and experimentally obtained values of more than a factor of four has been described before ${ }^{[226,366]}$. This observation applies to the $2 \mathrm{ElO}$ values, too. Compared to the $1 \mathrm{ElO}$ oscillation, the amplitudes of $2 \mathrm{EIO}$ 
resonators are significantly smaller, which is seen best for the maximal shear amplitude at $r=0 \mathrm{~mm}$ in Fig. 4-9. For 2ElOs, maximal amplitudes of $x_{0}^{\text {exp }}=0.7 \mathrm{~nm}$ (in air) and $x_{0}^{\text {exp }}=0.3 \mathrm{~nm}$ (with one quartz face immersed in water) are estimated. At the electrode edges $\left(r=r_{e l}\right)$, the shear oscillation has decayed to $5.8 \%$ (air) and $13.1 \%$ (water) of the maximal value, respectively. At the distance $r=r_{e l}+d(=3.75 \mathrm{~mm}$ ), i.e. at the electrode edge of the second, non-oscillating unit, the shear amplitude of the active resonator has decayed to $0.0002 \%$ (in air) and to $0.009 \%$ (in water) of the maximal amplitude. According to these calculations, motion of the quartz material due to an adjacent active resonator is negligible at a distance of $2 \mathrm{~mm}$, where the electrode edge of the inactive resonator is located.

The results from shear amplitude calculations cannot readily explain the observed coupling of the adjacent sensor spots. Hence, either the experimentally based equation underestimates the motion profile of the oscillating quartz material, or other not yet considered effects play a crucial role in the electromechanical coupling and interference of the resonators.

In the course of the calculations above, inconsistencies were observed in the trends of $x_{a v}$ and $x_{0}$ values for the theoretically and experimentally calculated values. This becomes evident by comparing them in Tab. 4-3. With decreasing $Q$-factors, the experimentally obtained, maximal shear amplitudes $x_{0}^{\text {exp }}$ decreased likewise (see Eq. (62)). However, for the theoretically obtained averaged and maximal shear amplitudes, $x_{a v}^{\text {theory }}$ and $x_{0}^{\text {theory }}$, there is a discrepancy. $x_{a v}^{\text {theory }}$ and $x_{0}^{\text {theory }}$ values are lower for the $1 \mathrm{ElO}$ oscillation in water $(Q=2500)$ than for the $2 \mathrm{ElO}$ oscillation in water $(Q=1600)$. This observation can be explained by a closer look at Eq. (61) that has been used to determine $x_{a v}$. Calculations for $1 \mathrm{EIO}$ and 2 ElO average amplitudes only differ in the denominator values $A$ and $R_{\text {tot }}$. Both values are related to each other but do not change to a similar extent. In the present case, the $A \cdot R_{\text {tot }}$ product for 1 EIO in water is bigger than for $2 \mathrm{ElO}$ in water, and shear amplitudes of the former resonator are consequently lower than of the latter. This is vice versa for oscillations of both resonators in air. For this reason, shear amplitude values from theoretical calculations (Eqs. (60) and (61)) cannot be used for comparing resonators of different electrode size.

Tab. 4-3 Used parameters for calculating the amplitude of shear oscillation $x_{0}$ in the center of ${ }_{1}$ ElOs and 2 ElOs under air and liquid (water) loading. The resonators were based on $5 \mathrm{MHz}$ AT-cut quartz disks $\left(\omega_{0}=2 \pi f_{0}=3.14 \cdot 10^{7} \mathrm{~Hz}, d_{q}=330 \mu \mathrm{m}\right) ; R_{t o t}$ and $X_{\text {tot }}$ are averaged from measured values $\left(R_{\text {tot }}=R_{q}+R_{L}, X_{\text {tot }}=\omega L_{q}+\operatorname{Im}\left(\underline{Z}_{L}\right), \operatorname{Im}\left(\underline{Z}_{L}\right)=\sqrt{\left|Z_{L}\right|^{2}-\left(R_{t o t}-R_{q}\right)^{2}}, Q=X_{\text {tot }} / R_{\text {tot }}\right)$; the amplitude of the driving voltage was set to $U_{0}=0.15 \mathrm{~V}$, and Gaussian distribution coefficients of $w^{\text {air }}=2.84$ and $w^{\text {water }}=2.03^{[365]}$ were used.

\begin{tabular}{|c|c|c|c|c|c|c|c|c|c|}
\hline $\begin{array}{l}\text { Type of } \\
\text { Resonator }\end{array}$ & $\begin{array}{l}r_{e l} / \\
\mathrm{mm}\end{array}$ & $\begin{array}{c}A / \\
\mathrm{mm}^{2}\end{array}$ & Load & $\begin{array}{l}R_{\text {tot }} / \\
\text { Ohm }\end{array}$ & $\begin{array}{c}X_{\text {tot }} / \\
\text { MOhm }\end{array}$ & $Q$ & $\begin{array}{c}x_{0}^{e x p} / \\
\mathrm{nm}\end{array}$ & $\begin{array}{c}x_{a v}^{\text {theory }} / \\
\mathrm{nm}\end{array}$ & $\begin{array}{c}x_{0}^{\text {theory }} / \\
\mathrm{nm}\end{array}$ \\
\hline \multirow[t]{2}{*}{ 1EIQ } & 3.00 & 28.27 & air & 100 & 1.5 & 15000 & 3.2 & 5.8 & 17.6 \\
\hline & & & water & 600 & 1.5 & 2500 & 0.5 & 1.0 & 2.3 \\
\hline \multirow[t]{2}{*}{$2 \mathrm{ElQ}$} & 1.75 & 9.62 & air & 450 & 1.5 & 3300 & 0.7 & 3.8 & 11.5 \\
\hline & & & water & 950 & 1.5 & 1600 & 0.3 & 1.8 & 4.2 \\
\hline
\end{tabular}

\section{Resonator Synchronization}

It was found that the oscillation disturbances of $2 \mathrm{EIO}$ resonators in air and in liquid vanish, when the two electrodes on the top side as well as the two electrodes on the bottom side of the disk are short-circuited, 
respectively (Fig. 4-4). This outcome provides evidence that the disturbances in the oscillations occur due to the presence of the second pair of electrodes/ sensor spot on the quartz surface. By shortcircuiting, the two formerly separate sensor spots ( $\mathrm{OCM}_{1}$ and $\mathrm{OCM} 2$ ) perform synchronous oscillation $(\mathrm{OCM} 1+2)$ with impedance spectra similar to that of $1 \mathrm{EIO}$ vibrations around resonance. However, the spectral properties and characteristic impedance values of short-circuited $2 \mathrm{EIO}$ resonators are considerably different with respect to classical $1 \mathrm{EIO}$ oscillation properties. Typical impedance and phase spectra of a $1 \mathrm{EIQ}(\cdots .$.$) and of a short-circuited 2 \mathrm{EIO}(--)$ oscillation in air are shown in Fig. 4-10 A and B. The spectrum of a perturbed, non-short-circuited $2 \mathrm{ElO}$ oscillation (---) is shown for comparison, too. The phase maximum of the short-circuited $2 \mathrm{ElO}$ in air exhibits a value of $\varphi_{\max } \approx 65^{\circ}$, which is $\sim 20^{\circ}$ lower than the value for the $1 \mathrm{EIO}$ oscillation $\left(\varphi_{\max } \approx 85^{\circ}\right)$. This significant higher acoustic damping is also reflected in higher $|Z|_{\text {min }}$ values for the $2 \mathrm{EIO}(\sim 550 \mathrm{Ohm})$ compared to the $1 \mathrm{EIO}(\sim 100 \mathrm{Ohm})$. These differences in impedance properties are due to differences in the electrode layout for a $1 \mathrm{EIO}$ and a $2 \mathrm{EIQ}$, and are readily explained by BVD equivalent circuit elements, whose expressions are given in Tab. 3-5. According to that, the resistance $R_{q}$ of the motional branch of the BVD equivalent circuit dominates the value of $|Z|_{\text {min }}\left(|Z|_{\text {min }}=R_{q} /\left(1-\left(\omega R_{q} C_{0}\right)^{2}\right) \approx R_{q}\right) \cdot R_{q}$ is inversely proportional to the electrode area (Eq. (30)), and in comparison to the classical 1 ElO, the electrode area of the two sensor spots on a $2 \mathrm{EIO}$ is decreased by a factor of $\sim 1.5\left(A_{1 \mathrm{EIO}}=28.3 \mathrm{~mm}^{2}\right.$ vs. $\left.2 \times A_{2 \mathrm{EIO}}=2 \times 9.6 \mathrm{~mm}^{2}=19.2 \mathrm{~mm}^{2}\right)$. Hence, the increase of $|Z|_{\min }$ can be mainly contributed to a reduction of the net electrode area and, thus, the active oscillation region. Since damping effects also arise from the finite dimensions and edges of the electrodes, the circumference-to-area ratio $C / A$ is a factor for the degree of damping, too. High values cause strong damping while lower values effect less damping. Applying this to $1 \mathrm{ElO}$ s and $2 \mathrm{ElQ}$, one obtains ratios of $C / A(1 \mathrm{EIO}) \approx 0.67 \mathrm{~mm}^{-1}$ and $C / A(2 \mathrm{ElO}) \approx 1.14 \mathrm{~mm}^{-1}$. Thus, electrodes of $2 \mathrm{ElO}$ cause bigger dissipation losses of the motional impedance than electrodes of 1 ElOs. Moreover, when shortcircuiting 2 ElOs four electrodes had to be contacted to the electronics instead of two with 1 ElOs, so that contact resistance of the former should be twice as high.

A

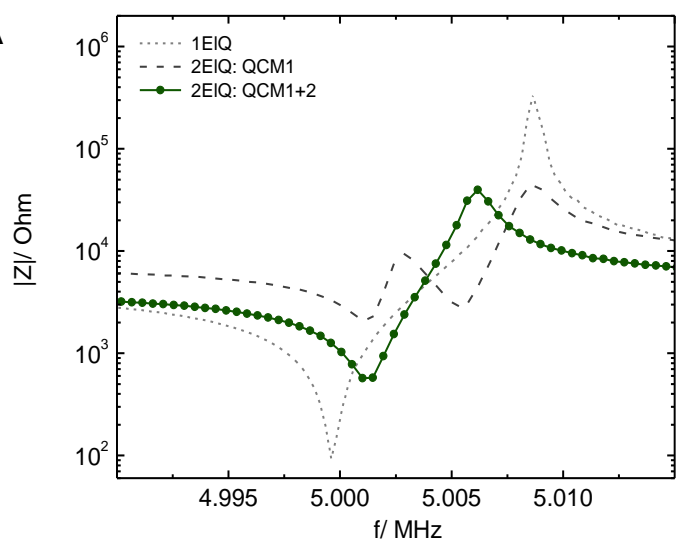

B

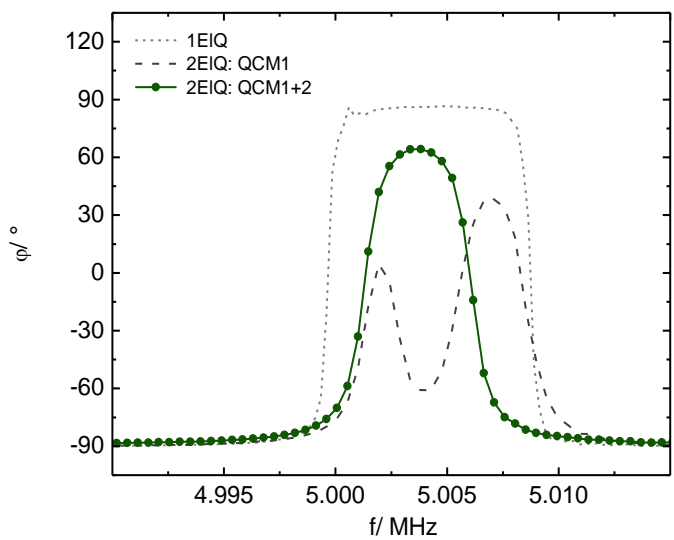

Fig. 4-10 Spectra of (A) the magnitude and (B) the phase of impedance, recorded in air of a typical $1 \mathrm{EIO}(\cdots \cdots)$, of the perturbed $\mathrm{OCM} 1 / \mathrm{OCM} 2$ of a $2 \mathrm{EIO}(---)$, and of a short-circuited $2 \mathrm{EIO}$ $(\mathrm{OCM} 1+2 ;-\bullet)$.

Despite the higher energy dissipation of $2 \mathrm{ElOs}$ compared to $1 \mathrm{ElOs}$, the short-circuited $2 \mathrm{EIO}$ could be applied for sensing adherent cells. The impedance spectra exhibit significant differences between the initial oscillation in culture medium and the oscillation of a quartz plate covered with a confluent cell layer

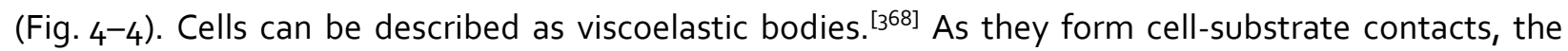


viscoelastic bodies are mechanically coupled with the OCM sensor surface. Besides a small nondissipative influence on the quartz oscillation, the cells exhibit a big dissipative contribution. This leads to a strong damping of the oscillation and significantly increased $|Z|_{\min }$ values for a cell-covered resonator with respect to cell-free conditions under medium.

\subsubsection{Decoupling of 2ElQ Resonators}

Different strategies of decoupling resonances are discussed in literature, regarding the effects of the design parameters electrode spacing ${ }^{[362,363]}$, electrode size ${ }^{[363]}$, positioning of electrodes ${ }^{\left[{ }^{[64} 4\right.}$, electrode thickness ${ }^{[362,364]}$, different coatings on electrodes ${ }^{[354]}$, variations in quartz thickness by mesa-shape and convex-shape designs, MOCMs with $\mathrm{x}$-axis inversion, or a successive oscillation of resonators ${ }^{[352,360]}$.

\section{Oscillation Properties of Decoupled 2EIQ Resonators}

For the post-fabrication decoupling of $2 \mathrm{ElO}$ microbalances the selective coating of only one sensor unit with a thin and rigid layer of polymer turned out to be successful. This strategy provided a loss-free decoupling of $2 \mathrm{ElO}$ interferences (cf. chapter 4.1.5, p. $90 \mathrm{ff}$.). The film consists of photoresist that was applied to the whole top quartz surface by spin coating. Half of the coating, i.e. photoresist on one resonator $(\mathrm{OCM} 2)$, was subsequently removed by photolithography and remaining photoresist was cured in a final hard baking process, resulting in a rigid film on the second resonator $\left(\mathrm{OCM}_{1}\right)$. According to Sauerbrey's relation (Eq. (23)), the resonance frequency (the whole resonance spectrum) of the coated resonator was shifted to lower values by $\sim 22 \mathrm{kHz}$, whereas the oscillation properties of the uncoated sensor spot remained unaffected. The rigid photopolymer (PhoP) layer causess an increase in the thickness of the quartz plate and consequently a decrease in the frequency (increase in the acoustic wavelength) for meeting resonance conditions, i.e. a standing acoustic wave between the surfaces of the oscillating material. This can be nicely seen in the exemplary spectra in Fig. 4-11 A and B. Starting from the perturbed impedance spectrum of $\mathrm{OCM}_{2}(--)$, the resonance of $\mathrm{OCM}_{1}(-)$ ) is shifted to lower frequencies by the rigid coating and, thus, is separated from the resonance of $\mathrm{OCM}_{2}(-\square)$. Due to the difference in thickness of $\mathrm{OCM}_{1}$ and $\mathrm{OCM}_{2}$, the resonance frequencies are sufficiently separated from each other and mutual perturbations are eliminated. The impedance data (magnitude and phase shift) of decoupled $\mathrm{OCM} 2$ almost completely overlap with that of the initially uncoated $2 \mathrm{ElQ}$, except for a narrow frequency region (4.990 to 5.005 MHz) where interference occurs (- - - vs. --- in Fig. 4-11 A, B). Corresponding Nyquist plots are shown in $S / 9 C$ (p. 265). Except for the resonance shift, the oscillations of separated $\mathrm{OCM} 1$ and $\mathrm{OCM} 2$ exhibit similar shapes of impedance magnitude and phase spectra with comparable values of the characteristic impedance quantities $|Z|_{\max },|Z|_{\min }$, and $\varphi_{\max }$ (cf. Tab. 4-1).

With respect to the 1 ElQ impedance spectra ( $\cdots . .$. in Fig. 4-11 A and B), the impedance spectra of the two separated $2 \mathrm{ElO}$ resonators are significantly more damped, with higher $|Z|_{\min }$, and lower $|Z|_{\max }$ and $\varphi_{\max }$ values, respectively. This also can be readily explained by the increased internal oscillation damping when the active oscillating area, i.e. the electrode size, is reduced, as discussed previously for synchronized $\mathrm{QCM} 1$ and $\mathrm{QCM}_{2}$ of an uncoated $2 \mathrm{ElQ}$. In comparison to the classical $1 \mathrm{ElQ}$, the electrode area of one resonator spot of a $2 \mathrm{EIO}$ is decreased by a factor of $2.9\left(A_{1 \mathrm{EIO}}=28.3 \mathrm{~mm}^{2} \mathrm{vs} . A_{2 \mathrm{ElO}}=9.6 \mathrm{~mm}^{2}\right)$. Just like $|Z|_{\text {min }}$, the parameter $|Z|_{\text {max }}$ is also dominated by the motional resistance $R_{q}$, according to its expression in Eq. (45), and thus by the electrode size (Eq. (30)). Since $|Z|_{\max }$ and $R_{q}$ are inversely related, a decrease in the electrode size is accompanied by a decrease in $|Z|_{\text {max }}$, just as it is observed for the 2ElOs compared to typical $1 \mathrm{ElOs}$ in Fig. 4-11 A. In the frequency region between the parallel and the serial resonance frequency, the inductance $L_{q}$ in the BVD equivalent circuit of the quartz dominates the 
oscillation properties. This is reflected in impedance phase values $\varphi>$ zero degrees. Values of $\varphi=90^{\circ}$ are almost reached with 1 ElOs in air, as they exhibit a large electrode size and low values of $R_{q}$. The effect of increasing $R_{q}$ values (in this case due to a reduced electrode size) on the maximal phase shift can be nicely seen in the Nyquist plot (cf. Fig. 3-8 D, p. 41). In the complex plane, the circle of the oscillation impedance is downwards shifted along the $\operatorname{Im}(\underline{Z})$-axis with increasing $R_{q}$ values. Thus, the maximal phase angle between real and imaginary part is shifted to lower values. However, the minimal phase of $\varphi=-90^{\circ}$ beyond resonance conditions is due to the parallel capacitance $C_{0}$ and not affected by $R_{q}$ changes.

A

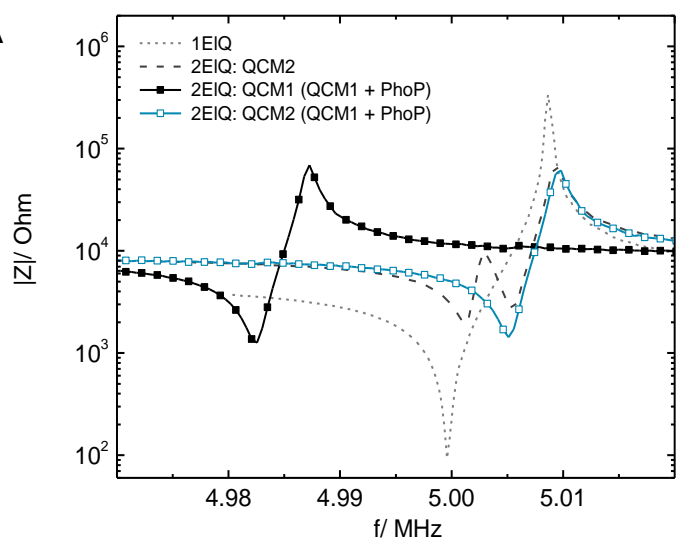

B

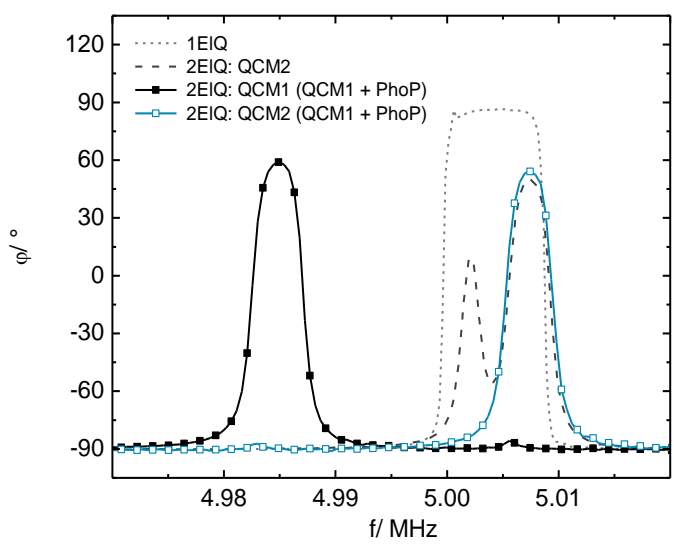

Fig. 4-11 Spectra of (A) impedance magnitude and (B) impedance phase, recorded in air of a typical 1 EIO $(\cdots \cdots)$, of the perturbed $\mathrm{OCM}_{1} / \mathrm{OCM}_{2}$ of a $2 \mathrm{EIO}(---)$, and of $\mathrm{OCM}_{2}(-\square)$ and $\mathrm{OCM}_{1}$ $(\rightarrow)$ of a 2 ElO with rigid loading on OCM1.

The changes in the oscillation properties are a result of electrode size reduction and can also be explained by a decreased quality factor $Q$ of the $2 \mathrm{EIO}$ resonators. The $Q$-factor is defined as the ratio of stored energy and the dissipated energy of the quartz oscillation:

$$
Q=\frac{1}{D}=\frac{X_{t o t}}{R_{t o t}}
$$

where $D$ is the dissipation factor, $X_{\text {tot }}$ represents the reactive properties of the quartz as well as any imaginary contributions of a load, and $R_{\text {tot }}$ stands for the total resistance of the quartz oscillation. ${ }^{[240]}$ For an unloaded quartz plate, $X_{\text {tot }}$ and $R_{\text {tot }}$ are given by the internal quartz properties $L_{q}$ and $R_{q}$. By inserting the expressions from Eq. (28) and Eq. (30), the quality factor is:

$$
Q=\frac{\omega L_{q}}{R_{q}}=\frac{\omega \rho_{q}}{r}=\frac{\omega \rho_{q} d_{q}^{2}}{\eta_{q} \pi^{2}}
$$

where $r$ is the damping constant ( $r=\eta_{q} \pi^{2} / d_{q}^{2}$, with $\eta_{q}$ as effective quartz viscosity). According to Eq. (64), the $Q$-factor depends on the effective viscosity of the quartz, which increases with decreasing electrode size. ${ }^{[288]}$ Thus, the $Q$-factor of 2 ElOs is lower than for ${ }_{1}$ ElOs, resulting in the previously described changes of the characteristic oscillation parameters. Experimental data deliver a $\sim 4.5$-fold lower $Q$-factor (in air) for the 2 EIQ compared to the 1 EIO resonator (Tab. 4-3). 
As discussed in the previous subsection, $Q$ is also directly linked with the amplitude of shear displacement (Eq. (62)). Consequently, the shear displacement in the center of a $2 \mathrm{EIO}$ resonator is lower than for a $1 \mathrm{ElO}$ and its Gaussian distribution is more damped at the electrode edges as well. Moreover, the decay length of the shear wave, which propagates vertically into the medium above the resonator, is expected to be shorter for smaller electrodes. Thus, a drawback of $2 \mathrm{EIO}$ sensors might be a shorter depth of penetration into the specimen (the cell layer) at the quartz surface.

\section{Oscillation Properties of Decoupled and Synchronized 2EIQ Resonators}

In another measurement approach the impedance spectra of the decoupled resonators $\mathrm{OCM}_{1}$ and $\mathrm{QCM}_{2}$ were measured simultaneously, by short-circuiting the electrodes on the top side and on the bottom side of the $2 \mathrm{EIO}$, respectively. This provided the impedances of both resonator units as a combined signal, $\underline{Z}_{O C M 1+2}$ by a single frequency sweep ( $\bullet-$ in Fig. 4-5). The observable discrepancy between the combined impedance signal and the impedance spectra of successively measured resonators can be assigned mainly to changes in the dielectric properties, i.e. the parallel capacitance $C_{0}$ of the quartz, by shortcircuiting $\mathrm{OCM}_{1}$ and $\mathrm{OCM}_{2}$. This becomes evident by a closer look the impedances at off-resonance frequencies $(4.96 \mathrm{MHz}$ and $5.03 \mathrm{MHz}$ in Fig. 4-5 B). There, the quartz behaves like a parallel-plate capacitor and the impedance is dominated by the static capacitance $C_{0}$ (see BVD model in theory section 3.3.2, p. 37 ff.). As the impedance decreases with increasing parallel capacitance (Eq. (32)) and as $C_{0}$ is directly proportional to the electrode area (Eq. (31)), impedance and electrode size are inversely correlated with each other. Typical impedance magnitude values of individual $2 \mathrm{EIO}$ resonators $\left(|Z|_{\mathrm{OCM} 1}\right.$ and $\left.|Z|_{\mathrm{OCM}_{2}}\right)$ and of short-circuited $2 \mathrm{EIO}$ resonators $\left(|Z|_{\mathrm{OCM}_{1+2}}\right)$ at the off-resonance frequencies $4.96 \mathrm{MHz}$ and $5.03 \mathrm{MHz}$ are listed in Tab. 4-4. The data confirm that the impedance of the short-circuited resonators $\left(|Z|_{O C M_{1+2}}\right)$ is reduced almost exactly by half with respect to the averaged impedance of the separately measured $\mathrm{QCM} 1$ and $\mathrm{OCM}_{2}\left(1 / 2\left(|Z|_{\mathrm{OCM}_{1}}+|Z|_{\mathrm{OCM}_{2}}\right)\right)$. This can be assigned to the doubling of the electrode area when synchronizing $\mathrm{OCM} 1$ and $\mathrm{OCM} 2$.

Tab. 4-4 Tapical impedance magnitude values for 2 ElO resonators ( $Q C M 1$ and $\mathrm{OCM}_{2}$ ), which were decoupled by the deposition of a rigid photoresist film on $\mathrm{OCM}_{1}$. Values are shown for individual $\left(|Z|_{O C M_{1}},|Z|_{O C M_{2}}\right)$ and for short-circuited $\left(|Z|_{O C M_{1+2}}\right)$ oscillations at off-resonance frequencies (4.96 MHz, 5.03 MHz).

\begin{tabular}{|c|c|c|c|c|}
\hline$f / \mathrm{MHz}$ & $\begin{array}{c}|Z|_{\mathrm{OCM}_{1}} \mid \\
\mathrm{kOhm}\end{array}$ & $\begin{array}{c}|Z|_{\mathrm{OCM}_{2}} \mid \\
\text { kOhm }\end{array}$ & $\begin{array}{c}1 / 2\left(|Z|_{\left.\mathrm{OCM}_{1}+|Z|_{\text {OCM2 }}\right) /}\right. \\
\mathrm{kOhm}\end{array}$ & $\begin{array}{l}|Z|_{\mathrm{OCM}_{1+2}} \mid \\
\mathrm{kOhm}\end{array}$ \\
\hline 4.96 & $7 \cdot 3$ & 8.3 & 7.8 & 3.9 \\
\hline 5.03 & 9.5 & 10.6 & 10.1 & 5.1 \\
\hline
\end{tabular}

The increase of $C_{0}$ by short-circuiting $\mathrm{OCM}_{1}$ and $\mathrm{OCM} 2$ also influences the characteristic impedance values near parallel resonance $\left(f_{p}, f_{|Z|_{\text {max }}},|Z|_{\text {max }}\right.$ ). This is reflected in a negative shift of both the

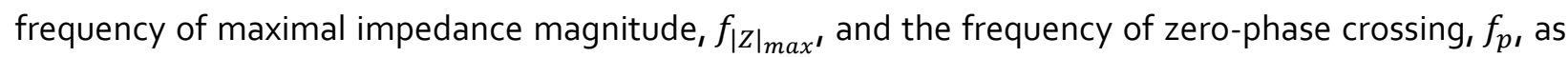
well as in a decrease of the maximal impedance magnitude $|Z|_{\max }$. The impact of $C_{0}$ changes on these parameters is readily apparent from their expression by electrical equivalents in Eqs. (39) - (45) (p. 42). For instance, the severe reduction of $|Z|_{\text {max }}$ by a factor of $\sim 4$ upon connecting resonators $(\sim 65 \mathrm{kOhm}$ $\rightarrow \sim 16 \mathrm{kOhm}$, see Fig. 4-5 B) is comprehensible, as $|Z|_{\max }$ is inversely related to the square of the static capacitance $C_{0}$ of the quartz material (Eq. (45)). Thus, doubling of the parallel-plate capacitor area by 
short-circuiting $\mathrm{OCM}_{1}$ and $\mathrm{OCM} 2$ results in changes of the maximal impedance magnitude by a factor of four, as observed in Fig. 4-5. At frequencies where the motional branches are resonant, the characteristic impedance values $\left(f_{|Z|_{\text {min }},},\left.Z\right|_{\text {min }}, f_{s}\right)$ are not affected by short-circuiting of electrodes. There, the piezoelectric properties of the quartz material dominate the impedance and dielectric properties, i.e. the static capacitance $C_{0}$, can be neglected. This is also reflected in the corresponding expressions of impedance parameters by electrical elements of the BVD equivalent circuit (Eqs. (38) - (44) in Tab. 3-5, p. 42). These observations, of the impact of electrode short-circuiting on impedance characteristics, also match well with previously presented simulations, which demonstrate the influence of different $C_{0}$ values on the shape and characteristic parameters of impedance spectra. ${ }^{[296]}$

The approach of short-circuiting resonators and simultaneously recording their resonance characteristics provides some considerable advantages over the successive measurements of individual microbalances. On the one hand, one can reduce the data acquisition time, as the frequency window does not have to be scanned anew for each sensor spot and as switching between ports for contacting the individual resonators can also be omitted. On the other hand, the effort of contacting the various electrodes on the quartz disc is minimized, as in total then only two contact pads are required: each one on the top and bottom surface for contacting simultaneously all short-circuited electrodes on each side. The shortcircuiting for example can be implemented by conduction paths at the edge of the disc. This is of great relevance, especially with regard to parallelization of sensor spots and miniaturization of the experimental setup and, therefore, could promote the development in these directions. However, the basic requirement in any case of simultaneous $\mathrm{OCM}$ measurements is the sufficient spectral separation of resonances of all oscillating units.

\section{Oscillation Properties of Decoupled 2 ElO Resonators under Liquid Loading}

When performing oscillations with the decoupled 2ElOs under liquid loading (Fig. 4-6, p. 93), a strong damping of the impedance spectra of both resonators was observed. This is due to the viscosity-density product of the liquid, which adds an additional resistance and inductance in series to the motional branch of the BVD equivalent circuit of each resonator (cf. Tab. 3-6 B, p. 44). The degree of damping is similar for $\mathrm{OCM}_{1}$ and $\mathrm{OCM}$, which is reflected in similar changes of characteristic impedance parameters (Tab. 4-1). This is not necessarily expected, since one resonator (OCM1) is covered with an additional polymer film and, thus, exhibits a different quartz-liquid interface compared to the other. Swelling of the polymer on $\mathrm{OCM}_{1}$ upon immersion in liquid and a consequently higher energy dissipation of the oscillation could lead to a change from a rigid into a viscoelastic polymer film. However, $\mathrm{OCM}_{1}$ and $\mathrm{QCM} 2$ exhibit similar impedance profiles after equilibration in cell culture medium over $24 \mathrm{~h}$. Hence, swelling of the polymer seems to be weak and the influence of the polymer on $\mathrm{OCM}_{1}$ oscillation properties under liquid can be considered negligible. The preserved rigidity of the photoresist on a $2 \mathrm{EIO}$ with liquid loading also becomes apparent in the stable resonance frequency difference between $\mathrm{OCM}_{1}$ and $\mathrm{OCM}_{2}$ and the persistent uncoupling of the resonators.

An effect that has to be considered especially for resonators immersed in an electrolyte is the occurrence of field fringing at the edges of the electrodes. ${ }^{[197,349,369-371]}$ They cause a stray capacitance additional to the static capacitance $C_{0}$ of the quartz and, thus, contribute to parallel resonance properties of a resonator as well as to the impedance off resonance. Field fringing at the electrode edges depends on the depolarization capabilities and, thus, on the dielectric properties of the quartz loading. Since the dielectric constant for an electrolyte is by far higher than for air, field-fringing of the quartz and, consequently, the effective parallel capacitance is larger in medium than in air. Moreover, covering the electrodes with an electrolyte solution will cause the formation of electrical double layers, which act as 
capacitors in addition to the parallel capacitance of the quartz plate. ${ }^{[296]}$ These effects become evident when comparing the impedance magnitude spectra of the 2 ElO resonators in air (Fig. 4-5, p. 91) and under medium (Fig. 4-6, p. 93) far from resonance and at parallel resonance frequencies. It has to be noted that parallel capacitance contributions arising from the $2 \mathrm{EIO}$ surface in contact with an electrolyte are more pronounced for the uncoated resonator than for the photoresist-coated resonator, reflected in almost $10^{4} \mathrm{Ohm}$ lower $|Z|_{\text {max }}$ values of $\mathrm{OCM}_{2}$ compared to $\mathrm{OCM}_{1}$ at parallel resonance frequencies. Whereas the resonators in air exhibit off-resonance impedances (at $4.96 \mathrm{MHz}$ ) of $7.3 \mathrm{kOhm} \mathrm{(OCM1}$ + PhoP) and $8.3 \mathrm{kOhm}(\mathrm{OCM} 2)$, the same oscillators loaded with medium for $24 \mathrm{~h}$ exhibit equal impedance magnitudes of $5.5 \mathrm{kOhm}$. The decrease is due to an increase in the parallel capacitance for both resonators on the $2 \mathrm{ElQ}$ upon covering one quartz plate face with medium. It is noticeable that the two resonators finally show equal values and the photoresist-related differences measured in air obviously vanished in medium. Thus, the potential dielectric contribution by the polymer is completely masked in medium by fringing of the electric field and electrical double layers at the quartz-electrolyte interface.
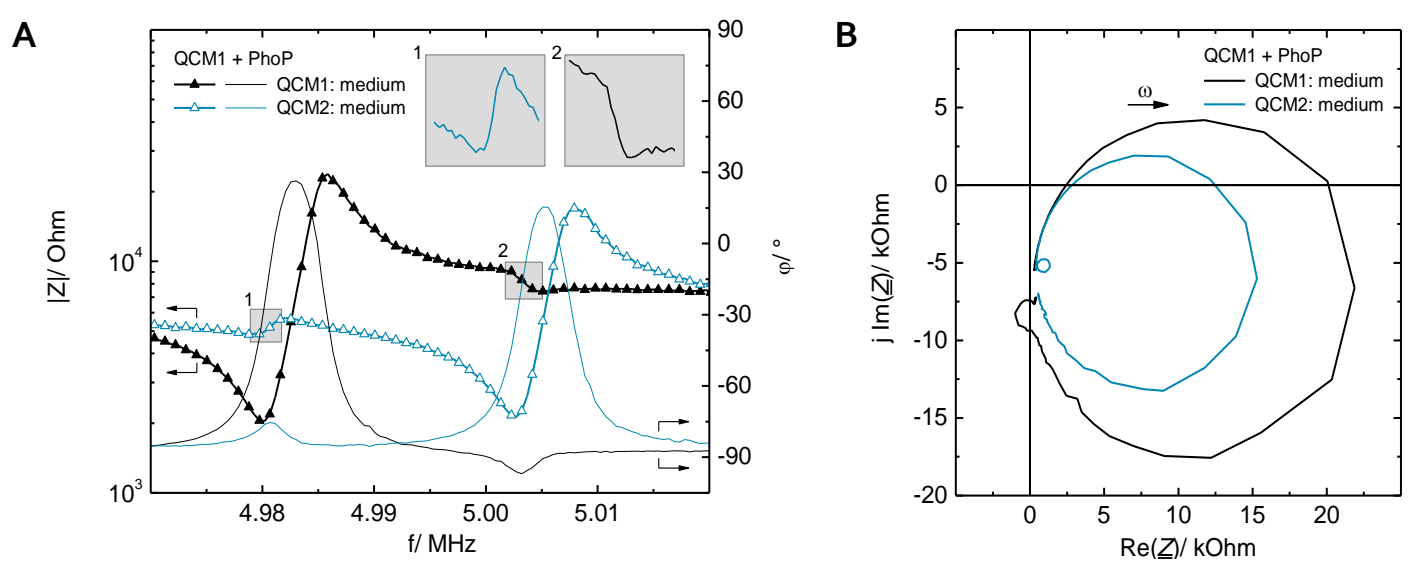

Fig. 4-12 (A) Combined plot of impedance magnitude and phase of $\mathrm{OCM}_{1}(-,-)$ and $\mathrm{OCM}_{2}$ $(-\Delta,-)$ of a $2 E l O$ with rigid loading on $\mathrm{OCM}_{1}$, oscillating under medium. (B) Nyquist representation of the impedance data shown in A.

The impedance spectra of the decoupled $2 \mathrm{ElO}$ oscillations in medium (Fig. 4-6) exhibit slight perturbations and secondary resonances, respectively, for both resonators (OCM1 and $\mathrm{OCM}_{2}$ ) in impedance magnitude and phase spectra. For a better analysis, impedance magnitude and phase spectra of both sensing units are merged in one diagram, shown in Fig. 4-12 A. For $\mathrm{OCM}_{1}(-)$ ) this unwanted resonance occurs at $\sim 20 \mathrm{kHz}$ upwards from serial resonance. This is exactly the frequency region where the non-coated $\mathrm{OCM}_{2}(--)$ exhibits resonance. The same effect is observed for the impedance spectrum of $\mathrm{OCM}$, about $20 \mathrm{kHz}$ downwards from its serial resonance, at frequencies where the photoresistcovered $\mathrm{OCM} 1$ resonance occurs. Hence, the decoupled resonators on the $2 \mathrm{ElQ}$ obviously again interfere with each other when the two resonators perform oscillations in medium. For analyzing oscillations of a quartz plate exposed to an electrolyte like cell culture medium also the electrical properties of the loading have to be considered in addition to the mechanical properties. Conductivity of the loading as well as related effects like acoustoelectrics, fringing fields and stray capacitances considerably codetermine the resonator properties. In the case of two (or more) adjacent resonators separated only by a small unelectroded quartz area $\left(A_{2 \mathrm{EIO}} / d \leq 9.6 \mathrm{~mm}^{2} / 2 \mathrm{~mm}\right)$, these effects have to be taken into account, too, in terms of their potential contribution to the electromechanical lateral coupling of the sensor spots. As 
already discussed above, the amplitude of motion is non-zero at the electrode edges and vibration further proceeds in the region of blank, not metallized quartz. This vibration generates charges at the surface of the quartz due to piezoelectricity, all along the acoustic wave. This polarizations on the quartz surface causes additional electric fields, dependent on the depolarization capabilities of the loading. If polarization of the faces is not completely compensated by the loading on the quartz, this again causes electromechanically induced vibration according to the inverse piezoelectric effect. The electric situation is different for the two resonators and their vicinity on a decoupled $2 \mathrm{ElO}$ exposed to medium. Due to a smaller dielectric constant of the polymer, surface charge compensation is assumed to be less pronounced for the photoresist-coated quartz area near QCM1 compared to the blank uncoated quartz area near $\mathrm{QCM} 2$ that is directly exposed to the conductive liquid. This would theoretically increase the effective electrode area of $\mathrm{OCM}_{1}$ and, thus, minimize the separation band between the two liquid-side electrodes. These assumed acoustoelectric effects as well as excess field fringing, stray capacitances and electric double layer formation could all affect the oscillation properties of $\mathrm{OCM}_{1}$ and $\mathrm{OCM}_{2}$. Finally, coupling of the sensing units is enabled by the electrolytic conductivity of the medium, what is prevented when the two electrodes on the topside of a $2 \mathrm{EIO}$ are not conductively connected via an electrolyte.

The perturbation of $\mathrm{OCM}_{2}$ at frequencies where $\mathrm{OCM} 1$ is resonant seems like a secondary resonance with a similar small and narrow increase of the phase $\left(\Delta \varphi \approx+10^{\circ}\right)$ and less pronounced serial and parallel resonances (Fig. 4-12 A, zoom-in No. 1), with respect to the fundamental resonance. However, the secondary resonance observed for $\mathrm{OCM}_{1}$ is remarkably different (Fig. 4-12 A, zoom-in No. 2). The $\mathrm{QCM}_{1}$ impedance magnitude does not really show a resonance-like profile in the resonance region of $\mathrm{OCM}_{2}$, but only a $\sim 2 \mathrm{kOhm}$ drop to a lower value, which remains constant when scanning to higher frequencies. Moreover, the phase shift drops to values below $-90^{\circ}\left(\Delta \varphi \approx-10^{\circ}\right)$, showing exactly the reverse behavior than observed for $\mathrm{QCM}$. Fig. 4-12 B shows the corresponding Nyquist plots of the decoupled mediumcovered $2 \mathrm{ElO}$ sensor spots. The oscillation impedances appear as circles in the Gaussian plane with a clockwise increase of the applied AC frequency (cf. Fig. 3-8 D, p. 41). Compared to the OCM1 circle, the circle of $\mathrm{OCM}_{2}$ is smaller in diameter, what is related to a slightly lower maximal impedance magnitude and maximal phase shift, respectively, also visible in the respective Bode plot (Fig. 4-12 A). These discrepancies in the impedance spectra of $\mathrm{OCM}_{1}$ and $\mathrm{OCM}_{2}$ in the resonance region of the other resonator are also visible in the Nyquist plot. Interferences are identified as additional small circles at frequencies lower $\left(\mathrm{OCM}_{2}\right)$ or higher $(\mathrm{OCM})$ than the respective fundamental resonance frequency (shortest vector in the Gaussian plane). The origin of this phenomenon is not understood yet and needs to be further investigated.

The interference effects that appear for the decoupled $2 \mathrm{EIO}$ upon exposing it to electrolyte solution are small and occur at frequencies that are sufficiently far from the corresponding fundamental resonances. Thus, the observed coupling does not affect the analysis of minimal impedance values. Nevertheless, interferences are detectable, revealing that the deposited rigid film on one resonator for decoupling of resonance frequencies should at least not be thinner.

In summary, decoupling of resonators on 2 ElOs was be successfully achieved by depositing a rigid mass, i.e. a thin layer of photoresist, on one resonator unit so that resonance frequencies of the two sensor spots were sufficiently separated. The degree of decoupling of resonances was even sufficient for quartz oscillations under loading with conducting liquid. Thus, prerequisites were fulfilled to apply 2 EIO plates with two individual readout spots for whole-cell biosensing. 


\section{Impact of Film Thickness on the Degree of Decoupling}

The findings presented in Fig. 4-7 allow the following conclusions regarding the thickness of deposited films and the degree of resonance shift and of decoupling of $\mathrm{OCM}_{1}$ and $\mathrm{QCM} 2$ resonances, respectively. A sufficient separation of resonances of the oscillating units on a $2 \mathrm{EIO}$ was obatained for all of the applied spin coating velocities, which correlated with deposited film thicknesses between 2.7 and $4.2 \mu \mathrm{m}$. Even the thinnest deposited film was still thick enough to decrease the resonance frequency of the coated sensor spot to values $(-15.4( \pm 0.2) \mathrm{kHz})$ which avoided a spectral overlap of its resonance region with the resonance of the uncoated resonator. However, a decrease in the film thickness is accompanied with a significant increase in the degree of interferences off resonance in the impedance spectra (cf. inset graphs in Fig. 4-7 A and C). When shifting the spectra of both resonators even closer to each other than the minimum obtained in the test series, to estimated values of $\Delta f_{|Z|_{\text {min }}} \approx-(5-10) \mathrm{kHz}$, interference near resonances are expected to occur. This would result in disturbed oscillation properties of the resonators $\mathrm{QCM}_{1}$ and $\mathrm{QCM} 2$, similar to the completely uncoated resonator (Fig. 4-2, p. 86), avoiding extraction of $|Z|_{\text {min }}$ values for monitoring impedance changes. Therefore, $3000 \mathrm{rpm}$ was set as the standard rotational speed for spin coating 2 ElOs with photopolymer, in order to maintain a sufficient separation of resonances required for their application as MOCM.

From OCM measurements and white light vertical scanning interferometry measurements of similary coated substrates, a correlation (Eq. (58)) was established that connects the frequency shift between $\mathrm{QCM}_{1}$ and $\mathrm{QCM}_{2}$ resonator on a $2 \mathrm{ElO}$ with the thickness of the deposited photoresist layer. This calibration allows to estimate the polymer height of $2 \mathrm{ElOs}$ that have been coated with the same photoresist via the determination of the resonance frequency shift $\Delta f_{|Z|_{\text {min }}}$ between coated and uncoated resonator. The correlation given in Eq. (58) should be valid for any concentration and viscosity of the same type of polymer solution in the linear range of the equation, since the final physical properties of the hard-baked and solvent-free photoresist layers, like e.g. density, hardeness, wettability, or swelling properties, should be similar. The only basic prerequisites are that the deposited layer forms a homogeneous and rigid film on the substrate and does not cause energy dissipation of the quartz oscillation. ${ }^{[349]}$ Hence, solvent has to be completely evaporated during hard-baking of the film and swelling of the deposited film in liquid or due to air humidity has to be avoided during OCM measurements.

The results further show that quartz disks had significantly larger resonance shifts and thicker films with the "old" than with the "new" photoresist, even though coating has been performed at the same spin coating velocity and conditions. A higher flow viscosity of the "old" photoresist due to solvent evaporation over time explains this. This causes a higher counterforce against the centrifugal force during spin coating, which results in a decrease of the speed of polymer spreading. The slower film spreading in combination with unchanged solvent evaporation rates finally results in thicker films after spin coating. The film thickness of $\sim 2.7 \mu \mathrm{m}$ at $4000 \mathrm{rpm}$ for the "new" photoresist agreed well with the predicted manufacturer's data. ${ }^{[61]}$ It has to be noted that spin coating of substrates with one and the same photoresist solution (same viscosity) and equal spin coating speed does not necessarily result in equally thick polymer layers, since the final thickness is also determined by the ambient conditions during coating. For instance, the ambient temperature, air humidity and air pressure affect the equilibrium between film spreading on the substrate surface and solvent evaporation during the spin coating process. Moreover, post coating photolithography and related processes influence the final height of the polymer, too. 


\subsubsection{Outlook}

Based on the established a two-spot QCM resonator, various further developments of the sensor layout are thinkable, like for instance an increase in the number of resonator spots, the integration of various surface properties and sensing capabilities on the substrate, and the implementation of additional measurement techniques for multiple cellular and chemical sensing.

At first, one could think of a development of the MOCM approach, by multiplying the number of sensor units on the $14 \mathrm{~mm} 5 \mathrm{MHz}$ AT-cut quartz disk, starting from the $2 \mathrm{ElOs}$ to quartz plates that contain for example four electrodes, as schematically illustrated in Fig. 4-13. For this, electrodes have to be further reduced in size, though this would be accompanied by a decrease in the quality factor and, thus, the sensitivity of each resonator. Moreover, one has to keep the problem of potential lateral mechanical coupling of OCM spots in mind, as described and discussed above (section 4.1) for the 2 ElO layout. A reduction in electrode size, while maintaining a maximum inter-electrode distance, could be sufficient for avoiding resonance coupling of adjacent sensor units. If not, much more effort would be necessary for coating the electrodes in order to obtain sensor spots with different thicknesses and resonance frequencies, respectively, as crucial for resonance decoupling.

A

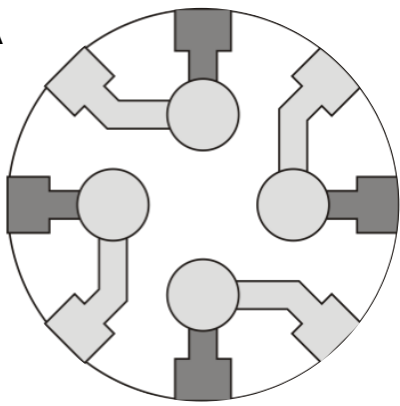

B

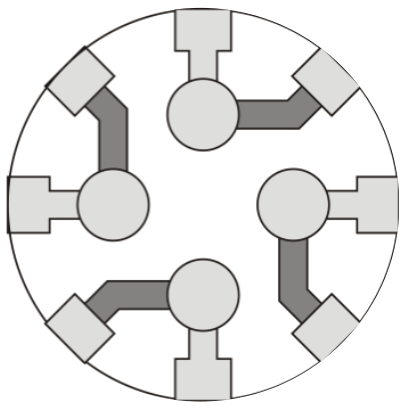

Fig. 4-13 (A) Top view and (B) bottom view of a possible MOCM layout on a circular quartz plate with four sensor spots that are individually addressable from the bottom side.

In general, the $2 \mathrm{ElOs}$ could be applied as gas-phase sensor as well as for chemosensing or bioanalytical applications in liquid phase. One resonator unit of the two-electrode layout could thereby be used as the actual OCM measurement unit, while the second OCM unit could serve as an internal, substrateintegrated reference sensor. To achieve this, different coatings could be applied on the measurement spot for selectively recognizing analytes in the sample or monitoring the interaction of biomolecules or whole cells with a modified substrate surface. The reference spot could be used for detecting non-specific sensor responses and, thus, to compensate interfering signals. In whole-cell biosensing applications of the OCM, probing the micromechanical interactions of cells with the sensor surface, a reference resonator could serve for monitoring the unaffected integrity and vitality of the cell layer, while the second resonator is open for investigating any kind of stimulus applied to the cells. However, this stimulus has to be located only at the "sensor-QCM" and avoided at the "control-OCM". For instance, this can be achieved by coating only one resonator with a substance or material of interest. Cell attachment and spreading properties on the coated sensor then could be investigated and compensated to the cell behavior on the uncoated reference resonator. This approach is just as well applicable for any locally immobilized substance or (bio)molecule. Besides coating of one resonator using chemi- or physisorption, any other approach with a localizable stimulus would be conceivable. This includes 
physical stress (pressure, temperature, electric fields, acceleration, radiation and waves of any energy, etc.) or in-situ generated local chemical or biochemical stress (e.g. reactive oxygen species, photocatalytically or electrochemically generated species). Stimulations and, thus, the field of possible cellular assays can be extended to (bio)chemically as well as pharmaceutically relevant molecules that are present in dissolved or suspended form. However, if one cell-covered resonator of the 2 ElQ should serve as a reference, one has to manage to stimulate the cell layer with lateral resolution only on one OCM spot. From the technical side a QCM chamber inset or a novel QCM chamber would be necessary for the 2 ElO substrates, dividing the quartz surface into two separated sensing compartments. By accomplishing a localized stimulation, sensing of micromechanical cell responses towards the stimulus in comparison to a reference could be performed with $2 \mathrm{ElOs}$ on the same substrate, simultaneously, with the same cell population and for similar conditions.

Besides the dual QCM sensing with or without using one resonator for reference measurements, further approaches in sensor development could address the integration of additional sensing functionalities onto the surface of the $2 \mathrm{ElO}$ substrate, and/ or the implementation of other measurement techniques. For instance, light microscopy is used by default as an independent reference and verification method or as a complementary technique for cell-sensing approaches that are not based on light microscopic cell investigations. By combining the OCM with a luminescence-based optode, one could take advantage of a fluorescence microscope and increase the information density from the system under investigation. For optode implementation, the surface of a quartz plate is (partially) coated with a polymer layer in which the chemical transducer, i.e. a luminescent molecule, is immobilized. Optical chemical sensing (OCS) of any analyte or changing chemical/ physical property to which the fluorescent probe is sensitive then can be performed in parallel to OCM sensing. Thereby, sensitivity and selectivity of the sensor can be triggered by the choice of the polymer material in which the dye is embedded. Thin film OCS systems have been presented for measuring $\mathrm{O}_{2}, \mathrm{CO}_{2}, \mathrm{pH} / \mathrm{H}^{+}$, glucose and temperature. ${ }^{[173,178,181,195]}$ Using multilayer systems and embedding the luminescent dyes in polymer beads even enables simultaneous multiple chemical sensing. Sensing of multiple measurands on a quartz plate would also be conceivable by various sensor spots that are sensitive towards different target variables. When seeding cells on a quartz plate with integrated OCS spots, the cells will grow on or around the sensor spots, depending on the cytocompatibility of the polymer, thus providing either subcellular or bulk measurements. Based on this, sensors for 6.1 Imaging the Frictional Heat Induced by High-Amplitude Quartz Oscillations (p. 223 ff.) as well as for 6.2 Imaging the Oxygen Consumption Rate (OCR) beneath Adherent Cells (p. 232 ff.) have been developed.

Electrical measurement techniques seem to be predestined for combination with OCM measurements, since MOCM substrates contain at least two electrodes on each face for exciting and recording quartz oscillations. However, for whole-cell biosensing applications, the applied voltage/ current must not be invasive for cellular structures. A widely used technique is the electric impedance sensing of cells, grown on substrate-integrated electrodes (cf. 3.4 Electric Cell-Substrate Impedance Sensing (ECIS)). These measurements provide the complex impedance, which is dominated by the morphological properties of the cells on the electrodes within a certain frequency region. Depending on the applied AC frequency, information on the coverage of electrodes with cells and the barrier resistance of the cell layer are available. The implementation of ECIS-like measurements on 2 ElO substrates and various cell-based assays of the OCM-ECIS double sensors are presented in the following Chapter 5 OCM-ECIS: Combined Viscoelastic and Dielectric Sensing of Cells. 


\section{Chapter 5 \\ OCM-ECIS: Combined Viscoelastic and Dielectric Sensing of Cells}

5.1 QCM-ECIS Sensor Layout and Characteristics

5.2 OCM-ECIS Biosensor for Cell Attachment and Spreading

5.3 OCM-ECIS Whole-Cell Biosensor

143

5.4 Discussion 
A combination of the two transducer principles QCM and electrochemical impedance spectroscopy (EIS) was applied in the past for in situ monitoring and analyzing solution properties ${ }^{[372]}$, degradation of thin polymer films ${ }^{[373]}$, formation of supported lipid bilayers ${ }^{[374]}$, and adsorption processes of proteins on bare ${ }^{[375]}$, polymer-coated ${ }^{[376]}$, and with platinum ${ }^{[377]}$ or copper ${ }^{[378]}$ electroplated $\mathrm{OCM}$ gold electrodes. In all studies, EIS data was measured between the top OCM electrode facing the electrolyte as working electrode and an external counter electrode (Platinum foil or carbon rod) that is introduced into the measurement cell and dips into the electrolyte. By default, a saturated calomel electrode (SCE) or $\mathrm{Ag} / \mathrm{AgCl}$ electrode was used as reference electrode. Janshoff and Wegener (1996) ${ }^{[219]}$ firstly presented the experimental approach of double-mode impedance analysis of adherent cell layers for biosensing applications. They introduced an additional low impedance platinum dipping electrode into the OCM measurement chamber, which enabled the combined EIS-mode and OCM-mode monitoring of a cell layer seeded on the electrode on the top side of the quartz disk. EIS in the context of recording and analyzing the impedance properties of cell-covered gold electrodes is also referred to as Electric CellSubstrate Impedance Sensing (ECIS) (section 3.4, p. 50 ff.), introduced by Giaever and Keese in 1991 [96]. By this hyphenated approach of QCM-ECIS double-mode impedance analysis, the " $1^{\text {st }}$ generation $\mathrm{OCM}$ " (simply a quartz disc sandwiched between two circular gold film electrodes) was technologically upgraded to what we name the " $2^{\text {nd }}$ generation $Q C M^{\prime}$. This is schematically illustrated in Fig. 5-1 A, B.

Combined OCM-ECIS sensors are capable of reporting on complementary information as viscoelastic and electrical properties of cell monolayers, with the latter allowing statements on the degree of cell coverage of the working electrode and the barrier resistance of cellular junctions. Double-mode impedance analysis was applied in various assay formats to test cell-cell and cell-substrate interactions. These comprised investigations on the cell adhesion and differentiation kinetics of different cell types $[22,206,236,267]$, adhesion properties of cells on structured growth substrates ${ }^{[236]}$, cell migration and wound healing ${ }^{[236]}$, properties of chemically fixated cells ${ }^{[268]}$, and studies on the cell layer responses to external stimuli like substances that affect the cell membrane ${ }^{[268]}$ or the actin cytoskeleton ${ }^{[267,268]}$. However, introducing an additional dipping electrode in the OCM chamber, for extending the $1^{\text {st }}$ generation QCM to $a 2^{\text {nd }}$ generation $\mathrm{QCM}$, entails some inconveniences. There is a complex experimental setup, combined with a reduced working space above the sensor. Moreover, the opening for the dipping electrode may be an entrance point for microorganisms. As long as the counter electrode is manually introduced for each experiment, its well-defined positioning above the WE is problematic, too. However, reproducible experimental conditions are prerequisites for the comparison of results obtained from different studies.

In this project a novel coplanar electrode layout integrated on a piezoelectric quartz disk is presented, which allows for double-mode (OCM-ECIS) impedance measurements like the $2^{\text {nd }}$ generation $\mathrm{OCM}$, however overcomes the drawbacks of a dipping electrode. This technological advancement upgrades the $2^{\text {nd }}$ generation OCM to the " $3^{\text {rd }}$ generation OCM" (Fig. 5-1 C). The technical realization of the two transducer principles in one experimental setup is addressed in section 5.1. There, the electrode designing and the implementation of OCM and ECIS measurements are presented (5.1.1). The surface characteristics as well as the QCM-mode and ECIS-mode performances of the fabricated sensor plates are shown for the standard experimental setup (5.1.2 - 5.1.5) and for variations of this (5.1.6). These dual whole-cell biosensors enable a multi-parametric, substrate-integrated, label-free, non-invasive, and realtime monitoring of adherent cells, providing complementary mechanical (viscoelastic) and electrical information (electrode coverage, barrier resistance) from the same cell layer at the same time. The gain and diversified application spectrum of this hyphenated approach are demonstrated in various cell-based assays, comprising cell attachment and spreading of different cells under various conditions (section 5.2) and cellular responses towards a diversity of external and internal stimuli (section 5.3). 
A

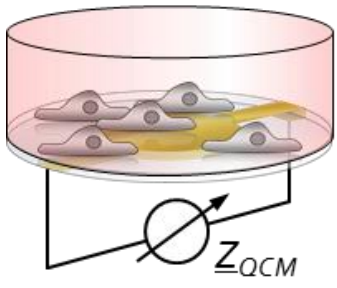

$1^{\text {st }}$ Generation
B

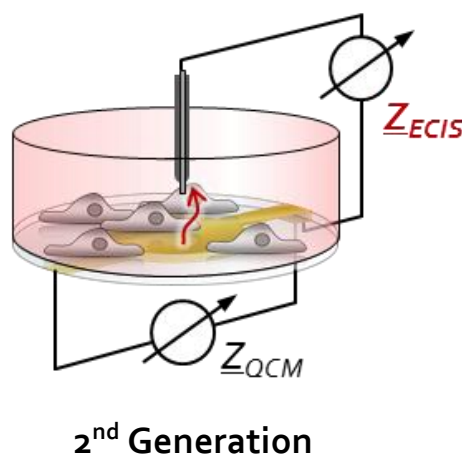

C

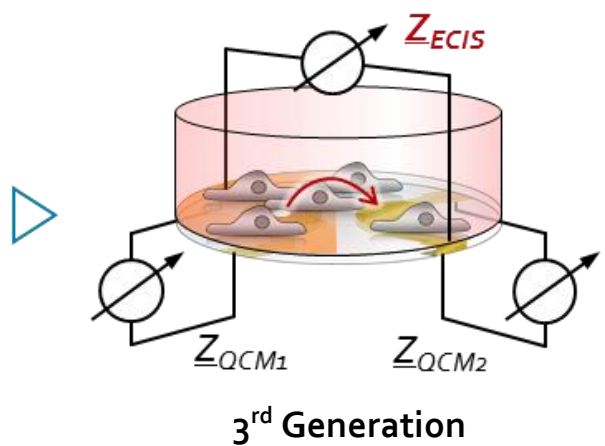

Fig. 5-1 Different QCM generations based on a circular $5 \mathrm{MHz}$ AT-cut quartz plate. (A) $1^{\text {st }}$ generation QCM: classical 1-Electrode QCM. (B) $2^{\text {nd }}$ generation QCM: classical 1-Electrode QCM extended by a dipping electrode for (QCM-ECIS) double-mode impedance analysis. (C) $3^{\text {rd }}$ generation OCM: 2-Electrode QCM (2EIO) layout with substrate-integrated coplanar electrodes for ECIS-mode measurements. Drawings are not to scale.

\subsection{QCM-ECIS Sensor Layout and Characteristics}

\subsubsection{Substrate-Integration of QCM-ECIS}

In order to overcome the disadvantages of former QCM-ECIS setups ( $2^{\text {nd }}$ generation OCM), a novel, substrate-integrated combination of both transducer principles was developed. This sensor design is based on the previously presented 2-Electrode MOCM platform (2EIQ), comprising two co-planar gold film electrodes integrated on either side of a quartz plate (Chapter 4: MOCM: Multiple Cytomechanic Sensing). By electrically connecting the electrodes on the sensing side via a conducting saline solution, one could measure the current flow in between, providing ECIS-like impedance information from the system on top of the sensor (cf. 3.4 Electric Cell-Substrate Impedance Sensing (ECIS), p. 50 ff.). As shown in the previous chapter, the bare, uncoated $2 \mathrm{EIO}$ resonators exhibit a lateral coupling of oscillations (cf. Fig. 4-2 and Fig. 4-3), which had to be eliminated by separating their resonance frequencies. Decoupling of resonances was successfully realized by depositing a rigid polymer layer on one half of the $2 \mathrm{EIO}$, i.e. one electrode on the sensing side of the quartz plate. Thereby, the acoustic thickness of the coated resonator unit was increased and consequently its resonance frequency was decreased, so that the resonance regions of the coated and uncoated resonators were sufficiently separated and coupling effects near the serial resonances were prevented (cf. subsection 4.1.5 Resonance Decoupling via Selective Rigid Coating, p. 90). Half-side coating of the top quartz surface was achieved by spin coating and photolithographic preparation steps, according to the procedure mentioned in 3.3.3 Experimental (p. 46) and illustrated in Fig. $3-11$. Since the deposited photoresist forms an insulating layer of approx. $3 \mu \mathrm{m}$ in thickness (cf. 4.2.2 Film Thickness Determination, p. 95 ff.), the coated electrode is shielded against charge transfer from the electrolyte side. However, impedances between the electrodes have to be minimized for ECIS-mode measurements, in order to be sensitive for impedance contributions originating from cells on the electrodes. The coated electrode could be made accessible for ECIS-like recordings, by implementing a small hole into the polymer film and thus establishing an electrical link between the two OCM topside-electrodes via the electrolyte. This photopolymer processing was 
performed in the course of the coating process, by using an adapted photolithography mask for the positive photoresist that exhibited a circular opening in the opaque area (cf. Fig. 3-12). After UV-light illumination through the mask, the illuminated photopolymer areas were developed and removed, resulting in a final electrode layout as exemplarily shown in Fig. 5-2. Thus, in the course of resonator coating for decoupling OCM resonances of $2 \mathrm{EIOs}$, it could additionally be implemented an electrode layout on the quartz surface that is in size and shape similar to commercial ECIS electrodes.

A
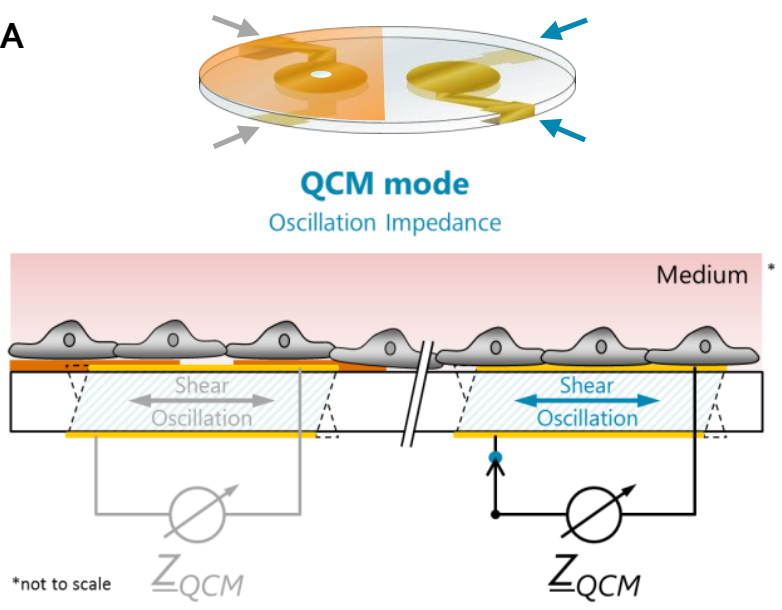

B

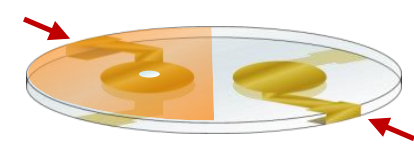

ECIS mode

Electrical Impedance

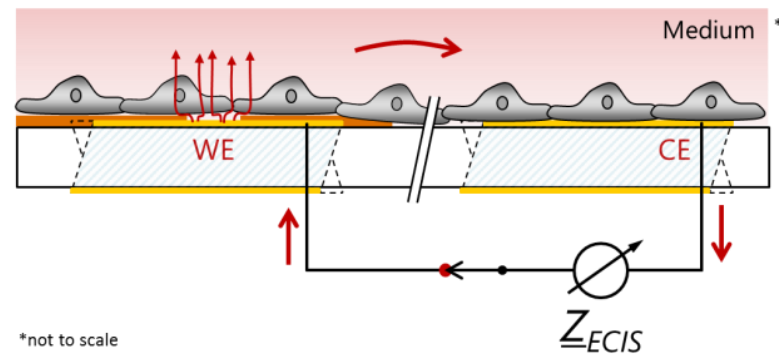

Fig. 5-2 Layout of the $3^{\text {rd }}$ generation OCM. Contacting different pairs of electrodes of a QCMECIS sensor allows for different sensing modes, which report on different cell layer properties. (A) QCM-mode: Electrode pairs sandwiching the quartz plate (indicated with gray and blue arrows) are contacted to excite and record the quartz oscillation, providing the oscillation impedance $\underline{Z}_{O C M}$ and reporting on cytomechanical changes. (B) ECIS-mode: Gold-film electrodes on the sensingside of the $2 \mathrm{ElO}$ are used for measuring the electrical impedance $\underline{Z}_{E C I S}$ of low-amplitude current flow through the system, reporting on morphological alterations within the cell layer.

On the basis of the $2 \mathrm{EIQ}$ development and by a modified surface structuring the transducer principles of QCM and ECIS could be combined and functionally coupled in one experimental setup (OCM-ECIS). The measurement chamber and hardware requirements for the technical implementation of both sensingmodes are illustrated in Fig. 3-18 (p. 57). Different pairs of electrodes can be electrically addressed from the bottom side of the quartz plate with a software-controlled relay, as schematically illustrated in sideview in Fig. 5-2. Each pair of gold-film electrodes sandwiching the quartz plate (Fig. 5-2 A) can be individually contacted to drive the shear oscillation of the respective resonator (OCM-mode). From the analysis of the resonance parameters one thus gets information on the cell layer mechanics close to the surface from two individual sensor spots. After switching the contacts to the two electrodes facing the cells and the medium (Fig. 5-2 B), ECIS-like data can be recorded (ECIS-mode), which enables the detection of cell morphology changes. Thus, using this hyphenated OCM-ECIS approach, a cell population seeded on the modified resonator surface can be monitored simultaneously and in real-time by ECIS- and OCM-mode measurements. This enables a multi-parametric analysis of cell layer properties and a time-resolved, micro-mechanical and dielectric characterization of the system under study.

By creating a smaller working electrode (WE) and a bigger counter electrode (CE) the sensitivity of ECIS measurements with respect to morphological changes of the cells was optimized. Since the smaller WE forms the bottleneck of current flow in the circuit, the system's impedance is very sensitive to impedance changes on top of this electrode, i.e. opening and closing current pathways through and around a 
confluent cell layer. Maximizing ECIS sensitivity by reducing the WE size, however, goes along with the measurement of a smaller fraction of the entire cell population. Hence, sensitivity and the average number of investigated cells have to be tailored to each assay and the respective focus of interest. The influence of different ECIS WE sizes and layouts on both OCM and ECIS sensing parameters were investigated. The different photolithography masks applied for $2 \mathrm{EIO}$ surface structuring are illustrated in Fig. 3-12 B-G (p. 47). The following subsections present the surface properties and the QCM-mode and ECIS-mode impedance characteristics of cell-free and cell-covered sensor surfaces of the standard sensor layout (5.1.2 - 5.1.5) that is used by default throughout this work and of sensors with particular ECIS electrode sizes and layouts (5.1.6).

\subsubsection{Standard QCM-ECIS Sensor Layout}

A standard surface structuring protocol of 2 ElOs was defined for $3^{\text {rd }}$ generation OCM (OCM-ECIS) sensors. This is schematically shown in Fig. 5-3. A 5 MHz AT-cut quartz plate with two pairs of electrodes (A) is spin coated with an insulating, positive photoresist at $3000 \mathrm{rpm}(\mathrm{B})$, according to the protocol described on p. 46. The thickness of the photoresist layer was found to be sufficient for the decoupling of the adjacent resonator units (cf. 4.2.1 Degree of Decoupling as a Function of Film Thickness, p. 93 ff.). For the default layout of the OCM-ECIS sensor surface a photolithography mask was applied during UV illumination $(C)$ that exhibits a circular opening of $0.5 \mathrm{~mm}$ in diameter in the center of the half-circular, opaque area (Fig. 5-4 A). After developing and a final hard-baking step, a 2 ElO was produced (D) capable for dual OCM-mode sensing, with two decoupled resonators due to a half-side photoresist coating, and for ECIS-mode recordings, with a small WE $\left(\varnothing_{\text {mask }}=0.5 \mathrm{~mm}\right)$ at the coated side and a $\sim 50$-fold larger CE $\left(\varnothing_{\text {Electrode }}=3.5 \mathrm{~mm}\right)$ at the uncoated side of the sensor surface.

A

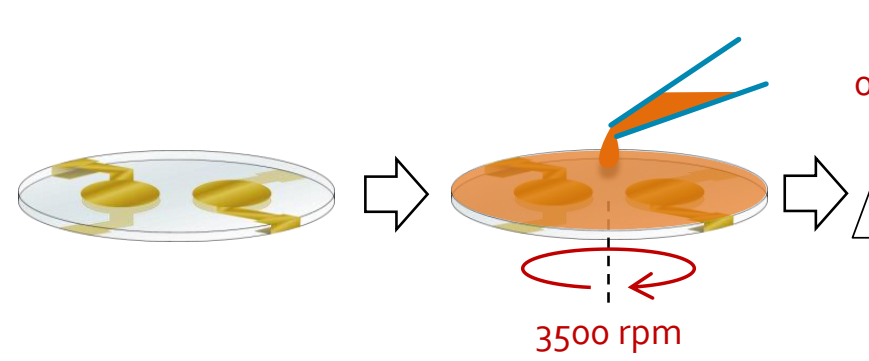

C

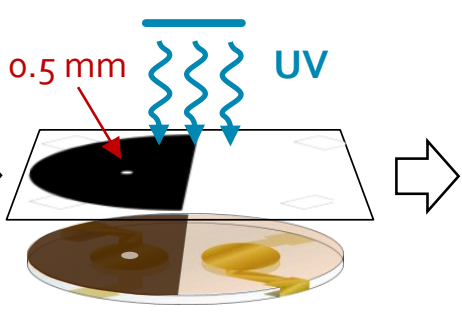

D

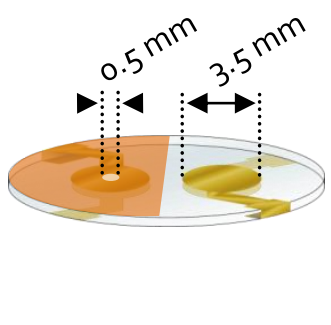

Fig. 5-3 Schematic illustration of the preparation process for a standard OCM-ECIS sensor, based on a blank $2 \mathrm{ElO}$ disk (A). Spin coating of an insulating, positive photoresist (B) was performed at $3000 \mathrm{rpm}$, followed by photolithographic structuring (C) through a mask with a circular opening of $\varnothing_{\text {mask }}=0.5 \mathrm{~mm}$ in the center of the half-circular opaque area (indicated with an arrow). This results in a final QCM-ECIS layout (D) with a small WE at the coated side and a bigger $\mathrm{CE}$ at the non-coated side of the $2 \mathrm{ElO}$ surface.

\subsubsection{Surface Characterization}

Since the area of the working electrode is a crucial parameter in ECIS-mode measurements (cf. 3.4.1 Basic Principles of the ECIS Technique, p. 50), the real area and average diameter of the WE was of interest. Therefore, the size and shape of the working electrode and the surface topography at the edges of the photoresist of the standard QCM-ECIS sensors were analyzed. A schematic drawing of the mask used for standard photolithographic structuring $\left(\mathrm{WE}-\varnothing_{\text {mask }}=500 \mu \mathrm{m}\right)$ is illustrated in Fig. 5-4 A, with a dashed red 
line indicating the alignment of the mask and the $2 \mathrm{EIO}$ plate during UV illumination. Fig. $5-4 \mathrm{~B}$ shows a photograph of the final QCM-ECIS sensor layout with a visible circular working electrode in the center of the coated electrode. The real size and the shape of the created WEs were determined microscopically, by phase-contrast imaging of the quartz plates and subsequent micrograph analysis with ImageJ software ( $\mathrm{NIH}_{\text {; USA }}{ }^{[330,331]}$. A bright mercury vapor lamp was applied as light source, capable of shining through the gold film electrodes. Fig. ${ }^{-}-4 \mathrm{C}$ shows the phase-contrast micrograph of the WE of a standard QCM-ECIS sensor, with a dotted line indicating its manually determined contour and area, respectively. All working electrodes produced in this work exhibited almost perfect circular shapes with well-defined sharp edges between the bare gold and the surrounding photoresist. The bare gold electrodes implemented in the photoresist coating exhibited a real average area of $A_{\text {real }}=2.05( \pm 0.03)$ $\cdot 10^{-3} \mathrm{~cm}^{2}$ (mean $\pm \mathrm{SEM} ; \mathrm{N}=7$ ). Assuming a circular shape, this correlates to an average WE diameter of $\varnothing_{\text {real }}=511( \pm 4) \mu \mathrm{m}$. The structuring of a WE on a polymer-covered $2 \mathrm{EIQ}$, by applying a photolithography mask with a circular hole of $\varnothing_{\text {mask }}=500 \mu \mathrm{m}$, thus resulted in $2.2( \pm 0.8) \%$ bigger WE diameters and 4.4 ( \pm 1.5$) \%$ bigger WE areas, respectively.

A

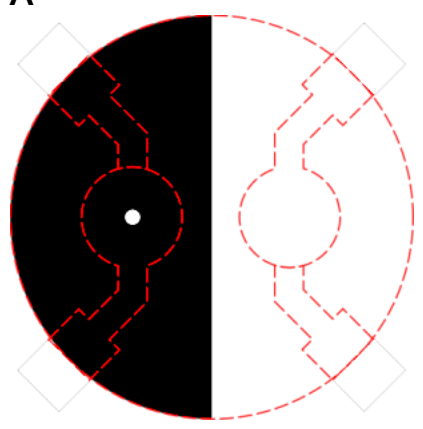

B

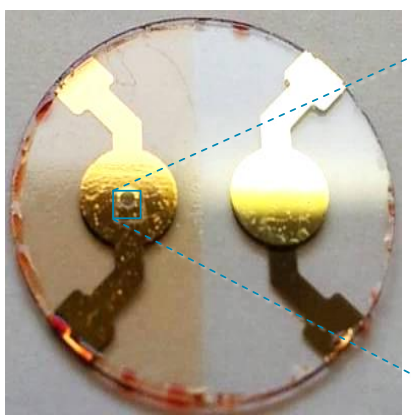

C

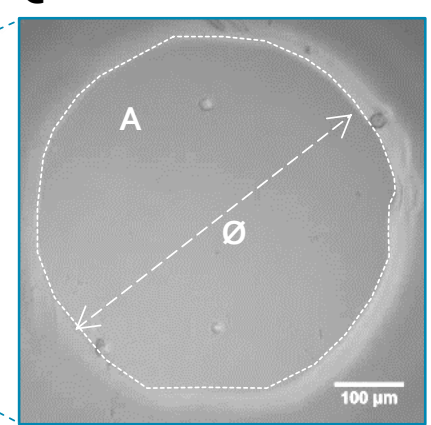

Fig. 5-4 (A) Photolithography mask for uncovering one half of a $2 \mathrm{ElO}$ quartz disk as well as a circular area in the center of the second electrode $\left(\varnothing_{\text {mask }}=500 \mu \mathrm{m}\right)$ from photoresist. Red dashed lines are not part of the mask and indicate the alignment and positioning of $2 \mathrm{ElO}$ and mask during UV illumination. (B) Photograph of a final OCM-ECIS sensor, structured with the mask in A. (C) Phase-contrast micrograph of a WE with a diameter defined to $500 \mu \mathrm{m}$ by the mask. Measuring of WE circumferences (dotted line) provided real averaged values of $\varnothing_{\text {real }}=511( \pm 4) \mu \mathrm{m}$ and $A_{\text {real }}=2.05( \pm 0.03) \cdot 10^{-3} \mathrm{~cm}^{2}($ mean $\pm \mathrm{SEM} ; \mathrm{N}=7)$.

Topographic information of the lithographically structured OCM-ECIS sensor surface were obtained by profilometry measurements. Investigations were performed by Fraunhofer EMFT (Munich, Germany), using a "Dektak IIA profilometer" from Sloan (now Veeco; Plainview, NY, USA). Fig. 5-5 A shows a 3-D profile plot of a OCM-ECIS sensor surface in a region of $\sim 5.5 \times 5.5 \mathrm{~cm}^{2}$ that holds the WE $\left(\varnothing_{\text {mask }}=500 \mu \mathrm{m}\right)$ and the polymer edge in the center of the quartz plate. The height profile is illustrated in pseudo-colors and the $z$-scale is stretched by a factor of $\sim 1000$ with respect to the scale in the xy-plane. For height profile analysis, an optical cross-section in the xz-plane was chosen, starting from the polymer-free quartz area and going across the polymer edge and the topographic depression of the WE (cf. red arrow in Fig. 5-5 B). The resulting height profile along the $x$-direction is illustrated in Fig. $5-5$ C. The z-axis was adjusted in scale to visualize small height differences compared to the larger lateral dimension. Photoresist thickness is $3 \mu \mathrm{m}$ on average. This matches well with the calculations presented in subsection 4.2.2 (p. 95 f.), which correlated the spin coating speed with the resulting film height on the substrate (Tab. 4-2). Stretching of the $\mathrm{z}$-axis in Fig. ${ }^{-5} \mathrm{C}$ causes a distorted representation. This might mislead to 
the assumption of a higher aspect ratio of the working electrode cavity than it actually is, and of almost a right angle between the photoresist layer and the blank quartz plate $(\alpha)$ or the electrode $(\beta, \gamma)$. The values of the respective angles were determined by calculating the slope of a fitting function in the last data point before rise, resulting in $\alpha=174.3^{\circ}, \beta=171.5^{\circ}$, and $\gamma=170.9^{\circ}$. Hence, the angles between substrate surface and photoresist layer are all in the order of $170-175^{\circ}$, reflecting flat rising and trailing edges of the polymer as well as a low aspect ratio of the structures.

A

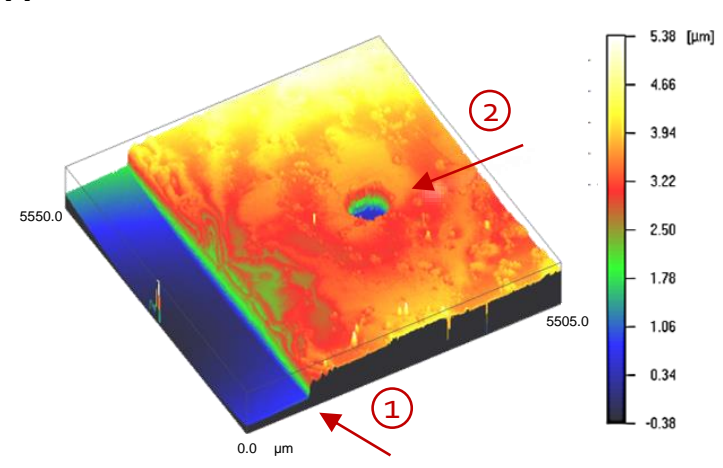

B

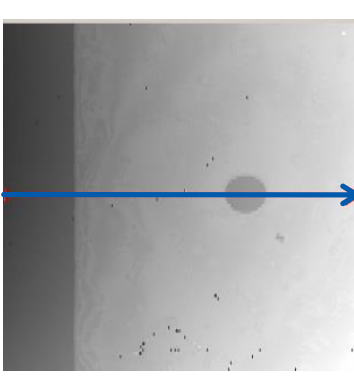

C

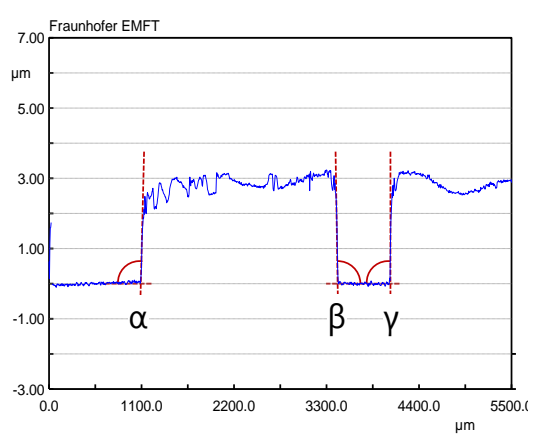

Fig. 5-5 (A) 3-D profile of a selected area of a QCM-ECIS sensor, comprising the photoresist edge in the center of the quartz plate (arrow (1)) and the circular cavity in the photoresist layer opening the working electrode (arrow (2)). (B) Scanning axis and direction (red arrow) for measuring a two-dimensional height profile, shown in the diagram right next to it (C). Angles between the baseline of the substrate surface and the photoresist flanks were determined to $\alpha=174.3^{\circ}, \beta=171.5^{\circ}$, and $\gamma=170.9^{\circ}$.

\subsubsection{QCM-mode: Piezoelectric Characterization of the Sensor}

The shear oscillation properties of both resonator units of a standard QCM-ECIS sensor layout (3000 rpm, $W E-\varnothing=0.5 \mathrm{~mm}$ ) were recorded and analyzed. Measurements were performed for the unloaded situation in dry air, under loading of the coated sensor side with serum-free cell-culture medium, and with cells adherently grown on the quartz surface of coated and uncoated QCM electrode. Spectra of the sensor exposed to medium ( $1.5 \mathrm{~mL}$ ) were recorded after an equilibration phase of $\sim 24 \mathrm{~h}$ and the oscillation properties of $\mathrm{OCM}_{1}$ and $\mathrm{OCM}_{2}$ of a cell-covered sensor were recorded the day after seeding MDCK-II cells $\left(450000\right.$ cells $/ \mathrm{cm}^{2}$ ), when cells have formed a confluent monolayer on the quartz surface.

Representative oscillation impedance spectra of the two resonators $\mathrm{OCM}_{1}$ (with PhoP coating) and $\mathrm{OCM}_{2}$ (uncoated) of a OCM-ECIS sensor at the respective loading conditions are summarized in Fig. 56. OCM-mode measurements were performed at 200 equidistant frequencies around the fundamental resonances of $\mathrm{QCM} 1$ and $\mathrm{QCM} 2$, from 4.97 to $5.02 \mathrm{MHz}$. Spectra of impedance magnitude (Fig. 5-6 A) and phase shift (Fig. 5-6 B) of $\mathrm{OCM}_{1}$ (filled symbols) and $\mathrm{OCM}_{2}$ (open symbols) are shown for the sensor in air $\left(-,,-\square^{-}\right)$, loaded with medium $(\triangle,-\Delta)$, and covered with a confluent cell layer $(\rightarrow-,-\infty)$. Fig. 5$6 \mathrm{C}$ represents the oscillation impedance data of $\mathrm{OCM}_{1}$ (air, medium, w/ cells) in the complex plane. In Fig. 5-6 D, the Nyquist diagram is extended to the third dimension (3-D-Nyquist), with AC frequency as $x$-axis, impedance real part $\operatorname{Re}(\underline{Z})$ as $y$-axis, and impedance imaginary part $\operatorname{Im}(\underline{Z})$ as z-axis. The projection of the 3-D curves to the yz-plane (Re( $\underline{Z})$ - $\operatorname{Im}(\underline{Z}))$ mirrors the 2-D representation of Fig. 5-6 C. 
A

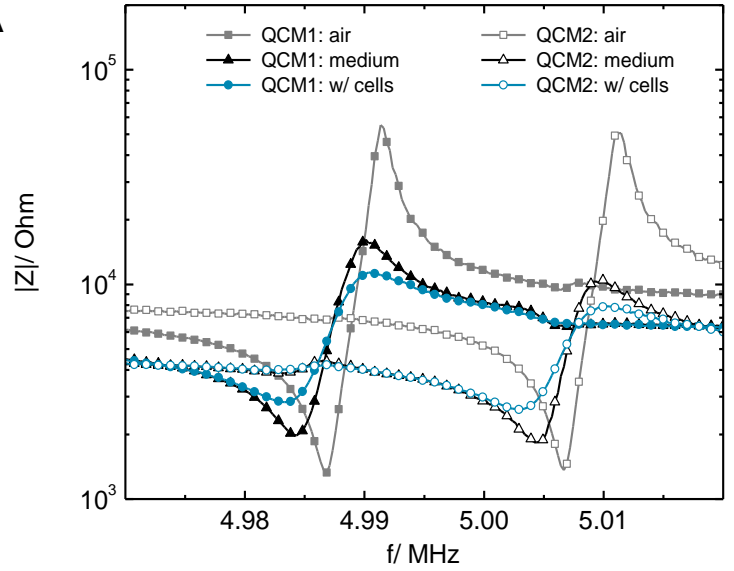

C

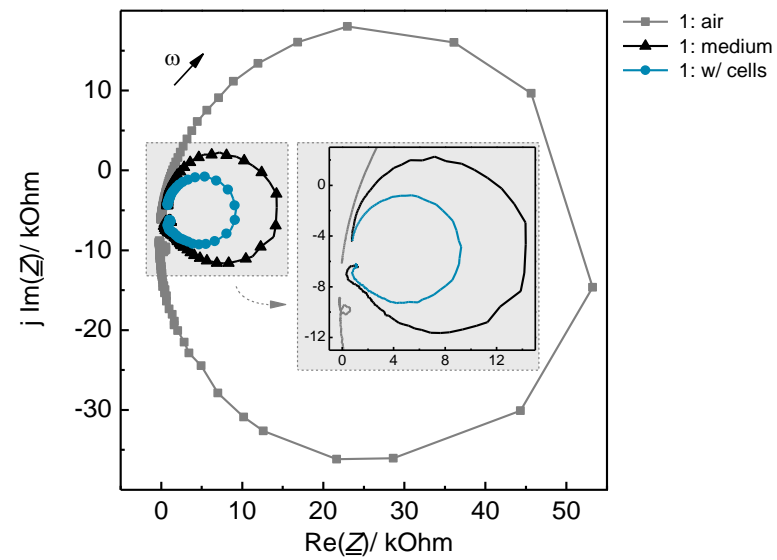

B

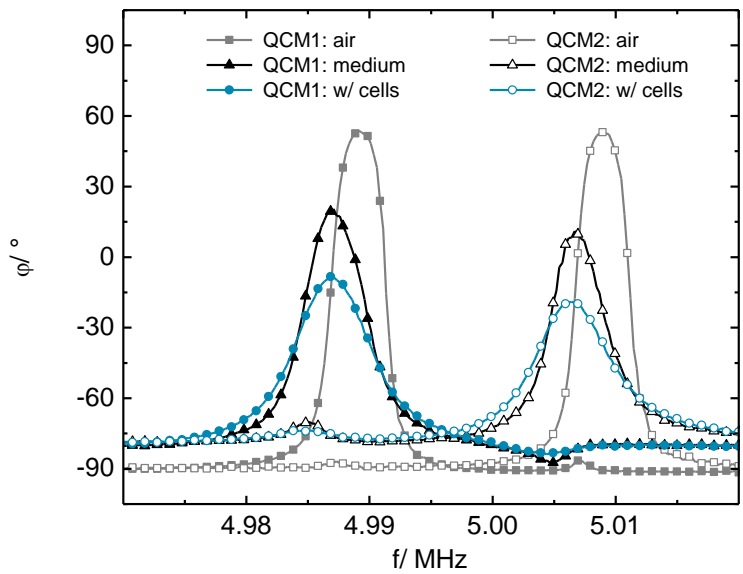

D

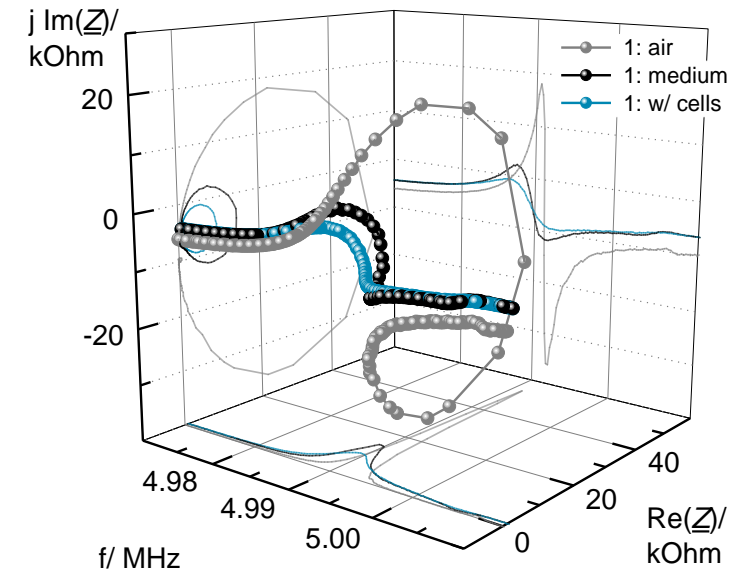

Fig. 5-6 Impedance spectra of a OCM-ECIS sensor in OCM-mode. Spectra of oscillation impedance magnitude (A) and phase (B) of $\mathrm{OCM}_{1}$ ( $\mathrm{w} / \mathrm{PhoP}$ coating; filled symbols) and $\mathrm{OCM}_{2}$ (w/o coating; open symbols) are shown for sensors in air $\left(-{ }^{-},-{ }^{-}\right)$, loaded with cell culture medium $(-,-\Delta-)$, and covered with a confluent layer of MDCK-II cells ( $w /$ cells; $-\rightarrow-,-0-)$. (C) $2-D$ and (D) 3-D complex (Nyquist) representation of $\mathrm{OCM}_{1}$ oscillations at the respective loading conditions. Impedance values were recorded at 200 equidistant frequencies around resonances, between $4.97 \mathrm{MHz}$ and $5.02 \mathrm{MHz}$. Data points were reduced for sake of clarity in A-C.

Resonances of $\mathrm{OCM}_{1}$ and $\mathrm{QCM} 2$ are well separated by $\sim 20 \mathrm{kHz}$ in any loading situation (cf. Tab. 5-1), which is due to the deposition of a rigid PhoP layer on $\mathrm{OCM}_{1}$. Impedance profiles of the two resonator units are similar except for the translation along the frequency axis. The changes in the $\mathrm{OCM}_{1}$ and $\mathrm{OCM}_{2}$ impedance spectra of a QCM-ECIS sensor in the course of medium loading are similar to the changes observed previously for the medium loading of a decoupled $2 \mathrm{EIO}$ plate, on which no WE for ECIS-mode measurements was implemented (cf. 4.1.5 Resonance Decoupling via Selective Rigid Coating, p. 90 ff.). That are, firstly, a strong damping of both resonators $\left(\Delta|Z|_{\min } \approx 700 \mathrm{Ohm}\right)$ and, secondly, a slight shift of both resonance curves to lower frequency values $\left(\Delta f_{|Z|_{\text {min }}} \approx-3 \mathrm{kHz}\right)$. Damping and frequency shift are both a consequence of the viscosity-density product of the Newtonian liquid, which is, in the BVD equivalent circuit, considered by an additional resistance and inductance element in series to the motional branch (cf. Tab. 3-6 B, p. 44). Thereby, the resistance accounts for energy dissipation (damping), and the inductive reactance reflects an additional mass layer of the liquid (frequency shift) oscillating synchronously and lossless with the resonator. Tab. 5-1 summarizes for the two resonator

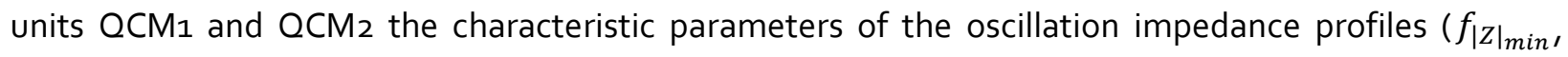
$\left.|Z|_{\text {min }}, f_{s,} f_{p}, f_{|Z|_{\text {max }}}|Z|_{\text {max }}, \varphi_{\max }\right)$ for the investigated loading conditions. It has to be noted that the 
parallel resonance parameters, $|Z|_{\max }$ and $\varphi_{\max }$ of the uncoated $\mathrm{OCM} 2$ respond stronger to medium loading than the photoresist-coated $\mathrm{OCM}_{1}$. The growth of cells on the sensor surface further increases the energy dissipation of the $\mathrm{OCM}_{1}$ and $\mathrm{OCM}_{2}$ oscillations, which is reflected in significantly damped impedance magnitude and phase profiles as well as in a broadening of the spectra (Fig. 5-6 A, B). The characteristic impedance parameters of both $2 \mathrm{ElO}$ microbalances change to a similar extent. Increased dissipation upon loading the OCM-ECIS surface with viscoelastic cell bodies is reflected in an increase of minimal impedance values $\Delta|Z|_{\min }$ by $\sim 850 \mathrm{Ohm}$ and a reduction of the maximal phase $\Delta \varphi_{\max }$ by $\sim-30^{\circ}$ (Tab. 5-1). Cell attachment and anchorage on the electrodes also caused equal resonance frequency changes of $\mathrm{OCM} 1$ and $\mathrm{OCM}_{2}$ in the order of $\Delta f_{|Z|_{\text {min }}} \approx-1 \mathrm{kHz}$ with respect to the cell-free vibrations in medium (Tab. 5-1). In the complex 2-D and 3-D Nyquist plots of the oscillation impedance profiles (Fig. 5-6 C, D) the mentioned parameter changes due to loading the resonators with cells are mirrored in a distinct contraction of the impedance circle and of the "resonance loop", respectively. The zoom-in diagram in Fig. 5-6 C additionally reveals a shift of the loop to bigger values along both the imaginary and the real axis, by loading the resonator with medium and cells. This shift represents increases in storage of oscillation energy as well as an increase in energy dissipation.

Tab. 5-1 Impedance characteristics of the two resonators, $\mathrm{QCM} 1$ and $\mathrm{OCM}$, on a $\mathrm{QCM}-\mathrm{ECIS}$ sensor, performing oscillations in air, $24 \mathrm{~h}$ after loading the top side with medium and after MDCK-II cells have formed a confluent monolayer on the sensor surface. QCM1 was coated with a film of photopolymer (PhoP) except for a small hole in the center $(\varnothing=0.5 \mathrm{~mm})$ in order to generate a WE for ECIS-mode measurements. Values were obtained by spline-interpolation of data points in the respective frequency regions.

\begin{tabular}{|c|c|c|c|c|c|c|c|c|}
\hline Resonator & Load & $\begin{array}{c}f_{|Z|_{\min }} l \\
\mathrm{MHz}\end{array}$ & $\begin{array}{c}|Z|_{\min } \mid \\
\mathrm{kOhm}\end{array}$ & $\begin{array}{c}f_{s} l \\
\mathrm{MHz}\end{array}$ & $\begin{array}{c}f_{p} / \\
\mathrm{MHz}\end{array}$ & $\begin{array}{c}f_{|Z|_{\max }} \mid \\
\mathrm{MHz}\end{array}$ & $\begin{array}{c}|Z|_{\max } \mid \\
\mathrm{kOhm}\end{array}$ & $\varphi_{\max } l^{\circ}$ \\
\hline \multirow[t]{3}{*}{$\mathrm{OCM}_{1}+\mathrm{PhoP}$} & air & 4.987 & 1.3 & 4.987 & 4.991 & 4.991 & 55.2 & 54 \\
\hline & medium & 4.984 & 2.0 & 4.985 & 4.989 & 4.990 & 16.2 & 21 \\
\hline & MDCK-II cells & 4.983 & 2.8 & - & - & 4.990 & 11.4 & -8 \\
\hline \multirow[t]{3}{*}{$\mathrm{OCM}_{2}$} & air & 5.007 & 1.4 & 5.007 & 5.011 & 5.011 & 50.7 & 53 \\
\hline & medium & 5.004 & 1.8 & 5.006 & 5.008 & 5.010 & 10.5 & 11 \\
\hline & MDCK-II cells & 5.003 & 2.6 & - & - & 5.010 & 7.9 & -19 \\
\hline
\end{tabular}

In each representation of the oscillation impedances a secondary resonance can be observed for $\mathrm{OCM}_{1}$ and $\mathrm{OCM}_{2}$ at frequencies where the respective other oscillator is resonant. This coupling effect has already been described and discussed in the previous chapter (4.1.5). It is most pronounced for quartz oscillations in air and vanishes with increasing damping. These side resonances appear far away from fundamental resonances and thus do not perturb the extraction of minimal impedance values for a timedependent analysis. The cells show similar mechanical interaction with the different surfaces of the two resonators, photoresist $(\mathrm{OCM})$ and gold $\left(\mathrm{OCM}_{2}\right)$, as evident from similar changes of the characteristic oscillation parameters (Tab. 5-1). In summary, it was demonstrated that the OCM-ECIS sensor in OCMmode is capable for reporting with two individual sensor spots on the mechanical interactions of the cells with their growth substrate. This provides the basis for applying the QCM-ECIS platform as sensor for cell attachment and spreading and as whole-cell biosensor for monitoring micromechanical (viscoelastic) alterations of an intact cell layer. 


\subsubsection{ECIS-mode: Electrochemical Characterization of the Sensor}

After recording the piezoelectric impedances of $\mathrm{OCM}_{1}$ and $\mathrm{OCM}_{2}$ in $\mathrm{OCM}$-mode (Fig. 5-2 A), the electrode contacts were switched to the electrodes on the upper side of the dual sensor (Fig. 5-2 B) in order to record in ECIS-mode the electrochemical impedance characteristics of the WE. The ECIS-mode capability of the sensor was tested across a wide frequency window from $1 \mathrm{~Hz}$ to $1 \mathrm{MHz}$. The electrical impedance properties described below were measured with the standard OCM-ECIS sensor layout (3000 $\mathrm{rpm}, \mathrm{WE}-\varnothing=0.5 \mathrm{~mm}$ ). Impedance spectra were recorded after equilibration of the coated sensor under $1.5 \mathrm{~mL}$ serum-free medium for $\sim 24 \mathrm{~h}$, and the day after seeding ( 450000 cells/ $\mathrm{cm}^{2}$ ) MDCK-II cells on the sensor, when cells have formed a confluent monolayer across the coated and uncoated electrode on the top side of the sensor.

In Fig. 5-7, the spectra of impedance magnitude (Fig. 5-7 A) and phase (Fig. 5-7 B) in ECIS-mode are illustrated for the electrodes immersed in serum-free medium (SFM; $-\Delta$ ) and for MDCK-II cell-covered electrodes $(\rightarrow)$. A 3 -D representation of the electrochemical impedance data with the three perpendicular axes, $f,|Z|$, and $\varphi$, is shown in $S / 10 A$ (p. 266). The experimental impedance spectra in Fig. 5-7 A and $B$ are very similar to the simulated impedance magnitude and phase spectra of cell-free and a cell-covered ECIS electrodes shown in Fig. 3-15 (p. 53). In the cell-free situation the impedance is dominated by the double layer capacitance of the $W E$ in the frequency range of $1-10^{4} \mathrm{~Hz}$. This is reflected in the constant negative slope of the impedance magnitude of $-1\left(\underline{Z}_{C}=-\mathrm{j} \cdot(\omega C)^{-1}\right)$ as well as in phase shift values of almost $-90^{\circ}$. Deviations from an ideal capacitive behavior are accounted for by describing the electrode as CPE in equivalent circuits. In the high frequency band $\left(f>10^{4} \mathrm{~Hz}\right)$ the current flow through the system is determined by the bulk resistance of the electrolyte (SFM), which is expressed by $|Z|$ converging to a constant, frequency independent level and by an convergence of the phase shift from $-90^{\circ}$ towards $0^{\circ}$. For cell-covered electrodes, the impedance situation is different. In the very high and very low frequency regions, the impedance is still dominated by the electrode capacitance $(f<10 \mathrm{~Hz})$ and the bulk resistance $\left(f>10^{5} \mathrm{~Hz}\right)$, respectively. However, the impedance is significantly increased inbetween by the presence of cells on the $W E$, which act as insulating particles. A capacitance and resistance in parallel, in series to the CPE of the WE and the bulk resistance, can approximately describe the impedance of a cell-covered WE (cf. spectral properties of an R\|C circuit in Tab. 3-4 B, p. 29). This is confirmed by the phase of the impedance. Scanning from low to high frequencies $(f>10 \mathrm{~Hz})$, the phase of the cell-covered sensor firstly deviates from $-90^{\circ}$ of the ideal electrode capacitance towards more positive values around $10^{2} \mathrm{~Hz}$, reflecting an increasing resistance contribution by the MDCK-II cells. Around $10^{3}-10^{5} \mathrm{~Hz}$, the membrane capacitance of the cells play a decisive role for current pathways through the cell layer, reflected in a phase drop towards $-90^{\circ}$ again, until at higher frequencies $\left(f>10^{5} \mathrm{~Hz}\right.$ ) the bulk resistance of the medium determines the entire impedance/ phase signal. The frequency region in which the cell layer affects impedance and the extent of its impedance contribution depends on the cell type. Critical properties are the tightness of intercellular junctions, the average radius of cell bodies, the cell-electrode distance, the specific resistivity of the subcellular electrolyte, and the membrane capacitance of the cells (cf. Fig. 3-17 and subsection 3.4.3). For instance, the ECIS data of MDCK-II cells on the QCM-ECIS sensor ( - in Fig. $5-7$ ) show impedance contributions by the cells across three orders of magnitude of frequency. Moreover, the MDCK-II cell layer increased the magnitude of impedance by a factor of almost six at the most sensitive frequency. Absolute values are listed in Tab. 52. A dotted line in Fig. 5-7 A, B indicates the sensitive frequency. It is determined from the normalized impedance spectrum $|Z|_{\text {norm }}(f)$ shown in Fig. 5-7D, representing the impedance spectrum of a cellcovered sensor divided by that of a cell-free sensor $\left(|Z|_{\text {norm }}=|Z|_{\mathrm{w} / \text { cells }} /|Z|_{\mathrm{w} / \mathrm{o} \text { cells }}\right)$. This provides a peak function with the sensitive frequency as the frequency where $|Z|$ changes most due to the cells 
$\left(f_{\text {sens }}(|Z|)=f\left(|Z|_{\text {norm }}^{\max }\right)\right)$. For the exemplary data, the peak maximum of $|Z|_{\text {norm }}$ was determined at $f_{\text {sens }}(|Z|)=2.5 \mathrm{kHz}$.

For a better and more specific analysis, the impedance was expressed by complex numbers. Thereby, the real part of the complex impedance includes all resistive contributions of the observed system and is expressed by an equivalent resistance, $\operatorname{Re}(\underline{Z})=R$. Since inductive elements are not present in the observed electrochemical system, the imaginary part of the impedance is expressed by an equivalent capacitance, $\operatorname{Im}(\underline{Z})=-(\omega C)^{-1}$. The representation of the complex impedance by the respective values of resistance $\left(-\square_{-},--\right)$and capacitance $\left(-0_{-},--\right)$) is shown in a frequency dependent manner for cell-free (open symbols) and cell-covered (filled symbols) electrodes in Fig. 5-7 C. A corresponding 3-D representation of the electrochemical resistance and capacitance data with the three perpendicular axes, $f, C$, and $R$, is shown in $S I 10 B$ (p. 266). The resistance and capacitance spectra that are extracted from the experimental electrical impedance (Fig. $5_{-7}$ C) show profiles similar to the simulated data presented above in Fig. 3-16 (p. 54). From the spectra in Fig. 5-7 C, one can see the most pronounced response of the resistance to the MDCK-II cell layer at lower frequencies $\left(f_{\text {sens }}(R)=200 \mathrm{~Hz}\right)$, whereas the capacitance is sensitive for the presence of MDCK-II cell membranes on the WE at elevated frequency levels $\left(f_{\text {sens }}(C)=32 \mathrm{kHz}\right)$. Fig. 5-7 D shows the respective spectra of normalized resistance $\left(R_{\text {norm }}=\right.$ $\left.R_{\mathrm{w} / \text { cells }} / R_{\mathrm{w} / \mathrm{ocells}}{ }^{-}-\right)$) and normalized capacitance $\left(C_{\mathrm{norm}}=C_{\mathrm{w} / \text { o cells }} / C_{\mathrm{w} / \text { cells }}{ }^{-{ }^{-}-}\right)$. The presence of a confluent MDCK-II cell layer on the WE causes a decrease in the capacitance by a factor of six and a 32fold increase in the resistance at the respective frequencies, with respect to the WE exposed to cell culture medium only. Absolute values of parameters are summarized in Tab. 5-2. Stray capacitances due to the wiring cause a drop of the capacitance at $f>\sim 50 \mathrm{kHz}$. Here, the experimental data deviate from the simulated curves, as parasitic effects have not been considered in the model calculations.

Fig. 5-7 E shows the ECIS data represented in the complex plane, with the linear axes $\operatorname{Re}(\underline{Z})$ and $-\mathrm{j}$. $\operatorname{Im}(\underline{Z})$. The imaginary axis is plotted inversely for a better presentation. The bright grey inset in Fig. 5$7 \mathrm{E}$ shows a zoom-in to the high frequency range to hide the CPE contribution of the WE, which dominates at low frequencies, and to visualize the impedance contribution of cells in the complex presentation. The cell-free sensor $(\leftarrow)$ ) exhibits impedance properties of a CPE with dominating capacitive contributions in series with the bulk resistance of the electrolyte (cf. Tab. 3-4A). Cells (MDCK-II) on the WE ( --$)$ add a R $\| C$ element in series to the serial CPE- $R_{\text {bulk }}$ circuit. This is noticeable in a knick in the curve at $f>300 \mathrm{~Hz}$ compared to the cell-free situation (see inset in Fig. 5-7 E), which reveals the semicircle of a $\mathrm{R} \| \mathrm{C}$ element in the complex plane (cf. Tab. 3-4 B). For $f<300 \mathrm{~Hz}$ the CPE dominates current flow, illustrated by a strong increase of the imaginary part. A further zoom-in to the maximal frequency (dark grey insert in Fig. 5-7 E) uncovers that the curves do not cross the origin of the complex plane. This reflects the constant real part contribution of the bulk resistance (offset in $\mathrm{x}$-axis direction; cf. Tab. $3-4 \mathrm{C}$ ) as well as stray capacitances arising from the wiring (offset in $y$-axis direction). Fig. 5-7 $\mathrm{F}$ extends this 2-D plot to a 3-D Nyquist plot by the introduction of the frequency $f$ as the third axis. All axes are perpendicular to each other and are presented in logarithmic scale. The projection of the curves to the complex plane (left back plane) mirrors a double-logarithmic presentation of the 2-D Nyquist plot shown in Fig. 5-7 E. The projection to the xy-plane (bottom plane) reflects the resistance spectra shown in Fig. ${ }_{5-7} C(\operatorname{Re}(\underline{Z})=R)$, and the projection in the xz-plane (right back plane) illustrates modified capacitance spectra $\left(\operatorname{Im}(\underline{Z})=-(\omega C)^{-1}\right)$. 
A

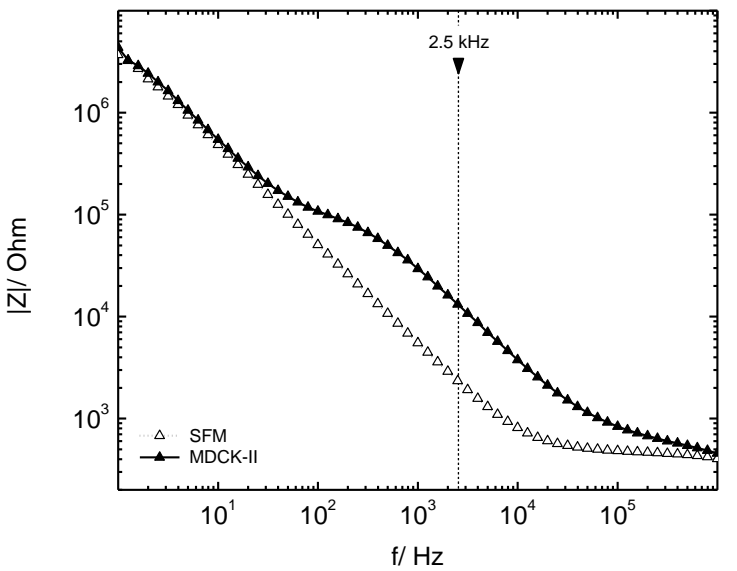

C

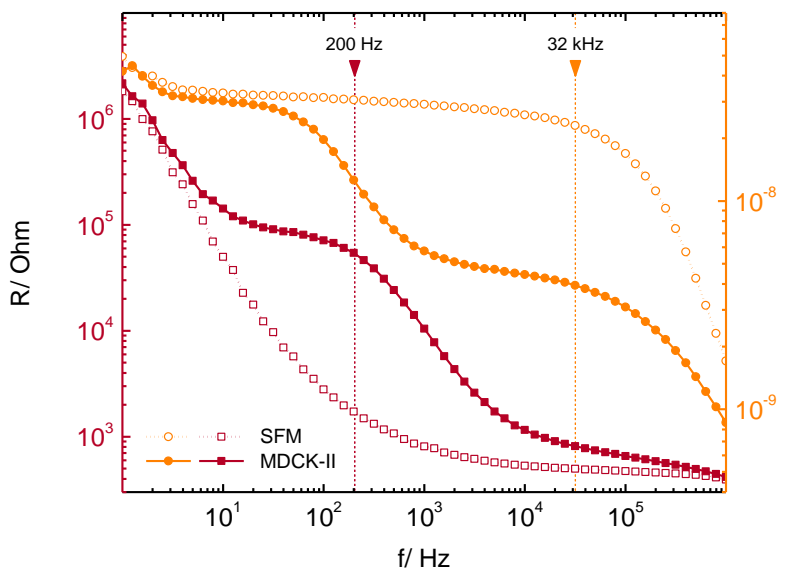

E

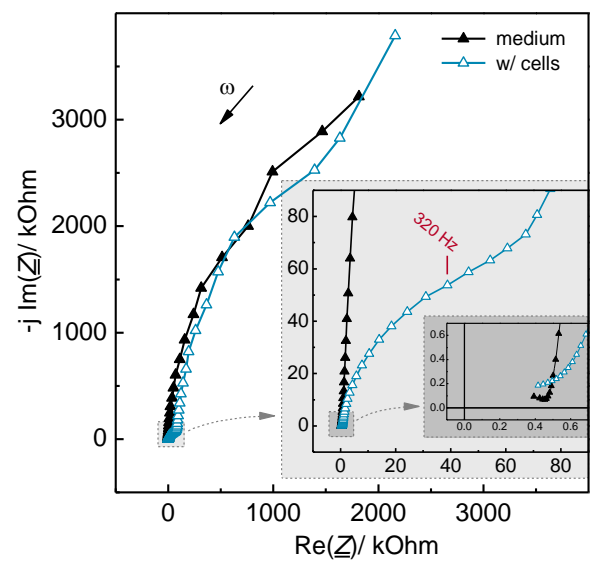

B
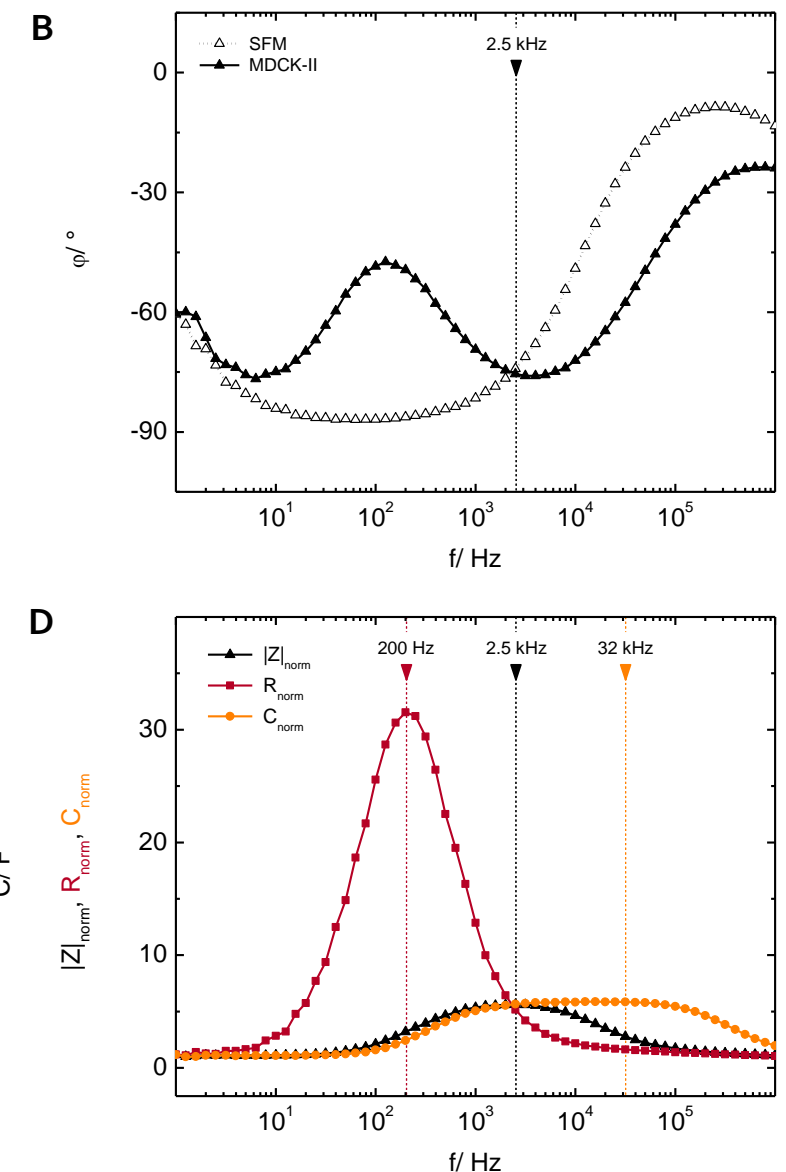

$\mathbf{F}$

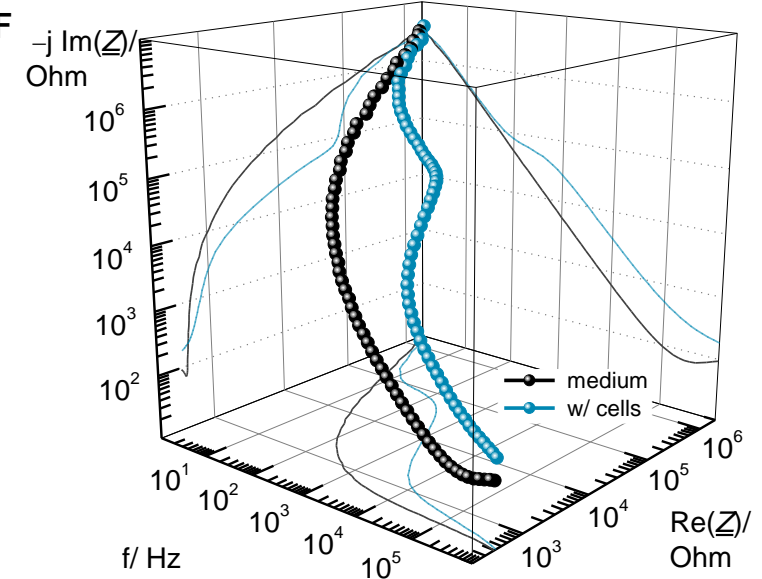

Fig. 5-7 Electrical impedance spectra of a OCM-ECIS sensor in ECIS-mode. (A) $|Z|(f)$ and (B) $\varphi(f)$ are shown for the sensor loaded with cell culture medium $(-\Delta)$ and covered with a confluent layer of MDCK-II cells $(\boldsymbol{-})$. The most sensitive frequency of maximal cell response of $|Z|$ is indicated $(2.5 \mathrm{kHz})$. (C) Spectra of equivalent resistance $\left(R(f) ;-\square^{-},-\right)$) and capacitance $(C(f)$; $\left.-0_{-},-\right)$were extracted from the real part $(\operatorname{Re}(\underline{Z})=R)$ and the imaginary part $(\operatorname{Im}(\underline{Z})=$ $-(\omega C)^{-1}$ ) of the complex impedance and are shown for the cell-free (open symbols) and the cellcovered (filled symbols) sensor. The most sensitive frequencies of $R$ and $C$ with respect to cell coverage of the WE are indicated ( $200 \mathrm{~Hz}$ and $32 \mathrm{kHz}$, respectively). (D) Normalized spectra of $|Z|$ $\left(-;|Z|_{\text {norm }}=|Z|_{\text {w/ cells }} /|Z|_{\text {w/o cells }}\right), R\left(-; R_{\text {norm }}=R_{\text {w/ cells }} / R_{\text {w/o cells }}\right)$ and $C\left(\rightarrow ; C_{\text {norm }}=\right.$ $\left.C_{\mathrm{w} / \text { o cells }} / C_{\mathrm{w} / \text { cells }}\right)$, corresponding to the spectra of cell-free/ cell-covered WEs in A and C. The peak maxima provide the most sensitive frequency of each parameter, as indicated. (E) 2-D and (F) 3-D Nyquist representation of the complex impedances. Note: the imaginary axis (z-axis) is displayed inverted, and axes in $E$ are linear while they are logarithmic in $F$. Impedance values were recorded at 61 equidistant frequencies between $1 \mathrm{~Hz}$ and $1 \mathrm{MHz}$. 
Tab. 5-2 Electrical impedance properties of a QCM-ECIS sensor (WE- $\varnothing=0.5)$, obtained from exemplary ECIS-mode recordings of electrodes immersed (for $24 \mathrm{~h}$ ) in cell culture medium, and covered with a confluent layer of MDCK-II cells. Values of $X=|Z|, R$, and $C$ are determined at the respective most sensitive frequencies $f_{\text {sens }}(X)$, where parameters change most upon MDCK-II cell layer formation. Normalized parameter values $X_{\text {norm }}$ are calculated according to $|Z|_{\text {norm }}=$ $|Z|_{\mathrm{w} / \text { cells }} /|Z|_{\mathrm{w} / \text { o cells, }} R_{\text {norm }}=R_{\mathrm{w} / \text { cells }} / R_{\mathrm{w} / \mathrm{ocells}}$ and $C_{\mathrm{norm}}=C_{\mathrm{w} / \mathrm{o} \text { cells }} / C_{\mathrm{w} / \text { cells }}$.

\begin{tabular}{lcrr}
\hline$X=$ & $|Z|$ & \multicolumn{1}{c}{$R$} & \multicolumn{1}{c}{$C$} \\
\hline$f_{\text {sens }}(X)$ & $2.5 \mathrm{kHz}$ & $200 \mathrm{~Hz}$ & $32 \mathrm{kHz}$ \\
$X$ (medium) & $2.3 \mathrm{kOhm}$ & $1.7 \mathrm{kOhm}$ & $23.0 \mathrm{nF}$ \\
$X$ (MDCK-Il cells) & $13.2 \mathrm{kOhm}$ & $54.3 \mathrm{kOhm}$ & $3.9 \mathrm{nF}$ \\
$X_{\text {norm }}$ & 5.7 & 32 & 5.9 \\
\hline
\end{tabular}

\subsubsection{QCM-ECIS Sensors: Systematic Variation of WE Sizes and Layouts}

In addition to the previously characterized default QCM-ECIS sensor, prototypes with various different working electrode sizes and numbers were designed in proof-of-concept studies, and their impedance properties in both QCM-mode and ECIS-mode recordings were analyzed. Photoresist was coated on $2 \mathrm{ElO}$ plates at a spin velocity of $3000 \mathrm{rpm}$ and structured subsequently with different WE sizes by photolithography, according to the masks depicted in Fig. 5-8 A1-D1. Photographs next to the masks in Fig. 5-8 show the processed sensors. WE-sizes of $\varnothing_{\text {mask }}=250 \mu \mathrm{m}\left(A_{\text {mask }}=0.5 \cdot 10^{-3} \mathrm{~cm}^{2} ;\right.$ Fig. 5-8 A 1$)$, $\varnothing_{\text {mask }}=1000 \mu \mathrm{m}\left(A_{\text {mask }}=7.9 \cdot 10^{-3} \mathrm{~cm}^{2} ;\right.$ Fig. 5-8 $\left.C_{1}\right)$, and $\varnothing_{\text {mask }}=2000 \mu \mathrm{m}\left(A_{\text {mask }}=31.4 \cdot 10^{-3} \mathrm{~cm}^{2} ;\right.$ Fig. 5$8 \mathrm{D} 1$ ) were prepared. For comparison purposes, $\mathrm{QCM}$ - and ECIS-mode impedance characteristics of the standard sensor layout $\left(\varnothing_{\text {mask }}=500 \mu \mathrm{m} ; A_{\text {mask }}=2.0 \cdot 10^{-3} \mathrm{~cm}^{2} ;\right.$ Fig. $5-8 \mathrm{~B} 1$ ) are considered, too. Additional to variations in the WE size, prototypes comprising more than one working electrode were prepared. Two different photomasks were used for uncovering an array of ten working electrodes with a defined diameters of each electrode of either $\varnothing_{\text {mask }}=250 \mu \mathrm{m}$ or $\varnothing_{\text {mask }}=500 \mu \mathrm{m}$. These lithography masks and photographs of prepared QCM-ECIS sensors are shown in Fig. 5-10 A1, B1.

Diagrams in Fig. 5-8 A2 - D2 show the oscillation impedance amplitudes of the two resonator units of typical QCM-ECIS sensors with different sizes of the WE on QCM1. Phase spectra, 3-D-Bode plots, and complex representation of the impedance characteristics is presented in SI 11 (p. 266). The oscillations of $\mathrm{QCM}_{1}$ and $\mathrm{OCM}_{2}$ on the $2 \mathrm{ElO}$ are well separated and resonances are sufficiently decoupled for all prototypes. By increasing the size of the $W E$, i.e. removing an increasing amount of photoresist mass from $\mathrm{OCM}_{1}$, the separation of $\mathrm{QCM}_{1}$ and $\mathrm{OCM}_{2}$ resonances decreases (Fig. 5-8 A-D). The differences in the frequency of minimal impedance $\Delta f_{|Z|_{\text {min }}}$ of the adjacent resonators are listed in Tab. 5-3. By an 8fold extension of the WE diameter, from $250 \mu \mathrm{m}$ to $2 \mathrm{~mm}, \Delta f_{|Z|_{\text {min }}}$ is decreased by more than $8 \mathrm{kHz}$. Increasing electrode diameters on $\mathrm{QCM}_{1}$ also increase the occurrence of the second resonance at the frequency where the uncoated microbalance $(\mathrm{OCM} 2)$ is resonant. Moreover, the main resonance of $\mathrm{OCM}_{1}$ moves closer towards the frequency region of this unwanted resonance, which is critical for mutual disturbances. For the tested sensor layouts, interference occurs neither in the parallel resonance region of $\mathrm{OCM} 1$ nor in the serial resonance region of $\mathrm{OCM}_{2}$, neither in the spectra of impedance amplitude (Fig. 5-8 A2-D2) nor in that of phase shift (S/ 11). The minimal resonance separation of $13.3 \mathrm{kHz}$ for the maximal WE-size $\left(\varnothing_{\text {mask }}=2000 \mu \mathrm{m} ;\right.$ Fig. $\left.5^{-8} \mathrm{D}\right)$ is just sufficient to maintain decoupling of $\mathrm{QCM}_{1}$ and $\mathrm{OCM} 2$ resonances. 
A1
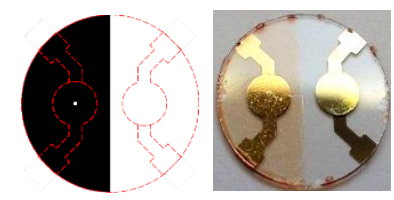

B1
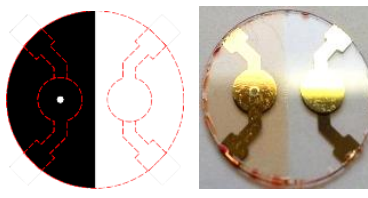

A2

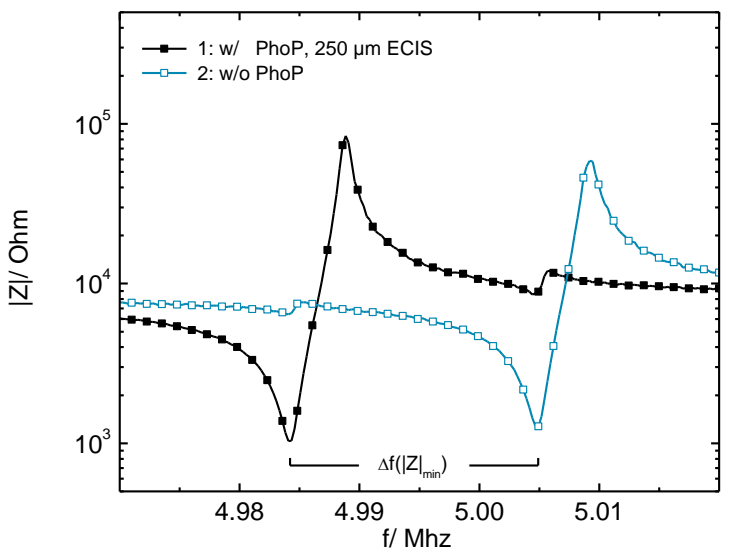

$\mathrm{C}_{2}$

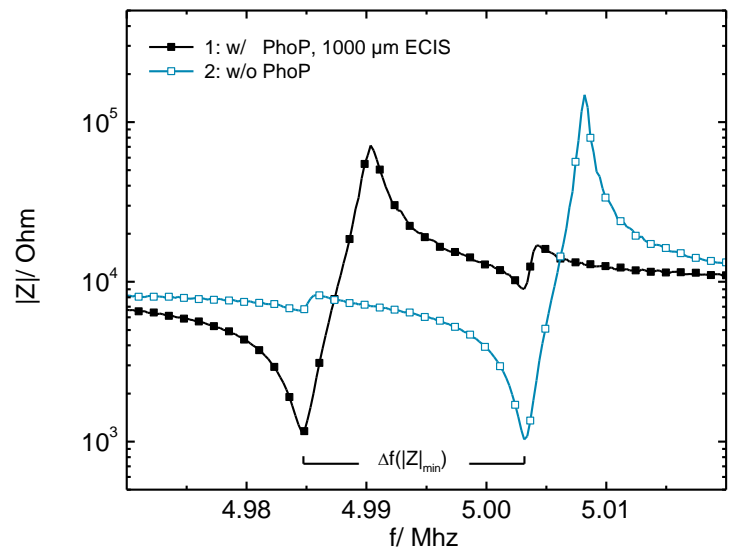

C1

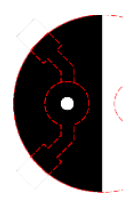

D1

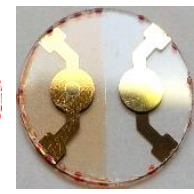

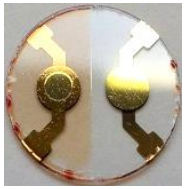

B2

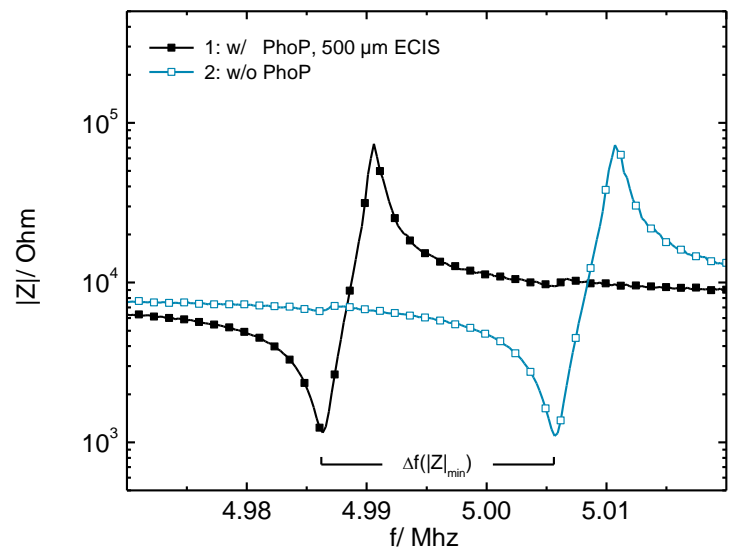

D2

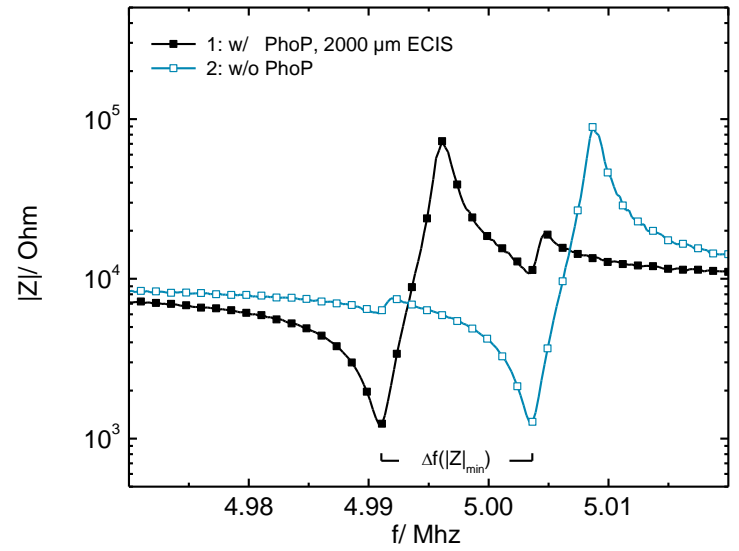

Fig. 5-8 OCM-mode characteristics of OCM-ECIS designs with different WE sizes. Sensors were prepared with WE diameters of (A) 250, (B) 500, (C) 1000, and (D) $2000 \mu \mathrm{m}$. For each layout, the used photomask, a photograph of the sensor, and corresponding spectra of the oscillation impedance magnitudes of photopolymer-coated $\mathrm{QCM} 1(-;$ w/ PhoP $)$ and of photopolymer-free $\mathrm{OCM}_{2}\left(-{ }^{-} ; \mathrm{w} / \mathrm{O}\right.$ PhoP) in air are shown. Data points were reduced for sake of clarity.

The electrochemical impedance characteristics (ECIS-mode) of the presented sensor layouts with increasing WE diameters are summarized in Fig. 5-9, by the spectra of (A) impedance magnitude, (B) normalized impedance magnitude, $(C)$ resistance, $(D)$ normalized resistance, $(E)$ capacitance, and $(F)$ normalized capacitance for WE diameters of $250 \mu \mathrm{m}\left(-\square_{-},--\right)$, $\left.500 \mu \mathrm{m}(-\Delta-,-)^{-}\right), 1000 \mu \mathrm{m}(-0-,-\bullet)$, and $2000 \mu \mathrm{m}(\cdot \nabla-,-\rightarrow)$. Impedance spectra of the sensors were recorded $\sim 24 \mathrm{~h}$ after adding medium into the measurement chamber (w/o cells; open symbols), whereas sensors covered with a confluent layer of MDCK-II cells ( $\mathrm{w} /$ cells; filled symbols) were studied the day after seeding $450000 \mathrm{cells} / \mathrm{cm}^{2}$. Impedance magnitude spectra of all systems exhibit typical curve shapes in the cell-free situation, with a slope of about minus one in the low frequency band and a straight line parallel to the $x$-axis at higher frequencies. This electrical behavior is equal to that of an electrical equivalent circuit of a resistance and a CPE in series and reflects the typical properties of an electrode immersed in an electrolyte. The increase of the WE size expands the dominance of the resistance contribution towards lower frequency values 
(Fig. 5-9 D), causing a likewise shift of the entire impedance spectra. In the course of this, the impedance magnitude is reduced in the low frequency region, reflecting an increasing capacitive and a decreasing resistive contribution of the CPE. This is evident, since a bigger electrode area $A$ increases the capacitance according to $C=\varepsilon_{0} \varepsilon_{r} A / d$.

A

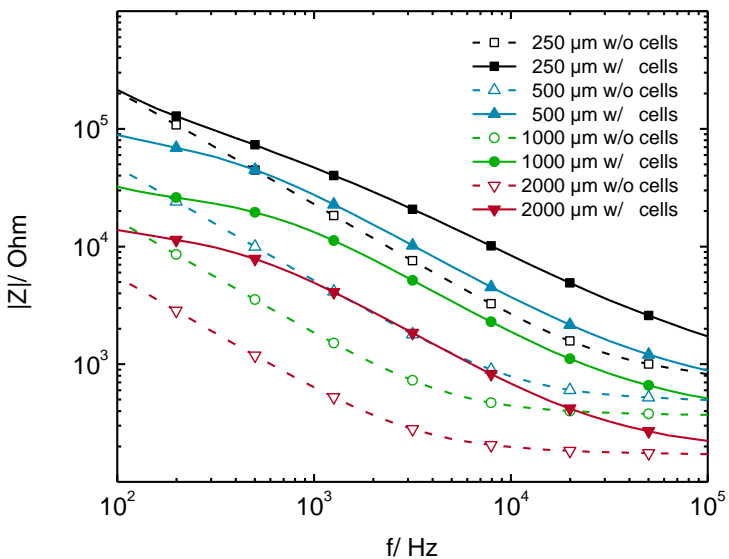

C

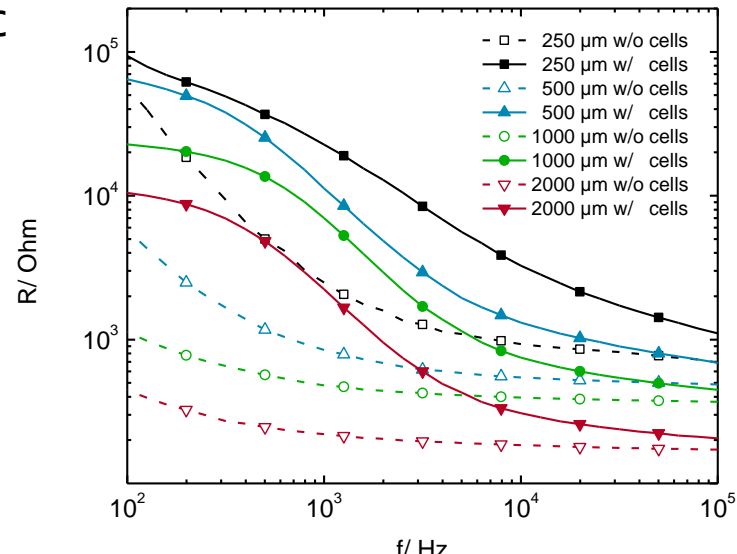

E

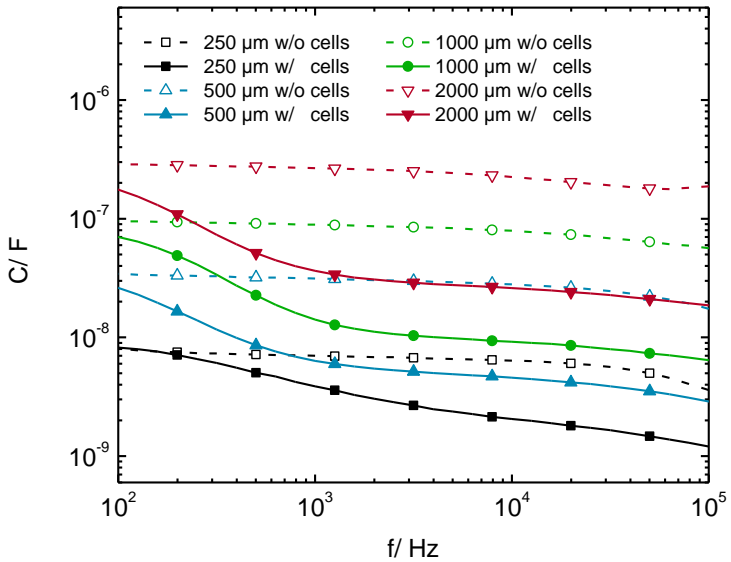

B

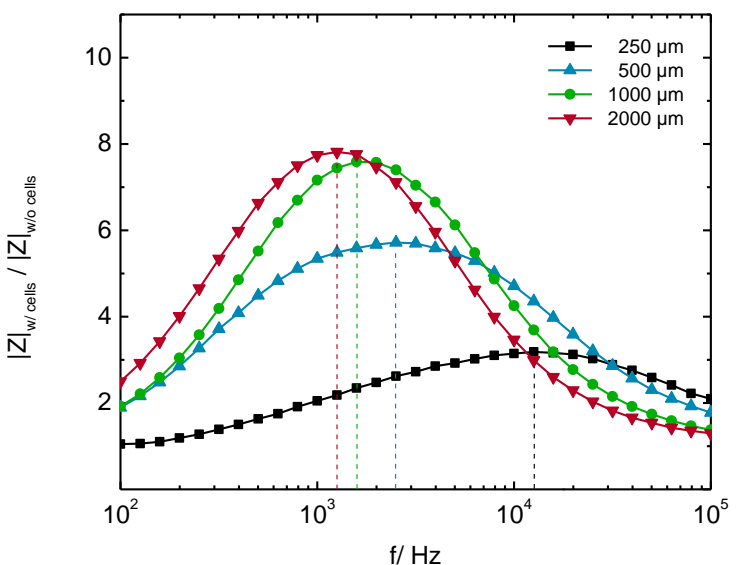

D

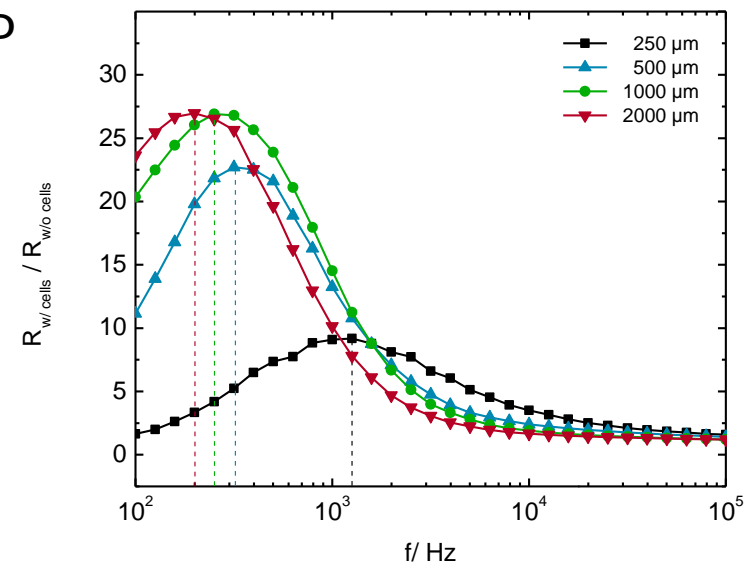

$\mathbf{F}$

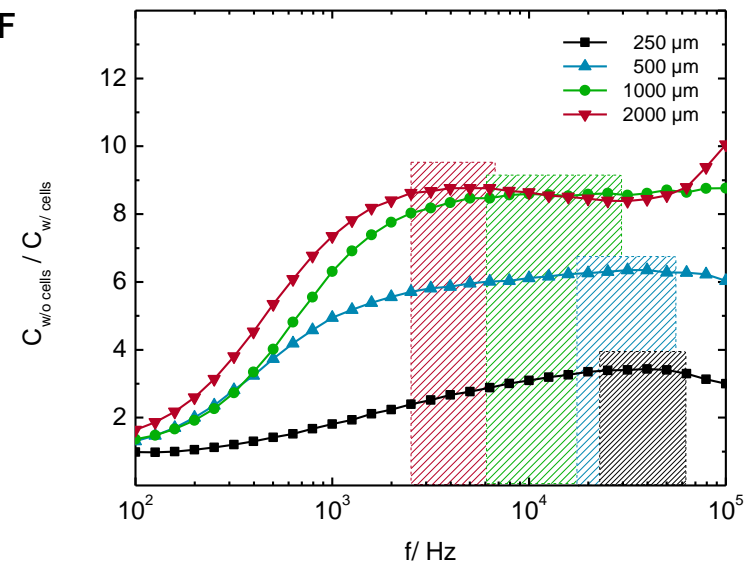

Fig. 5-9 ECIS-mode characteristics of various QCM-ECIS sensor designs with WE diameters of

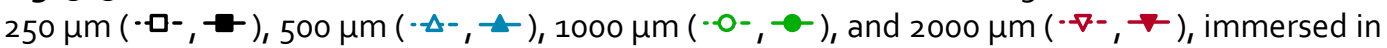
medium (w/o cells; open symbols) and covered with a MDCK-II cell layer ( $w /$ cells; filled symbols). Spectra are shown for absolute values (left-hand side) and normalized values (right-hand side) of (A), (B) impedance magnitude, (C), (D) resistance, and (E), (F) capacitance. Dashed lines and areas indicate frequencies of maximal sensitivity of the respective quantity. Data points were reduced for sake of clarity. 
The presence of cells on the electrodes significantly changes the impedance characteristics of the sensors with an additional impedance contribution at frequencies between $100 \mathrm{~Hz}$ and $100 \mathrm{kHz}$. The frequency band of the cell layer-related influence on the impedance magnitude (Fig. 5-9 A) thereby depends on the WE size. With increasing diameter of the WE it shifts to lower values. This affects the frequency of maximal cell response as reflected in the normalized spectra of impedance magnitude $\left(|Z|_{\mathrm{w} / \text { cells }} /|Z|_{\mathrm{w} / \mathrm{o} \mathrm{cells}}\right)$ in Fig. 5-9 B. The peak frequency (indicated with dashed lines) shifts to lower values with increasing WE area, from $\sim 10^{4} \mathrm{~Hz}$ for the $\varnothing_{\text {mask }}=250 \mu \mathrm{m}$ electrodes $\left(\rightarrow\right.$ ) to $\sim 10^{3} \mathrm{~Hz}$ for the $\varnothing_{\text {mask }}=2000 \mu \mathrm{m}$ electrodes $(\rightarrow)$. Averaged values of the most sensitive frequencies of the different sensor layouts are listed inTab. 5-3.

Spectra of resistance (Fig. 5-9 C, D), representing the real parts of the complex impedances, depend similarly on the WE size like the spectra of impedance magnitude. Compared to impedance, resistance is more sensitive for the cell layer at lower frequencies $\left(f<10^{4} \mathrm{~Hz}\right)$. By enlarging the WE area, the lowfrequency resistance decreases and the frequency region being sensitive for cells is shifted to lower values (Fig. 5-9 D). This trend also applies for the capacitance spectra, whereas the absolute capacitance values increase likewise with the WE size (Fig. 5-9 E, F). In contrast to the peak-shaped spectra $|Z|_{\text {norm }}$ and $R_{\text {norm, }}$ spectra of normalized capacitance exhibit a sigmoidal curve and thus a broad region of frequencies with similar sensitivity. Mean values of sensitive frequencies of impedance, resistance, and capacitance are summarized in Tab. 5-3 for all QCM-ECIS sensors under test.

Tab. 5-3 Influence of the WE dimension on the resonance separation $\Delta f_{|Z|_{\min }}$ between $\mathrm{QCM}_{1}$ and $\mathrm{QCM}_{2}$ in $\mathrm{QCM}$-mode and on the most sensitive frequencies $f_{\text {sens }}(|Z|, R, C)$ in $\mathrm{ECIS}$-mode $($ mean $\pm \mathrm{SEM})$. Specified values of WE diameter $\left(\mathrm{WE}-\varnothing_{\text {mask }}\right), \mathrm{WE}$ area $\left(\mathrm{WE}-A_{\text {mask }}\right)$, and of the percentage of PhoP coverage on the entire electrode area of $\mathrm{OCM}_{1}\left(\varnothing_{\text {Electrode }}=3.5 \mathrm{~mm}\right)$ are calculated from photomask dimensions.

\begin{tabular}{rrccccccc}
\hline $\begin{array}{c}\text { WE- } \varnothing_{\text {mask }} / \\
\mu \mathrm{m}\end{array}$ & $\begin{array}{c}\text { WE- } A_{\text {mask }} / \\
\mathrm{cm}^{2}\end{array}$ & $\begin{array}{c}\text { PhoP Coverage } \\
\text { on QCM } / \%\end{array}$ & $\begin{array}{c}\Delta f_{|Z| \text { min }} / \\
\mathrm{kHz}\end{array}$ & $\mathrm{N}$ & $\begin{array}{c}f_{\text {sens }}(|Z|) / \\
\mathrm{kHz}\end{array}$ & $\begin{array}{c}f_{\text {sens }}(R) / \\
\mathrm{kHz}\end{array}$ & $\begin{array}{c}f_{\text {sens }}(C) / \\
\mathrm{kHz}\end{array}$ \\
\hline 250 & $0.5 \cdot 10^{-3}$ & 99.5 & $-21.6 \pm 0.3$ & 5 & $15 \pm 2$ & $1.5 \pm 0.5$ & $61 \pm 11$ & 3 \\
500 & $2.0 \cdot 10^{-3}$ & 98.0 & $-19.70 \pm 0.14$ & 100 & $3.4 \pm 0.3$ & $0.29 \pm 0.02$ & $41 \pm 8$ & 18 \\
1000 & $7.9 \cdot 10^{-3}$ & 91.8 & $-18.7 \pm 0.4$ & 4 & $2.3 \pm 0.5$ & $0.28 \pm 0.06$ & $24 \pm 13$ & 3 \\
2000 & $31.4 \cdot 10^{-3}$ & 67.3 & $-13.3 \pm 0.5$ & 4 & $1.6 \pm 0.2$ & $0.26 \pm 0.03$ & $6 \pm 1$ & 3 \\
$10 \times 250$ & $4.9 \cdot 10^{-3}$ & 94.9 & -20.9 & 1 & - & - & - & - \\
$10 \times 500$ & $19.6 \cdot 10^{-3}$ & 79.6 & -17.6 & 1 & - & - & - & - \\
\hline
\end{tabular}

Another proof-of-principle approach aimed to introduce more than one working electrode in the photoresist of the coated gold electrode. This follows the architecture of commercial $8 \mathrm{~W}_{10} \mathrm{E}^{\mathrm{TM}} \mathrm{ECIS}$ electrodes (Applied BioPhysics; Troy, NY, USA) with ten working electrodes integrated in one measurement chamber. This makes the OCM-ECIS sensors capable for performing ECIS-mode monitoring of a bigger fraction of the cell population and over a larger region of the substrate. Two different sensors were designed, by applying photomasks comprising a 3-4-3 WE array with a diameter of each electrode of either $\varnothing_{\text {mask }}=250 \mu \mathrm{m}$ or $\varnothing_{\text {mask }}=500 \mu \mathrm{m}$. Masks for lithography are shown in Fig. 5$10 \mathrm{~A} 1, \mathrm{~B} 1$ together with photographs of prepared $\mathrm{QCM}-\mathrm{ECIS}$ sensors. 
Impedance spectra of $\mathrm{OCM}_{1}(-)$ ) and $\mathrm{OCM}_{2}(-\square)$ shear oscillations of both multi-WE sensors are similar in shape to that recorded for the default OCM-ECIS layout (cf. Fig. 5-6). Differences in the spectral properties only appear in a smaller resonance shift between the adjacent QCM spots. The resonance of $\mathrm{OCM}_{1}$ shifts to higher values as observed during processing for different WE arrays. This is in compliance with Sauerbrey (Eq. (23)). Tab. 5-3 lists for the different OCM-ECIS sensors with ten WEs under test the values of the integrated WE area, the degree of $\mathrm{PhOP}$ coverage on $\mathrm{OCM}_{1}$, and the resulting separations $\Delta f_{|Z|_{\text {min }}}$ of $\mathrm{QCM} 1$ and $\mathrm{QCM} 2$ resonances.

A1

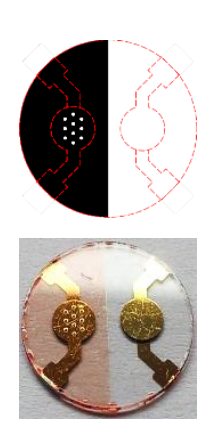

B1

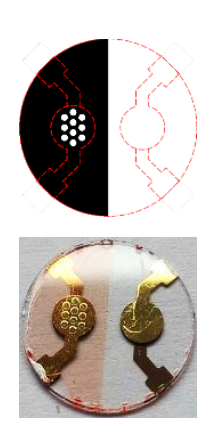

A2

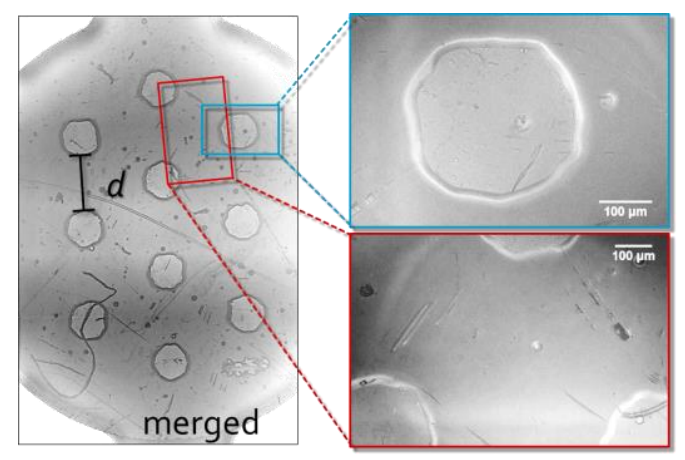

B2

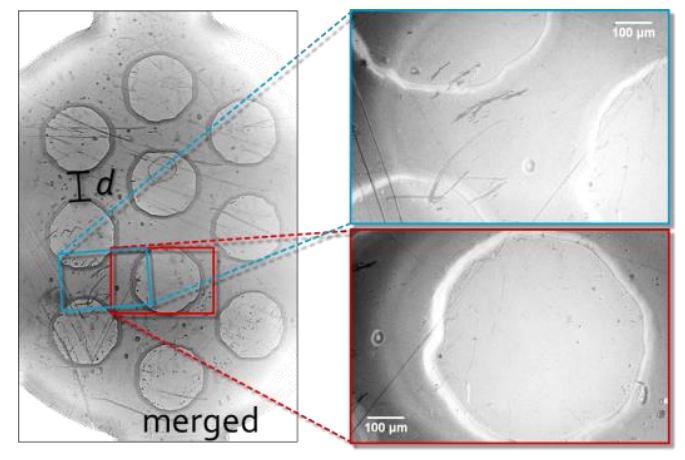

$\mathrm{A}_{3}$

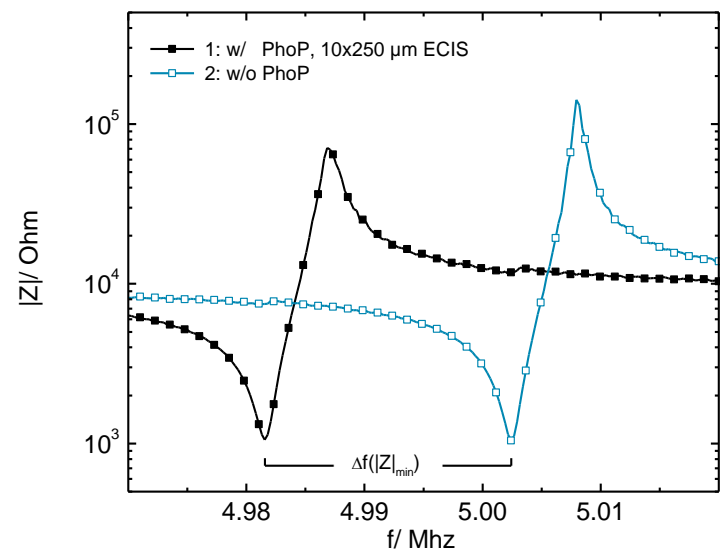

B3

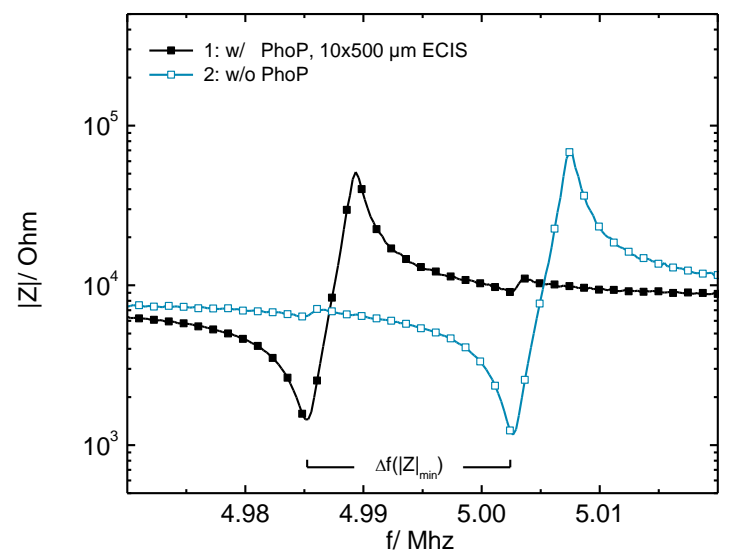

Fig. 5-10 OCM-mode characteristics of OCM-ECIS prototypes comprising ten WEs with diameters of each electrode of either (A) $\varnothing_{\text {mask }}=250 \mu \mathrm{m}$ or (B) $\varnothing_{\text {mask }}=500 \mu \mathrm{m}$. For each sensor layout are shown: $\left(A_{1}\right),\left(B_{1}\right)$ the photomask applied and a photograph of a processed $2 E_{1} Q_{1}\left(A_{2}\right)$, (B2) micrographs of the WE region, and $\left(A_{3}\right),\left(B_{3}\right)$ the corresponding impedance magnitude spectra of photopolymer-coated OCM1 $\left(-;\right.$ w/ PhoP) and photopolymer-free $\mathrm{OCM}_{2}\left(-{ }_{-}-;\right.$w/o $P h o P$ ) in air. Data points were reduced for sake of clarity. Overview micrographs in $A_{2}$ and $B 2$ were merged from three individual pictures; insets show magnifications of selected areas. The scale bar corresponds to $100 \mu \mathrm{m}$ and $d$ indicates the distance between electrode openings.

Phase-contrast micrographs in Fig. 5-10 show the polymer-coated electrode on the QCM-ECIS sensor surface with circular openings of either $250 \mu \mathrm{m}$ (Fig. 5-10 A2) or $500 \mu \mathrm{m}$ (Fig. 5-10 B2) in diameter at ten positions. Overview images were assembled from three individual micrographs, taken with objectives of $4 \times$ magnification. Insets show details with a higher magnification factor, revealing deviations from 
circularity and warp edges of the WEs. These abnormalities are more pronounced for the smaller openings. Analysis of micrographs provided values of the integrated WE area of $A_{\text {real }}=6.2( \pm 0.2)$ $\cdot 10^{-3} \mathrm{~cm}^{2}(\mathrm{~N}=5)$ and of $A_{\text {real }}=20.5( \pm 0.3) \cdot 10^{-3} \mathrm{~cm}^{2}(\mathrm{~N}=7)$ for sensors prepared with the $10 \times 250 \mu \mathrm{m}$ and $10 \times 500 \mu \mathrm{m}$ masks, respectively. These values correspond to average WE diameters of $\varnothing_{\text {real }}=280( \pm 4) \mu \mathrm{m}\left(\varnothing_{\text {mask }}=250 \mu \mathrm{m}\right)$ and $\varnothing_{\text {real }}=511( \pm 4) \mu \mathrm{m}\left(\varnothing_{\text {mask }}=500 \mu \mathrm{m}\right)$. Average distances $d$ between adjacent WEs are measured to $d_{\text {real }}=261( \pm 3) \mu \mathrm{m}\left(\mathrm{N}=6 ; d_{\text {mask }}=300 \mu \mathrm{m} ;\right.$ Fig. 5-10 A2) and $d_{\text {real }}=465( \pm 4) \mu \mathrm{m}\left(\mathrm{N}=11 ; d_{\text {mask }}=500 \mu \mathrm{m} ;\right.$ Fig. 5-10 B2). Hence, WE diameters prove to be bigger and inter-electrode separations turn out to be smaller than actually defined by the photomasks.

The electrochemical characteristics (ECIS-mode) of the ECIS-OCM sensors comprising ten working electrodes presented (Fig. 5-10 A1, B1) were analyzed as well. Diagrams in Fig. 5-11 show spectra of (A) absolute and (B) normalized impedance magnitude of sensors with WE-diameters of $250 \mu \mathrm{m}\left(\cdot-\square_{-},-\mathbf{-}_{-}\right)$ and $500 \mu \mathrm{m}\left(-\Delta_{-},-\right)$, incubated in cell culture medium (open symbols) and covered with a confluent layer of MDCK-II cells (filled symbols). Compared to each other, the bigger working electrode area translates the impedance spectrum to lower values along the frequency axis (Fig. 5-11 B). Differences are most pronounced in the low frequency region, where the electrode properties determine the overall impedance. The bigger the total working electrode area the lower is the impedance to current flow across the electrode into the adjacent medium. The increase of the WE dimensions shifts the sensitive frequency for cells to lower values (dashed line in Fig. 5-11 B), as it has already been determined above for the single-WE sensors with various WE sizes (cf. Fig. 5-8). For corresponding spectra of absolute and normalized resistance and capacitance see $S / 12$.

A

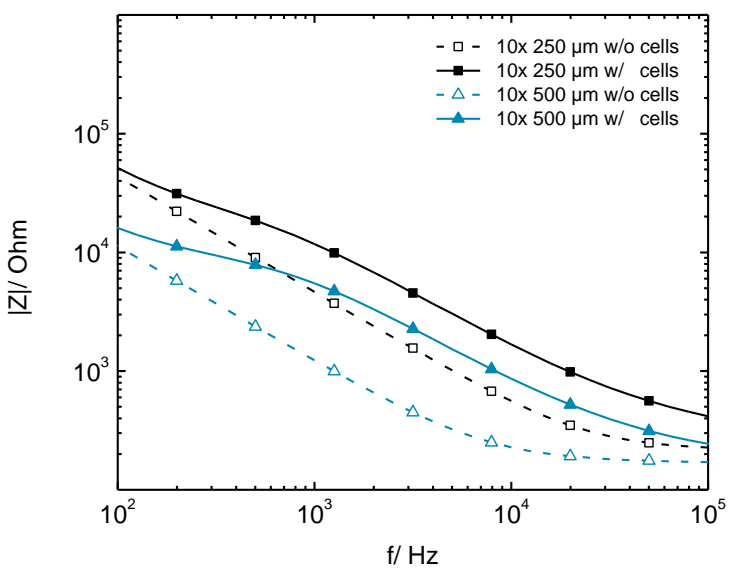

B

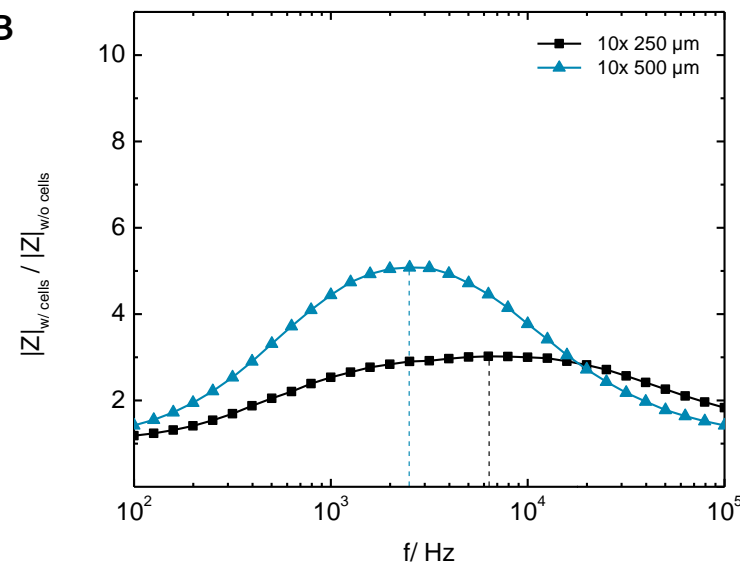

Fig. 5-11 ECIS-mode characteristics of OCM-ECIS prototypes comprising ten WEs with diameters of each electrode of either $\varnothing_{\text {mask }}=250 \mu \mathrm{m}(-\square-,--)$ or $\varnothing_{\text {mask }}=500 \mu \mathrm{m}\left(\cdot-\Delta_{-},--\right)$. (A) Absolute values of impedance magnitude of the senors exposed to medium (open symbols) and covered with a confluent MDCK-II cell layer (filled symbols). (B) Normalized impedance spectra indicate the most sensitive frequency for cell monitoring (dashed lines). Data points were reduced for sake of clarity. 


\subsection{QCM-ECIS Biosensor for Cell Attachment and Spreading}

The impedance analyses of OCM-mode and ECIS-mode recordings of the $3^{\text {rd }}$ generation OCM (5.1.4 and 5.1.5) convincingly demonstrated its capability of discriminating a cell-free and a cell-covered sensor in mechanical and electrical respects, as the characteristic sensor parameters respond very sensitive to the attachment of cells in both modes of measurement. For instance, the minimal impedance of quartz oscillation increases by $\Delta|Z|_{\text {min }} \approx 850 \mathrm{Ohm}$ upon coverage of the sensor surface with a confluent monolayer of MDCK-II cells, which is a signal increase by $\sim 45 \%$ with respect to the cell-free value under medium loading (cf. Tab. 5-1). In the course of this experiment, the magnitude of electrical impedance between WE and CE changes by $\Delta|Z| \approx 7.8 \mathrm{kOhm}(\sim 600 \%$ signal increase) at the most sensitive frequency of $5 \mathrm{kHz}$ (cf. Tab. 5-3). Split into real and imaginary part, MDCK-Il cells on the sensor alter the resistance at $316 \mathrm{~Hz}$ by $\Delta R \approx 33 \mathrm{kOhm}(\sim 2400 \%$ signal increase), and the capacitance at $31.2 \mathrm{kHz}$ by $\Delta C \approx-19.7 \mathrm{nF}(\sim-88 \%$ signal decrease) (cf. Tab. 5-3). Thus, the OCM-ECIS sensor allows for sensitive, multi-parametric, substrate-integrated, label-free, and quasi-simultaneous sensing of adherent cells. Since both transducer principles are non-invasive on cells, mechanical (viscoelastic) and electrical properties (electrode coverage, barrier resistance) of the same cell layer can be monitored in real-time, providing time-resolved information on establishing, disassembling, and modifying cell-cell and cellsubstrate interactions.

Combined OCM- and ECIS-mode recordings were applied for investigating the absolute and kinetic viscoelastic and electrical characteristics of different cell lines in the course of cell-substrate and cell-cell interactions on the QCM-ECIS sensors developed here. Since the coated photopolymer layer on the sensing side of the sensor is expected to swell upon immersion with liquid ${ }^{[236]}$, the sensor surfaces were exposed to serum-free cell culture medium (SFM) and equilibrated for $\sim 20 \mathrm{~h}$ before the seeding of cells. This procedure was meant to decouple impedance alterations due to the equilibration from cell signals arising in the course of an experiment. The influence of SFM incubation on the OCM-ECIS sensor responses in QCM- and ECIS-mode recordings is addressed firstly (5.2.1), followed by time-resolved cell adhesion studies. The investigations comprise the attachment and spreading of MDCK-II cells (5.2.2) and NRK cells (5.2.3) under standard cell culture conditions. MDCK-II cells are studied additionally in modified culture medium (5.2.4). The setup illustrated in Fig. 3-18 (p. 57) was used for the cell-based assays. All studies were performed with the standard layout of the OCM-ECIS sensor. This is a 2 EIQ whose one resonator unit was coated with photoresist of $\sim 3 \mu \mathrm{m}$ in thickness. A circular opening was introduced in the center of the coating to uncover a defined fraction of the covered gold electrode (Fig. 5-4). The bare, small area of the electrode serves as WE for ECIS-mode impedance analysis, vis-à-vis to the uncoated electrode of the second resonator unit which serves as the CE (Fig. 5-2 B). The WE diameter was experimentally determined and averaged out to 511 ( \pm 4 ) $\mu \mathrm{m}$ (cf. 5.1.1).

\subsubsection{Sensor Preincubation with Culture Medium}

Double-mode impedance recordings in the forerun of a cell experiment monitored moisture expansion of photopolymer (PhoP) on the OCM-ECIS sensor surface. Sensors were immersed with $1.5 \mathrm{~mL}$ of SFM under sterile conditions, measurement chambers were placed in a cell-culture incubator $\left(37^{\circ} \mathrm{C}, 5 \% \mathrm{CO}_{2}\right)$, and impedance measurements were directly started afterwards to follow polymer swelling. Fig. 5-12 A 
represents $\mathrm{OCM}$-mode measurements, depicting average time courses of minimal impedance changes, $\Delta|Z|_{\min }$ (mean $\pm \mathrm{SEM} ; \mathrm{N} \geq 24$ ), for the two resonator units $\mathrm{QCM} 1$ (w/ PhoP; black curve) and $\mathrm{OCM} 2$ (w/o PhoP; blue curve). Fig. 5-12 $\mathrm{B}$ shows average resistance and capacitance changes, $\Delta R$ and $\Delta C$ (mean $\pm \mathrm{SEM} ; \mathrm{N}=11$ ), extracted from ECIS-mode recordings. Values are referenced to initial values, obtained from the first impedance spectrum after adding SFM.

An initial drop by $\Delta|Z|_{\text {min }} \approx-50 \mathrm{Ohm}$ within $2-6 \mathrm{~h}$ and a subsequent constant value of $|Z|_{\text {min }}$ for at least $16 \mathrm{~h}$ characterize the time course of the shear oscillation impedance after exposing one face of the sensor to SFM. Marginal differences in the signal changes of photopolymer-coated $\mathrm{OCM}_{1}$ and uncoated $\mathrm{OCM}_{2}$ appear in the kinetics of the initial drop and in the final values after $18 \mathrm{~h}$ of incubation. $\Delta|Z|_{\min }$ of $\mathrm{QCM}$ reaches equilibrium within $2 \mathrm{~h}$, whereas this takes slightly longer for $\mathrm{OCM}$. After equilibration, $|Z|_{\min }$ of OCM1 is $-60( \pm 10)$ Ohm $(N=27)$ lower and $|Z|_{\text {min }}$ of $\mathrm{OCM} 2$ is $-30( \pm 10) \mathrm{Ohm}(\mathrm{N}=24)$ lower than initial values. With respect to absolute values, $|Z|_{\min }$ is decreased by $-2.9( \pm 0.6) \%(\mathrm{OCM})$ and $-1.7( \pm 0.5) \%(\mathrm{OCM} 2)$, respectively. Hence, damping of the shear oscillations is slightly reduced to a similar extent for both resonators in the course of SFM incubation. Exposure of the QCM-ECIS sensor to SFM for $18 \mathrm{~h}$ results in averaged absolute values of $|Z|_{\min }(\mathrm{OCM})=1890( \pm 30) \mathrm{Ohm}$ and $|Z|_{\min }(\mathrm{OCM} 2)=1830( \pm 30) \mathrm{Ohm}$, respectively. Hence, the oscillation damping of microbalances is by $\sim 60$ Ohm just slightly higher for OCM1 coated with PhoP.

A

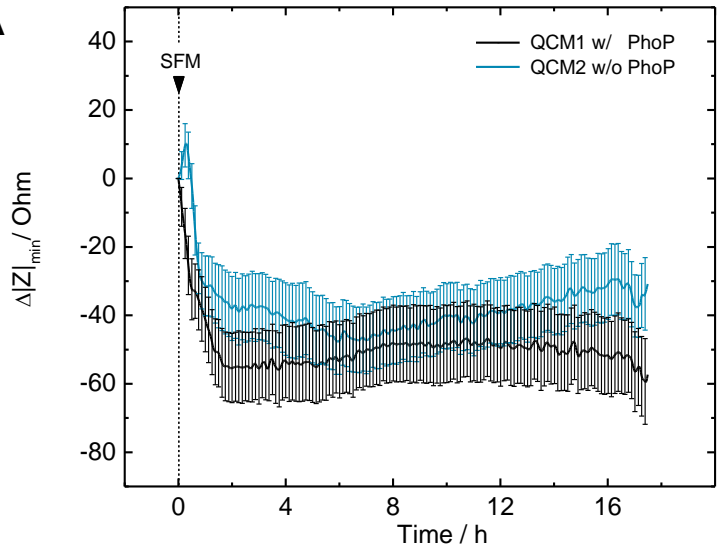

B

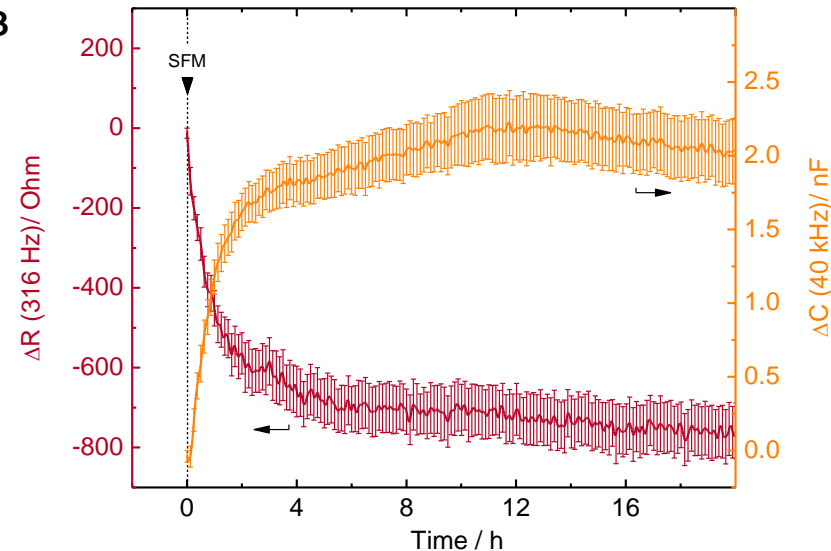

Fig. 5-12 (A) Changes in the minimal impedance of $\mathrm{OCM}_{1}$ and $\mathrm{OCM}_{2}$ oscillations and (B) electrochemical impedance changes upon immersing the standard OCM-ECIS sensor with SFM. (A) Averaged time courses of $\Delta|Z|_{\min }(\operatorname{mean} \pm \mathrm{SEM})$ for $\mathrm{OCM} 1$ (w/PhoP; black curve; $\mathrm{N}=27$ ) and $\mathrm{OCM} 2$ (w/o PhoP; blue curve; $\mathrm{N}=24$ ). The impedance changes within $18 \mathrm{~h}$ by $\Delta|Z|_{\min }(\mathrm{QCM})=-60( \pm 10) \mathrm{Ohm}$ and $\Delta|Z|_{\min }(\mathrm{QCM})=-30( \pm 10) \mathrm{Ohm}$, resulting in constant final values of $|Z|_{\min }(\mathrm{OCM})=1890( \pm 30) \mathrm{Ohm}$ and $|Z|_{\min }(\mathrm{OCM})=1830( \pm 30) \mathrm{Ohm}$. (B) Averaged time courses of resistance change $\Delta R$ at $316 \mathrm{~Hz}$ (red curve) and capacitance change $\Delta C$ at $40 \mathrm{kHz}$ (orange curve) upon immersion of the sensor with $\mathrm{SFM}$ (mean $\pm \mathrm{SEM} ; \mathrm{N}=11$ ). Equilibration is reached after $\sim 8 \mathrm{~h}$, with final signal changes of $\Delta R=-750( \pm 60) \mathrm{Ohm}$ and $\Delta C=$ $2.1( \pm 0.2) \mathrm{nF}$. Final stationary values are $R=1390( \pm 60) \mathrm{Ohm}$ and $C=22.1( \pm 0.7) \mathrm{nF}(t=18 \mathrm{~h})$.

Sensor incubation with SFM shows more pronounced impedance responses in the electrochemical recordings (Fig. 5-12 B). Resistance at $316 \mathrm{~Hz}$ (red curve) and capacitance at $40 \mathrm{kHz}$ (orange curve) strongly change within $\sim 8 \mathrm{~h}$ after SFM addition with similar kinetics and opposing trends. In this period, low-frequency resistance decreases by $\Delta R=-750( \pm 60) \mathrm{Ohm}\left(\Delta R / R_{0}=-35( \pm 3) \%\right)$ and high-frequency 
capacitance rises by $\Delta C=2.1( \pm 0.2) \mathrm{nF}\left(\Delta C / C_{0}=+10( \pm 1) \%\right)$. Final stationary values of $R=1390( \pm 60)$ Ohm and $C=22.1( \pm 0.7) \mathrm{nF}$ on average are reached after $18 \mathrm{~h}$ of SFM incubation.

These distinct changes in oscillation impedances and especially in electrochemical impedance properties of QCM-ECIS sensors illustrate the impact of SFM incubation on sensor responses and the need for its preincubation for at least $6 \mathrm{~h}$ in the forerun of cell attachment and spreading studies.

\subsubsection{MDCK-II Cell Attachment and Spreading}

Attachment and spreading of MDCK-II cells was investigated in time-resolved double-mode impedance measurements by QCM-ECIS sensors. The day after incubating the quartz surface with SFM ( $t \geq 20 \mathrm{~h})$ medium was replaced by $1.5 \mathrm{~mL}$ MDCK-II cell suspension of $\sim 350000$ cells/ $\mathrm{mL}$ (seeding to confluence). Motional impedance spectra of resonators loaded with SFM and with a confluent MDCK-II cell layer have been presented and discussed above (Fig. 5-6, p. 118). Spectra of impedance magnitude, resistance, and capacitance from ECIS-mode recordings of a cell-free and a MDCK-II cell-covered sensor are shown in Fig. 5-7 (p. 122) and the respective characteristics are discussed in the accompanying text passages.

The time courses of minimal impedance change $\Delta|Z|_{\text {min }}$ during MDCK-II cell attachment and spreading are shown in Fig. 5-13 A. Averaged traces (mean \pm SEM) are depicted separately for recordings with $\mathrm{OCM}_{1}\left(\mathrm{w} / \mathrm{PhoP} ;-_{-}^{-} ; \mathrm{N}=31\right.$ ) and $\mathrm{OCM}_{2}$ (w/o PhoP; ${ }^{--} ; \mathrm{N}=24$ ), and for pooled data sets of $\mathrm{OCM}_{1}$ and $\mathrm{OCM} 2$ recordings ( $-; \mathrm{N}=55$ ). Curves are referenced in time and $|Z|_{\min }$ values to the last data point before cell addition. After $\geq 20 \mathrm{~h}$ of incubation with SFM, average starting values of $|Z|_{\min }\left(t_{0}\right)$ are $1890( \pm 20) \mathrm{Ohm}$ for polymer-coated $\mathrm{OCM}$ resonators and $1810( \pm 30) \mathrm{Ohm}$ for uncoated $\mathrm{OCM} 2$ resonators (mean $\pm \mathrm{SEM} ; \mathrm{N}=40$ ). Hence, initial minimal impedance values are slightly but significantly lower by $\sim 80 \mathrm{Ohm}$ for the uncoated resonator surfaces. Tab. 5-4 summarizes the characteristic shear displacement parameters of separate and pooled data sets of $\mathrm{OCM} 1$ and $\mathrm{OCM} 2$ in the course of MDCK-II attachment and spreading.

$\Delta|Z|_{\text {min }}$ starts to increase instantaneously after replacing SFM with the cell suspension (Fig. $5-13$ A). As an indicator for the kinetics of cell attachment in QCM-mode, times $\left(t_{m}\right)$ and values of maximal slope $m=\Delta|Z|_{\min } / \Delta t$ were determined. Values of $t_{m}$ were obtained from the maximum of the first derivative of the time courses and the maximal slopes were calculated by linear regression of nine data points around $t_{m}$. For both resoantors a maximal slope of $m \approx 16 \mathrm{Ohm} \cdot \mathrm{min}^{-1}$ occurs $30 \mathrm{~min}$ after seeding cells to confluence. The slope is slightly higher for recordings with resonator $\mathrm{OCM}_{1}$ compared to that with $\mathrm{OCM}_{2}$ (Tab. 5-4). A second parameter reporting on the kinetics of mechanical cell-substrate interactions is time $t_{1 / 2}$, when half of the final impedance change $\Delta|Z|_{\min }\left(t_{1}\right)$ is reached $\left(t_{1 / 2}=t\left(1 / 2 \cdot \Delta|Z|_{\min }\left(t_{1}\right)\right)\right)$. This period is by $t_{1 / 2}=40 \mathrm{~min}$ similar for the two microbalances on the sensor. At $t^{\prime} \approx 190 \mathrm{~min}$ after seeding, $\Delta|Z|_{\min }$ converges to a first plateau of $750( \pm 10) \mathrm{Ohm}(\mathrm{OCM} 1)$ and $700( \pm 10) \mathrm{Ohm}(\mathrm{QCM} 2)$, followed by a second increase to a transient maximum of $940( \pm 20) \mathrm{Ohm}(\mathrm{OCM})$ and $850( \pm 20) \mathrm{Ohm}$ $(\mathrm{OCM})$ at time $t^{\prime \prime} \approx 490 \mathrm{~min}$. It is noticeable that $\Delta|Z|_{\text {min }}$ of $\mathrm{OCM}_{1}$ takes approximately 20 min longer to reach this maximum (Tab. 5-4). Afterwards, oscillation damping of $\mathrm{OCM}_{1}$ and $\mathrm{OCM} 2$ decline equally by $\sim 50 \mathrm{Ohm}$ within $4 \mathrm{~h}$ to stationary values $(t \geq 12 \mathrm{~h})$ and remain constant for at least $8 \mathrm{~h}$. At the end of MDCK-II attachment and spreading $\left(t_{1}=20 \mathrm{~h}\right),|Z|_{\min }$ values are increased by $890( \pm 10) \mathrm{Ohm}(\mathrm{OCM})$ and $800( \pm 20) \mathrm{Ohm}(\mathrm{OCM} 2)$, resulting in final absolute values of $2780( \pm 20) \mathrm{Ohm}(\mathrm{OCM})$ and 2620 ( \pm 20$)$ Ohm (OCM2). $\Delta|Z|_{\text {min }}$ time courses are similar in shape for $\mathrm{OCM}_{1}$ and $\mathrm{OCM} 2$ recordings (Fig. 5-13 A). However, the extent of signal increase is significantly lower for the $\mathrm{QCM} 2$ resonator, with an offset of $\sim 50-90 \mathrm{Ohm}$ all along the investigated period. Hence, photoresist coating has a marginal but significant influence on the sensor response. 
A

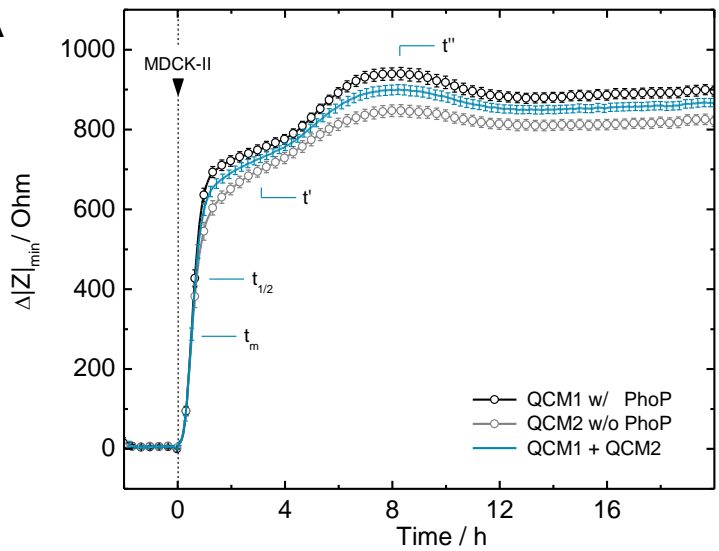

C

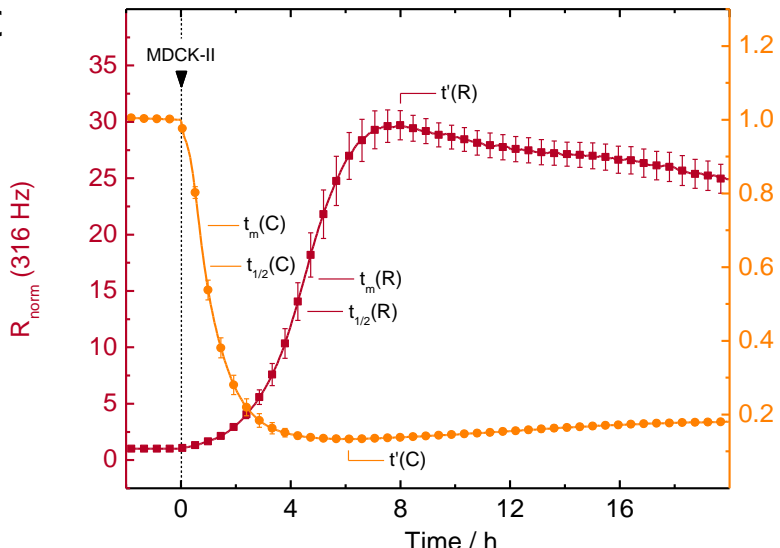

E

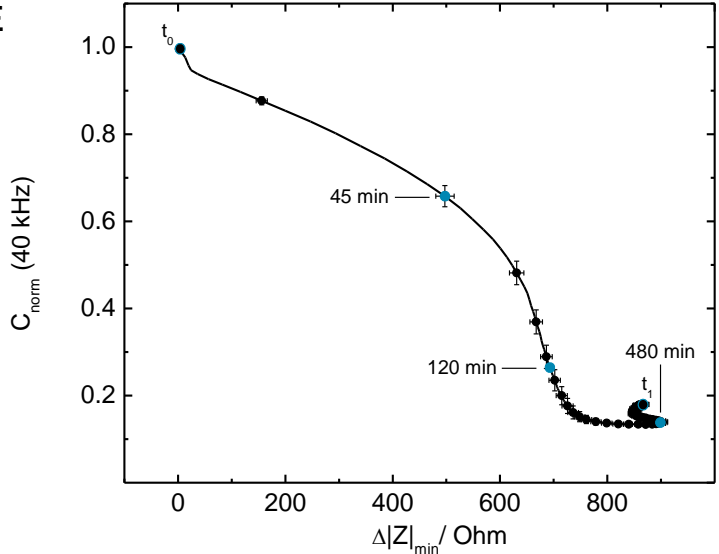

B

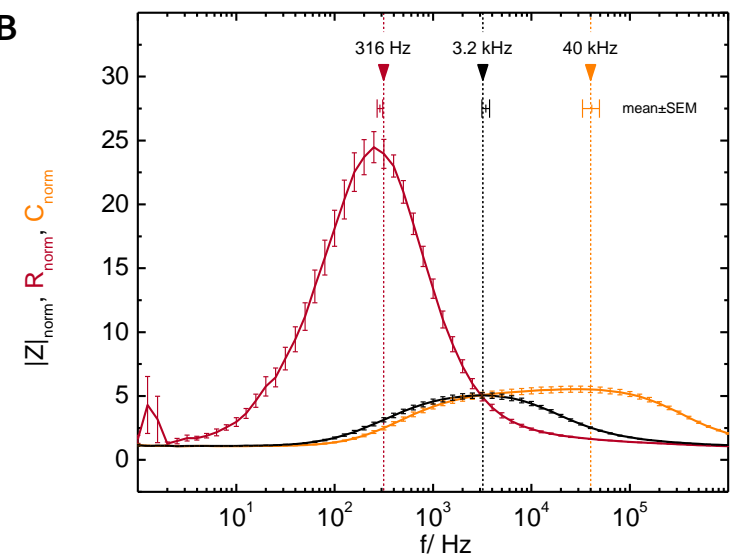

D

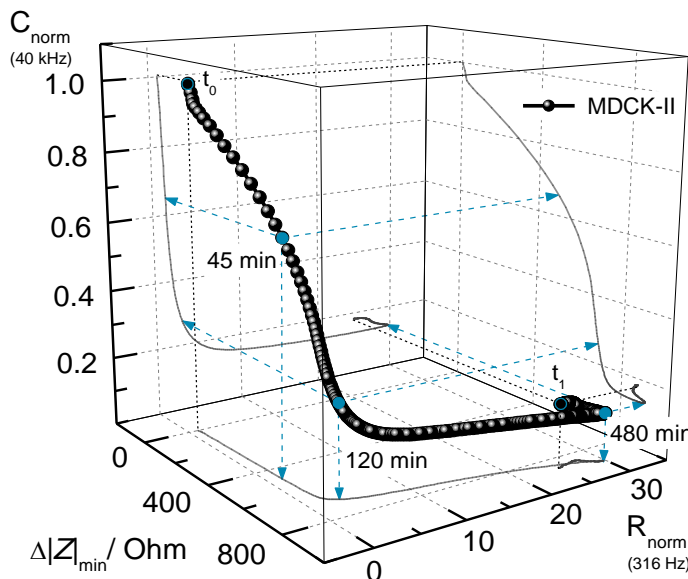

$\mathbf{F}$

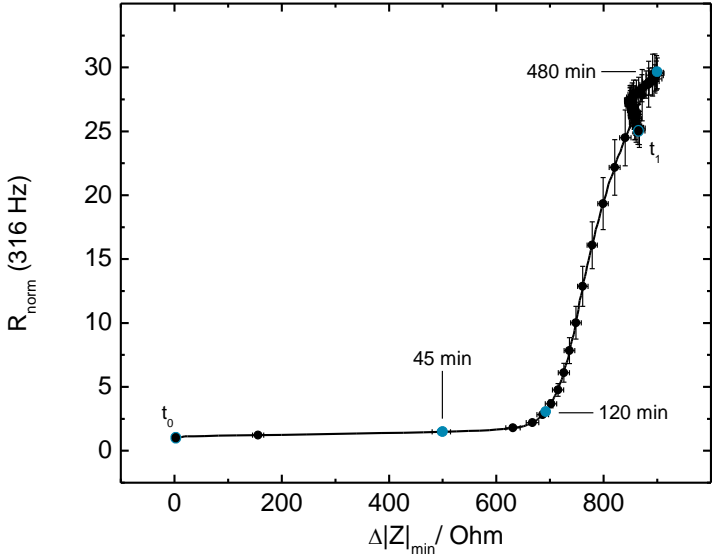

Fig. 5-13 OCM-ECIS sensor responses during MDCK-II cell attachment and spreading. (A) Time courses of minimal impedance change, $\Delta|Z|_{\text {min }}$, of resonators' shear displacement. Curves are averaged from individual traces (mean $\pm \mathrm{SEM}$ ) and displayed separately for $\mathrm{OCM}_{1}\left(\mathrm{w} / \mathrm{PhoP}_{;}-\mathrm{O}_{\text {; }}\right.$; $\mathrm{N}=31$ ) and $\mathrm{OCM}_{2}$ (w/o PhoP; $-\mathrm{O}_{-} ; \mathrm{N}=24$ ) and for combined $\mathrm{OCM}_{1}$ and $\mathrm{OCM}_{2}$ impedance data sets $(-; \mathrm{N}=55)$. The dashed line indicates cell inoculation at time zero. Absolute starting values $|Z|_{\min }\left(t_{0}\right)$ amount to $1890( \pm 20) \mathrm{Ohm}(\mathrm{OCM})$, $1810( \pm 30) \mathrm{Ohm}\left(\mathrm{OCM}_{2}\right.$; both $\left.\mathrm{N}=40\right)$, and $1850( \pm 20) \mathrm{Ohm}(\mathrm{OCM} 1+\mathrm{OCM} 2 ; \mathrm{N}=80)$. (B) Averaged normalized spectra of impedance magnitude $(-)$, resistance $(-)$, and capacitance $(-)$, calculated from ECIS-mode responses of cell-free (medium-loaded) and MDCK-II cell-covered sensor surfaces, according to $|Z|_{\text {norm }}=$ $|Z|_{\mathrm{w} / \text { cells }} /|Z|_{\mathrm{w} / \text { c cells }}, R_{\mathrm{norm}}=R_{\mathrm{w} / \text { cells }} / R_{\mathrm{w} / \mathrm{o} \mathrm{cells} \text {, }}$ and $C_{\text {norm }}=C_{\mathrm{w} / \text { c cells }} / C_{\mathrm{w} / \text { cells }}$ (mean $\pm \mathrm{SEM} ; \mathrm{N}=18$ ). Spectra indicate most sensitive sampling frequencies $f_{\text {sens }}$ of $316 \mathrm{~Hz}$ for $R, 3.2 \mathrm{kHz}$ for $|Z|$, and $40 \mathrm{kHz}$ for $C$. (C) Time courses of $R_{\text {norm }}\left(316 \mathrm{~Hz}_{i}{ }^{-}\right)$) and $C_{\text {norm }}\left(40 \mathrm{kHz}_{i}{ }^{--}\right)$during MDCK-II attachment and spreading. Curves are averaged from $\mathrm{N}=18$ individual traces (mean $\pm \mathrm{SEM}$ ). Absolute starting values of QCM-ECIS sensors immersed with SFM are $R\left(t_{0}\right)=1430( \pm 50) \mathrm{Ohm}$ and $C\left(t_{0}\right)=20.5( \pm 0.8) \mathrm{nF}$. For averaging multiple curves, each time course was firstly referenced 
in time to the last values before cell inoculation. Traces were subsequently provided with an uniform timescale in a time frame of $2000 \mathrm{~min}$ by a linear interpolation of the data with 1000 points in total. Finally, the measured quantity was averaged at each time and the standard error of the mean was calculated from the population of individual curves. (D) 3-D and (E), (F) 2-D impedance plots, merging the time courses of characteristic impedance parameters from recordings in QCM-mode $\left(\Delta|Z|_{\text {min }}\right)$ and ECIS-mode $\left(C_{\text {norm }}, R_{\text {norm }}\right)$ during MDCK-II cell attachment and spreading. Traces exhibit 430 equidistant data points between $t_{0}=0 \mathrm{~min}$ and $t_{1}=1200 \mathrm{~min}$. Characteristic times are indicated. $\Delta|Z|_{\text {min }}$ values were used from pooled $\mathrm{QCM}_{1}$ and $\mathrm{QCM} 2$ data $(\mathrm{N}=55), R_{\text {norm }}$ and $C_{\text {norm }}$ values were averaged from $\mathrm{N}=18$ individual measurements. Data points displayed in $A, C, E$, and $F$ were reduced for sake of clarity.

Combined impedance data of $\mathrm{OCM} 1$ and $\mathrm{OCM}_{2}$ shear displacements provide an averaged time course of the mechanical interaction of MDCK-II cells with the OCM-ECIS sensor surface and cytomechanical alterations during cell layer formation. Since curve shape and time-dependent characteristics of $\Delta|Z|_{\text {min }}$ are similar for both resonators and they only differ to a moderate extent in the signal height, it is fair to pool data sets. This increases the number of data points by the factor of two, allowing for a more accurate averaging of the final values as well as of the time-resolve signal changes arising from the cells (Tab. 54). Hence, damping of $\mathrm{OCM}_{1}$ and $\mathrm{OCM}_{2}$ shear displacement by an established confluent monolayer of MDCK-II cells is estimated to an averaged value of $\Delta|Z|_{\min }\left(t_{1}\right)=850( \pm 10)$ Ohm (mean $\pm \mathrm{SEM} ; \mathrm{N}=80$ ). Moreover, pooling of data provides an averaged maximal rate of impedance change of $\Delta|Z|_{\text {min }} /$ $\Delta t \approx 16.61( \pm 0.06) \mathrm{Ohm} \cdot \mathrm{min}^{-1}$ at $t_{m} \approx 30 \mathrm{~min}$, a first plateau of $\Delta|Z|_{\min }=730( \pm 10) \mathrm{Ohm}$ at time $t^{\prime} \approx 190 \mathrm{~min}$, and a transient maximum of $\Delta|Z|_{\text {min }}=900( \pm 10)$ Ohm at $t^{\prime \prime} \approx 490 \mathrm{~min}$ after cell inoculation (mean $\pm \mathrm{SEM} ; \mathrm{N}=55$ ).

Tab. 5-4 Characteristic shear oscillation parameters (QCM-mode) of resonators $\mathrm{OCM}_{1}$ and $\mathrm{OCM}_{2}$ in the time course of MDCK-II cell attachment and spreading on the OCM-ECIS sensor surface. Values of separate $\left(\mathrm{QCM}_{1}, \mathrm{QCM}_{2}\right)$ and pooled $\left(\mathrm{OCM}_{1}+2\right)$ impedance data of resonators are shown. Mean starting values are obtained by averaging absolute $|Z|_{\text {min }}$ values of data sets at time zero $\left(t_{0}\right)$. Maximal slopes $m=\Delta|Z|_{\min } / \Delta t$ were calculated by linear regression around $t_{m}( \pm 4$ data points) considering standard errors (instrumental weighting). Mean $\Delta|Z|_{\min }$ values are obtained from averaged time curves at the respective times of the first plateau $\left(t^{\prime}\right)$, of maximal $\Delta|Z|_{\text {min }}$ signal $\left(t^{\prime \prime}\right)$, and at the end of investigations $\left(t_{1}=20 \mathrm{~h}\right) \cdot t_{1 / 2}$ marks the point in time of half of the final $\Delta|Z|_{\min }$ change. (mean $\pm \mathrm{SEM} ; \mathrm{N}={ }^{\mathrm{a})} 40,{ }^{\mathrm{b})} 80,{ }^{\mathrm{c})} 31,{ }^{\mathrm{d})} 24,{ }^{\mathrm{e})} 55$ ).

\begin{tabular}{|c|c|c|c|c|c|c|c|c|c|c|}
\hline & $\begin{array}{c}|Z|_{\min }\left(t_{0}\right) / \\
\mathrm{Ohm}\end{array}$ & $\begin{array}{l}t_{m} / \\
\min \end{array}$ & $\begin{array}{c}m=\Delta|Z|_{\min } / \Delta t \mid \\
\text { Ohm } \cdot \min ^{-1}\end{array}$ & $\begin{array}{c}t^{\prime} / \\
\min \end{array}$ & $\begin{array}{c}\Delta|Z|_{\min }\left(t^{\prime}\right) / \\
\text { Ohm }\end{array}$ & $\begin{array}{l}t^{\prime \prime} / \\
\min \end{array}$ & $\begin{array}{c}\Delta|Z|_{\min }\left(t^{\prime \prime}\right) / \\
\mathrm{Ohm}\end{array}$ & $\begin{array}{l}t_{1 / 2} l \\
\min \end{array}$ & $\begin{array}{l}t_{1} / \\
\min \end{array}$ & $\begin{array}{c}\Delta|Z|_{\min }\left(t_{1}\right) / \\
\text { Ohm }\end{array}$ \\
\hline $\mathrm{QCM}_{1}$ & $\left.1890( \pm 20)^{a}\right)$ & 30 & $\left.17.50( \pm 0.07)^{c}\right)$ & 190 & $750( \pm 10)^{c)}$ & 500 & $940( \pm 20)^{c)}$ & 40 & 1200 & $890( \pm 10)^{a)}$ \\
\hline $\mathrm{QCM}_{2}$ & $1810( \pm 30)^{a)}$ & 30 & $15.0( \pm 0.1)^{d)}$ & 190 & $700( \pm 10)^{d)}$ & 480 & $850( \pm 20)^{d)}$ & 40 & 1200 & $\left.800( \pm 20)^{a}\right)$ \\
\hline $\mathrm{QCM}_{1+2}$ & $\left.1850( \pm 20)^{b}\right)$ & 30 & $16.61( \pm 0.06)^{e)}$ & 190 & $730( \pm 10)^{e)}$ & 490 & $\left.900( \pm 10)^{e}\right)$ & 40 & 1200 & $850( \pm 10)^{b)}$ \\
\hline
\end{tabular}

Fig. 5-13 B shows averaged normalized spectra and the most sensitive frequencies of impedance magnitude, resistance, and capacitance, calculated from electrochemical impedance measurements of the OCM-ECIS sensor exposed to cell-culture medium and covered with a confluent layer of MDCK-II cells (mean $\pm \mathrm{SEM} ; \mathrm{N}=18$ ). Averaging the most sensitive frequencies of $|Z|, R$, and $C$ from 18 individual recordings provides $f_{\text {sens }}(|Z|)=3.4( \pm 0.3) \mathrm{kHz}, f_{\text {sens }}(R)=0.29( \pm 0.02) \mathrm{kHz}$ and $f_{\text {sens }}(C)=41( \pm 8) \mathrm{kHz}$. Mean values and standard error bars ( \pm 1 SEM) of the most sensitive frequencies are indicated above the peaks in Fig. 5-13 B. Arrows and dotted lines in Fig. 5-13 B mark the sampling frequencies that are closest 
to the respective calculated mean sensitive frequency. For time-dependent analysis of electrochemical impedance parameters, frequencies of $316 \mathrm{~Hz}(R), 3.2 \mathrm{kHz}(|Z|)$ and $40 \mathrm{kHz}(C)$ were chosen. The time courses of $R_{\text {norm }}$ at $316 \mathrm{~Hz}(--)$ and $C_{\text {norm }}$ at $40 \mathrm{kHz}(--)$ during the processes of MDCK-II attachment and spreading are shown in the merged plot in Fig. $5^{-13} \mathrm{C}$, normalized to the time of adding cells.

The capacitance, starting at $20.4( \pm 0.8) \mathrm{nF}$ at cell-free conditions, instantaneously decreases with cell inoculation and exhibits a maximal capacitance reduction rate of $\sim-12 \mathrm{nF} \cdot \mathrm{h}^{-1}$ at $t_{m}(C) \approx 40 \mathrm{~min}$ (Tab. 55). Roughly ten minutes later, $C$ reaches half the overall signal change obtained at the end of the experiment $\left(t_{1 / 2} \approx 50 \mathrm{~min}\right)$. After $\sim 2 \mathrm{~h}$ of almost linear decrease capacitance values approximate within four hours to a minimum of $\sim 14 \%$ of initial values at $t^{\prime}(C) \approx 370 \mathrm{~min}$. Values slightly recover afterwards, resulting in an averaged final value of $C\left(t_{1}\right)=3.7( \pm 0.2) \mathrm{nF}\left(C_{\text {norm }}\left(t_{1}\right)=0.180( \pm 0.007)\right)$ at the end of the experiment $\left(t_{1}=20 \mathrm{~h}\right)$. Resistance values at $316 \mathrm{~Hz}$ exhibit a contrary time course. However, within the first period of $\sim 2 \mathrm{~h}$ after seeding of cells resistance rises only marginally. After this time lag, $R_{\text {norm }}$ strongly increases within $4-5 \mathrm{~h}$ hours, showing a rise to half of its final values at $t_{1 / 2}(R) \approx 250 \mathrm{~min}$ and a maximal slope of $\Delta R / \Delta t=11.9( \pm 0.4) \mathrm{kOhm} \cdot \mathrm{h}^{-1}$ at $t_{m}(R) \approx 270 \mathrm{~min}$. Resistance reaches a transient maximum at $t^{\prime}(R) \approx 480 \mathrm{~min}$, with values being $\sim 2900( \pm 100) \%$ of the cell-free sensor resistance (Tab. 5-5). Subsequently, $R_{\text {norm }}$ slightly declines and converges to a final relative resistance of $R_{\text {norm }}\left(t_{1}\right)=25( \pm 1)$ and an absolute value of $R\left(t_{1}\right)=35( \pm 1) \mathrm{kOhm}$ at $t_{1}=20 \mathrm{~h}$, respectively.

Tab. 5-5 Characteristic electrochemical impedance parameters (ECIS-mode) along the time course of MDCK-II cell attachment and spreading on the QCM-ECIS sensor surface. Calculated averaged sensitive frequencies $\left(f_{\text {sens }}^{\text {calc }}\right)$ and appropriate sampling frequencies $\left(f_{\text {sens }}^{\text {used }}\right)$ used for time-dependent analysis of resistance $(R)$ and capacitance $(C)$ are listed. Absolute and normalized mean values of $R$ and $C$ are obtained from respective averaged curves at times of cell inoculation $\left(t_{0}\right)$, of maximal signal change $\left(t^{\prime}(Y), Y=R, C\right)$, and at the end of experiments $\left(t_{1}=20 \mathrm{~h}\right)$. Maximal slopes $m=\Delta Y / \Delta t$ were calculated by linear regression around $t_{m}( \pm 4$ data points) considering standard errors (instrumental weighting). $t_{1 / 2}(Y)$ marks the points in time when half of the final parameter values are attained. (mean $\pm \mathrm{SEM} ; \mathrm{N}=18$ ).

\begin{tabular}{|c|c|c|c|c|c|c|c|c|c|c|}
\hline & $\begin{array}{c}f_{\text {sens }}^{\text {calc }} / \\
\mathrm{Hz}\end{array}$ & $\begin{array}{c}f_{\text {sens }}^{\text {used }} / \\
\mathrm{Hz}\end{array}$ & $\begin{array}{c}Y\left(t_{0}\right) / \\
{[Y]}\end{array}$ & $\begin{array}{l}t_{m} / \\
\min \end{array}$ & $\begin{array}{c}m=\Delta Y / \Delta t \\
{[Y] \cdot \mathrm{h}^{-1}}\end{array}$ & $\begin{array}{c}t^{\prime} / \\
\min \end{array}$ & $\begin{array}{c}Y_{\text {norm }}\left(t^{\prime}\right) / \\
\%\end{array}$ & $\begin{array}{l}t_{1 / 2} / \\
\min \end{array}$ & $\begin{array}{c}t_{1} / \\
\min \end{array}$ & $\begin{array}{c}Y_{\text {norm }}\left(t_{1}\right) / \\
\%\end{array}$ \\
\hline$Y=R$ & $290( \pm 20)$ & 316 & $\begin{array}{c}1430( \pm 50) \\
\text { Ohm }\end{array}$ & 270 & $\begin{array}{c}11.9( \pm 0.4) \\
\mathrm{kOhm} \cdot \mathrm{h}^{-1}\end{array}$ & 480 & $2900( \pm 100)$ & 250 & 1200 & 2500 \\
\hline$Y=C$ & $41( \pm 8) \cdot 10^{3}$ & $40 \cdot 10^{3}$ & $\begin{array}{c}20.4( \pm 0.8) \\
n F\end{array}$ & 40 & $\begin{array}{c}-12.2( \pm 0.1) \\
\mathrm{nF} \cdot \mathrm{h}^{-1}\end{array}$ & 370 & $13.6( \pm 0.5)$ & 50 & 1200 & $18.0( \pm 0.7)$ \\
\hline
\end{tabular}

Fig. 5-13 $D$ visualizes correlations between the characteristic shear oscillation (QCM-mode) and electrochemical (ECIS-mode) parameters along the course of MDCK-II attachment and spreading, by a 3-D QCM-ECIS impedance plot with the axes $\Delta|Z|_{\min }(\mathrm{x}), R_{\mathrm{norm}}(\mathrm{y})$, and $C_{\text {norm }}$ (z). Fig. 5-13 E, Fillustrate in 2-D plots the correlation of the QCM parameter $\Delta|Z|_{\min }$ with the ECIS parameters $C_{\text {norm }}$ and $R_{\text {norm }}$ respectively, thus allowing for a more detailed view on the $x z$ and $x y$ projection planes of the 3-D curve in Fig. 5-13 D. A 2-D diagram of $C_{\text {norm }}$ vs. $R_{\text {norm }}$ and 3-D plots of two of the three impedance parameters as a function of time ( $C_{\text {norm }}$ vs. $R_{\text {norm }}$ vs. $t, C_{\text {norm }}$ vs. $\Delta|Z|_{\min }$ vs. $t$, and $R_{\text {norm }}$ vs. $\Delta|Z|_{\min }$ vs. $t$ ) are illustrated in $S / 13$ (p. 268). The diagrams in Fig. 5-13 D-F show 430 equidistant data points between $t_{0}=0 \mathrm{~min}$ and $t_{1}=1200 \mathrm{~min}$. Start and end values as well as selected, characteristic times $t=45,120$, and $480 \mathrm{~min}$ are highlighted in the diagrams. In the 3-D plot, arrows indicate the projection lines of these data 
points to the corresponding points in the planes. The 3-D trace (Fig. 5-13 D) as well as the 2-D projections (Fig. 5-13 E, F) reveal a four-phasic time course. From $t_{0}$ to $t \approx 120 \mathrm{~min}, C_{\text {norm }}$ vs. $\Delta|Z|_{\text {min }}$ changes (Fig. 5-13 E) can be roughly divided into two linear regions with different slopes. After an initial short drop of $C_{\text {norm }}$ by $\sim 5 \%, \Delta|Z|_{\min }$ and $C_{\text {norm }}$ change with very similar kinetics for $\sim 45 \mathrm{~min}$ (1 $1^{\text {st }}$ phase). Afterwards, $\Delta|Z|_{\min }$ approximates a tempory saturation, whereas $C_{\text {norm }}$ unabatedly decreases further ( $2^{\text {nd }}$ phase). Within this first two hours after adding the cell suspension both parameters perform about $85 \%$ of their overall signal change, whereas changes of $R_{\text {norm }}$ are marginal in this period (Fig. 5-13 F). After this time lag, $R_{\text {norm }}$ starts to strongly increase for $\sim 6 \mathrm{~h}$ ( $3^{\text {rd }}$ phase). $C_{\text {norm }}$ slightly decreases further within this phase, converging to its minimum after four hours (Fig. 5-13 E), and $\Delta|Z|_{\text {min }}$ rises after the tempory plateau to the transient maximum, which coincides with the maximum of $R_{\text {norm }} 480$ min after cell inoculation (Fig. 5-13 F). After passing the maxima, all parameters slightly recover in the following $12 \mathrm{~h}$ ( $4^{\text {th }}$ phase). Thereby, regresses of $\Delta|Z|_{\min }$ and $R_{\text {norm }}$ exhibit the same dependency as in the rising phase. This becomes visible in Fig. 5-13 F, where the curve runs almost exactly the same way back after having reached the maximum ( $480 \mathrm{~min} \rightarrow t_{1}$ ). By contrast, changes of $C_{\text {norm }}$ vs. $\Delta|Z|_{\text {min }}$ and $C_{\text {norm }}$ vs. $R_{\text {norm }}$ in the recovery phase differ from the traces in the rising phase before reaching maxima of $\Delta|Z|_{\min }$ and $R_{\text {norm }}$ respectively. The recovery of $R_{\text {norm }}$ is little more pronounced with respect to the reduction of electrochemical capacitance $(S / 13 A)$, which in turn recovers slightly more compared to the minimal oscillation impedance (Fig. 5-13 E).

\subsubsection{NRK Cell Attachment and Spreading}

Besides studies of MDCK-II cell attachment and spreading, NRK cells were studied as a second epithelial model cell line by double-mode impedance analysis using the presented novel OCM-ECIS sensors. In the forerun of cell attachment studies, sensors were equilibrated under SFM loading for $t \geq 20 \mathrm{~h}$ (cf. 5.2.1 and Fig. 5-12). For investigations, NRK cells were seeded to confluence by replacing SFM on the sensor surface with $1.5 \mathrm{~mL}$ cell suspension of $\sim 350000$ cells $/ \mathrm{mL}$. Oscillation and electrochemical impedance spectra of the sensor loaded with medium and covered with a confluent layer of NRK cells are presented in $S / 14$ and $S / 15$ (p. 268 f.). Fig. 5-14 depicts a summary of time courses of the characteristic impedance parameters $\Delta|Z|_{\min }\left(\mathrm{OCM}\right.$-mode), $R_{\text {norm }}$ and $C_{\text {norm }}$ (both ECIS-mode) during the processes of NRK cell attachment and spreading on the sensor surface.

Fig. 5-14 A shows averaged traces of shear oscillation impedance minima (mean $\pm S E M$ ) of individual resonator units, $\mathrm{OCM}_{1}\left(\mathrm{w} / \mathrm{PhoP} ;{ }^{-0-} ; \mathrm{N}=16\right.$ ) and $\mathrm{OCM}_{2}$ (w/o PhoP; $\left.{ }^{-0} ; \mathrm{N}=16\right)$, and of pooled $\mathrm{QCM}_{1}$ and $\mathrm{OCM} 2$ impedance data sets $(-; \mathrm{N}=32)$. Curves are referenced to the point of cell inoculation in time and $|Z|_{\text {min }}$. Characteristic impedance values and times of the curves are listed in Tab. 5-6. After $t \geq 20 \mathrm{~h}$ of SFM incubation, starting values of the minimal impedance $|Z|_{\min }\left(t_{0}\right)$ amount to $1900( \pm 30) \mathrm{Ohm}$ $(\mathrm{OCM}), 1800( \pm 30) \mathrm{Ohm}(\mathrm{OCM} 2)$, and $1850( \pm 20) \mathrm{Ohm}(\mathrm{OCM} 1+2)$. By exchanging SFM with the NRK cell suspension, $\Delta|Z|_{\min }$ starts to increase immediately and reaches $90 \%$ of the entire signal change within the first six hours. After this initial steep increase, $\Delta|Z|_{\min }$ approximates a stationary value of $800( \pm 20)$ Ohm on average $\left(\mathrm{OCM}_{1}+2 ; \mathrm{N}=32\right)$ after $20 \mathrm{~h}$. The kinetics of the signal is characterized by a mean maximal slope of $m=6.97( \pm 0.07) \mathrm{Ohm} \cdot \mathrm{min}^{-1}\left(\mathrm{OCM}_{1}+2\right)$ at time $t_{m} \approx 40 \mathrm{~min}$, and by a halfmaximal signal increase attained at $t_{1 / 2} \approx 80 \mathrm{~min}$ after seeding cells (Tab. 5-6). The traces of photoresistcoated resonator $\mathrm{OCM}_{1}$ and uncoated resonator $\mathrm{OCM} 2$ thereby slightly differ in their $\Delta|Z|_{\text {min }}$ signal responses. Differences are similar to that observed in MDCK-II attachment studies (Fig. 5-13), with the $\Delta|Z|_{\min }$ increase being slightly faster and by $\sim 80$ Ohm bigger for OCM1 compared to OCM2 (Tab. 5-6). 
A
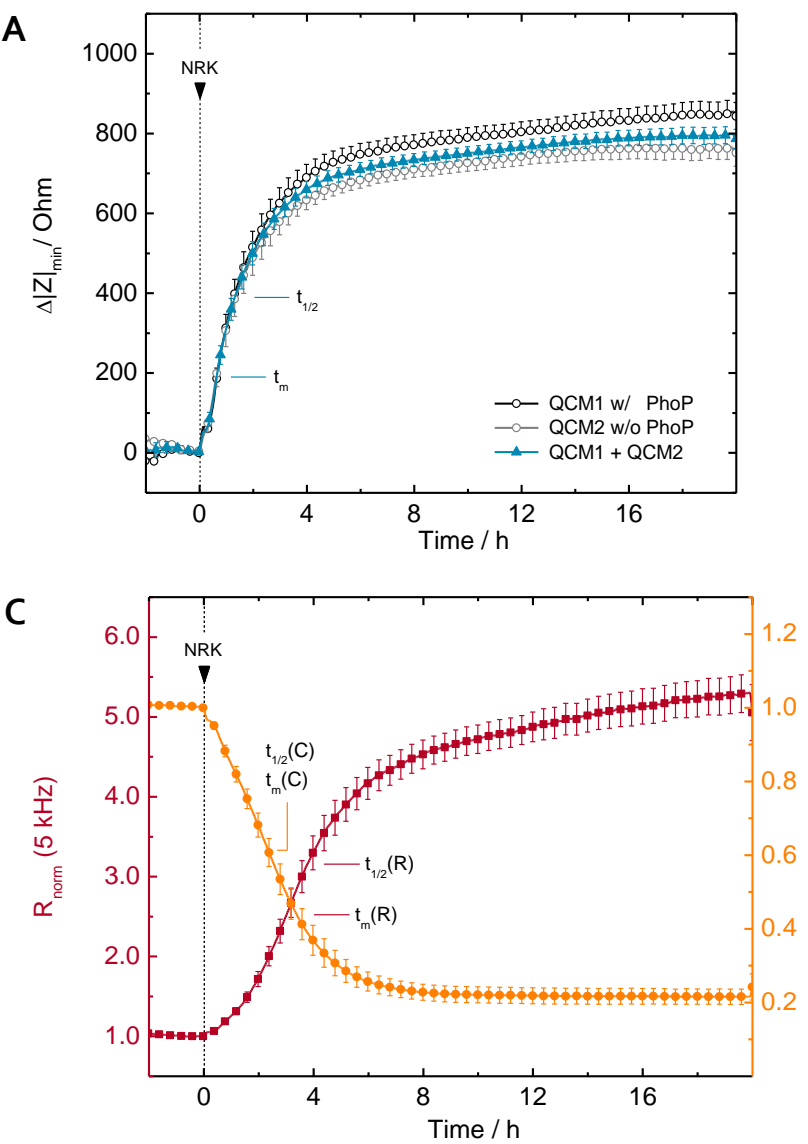

E

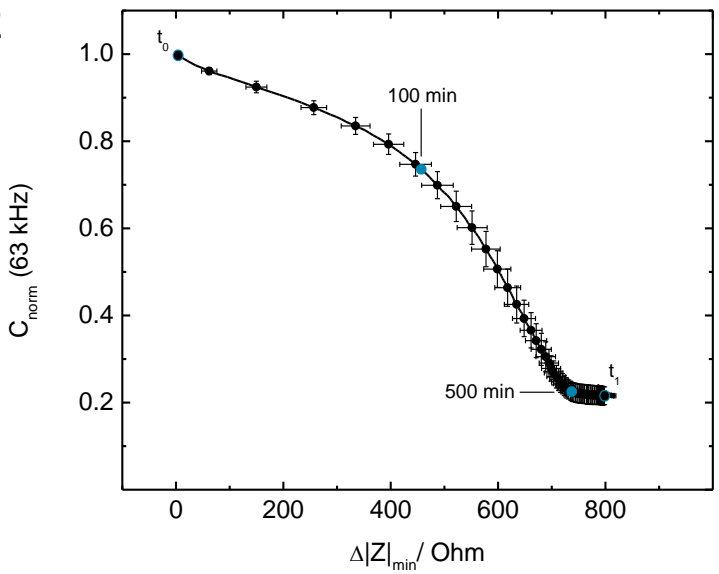

B

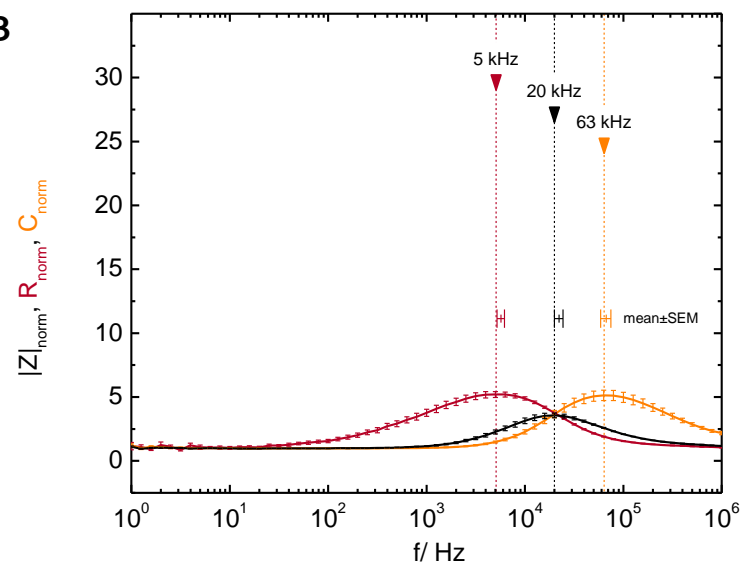

D

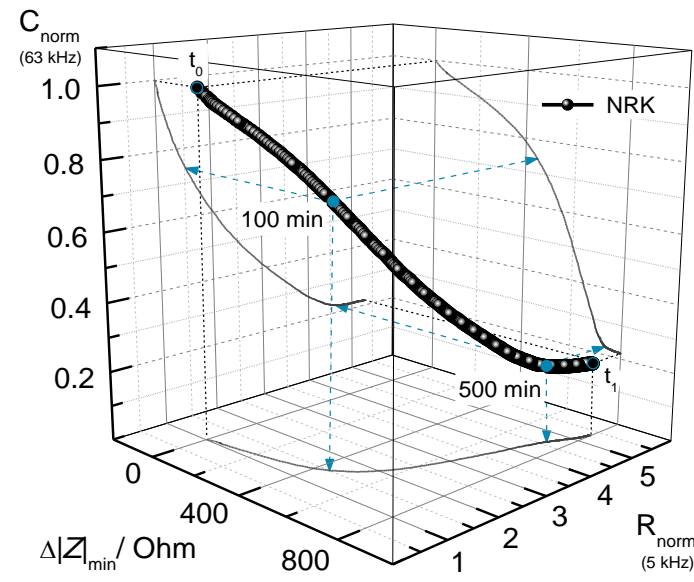

$\mathbf{F}$

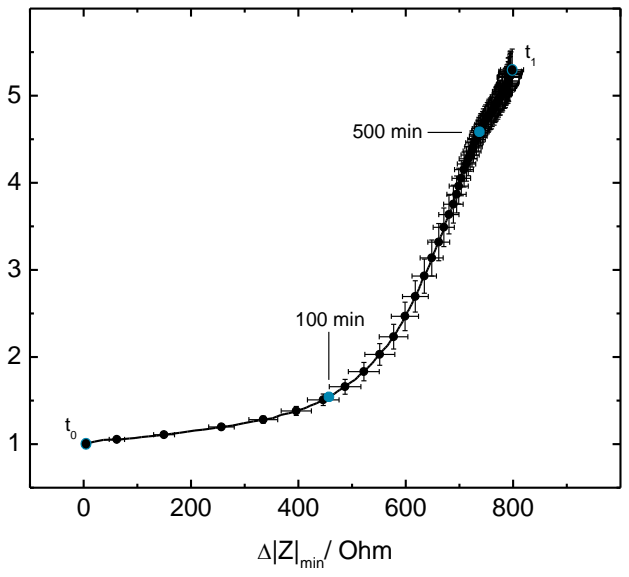

Fig. 5-14 OCM-ECIS sensor responses during NRK cell attachment and spreading. (A) Averaged time courses (mean \pm SEM) of changes in the minimal impedance $\Delta|Z|_{\min }$ of the shear displacement of resonators are displayed separately for $\mathrm{OCM}_{1}(\mathrm{w} / \mathrm{PhoP} ;-\mathrm{O}-; \mathrm{N}=16)$ and $\mathrm{OCM}_{2}$ (w/o PhoP; $-\mathrm{O}_{-} ; \mathrm{N}=16$ ), as well as for pooled data sets of $\mathrm{QCM} 1$ and $\mathrm{OCM}_{2}$ impedance changes $(-; N=32)$. Absolute values of $|Z|_{\min }$ at the time of adding cells $\left(t_{0}\right)$ amount to $1900( \pm 30) \mathrm{Ohm}$ $(\mathrm{OCM}), 1800( \pm 30) \mathrm{Ohm}(\mathrm{QCM} 2 ;$ both $\mathrm{N}=20)$, and $1850( \pm 20) \mathrm{Ohm}\left(\mathrm{OCM}_{1}+2 ; \mathrm{N}=40\right)$. (B) Averaged normalized spectra of impedance magnitude $\left(-;|Z|_{\text {norm }}=|Z|_{\mathrm{w} / \text { cells }} /|Z|_{\text {w/o cells }}\right)$, resistance $\left(-; R_{\mathrm{norm}}=R_{\mathrm{w} / \text { cells }} / R_{\mathrm{w} / \mathrm{o} \text { cells }}\right)$, and capacitance $\left(-; C_{\mathrm{norm}}=C_{\mathrm{w} / \mathrm{o} \text { cells }} / C_{\mathrm{w} / \text { cells }}\right)$, calculated from ECIS-mode spectra of cell-free (medium-loaded) and NRK cell-covered sensors (mean $\pm \mathrm{SEM} ; \mathrm{N} \geq 14$ ). Spectra indicate the most sensitive sampling frequencies $f_{\text {sens }}$ of $5 \mathrm{kHz}$ for $R, 20 \mathrm{kHz}$ for $|Z|$, and $63 \mathrm{kHz}$ for $C$. (C) Time courses of $R_{\text {norm }}\left(5 \mathrm{kHz}^{-{ }^{-}}\right)$) and $C_{\text {norm }}\left(63 \mathrm{kHz}^{-{ }^{-}-}\right)$ during NRK attachment and spreading. Curves are averaged from $\mathrm{N}=14$ individual traces (mean $\pm \mathrm{SEM}$ ). Absolute starting values of cell-free QCM-ECIS sensors immersed with SFM are $R\left(t_{0}\right)=680( \pm 20) \mathrm{Ohm}$ and $C\left(t_{0}\right)=11( \pm 1) \mathrm{nF}$. Averaging of curves was performed according to the caption of Fig. 5-13. (D) 3-D and (E), (F) 2-D impedance plots, merging the time courses of 
characteristic impedance parameters from recordings in QCM-mode $\left(\Delta|Z|_{\text {min }}\right)$ and ECIS-mode $\left(C_{\text {norm }}, R_{\text {norm }}\right)$ during NRK cell attachment and spreading. Traces exhibit 600 equidistant data points between $t_{0}=0 \mathrm{~min}$ and $t_{1}=1200 \mathrm{~min}$. Characteristic times are indicated. $\Delta|Z|_{\min }$ values were used from pooled $\mathrm{OCM} 1$ and $\mathrm{OCM} 2$ data sets. Data points displayed in $\mathrm{A}, \mathrm{C}, \mathrm{E}$, and $\mathrm{F}$ were reduced for sake of clarity.

Values of $|Z|_{\min }$ at the end of SFM incubation are equal to the previously shown MDCK-II experiments (cf. Tab. 5-4), confirming consistent starting conditions of the sensor for cell attachment studies. This enables the comparison of parameter time courses obtained from the different cell lines. Moreover, merging the population of data of MDCK-II and NRK studies enables a more accurate estimation of $|Z|_{\text {min }}$ starting values. Doing so, following reference values are obtained for $\mathrm{OCM}-\mathrm{ECIS}$ sensors covered with serum-free cell culture medium: $1890( \pm 20) \mathrm{Ohm}$ for $\mathrm{OCM}_{1}(\mathrm{~N}=60), 1810( \pm 20) \mathrm{Ohm}$ for QCM2 $(N=60)$, and $1850( \pm 10)$ Ohm for pooled $Q_{1} C_{1}$ and $Q C M 2$ responses $\left(Q_{C M}+2 ; N=120\right)$.

Tab. 5-6 Characteristic shear oscillation parameters (QCM-mode) of resonators $\mathrm{OCM} 1$ and $\mathrm{QCM} 2$ along the course of NRK cell attachment and spreading on OCM-ECIS sensors. Values of separate $\left.\left(\mathrm{OCM}_{1}, \mathrm{QCM}\right)_{2}\right)$ and pooled $\left(\mathrm{OCM}_{1}+2\right)$ impedance data sets of resonators are shown. Mean starting values are obtained by averaging absolute $|Z|_{\text {min }}$ values of data sets at time zero $\left(t_{0}\right)$. Final $\Delta|Z|_{\min }$ values are obtained from averaged time curves at the end of experiments $\left(t_{1}=20 \mathrm{~h}\right)$. Maximal slopes $m=\Delta|Z|_{\min } / \Delta t$ were calculated by linear regression around $t_{m}( \pm 4$ data points) considering standard errors (instrumental weighting). $t_{1 / 2}$ marks the time of halfmaximal $\Delta|Z|_{\min }$ increase. (mean $\left.\pm \mathrm{SEM} ; \mathrm{N}={ }^{\mathrm{a})} 20,{ }^{\mathrm{b})} 40,{ }^{\mathrm{c})} 16,{ }^{\mathrm{d}}\right) 32$ ).

\begin{tabular}{lcccccc}
\hline & $\begin{array}{c}|Z|_{\min }\left(t_{0}\right) / \\
\text { Ohm }\end{array}$ & $\begin{array}{c}t_{m} / \\
\min \end{array}$ & $\begin{array}{c}m=\Delta|Z|_{\min } / \Delta t / \\
\text { Ohm } \cdot \mathrm{min}^{-1}\end{array}$ & $\begin{array}{c}t_{1 / 2} / \\
\min \end{array}$ & $\begin{array}{c}t_{1} / \\
\min \end{array}$ & $\begin{array}{c}\Delta|Z|_{\min }\left(t_{1}\right) / \\
\text { Ohm }\end{array}$ \\
\hline OCM1 & $1900( \pm 30)^{\mathrm{a})}$ & 40 & $7.44( \pm 0.09)^{\mathrm{c})}$ & 90 & 1200 & $850( \pm 30)^{\mathrm{c})}$ \\
OCM2 & $1800( \pm 30)^{\mathrm{a})}$ & 40 & $6.68( \pm 0.07)^{\mathrm{c})}$ & 80 & 1200 & $770( \pm 30)^{\mathrm{c})}$ \\
OCM1+2 & $1850( \pm 20)^{\mathrm{b})}$ & 40 & $6.97( \pm 0.07)^{\mathrm{d})}$ & 80 & 1200 & $800( \pm 20)^{\mathrm{d})}$ \\
\hline
\end{tabular}

Fig. 5-14 B illustrates averaged normalized spectra of impedance magnitude (black curve), resistance (red curve) and capacitance (orange curve), obtained from ECIS-mode recordings of the OCM-ECIS sensors exposed to SFM only and covered with a confluent NRK cell layer (mean $\pm S E M ; N \geq 14$ ). Exemplary spectra of absolute values of $|Z|, R$, and $C$ for both cell-free and NRK cell-covered QCM-ECIS sensors are shown in $S / 15$ (p. 269). The peak maxima of averaged curves in Fig. 5-14 B indicate the most sensitive recording frequencies of the parameters for NRK cells. Mean values and standard errors of sensitive frequencies are shown above the curves' maxima and are calculated to $f_{\text {sens }}(|Z|)=22( \pm 2) \mathrm{kHz}$, $f_{\text {sens }}(R)=5.7( \pm 0.5) \mathrm{kHz}$, and $f_{\text {sens }}(C)=67( \pm 8) \mathrm{kHz}$. Dotted lines mark the recording frequencies, which are closest to the calculated values and which thus were chosen for the time-dependent analysis of the corresponding parameter changes. These frequencies for NRK cells are: $5 \mathrm{kHz}(R), 20 \mathrm{kHz}(|Z|)$ and $63 \mathrm{kHz}(C)$. Fig. 5-14 C displays the averaged time courses of $R_{\text {norm }}(-)$ ) and $\left.C_{\text {norm }}(-)^{-}\right)$at the respective frequencies in a combined plot during the processes of NRK cell attachment and spreading (mean $\pm \mathrm{SEM}$; $\mathrm{N}=14)$. The values are normalized to the point of cell inoculation $\left(t_{0}\right)$. Parameters and characteristic values of $R_{\text {norm }}$ and $C_{\text {norm }}$ time courses are listed in Tab. 5-7. The cell free QCM-ECIS sensor, incubated for at least $20 \mathrm{~h}$ with SFM, exhibits a capacitance of $11( \pm 1) \mathrm{nF}$ at $63 \mathrm{kHz}$. It linearly decreases immediately with cell seeding, attaining a maximal slope $m=\Delta C / \Delta t$ of $-{ }^{\circ} 2.1 \mathrm{nF} \cdot \mathrm{h}^{-1}$ after $t_{m}(C) \approx 140 \mathrm{~min}$, which also marks the time of $50 \%$ of the entire signal change $\left(t_{1 / 2}(C)\right)$. The capacitance of the NRK cell-covered 
WE is reduced to $22( \pm 2) \%$ on average with respect to the starting capacitance at the end of cell attachment and spreading $\left(t_{1}=1200 \mathrm{~min}, C\left(t_{1}\right)=2.3( \pm 0.4) \mathrm{nF}\right)$. The time course of $R_{\text {norm }}$ exhibits a sigmoidal shape, starting with a slow increase of the slope within the first $1-2 h$, followed by a linear rise for $\sim 2 \mathrm{~h}$ and convergence to stationary values afterwards. The resistance change from initial cell-free values of $R\left(t_{0}\right)=680( \pm 20)$ Ohm to final values of $R\left(t_{1}\right)=3600( \pm 200)$ after NRK cell layer formation reflects an overall $R_{\text {norm }}$ increase by $530( \pm 20) \%$. $R_{\text {norm }}$ exhibits a maximal slope of $\sim 600 \mathrm{Ohm} \cdot \mathrm{h}^{-1}$ $\left(t_{m} \approx 180 \mathrm{~min}\right)$ in the linear range (Tab. 5-7). The $50 \%$ value of the entire increase is attained at $t_{1 / 2} \approx 230 \mathrm{~min}$.

Tab. 5-7 Characteristic electrochemical impedance parameters (ECIS-mode) along the course of NRK cell attachment and spreading on OCM-ECIS sensors. Calculated averaged sampling frequencies $\left(f_{\text {sens }}^{\text {calc }}\right)$ and appropriate sampling frequencies $\left(f_{\text {sens }}^{\text {used }}\right)$, which were used for timedependent analysis of resistance $(R)$ and capacitance $(C)$, are listed. Absolute and normalized mean values of $R$ and $C$ are obtained from averaged curves at points of cell inoculation $\left(t_{0}\right)$ and at the end of experiments $\left(t_{1}=20 \mathrm{~h}\right)$. Maximal slopes $m=\Delta Y / \Delta t(Y=R, C)$ were calculated by linear regression around $t_{m}$ ( \pm 4 data points) considering standard errors (instrumental weighting). $t_{1 / 2}(Y)$ marks the time when half of the final signals are attained. (mean $\pm S E M ; N \geq 14$ ).

\begin{tabular}{ccccccccc}
\hline & $\begin{array}{c}f_{\text {sens }}^{\text {calc }} \mathrm{k} \\
\mathrm{kHz}\end{array}$ & $\begin{array}{c}f_{\text {sens }}^{\text {usen }} \mathrm{kHz} \\
\mathrm{kHz}\end{array}$ & $\begin{array}{c}Y\left(t_{0}\right) / \\
{[Y]}\end{array}$ & $\begin{array}{c}t_{m} / \\
\mathrm{min}\end{array}$ & $\begin{array}{c}m=\Delta Y / \Delta t \\
{[Y] \cdot \mathrm{h}^{-1}}\end{array}$ & $\begin{array}{c}t_{1 / 2} / \\
\mathrm{min}\end{array}$ & $\begin{array}{c}t_{1} / \\
\min \end{array}$ & $\begin{array}{c}Y_{\text {norm }}\left(t_{1}\right) / \\
\%\end{array}$ \\
\hline$Y=R$ & $5.7( \pm 0.5)$ & 5 & $\begin{array}{c}680( \pm 20) \\
\mathrm{Ohm}\end{array}$ & 180 & $\begin{array}{c}594( \pm 6) \\
\mathrm{Ohm} \cdot \mathrm{h}^{-1}\end{array}$ & 230 & 1200 & $530( \pm 20)$ \\
$Y=C$ & $67( \pm 8)$ & 63 & $\begin{array}{c}11( \pm 1) \\
\mathrm{nF}\end{array}$ & 140 & $\begin{array}{c}-2.09( \pm 0.02) \\
\mathrm{nF} \cdot \mathrm{h}^{-1}\end{array}$ & 140 & 1200 & $22( \pm 2)$ \\
\hline
\end{tabular}

Fig. 5-14 D shows a 3-D QCM-ECIS impedance plot of the NRK attachment and spreading process, which combines in one diagram alterations of the three characteristic sensing parameters $\Delta|Z|_{\text {min }}$ (OCMmode), $R_{\text {norm }}$ and $C_{\text {norm }}$ (both ECIS-mode). Fig. 5-14 E, F illustrate in 2-D plots the correlation of $\Delta|Z|_{\min }$ with $C_{\text {norm }}$ and $R_{\text {norm }}$, respectively, and thus allow for a more detailed view on the xz and xy projection planes of the 3-D curve in Fig. 5-14 D. A 2-D diagram of $C_{\text {norm }}$ vs. $R_{\text {norm }}$ is illustrated in SI 16 A (p. 270). Those 2-D diagrams extended to 3-D diagrams by the integration of the time as third axis $\left(C_{\text {norm }}\right.$ vs. $R_{\text {norm }}$ vs. $t, C_{\text {norm }}$ vs. $\Delta|Z|_{\min }$ vs. $t$, and $R_{\text {norm }}$ vs. $\Delta|Z|_{\min }$ vs. $\left.t\right)$ are shown in $S / 16 B, C, D$. They comprise in two projection planes the time courses of parameters, as shown in Fig. 5-14 A, $C$, and the correlation of the respective two parameters in the third plane of projection. Fig. 5-14 D summarizes the interrelations of $\Delta|Z|_{\text {min }}, R_{\text {norm }}$ and $C_{\text {norm }}$ in one diagram. Each point in the spatial curve is a function of these parameters and represents a specific point in time between seeding cells $\left(t_{0}\right)$ and the end of investiagtions $\left(t_{1}=20 \mathrm{~h}\right)$. Projections of this curve reveal in all three planes a more or less simultaneous change of parameters. This disallows for a clear breakdown of curves into parts and thus a separation of NRK attachment and spreading into individual phases and structural processes (which influence either cell mechanics or electric cell properties). Different phases can be estimated the most from the projection to the xz-plane ( $C_{\text {norm }}$ vs. $\Delta|Z|_{\text {min }}$ ) in Fig. 5-14 D and in Fig. 5-14 E, respectively. In a first phase after seeding NRK cells $\left(t_{0} \leq t \leq 100 \mathrm{~min}\right)$, there is a simultaneous change in $\Delta|Z|_{\text {min }}$ and $C_{\text {norm. }}$. While the capacitane further decreases linearly around $t \approx 120 \mathrm{~min}$ (cf. Fig. 5-14 C), the kinetics of the $\Delta|Z|_{\text {min }}$ increase slow down (cf. Fig. 5-14 A). This results in a second phase (100 $\mathrm{min} \leq t \leq 500 \mathrm{~min}$ ) with a steeper negative slope of the $C_{\text {norm }}$ vs. $\Delta|Z|_{\min }$ curve. The reduced increase of $\Delta|Z|_{\min }$ and the maximal increasing rate of $R_{\text {norm }}$ at about $2-3 \mathrm{~h}$ after cell inoculation also cause a (this time positive) 
slope increase in Fig. 5-14 F. Roughly eight hours after seeding NRK cells (500 $\min \leq t \leq t_{1}$ ), $C_{\text {norm }}$ reaches stationary values, while there is a continuing slight increase in $\Delta|Z|_{\min }$ (Fig. 5-14 E) and in $R_{\text {norm }}$ (Fig. 5${ }_{14} \mathrm{~F}, \mathrm{~S} / 16 \mathrm{~A}$ ). This period marks the third and last phase in the recorded mechanical and electrical properties of the QCM-ECIS sensor during NRK cell settlement and monolayer formation.

\subsubsection{Cell Attachment and Spreading in Absence of $\mathrm{Ca}^{2+}$ and $\mathrm{Mg}^{2+}$}

Critical processes of epithelial cell attachment and monolayer formation on a growth substrate are the anchorage of cells to the substratum (cell-matrix adhesion) and the mechanical connection of individual adjacent cells to a functional and structural cellular unit (cell-cell adhesion). There are two main transmembrane glycoprotein structures mediating cell adhesion: integrins and cadherins. Integrins (heterodimers with an $\alpha$ and $\beta$ subunit; for reviews see ${ }^{[379,380]}$ ) are located in the basal membrane and predominantly connect the intracellular cytoskeleton with the underlying growth substrate and the extracellular matrix (ECM) by focal adhesions (FAs) and hemidesmosomes (HDs). ${ }^{\left[{ }^{381-384]}\right.}$ FAs are linked intracellularly to the actin cytoskeleton and the integrins act extracellularly as cell surface receptors, binding in a $\mathrm{Ca}^{2+}$ and $\mathrm{Mg}^{2+}$ dependent manner ${ }^{[385]}$ to glycoprotein ligands like fibronectin (FN), laminin and collagen in the ECM. ${ }^{[386]}$ The major role for epithelial intercellular anchorage play the cadherins, the calcium-dependent cell adhesion molecules, which are structural elements of apically located zonula adherens (adherens junctions, AJs) and more basally located macula adherens (desmosomes). They join together the actin microfilaments $(\rightarrow \mathrm{AJ}$ ) and intermediate filaments ( $\rightarrow$ desmosomes) of neighboring cells. ${ }^{\left[{ }^{386-388]}\right.}$ Besides the anchoring junctions, epithelial plasma membranes are laterally connected via communicating junctions (gap junctions, GJs) and sealing junctions (tight junctions, TJs). ${ }^{[389,390]} \mathrm{TJ}$, also referred to as zonula occludens (ZO), are located above desmosomes and AJs and form a tight diffusion barrier for macromolecules between neighboring cells. They are directly linked to the cytoskeletal actin via ZO proteins. The structural and functional integrity of TJs in epithelial cells depends indirectly on extracellular $\mathrm{Ca}^{2+}$, as a result of calcium-sensitivity of structures in the vicinity of ZO. ${ }^{[345,346,391]}$

The influence of the bivalent cations $\mathrm{Ca}^{2+}$ and $\mathrm{Mg}^{2+}$ on the adhesion properties of MDCK-II cells was investigated, applying the dual OCM-ECIS sensor presented in this work (cf. chapter 5.1). The multiparametric recordings provide concurrent time-resolved insight to cytomechanics, cell morphology, and barrier resistance of TJs during cell attachment and spreading processes. Sensor substrates were precoated with $200 \mu \mathrm{L} \mathrm{FN}\left(25 \mu \mathrm{g} / \mathrm{mL}\right.$ in Earles' balanced salt solution without $\mathrm{Ca}^{2+}$ and $\left.\mathrm{Mg}^{2+}\left(\mathrm{EBSS}^{--}\right)\right)$for $24 \mathrm{~h}$ at $37^{\circ} \mathrm{C}$ and $5 \% \mathrm{CO}_{2}$. FN-coated substrates were washed three times with EBSS ${ }^{--}$buffer, before seeding cells to confluence ( 450000 cells $/ \mathrm{cm}^{2}$ ) in $\mathrm{EBSS}^{--}$buffer supplemented with physiological concentrations of either $\mathrm{Ca}^{2+}\left(1.8 \mathrm{mM}^{2} \mathrm{EBSS}^{+-}\right)$, or $\mathrm{Mg}^{2+}\left(0.81 \mathrm{mM}^{2} \mathrm{EBSS}^{-+}\right)$or both $\left(\mathrm{EBSS}^{++}\right)$.

Fig. 5-15 A shows the averaged $\Delta|Z|_{\text {min }}$ time courses from QCM-mode recordings of MDCK-II cells seeded on the sensor in buffer containing both $\mathrm{Ca}^{2+}$ and $\mathrm{Mg}^{2+}(--)$, or $\mathrm{Ca}^{2+}$ only $(--)$, or $\mathrm{Mg}^{2+}$ only $\left(-\square_{-}\right)$ (pooled data sets of $\mathrm{OCM} 1$ and $\mathrm{OCM}_{2}$; mean $\pm \mathrm{SEM}_{;} \mathrm{N}=4$ ). Characteristic parameter values of time courses are summarized in $S / 17$ (p. 271). The $\Delta|Z|_{\text {min }}$ attachment and spreading curves of MDCK-II cells inoculated in $\mathrm{Ca}^{2+}$-containing buffer, either alone or in combination with $\mathrm{Mg}^{2+}$, exhibit a biphasic shape (filled symbols in Fig. 5-15 A), which is similar to the experiments under complete cell culture medium (cf. Fig. 5-13). However, in $\mathrm{Ca}^{2+}$-free buffer ( $\mathrm{Mg}^{2+}$ only; open symbols) the $\Delta|Z|_{\min }$ time course shows a steep single-phase increase to a constant value within $\sim 1.5 \mathrm{~h}$. The transient maxima in both biphasic time courses occur at the same point of time $\left(t^{\prime \prime} \approx 8 \mathrm{~h}\right)$ as observed for MDCK-II in complete medium. The zoom-in to the initial stages of cell-substrate interaction ( $t \leq 2 \mathrm{~h}$ ) in Fig. 5-15 A indicates slightly slower 
MDCK-II attachment kinetics in the absence of $\mathrm{Mg}^{2+}$. This is quantified by the parameter $t_{1 / 2}$, which is the time for attaining half of the final signal changes. This period is delayed for $\mathrm{Mg}^{2+}$-free buffer by $\sim 10 \mathrm{~min}\left(t_{1 / 2}\left(\mathrm{Ca}^{2+}\right) \approx 45 \mathrm{~min}\right)$ compared to cell attachement in buffer containing $\mathrm{Mg}^{2+}\left(t_{1 / 2}\left(\mathrm{Ca}^{2+}+\mathrm{Mg}^{2+}\right) \approx\right.$ $\left.t_{1 / 2}\left(\mathrm{Mg}^{2+}\right) \approx 35 \mathrm{~min}\right)$. Final $\Delta|Z|_{\min }$ values $\left(t_{1}=20 \mathrm{~h}\right)$ are similar for the three investigated buffer compositions, all exhibiting a signal increase by approximately $800 \mathrm{Ohm}$ (cf. table in S/ 17).

Fig. 5-15 B shows electrochemical resistance and capacitance spectra from ECIS-mode recordings of the OCM-ECIS sensor after FN coating (---) and $20 \mathrm{~h}$ after inoculating MDCK-II cells on the QCM-ECIS sensor in $\mathrm{EBSS}^{--}$buffer containing either $\mathrm{Ca}^{2+}$ and $\mathrm{Mg}^{2+}(-)$, or $\mathrm{Ca}^{2+}$ only $\left(-{ }^{-}\right)$, or $\mathrm{Mg}^{2+}$ only $(-\square)$. From these data one can conclude different sensitivities and different values for the most sensitive frequencies for cells in the presence and in the absence of $\mathrm{Ca}^{2+}$ for both $R$ and $C$. If $\mathrm{Ca}^{2+}$ is present, either alone or together with $\mathrm{Mg}^{2+}$, the resistance and capacitance are most sensitive to MDCK-II cell attachment at $f_{\text {sens }}^{\text {calc }}(R)=570( \pm 30) \mathrm{Hz}$ and $f_{\text {sens }}^{\text {calc }}(C)=100( \pm 10) \mathrm{kHz}$, respectively. These frequencies of both $R$ and $C$ are shifted to higher frequencies in buffer lacking $\mathrm{Ca}^{2+}$ ( $-\square-$ in Fig. 5-15 $\mathrm{B} ; f_{\text {sens }}^{\text {calc }}(R)=10.2( \pm 0.5) \mathrm{kHz}$, $\left.f_{\text {sens }}^{\text {calc }}(C)=290( \pm 40) \mathrm{kHz}\right)$. The corresponding normalized spectra of impedance, resistance, and capacitance in presence and absence of $\mathrm{Ca}^{2+}$ are illustrated in $\mathrm{SI} 18$ (p. 272). For time-resolved impedance analysis of cell attachment and spreading, $C$ at $100 \mathrm{kHz}$ and $R$ at $630 \mathrm{~Hz}$ were extracted from frequency scans. The resulting exemplary time courses are shown in Fig. ${ }^{-15} \mathrm{C}$ for the capacitance and in Fig. 5$15 \mathrm{D}$ for the resistance.

An initial drop in the capacitance within $2 \mathrm{~h}$ after cell inoculation reports on attachment and spreading of the cells on the substrate for the three applied buffer compositions. The zoom-in graph in Fig. 5-15 $\mathrm{C}$, showing the capacitance changes within the first two hours after seeding the cells, reveals slightly slower attachment kinetics for MDCK-II cells in a $\mathrm{Mg}^{2+}$-free buffer $(-\infty)$ compared to buffers supplemented with $\mathrm{Mg}^{2+}(--,-)$. In the absence of $\mathrm{Mg}^{2+}$ there is a delay in the capacitance drop, with both, the time of half of the final signal change $t_{1 / 2}$ and the time of maximal spreading rate $t_{m}$, appearing $\sim 10$ min later compared to the capacitance traces of cell attachment in $\mathrm{Mg}^{2+}$-enriched buffer (see table in S/ 17). However, spreading rates $m=\Delta C / \Delta t$ are similar for buffers with and without $\mathrm{Mg}^{2+}\left(\sim-5.1 \mathrm{nF} \cdot \mathrm{h}^{-1} ; S / 17\right)$. In the presence of $\mathrm{Ca}^{2+}$, either alone $(--)$ or in the co-presence of $\mathrm{Mg}^{2+}(-)$, the entire capacitance

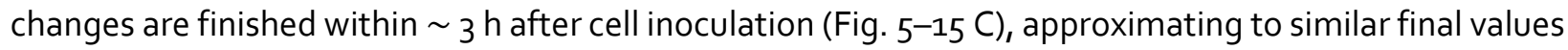
at the end of experiments $\left(t_{1}=20 \mathrm{~h}\right.$ ). The seeding of MDCK-II cells under $\mathrm{Ca}^{2+}$-free conditions ( $-{ }^{-}$) causes an initial capacitance drop within two hours similar to the control buffer containing both $\mathrm{Ca}^{2+}$ and $\mathrm{Mg}^{2+}(-)$. However, values recover afterwards in the direction of the cell-free sensor to $\sim 150 \%$ higher values (at $t_{1}=20 \mathrm{~h}$ ) compared to calcium-containing conditions (Fig. 5-15 C).

Electrochemical resistance values (Fig. 5-15 D) start to increase after a time delay of $\sim 1 \mathrm{~h}$ in a sigmoidal shape for the three different buffer conditions. In the presence of $\mathrm{Ca}^{2+}(-,-\rightarrow) R$ strongly increases by $\sim 30 \mathrm{kOhm}$ to constant values within $6 \mathrm{~h}$ after seeding cells. In the presence of $\mathrm{Mg}^{2+}$ only ( $-\square-$ ), however, $R$ increases considerably less by $\sim 2.5 \mathrm{kOhm}$ within three hours, followed by a recovery to almost starting values until the end of experiments $\left(t_{1}=20 \mathrm{~h}\right.$ ). Final resistance values are by $\sim 89 \%$ lower without than with $1.8 \mathrm{mM}$ extracellular calcium. In presence of $\mathrm{Ca}^{2+}$ either alone or in co-presence of $\mathrm{Mg}^{2+}$ halfmaximum signal changes are attained simultaneously at $t_{1 / 2}=240 \mathrm{~min}$. In the presence of $\mathrm{Ca}^{2+}$ only maximal slopes $m=\Delta R / \Delta t$ occure somewhat earlier and are slightly steeper $\left(t_{m}=225 \mathrm{~min}, \Delta R / \Delta t=\right.$ $\left.6.8( \pm 0.2) \mathrm{kOhm} \cdot \mathrm{h}^{-1}\right)$ than in the co-presence of $\mathrm{Mg}^{2+}\left(t_{m}=245 \mathrm{~min}, \Delta R / \Delta t=5.0( \pm 0.2) \mathrm{kOhm} \cdot \mathrm{h}^{-1}\right)$. 
A

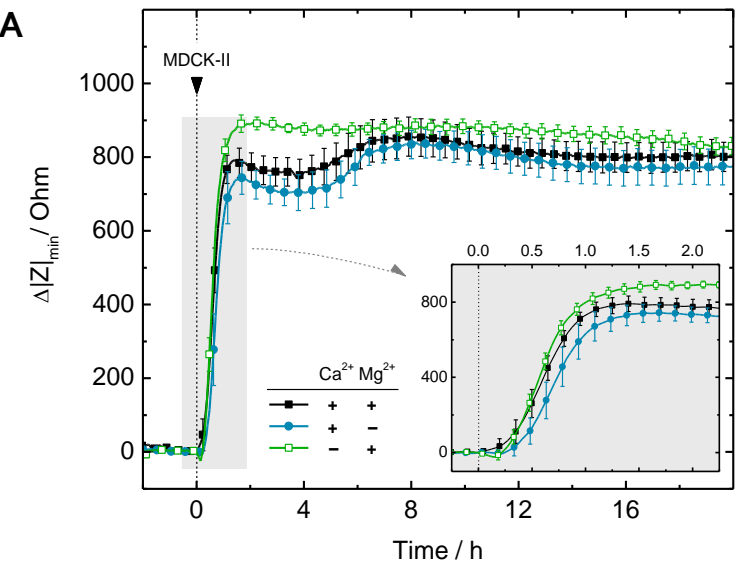

C

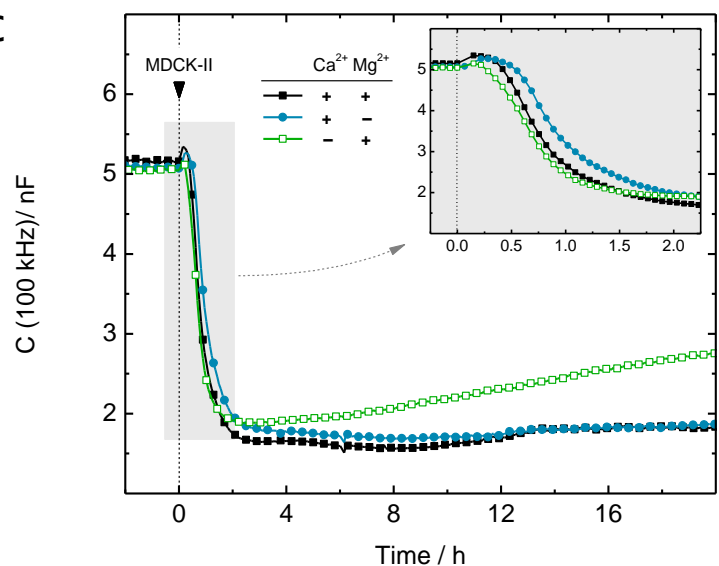

B

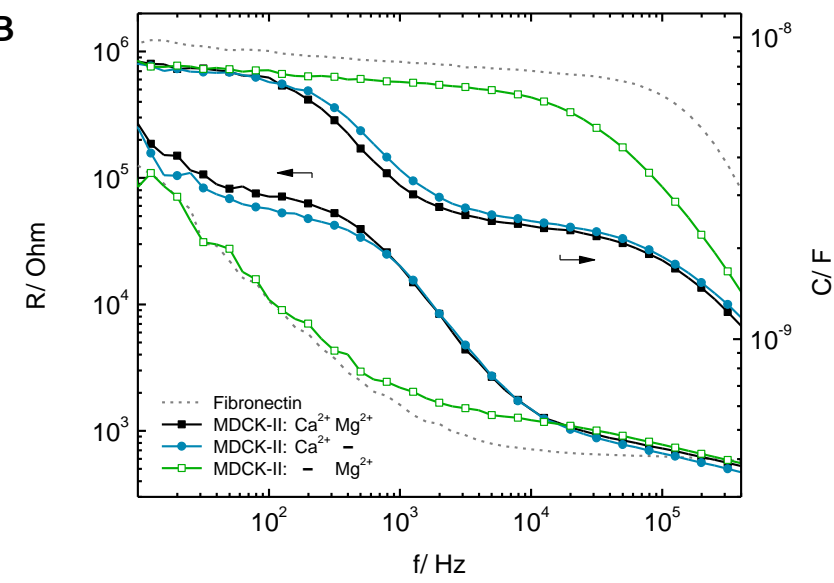

D

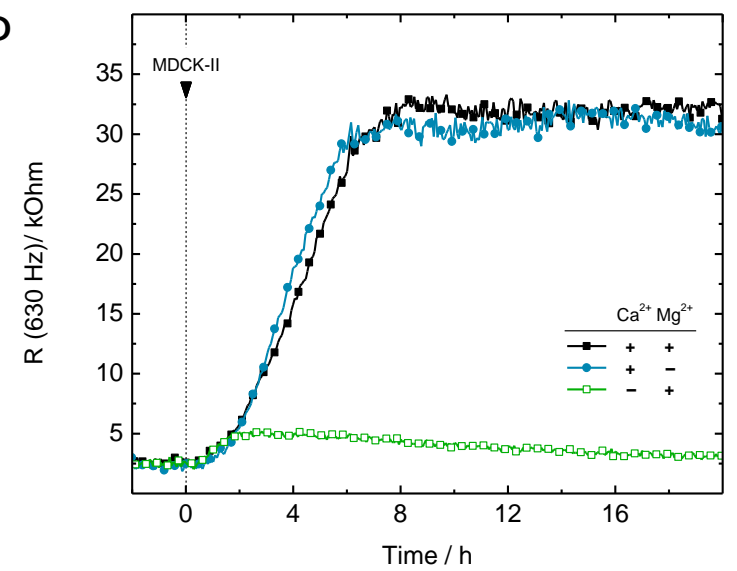

Fig. 5-15 OCM-ECIS sensor responses during MDCK-II cell attachment and spreading on FN-

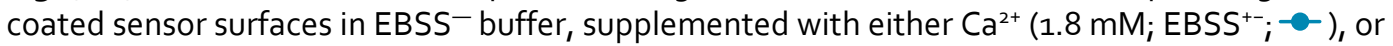
$\mathrm{Mg}^{2+}\left(0.81 \mathrm{mM} \mathrm{EBSS}^{-+} ;-{ }^{-}\right)$, or both $\left(\mathrm{EBSS}^{++} ;-{ }^{-}\right)$. (A) Averaged $\Delta|Z|_{\min }$ time courses from $\mathrm{OCM}$-mode recordings (pooled data sets of $\mathrm{OCM} 1$ and $\mathrm{QCM} 2$; mean $\pm \mathrm{SEM}_{i} \mathrm{~N}=4$ ). (B) Resistance $(R)$ and capacitance $(C)$ spectra from ECIS-mode recordings of the FN-coated sensors in EBSS ${ }^{--}$ buffer (--) and $20 \mathrm{~h}$ after seeding cells under the various buffer conditions. Most sensitive sampling frequencies for MDCK-II cells seeded in $\mathrm{Ca}^{2+}$-containing buffer were determined to $630 \mathrm{~Hz}$ for $R$ and $100 \mathrm{kHz}$ for $C$. (C), (D) Exemplary time courses of the ECIS-mode capacitance and resistance during MDCK-II cell attachment and spreading in EBSS ${ }^{++}$, EBSS ${ }^{+-}$and EBSS ${ }^{-+}$. Absolute values of $|Z|_{\text {min }}, R$, and $C$ at the time of cell inoculation ( $t_{0} ;$ marked with dashed line), signal changes after $t_{1}=20 \mathrm{~h}$ as well as characteristic curve parameters $\left(t_{m}, m=Y / \Delta t\right.$ $\left(Y=\Delta|Z|_{\min }, R, C\right)$, and $\left.t_{1 / 2}\right)$ are listed in table $S / 17$. Data points displayed are reduced for sake of clarity.

In order to support the QCM-ECIS data with independent data from a second method and to confirm morphological and cell structure properties concluded from these measurements, MDCK-II cells were investigated microscopically in an independent test series under similar conditions. Circular glass plates were coated with $25 \mu \mathrm{g} / \mathrm{mL} \mathrm{FN}$ in EBSS $^{--}$for $20 \mathrm{~h}$ and subsequently washed three times with EBSS ${ }^{--}$. MDCK-II cells ( 450000 cells $/ \mathrm{cm}^{2}$ ) were seeded in either EBSS ${ }^{+-}$, or EBSS ${ }^{-+}$, or EBSS ${ }^{++} .24 \mathrm{~h}$ hours after cell inoculation, the substrates were investigated by phase-contrast microscopy and, after cytochemical staining, by confocal fluorescence microscopy. Fig. 5-16 depicts micrographs of MDCK-II cells seeded in EBSS ${ }^{--}$supplemented with $\mathrm{Ca}^{2+}$ and $\mathrm{Mg}^{2+}$ (Fig. 5-16 A), with $\mathrm{Ca}^{2+}$ only (Fig. 5-16 B), and with $\mathrm{Mg}^{2+}$ only (Fig. 5-16 C). They show cells in phase-contrast $\left({ }^{*}{ }_{1} * * A, B, C\right)$, stained for live/ dead cells (*2), and triple stained for DNA $\left({ }^{*} 3\right), \mathrm{ZO}-1\left({ }^{*}\right)$ and actin $\left({ }^{*} 5\right)$. Micrograph overlays of the triple staining are illustrated in SI 19. 


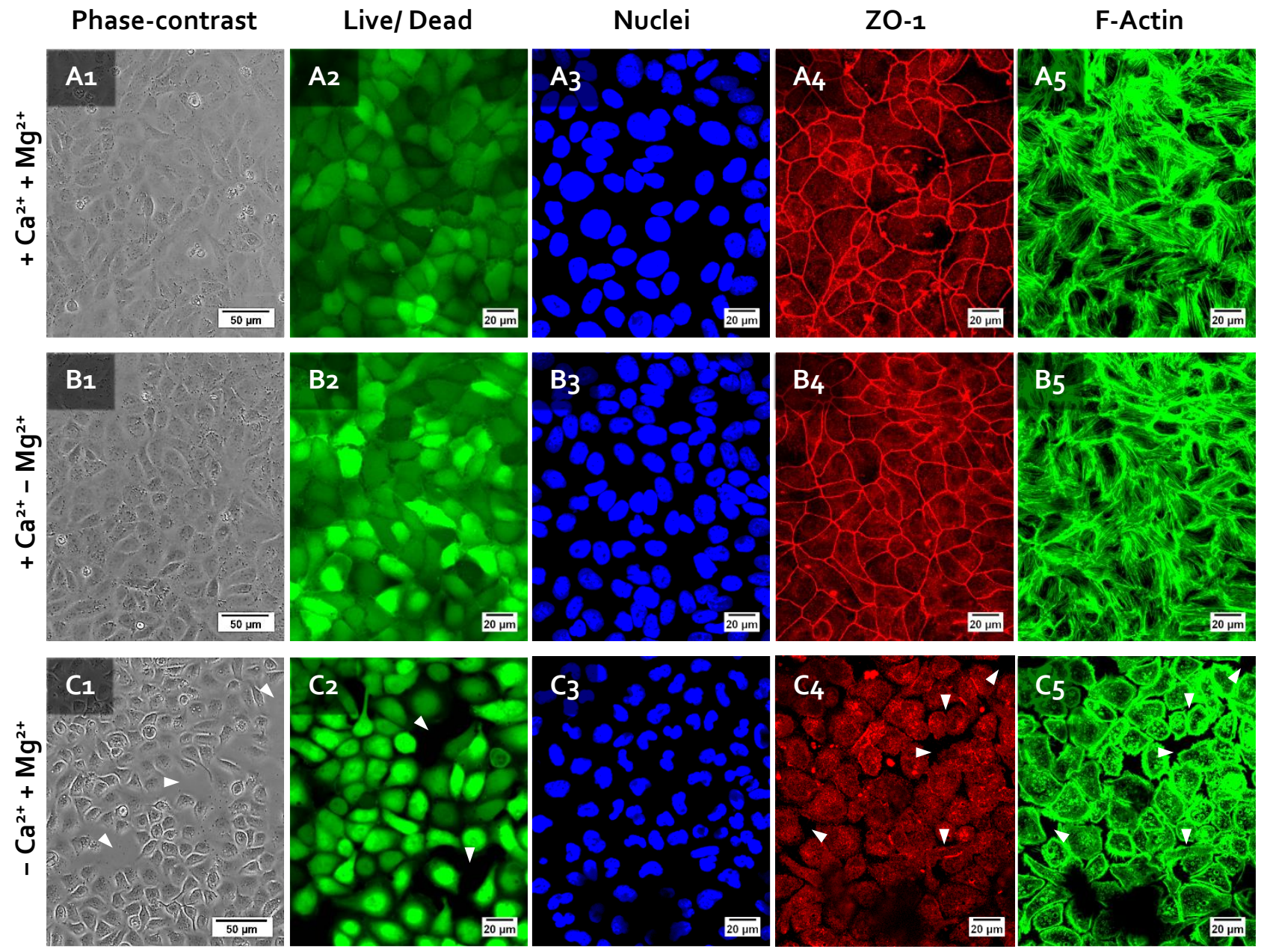

Fig. 5-16 Phase-contrast (1) and confocal fluorescence micrographs (2) - (5), taken $24 \mathrm{~h}$ after cells were seeded on $\mathrm{FN}$-coated glass substrates in the presence of physiological extracellular concentrations of (A) $\mathrm{Ca}^{2+}$ and $\mathrm{Mg}^{2+},(\mathrm{B}) \mathrm{Ca}^{2+}$ only, and (C) $\mathrm{Mg}^{2+}$ only. Cells were stained for (2) live/ dead, (3) nuclei, (4) ZO-1, and (5) f-actin. Overlays of the triple staining $(3+4+5)$ are illustrated in $\mathrm{SI}$ 19. Arrows indicate cell-free areas.

Phase-contrast micrographs of cells seeded in calcium-containing medium show confluent layers of spread cells on the substrates, exhibiting typical cobblestone-like morphologies (Fig. 5-16 A1, B1). The live/ dead staining shows a continuous green fluorescence (live stain) across the cell network and the absence of red fluorescent nuclei (dead stain), confirming vitality of individual cells and the integrity of the cell layer (Fig. 5-16 A2, B2). As it is distributed across the entire cytoplasm, the green fluorescence also unveils the cell dimensions, and thus provides information about the spreading of the cells as well as about close contacts without gaps of plasma membranes of neighboring cells. However, the morphological situation is different for cells inoculated on FN-coated glass substrates in the absence of $\mathrm{Ca}^{2+}$. Though phase-contrast images (Fig. 5-16 $\mathrm{C}_{1}$ ) and confocal fluorescence micrographs of live/ dead stained cells (Fig. 5-16 C2) show growth substrates densely covered with vital cells, the cells are not or only marginally spread on the substrate and remain attached in round shape. Moreover, the cell layer exhibits bigger inter-cellular gaps, which indicate the absence of cell-cell junctions (AJs, desmosomes, GJs, and TJs), as well as some cell-free areas (indicated by arrows in Fig. 5-16 C), which give evidence on either failed cell adhesion or the detachment of individual cells from the surface (maybe during the staining procedure). 
Confocal fluorescence micrographs after triple staining of nuclei, ZO-1, and actin reveal almost similar structural characteristics for cells, independent on whether $\mathrm{Mg}^{2+}$ is co-present to $\mathrm{Ca}^{2+}$ or not during culturing. However, there are significant differences to these observations, when the extracellular medium lacks $\mathrm{Ca}^{2+}$ and contains $\mathrm{Mg}^{2+}$ only. The nuclei undergo a transformation from a spheroidal shape in the presence of $\mathrm{Ca}^{2+}$ (Fig. 5-16 $\mathrm{A}_{3}, \mathrm{~B}_{3}$ ) to a mostly horseshoe-like shape in the absence of $\mathrm{Ca}^{2+}$ (Fig. 5$16 C_{3}$ ). The fluorescence of the immunostained TJ protein ZO- 1 is discretely localized around each cell in the presence of calcium (Fig. 5-16 A4, B4), defining close and distinct cell-cell contacts at the $Z O$ and indicating a continuous network of intercellular TJs. In contrast, the ZO-1 immunofluorescence of cells cultured in calcium-free conditions is delocalized throughout the cytoplasm without any localization at the cell boundaries between neighboring cells (Fig. 5-16 $C_{4}$ ). The diffuse ZO-1 distribution, however, allows to determine the spreading area and the boundaries of individual cells, which discloses intercellular gaps and, thus, the absence of intercellular junctions (indicated by - in Fig. $5-16 C_{4}$ ).

Since AJs and TJs are connected to the intracellular actin cytoskeleton and epithelial TJ permeability is known to be regulated by alterations of the actin cytoskeleton ${ }^{[392]}$, fibrous actin was stained by fluorescence-labeled phalloidin and investigated microscopically in this test series. Actin is co-localized with ZO around the cell periphery and, as long as $\mathrm{Ca}^{2+}$ is present, MDCK-II cells also form distinctive cellspanning actin stress fibers (Fig. 5-16 $\mathrm{A}_{5}, \mathrm{~B}_{5}$ ). In cells inoculated in $\mathrm{Ca}^{2+}$-free buffer, supplemented with $\mathrm{Mg}^{2+}$ only, $\mathrm{f}$-actin is localized in thick bundles along the cell boundary of individual cells and stress fibers within single cells or spanned across adjacent cells are not formed at all (Fig. 5-16 C5). Instead, f-actin is disassembled and localized in punctual aggregations, which are distributed across the entire cytoplasm. Moreover, gaps are clearly visible between the plasma membranes of neighboring cells ( $\boldsymbol{D}$ in Fig. 5$16(5)$, providing evidence of rounding up of cells, disabled formation of cellular junction, suppressed TJ closing and, thus, separation of individual cells from the cellular network due to the absence of extracellular calcium.

\subsection{QCM-ECIS Whole-Cell Biosensor}

In this thesis the QCM-ECIS sensor with a confluent cell layer on it (Whole-Cell Biosensor) was tested with different cell lines in a multitude of stimulation assays, focusing on kinetic differences of cell responses in mechanical (viscoelastic) and passive electrical properties. The investigations comprise the analysis of cell responses upon extracellular (5.3.1 Cytochalasin D, 5.3.2 Glutaraldehyde/ Paraformaldehyde) and intracellular (5.3.3 8-CPT-CAMP) stimulations, as well as a proof-of-concept of the in situ electroporation capabilities of cells on the surface of the developed OCM-ECIS sensors (5.3.4 ELPO-ECIS-OCM). For experiments the setup illustrated in Fig. 3-18 (p. 57) was used, unless mentioned otherwise in the description of the respective experiment. All studies were performed with the standard layout of the QCM-ECIS sensor. This is a $2 \mathrm{EIQ}$, half-side (one resonator unit) coated with photoresist of $\sim 3 \mu \mathrm{m}$ in thickness, comprising a circular cavity in the center of the covered gold electrode. This bared, small area of the electrode serves as WE for ECIS-mode impedance analysis, vis-à-vis to the uncoated electrode of the second resonator unit, which serves as the CE (Fig. 5-3 D, Fig. 5-4). The WE diameter was experimentally determined to $511( \pm 4) \mu \mathrm{m}(5.1 .1)$. 


\subsubsection{Degradation of the Actin Cytoskeleton}

The actin cytoskeleton network is known to be important for the mechanical properties of cells ${ }^{[393-396]}$ as well as for the junctional permeability of epithelial cell layers ${ }^{[392,397,398]}$. There is a dynamic assembling and disassembling process of the filamentous actin structures in living cells. The fungal toxin cytochalasin $D(C D)$ is a well known membrane-permeable drug that specifically blocks the growing end of $\mathrm{f}$-actin and thus inhibits polymerization, while it does not affect depolymerization at the opposite end of actin filaments. This consequently leads to a disassembly of the actin cytoskeleton and ends up in the aggregation of monomeric actin molecules. ${ }^{[399-403]}$ From AFM [395,396,403-408] and other rheological studies of cytochalasin treated cells (Micropipette Aspiration [409-413], Optical Tweezer/ Laser Trap ${ }^{[414]}$, Magnetic Twisting Cytometry ${ }^{[415-420]}$, Magnetic Trap ${ }^{[421]}$, Uniaxial Stress-Strain Testing ${ }^{[394]}$, Micro Tensile Tester ${ }^{[422]}$, Scanning Acoustic Microscopy ${ }^{[423,424]}$, Cell Poker ${ }^{[425]}, \mathrm{SICM}^{[426]}$ ), this was correlated with a decrease in the stiffness of individual cells and the entire cell layer, respectively. In literature, also a cytochalasinmediated increase in the transepithelial permeability and opening of intercellular TJs [97,116,397,398,427-432] have been reported.

The influences of actin cytoskeleton disruption by $\mathrm{cD}$ on cellular mechanics close to the substrate as well as on cell shape and cellular junction permeability were simultaneously investigated by means of timeresolved QCM-ECIS measurements. The course of a typical CD experiment is shown in Fig. 5-17 by the time courses of the characteristic QCM-mode parameter $\left(\Delta|Z|_{\min } ;\right.$ Fig. 5-17 A) and ECIS-mode parameters ( $R_{\text {norm }}, C_{\text {normi }}$ Fig. 5-17 C), normalized to the point of cell inoculation (" $\nabla$ MDCK-II"). MDCKII cells were seeded on the OCM-ECIS sensors ( 450000 cells/ $\mathrm{cm}^{2}$ ) and grown to confluence for $24 \mathrm{~h}$. In cases where OCM- and ECIS-mode responses were not monitored during cell attachment and spreading, individual spectra were recorded in both operation modes before and $24 \mathrm{~h}$ after seeding cells. Afterwards, $1.9 \mu \mathrm{L} \mathrm{cD}$ solution (dissolved in DMSO) were premixed with culture medium and carefully added to the measurement vessel (" $\nabla C D$ " in Fig. 5-17 A, C), to obtain final concentrations of $0.1,1.0$, and $5.0 \mu \mathrm{M}$ in the cell culture medium. Negative control cells (-ctrl) were exposed to $1.9 \mu \mathrm{L}$ DMSO in $1.5 \mathrm{~mL}$ medium. The DMSO concentration in all experiments thus amounted to $0.13 \%(\mathrm{v} / \mathrm{v})$. For reference (+ctrl), $0.012 \%(v / v)$ Saponin was added to the cells after cD treatments in order to perforate cell membranes and to induce cell detachment from the sensor surface.

The plots next to the time courses in Fig. 5-17 show (in 3-D representation) the changes of QCM spectra (Fig. 5-17 B; $f$ vs. $|Z|$ vs. $\varphi$ ) and ECIS spectra (Fig. $5^{-17} \mathrm{D}_{i} f$ vs. $R$ vs. $C$ ) in the course of a typical cD experiment. Selected spectra are shown for (1) the cell-free sensor in SFM, (2) the cell-covered sensor ( $24 \mathrm{~h}$ after seeding cells to confluence), cells exposed to $5 \mu \mathrm{M} \mathrm{cD}$ for (3) $30 \mathrm{~min},(4) 2 \mathrm{~h},(5) 4 \mathrm{~h}$, and (6) cells after Saponin treatment. Corresponding 2-D spectra (QCM: $|Z|(f), \varphi(f) ; \mathrm{ECIS:}|Z|(f), \varphi(f), R(f)$, $C(f)$ ) are illustrated in $S I 2 O$ (p. 273). The 3-D loop of the oscillation impedance is strongly contracted in $y$-axis $(|Z|)$ and z-axis $(\varphi)$ direction due to attachment and spreading of MDCK-Il cells $(1 \rightarrow 2$ in Fig. 517 B). Upon $C D$ treatment this loop expands again equally in these directions, by $\sim 1 / 3$ of the cellular impedance signal $(2 \rightarrow 5)$. In the presented case, stationary OCM spectra are attained within 30 min after adding $5.0 \mu \mathrm{M} \mathrm{CD}$. Subsequent plasma membrane perforation causes almost complete reduction of the acoustic load impedance towards the initial, cell-free situation $(5 \rightarrow 6)$. The electrochemical impedance spectra (Fig. 5-17 D) show changes in both the resistive and the capacitive part. The most sensitive frequency of either parameter $(R: 316 \mathrm{~Hz}, C: 40 \mathrm{kHz})$ is indicated in the respective projection plane. After the initial capacitance decrease and resistance increase upon cell layer formation $(1 \rightarrow 2)$, both values go in opposite direction again by exposing cells to $5.0 \mu \mathrm{M} \mathrm{CD}(2 \rightarrow 5)$. The signal change in capacitance is 
slower compared to the oscillation impedance change, reflected in further spectral changes after $0.5 \mathrm{~h}$ of cD incubation.

From the time courses in Fig. 5-17 A and C and the spectral breakdown in Fig. 5-17 B and D, one can see different impacts of actin cytoskeleton disintegration on the oscillation impedance $\left(\Delta|Z|_{\min }\right)$, paracellular current pathways at low-frequency $\left(R_{\text {norm }}\right)$, and transcellular current pathways at highfrequency $\left(C_{\text {norm }}\right)$, in both the kinetics and the degree of the effect. These are analyzed and discussed below for various concentrations of cytochalasin $D$.

A

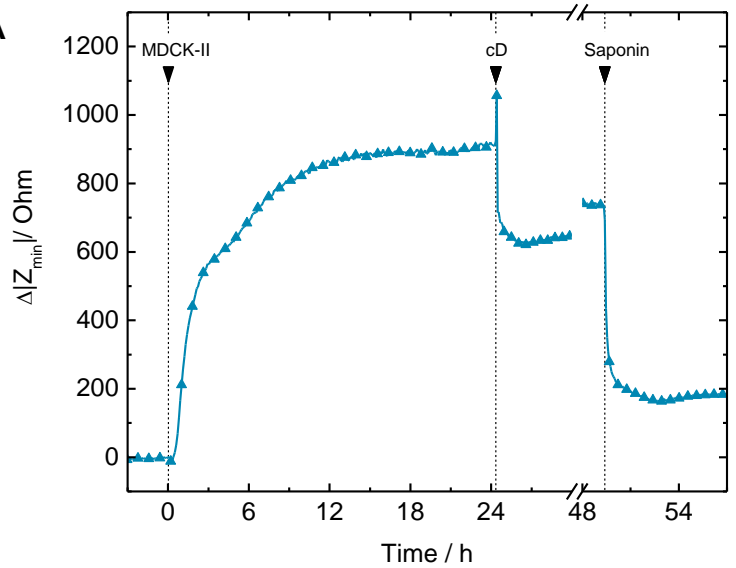

C

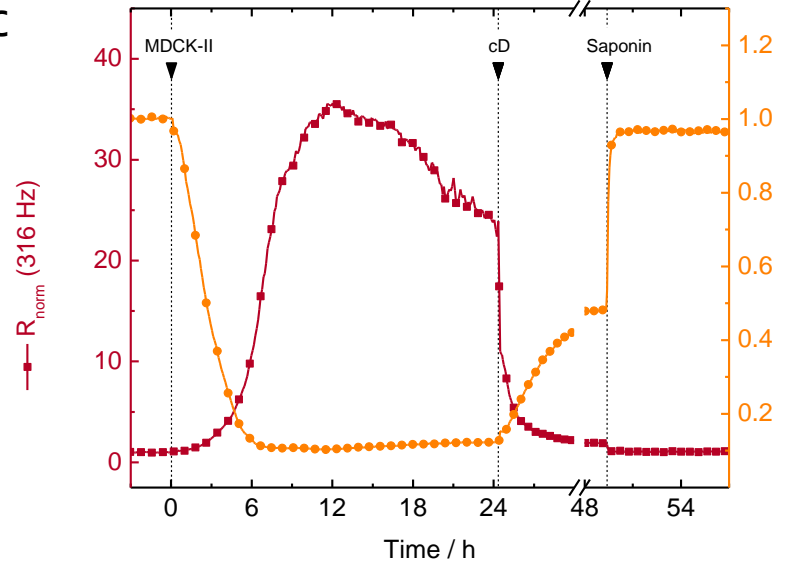

B

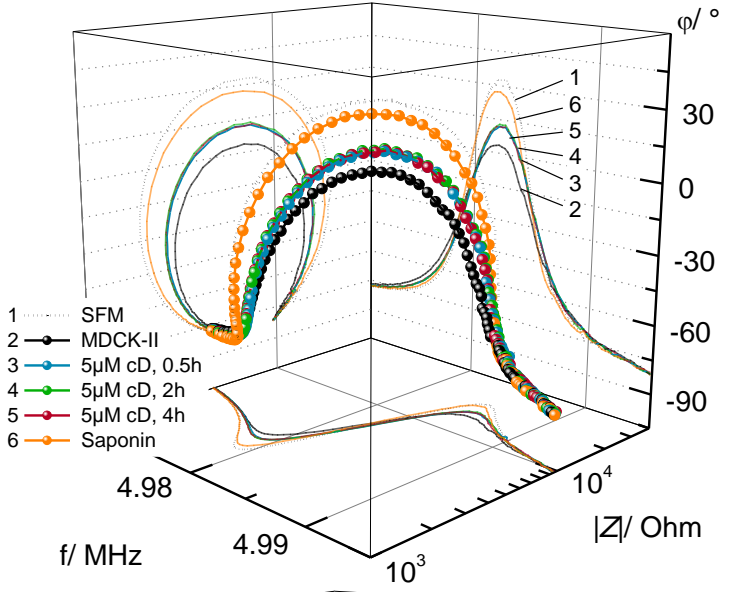

D

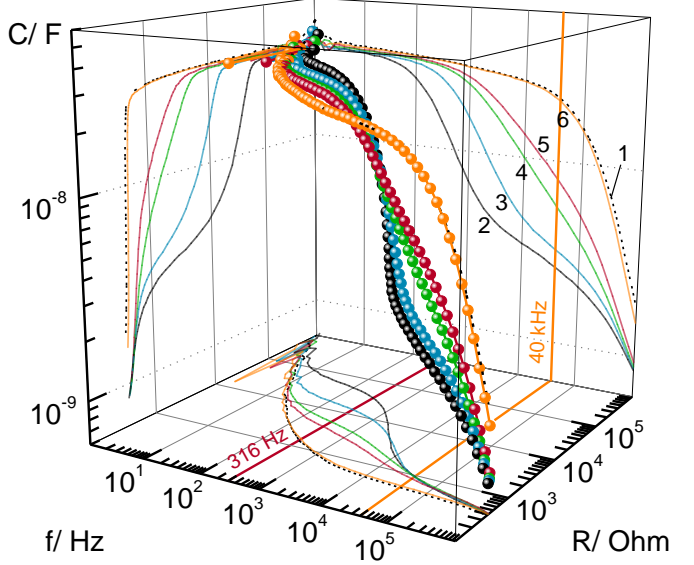

Fig. 5-17 Exemplary QCM-ECIS measurement of a confluent MDCK-II cell layer treated with the actin cytoskeleton affecting drug cytochalasin $D(C D)$ in $5 \mu \mathrm{M}$ concentration. Diagrams display the time courses (left hand side) and the spectra (in 3-D) at defined time points (right hand side) of CD experiments, obtained from recordings in (A), (B) OCM-mode and (C), (D) ECIS-mode. The time courses of $\Delta|Z|_{\text {min }}, R_{\text {norm }}$ (at $316 \mathrm{~Hz}$ ), and $C_{\text {norm }}$ (at $40 \mathrm{kHz}$ ) are normalized to the last value before seeding cells. Arrows mark the points of additions. Saponin treatment after $C D$ experiments serves as positive control. 3-D spectra of (B) OCM and (D) ECIS parameters are shown for (1) the cell-free sensor in SFM, (2) the sensor covered with a confluent MDCK-Il cell layer, cells on the sensor exposed to $5 \mu \mathrm{M} \mathrm{CD}$ for (3) $0.5 \mathrm{~h} /(4) 2 \mathrm{~h} /(5) 4 \mathrm{~h}$, and (6) cells after Saponin treatment. The most sensitive frequency of either parameter $(R: 316 \mathrm{~Hz}, C: 40 \mathrm{kHz})$ is indicated in the respective projection plane. Corresponding 2-D spectra $(|Z|(f), \varphi(f), R(f), C(f))$ are illustrated in $S / 20$. Data points displayed in $\mathrm{A}$ and $\mathrm{C}$ are reduced for sake of clarity.

Fig. 5-18 show averaged time courses of $\Delta|Z|_{\min }$ (Fig. 5-18 A), $R_{\text {norm }}$ at $316 \mathrm{~Hz}$ (Fig. 5-18 C), and $C_{\text {norm }}$ at $40 \mathrm{kHz}$ (Fig. 5-18 E), normalized to the point of adding either $C D$ in various concentrations ( $0.1 \mu \mathrm{M}:-$, $1 \mu \mathrm{M}:-, 5 \mu \mathrm{M}: \rightarrow)$ ) or equivalent amounts of DMSO only $(-\mathrm{ctrl} ;-)$ ) to the cell culture medium 
(mean $\pm \mathrm{SEM}$ ). In each diagram, a grey horizontal line indicates the mean cell-free value, determined in each experiment after sensor preincubation with SFM and before seeding cells. The bar graphs next to the time courses in Fig. 5-18 display the mean signal changes of parameters after $4 \mathrm{~h}$ of cell treatment with $0.1 \mu \mathrm{M}$ ( $/ Z$ ) , $1 \mu \mathrm{M}$ ( time-resolved monitoring and endpoint measurements. In the case of $0.1 \mu \mathrm{M} C D$, parameters did not reach constant levels within $4 \mathrm{~h}$. Hence, in addition to these transient values, the final stationary values attained after $\sim 12 \mathrm{~h}$ of $0.1 \mu \mathrm{M}$ cD incubation ( ) are shown in diagrams, too. Diagrams also include values after DMSO addition only (-ctrl; $\mathbf{Z})$, after Saponin treatment ( $+\mathrm{ctrl} ; \mathrm{MV})$, as well as values of the cell-free sensor immersed in SFM $(+c$ trl; $\square)$. Mean values of the MDCK-II cell-covered sensor amount to: $|Z|_{\min }=2760( \pm 30) \mathrm{Ohm}(\mathrm{N}=28), R_{316 \mathrm{~Hz}}=36( \pm 4) \mathrm{kOhm}(\mathrm{N}=14)$, and $C_{40 \mathrm{kHz}}=3.0( \pm 0.3) \mathrm{nF}$ $(\mathrm{N}=14)$. Absolute mean values of the cell-free sensors immersed in SFM are: $|Z|_{\text {min }}=1900( \pm 30) \mathrm{Ohm}$ $(\mathrm{N}=28), R_{316 \mathrm{~Hz}}=3.3( \pm 0.7) \mathrm{kOhm}(\mathrm{N}=14)$, and $C_{40 \mathrm{kHz}}=15.4( \pm 1.5) \mathrm{nF}(\mathrm{N}=14)$. Related to the values of cell-covered sensors, these cell-free values serve as a reference level in the bar plots in Fig. $5-18(\square)$.

Under conditions of negative control, neither the piezoelectric nor the electrochemical parameters are significantly affected within the investigated time period (Fig. 5-18 A, C, E: - ) , reflected in values of $\Delta|Z|_{\text {min }}=-2( \pm 17) \mathrm{Ohm}(\mathrm{N}=14), R_{\text {norm }}=1.01( \pm 0.04)(\mathrm{N}=3)$, and $C_{\text {norm }}=1.04( \pm 0.01)(\mathrm{N}=3), 4 \mathrm{~h}$ after DMSO addition. From time-resolved data, an instantaneous steep decrease in $\Delta|Z|_{\min }$ and $R_{\text {norm }}$ and a comparatively slower but continuous increase in $C_{\text {norm }}$ becomes evident upon injection of cytochalasin D into the measurement chamber. $A c D$ concentration of $0.1 \mu \mathrm{M}$ causes a little but significant decrease in both the oscillation impedance (OCM-mode; Fig. 5-18 A) and the electrochemical resistance (ECISmode; Fig. 5-18 C) in the early stages after addition, whereas the high-frequency capacitance from ECISmode recordings remains unaffected (Fig. 5-18 E). $4 \mathrm{~h}$ after adding $0.1 \mu \mathrm{M} \mathrm{cD}$ to the cells, $\Delta|Z|_{\text {min }}$ is reduced by $66( \pm 15) \mathrm{Ohm}(\mathrm{N}=6)$ and $R_{\text {norm }}$ has dropped to $0.66( \pm 0.14)(\mathrm{N}=3)$ with respect to starting values. However, sensor responses recover again in the course of the experiment. Within $12 \mathrm{~h}$ after adding $C D$, almost initial parameter values are reached, with $\Delta|Z|_{\min }=-13( \pm 18) \mathrm{Ohm}(\mathrm{N}=7)$, $R_{\text {norm }}=0.88( \pm 0.02)(\mathrm{N}=4)$, and $C_{\text {norm }}=1.1( \pm 0.1)(\mathrm{N}=4)$.

Applying of at least ten times higher $\mathrm{CD}$ concentrations to MDCK-II cell layers results in a persistent disruption of both the mechanical stability (Fig. 5-18 A) and the paracellular barrier resistance (Fig. 5$18 \mathrm{C}$ ) of the cells. In contrast to $0.1 \mu \mathrm{M} \mathrm{CD}, C_{\text {norm }}$ changes, too, upon incubation of MDCK-II cells with either $1.0 \mu \mathrm{M}$ or $5.0 \mu \mathrm{M} \mathrm{CD}$ (Fig. 5-18 E). By applying these amounts to the cells, $\Delta|Z|_{\text {min }}, R_{\text {norm }}$ and $C_{\text {norm }}$ rapidly change and reach constant values within a period of $\sim 60-90 \mathrm{~min}$. There is a concentration dependent effect on all parameters. The higher the $C D$ concentration is the faster are the kinetics and the bigger are the absolute values of parameter changes. Four hours after adding $1.0 \mu \mathrm{M} \mathrm{CD},|Z|_{\text {min }}$ is decreased by $190( \pm 40) \mathrm{Ohm}(\mathrm{N}=8), R_{\text {norm }}$ is reduced to $21( \pm 3) \%(\mathrm{~N}=4)$, and $C_{\text {norm }}$ is increased to $160( \pm 30) \%(N=4)$, with respect to pre-treatment values. When exposing the MDCK-II cell layer to $5.0 \mu \mathrm{M} c D$ for $4 \mathrm{~h}$, effects are even bigger, with $\Delta|Z|_{\text {min }}=-250( \pm 30)$ Ohm $(\mathrm{N}=8), R_{\text {norm }}=12( \pm 2) \%$ $(\mathrm{N}=4)$, and $C_{\text {norm }}=230( \pm 30) \%(\mathrm{~N}=4)$. Hence, by treating cells with $5.0 \mu \mathrm{M} \mathrm{cD}, R_{\text {norm }}$ reaches values of the cell-free sensor $\left(R_{\mathrm{w} / \mathrm{o} \mathrm{cells}} / R_{\mathrm{w} / \text { cells }}=12( \pm 4) \%(\mathrm{~N}=14)\right)$.

Saponin treatment subsequent to $\mathrm{CD}$ stimulation systematically destroys the cellular mechanics and, thus, to a very high degree its load impedance contribution $\left(\Delta|Z|_{\min }=-620( \pm 30) O \mathrm{Om} ; \mathrm{N}=27\right)$. However, values do not completely reach the level of the cell-free sensor $\left(\Delta|Z|_{\min }=-860( \pm 20) O \mathrm{Om}\right.$; $\mathrm{N}=28$ ). Similar effects can be seen in $C_{\text {norm }}$ values (Fig. 5-18 F). $C_{\text {norm }}$ attains almost cell-free values $(5.2( \pm 0.4) ; \mathrm{N}=14)$ after exposing cells to Saponin $(4.7( \pm 0.4) ; \mathrm{N}=14)$. 
A

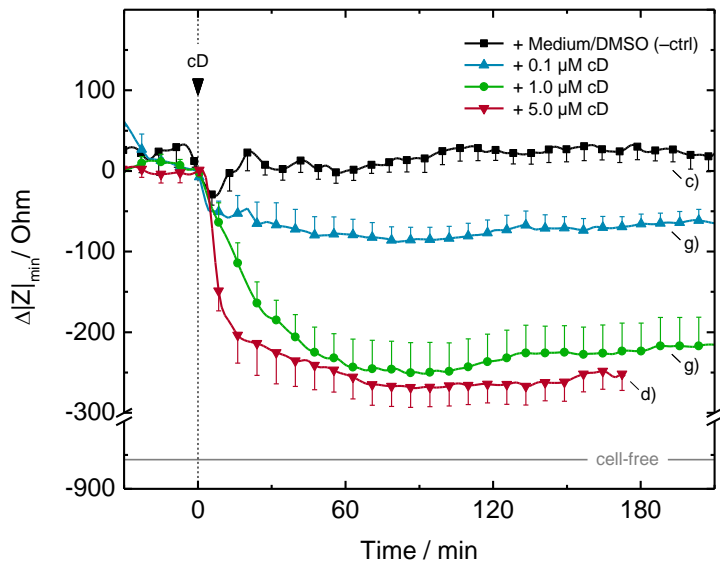

C

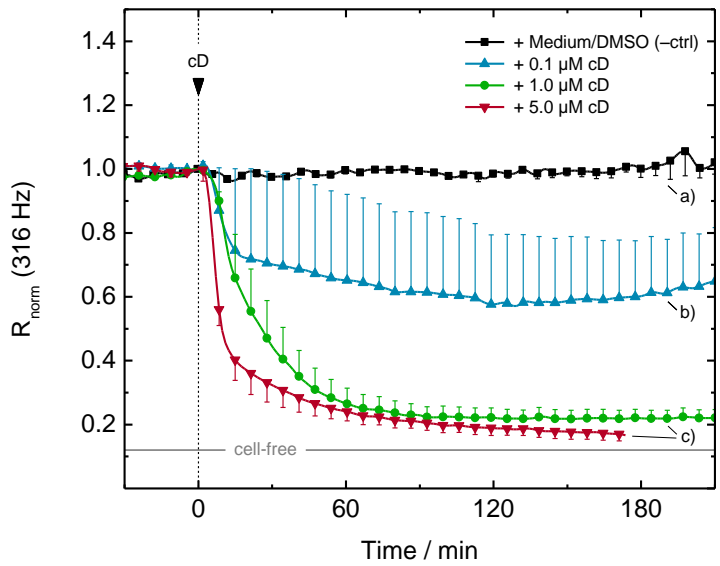

$E$

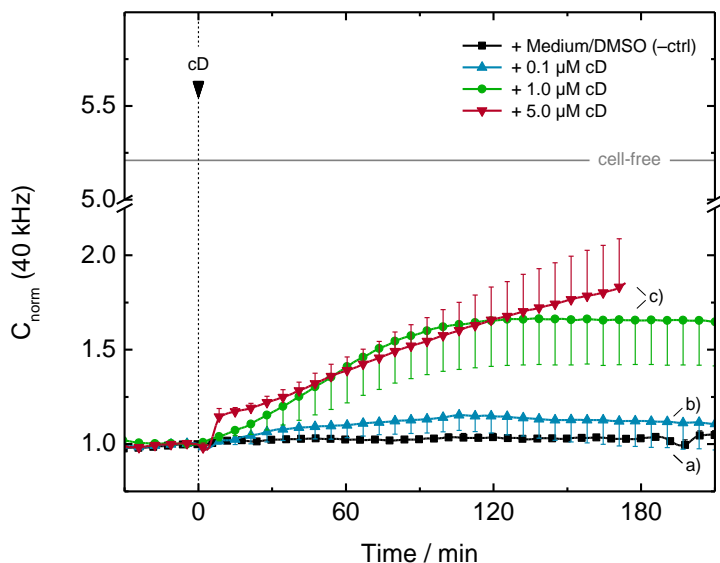

B

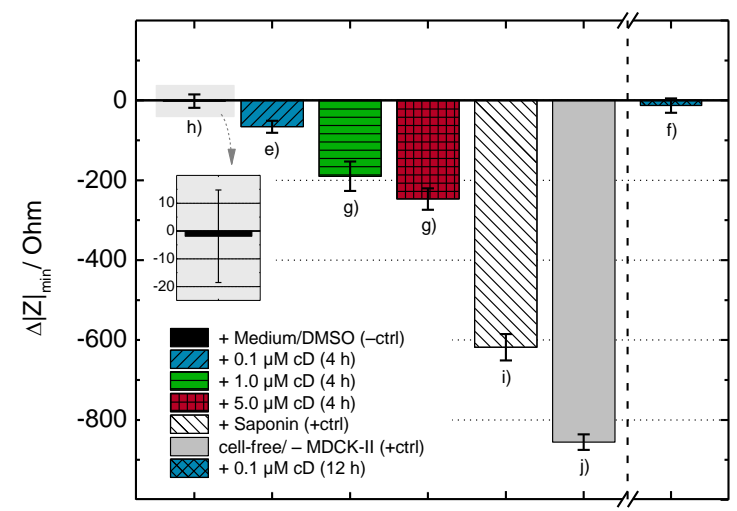

D

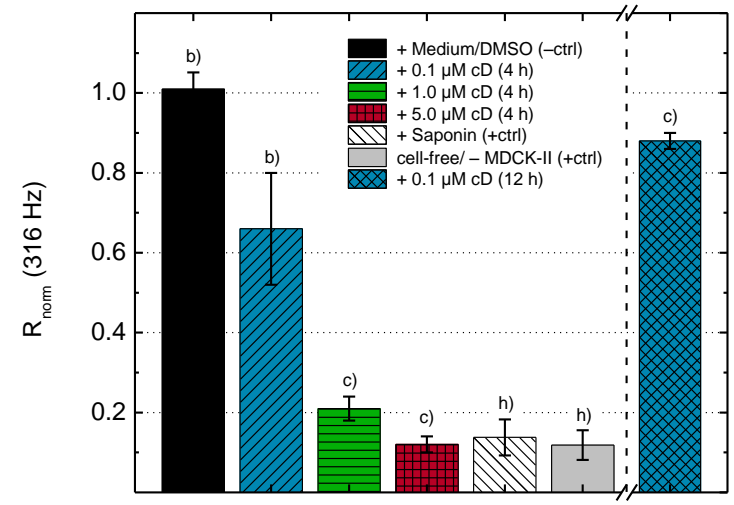

$\mathbf{F}$

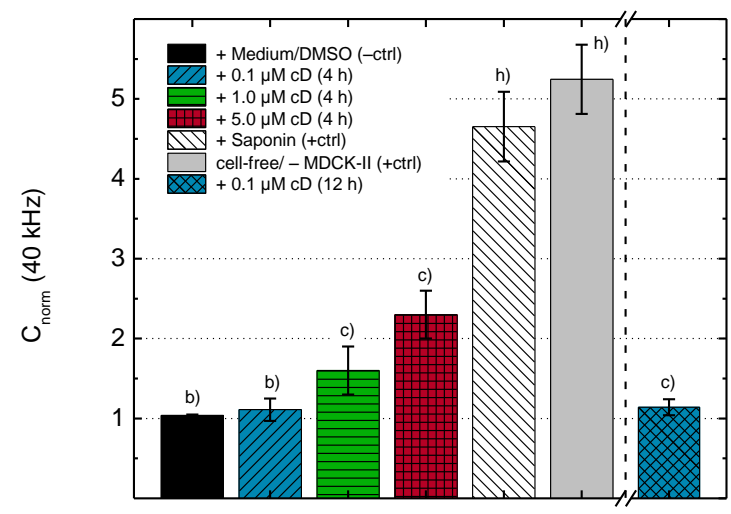

Fig. 5-18 OCM-ECIS sensor responses during treatment of a confluent MDCK-II cell layer with different concentrations of cytochalasin $D(C D)$. Averaged time courses and bar graphs, of mean values $4 \mathrm{~h}$ after treatment, of (A), (B) $\Delta|Z|_{\min }$ (C), (D) $R_{\text {norm }}$ at $316 \mathrm{~Hz}$, and (E), (F) $C_{\text {norm }}$ at $40 \mathrm{kHz}$

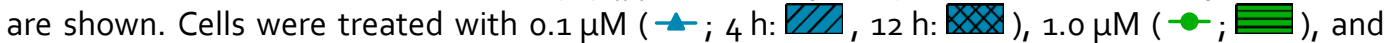
$5.0 \mu \mathrm{M}(\rightarrow ;-\mathrm{CD}$. Cell culture medium contains $0.13 \%(\mathrm{v} / \mathrm{v})$ DMSO after CD addition. For negative control $(-\mathrm{ctrl})$, cells were exposed to $0.13 \%(\mathrm{v} / \mathrm{v})$ DMSO without $\mathrm{CD}(-;-$ ) Parameter values after Saponin treatment ( $\mathrm{MIV}$ ) and values of the cell-free sensor immersed in SFM only ( $\square$ ) serve as a reference level (+ctrl). Grey horizontal lines in $A, C$, and $E$ indicate mean values of cell-free sensors. $\Delta|Z|_{\text {min }}$ values in $A$ and $B$ are pooled data from $\mathrm{OCM}_{1}$ and $\mathrm{OCM}_{2}$ recordings. Displayed data points are reduced for sake of clarity. (mean $\pm \mathrm{SEM} ; \mathrm{N}={ }^{\mathrm{a})} 2,{ }^{\mathrm{b})} 3,{ }^{\mathrm{c}} 4,{ }^{\mathrm{d}}{ }_{5}$, e) $6,{ }^{\text {f) }} 7,{ }^{\text {g) }} 8,{ }^{\text {h) }} 14,{ }^{\text {i) }} 27,{ }^{\mathrm{j}} 28$ ).

These QCM-ECIS findings and conclusions were verified by morphological studies of the actin cytoskeleton conditions under the influence of various concentrations of cD. MDCK-II cells were seeded 
to confluence on circular glass substrates ( 450000 cells/ $\mathrm{cm}^{2}$ ) and exposed to $\mathrm{CD} 24 \mathrm{~h}$ later. Exposures of cells to $\mathrm{CD}$ were terminated either $1 \mathrm{~h}$ or $25 \mathrm{~h}$ after adding the drug. The cells were subsequently fixated, permeabilized, and stained for f-actin with TRITC-labeled phalloidin (cf. Tab. 3-11, p. 79) for microscopic studies. Fig. 5-19 shows the respective micrographs, obtained from MDCK-II cells incubated with (A) $0 \mu \mathrm{M} \mathrm{CD}$ (negative control/ -ctrl), (B) $0.1 \mu \mathrm{M} \mathrm{CD},(C) 1.0 \mu \mathrm{M} \mathrm{CD}$, and (D) $5.0 \mu \mathrm{M} \mathrm{CD}$. Cells of negative control were exposed to $0.13 \%(v / v)$ DMSO without $c D$. Micrographs were taken either $1 \mathrm{~h}\left(\mathrm{~A}_{1}-\mathrm{D}_{1}\right)$ or $25 \mathrm{~h}\left(\mathrm{~A}_{2}-\mathrm{D}_{2}\right)$ after adding DMSO or $\mathrm{CD}$ to the confluent cell layers.

In the absence of $c D$ (Fig. 5-19 $A_{1}, A_{2}$ ), fluorescence of TRITC-phalloidin shows actin localization in two main structures: the actin cortex along the cell perimeter that is involved in intercellular adherens junctions (AJ), and actin stress fibers that interconnect integrins of focal adhesion sites (FA) at the basal cell membrane. There is no alteration in actin microfilament structures, when MDCK-II cells have been exposed to DMSO for $1 \mathrm{~h}$ (Fig. 5-19 $\mathrm{A}_{1}$ ) or for $25 \mathrm{~h}$ (Fig. 5-19 $\mathrm{A}_{2}$ ). Hence, the applied DMSO concentration in $C D$ experiments does not affect cellular morphology and $f$-actin structures. After incubating cells with $0.1 \mu \mathrm{M} \mathrm{CD}$ for $1 \mathrm{~h}$ (Fig. 5-19 B1), one can spot a slight reduction in the amount and length of stress fibers as well as reduced staining of actin microfilament bundles along the cell boundaries (indicated by - in Fig. $5^{-19} \mathrm{~B} 1$ ). Further depletion of stress fibers and punctual aggregation of monomeric actin can be observed after 25 h of cell exposure to $0.1 \mu \mathrm{M} \mathrm{CD}(-$ in Fig. 5-19 B2). However, the cells remain attached to the substrate surface in a spread shape and still form a tight and confluent monolayer.

When applying the ten-fold higher $\mathrm{CD}$ concentration, this has a more drastic effect on the actin cytoskeleton and, as a consequence, also on the cell morphology. One hour of incubating cells with 1.0 $\mu \mathrm{M} \mathrm{cD}$ causes a strong depolymerization of $\mathrm{f}$-actin in stress fibers and in the actin cortex. Partly depolymerized actin fibers are aggregated in large clusters, scattered within the cytoplasm and localized along the contact area of adjacent cells ( - in Fig. 5-19 C 1 ). Exposure of cells to $1.0 \mu M$ cD for 25 h causes further disassembly of these large clumps into finely distributed aggregations of actin monomers ( $\boldsymbol{D}$ in Fig. 5-19 (2). Moreover, the actin clusters observable along cell junctions after $1 \mathrm{~h}$ of $1.0 \mu \mathrm{M} \mathrm{CD}$ treatment disappear completely after $25 \mathrm{~h}$ incubation time. By contrast, opening of cellular junctions and the formation of small intercellular gaps are noticeable in micrographs ( $>$ in Fig. 5-19 C2).

Treating cells with $5.0 \mu \mathrm{McD}$ causes similar actin cytoskeleton alterations and cell morphological effects as described for $1.0 \mu \mathrm{M} \mathrm{CD}$. The micrograph in Fig. 5-19 D1 reveals a degradation of $\mathrm{f}$-actin into clumplike aggregates, scattered in the cytoplasm and along the former actin belt around the cells $(\boldsymbol{D})$. However, incubating cells with $5.0 \mu \mathrm{M} \mathrm{CD}$ for $25 \mathrm{~h}$ leads to the formation of larger intercellular openings than with 1.0 $\mu \mathrm{M} \mathrm{CD}$ at the same time (Fig. 5-19 D2). Moreover, one can observe even cell-free areas in the formerly confluent cell layer ( $>$ in Fig. 5-19 D2).

These morphological findings match very well with the results from the previously presented QCM-ECIS measurements of cellular stiffness and cell shape properties (cf. Fig. 5-18). Those reported on a moderate loss of mechanical stiffness and a slight decrease in the paracellular resistance (opening of cellular junctions), however no detachment of cells from the sensor surface, upon treating cells with $0.1 \mu \mathrm{M} \mathrm{CD}$ for 1 or $25 \mathrm{~h}$. When cells have been exposed to 1.0 and $5.0 \mu \mathrm{McD}$, however, a significant opening of cellcell contacts, an increasing fraction of cell-free areas on the WE as well as a pronounced stiffness loss of MDCK-II cells were sensitively detected and followed over time by means of the parameters of OCMmode and ECIS-mode recordings. 
$1 \mathrm{~h}$
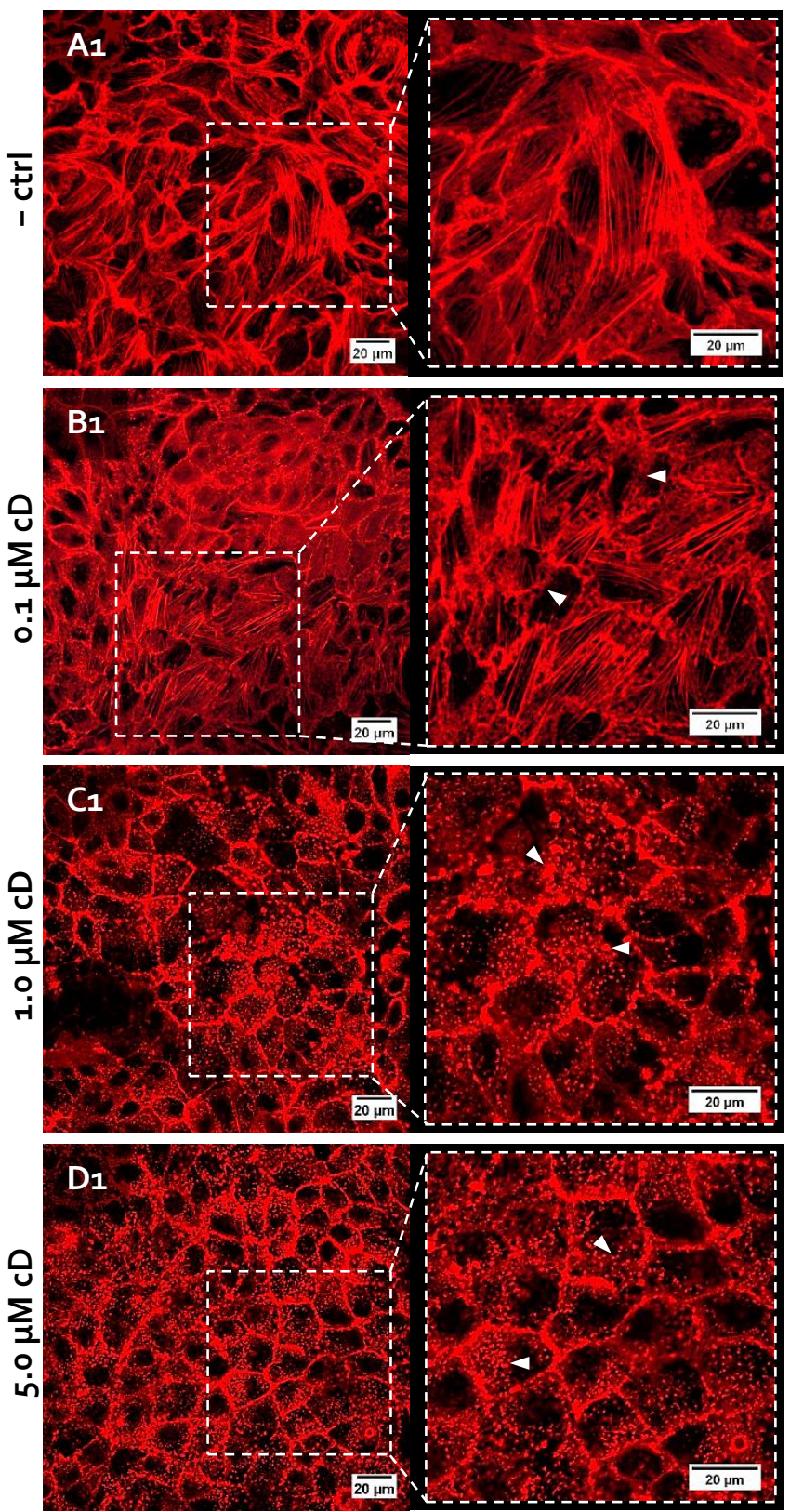

$25 \mathrm{~h}$
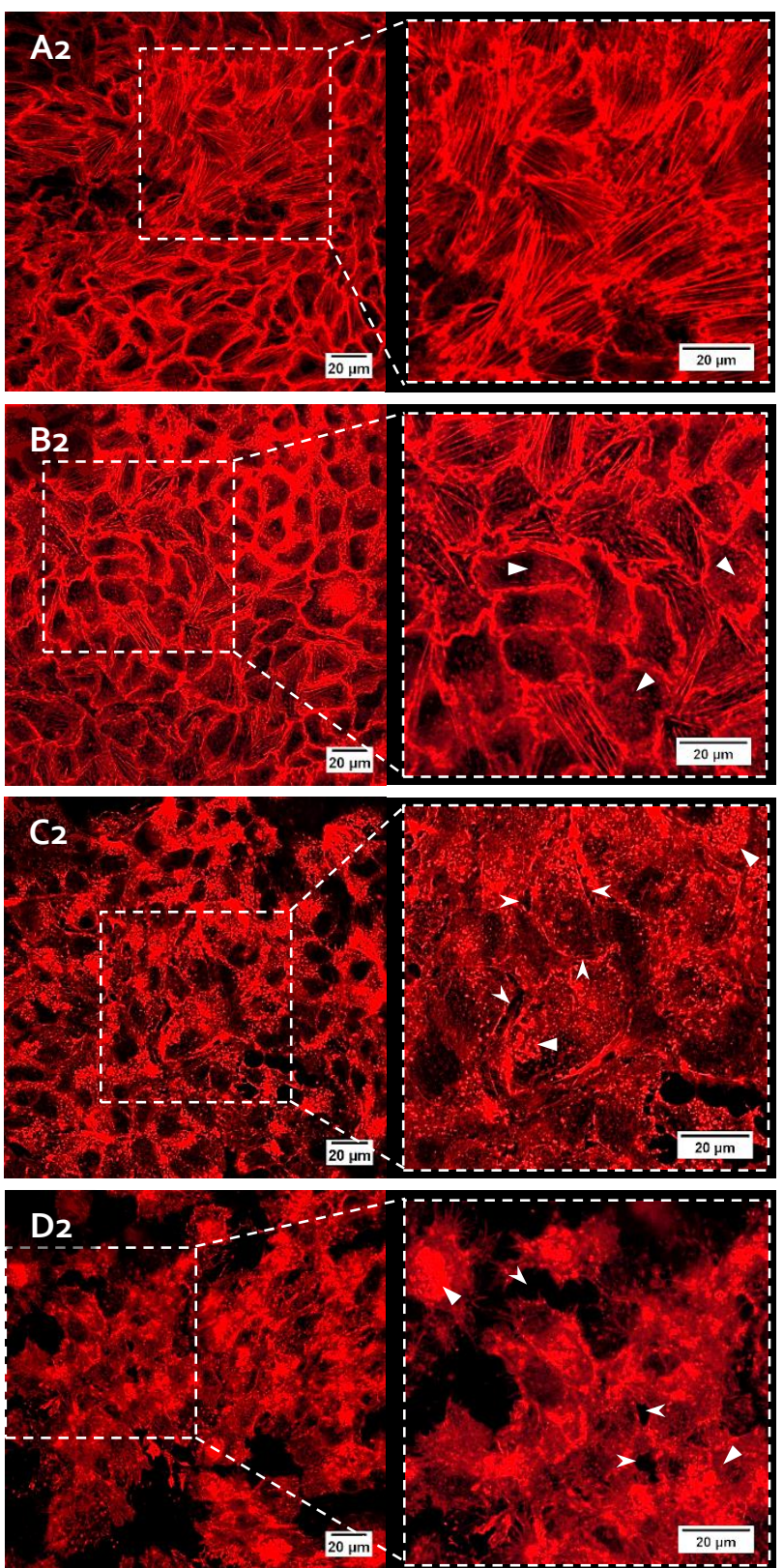

Fig. 5-19 Confocal fluorescence micrographs of MDCK-II cells exposed to various concentrations of cytochalasin $D(C D)$ and subsequently stained for $f$-actin (TRITC-phalloidin). Cells were treated with (A) o $\mu \mathrm{McD}$ (negative control/ -ctrl), (B) $0.1 \mu \mathrm{McD}$, (C) $1.0 \mu \mathrm{M} \mathrm{cD}$, and (D) $5.0 \mu \mathrm{M} \mathrm{CD}$. Cells of negative control were exposed to $0.13 \%(\mathrm{v} / \mathrm{v})$ DMSO without $\mathrm{CD}$. Micrographs were taken either $1 h\left(A_{1}-D_{1}\right)$ or $25 h\left(A_{2}-D_{2}\right)$ after treatment with $C D$.

\subsubsection{Cell Stiffening using Chemical Fixatives}

OCM-ECIS measurements provided complementary information on cell mechanics and cell shape upon degradation of the actin cytoskeleton and associated softening of the cells by $\mathrm{CD}$ treatment. In order to further investigate the complementarity of OCM and ECIS signals and, thus, to demonstrate the advantages of simultaneous measurements, the mechanical properties of MDCK-II cells were systematically stiffened by cross-linking all cellular protein with the chemical fixatives glutaraldehyde (GA) and paraformaldehyde (PFA). The fixation of cells is a standard cytochemical method in cell-based 
assays before fluorescence staining of specific cellular proteins or organelles for their microscopic analysis. It is assumed that this preparation step for cytological samples just "freezes" the structural state of the cells by cross-linking all cellular protein at the moment of adding the fixative. The aldehydes glutaraldehyde (GA) and paraformaldehyde (PFA) are often used for this. It is known from AFM ${ }^{\left[404,408,433^{-}\right.}$ ${ }^{439]}$, SPR ${ }^{[440]}$ as well as from previuos OCM studies applying the classical 1 ElOs ${ }^{[22,246,268]}$ that there are drastic and rapid increases in cellular stiffness (Young's modulus), in the refractive index, and in the damping of the microbalance oscillation upon cell fixation. In this thesis, the OCM-ECIS sensors were used for monitoring in real-time the responses of cultured MDCK-II cells when adding various concentrations of GA and PFA.

Fig. 5-20 illustrates the course of a typical cell fixation experiment, by means of parameter time courses from QCM-mode measurements ( $\Delta|Z|_{\text {minin }}$ Fig. 5-20 A) and ECIS-mode measurements $\left(R_{\text {norm }}, C_{\text {norm }}\right.$; Fig. 5-20 C). The curves are normalized to the last value before seeding MDCK-II cells to confluence on the sensor (" $\nabla$ MDCK-II"). The day after cell inoculation, the culture medium was exchanged against $1.5 \mathrm{~mL} \mathrm{PBS}^{++}$buffer (supplemented with $1 \mathrm{~g} / \mathrm{L}$ glucose) in order to avoid cross-reactions of the fixatives with ingredients of the cell culture medium (" $\nabla \uparrow M e d . \downarrow \mathrm{PBS}^{++\prime \prime}$ ). When signals indicated establishment of equilibrium after the medium exchange, $150 \mu \mathrm{L}$ of the buffer were replaced by $150 \mu \mathrm{L}$ of a tenfold concentrated fixative solution (in $\mathrm{PBS}^{++}$). In the exemplary experiment shown in Fig. 5-20, cell proteins were cross-linked with a final GA concentration of $0.25 \%(\mathrm{v} / \mathrm{v})($ " $\nabla \mathrm{GA}$ "). When $\mathrm{OCM}$ and ECIS parameters have attained stationary levels after cross-linking, the cell layers were additionally treated with Saponin, in order to analyze the effect of membrane permeabilization after cell fixation on both the mechanical and electrical properties of the cell layer.

The plots right next to the time courses in Fig. 5-20 show 3-D representations of OCM spectra (Fig. 5$20 \mathrm{~B}_{i} f$ vs. $|Z|$ vs. $\varphi$ ) and ECIS spectra (Fig. $5-20 \mathrm{D}_{i} f$ vs. $R$ vs. $C$ ) at various points of the experiment. Spectra are shown for (1) the cell-free OCM-ECIS sensor equilibrated in SFM, (2) the MDCK-II cell-covered sensor, cells treated with $0.25 \%$ (v/v) GA for (3) $5 \mathrm{~min} /(4) 10 \mathrm{~min} /(5) 180 \mathrm{~min}$, and (6) fixated cells after Saponin treatment. Corresponding 2-D spectra (OCM: $|Z|(f), \varphi(f) ; \mathrm{ECIS:}|Z|(f), \varphi(f), R(f), C(f)$ ) are illustrated in $S I 21$ (p. 274). The 3-D loop of the oscillation impedance, contracted in the course of cell attachment and spreading, is further damped in phase shift and impedance magnitude upon fixation of the cells by additional $50-100 \%$ of the actual cell signal ( $2 \rightarrow 5$ in Fig. 5-20 B). Saponin treatment subsequent to cell layer fixation degrades the cell membranes. However, this causes no reduction in the damping of the OCM impedance $(5 \rightarrow 6)$. The situation is different for ECIS-mode parameters. There is only a slight reduction in the low-frequency resistance and capacitance values at higher frequencies even show negligible changes due to the addition of GA (Fig. 5-20 D). The most sensitive frequency of either parameter for MDCK-II cells $(R: 316 \mathrm{~Hz}, C: 40 \mathrm{kHz})$ is indicated in the respective plane of projection in Fig. 5-20 D. When the cells are perforated by Saponin after protein cross-linking, resistance and capacitance values go back to almost cell-free levels ( $5 \rightarrow 6$ in Fig. $5-20 \mathrm{D})$.

Hence, one can spot very distinct differences in the acoustic and electrochemical impedance spectra as well as in the courses of $\Delta|Z|_{\text {min }}, R_{\text {norm }}$ and $C_{\text {norm }}$ responses in both the kinetics and the extent of signal changes upon cross-linking of all cellular protein as well as subsequent cell permeabilization. These are analyzed and discussed in detail below for various concentrations of GA and PFA. 
A

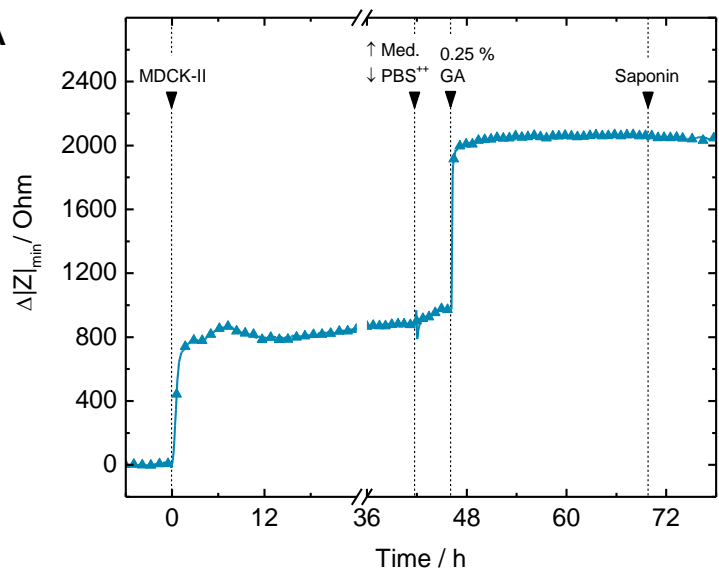

C

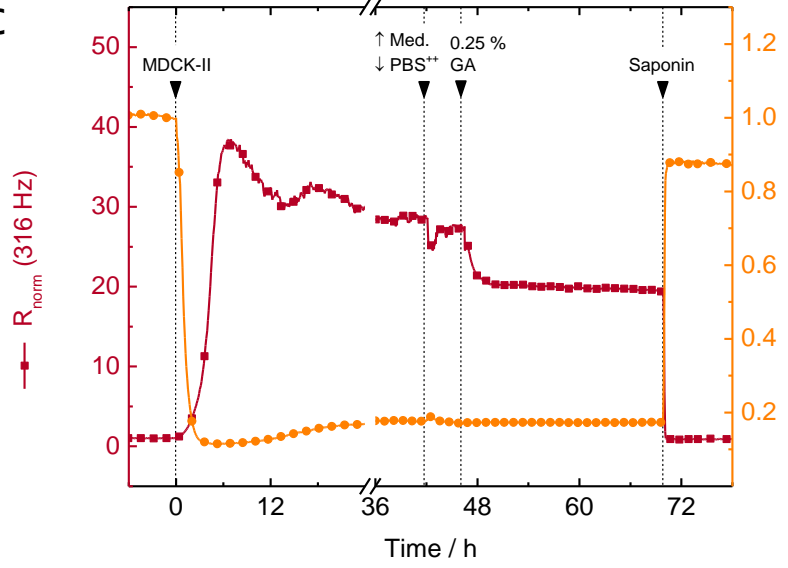

B

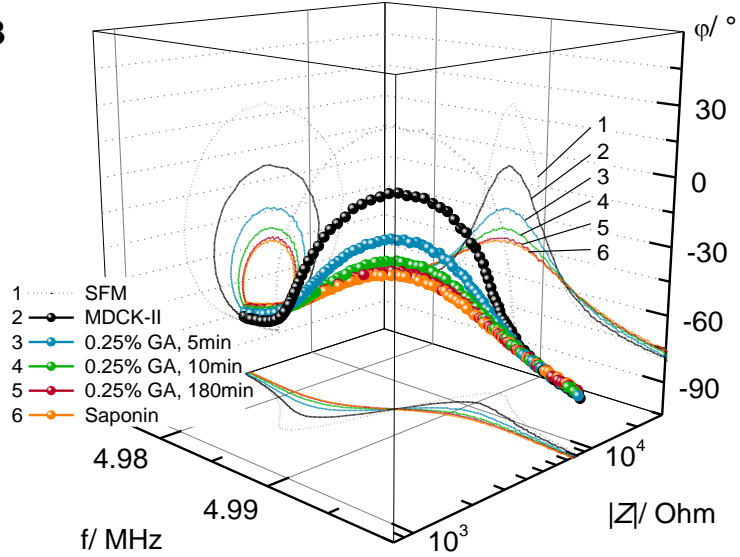

D

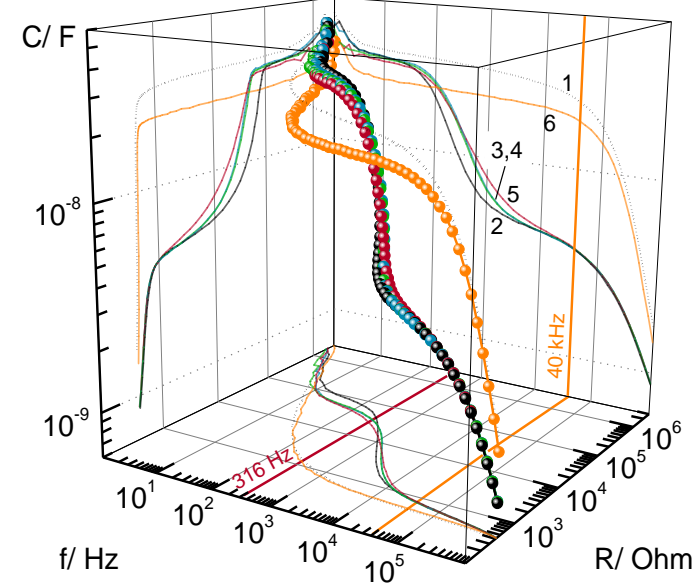

Fig. 5-20 Exemplary OCM-ECIS measurement of MDCK-II cells seeded on QCM-ECIS substrates and subsequently treated with the protein cross-linking agent glutaraldehyde (GA). Diagrams display the time courses (left) and the spectra (in 3-D) at defined points of the experiment (right), obtained from (A), (B) OCM-mode and (C), (D) ECIS-mode recordings. The time courses of $\Delta|Z|_{\text {min, }} R_{\text {norm }}$ (at $316 \mathrm{~Hz}$ ), and $C_{\text {norm }}$ (at $40 \mathrm{kHz}$ ) are normalized to the last value before cell inoculation. Arrows mark the points of seeding the cells ("MDCK-II"), of exchanging medium against $\mathrm{PBS}^{++}$buffer ("TMed., $\downarrow \mathrm{PBS}^{++"}$ ), of adding "0.25\% GA", and of finally permeabilizing cells with "Saponin". 3-D impedance spectra of (B) OCM and (D) ECIS parameters are shown for the sensor (1) $24 \mathrm{~h}$ after SFM incubation, (2) $24 \mathrm{~h}$ after seeding cells to confluence, (3) $5 \mathrm{~min} /(4) 10 \mathrm{~min} /(5) 180 \mathrm{~min}$ after $0.25 \% \mathrm{GA}$ injection, and (6) after adding Saponin. The most sensitive frequency of either parameter $(R: 316 \mathrm{~Hz}, C: 40 \mathrm{kHz})$ is indicated in the respective projection plane. Corresponding 2-D spectra $(|Z|(f), \varphi(f), R(f), C(f))$ are illustrated in $S / 21$. Data points displayed in $\mathrm{A}$ and $\mathrm{C}$ are reduced for sake of clarity.

Fig. 5-21 summarizes the parameter changes obtained from a variety of OCM-ECIS replicate measurements of a confluent MDCK-II cell layer upon treatment with GA and PFA. Time-resolved alterations of $\Delta|Z|_{\min }$ (Fig. 5-21 A), $R_{\text {norm }}$ (Fig. 5-21 C), and $C_{\text {norm }}$ (Fig. 5-21 E) were examined for cell treatments with $0.025(-), 0.1(-\rightarrow)$, and $0.25 \%(\mathrm{v} / \mathrm{v}) \mathrm{GA}(\rightarrow)$, and $1 \%(\mathrm{w} / \mathrm{v})$ PFA $(\rightarrow)$ (mean \pm SEM). For negative control (-ctrl), $\mathrm{PBS}^{++}$buffer was exchanged (- - ). All time courses are set to time zero at the point of adding the fixative and replacing the buffer, respectively $(\boldsymbol{\nabla})$. Values of the cell-free sensor immersed in SFM serve as a reference level (+ctrl) and are indicated by a grey horizontal line in Fig. 5-21 $A, C$, and $E$. The bar graphs next to the time-resolved data display average signal changes (mean $\pm \mathrm{SEM}$ ) of parameters after $3 \mathrm{~h}$ exposure of the cells to various concentrations of GA (0.025\%: $Z / Z, 0.1 \%$ : $0.25 \%$ : and 1 \% PFA ( $)$ ) as well as values of negative ( $\square$ ) and positive ( $\square$ ) controls. These plots include data from time-resolved monitoring experiments as well as from endpoint measurements. 
In Fig. 5-21 B, D, and F, parameter values after Saponin treatment are exemplary shown for the cell layer that has been fixated with $0.25 \%$ GA before ( data from both microbalance sensor spots of the OCM-ECIS sensors. Absolute mean values of the cellfree sensor immersed in SFM amount to $|Z|_{\text {min }}=1860( \pm 20) \mathrm{Ohm}(\mathrm{N}=42), R_{316 \mathrm{~Hz}}=2.8( \pm 0.4) \mathrm{kOhm}$ $(\mathrm{N}=21)$, and $C_{40 \mathrm{kHz}}=14.9( \pm 1.1) \mathrm{nF}(\mathrm{N}=21)$. Average starting values of the MDCK-Il cell-covered sensor before fixation are $|Z|_{\min }=2740( \pm 30) \mathrm{Ohm}(\mathrm{N}=42), R_{316 \mathrm{~Hz}}=33( \pm 3) \mathrm{kOhm}(\mathrm{N}=21)$, and $C_{40 \mathrm{kHz}}=3.05$ $( \pm 0.19) \mathrm{nF}(\mathrm{N}=21)$ (mean $\pm \mathrm{SEM})$. The relations of cell-free to cell-covered values serve as a reference level in Fig. 5-21 B, D, and $F(\square)$. Negative control cells show almost no response upon buffer exchange. After three hours, one can observe only slight changes of $\Delta|Z|_{\min }=120( \pm 20) \mathrm{Ohm}$ $($ mean $\pm \mathrm{SEM} ; \mathrm{N}=14), R_{\text {norm }}=0.93( \pm 0.04)$, and $C_{\text {norm }}=1.00( \pm 0.01)($ both $\mathrm{N}=7)$.

$\Delta|Z|_{\text {min }}$ steeply increases within a few minutes, starting immediately upon injecting GA to the measurement vessel of the cell-covered sensor plate (Fig. 5-21 A). After $3 \mathrm{~h}$ of GA incubation, $|Z|_{\text {min }}$ is increased by $710( \pm 20)$ Ohm for $0.025 \%$ GA, by 950 ( \pm 60 ) Ohm for $0.1 \%$ GA, and by $1030( \pm 50)$ Ohm for $0.25 \% \mathrm{GA}$ (mean $\pm \mathrm{SEM} ; \mathrm{N}=10$ ) (Fig. 5-21 B). The higher the concentration of $\mathrm{GA}$ is, the faster is the increase and the earlier stationary levels of $|Z|_{\min }$ are reached (Fig. 5-21 A). However, final levels are similar for all concentrations, as long as the duration of GA incubation is sufficiently long. For instance, $|Z|_{\min }$ is finally increased by $1050( \pm 30)$ Ohm after incubating cells with $0.025 \%$ GA for $\sim 65 \mathrm{~h}$ (data not shown). Here, $90 \%$ of the overall signal change are attained within approximately $20 \mathrm{~h}$ of incubation, whereas the remaining $10 \%$ of $\Delta|Z|_{\min }$ increase, however, take more than twice as long. By defining $\Delta|Z|_{\min }=1050( \pm 30) \mathrm{Ohm}$ as the stationary, maximal end value attained after protein cross-linking of cellular protein with $\mathrm{GA}$, the duration $t_{1 / 2}$ required for half-maximal $\Delta|Z|_{\min }$ increase $\left(t_{1 / 2} \stackrel{\text { def }}{=} t\left(\Delta|Z|_{\min }\right.\right.$ $=525 \mathrm{Ohm})$ ) serves as a kinetic parameter describing the time courses for various concentrations of GA. Applying this to the time courses in Fig. $5^{-21} \mathrm{~A}$, one obtains $t_{1 / 2}=3.8 \mathrm{~min}$ for $0.25 \% \mathrm{GA}, t_{1 / 2}=6.6 \mathrm{~min}$ for $0.1 \% \mathrm{GA}$, and $t_{1 / 2}=61.1 \mathrm{~min}$ for $0.025 \% \mathrm{GA}$. As further kinetic parameters the time $t_{m}$ of the steepest increase and the slope $m=\Delta|Z|_{\min } / \Delta t$ at this point (by linear regression around $t_{m}$ considering SEM) were extracted from the time-resolved data. This provides $t_{m}=3.3 \mathrm{~min}$ and $m=128( \pm 6)$ Ohm. $\mathrm{min}^{-1}$ for $0.25 \%, t_{m}=3.9 \mathrm{~min}$ and $m=79( \pm 4) \mathrm{Ohm} \cdot \mathrm{min}^{-1}$ for $0.1 \%$, and $t_{m}=6.1 \mathrm{~min}$ and $m=28 \mathrm{Ohm} \cdot \mathrm{min}^{-1}$ for $0.025 \% \mathrm{GA}$. By adding PFA to the cell layer, $\Delta|Z|_{\min }$ rises to $680( \pm 20) \mathrm{Ohm}$ $(\mathrm{N}=4)$ within $3 \mathrm{~h}$ (Fig. $\left.5^{-21} \mathrm{~B}\right)$, and to stationary end values of $800( \pm 40) \mathrm{Ohm}(\mathrm{N}=6)$ after $\sim 18 \mathrm{~h}$ of exposure time (data not shown). Related to this final value, the time $t_{1 / 2}$ of half-maximal $\Delta|Z|_{\text {min }}$ change $\left(t_{1 / 2} \stackrel{\text { def }}{=} t\left(\Delta|Z|_{\min }=400 \mathrm{Ohm}\right)\right.$ is determined to $18.6 \mathrm{~min}$ for cell-fixation with $1 \%$ PFA. The signal response exhibits a biphasic shape, with a steep increase within the first $8 \mathrm{~min}$ after PFA inoculation $\left(t_{m}=1.5 \mathrm{~min}\right.$ and $\left.m=90 \mathrm{Ohm} \cdot \mathrm{min}^{-1}\right)$, followed by a slowed down but steady increase for hours to final levels. As already mentioned for the exemplary $\Delta|Z|_{\text {min }}$ curve in Fig. 5-20 A, Saponin treatment after chemical fixation of all cellular protein does not change oscillation impedance values anymore. This is the case for all applied concentrations of GA. In Fig. 5-21 B, this is exemplarily shown for cell layers fixated with $0.25 \%$ GA followed by Saponin incubation ( which provides final $\Delta|Z|_{\text {min }}$ values of $1080( \pm 50)$ Ohm $(\mathrm{N}=10)$.

The electrochemical resistance instantaneously drops within 5 min after adding PBS ${ }^{++}$(-ctrl) or GA to the cells (Fig. $5-21 \mathrm{C}$ ). In this period, $R_{\text {norm }}$ is decreased to $\left.0.88( \pm 0.01)^{\mathrm{b}}\right)$ for negative control cells, to $0.688( \pm 0.005)^{\mathrm{a})}$ by $0.025 \% \mathrm{GA}$, to $0.78( \pm 0.05)^{\mathrm{a})}$ by $0.1 \% \mathrm{GA}$, and to $0.85( \pm 0.02)^{\mathrm{a})}$ by $0.25 \% \mathrm{GA}$ (mean $\pm \mathrm{SEM} ; \mathrm{N}={ }^{\text {a) }} 2,{ }^{\mathrm{b}}$ ) $)$. Afterwards, $R_{\text {norm }}$ recovers again for control cells and further decreases slightly for $G A$ treated cells. Fig. 5-21 D shows stationary signal levels, attained at $2-3 \mathrm{~h}$ after GA addition. Final $R_{\text {norm }}$ values $(t=3 \mathrm{~h})$ amount to $0.51( \pm 0.06)$ for $0.025 \% \mathrm{GA}, 0.65( \pm 0.06)$ for $0.1 \% \mathrm{GA}$, and $0.78( \pm 0.03)$ for $0.25 \% \mathrm{GA}(\mathrm{N}=5)$. Thus, cross-linking of cellular proteins affects the resistive 
properties of the cell layer. It is remarkable that the effect is inversely proportional to the concentration of the fixative, with smaller decreases of the resistance at higher GA concentrations.

A

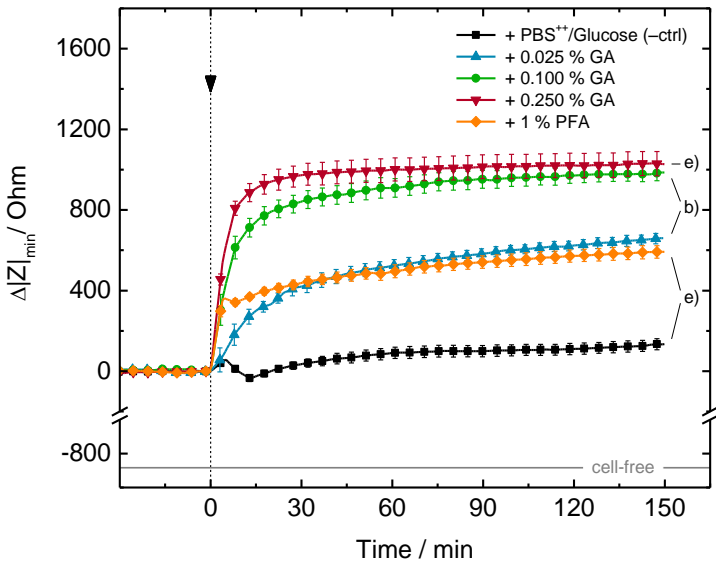

C

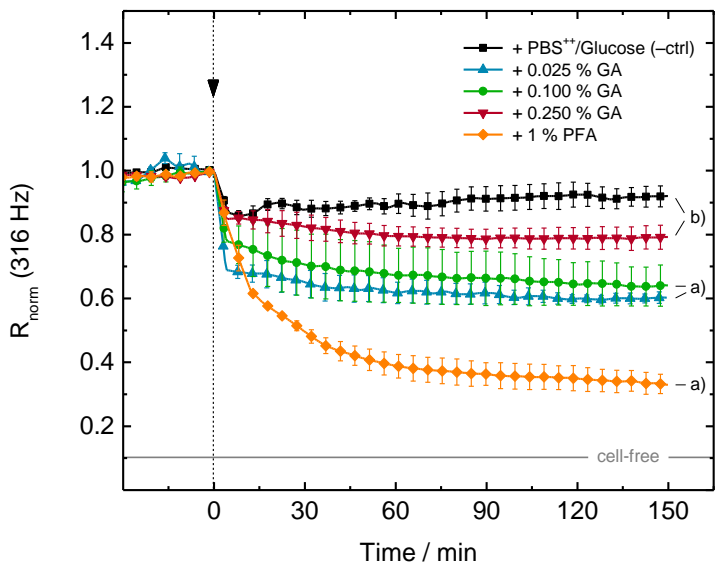

E

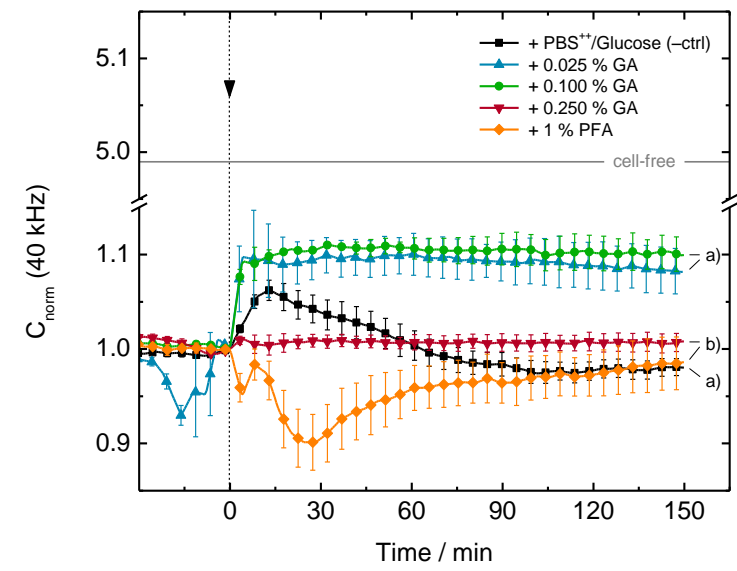

B

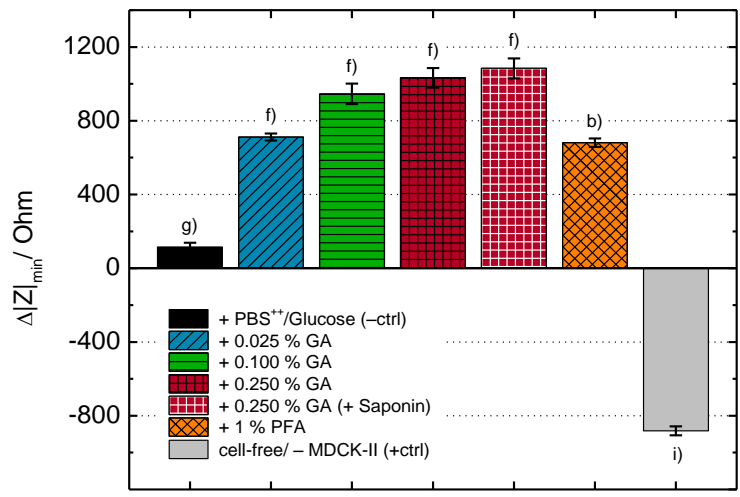

D

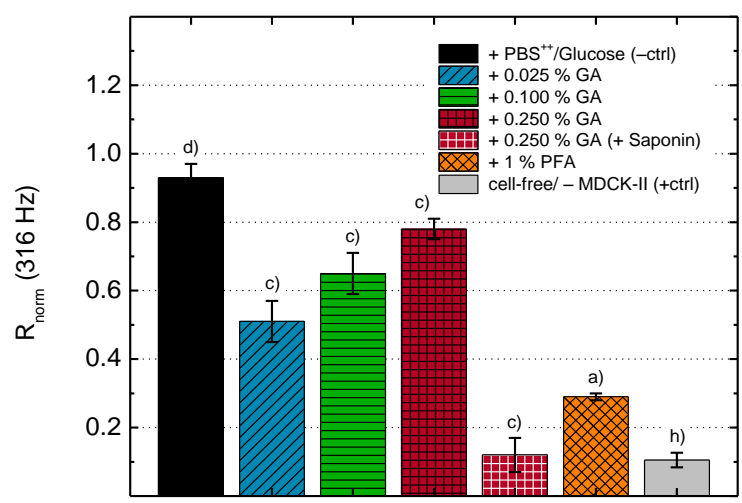

$\mathbf{F}$

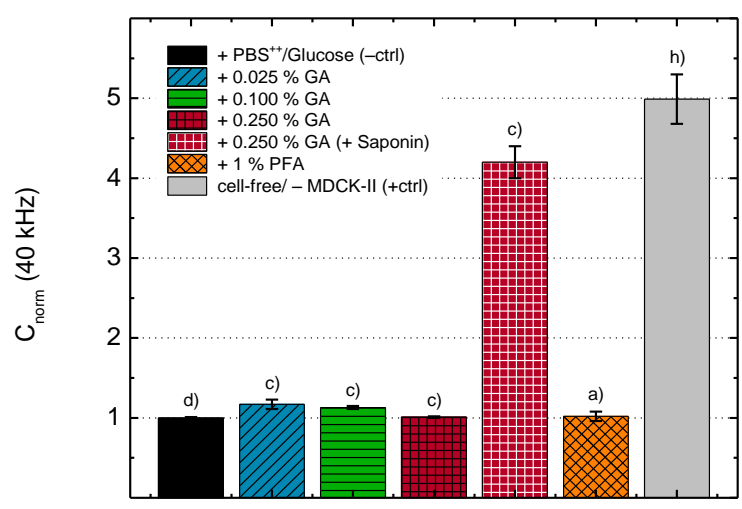

Fig. 5-21 OCM-ECIS sensor responses upon treatment of a confluent MDCK-II cell layer with different concentrations of glutaraldehyde (GA) and paraformaldehyde (PFA). Averaged time courses (left) and bar graphs of mean values $3 \mathrm{~h}$ after treatment (right) of (A), (B) $\Delta|Z|_{\text {min }}$ (C), (D) $R_{\text {norm }}$ at $316 \mathrm{~Hz}$, and (E), (F) $C_{\text {norm }}$ at $40 \mathrm{kHz}$ are shown. Cells were exposed to $0.025 \% \mathrm{GA}(-$; VIA ), $0.100 \%$ GA $(\rightarrow-$; control (-ctrl), PBS ${ }^{++}$buffer was exchanged $(-;-$). Values of the cell-free sensor immersed in SFM only $(\square)$ serve as a reference level (+ctrl) and are indicated by a grey horizontal line in $A$, $C$, and E. Parameter values after Saponin treatment are exemplary shown for the cell layer that has been previously fixated with $0.25 \% \mathrm{GA}$ (册). $\Delta|Z|_{\text {min }}$ values in $\mathrm{A}$ and $\mathrm{B}$ are obtained from pooled $\mathrm{OCM}_{1}$ and $\mathrm{QCM}_{2}$ data sets. Displayed data points of time courses are reduced for sake of clarity. $\left(\text { mean } \pm \mathrm{SEM} ; \mathrm{N}={ }^{\mathrm{a})} 2,{ }^{\mathrm{b})} 4,{ }^{\mathrm{c})} 5,{ }^{\mathrm{d})} 7,{ }^{\mathrm{e})} 8,{ }^{\mathrm{f}}{ }_{10},{ }^{\mathrm{g}}\right)_{14},{ }^{\mathrm{h}}{ }_{21},{ }^{\mathrm{i}} 42$ ). 
When applying $1 \%$ PFA instead of GA for protein cross-linking, the decrease of $R_{\text {norm }}$ is significantly different. There is no biphasic but a continuous decrease in the resistance at $316 \mathrm{~Hz}$. The total decrease of $R_{\text {norm }}$ is significantly slower for PFA ( $t_{1 / 2}=11.3 \mathrm{~min}$ ) than for GA treatments ( $\left.t_{1 / 2} \approx 3 \mathrm{~min}\right)$. However, the resistance reduction is remarkably bigger upon PFA- compared to GA-mediated cell fixation. $R_{\text {norm }}$ is reduced to $0.29( \pm 0.01) 3 \mathrm{~h}$ after adding PFA. Stationary levels of $R_{\text {norm }}=0.31( \pm 0.05)$ are attained after $18 \mathrm{~h}$ of PFA incubation ( $\mathrm{N}=3$ ). Membrane permeabilization by Saponin after cross-linking cellular proteins causes a complete reduction of the electrical resistance at $316 \mathrm{~Hz}$. This is exemplarily shown in Fig. 5-21 D for cell layers previously fixated with $0.25 \%$ GA. After Saponin treatment, $R_{\text {norm }}$ drops to $0.12( \pm 0.05)(\mathrm{N}=5)$, which is very close to values of the cell-free reference $\left(R_{\text {norm }}=0.11( \pm 0.02) ; \mathrm{N}=21\right)$.

In contrast to responses of oscillation impedance and electrochemical resistance, changes of the electrochemical capacitance at $40 \mathrm{kHz}$ upon cell fixation are very low and almost negligible (Fig. 5-21 $\mathrm{E}$, F). Capacitance values slightly increase to stationary levels within $\sim 5 \mathrm{~min}$ at GA concentrations of $0.025 \%\left(C_{\text {norm }}=1.17( \pm 0.06)\right)$ and $0.1 \%\left(C_{\text {norm }}=1.13( \pm 0.02)\right)$, whereas $C_{\text {norm }}$ remains at initial values upon fixation for $0.25 \% \mathrm{GA}\left(C_{\text {norm }}=1.01( \pm 0.01)\right)($ mean $\pm S E M ; N=5)(F i g .5-21 F)$. This is likewise observable for PFA-fixated cells, exhibiting $C_{\text {norm }}=1.02( \pm 0.06)$ after $3 \mathrm{~h}$ and $C_{\text {norm }}=1.01( \pm 0.04)$ after $18 \mathrm{~h}$ of exposure $(\mathrm{N}=3)$. Hence, there is no or almost no change in the time courses of high-frequency current through the cell layer due to protein cross-linking by either GA or PFA. Saponin treatment of the cell layers after protein cross-linking causes a steep increase of $C_{\text {norm }}$ to almost levels of the cell-free sensor $\left(C_{\text {norm }}=5.0( \pm 0.3) ; \mathrm{N}=21\right.$ ) (cf. Fig. 5-20 C). This is again exemplarily shown in the bar plot in Fig. 5-21 $\mathrm{F}$ for cell layers fixated by $0.25 \% \mathrm{GA}$ prior to Saponin exposure (埥). Capacitance values are drastically increased to $C_{\text {norm }}=4.2( \pm 0.2)(N=5)$, reflecting the opening of transcellular current pathways due to membrane perforation. Cellular fragments and membrane structures not washed away and remaining on the WE cause capacitances to not completely reach values of the cell-free sensor.

\subsubsection{Stimulating Intracellular Signaling Cascades}

Previous sections presented the application of the newly developed OCM-ECIS sensors for either cell attachment and spreading assays of two different cell lines and at various conditions or for assays testing the influence of xenobiotics on the viscoelastic and electric properties of confluent cell layers. Both seeding of cells on QCM-ECIS sensor surfaces and the drastic impacts of cytoskeleton disrupting drugs or protein cross-linking agents on cellular structures were found to be associated with profound electrical and mechanical changes at the substrate surface and, thus, considerable OCM and ECIS sensor responses. Besides these massive interventions into specific structural elements, cell layers were nondestructively stimulated by increased intracellular concentrations of adenosine $3^{\prime}, 5^{\prime}$-cyclic monophosphate (cAMP).

Cyclic AMP is a well-known second messenger involved in intracellular signal transduction, which is essential in the phosphorylation of various proteins via the CAMP-dependent protein kinase (Protein kinase A, PKA). PKA (de)activates various proteins important in metabolism, including the glycogen

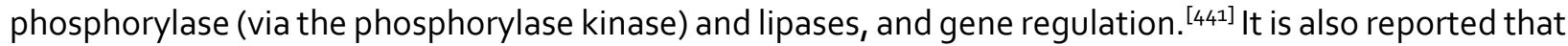
the cell permeable CPT-cAMP stimulates ion-channel activation. ${ }^{[422]}$ Various studies demonstrated that TJ permeability and transepithelial barrier resistance as well as changes in the actin cytoskeleton structures are regulated by cAMP-dependent mechanisms. With increased intracellular cAMP a tightening of intercellular junctions was found for some cells ${ }^{[314,429,443-450]}$ whereas a decrease of the transepithelial barrier and an increased TJ permeability was found for others [451-453]. The actin cytoskeleton is suggested to play an important role in cAMP-regulated epithelial permeability. Duffey et 
al. ${ }^{[455]}$ and Bensch et al. ${ }^{[454]}$ found changes in microfilament structures of epithelial cells and Stelzner et al. ${ }^{[446]}$ reported on a quantitative rise of $\mathrm{f}$-actin after increasing the intracellular CAMP concentration. Ohta et al. ${ }^{[455]}$ showed that PKA-induced phosphorylation of monomeric actin reduces its polymerizability and cell migration and the underlying actin cytoskeleton dynamics are regulated via CAMP/ PKA as well ${ }^{\left[45^{6]}\right.}$. However, there is no general rule of structural and accompanying electrical and/ or mechanical cell responses as a consequence of changes in the cAMP concentration. Changes of cytomechanics (cytoskeleton), cell morphology, and cell-adhesion properties may turn out differently and presumably depend on the current cellular state, the cell cycle, the origin of the tissue, and the species.

The developed QCM-ECIS sensors allow for testing the indirect effects of CAMP on both mechanical and electrical properties, simultaneously, non-invasively, and in time-resolved manner. OCM-ECIS whole-cell biosensing of the membrane permeable CAMP-analogue 8-CPT-CAMP (8-(4-Chlorophenylthio)-CAMP) was performed on confluent NRK cell layers.

Fig. 5-22 illustrates typical time courses of the OCM parameter $\Delta|Z|_{\min }$ (Fig. 5-22 A), and of the ECISmode parameters $R_{\text {norm }}\left(-\right.$ in Fig. 5-22 C) and $C_{\text {norm }}(--$ in Fig. 5-22 C) upon seeding NRK cells on the QCM-ECIS sensor substrates (" $\nabla$ NRK") and stimulation of the confluent cell layer with 8-CPT-cAMP the day after ("V $\downarrow 100 \mu \mathrm{M}$ CPT-cAMP"). In this test series, the most sensitive frequencies of the sensors to NRK cells in ECIS-mode were determined to $5 \mathrm{kHz}$ for the resistance and to $50 \mathrm{kHz}$ for the capacitance. Time courses are normalized to the last values before cell inoculation. Time courses also show cell responses to the subsequent withdrawal of CPT-CAMP, $\sim 7 \mathrm{~h}$ after addition, achieved by completely exchanging the medium above the cell layer against medium lacking the second messenger analogue (" $\nabla \uparrow 100 \mu \mathrm{M}$ CPT-CAMP"). The plots on the right hand side in Fig. 5-22 illustrate in a 3-D representation the impedance and phase shift spectra from OCM-mode recordings (Fig. 5-22 B), and the resistance and capacitance spectra from ECIS-mode recordings (Fig. 5-22 D) at selected points of the experiment. These are indicated in the time courses in Fig. 5-22 A, C. Spectra are shown for (1) the cell-free QCMECIS sensor immersed in SFM, (2) the NRK cell-covered sensor, and cells treated with $100 \mu \mathrm{M}$ 8-CPTcAMP for (3) $10 \mathrm{~min},(4) 30 \mathrm{~min},(5) \mathrm{h}$, and (6) $4 \mathrm{~h}$. Corresponding 2-D spectra (OCM: $|Z|(f), \varphi(f)$; ECIS: $|Z|(f), \varphi(f), R(f), C(f))$ are shown in $S / 22$ (p. 275). The time courses and spectra in Fig. 5-22 show distinct signal responses in both recording modes QCM and ECIS to the intracellular stimulation of cells with CPT-CAMP. Within $\sim 15$ min after its addition, values of $|Z|_{\min }$ and $R_{\text {norm }}$ (at $5 \mathrm{kHz}$ ) drop and $C_{\text {norm }}$ values (at $50 \mathrm{kHz}$ ) steeply increase first. In QCM and ECIS impedance spectra, this is reflected by the slight backward shift of curves towards cell-free sensor characteristics ( $2 \rightarrow 3$ in Fig. 5-22 B, D). However, these initial responses of all parameters are overcompensated within the following $\sim 30 \mathrm{~min} .|Z|_{\text {min }}, R_{\text {norm }}$, and $C_{\text {norm }}$ exhibit more pronounced long-term responses in the opposite direction and approximate stationary values within $\sim 1-4 \mathrm{~h}$ after injecting CPT-CAMP. In the 3-D OCM spectra (Fig. 5-22 B), the increase of oscillation damping is reflected by an increase of $|Z|_{\min }$ and a reduction of $|Z|_{\max }$ and $\varphi_{\max }$ and thus by an increased contraction of the resonance loop $(3 \rightarrow 6)$. The continuous decrease of $R_{\text {norm }}$ and increase of $C_{\text {norm }}$ is represented by a shift along the axis of the most sensitive frequencies in the 3-D ECIS spectra ( $3 \rightarrow 6$ in Fig. 5-22 D).

Hence, there are considerable cytomechanical and cell morphology changes due to the increased cAMP concentration in the cells. The time courses of the typical experiment also indicate the reversibility of cAMP-mediated stimulations of the mechanical and electrical properties of the cell layer. The kinetics 
and degree of $|Z|_{\text {min }}, R_{\text {norm }}$ and $C_{\text {norm }}$ responses of NRK cells towards $100 \mu$ M 8-CPT-CAMP stimulation and subsequent relaxation are described and quantified below on the basis of repeated experiments.

A

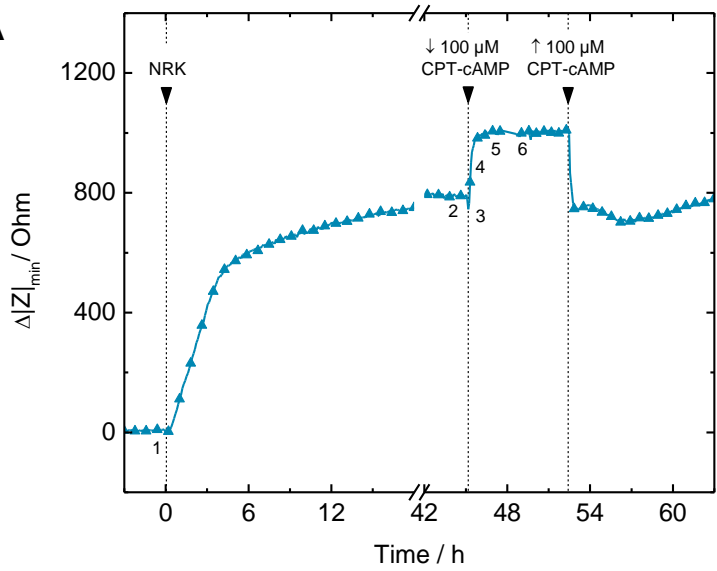

C

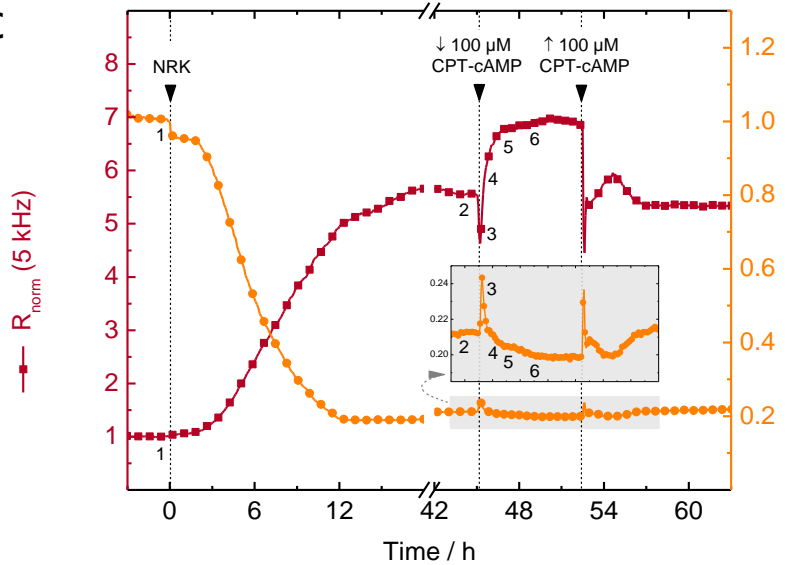

B

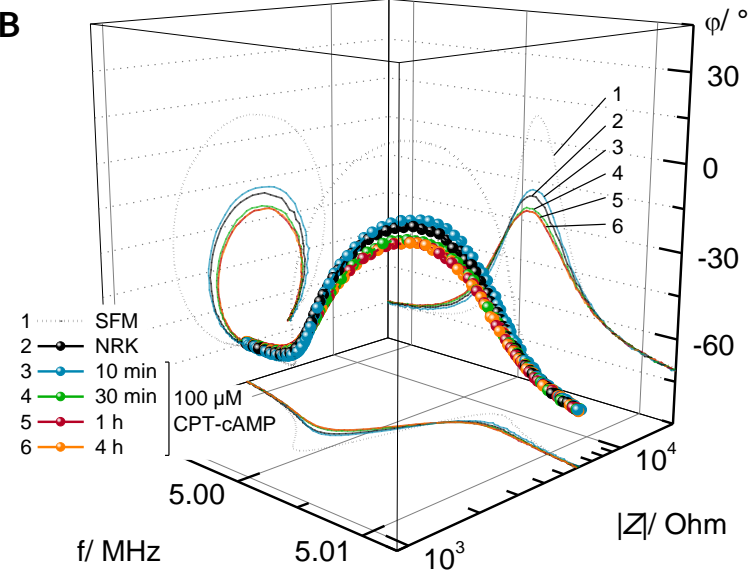

D

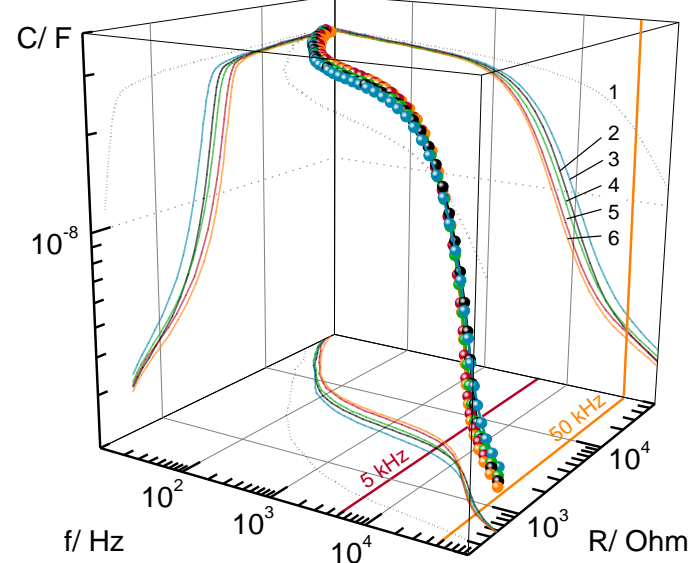

Fig. 5-22 Typical OCM-ECIS measurement of NRK cells seeded on OCM-ECIS substrates and subsequently treated with the membrane permeable cAMP-analogue 8-CPT-cAMP. Diagrams display the time courses (left) and the spectra (in 3-D) at defined points of the experiment (right), obtained from (A), (B) OCM-mode and (C), (D) ECIS-mode recordings. The time courses of $\Delta|Z|_{\min }, R_{\text {norm }}$ (at $5 \mathrm{kHz}$ ), and $C_{\text {norm }}$ (at $50 \mathrm{kHz}$ ) are normalized to the last value before cell inoculation. Arrows $(\boldsymbol{\nabla})$ mark the points of seeding the cells ("NRK"), of adding "100 $\mu \mathrm{M}$ CPTCAMP", and of finally exchanging medium containing CPT-CAMP against CPT-CAMP-free medium ("†100 $\mu \mathrm{M}$ CPT-CAMP"). 3-D impedance spectra of (B) OCM and (D) ECIS parameters are shown for the sensor (1) $20 \mathrm{~h}$ after SFM incubation, (2) $24 \mathrm{~h}$ after seeding cells to confluence, (3) $10 \mathrm{~min} /$ (4) $30 \mathrm{~min} /(5) \mathrm{h}$ and (6) $4 \mathrm{~h}$ after $100 \mu \mathrm{M}$ CPT-CAMP addition. These points are indicated in the time courses on the left. The most sensitive frequency of either parameter $(R: 5 \mathrm{~Hz}, C: 50 \mathrm{kHz})$ is indicated in the respective projection plane of the 3-D plots. Corresponding 2-D spectra $(|Z|(f)$, $\varphi(f), R(f), C(f))$ are illustrated in $S / 22$. Data points displayed in $\mathrm{A}$ and $\mathrm{C}$ are reduced for sake of clarity.

Fig. 5-23 summarizes the mean OCM-ECIS sensor responses obtained from a variety of replicate stimulation/ withdrawal experiments of NRK cells with $100 \mu \mathrm{M}$ CPT-CAMP (mean \pm SEM). Time courses of $\Delta|Z|_{\min }$ (Fig. 5-23 A), $R_{\text {norm }}$ at $5 \mathrm{kHz}$ (Fig. 5-23 C), and $C_{\text {norm }}$ at $50 \mathrm{kHz}$ (Fig. 5-23 E) are shown for cell stimulations (colored curves) and for an unstimulated reference (-ctrl; black curves). The time courses showing stimulation experiments are normalized to the last data point before injecting either $150 \mu \mathrm{L}$ of a 10-fold concentrated CPT-cAMP stock solution (dissolved in cell culture medium) or $150 \mu \mathrm{L}$ medium ( $\hat{=}-\mathrm{ctrl})$ to $1.35 \mathrm{~mL}$ medium in the measurement vessel. The time courses of CPT-cAMP withdrawal 
experiments are normalized in time to the point of complete medium exchange, and in the parameter value to the last data point before CAMP stimulation ( $\boldsymbol{\nabla}$ in Fig. 5-23 A, C, E). Values of the cell-free sensor immersed in SFM only $\left(|Z|_{\min }=1820( \pm 40) \mathrm{Ohm}(\mathrm{N}=20), R_{5 \mathrm{kHz}}=630( \pm 20) \mathrm{Ohm}(\mathrm{N}=7)\right.$, and $C_{50 \mathrm{kHz}}$ $=13.6( \pm 0.9) \mathrm{nF}(\mathrm{N}=7))$ serve as a reference $(+\mathrm{ctrl})$. They are indicated by grey horizontal lines in Fig. 5$23 \mathrm{~A}, \mathrm{C}, \mathrm{E}$. The bar graphs next to the time courses include data from time-resolved experiments as well as from endpoint measurements. They display average signal changes of $\Delta|Z|_{\text {min }}$ (Fig. 5-23 B), $R_{\text {norm }}$ (Fig. 5-23 D), and $C_{\text {norm }}$ (Fig. 5-23 F), after $4 \mathrm{~h}$ of cell exposure to $100 \mu \mathrm{M} \mathrm{CPT-cAMP} \mathrm{(} \square, \square, \square$ ),

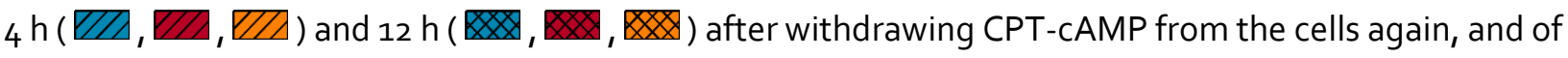
unstimulated ( $\mathbf{})$ and cell-free $(\square)$ references.

Initially after adding CPT-cAMP to the cells, $|Z|_{\min }$ drops from $2720( \pm 25) \mathrm{Ohm}(\mathrm{N}=20)$ within 8 min by $29( \pm 11)$ Ohm $(N=12)(-$ in Fig. $5-23 A)$. For electrochemical parameters, the maximum of this initial response peak is observed at $t=10 \mathrm{~min}$. At this point, the resistance ( - in Fig. $5-23 \mathrm{C}$ ) is reduced to $84( \pm 2) \%(\mathrm{~N}=4)$ of starting values $\left(R_{5 \mathrm{kHz}}\left(t_{0}\right)=3.72( \pm 0.14) \mathrm{kOhm} ; \mathrm{N}=7\right)$, and the capacitance ( - - in Fig. $5-23 \mathrm{E})$ is increased to $114( \pm 2) \%(\mathrm{~N}=4)$ with respect to starting values $\left(C_{50 \mathrm{kHz}}\left(t_{0}\right)=2.65( \pm 0.13) \mathrm{nF}\right.$; $N=7)$. Parameter values recover after these fast responses and overcompensate the initial spikes, passing starting levels again $15 \mathrm{~min}\left(\Delta|Z|_{\min }\right), 20 \mathrm{~min}\left(R_{\text {norm }}\right)$, and $45 \mathrm{~min}\left(C_{\text {norm }}\right)$ after adding CPT-CAMP. The recovery of signals and the long-term changes of parameters to stationary levels, respectively, occur with different kinetics. $|Z|_{\min }$ exhibits a maximal slope of $m=\Delta|Z|_{\min } / \Delta t=7.0( \pm 0.5) \mathrm{Ohm} \cdot \mathrm{min}^{-1}$ $(\mathrm{N}=12)$ at $t_{m}=20 \mathrm{~min}$, whereas the maximal slopes of $R_{\text {norm }}$ and $C_{\text {norm }}$ occur slightly earlier at $t_{m}=15 \mathrm{~min}$. There, $R$ increases with $m=\Delta R / \Delta t=53.7( \pm 1.9) \mathrm{Ohm} \cdot \mathrm{min}^{-1}(\mathrm{~N}=4)$ and $C$ decreases with $m=\Delta C / \Delta t=-24.2( \pm 0.7) \mathrm{pF} \cdot \mathrm{min}^{-1}(\mathrm{~N}=4)$.

Time points $t_{1 / 2}$ of half-maximal signal changes differ much clearer for the analyzed parameters. The mechanical changes at the sensor interface due to the addition of CPT-CAMP are finished much faster $\left(t_{1 / 2}\left(\Delta|Z|_{\text {min }}\right)=25 \mathrm{~min}\right)$ than alterations of the dielectric cell layer properties. Half-maximal changes in $R_{\text {norm }}$ are attained within $t_{1 / 2}=45 \mathrm{~min}$, whereas it takes up to $t_{1 / 2}=105 \mathrm{~min}$ for $C_{\text {norm }}$ values to reach this point (cf. Fig. 5-23 A, C, E). Hence, the different parameters likely report on different, asynchronous cellular reactions and processes occurring due to the stimulation. Four hours after adding CPT-CAMP to the NRK cell layer stationary signals are reached, which amount to $\Delta|Z|_{\min }=171( \pm 9) \mathrm{Ohm}(\mathrm{N}=12)$, $R_{\text {norm }}=123.5( \pm 1.3) \%(\mathrm{~N}=4)$, and $C_{\text {norm }}=93.4( \pm 0.9) \%(\mathrm{~N}=4)$. Signals of unstimulated reference cells do not show any of the responses described for stimulated cells, exhibiting stable signals in $|Z|_{\text {min }}(\star-$ in Fig. 5-23 A), $R_{\text {norm }}\left(-\right.$ in Fig. 5-23 C), and $C_{\text {norm }}(-$ in Fig. 5-23 E) throughout the entire period of monitoring. Reference values at $4 \mathrm{~h}$ after $0.15 \mathrm{~mL}$ medium exchange are $\Delta|Z|_{\min }=1.4( \pm 7.7) \mathrm{Ohm}$ $(\mathrm{N}=10), R_{\text {norm }}=100( \pm 4) \%(\mathrm{~N}=4)$, and $C_{\text {norm }}=101.0( \pm 1.0) \%(\mathrm{~N}=4)$.

Withdrawing of previously injected CPT-CAMP, by completely exchanging the medium in the measurement vessel, causes a rapid recovery of signals to parameter levels as before the stimulation (Fig. 5-23 A, C, E). After removing the stimulant, there are considerable fluctuations in the values for $\sim 4 \mathrm{~h}$. This can be observed especially for the electrochemical sensor parameters. After $4 \mathrm{~h}$, these approximate values of $R_{\text {norm }}=96.1( \pm 1.1) \%$ and $C_{\text {norm }}=102( \pm 2) \%$ (both $\mathrm{N}=3$ ). These values only slightly change afterwards and attain stable levels of $R_{\text {norm }}=94.2( \pm 0.6) \%$ and $C_{\text {norm }}=103.8( \pm 1.1) \%$ (both $\mathrm{N}=3$ ) after $12 \mathrm{~h}$ (Fig. 5-23 D, E). Within $4 \mathrm{~h}$ after CPT-cAMP removal, $\Delta|Z|_{\text {min }}$ decreases by $-42( \pm 16) \mathrm{Ohm}(\mathrm{N}=8)$ little but significantly below the value before stimulation. It recovers afterwards within further $4-8 \mathrm{~h}$ to almost exactly the starting value, exhibiting $\Delta|Z|_{\min }=2( \pm 21) \mathrm{Ohm}(\mathrm{N}=6)$ after $12 \mathrm{~h}$ (Fig. 5-23 B). 
A

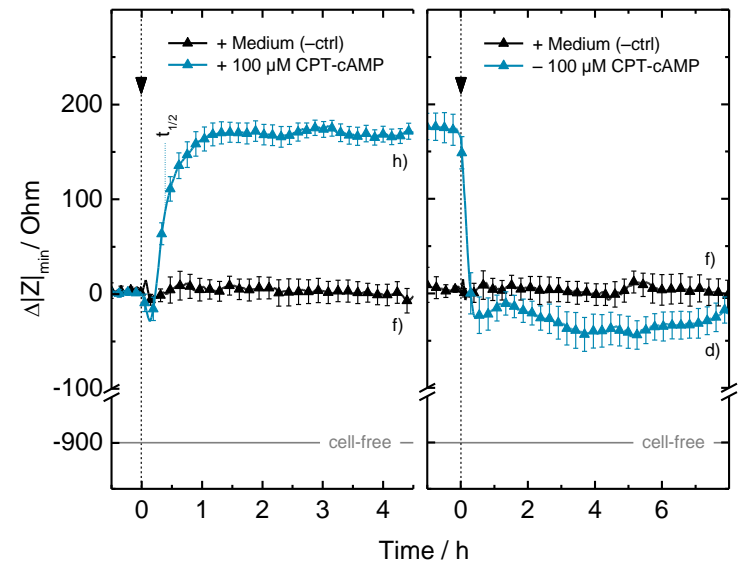

C

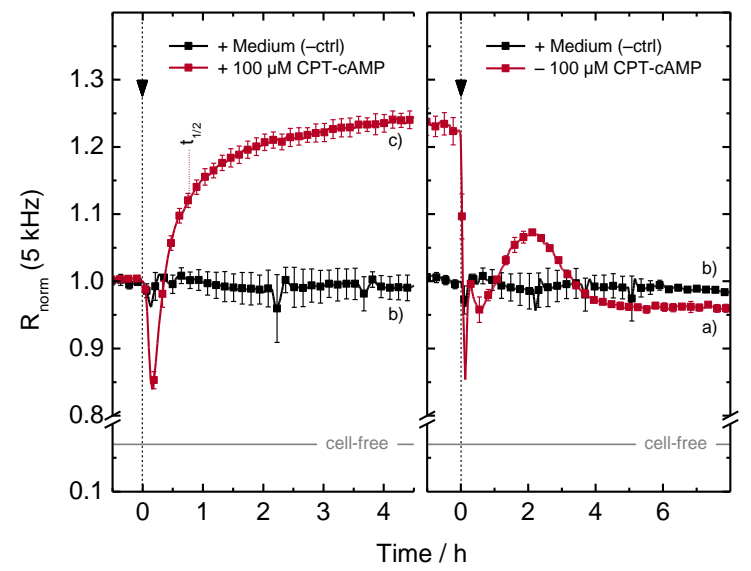

E

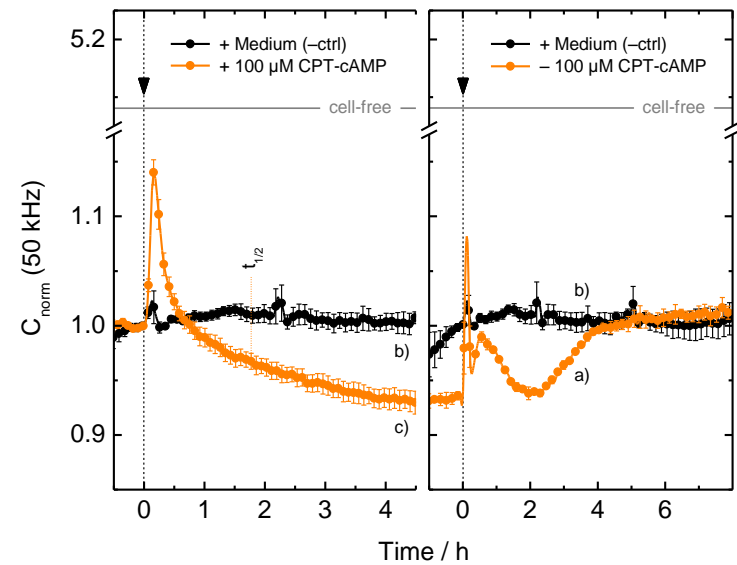

B

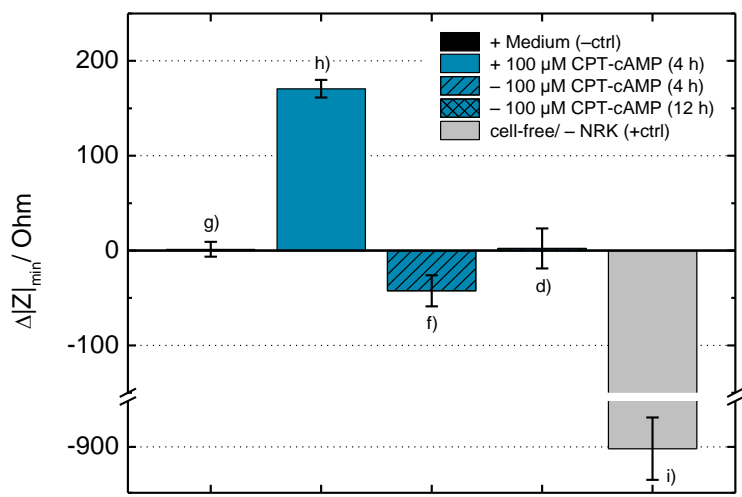

D

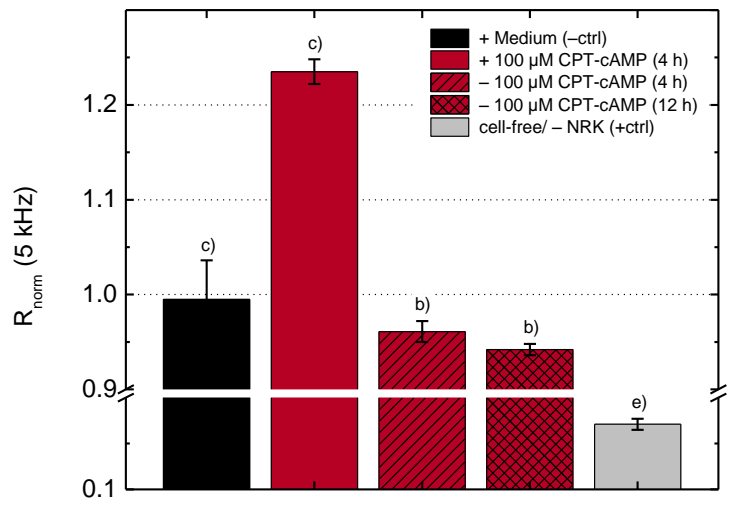

$\mathbf{F}$

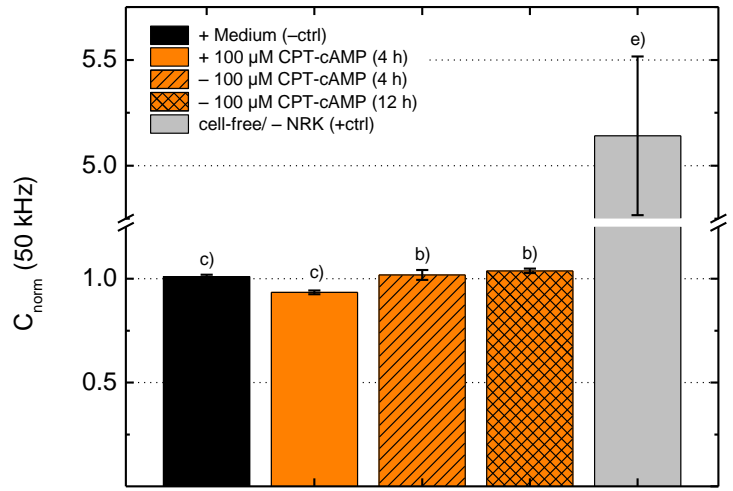

Fig. 5-23 OCM-ECIS sensor responses after injecting $100 \mu \mathrm{M}$ of the membrane permeable 8-CPT-cAMP to a confluent NRK cell layer. Averaged curves show time-resolved changes of (A) $\Delta|Z|_{\text {min }}$ (C) $R_{\text {norm }}$ at $5 \mathrm{kHz}$, and (E) $C_{\text {norm }}$ at $50 \mathrm{kHz}$ for stimulated cells $(-,--,-\infty)$ and for unstimulated references cells $(-,-\rightarrow,-\infty)$, normalized to the last value before treatment. Time courses next to that illustrate averaged parameter changes upon removing CPT-CAMP from the cells again. For negative controls (-ctrl), medium was exchanged against stimulant-free medium. Values of the cell-free sensor, immersed in SFM only, serve as a reference (+ctrl) and are indicated by grey horizontal lines in $\mathrm{A}, \mathrm{C}$, and E. Points $t_{1 / 2}$ of half-maximal signal changes are indicated The bar graphs next to the time courses show mean values at selected points of the experiment. They contain data from time-resolved monitoring and endpoint measurements of (B) $\Delta|Z|_{\text {min }}$ (D) $R_{\text {norm }}$ and (F) $C_{\text {norm }}$. Selected points are $4 \mathrm{~h}$ after adding CPT-CAMP $(\square, \square, \square)$ or medium only $(\square)$, and $4 \mathrm{~h}(\mathbb{Z}, \mathbb{Z}, \mathbb{Z}, \mathrm{Z})$ ) and $12 \mathrm{~h}$ ( ) after withdrawing CPT-cAMP from the cells. Values of the cell-free sensor (+ctrl) are displayed, too $(\square) . \Delta|Z|_{\min }$ values are obtained from pooled $\mathrm{OCM}_{1}$ and $\mathrm{OCM}_{2}$ data sets. Data points in time courses are reduced for sake of clarity. $\left(\right.$ mean $\pm \mathrm{SEM} ; \mathrm{N}={ }^{\mathrm{a})} 2,{ }^{\mathrm{b}} 3{ }_{3}{ }^{\mathrm{cl}} 4,{ }^{\mathrm{d})} 6,{ }^{\mathrm{e})} 7,{ }^{\mathrm{f}} 8,{ }^{\mathrm{g}}{ }_{10},{ }^{\mathrm{h})} 12,{ }^{\mathrm{i}} 20$ ). 


\subsubsection{Electric Manipulation and Double-mode Impedance Analysis (ELPO-ECIS-QCM)}

The exposure of cells to an invasive electrical field and the accompanying manipulation is a biophysical approach, used in many fields of cellular research. Besides the electric field-mediated dielectric polarization of cells, applied for rotation and translation of suspended cells ${ }^{[457]}$, non-invasive electric fields were also applied for the reorientation and elongation of anchored cells as well as for assays on electrotaxis, the phenomenon of electric field-driven migration of adherent cells ${ }^{[458,459]}$. Coplanar electrode layouts like in the commercial ECIS substrates, which are similar to that of the sensors presented in this work, have been applied in many studies for the specific, regioselective in situ electroporation (ELPO), electrofusion, and electrical wounding of cells on the working electrode by AC pulses. ${ }^{[7,99,118-120]}$ These coplanar electrodes allow for instantaneous monitoring of cell properties after the application of short electric field pulses, by non-invasive impedance analysis. Hence, the growth substrate-integrated coplanar electrodes provide a powerful and sensitive system for identifying and controlling the best electrical pulse parameters for each approach and for various adherent cell lines. ${ }^{[7]}$ Moreover, post-pulse non-invasive monitoring of cell viability, cell shape alterations, cell vitality/ motility, and barrier resistance of the cell layer by impedance analysis (ELPO-ECIS) provides an actuatorsensor combination for a multitude of wound healing, cell stimulation, cytotoxicity, and drug delivery assays. ${ }^{[7,99,199,312,460-462]}$ Electroporation in the presence of high extracellular concentrations of any substance or drug allows molecules to diffuse along the concentration gradient into the cells during the period of pore openings. This enables the direct and not membrane-enclosed presence of molecules that are actually not membrane-permeable in the cytoplasm of living cells. A brief introduction into the ELPO principle is given in 3.4.5 (p. 59 ff.). On the basis of the newly developed OCM-ECIS sensors it is now possible to monitor not only the dielectric changes on the working electrode but also changes in the micromechanical properties close to the sensor surface. The combination of ELPO with the QCM-ECIS sensors merges the benefits of regioselective electroporation, electrofusion, and electric wounding with the advantages of a multi-parametric cell-based monitoring system.

In a proof of concept study the newly developed OCM-ECIS sensor (section 5.1) was tested for its capability of electrical field pulse actuation and instantaneous, combined label-free ECIS and QCM sensing of adherently grown cell layers (ELPO-ECIS-OCM). The combination of in situ electroporation of cells and subsequent analysis of cellular mechanics by means of OCM measurements has not been reported in literature before. For ELPO-ECIS-OCM measurements, the experimental setup for sensing (Fig. 3-18) was extended by an oscilloscope and a frequency generator as AC voltage source for electric field pulse applications. The setup is illustrated in Fig. 3-20 (p. 61). For the electrical actuation-sensing experiments the standard OCM-ECIS sensor layout (3000 rpm, WE- $\varnothing=0.5 \mathrm{~mm}$; cf. section 5.1.2) was applied, which also was used in all cell-based OCM-ECIS assays presented above. Confluent NRK cell layers on the substrates were exposed to plasma membrane-impermeable, FITC-labeled dextran molecules (FITC-dextran; $\mathrm{M}=250 \mathrm{kDa}$ ). The cell layer properties upon FITC-dextran addition as well as after electrical pulsing were continuously analyzed by OCM- and ECIS-mode recordings. Moreover, the loading efficiency of cells with FITC-dextran molecules by ELPO was routinely analyzed by fluorescence microscopic investigations of the electroporated cells. 
A

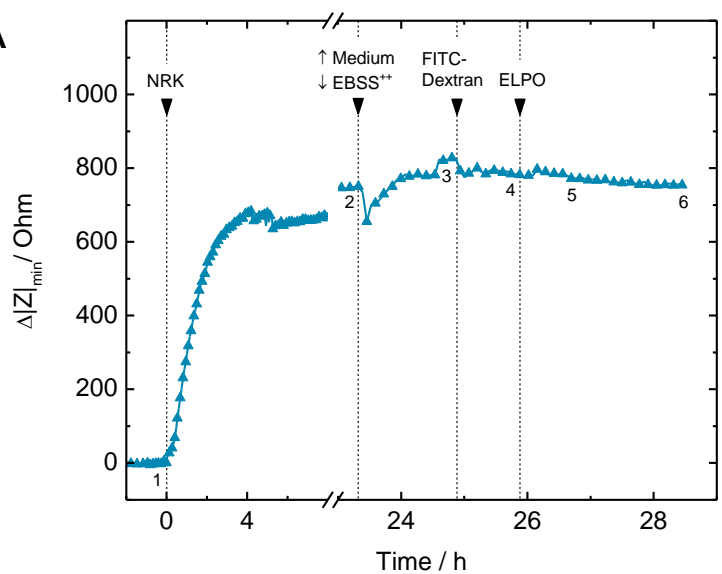

C

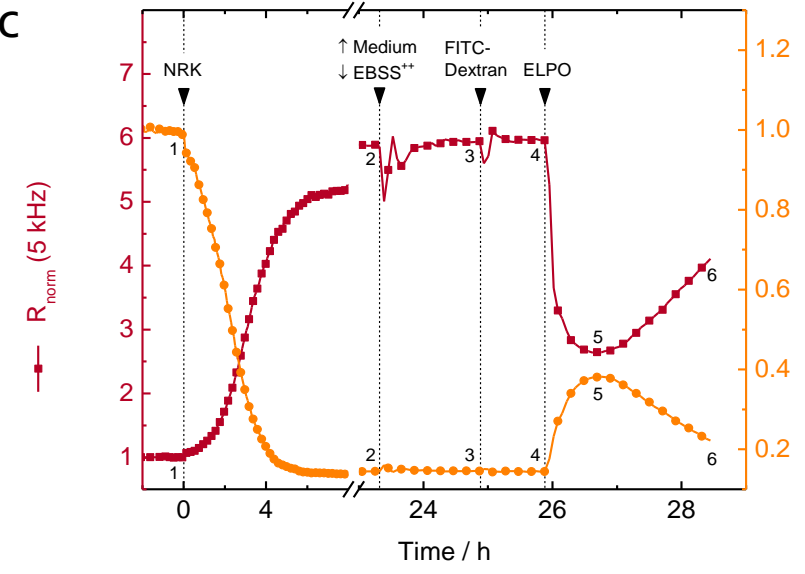

B

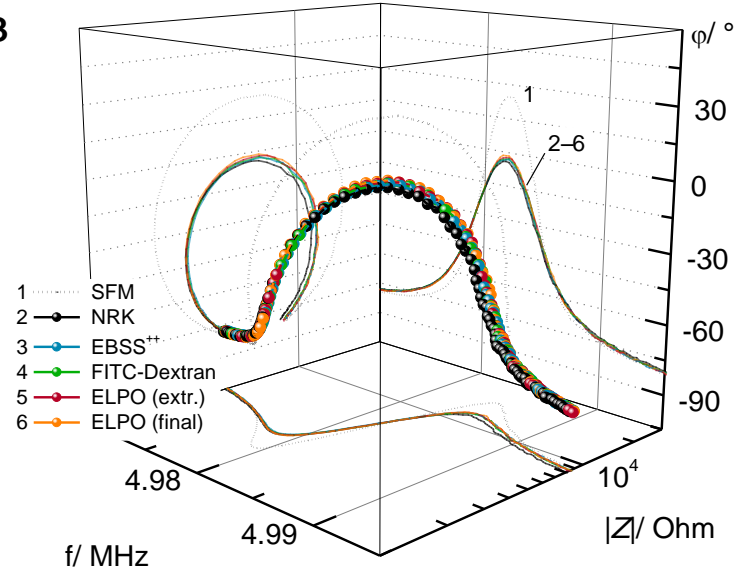

D

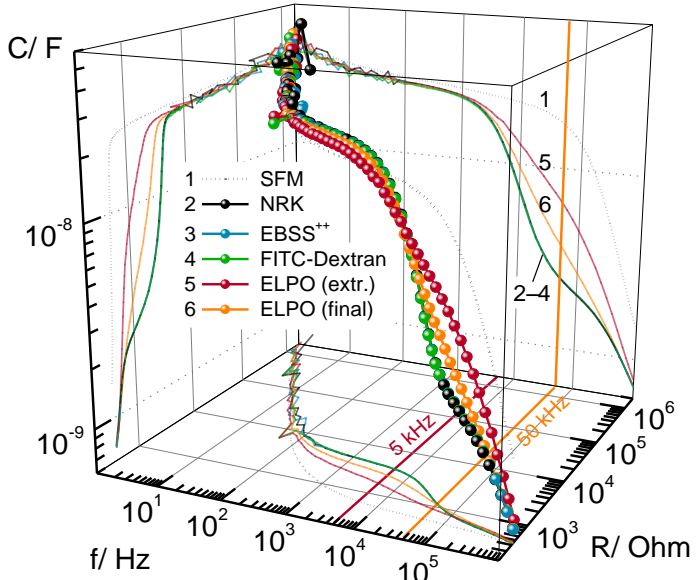

Fig. 5-24 Typical ELPO-ECIS-OCM experiment of NRK cells cultured on standard OCM-ECIS substrates in the presence of membrane-impermeable, FITC-labeled dextran (250 kDa). Diagrams display the time courses (left) and the spectra (in 3-D) at defined points of the experiment (right), obtained from (A), (B) OCM-mode recordings of $\mathrm{OCM} 1$ and (C), (D) ECIS-mode recordings. The time courses of $\Delta|Z|_{\text {min, }} R_{\text {norm }}$ (at $5 \mathrm{kHz}$ ), and $C_{\text {norm }}$ (at $50 \mathrm{kHz}$ ) are normalized to the last value before cell inoculation. Arrows ( $\boldsymbol{\nabla})$ mark the points of seeding the cells ("NRK"), of exchanging cell culture medium against EBSS ${ }^{++}$buffer (" $\uparrow$ Medium $\downarrow$ EBSS $^{++\prime}$ ), of adding extracellularly $1 \mathrm{~g} \mathrm{~L}^{-1}$ FITC-dextran, and of applying an AC pulse ("ELPO") of defined magnitude ( $4 \mathrm{~V})$, duration (200 ms), and frequency (40 kHz). 3-D impedance spectra of (B) QCM1 and (D) ECIS parameters are shown for the sensor (1) $20 \mathrm{~h}$ after SFM incubation, (2) $23 \mathrm{~h}$ after seeding cells to confluence, (3) after EBSS ${ }^{++}$equilibration, (4) after FITC-dextran addition, (5) at extreme values of parameter changes $\sim 1 / 2-1 \mathrm{~h}$ after ELPO, and (6) at final recording values $\sim 2^{1 / 2} \mathrm{~h}$ after ELPO. Corresponding 2-D spectra $(|Z|(f), \varphi(f), R(f), C(f))$ are illustrated in $S / 23$. Data points displayed in $A$ and $C$ are reduced for sake of clarity.

Fig. 5-24 illustrates a typical ELPO-ECIS-OCM experiment, by means of the time courses of the OCMmode parameter $\Delta|Z|_{\text {min }}$ of OCM1 (Fig. 5-24 A) and of the ECIS-derived parameters $R_{\text {norm }}$ and $C_{\text {norm }}$ (Fig. 5-24 C). OCM- and ECIS-mode recordings continuously monitored the mechanical and electrochemical properties of the cell layer throughout the entire experiment. The diagrams next to the time traces illustrate in 3-D representation the underlying spectra of parameters at selected points of the experiment. Numbers at the time courses mark the points in time that correspond to the spectra shown in Fig. 5-24 B, D. The sensor has been pre-incubated with SFM for $20 \mathrm{~h}$ for equilibration purposes (1). At time zero, SFM was exchanged against a NRK cell suspension of $\sim 350000$ cells $\mathrm{mL}^{-1}$ (" $\nabla$ NRK"). The day after seeding, when cells have formed a confluent monolayer (2), the cell culture medium was replaced by $800 \mu \mathrm{L}$ of pre-warmed EBSS ${ }^{++}$buffer (" $\nabla \uparrow$ Medium $\downarrow$ EBSS $^{++\prime}$ ). After signal equilibration in EBSS ${ }^{++}$for 
roughly two hours (3), $400 \mu \mathrm{L}$ of buffer were removed from the measurement vessel and replaced by $400 \mu \mathrm{LBSS}^{++}$buffer containing FITC-dextran $(250 \mathrm{kDa})$, resulting in a final concentration of $1 \mathrm{~g} \mathrm{~L}^{-1} \mathrm{FITC}$ dextran. Only marginal signal changes in QCM and ECIS parameters can be observed upon cell exposure to $1 \mathrm{~g} \mathrm{~L}^{-1}$ extracellular FITC-dextran. After equilibration (4), the conducting wires of the two microbalance electrodes facing the cells were connected to the frequency generator (cf. Fig. 3-20, p. 61) and an AC pulse of defined magnitude, duration, and frequency was manually applied to the cells attached to the working electrode (" $\nabla$ ELPO"). After pulsing, the respective cables were replugged and dual impedance sensing in QCM- and ECIS-mode was instantaneously continued. The electrical properties of the cell layer show immediate and drastic changes after pulse application (Fig. $5-24 \mathrm{C}$ ), whereas no significant effect can be observed in the damping of quartz oscillations of $\mathrm{QCM}_{1}$ (Fig. 5-24 A). This indicates a considerable change in cell morphology due to the electroporation pulse, however the cellular stiffness close to the substrate remains unaffected. Values of $R_{\text {norm }}$ and $C_{\text {norm }}$ reach extreme values of signal changes within $1 / 2-1 \mathrm{~h}$ after ELPO (5), and recover afterwards in the direction of values before ELPO (6).

Fig. 5-25 shows averaged, time-resolved $\Delta|Z|_{\text {min }}$ responses (Fig. 5-25 A) and electrochemical $R_{\text {norm }}$ and $C_{\text {norm }}$ time courses (Fig. 5-25 B, C) (mean \pm SEM; N = 2), recorded from NRK cell-covered OCM-ECIS sensors before and immediately after AC pulse application (" $\nabla$ ELPO"). The displayed OCM curves in Fig. 5-25 A show separate data sets of $\mathrm{OCM}_{1}$ and $\mathrm{OCM} 2$ recordings, since the voltage drops exclusively across the membrane of cells that are attached to the small WE of the coated QCM1 resoantor. After ELPO, the cell populations on $\mathrm{OCM}_{1}$ and $\mathrm{OCM}_{2}$ can be, therefore, no more considered as equal. The most sensitive frequencies of the ECIS-derived resistance and capacitance for NRK cells on OCM-ECIS substrates were determined to $5 \mathrm{kHz}$ and $50 \mathrm{kHz}$, respectively (indicated in the planes of the $3-\mathrm{D}$ spectra in Fig. 5-24 D). The time courses in Fig. 5-25 are referenced in time and magnitude to the last data point before ELPO. The cells were prepared according to the procedure of the typical measurement described above in the forerun of electroporation. Cells were electroporated in the presence of $1 \mathrm{~g} \mathrm{~L}^{-1}$ extracellular FITC-dextran $\left(250 \mathrm{kDa}\right.$ ) with a $40 \mathrm{kHz}$ AC pulse of $200 \mathrm{~ms}$ in duration and of either $2 \mathrm{~V}_{\mathrm{rms}}(-)$ or $4 \mathrm{~V}_{\mathrm{rms}}$ $(-)$ in magnitude $\left(V_{\text {rms }}=\right.$ root mean square voltage). Cells that were exposed to FITC-dextran without subsequent ELPO serve as negative control (-ctrl; - in Fig. 5-25 A $-\mathrm{C})$. Absolute mean values $( \pm \mathrm{SEM})$ of QCM and ECIS parameters at the point before pulse application amount to $|Z|_{\min }=2550( \pm 50) \mathrm{Ohm}$ $(\mathrm{N}=16), R_{5 \mathrm{kHz}}=3.27( \pm 0.16) \mathrm{kOhm}(\mathrm{N}=7)$, and $C_{50 \mathrm{kHz}}=3.06( \pm 0.17) \mathrm{nF}(\mathrm{N}=7)$. Values of the cell-free sensor, related to the respective cell-covered value, serve as a reference $(+\mathrm{ctrl})$. These are indicated by grey horizontal lines in the diagrams Fig. 5-25 A - C and amount to $\Delta|Z|_{\min }=-730( \pm 40) \mathrm{Ohm}(\mathrm{N}=12)$, $R_{\text {norm }}=21.4( \pm 3.1) \%(\mathrm{~N}=6)$, and $C_{\text {norm }}=560( \pm 70) \%(\mathrm{~N}=6)$.

The time courses in Fig. 5-25 A show that the minimal impedance of the $\mathrm{OCM}_{1}$ oscillation is not significantly altered within $2.5 \mathrm{~h}$ after ELPO, with respect to cells on OCM1 in control experiments (- -) as well as with respect to cells of the same population that, however, are attached to the $\mathrm{QCM} 2$ resonator $(--,-\infty)$. By contrast, the pore formation of cells on the working electrode has a big impact on the ECIS parameters $R_{\text {norm }}$ (Fig. 5-25 B) and $C_{\text {norm }}$ (Fig. 5-25 C). After applying the short electric AC field with $2 \mathrm{~V}_{\text {rms }}$, resistance values immediately drop by $\sim 20 \%$ and the capacitance of the cell layer instantaneously increases by $\sim 20 \%$ on average (see inset in Fig. 5-25 C) within $\sim 10$ min. Afterwards, $R_{\text {norm }}$ and $C_{\text {norm }}$ completely recover again within $2.5 \mathrm{~h}$ to values of the confluent cell layer as before pulsing. ELPO of NRK cells on the OCM-ECIS substrates with a $4 \mathrm{~V}_{\text {rms }}$ pulse causes even stronger, transient impacts on the cell layer dielectrics. $R_{\text {norm }}$ decreases to $\sim 40 \%$ and $C_{\text {norm }}$ increases to $\sim 260 \%$ on average, with respect to initial values. However, these maximum and minimum values are attained later compared to the $2 \mathrm{~V}_{\text {rms }}$ pulse, at roughly 60 min after pulse application, and the recovery of signals in the direction of initial values takes significantly longer $(t \geq 2.5 \mathrm{~h})$ for the higher pulse amplitude. 
A
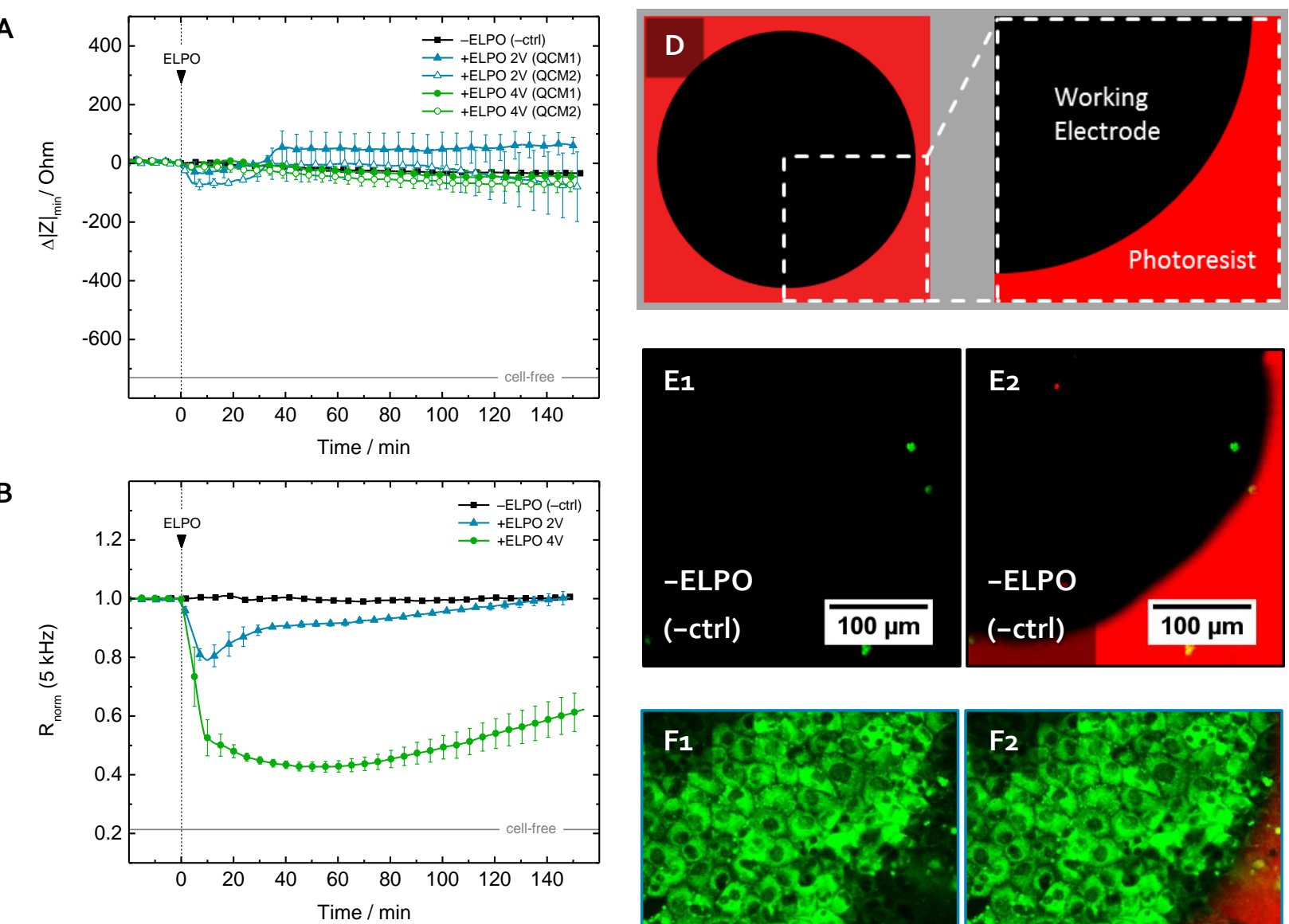

C
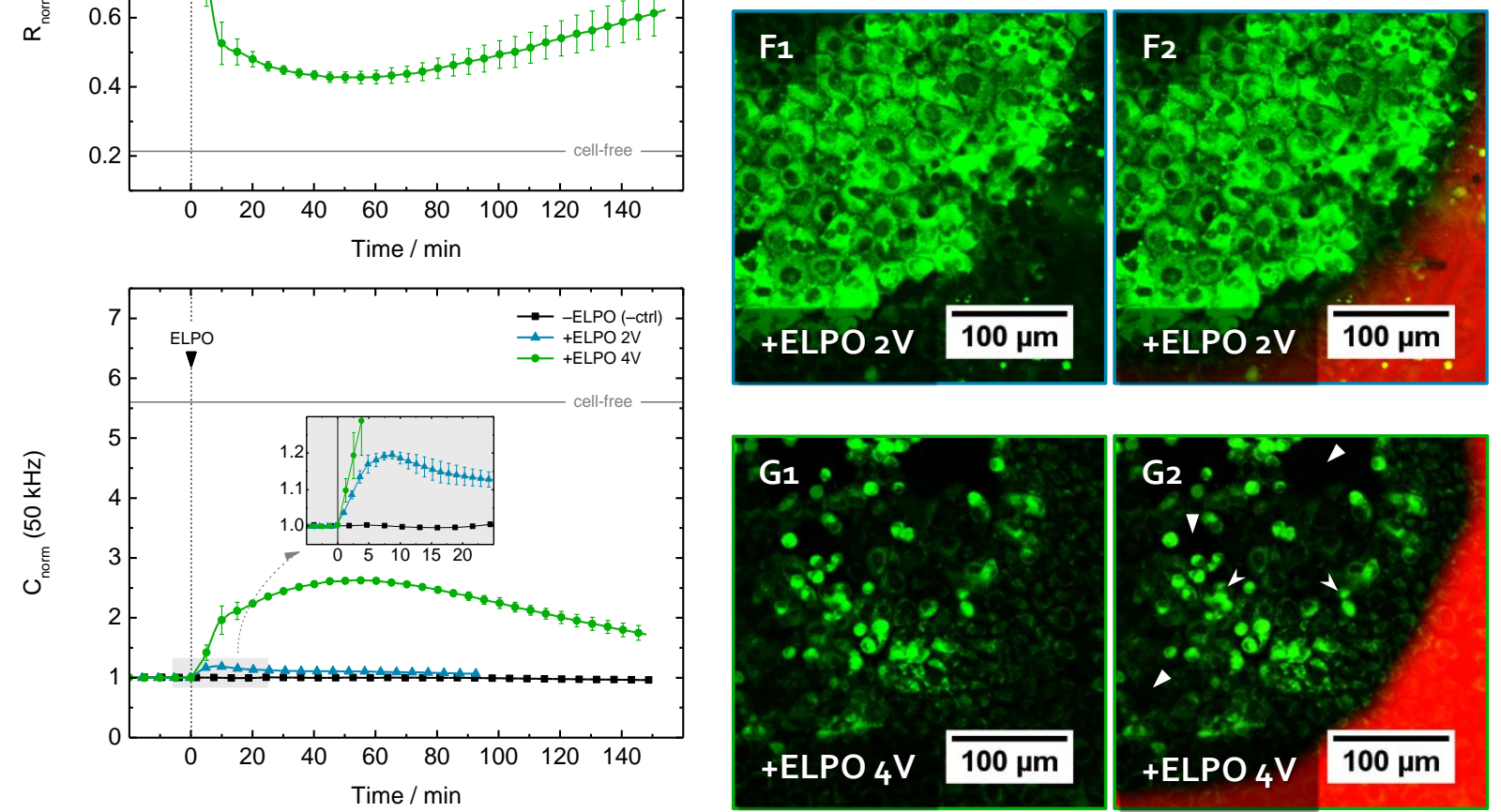

Fig. 5-25 QCM-ECIS sensor responses after electroporation (ELPO) of NRK cells in the presence of $1 \mathrm{~g} \mathrm{~L}^{-1}$ FITC-labeled dextran $(250 \mathrm{kDa}$ ). Averaged curves (mean $\pm \mathrm{SEM} ; \mathrm{N}=2$ ) show timeresolved changes of (A) $\Delta|Z|_{\text {min, }}(\mathrm{B}) R_{\text {norm }}$ at $5 \mathrm{kHz}$, and (C) $C_{\text {norm }}$ at $50 \mathrm{kHz}$, of cells exposed to an $40 \mathrm{kHz}$ AC pulse of $200 \mathrm{~ms}$ and $2 \mathrm{~V}_{\mathrm{rms}}$ ("+ELPO $2 \mathrm{~V}^{\prime \prime} ;-$ ) or $4 \mathrm{~V}_{\mathrm{rms}}$ ("+ELPO $4 \mathrm{~V}^{\prime \prime} ;-{ }^{-}$), and of negative control cells that were not electroporated ("-ELPO (-ctrl)"; - -). The time courses are normalized to the last data point before ELPO. Values of the cell-free sensor, immersed in SFM only, serve as a reference (+ctrl) and are indicated by a grey horizontal line. $\Delta|Z|_{\text {min }}$ time courses are shown separately for data sets from $\mathrm{QCM}_{1}$ (filled symbols) and $\mathrm{QCM}_{2}$ (open symbols) recordings, since the electric manipulation of a fraction of cells only occurs on $\mathrm{QCM}_{1}$ but not on $\mathrm{QCM}$ 2. Data points in the time courses are reduced for sake of clarity. Fluorescence micrographs show the green fluorescent FITC-dextran injected into the cells in the lower right quadrant of the working electrode (D): (E) without ELPO (-ctrl) and after ELPO with (F) 2 V and (G) 4 V. Cells were washed after ELPO to remove extracellular FITC-dextran. The red fluorescent photoresist indicates the boundary of the working electrodes. 
At the end of OCM-ECIS monitoring, the cells on the $2 \mathrm{EIOs}$ were gently washed three times with $\mathrm{EBSS}^{++}$ buffer in order to remove extracellular FITC-dextran. Afterwards, the measurement chamber was disassembled, and the sensor plate was placed in a petri dish. It was carefully flooded with $2 \mathrm{~mL} \mathrm{PBS}{ }^{++}$ buffer again and the fluorescence of electroporated, intracellular FITC-dextran was analyzed with a fluorescence microscope. Typical micrographs of cells electroporated on the ECIS working electrode of QCM-ECIS sensor disks are shown on the right hand side of Fig. 5-25. Green channel images (Fig. 5$25 \mathrm{E}_{1}-\mathrm{G}_{1}$ ) depict the fluorescence intensity of FITC-labeled dextran molecules inside the cells, and composite green and red channel micrographs (Fig. 5-25 E2-G2) additionally visualize the area of the working electrode as circular hole in the insulating red fluorescent photoresist. Images of not electroporated negative control cells (Fig. 5-25 E) demonstrate that FITC-dextran is taken up by the cells in no way of passive diffusion, active membrane transport, or endocytosis during the incubation time. However, if cells were electroporated with $40 \mathrm{kHz} A C$ voltage pulses of either $2 \mathrm{~V}_{\text {rms }}$ (Fig. 5-25 F) or $4 \mathrm{~V}_{\text {rms }}$ (Fig. 5-25 G) in magnitude for $200 \mathrm{~ms}$, strong fluorescence of intracellular FITC-dextran is observable. Moreover, one can see that loading of the cells occurs exclusively in the area where cells are in direct contact to the gold working electrode. Cells in the periphery of the WE remain non-fluorescent and, thus, were not electroporated. Moreover, fluorescence micrographs disclose differences in the electroporated cell layers, depending on the magnitude of the applied AC pulse. While cells treated with $2 \mathrm{~V}_{\text {rms }}$ show a homogeneous fluorescence across the entire working electrode, the cell layers exposed to $4 \mathrm{~V}_{\text {rms }}$ exhibit cell-free holes ( $\boldsymbol{D}$ in Fig. $5-25 G_{2}$ ) as well as rounded cells with bright fluorescence $(>$ ). Hence, the $4 \mathrm{~V}_{\text {rms }}$ pulse seems to be too strong and invasive, i.e. for a significant fraction of cells the generated pores in the plasma membrane are too big and too many, to ensure resealing and cell viability after ELPO.

The lasting invasiveness of the $4 \mathrm{~V}_{\text {rms }}$ pulse and its rather wounding than electroporation impact on the cells becomes evident by the micrographs in Fig. 5-26 of another experiment. Here, most of the cells on the working electrode detached or were washed away from the surface after the $4 \mathrm{~V}_{\text {rms }}$ pulse. Cells in the periphery of the working electrode remain stably attached and spread on the surface. This becomes apparent in the phase-contrast image in Fig. 5-26 C, which shows a confluent, unaffected cell layer outside the working electrode (indicated by a dashed line). Hence, the integrity of most cells on the working electrode is either immediately destroyed by pulsing with $4 \mathrm{~V}_{\mathrm{rms}}$, or at least weakened to such an extent that the cells die and detach from the surface after ELPO.
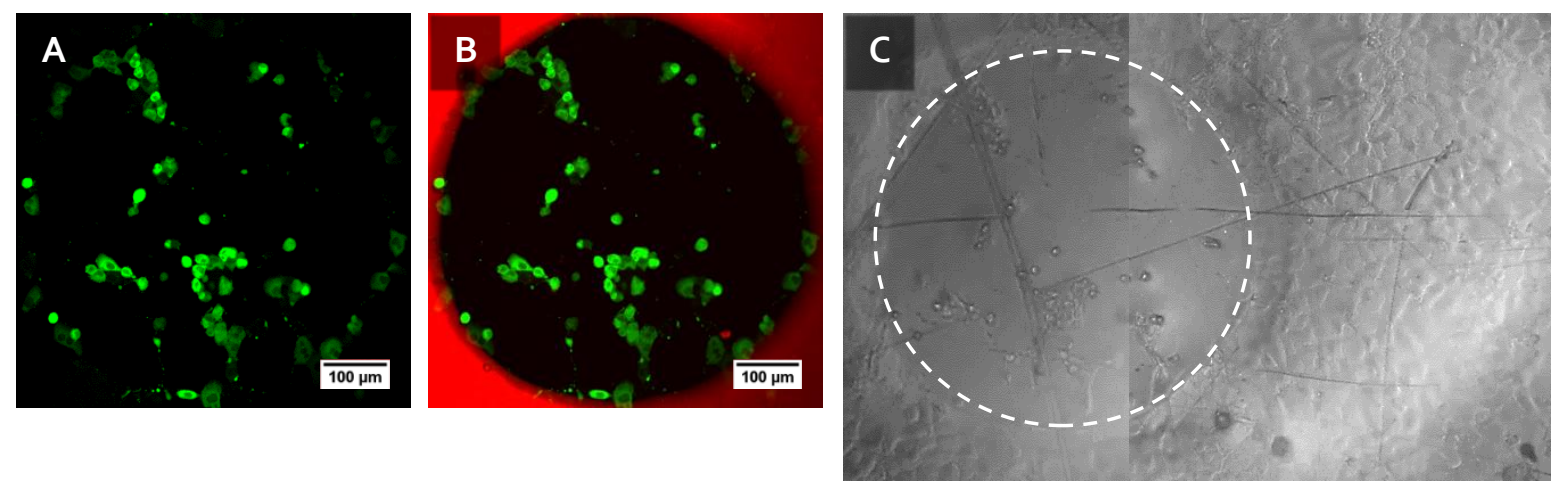

Fig. 5-26 (A), (B) Fluorescence micrographs show the green fluorescent FITC-dextran taken up by the cells on the WE after ELPO with $4 \mathrm{~V}_{\mathrm{rms}}$. Cells were washed after ELPO to remove extracellular FITC-dextran. The red fluorescent photoresist indicates the boundary of the WE. Areas of no green fluorescence report on cells disintegrated or detached from the surface from pulsing with $4 \mathrm{~V}_{\text {rms }}$. This is confirmed by the phase-contrast micrograph in (C), showing the largely cell-free WE (indicated by a dashed line) and an unaffected confluent cell layer around it. 


\subsection{Discussion}

In this chapter a novel dual sensor, combining the two sensor principles QCM and ECIS in one experimental setup, was presented. The development process from $2 \mathrm{ElO}$ s to the $3^{\text {rd }} \mathrm{gen}$. QCM and the sensor characteristics will be discussed below. This comprises the design of a new electrode layout on piezoelectric quartz disks for the substrate-integration of ECIS (5.4.1), the sensor surface properties after spin coating and photolithography, as well as the sensor performances in QCM-mode (5.4.2) and ECISmode (5.4.3) compared to the $2^{\text {nd }}$ gen. OCM. The effect of medium preincubation on the sensor response (5.4.4) and the dual sensor signals in a variety of cell-based assays, like in the course of cell attachment and spreading (5.4.5 and 5.4.6) or upon different stimuli on established cell layers (5.4.7-5.4.10), are discussed as well. The time-resolved, non-invasive dual impedance recordings provided a multitude of complementary information and a more detailed view on the physiological behavior and the reactions of cells, without the need for any type of labeling. It could be demonstrated the enormous power and potential of the novel QCM-ECIS sensors, being able to uncover coherent, time-delayed, or isolated mechanical and morphological changes within a cell layer; information that has not been accessible until now.

\subsubsection{Electrode Layout}

Starting from classical QCM (1 $1^{\text {st }}$ generation QCM) Janshoff and Wegener (1996) ${ }^{[219]}$ developed what we call " $2^{\text {nd }}$ generation $\mathrm{OCM}$ ", which allows for combined OCM and ECIS measurements of cells by the introduction of an electrode dipping from the top into the measurement vessel (cf. Fig. 5-1 A, B). This technological upgrade allows in various assay formats for the multi-parametric monitoring of cells cultured on QCM substrates. ${ }^{[22,206,236,267,268]}$ However, the additional wire electrode of the $2^{\text {nd }}$ gen. $O C M$ holds some disadvantages. Since the Pt electrode is not an integral, fixed part of the OCM measurement chamber, it has to be manually mounted for each experiment. An exact and consistent positioning and geometrical arrangement of the dipping CE above the WE for ECIS-like cell investigations is very critical. Reproducible experimental conditions, however, are crucial for the comparison and averaging of impedance data obtained from independent experiments. The additional electrode on top of the resonator also limits the working space above the measurement cell. Thus, it impedes substance injections and liquid removal, and one runs the risk of interfering with the measuring system during a running experiment. The platinum wire electrode may also be a steric hindrance for measurement chambers that are designed for microscopic studies in parallel to OCM-ECIS monitoring, which require unhindered access of objective and light source to the specimen. The dipping electrode and the opening for its introduction in the $2^{\text {nd }}$ gen. OCM measurement chamber introduces additional weak points for contaminations. These points were the motivation for the development of the OCM-ECIS approach and the design of a novel electrode layout, in order to advance the performance and the robustness of this hyphenated approach for cell-based assays. The development of the 2-Electrode quartzes (2ElO; cf. Chapter 4 ) provided the basis for this. The coplanar electrodes on the 2 ElOs were utilized for substrateintegrated impedance analysis of cells grown on the piezoelectric quartz plates, applying one electrode as the counter electrode (CE) and the other one as the working electrode (WE). This superseded the disadvantageous Pt wire electrode from the top. By spin coating and photolithography of a photoresist, a well-defined, small WE was created so that we ended up at an electrode layout similar to that on conventional ECIS substrates ${ }^{[463]}$ (Fig. 5-1 C). With these final sensors, comprising two independent, decoupled TSM resonator spots for QCM measurements and substrate-integrated electrodes for ECIS, a 
novel generation of dual sensing on quartz disks is presented, which we call the " 3 rd generation OCM" (Fig. 5-2).

The diameter of the working electrode for ECIS was set to $500 \mu \mathrm{m}$ by default. On the one hand, the ultimate goal of photoresist coating on $\mathrm{OCM}_{1}$ was the decoupling of the resonances of the two adjacent microbalances on a $2 \mathrm{EIO}$ sensor (studies on this, see section 4.1). Removal of big areas of photoresist again, for the generation of a large working electrode for ECIS-mode measurements, counteracts this. Moreover, high electric potentials across cell membranes for elevated field applications like electroporation and wounding of cells are easier to achieve with smaller electrodes, like that with 250 or $500 \mu \mathrm{m}$ in diameter (cf. discussion below in 5.4.10). On the other hand, experiments show - contrary to the actual experiences and expectations - increasing sensitivities in $|Z|, R$, and $C$ with increasing WE areas (cf. Fig. 5-9 and discussion below in 5.4.3). Hence, this would make substrates with WE diameters of 1 or $2 \mathrm{~mm}$ to the sensors of choice. Another practical drawback of the smallest WEs investigated $(250 \mu \mathrm{m})$ are limitations by the photolithographic system used for surface structuring. Though such small WEs were successfully fabricated, they show indistinct circularity and fringed edges of the photoresist. Consequently, no consistent WE size could be prepared, prohibiting reproducible and comparable impedance measurements. ECIS working electrodes of $500 \mu \mathrm{m}$ in diameter were prepared without limitations and showed similar sensitivities as the bigger WEs, but in turn exhibit an acceptable size for applications with elevated electrical fields. Hence, this was chosen as the working electrode size of the standard OCM-ECIS sensors.

With the photolithography mask defining a WE diameter of $500 \mu \mathrm{m}$, real dimensions of $\varnothing_{\text {real }}=$ $511( \pm 4) \mu \mathrm{m}$ and $A_{\text {real }}=2.05( \pm 0.03) \cdot 10^{-3} \mathrm{~cm}^{2}$ (mean $\pm \mathrm{SEM} ; \mathrm{N}=7$ ) were achieved. The slightly bigger size can be traced back to an undercut of the UV light at the edges of the lithographic mask during photolithography. Thus, a bigger area than actually defined is illuminated and, consequently, removed during development of the positive photoresist. Reasons for this could be that substrate and lithography mask are not close enough to each other during illumination and/ or the limited marginal sharpness of the printed lithography mask because of a too low resolution of the ink-jet printer.

In cases of a confluent monolayer of cells on $2 \mathrm{ElO}$, and assuming an average projected area of (MDCK-II) cells of $\sim 180 \pm 10 \mu \mathrm{m}^{2}[215]$, approximately 55000 cells are attached directly or indirectly to the electrodes of the QCM resonator spots $\left(\varnothing_{\text {Electrode }}=3.5 \mathrm{~mm}\right.$ ). The real number of measured cells by QCM-mode recordings is even somewhat bigger, since the shear oscillation is non-zero at the edges of the electrodes and cells attached to the surface at these regions contribute to the damping of the shear oscillation, too. Each OCM spot of the $3^{\text {rd }}$ generation OCM integrates only over one third of the cells compared to the classical single-spot $5 \mathrm{MHz}$ quartzes of the $1^{\text {st }}$ or $2^{\text {nd }}$ generation $\mathrm{QCM}\left(\varnothing_{\text {Electrode }}=6 \mathrm{~mm}\right.$ ). However, the fraction of measured cells by one resonator of $2 \mathrm{ElOs}$ is sufficiently high to obtain representative and reliable information on the mechanical situation of the entire cell population. Moreover, the two individual sensor spots on $3^{\text {rd }}$ gen. OCM quartz plates allow to independently sense cytomechanical information from two separate fractions of cells within the whole cell layer. Hence, there is a duplication of the OCM readout in any experiment, doubling the amount of data as well. The presence of two measurement spots on the quartz disks also enables the development of completely new and innovative approaches for cell-based assays. This will be addressed in the outlook section (5.4.12) of this discussion. By applying the standard WE layout of the $3^{\text {rd }}$ generation OCM for ECIS-mode recordings, the electrochemical impedance information is recorded and averaged from more than 1000 cells within a confluent monolayer. This has proven to be a good compromise between a sufficiently high sensitivity, for the detection of cell morphological alterations, and an adequately large number of measured cells, for obtaining a signal being representative for the whole population of cells. 


\subsubsection{QCM-mode: Piezoelectric Characterization of the Sensor}

\section{$3^{\text {rd }}$ Generation QCM: Influence of Partial Removal of Photoresist from 2ElOs on QCM Impedance Properties}

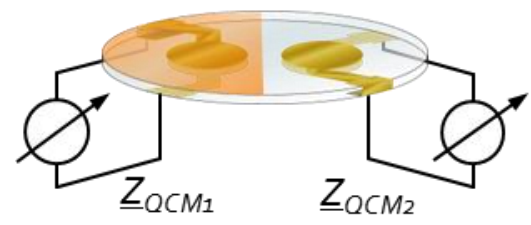

2EIQ
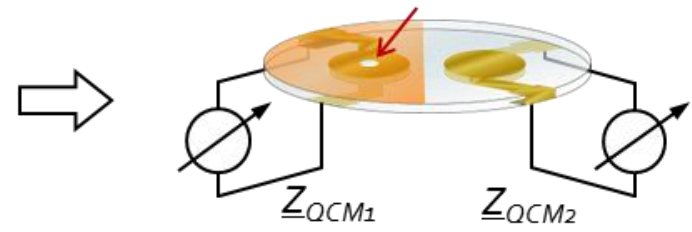

$3^{\text {rd }}$ generation QCM

The introduction of a circular cavity into the photoresist of a coated $2 \mathrm{ElO}$ resonator $(\mathrm{OCM} 1)$, for generating the WE for additional ECIS-like measurements, has no negative influence on the OCM properties, i.e. the shear oscillation impedance near resonance, of both $2 \mathrm{EIO}$ resonators. There is only a slight reduction of the resonance separation between $\mathrm{OCM}_{1}$ and $\mathrm{QCM}_{2}$ for ${ }^{\text {rd }}$ generation $\mathrm{OCM}$ sensors compared to $2 \mathrm{ElOs}$ without the ECIS electrode. This is due to the removal of a certain mass of photoresist from resonator $\mathrm{OCM}$, which increases its resonance frequency according to Saverbrey (cf. Eq. (23)). The resonance separation between the uncoated microbalance $\left(\mathrm{OCM}_{2}\right)$ and the coated microbalance $(\mathrm{OCM})$ depends on the size of the uncovered area in the coating of the latter, and decreases with increasing WE dimensions (cf. Fig. 5-8, p. 124).

Enlarging the WE size and, thereby, bringing the resonances of the adjacent resonators closer to each other causes the occurence of more pronounced side resonances, too. These secondary resonances in the impedance spectra of $2 \mathrm{ElO}$ microbalances are due to the lateral coupling of the adjacent OCM units, which has been described and discussed in the previous chapter (4.3.2 Decoupling of 2ElO Resonators > Oscillation Properties of Decoupled 2 ElO Resonators under Liquid Loading

, p. 105 ff.). However, it is striking to note that the side resonance of the coated $\mathrm{QCM}_{1}$ increases with an increasing size of the WE on it, while the side resonance of the uncoated $\mathrm{OCM} 2$ resonator is invariant and seems not to depend on the WE size on $\mathrm{OCM}_{1}$ (Fig. 5-8). Hence, besides lateral coupling of resonators, affecting the impedance spectra of both to a similar extent, a second effect is suggested, which only influences the spectral properties of $\mathrm{OCM}_{1}$ but not that of $\mathrm{OCM}_{2}$. Uncovering a circular WE in the center of the polymer-coated $\mathrm{OCM} 1$ resonator actually generates a resonator unit with two regions of different thickness - one with PhoP coating and the other without - differing by $\sim 3 \mu \mathrm{m}$. Thus, two different frequencies fulfill resonance conditions for a standing acoustic wave at $\mathrm{OCM}_{1}$, between the faces of the quartz on the one hand and between the bottom side of the quartz and the polymer surface on $\mathrm{OCM} 1$ on the other hand. With an increasing hole in the photopolymer, the fraction of the uncoated electrode increases and, thus, the fraction of the $\mathrm{OCM}_{1}$ unit that is resonant at frequencies where the completely uncoated $\mathrm{OCM} 2$ exhibits resonance. Together with the side resonance arising from coupling with $\mathrm{OCM} 2$, this causes a significant resonance peak of $\mathrm{OCM}_{1}$ at the frequency of $\mathrm{OCM}_{2}$ resonance (cf. Fig. 5-8 D). By contrast, the $\mathrm{OCM} 2$ unit is of uniform thickness. Thus, there is only one distinct resonance in the spectra of $\mathrm{OCM}$, accompanied by a comparatively small side resonance at the resonance frequency of the polymer-coated $\mathrm{QCM} 1$.

Overall, the impact of photoresist removal from the center of $\mathrm{OCM}_{1}$ on its impedance spectra is much less than expected. For the standard ECIS-OCM sensor layout comprising an ECIS working electrode of 
$500 \mu \mathrm{m}$ in diameter, neither two distinct resonances for $\mathrm{OCM}_{1}$ nor perturbations in the shape of the resonance curves of $\mathrm{OCM}_{1}$ and $\mathrm{OCM} 2$ are observed (cf. Fig. 5-6A-D, p. 118), even though the photoresist is removed from the center of the resonator, where the oscillation amplitudes and thus mass sensitivity are maximal. Even more surprising is that the introduction of ten holes (working electrodes) of either 250 or $500 \mu \mathrm{m}$ in diameter into the photoresist across the entire electrode area has obviously no perturbing influence on the oscillation properties of the resonator (cf. Fig. 5-10, p. 127). One can only observe the comprehensible resonance approximation of $\mathrm{OCM}_{1}$ and $\mathrm{OCM}_{2}$ due to the mass reduction on $\mathrm{OCM}_{1}$ (cf. Tab. 5-3). Similar findings have also been reported previously by Michaelis (2010), who investigated the influence of position and size ( $0.8 \%$ and $29.4 \%$ of the electrode area) of these cavities in the photoresist coating on the resonance properties of $1^{\text {st }}$ generation $\mathrm{OCM} .^{[236]}$ Perturbations in the impedance spectra of $3^{\text {rd }}$ generation OCM resonators were also expected due to a lateral coupling of the adjacent resonators with similar resonance conditions, i.e. $\mathrm{QCM} 2$ without PhoP coating and the region of $\mathrm{QCM} 1$ where PhoP was removed, as it was observed for the not partially coated, non-decoupled $2 \mathrm{ElO} s$ (cf. Fig. 4-2; p 86). Even a maximization of a single circular cavity on $\mathrm{QCM}_{1}$ to a diameter of $2 \mathrm{~mm}$, which means an uncovering of $\sim 33 \%$ of the electrode area (cf. Tab. $5-3$, p. 126), has no perturbing influence on the shape of the impedance spectra of both resonators. There is only a slight increase in the resonance peak of the uncovered electrode in the spectra of OCM1. However, this resonance is - in view of the percentage of uncovered electrode area - much less pronounced than the resonance that is related to the polymer-coated fraction of the resonator (Fig. 5-8 D). The complete understanding of the coupling effect of adjacent resonators on $3^{\text {rd }}$ generation QCM sensors and the influence of non-uniform resonator thickness on the spectral properties of the OCM units require further and more detailed investigations. The general QCM-mode impedance characteristics of the OCM-ECIS sensor platform, however, have proven to be well suited for extracting the magnitude of minimal impedance for the analysis of oscillation damping upon any type of viscous or viscoelastic loading on the substrate.

\section{$3^{\text {rd }}$ Generation QCM compared to $1^{\text {st }} / 2^{\text {nd }}$ Generation $Q C M$}

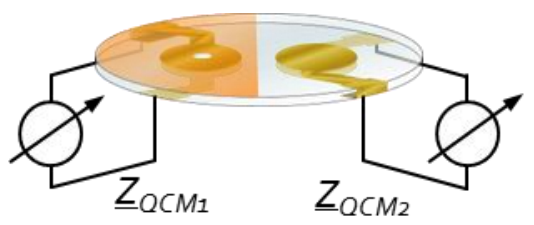

$3^{\text {rd }}$ generation $\mathrm{QCM}$

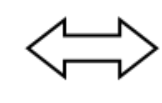

$1^{\text {st }} / 2^{\text {nd }}$ generation OCM

The oscillation impedances and spectral resonance characteristics of the dual OCM array (2EIO) compared to the classical single $\mathrm{OCM}\left(1 \mathrm{EIO}, 1^{\text {st }} / 2^{\text {nd }}\right.$ generation $\left.\mathrm{OCM}\right)$ in air have been discussed in the previous chapter of the MOCM development (4.3.2 Decoupling of 2ElO Resonators > Oscillation Properties of Decoupled 2 ElO Resonators, p. $102 \mathrm{ff}$.). Reducing the resonator size from the classical $1 \mathrm{EIO}$ to the $2 \mathrm{EIO}$ by $\sim 40 \%$ in diameter ( $65 \%$ in area) was accompanied by a distinct increase of shear oscillation damping and energy dissipation, respectively (cf. --- vs. - - - and - in Fig. 4-10 E, F, p. 101). This is not significantly altered by the integration of an ECIS working electrode on the photoresist-coated $\mathrm{QCM}_{1}$ resonator (see subsection 3rd Generation OCM: Influence of Partial Removal of Photoresist from 2 ElOs on OCM Impedance Properties above). In Fig. 5-27, the resonance curves of a $3^{\text {rd }}$ generation OCM (filled symbols) and of a single, $1^{\text {st }} / 2^{\text {nd }}$ generation $\mathrm{OCM}$ (open symbols) are compared. Here, the impedance spectra of a $3^{\text {rd }}$ generation OCM sensor are exemplarily shown for the photopolymer-coated $\mathrm{OCM}_{1}$ sensor spot, which includes an uncovered area of $500 \mu \mathrm{m}$ in diameter in the center as WE for ECIS. 
A

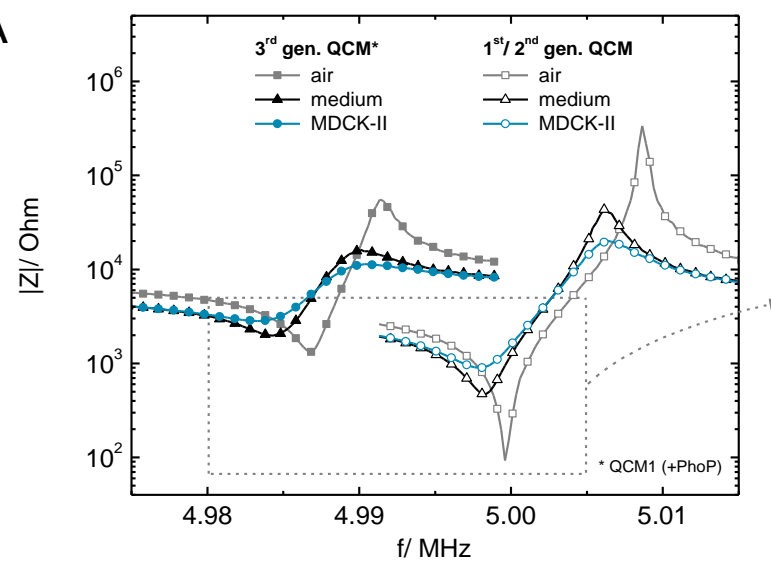

C

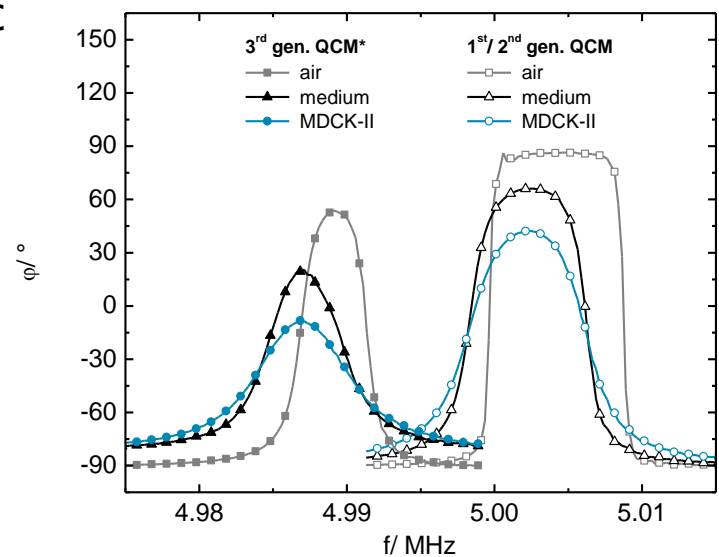

B

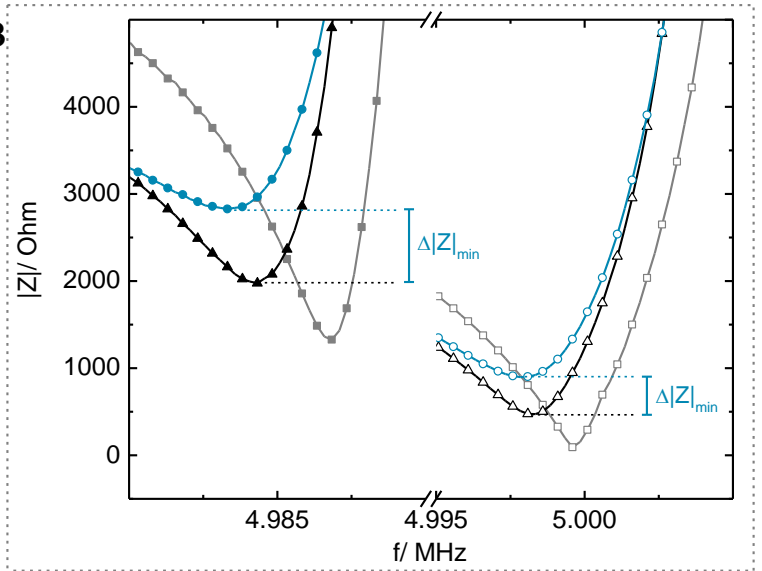

D

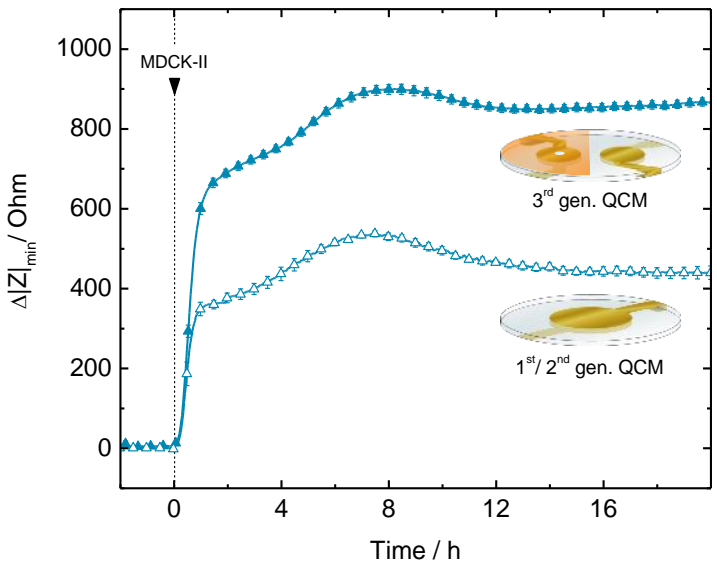

Fig. 5-27 OCM-mode impedance characteristics of the novel $2 \mathrm{ElO}$ microbalances with substrate-embedded ECIS electrodes ( $3^{\text {rd }}$ generation $\mathrm{OCM}$; filled symbols) and of classical 1 EIOs $\left(1^{\text {st }} / 2^{\text {nd }}\right.$ generation $\mathrm{OCM}$; open symbols). Spectra of (A), (B) impedance magnitude and (C) phase shift show differences of resonators oscillating in air $(-,-,-)$, under medium loading $\left(\triangle-,-\Delta_{-}\right)$, and when MDCK-II cells were grown to confluence on the sensors $(-\infty-,-\infty)$. Impedance characteristics of $3^{\text {rd }}$ gen. $Q C M$ are exemplarily shown for $\mathrm{OCM}_{1}$. The $\mathrm{OCM}_{1}$ resonator is coated with a thin film of rigid, insulating photopolymer except for a small hole in the center which forms the WE for ECIS-mode measurements (WE- $\varnothing=0.5 \mathrm{~mm}$ ). 2 EIO resonators show higher damping for any loading condition due to the reduced electrode size. However, $\Delta|Z|_{\min }$ and $\Delta \varphi_{\max }$ changes are more pronounced for $2 \mathrm{ElO}$ s than for 1 ElOs, if the sensors are loaded with either cell culture medium or a confluent cell layer. This becomes obvious by the zoom-in into the resonance region of $|Z|$, shown in $B$, and in the time-resolved $\Delta|Z|_{\min }$ curve in $D$, illustrating the mechanical interaction of MDCK-II cells during attachment and spreading with the OCM sensor surface of a $2 \mathrm{EIO}(-)$ and a $1 \mathrm{ElO}\left(-\Delta^{-}\right)$substrate. Data points were reduced for sake of clarity.

In air, the minimal impedance magnitude and, thus, oscillation damping is by $\sim 1000$ Ohm higher for 2 ElO ( - ) compared to 1 ElO disks ( $-\square^{-}$) (Fig. 5-27 A; magnified in Fig. 5-27 B). The phase shift lies by $\sim 30^{\circ}$ lower for the $2 \mathrm{EIO}$ resonators with reduced electrode size than for the $1 \mathrm{EIQ}$, reflecting an increased dissipation of energy as well (Fig. 5-27 C). The impedance characteristics of the shear oscillations of 1 ElOs and $3^{\text {rd }}$ generation $\mathrm{OCM}$ sensors do not only differ in air but also change to significantly different extents when loading them with cell culture medium or seeding cells on those quartz plates.

If exposed to cell culture medium, the resonance frequency $f_{|Z|_{\min }}$ shifts by $-2.35( \pm 0.04) \mathrm{kHz}(\mathrm{N}=10)$ for $2 \mathrm{ElO}$ resonators and under similar experimental conditions by $-1.30( \pm 0.05) \mathrm{kHz}(\mathrm{N}=4)$ for $1 \mathrm{ElO}$ resonators, with respect to the resonance of the respective oscillators in air (mean \pm SEM). Hence, the 
spectra of the dual, $3^{\text {rd }}$ generation $\mathrm{OCM}$ are by a factor of $\sim 1.8$ stronger shifted to lower frequencies upon viscous loading than that of the single, $1^{\text {st }} / 2^{\text {nd }}$ generation OCM.

In the course of MDCK-II cell layer formation, the serial resonances of $2 \mathrm{EIQs}$ and $1 \mathrm{EIQs}$ are further shifted to lower frequencies by $-0.96( \pm 0.05) \mathrm{kHz}(\mathrm{N}=10)$ and $-0.28( \pm 0.07) \mathrm{kHz}(\mathrm{N}=4)$, respectively. Hence, the resonance frequency response upon seeding cells is again by a factor of $\sim 3.4$ stronger for $3^{\text {rd }}$ compared to $1^{\text {st }} / 2^{\text {nd }}$ generation OCM resonators. This rather small frequency shift of both single and dual microbalances upon seeding cells might be counterintuitive at a first glance, since the attachment of cells is expected to drastically increase the mass on the resonators. According to the Saverbrey equation (Eq. (23)) this should go along with a more pronounced decrease of the resonance frequencies. A cell layer cannot be treated like a uniform and rigid layer that oscillates synchronously with the quartz material, which however is one basic prerequisite for the validity of the Saverbrey equation and its application for the QCM-based microweighing. Cells rather have to be considered as viscoelastic bodies. The first molecular layers of focal adhesion sites and of the extracellular matrix of cells on a resonator indeed oscillate synchronously with the quartz material. Thus, cells increase to some extent the lossless vibrating mass on the microbalance and thereby cause the observed Saverbrey-related frequency shift. However, the viscous and elastic properties are dominating the loading characteristics of the cell layer. This is reflected in a strong increase of the motional impedance and of energy dissipation, respectively, when seeding cells on the QCM ( $\bullet-$ in Fig. 5-27 A, B).

Loading a $3^{\text {rd }}$ generation microbalance with cell culture medium, this increases the motional impedance $|Z|_{\text {min }}$ of either resonator by $\sim 650$ Ohm with respect to the oscillations in air ( $\downarrow$ in Fig. 5-27 A, B). This is by 1.7 -fold more compared to the minimal impedance increase of a single OCM resonator $\left(\Delta|Z|_{\min } \approx 380 \mathrm{Ohm} ;-\Delta\right.$ in Fig. 5-27 A, B). Seeding MDCK-II cells to confluence on the quartz disks causes a further increase of the minimal impedance by $\sim 850 \mathrm{Ohm}$ for the novel $2 \mathrm{ElOs}(-\rightarrow-$ in Fig. $5-27 \mathrm{~A}$, B) and by $\sim 430 \mathrm{Ohm}$ for a classical 1 EIO (-o- in Fig. 5-27 A, B). Hence, the smaller resonators show a roughly 2 -fold stronger oscillation damping in parameter $|Z|_{\text {min }}$, which is used for monitoring and analyzing cytomechanics. The time-resolved increases of $|Z|_{\text {min }}$ upon MDCK-II cell layer formation are compared in Fig. 5-27 D for the two different generations of quartz disks. This comparison reveals that the kinetics and the characteristic points of MDCK-II attachment and spreading derived by OCM readings are equal for both sensor types, meaning that the signals measured report on similar mechanical alterations taking place at the cell-quartz interface. However, the absolute signal change is much more pronounced for the newest generation of OCM sensors ( $\downarrow$ in Fig. 5-27 D). This is equivalent to a higher sensitivity for monitoring small alterations in cytomechanics. The bigger signal changes of $2 \mathrm{ElO} s$ compared to 1 ElOs is likewise reflected in the shifts of the phase angle of impedance upon loading the microbalances. For both, exposing the sensors to medium and growing cells to confluence on one of the faces, $\Delta \varphi_{\max }$ is reduced by about $-30^{\circ}$ for a $2 \mathrm{ElO}$. The phase of a $1 \mathrm{ElO}$ is shifted by only approximately $-20^{\circ}$ in either case of loading.

The stronger responses in frequency shift, impedance magnitude, and phase shift of the dual ( $3^{\text {rd }}$ generation) compared to the single $\left(1^{\text {st }} / 2^{\text {nd }}\right.$ generation) $\mathrm{QCM}$ upon viscous or viscoelastic loading may be due to a mutual reinforcement of effects on MOCMs. Shen et al. ${ }^{\left[{ }^{663}\right]}$ reported on (theoretical) frequency interferences of two adjacent microbalances. These cause a resonance frequency shift of resonator No. 1, solely due to a mass loading exclusively on resonator No. 2. It was shown by simulations that the mutual influence of neighboring microbalances is pronounced for (i) low ratios of resonator distance to disk height, (ii) low ratios of OCM diameter to disk height, and (iii) QCM array layouts where the resonators have a similar width. Except for the last, all geometrical requirements are largely met in order to prevent stronger interferences of the two resonators of $3^{\text {rd }}$ generation OCMs. However, the equal size of the OCM 
spots increases the probability of frequency coupling. Hence, coupling of OCMs may not only cause the small side resonances observed for both resonators at the resonance frequency of the other. The calculations and the observed stronger responses of the dual compared to the single $\mathrm{OCM}$ also indicate interferences of the main resonances. This would mean that mechanical changes on $\mathrm{OCM}_{1}$, e.g. if cells interact with the surface, may also affect the characteristic oscillation parameters of $\mathrm{OCM} 2$ and vice versa. This hypothesis based on theoretical simulations is substantiated by experimental data, which are illustrated in Fig. 5-28 A, B. One of the two resonators ( $(\mathrm{CCM} 1)$ of a $2 \mathrm{EIO}$ was systematically loaded with viscous, highly damping silicone grease, while the other one (OCM2) was not loaded. This causes significant changes in the resonance properties of both the loaded $\mathrm{OCM}_{1}(-)$ and the actually unloaded $\mathrm{OCM}_{2}\left(-\Delta_{-}\right)$, compared to the respective oscillation impedance spectra of OCM1 $(-)$) and $\mathrm{OCM}_{2}(-\square)$ in air. This demonstrates that there is a crosstalk between the $2 \mathrm{ElO}$ resonators and that the resonance properties $\left(f_{|Z|_{\text {min }}}|Z|_{\text {min, }}\right.$ and $\left.\varphi_{\max }\right)$ of $\mathrm{OCM} 2$ are affected by the loading situation on $\mathrm{QCM} 1$. However, the crosstalk between the microbalances seems to be slightly different, as the $\mathrm{OCM}_{1}$ responds somewhat less to a loading on $\mathrm{OCM} 2$ than vice versa (data not shown). This may be the reason why the $\Delta|Z|_{\text {min }}$ responses to MDCK-II and NRK cell attachment and spreading are somewhat ( $~ 80-90 \mathrm{Ohm})$ more pronounced for $\mathrm{QCM}_{1}$ (w/ PhoP) compared to QCM2 (w/o PhoP) (MDCK-Il: cf. Fig. 5-13 A and Tab. 5-4, p. 132 f.; NRK: cf. Fig. 5-14 A and Tab. 5-6, p. 136 f.). It is assumed that the photopolymer coating on $\mathrm{OCM} 1$ causes the frequency coupling not to be equal in both directions. However, this has to be further investigated for clarification of the phenomenon.

A

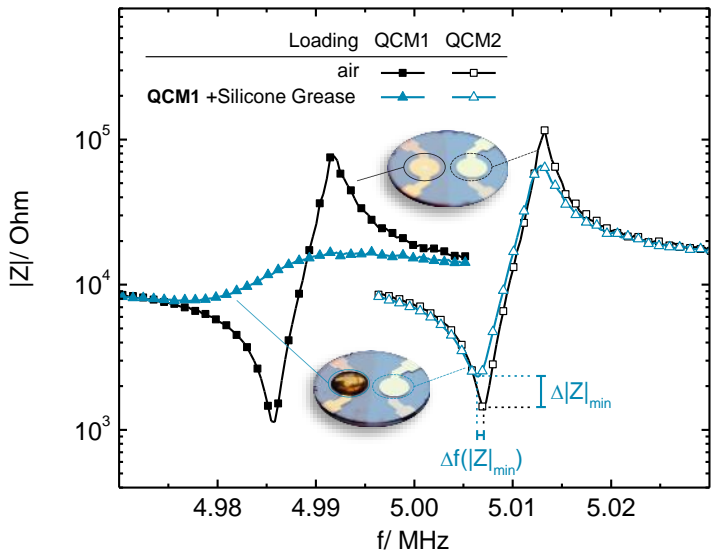

B

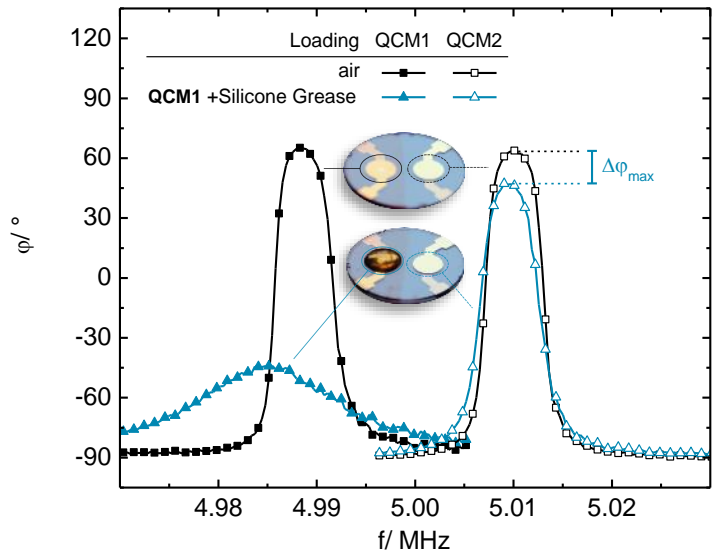

Fig. 5-28 (A) Impedance magnitude and (B) impedance phase spectra of $\mathrm{OCM}_{1}$ (filled symbols) and $\mathrm{OCM} 2$ (open symbols) oscillations of a $2 \mathrm{ElO}$, when both resonators operate in air (black squares) and when $\mathrm{OCM}_{1}$ is systematically loaded with highly damping silicone grease while $\mathrm{OCM} 2$ is not loaded (blue triangles). Loading of $\mathrm{OCM} 1$ affects the oscillation properties $\left(f_{|Z|_{\text {min }}}\right.$ $|Z|_{\min }$, and $\varphi_{\max }$ ) of both the loaded $\mathrm{OCM} 1$ and the actually unloaded $\mathrm{OCM} 2$.

One might consider the frequency coupling of adjacent microbalances as a disadvantage, as it reduces the sensitivity of the sensor spots and as the QCMs cannot be considered completely independent and isolated. However, to our purposes - we do not want to measure different properties but the same at different spots within the cell layer with both resonators - this turned out to be even advantageous. Since the microbalances in the presented experimental setup are exposed to the same medium, they detect the adhesion of cells not only on their own surface but also additionally, to some extent, the cells attaching to the adjacent resonator due to the frequency coupling. This causes a strengthening of oscillation damping and thus a sensor-integrated amplification of the monitored signal. This effect could 
explain, at least to a certain degree, why the microbalances of the novel generation respond much more pronounced in frequency shift, phase angle decrease, and impedance magnitude increase upon cell attachment and spreading compared to the isolated single OCM. The $3^{\text {rd }}$ generation OCM exhibits a roughly two-fold improved sensitivity compared to the $1^{\text {st }} / 2^{\text {nd }}$ generation $\mathrm{OCM}$ and thus allows for a more sensitive detection of smaller changes in the micromechanical properties of a cell layer at the quartz-liquid interface.

Hence, despite the reduction of the resonator size and the accompanied increase of energy dissipation, recordings with the $3^{\text {rd }}$ generation $\mathrm{OCM}$ show significant changes in characteristic quartz oscillation properties upon cell settlement and, therefore, enable to monitor the mechanical properties of cells and cell-substrate contacts. Moreover, OCM-mode impedance measurements demonstrated an even higher sensitivity for the $\Delta|Z|_{\text {min }}$ signal of the $2 \mathrm{ElO}$ microbalances compared to the classical $1 \mathrm{EIOs.}$

\subsubsection{ECIS-mode: Electrochemical Characterization of the Sensor}

\section{3rd Generation QCM: Influence of Partial Removal of Photoresist from 2ElOs on ECIS Impedance Properties}

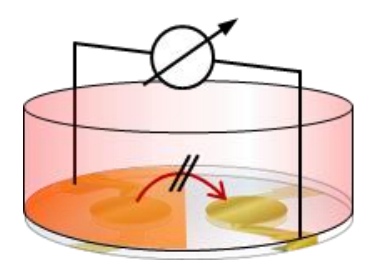

2EIO

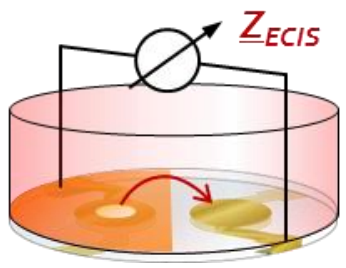

$3^{\text {rd }}$ generation QCM

The double-electrode quartzes (2EIOs), designed and developed for multichannel OCM measurements (Chapter 4 MOCM: Multiple Cytomechanic Sensing) were advanced to the $3^{\text {rd }}$ generation OCM with substrate-integrated ECIS-mode recording capability. This was realized by the photolithographic introduction of a defined hole into the photoresist of the coated electrode on the top side of resonator OCM1. Various sizes of this WE were tested for their influence on the spectral properties of electrochemical impedance readings, and the corresponding sensitivity of impedance with respect to the attachment and spreading of epithelial cells. As mentioned above, the WE diameter was set to $500 \mu \mathrm{m}$ in diameter by default, as a compromise between high sensitivity, consistent manufacturability, preservation of OCM decoupling, and usability for elevated field applications.

The observation that the sensitivities of ECIS parameters decrease with decreasing size of the WE (cf. Fig. 5-9) is counterintuitive at first glance, since the impedance contribution by the electrode-electrolyte interface and by cells attached to the WE increasingly dominate the constriction resistance of the bulk electrolyte with the WE size becoming smaller. ${ }^{[96]}$ Experimental studies confirmed a higher impedance sensitivity for cells on smaller WEs. ${ }^{[236]}$ This also becomes apparent in the simulated impedance spectra for different WE diameters shown in Fig. 5-46 (p. 215). The working electrode of the $3^{\text {rd }}$ generation QCM setup is limited in size not by the dimension of the gold electrode itself but by a defined hole in an insulating photopolymer (PhoP) on a larger gold electrode, which is $6 \mathrm{~mm}$ in diameter. Hence, a significant fraction of the electrode area is covered with photoresist in parallel to the blank working electrode. Electrochemically speaking, this area $A$ presents a capacitor, which contributes to the entire 
impedance properties of the system. The photoresist on the gold electrode can be considered as a parallel plate capacitor with a capacitance $C$ of:

$$
C=\frac{\varepsilon_{0} \varepsilon_{r} A}{d}
$$

where $\varepsilon_{0}$ is the electric permittivity of the vaccum, $\varepsilon_{r}$ is the relative dielectric constant of the layer of photoresist, and $d$ represents the distance between the polarizable faces and the thickness of the dielectric material, respectively. If the area fraction of photoresist-coated electrode compared to the uncoated electrode is big, the parasitic capacitance is expected to significantly influence the impedance properties of the system. This assumption was proven by simulations, in which the impedance characteristics of a typical ECIS electrode system with and without cells was calculated (according to the model presented in 3.4 .3$, p. 55 ff.) one time without and the other time with a parasitic capacitance $C_{p}$ arising from a photoresist-covered electrode. The results are illustrated in Fig. 5-29. The impedance spectra in Fig. 5-29 A indicate a reduced impedance increase from cell-free (open symbols) to cellcovered ECIS electrodes (filled symbols), if a parallel capacitance of $0.5 \mathrm{nF}$ is considered ( $\leftarrow$ vs. $-\square$ ). This is confirmed by the normalized impedance spectra in Fig. 5-29 B, illustrating a distinct reduction of the sensitivity of the electrochemical impedance for a cell layer on a small working electrode $\left(\Delta|Z|_{\text {norm }}\right)$, due to parasitic capacitance contributions.

A

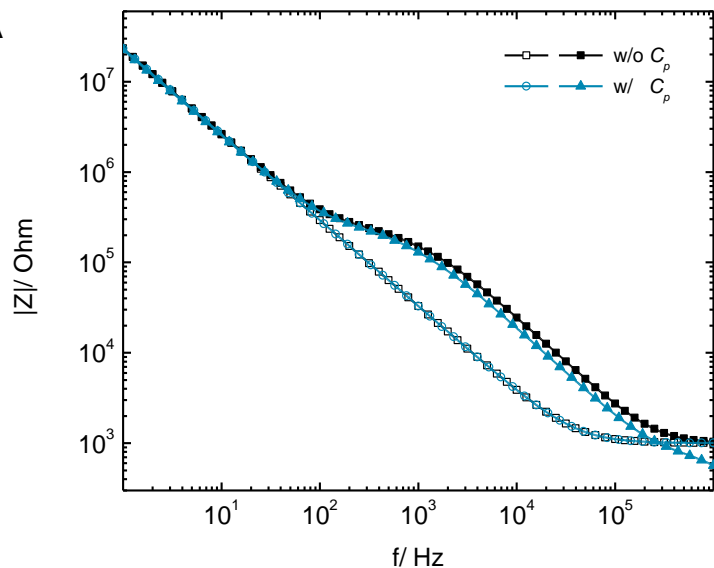

B

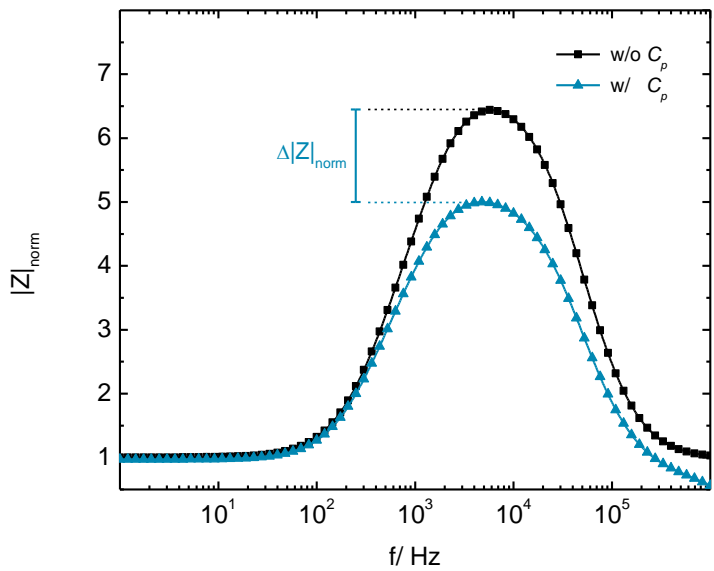

Fig. 5-29 (A) Simulated impedance spectra of typical ECIS electrodes without cells (open symbols) and covered with cells (filled symbols), considering an additional capacitance $C_{p}$ in parallel (blue) or not (black). (B) Corresponding normalized impedance spectra, indicating the loss in sensitivity of impedance recordings for cells $\left(\Delta|Z|_{\text {norm }}\right)$ around $10^{4} \mathrm{~Hz}$ due to $C_{p}$. Impedance spectra were calculated according to the model in 3.4 .3 (p. $55 \mathrm{ff}$.), using parameter values: $R_{b}=60 \Omega \cdot \mathrm{cm}^{2}, \alpha=18 \Omega^{1 / 2} \cdot \mathrm{cm}, C_{m}=3 \mu \mathrm{F} \cdot \mathrm{cm}^{-2}, R_{\text {bulk }}=1000 \Omega, n=0.95, A_{\text {Electrode }}=5 \cdot 10^{-4} \mathrm{~cm}^{2}$, and $C_{p}=0.5 \mathrm{nF}\left(\varepsilon_{\mathrm{PhOP}}=2, A_{\mathrm{PhOP}}=0.785 \mathrm{~cm}^{2}, d=3 \mu \mathrm{m}\right)$.

The simulation of ECIS data illustrated in Fig. 5-29 uncovers the trend of a decreasing impedance sensitivity to cells with an increasing fraction of the electrode area coated with photoresist. In cases of a constant dimension of the gold electrode and of varying WE diameters, like presented for 2 ElOs in Fig. 59, this can, at least to some extent, explain the decreasing sensitivity with decreasing WE dimensions. The model calculations in Fig. 5-29 include approximations and assumptions for the applied parameter values. If adapted to the real electrode dimension of the 2 ElOs, $C_{p}$ values are $\sim 10$-fold smaller and, thus, parasitic effects in impedance spectra are decreased accordingly. This means that the parallel 
capacitance arising from the PhoP cannot completely explain the strong decrease in sensitivity, which is observed for the OCM-ECIS sensors with a WE diameter of $250 \mu \mathrm{m}$. Further negative effects have to be considered, too. A clue may be the fact that parasitics have a bigger impact the higher the load impedance of a system is. The load impedance is biggest for the smallest electrode size, as it can nicely be seen by the black curves in Fig. 5-9 A. Parasitic capacitances arising from the electrode contacts and wiring between the measurement chamber and impedance analyzer, therefore, reduce disproportionately strong the impedance of a cell-covered, small electrode. An unwanted and random detachment of few cells could also be the reason for a comparatively low impedance of the smaller electrode in the cell-covered state. This is directly correlated with a reduced peak of the normalized impedance and, thus, with an apparent lower sensitivity.

\section{$3^{\text {rd }}$ Generation OCM compared to $2^{\text {nd }}$ Generation OCM}

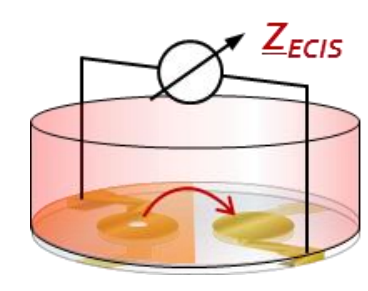

$3^{\text {rd }}$ generation $\mathrm{QCM}$

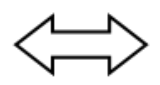

In ECIS-mode, the electrochemical properties of the $3^{\text {rd }}$ generation OCM (2EIO) are similar but not equal to the dielectric impedance characteristics of the $2^{\text {nd }}$ generation $\mathrm{OCM}(1 \mathrm{EIO})$ with a similar WE size, which were presented by Michaelis ${ }^{[236]}$. The frequency range in which the impedance of the system is sensitive to the attachment of MDCK-II cells is somewhat broader for the $2 \mathrm{EIO}(25 \mathrm{~Hz}-1 \mathrm{MHz})$ than for the $1 \mathrm{EIO}$ with the additional dipping electrode $(80 \mathrm{~Hz}-0.3 \mathrm{MHz})$. The most sensitive frequency $f_{\text {sens }}(|Z|)$ lies at $3.4( \pm 0.3) \mathrm{kHz}$ (mean $\pm \mathrm{SEM} ; \mathrm{N}=18$ ) for the former system compared to $5 \mathrm{kHz}$ for the latter. Though the most sensitive frequencies for MDCK-II cells are equal in capacitance $(40 \mathrm{kHz})$ and similar in resistance, with $290( \pm 20) \mathrm{Hz}$ for the coplanar and $250 \mathrm{~Hz}$ for the vertical ECIS-electrode arrangement, the absolute parameter values differ to some extent at similar frequencies. Upon medium loading, the $2^{\text {nd }}$ generation QCM exhibits capacitance and resistance values of $C_{40 \mathrm{kHz}}\left(t_{0}\right)=7.20( \pm 0.14) \mu \mathrm{F} \cdot \mathrm{cm}^{-2}$ and $R_{250 \mathrm{~Hz}}\left(t_{0}\right)=$ $2.97( \pm 0.06) \mathrm{kOhm}(\text { mean } \pm \mathrm{SEM} ; \mathrm{N} \geq 3)^{[236]}$, whereas for the $3^{\text {rd }}$ generation $\mathrm{OCM}$ these values amount to $C_{40 \mathrm{kHz}}\left(t_{0}\right)=10.0( \pm 0.4) \mu \mathrm{F} \cdot \mathrm{cm}^{-2}$ and $R_{250 \mathrm{~Hz}}\left(t_{0}\right)=1.64( \pm 0.06) \mathrm{kOhm}$ (mean $\pm \mathrm{SEM} ; \mathrm{N}=18$ ). Hence, starting values of electrochemical capacitance and resistance are somewhat higher and distinctly lower, respectively, for the coplanar electrode layout of the newly developed OCM-ECIS sensors. However, the observed differences in the impedance parameters of the cell-free sensors are hardly surprising, since the electrode layout and arrangement of $3^{\text {rd }}$ and $2^{\text {nd }}$ generation OCM are considerably different. Even though the nominal size of the working electrode is similar in both systems, the fabrication processes of spin coating and photolithography of the photoresist on the quartz plates also vary to some extent, which probably results in differences in the layer thickness, the sharpness of the photoresist borders, and probably also in the final electrode size.

Michaelis reported on a capacitance drop to $22 \%$ (at $40 \mathrm{kHz}$ ) and a resistance increase to $1700 \%$ (at $250 \mathrm{~Hz}$ ) with respect to starting values, when MDCK-II cells are grown to confluence on the $2^{\text {nd }}$ generation OCM. ${ }^{[236]}$ By contrast, seeding similar densities of MDCK-II cells on substrates of the standard $3^{\text {rd }}$ generation OCM results in a capacitance decreases to $18.0( \pm 0.7) \%$ (at $40 \mathrm{kHz}$ ) and a resistance increases to $2400( \pm 120) \%$ (at $250 \mathrm{~Hz}$ ). Moreover, signal changes of $C$ (at $40 \mathrm{kHz}$ ) and $R$ (at $5 \mathrm{kHz}$ ) of $17 \%$ and 
$\sim 600 \%$, respectively, are presented for NRK cell layer formation on the $2^{\text {nd }}$ generation $\mathrm{OCM}{ }^{[236]}$. These are similar to the results measured on sensors of the $3^{\text {rd }}$ generation OCM (signal changes of $21( \pm 4) \%$ in resistance and of $530( \pm 20) \%$ in capacitance) that are presented above in this thesis. The magnitudes of ECIS parameter changes upon cell attachment and spreading are similar, although not equal, for the newest generation of OCM sensors compared to the $2^{\text {nd }}$ generation OCM. This indicates that the measured signals report on similar electrochemical properties of the established cell layers. However, the values obtained from $2^{\text {nd }}$ generation $\mathrm{OCM}$ recordings can serve as orientation only, since there are too many variations in the measurement setup, the passage number of cells, the times of medium exchange, and due to experimenter- and laboratory-related differences in the cell culture and experimental implementation.

Hence, $3^{\text {rd }}$ generation QCM sensors exhibit a very pronounced sensitivity for electrochemical changes on the working electrode and, thus, ECIS-mode capability for analyzing the course of cell attachment and spreading. This is evident from the fact that cells with low barrier functionality, like NRKs, can be detected with high sensitivity, too. Moreover, the ECIS parameters also allow a distinction between various cell lines (cf. Fig. 5-30, p. 177), in both the kinetics and the overall signal change.

\subsubsection{Sensor Preincubation}

Prior to any cell-based assay the $2 \mathrm{ElO}$ sensors were equilibrated under serum-free medium for about $20 \mathrm{~h}$. After adding SFM to the sensor plates, the instantaneous time-resolved monitoring by OCM and ECIS readings uncovered non-negligible signal changes within about six hours (cf. Fig. 5-12, p. 130). In this period, the shear oscillation impedance decreases by $\sim 50 \mathrm{Ohm}$, no matter if the microbalance is coated with photoresist or not. Hence, the observed signal change cannot be traced back to any effect arising from the polymer coating, as for example swelling of the polymer layer and an accompanied increase of its viscoelasticity. Moreover, a viscoelasticity increase of an initially rigid microbalance loading is expected to rather increase than to decrease the motional impedance due to viscous damping. It seems likely that the $|Z|_{\text {min }}$ decrease is based on a temperature-related effect. The measurement chamber is filled with SFM at room temperature and is subsequently placed into the incubator for equilibration at $37^{\circ} \mathrm{C}$. The measurement chamber and the containing liquid consequently approach the temperature inside the incubator during sensor preincubation. This temperature increase of approximately $15^{\circ} \mathrm{C}$ goes along with a decrease in the dynamic viscosity of the serum-free salt solution, according to the equation of Andrade ${ }^{[464,465]}$. This again is directly correlated with a decrease in the density-viscosity product and, thus, with a decrease of the load impedance of the Newtoninan liquid on the microbalances (cf. Tab. 3-6 B, p. 44). The temperature-related effect also applies to the measured electrochemical parameter changes during the incubation period. It is well-known that the conductance of a liquid, among other things, depends on the mobility of ions, which is a function of temperature. The conductance increases by approximately $2 \%$ per $1^{\circ} \mathrm{C}$ of temperature increase. ${ }^{[466]}$ In ECIS-mode measurements at high frequencies the impedance real part reflects the bulk resistance of the system, which is the reciprocal value of the conductance. By analyzing the resistance change at $40 \mathrm{kHz}$ a conductance increase of $\sim 30 \%$ was estimated during medium incubation of the sensor surface. According to the correlation mentioned before, such an increase would be achieved by increasing the temperature of the medium by $\sim 15^{\circ} \mathrm{C}$. One gets exactly this temperature gradient during the equilibration phase, when assuming an average room temperature of $22^{\circ} \mathrm{C}$ at the beginning and final $37^{\circ} \mathrm{C}$ inside the incubator chamber. Hence, these considerations provide a strong hint that the observed signal changes are due to a temperature effect and the related changes in both viscosity and conductance 
of the liquid loading. A reduction of the equilibration phase, thus, could be achieved by a pre-warming of the whole measurement chamber as well as of the liquid used for preincubation of the sensor surface. From the signal changes in Fig. 5-12, an incubation time of six hours seems to be sufficient when starting at room temperature.

This influence of the temperature on the fluid properties and, thus, on the measured signals in both recording modes, QCM and ECIS, clearly illustrates the necessity for a temperature control in experiments. This is even more important in cell-based assays, considering that the cellular activity and cellular reactions to certain stimuli also strongly depend on the ambient temperature. In order to prevent interferences in the signals due to temperature effects as far as possible, it is essential to prewarm liquids with stimulants to $37^{\circ} \mathrm{C}$ before addition. Moreover, it is advised to keep the time of incubator opening and injection as short as possible and to use small incubators that can compensate for temperature fluctuations quickly.

\subsubsection{Cell Attachment and Spreading}

OCM-ECIS recordings, after seeding cells on the newly developed double-sensors, simultaneously provided complementary and time-resolved information on micro-mechanical as well as passive electrical alterations close to the sensor surface. The obtained signals can be related to physiological processes taking place during the attachment and spreading of MDCK-II and NRK cells. The recordings uncovered significant differences in the time courses, final values, and in mutual dependencies of the OCM and ECIS parameters for both cell types under study. This information enables deeper insights into physiological processes during cell layer formation. The simultaneous OCM- and ECIS-mode monitoring of cells therefore also provides a multiparametric database that might be used for cell profiling. This has not been accessible to now by label-free and non-invasive approaches.

\section{General QCM and ECIS Profiles of Cell Attachment and Spreading}

The initial, steep increase of $\Delta|Z|_{\min }$ within $\sim 4 \mathrm{~h}$ after cell seeding (Fig. 5-13 A, p. 132; Fig. 5-14 A, p. 136) reflect the mechanical interactions of a gradual number of cells with the sensor surface until the entire resonator is covered with cells. The spherical cells settle down to the surface of the sensor by sedimentation. When they are close enough to it $\left(\sim 50 \mathrm{~nm}{ }^{[467]}\right)$, cells begin to interact with the substrate surface by the integrin-mediated formation of initial focal adhesion sites and hemidesmosomes. ${ }^{[381-384]}$ This is followed by spreading of the cells on the growth substrate and the establishment of further cellsubstrate contacts going along with an increasing contact area. Thus, an increasing number of viscoelastic cell bodies anchors onto the sensor surface over time. Since the distance of cells anchored to the surface is smaller than the decay length of the shear acoustic wave of the resonators, damping of resonators' shear oscillation increases in the course of cell sedimentation and adhesion. Hence, cells that are closely attached to the quartz surface affect the load impedance of the microbalances, reflected in an increase of the impedance magnitude $|Z|_{\text {min }}$ near series resonance. The elementary processes of mechanical cell-substrate interaction are similar for both cell lines under study.

An almost simultaneous but contrary time course to $\Delta|Z|_{\text {min }}$ can be observed for the high-frequency capacitance $C_{\text {norm }}$ (at $\sim 30-100 \mathrm{kHz}$ ) from electrochemical (ECIS) recordings ( -0 - in Fig. $5-13 \mathrm{C}$ and Fig. 5-14 C). The immediate and strong decline of capacitance values, without any time lag after replacing medium with cell suspension, reflects the linear increase of electrode coverage with lipid double-layer membranes and, thus, settlement of cells onto the sensor (working electrode) surface. Capacitances approximate stationary values about four to six hours after cell inoculation, marking the 
point when the whole sensor surface is covered with a close and dense layer of cells. The kinetics of capacitance changes agree well with the $\Delta|Z|_{\text {min }}$ signals, which converge to stationary values in a similar time frame and report on a sensor surface that is completely loaded with viscoelastic cell bodies. Once the working electrode is covered with a confluent cell layer there is no further decrease in $C_{\text {norm }}$ values, since MDCK-II and NRK cells form a well-defined two-dimensional monolayer without growing above each other and forming a multi-layered, three-dimensional system. Hence, high-frequency capacitance from ECIS is a direct and representative measure for the number of spread cells on the substrate surface. Roughly $2 \mathrm{~h}$ ( $5 \mathrm{~h}$ ) after seeding MDCK-II (NRK) cells already $90 \%$ of the overall capacitance decrease has taken place and $\Delta|Z|_{\min }$ has reached already $80 \%(\sim 90 \%)$ of the entire signal increase, both of which report on a sensor surface that is almost completely covered with MDCK-II (NRK) cells.

After these initial phases of cell attachment that are sensitively monitored in changes of $|Z|_{\min }$ and $C_{\text {norm }}$ values, the low-frequency resistance $R_{\text {norm }}$ (at $\sim 0.1-10 \mathrm{kHz}$ ) starts to increase slowly and slightly for the NRK and faster and stronger for the MDCK-II cell layer ( - in Fig. $5^{-13} C$ and Fig. $5^{-14} C$ ). After attachment and spreading, epithelial cells start with the formation of intercellular anchoring junctions (adherens junctions, desmosomes), communication junctions (gap junctions), and sealing junctions (tight junctions). ${ }^{\left[{ }^{86-390]}\right.}$ By these, above all by the TJs, the paracellular current pathways (beneath and around cell bodies) are narrowed down, which gives rise to an increase in $R_{\text {norm }}$ at low frequencies. The appearance and tightness of sealing junctions, and the associated transcellular barrier resistance, thereby, depend on the cell line. After a strong increasing phase within $\sim 8 \mathrm{~h}$ after seeding either MDCK-II or NRK cells, the resistance approximates stationary values that remain constant for the rest of the investigation period.

The parameters extracted from combined OCM-ECIS monitoring during the MDCK-II and NRK cell attachment $\left(\Delta|Z|_{\min }, C_{\text {norm }}\right.$ and $\left.R_{\text {norm }}\right)$ all remain at quite constant levels for at least ten hours, after crossing maximal values $t \geq 10 \mathrm{~h}$ after seeding the cells. These observations report on the establishment of a confluent, stable, mechanically and electrically equilibrated, two-dimensional layer of MDCK-II or NRK cells on the OCM-ECIS sensors.

\section{Different QCM and ECIS Profiles for MDCK-II and NRK Cell Adhesion}

Significant sifferences in the kinetics as well as in the extent of OCM-mode and ECIS-mode parameter alterations are evident between MDCK-II and NRK cells during their attachment and spreading processes on the $3^{\text {rd }}$ gen. OCM sensor surface. For a better comparison the time courses of $\Delta|Z|_{\text {min }}, R_{\text {norm }}$ and $C_{\text {norm }}$ of both cell types during cell layer formation are directly compared in one figure in Fig. $5-30$. It has to be noted that in this plot the time courses of $R_{\text {norm }}$ and $C_{\text {norm }}$ are presented not at identical frequencies for NRK and MDCK-II investigations but at the respective most sensitive frequency of each parameter for each cell line (cf. sensitivity plots in Fig. 5-13 B and Fig. 5-14 B). The differences between the cell types also become apparent by comparing the characteristic points in time and the analysis parameters, which were extracted from the respective QCM/ ECIS time courses and which are listed in Tab. 5-4/ Tab. 5-5 (MDCK-II) and Tab. 5-6/ Tab. 5-7 (NRK).

For NRK cells the maximal slope in $\Delta|Z|_{\min }\left(m=6.97( \pm 0.07) \mathrm{Ohm} \cdot \mathrm{min}^{-1}\right)$ is less than a half and the time needed for half of the final $\Delta|Z|_{\text {min }}$ increase $\left(t_{1 / 2} \approx 80 \mathrm{~min}\right)$ is retarded by $\sim 40 \mathrm{~min}$ with respect to MDCK-II cells $\left(m \approx 16.61( \pm 0.06) \mathrm{Ohm} \cdot \mathrm{min}^{-1}, t_{1 / 2} \approx 40 \mathrm{~min}\right.$; cf. $\_$vs. $-\Delta-$ in Fig. 5-30). Almost equal $t_{1 / 2}$ values have been reported by Michaelis (2010) ${ }^{[236]}$ for MDCK-II and NRK cell attachment studies on $2^{\text {nd }}$ gen. OCM sensors. Final $\Delta|Z|_{\text {min }}$ values of the NRK cell layer $\left(t_{1}=20 \mathrm{~h}\right)$ are on average by $\sim 50 \mathrm{Ohm}$ below the level of the established MDCK-II cell layer. Hence, the time-resolved OCM measurements 
uncover a clear difference in the kinetics of the initial mechanical interactions of the two cell types with the resonator surface. The curve shape of $\Delta|Z|_{\min }$ is strikingly different, too. While it shows a singlephase increase during NRK cell attachment, it holds a biphasic increase for MDCK-II cells. In detail, MDCK-II cells feature a second increase of $|Z|_{\min }$ to a transient maximum ( 900 Ohm), after the initial increase to $\sim 750 \mathrm{Ohm}$ within $\sim 3 \mathrm{~h}$, which is reached after $\sim 8 \mathrm{~h}$ and which recovers afterwards to final stationary values $(\sim 850 \mathrm{Ohm})$ within about $4 \mathrm{~h}$. This characteristics have been described before for MDCK-II cells and are assigned to a rearrangement of the actin cytoskeleton after attachment and spreading of the cells. ${ }^{[22,236,468]}$ The cortical actin network and stress fibers are known as main contributors to the membrane tension, the mechanical properties and stiffness of cells. ${ }^{[469-471]}$ Thus, alterations in the actin structures of anchored MDCK-II cells cause micromechanical stiffness changes within the cell bodies that affect the shear oscillation properties of microbalances. However, NRK cells do not alter cytomechanics like MDCK-II cells do after having attached to the substrate, at least not to an extent and in a time frame that can be observed by the OCM recordings.

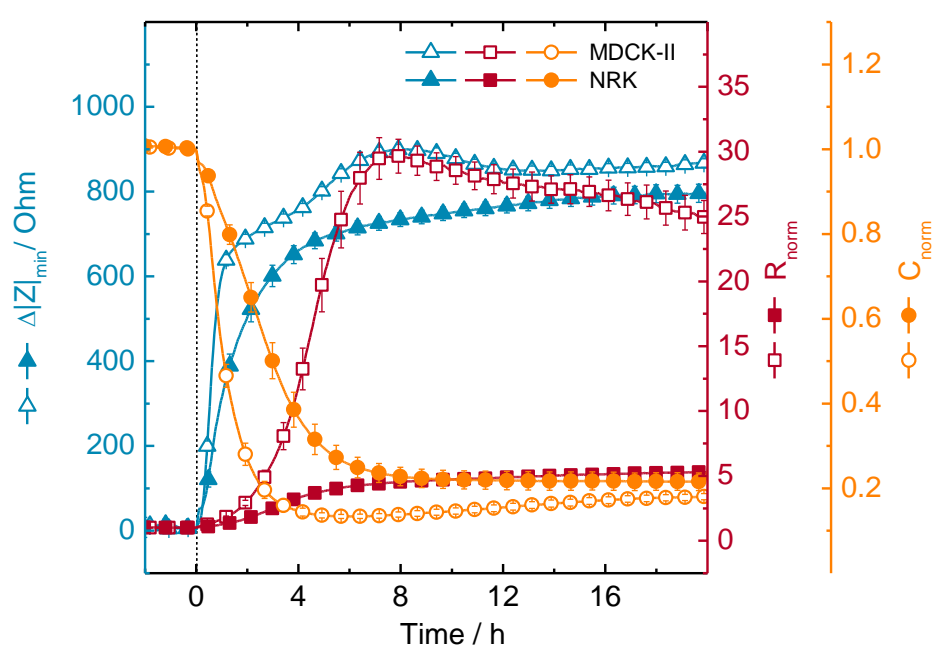

Fig. 5-30 Comparison of MDCK-II (open symbols) and NRK (filled symbols) cell attachment and spreading on $3^{\text {rd }}$ gen. QCM sensor surfaces by the time courses of parameters from recordings in QCM-mode $\left(\Delta|Z|_{\text {min }}\right.$ : triangles) and ECIS-mode ( $R_{\text {norm }}$ : squares; $C_{\text {norm }}$ : circles). $R_{\text {norm }}$ and $C_{\text {norm }}$ are displayed at the most sensitive frequency of each cell line, which are $316 \mathrm{~Hz}$ and $40 \mathrm{kHz}$ for MDCK-II cells, and $5 \mathrm{kHz}$ and $63 \mathrm{kHz}$ for NRK cells, respectively. Data points were reduced for the sake of clarity. (mean $\pm \mathrm{SEM} ; \mathrm{N} \geq 14$ ).

Besides the slower kinetics of the mechanical cell-substrate interactions, NRK cell attachment also causes a six-fold slower decrease rate (maximal slope) of the high-frequency capacitance $(m=\Delta C / \Delta t=$ $\left.-{ }^{\circ} 2.1 \mathrm{nF} \cdot \mathrm{h}^{-1}\right)$ compared to MDCK-II cell attachment $\left(m=-12 \mathrm{nF} \cdot \mathrm{h}^{-1}\right)$. Moreover, NRK cells attain the $50 \%$ value of the final $C_{\text {norm }}$ level about $11 / 2 \mathrm{~h}$ later $\left(t_{1 / 2}(C) \approx 140 \mathrm{~min}\right)$ than MDCK-Il cells $\left(t_{1 / 2}(C) \approx 50 \mathrm{~min}\right.$; cf. -0 vs. $-0-$ in Fig. 5-30). Compared to these findings, a similar $t_{1 / 2}$ value of the capacitance decrease for NRK cells and a $30 \mathrm{~min}$ retarded $t_{1 / 2}$ value for MDCK-II cells have been reported by Michaelis (2010) ${ }^{[236]}$ for cell adhesion on $2^{\text {nd }}$ gen. OCM sensors. The decrease rate $m$ of the capacitance reflects the gradual coverage of the working electrode with cell membrane and, thus, is a direct measure of the spreading rate of cells. It has been previously used for the quantitative analysis of MDCK-II cell spreading on ECIS electrodes that were precoated with different proteins. ${ }^{[89]}$ Frisch and Thoumine presented in 2002 a model for the prediction of cell spreading kinetics. ${ }^{[472]}$ According to this, the kinetics of cell 
spreading is directly proportional to the ratio of adhesion energy $E_{\text {adh }}$ and the cortical tension of the cell membrane $\sigma_{\text {mem }}$ :

$$
m \propto E_{\mathrm{adh}} / \sigma_{\mathrm{mem}}
$$

The spreading rate extracted from ECIS measurements, thus, can be applied as a relative measure for the energy that is necessary for cell adhesion on various biomaterial surfaces, provided that one uses the same cell type under consistent experimental conditions and assuming a constant cortical membrane tension. Cell-based OCM studies have been used in the past for testing the biocompatibility of various polymer surfaces, by analyzing characteristic shear oscillation parameters during cell adhesion on polymer-coated quartz plates. ${ }^{[236]}$ The combined OCM and ECIS measurements, therefore, provide an additional valuable and independent analysis parameter for quantifying the cell-substrate interactions and the cytocompatibility of substrate materials, by means of the spreading rate. It should be noted here that for insulating coatings however only OCM-mode measurements are possible. The significantly lower spreading rate and the retarded $t_{1 / 2}$ value of NRK cells both report on a slower rate of cell adhesion on the OCM-ECIS sensor surface compared to MDCK-II cells. This confirms the above-mentioned observations made by OCM measurements. Reasons for the different adhesion kinetics may arise from cell type-specific differences in (i) the cell density and diameter, (ii) the pre-immobilization of adhesionpromoting proteins, (iii) the number of specific receptor molecules expressed on the cell surface for these proteins, (iv) the kinetics and the degree of focal adhesion formation, ( $v$ ) the cortical tension of the plasma membrane that counteracts cell spreading, or (vi) cooperative effects of attached cells, which promote the attachment of further cells in their proximity. In the final stages of monolayer formation $\left(t_{1}=20 \mathrm{~h}\right)$, the normalized capacitances of both cell types approximate almost similar values, indicating a comparable amount of membrane coverage on the surface and the confluence of cell layers, respectively.

The biggest and most evident difference between the two cell types is reflected in the time traces of lowfrequency resistance. Roughly two hours after seeding cells, $R_{\text {norm }}$ values drift apart for sensor surfaces inoculated with NRK and MDCK-II cells ( - vs. - - in Fig. 5-30). While $R_{\text {norm }}$ increases comparatively little by a factor of five for a confluent NRK cell layer, it increases on average up to 25 -fold - transiently even up to 30 -fold - higher values than the cell-free electrode for a confluent layer of MDCK-II cells. This is due to the formation of close tight junctions between adjacent MDCK-II cells, being a tight intercellular diffusion barrier for the transepithelial transport of macro-molecules and for low-frequency current flow as well. ${ }^{[473-478]}$ The barrier resistance of MDCK-II layers, thus, is much higher compared to leakier cell layers like NRK, which originates in the latter case solely from the intercellular and the cell-substrate constrictions and by the non-sealing adherens junctions and desmosomes. Absolute capacitance/ resistance values and changes, however, have to be compared with caution, since the readout frequencies of parameters differ for the two cell lines. The frequencies chosen for time-resolved analyses reflect the frequencies where the capacitance/ resistance responds with a maximal signal change upon the attachment and spreading of cells (value at $t=20 \mathrm{~h}$ ) with respect to the cell-free value before seeding $(t=o \mathrm{~h}$ ). The most sensitive frequencies themselves, thereby, also represent valuable analysis parameters to distinguish cell types and to characterize cell layer properties.

\section{Correlation of QCM and ECIS Data}

The differences in QCM- and ECIS-mode responses of MDCK-II and NRK cells also become apparent in the 3-D QCM-ECIS impedance plot in Fig. 5-31 A. This way of representation allows for a distinct discrimination of cell types on the basis of the three parameters $\Delta|Z|_{\text {min }}, R_{\text {norm }}$ and $C_{\text {norm }}$ in a single 
diagram. It has to be noted that in this plot the time courses of $R_{\text {norm }}$ and $C_{\text {norm }}$ are presented not at identical frequencies for NRK and MDCK-II cells, but at the respective most sensitive frequency of each parameter for each cell line (cf. sensitivity plots in Fig. 5-13 B and Fig. 5-14 B). A confluent NRK cell layer covering the OCM-ECIS sensor surface causes a comparatively small increase in $R_{\text {norm }}$ and, consequently, a small expansion of the $3-D$ curve in y-axis direction (blue curve). By contrast, MDCK-Il cells (black curve) possess a strong expansion of the trace into the $y$-dimension. Starting from equal parameter values $\left(\Delta|Z|_{\min }\left(t_{0}\right)=0 ; R_{\text {norm }}\left(t_{0}\right)=C_{\text {norm }}\left(t_{0}\right)=1\right)$, final values of confluent NRK and MDCK-II cell layers (at $\left.t_{1}=20 \mathrm{~h}\right)$ are located in different regions of the $\Delta|Z|_{\text {min }}-R_{\text {norm }}-C_{\text {norm }}$ parameter space. Besides the discrimination of cell types at a certain point of time in the 3-D parameter space, the time-resolved recording of parameters additionally enables to analyze the entire curve shape - in the space or projected on the different planes - for identifying differences between cell types. Hence, Fig. 5-31 A reveals a characteristic and unique "OCM-ECIS signature" for MDCK-II and NRK cells between the points of cell seeding and an established confluent cell monolayer on the QCM-ECIS sensors. The projections of the 3-D curve to the planes allow for a more detailed analysis of parameter dependencies. The respective 2-D plots of $C_{\text {norm }}$ vs. $\Delta|Z|_{\text {min }}, R_{\text {norm }}$ vs. $\Delta|Z|_{\text {min }}$ and $C_{\text {norm }}$ vs. $R_{\text {norm }}$ are illustrated in Fig. 5-31 B, C, and D.
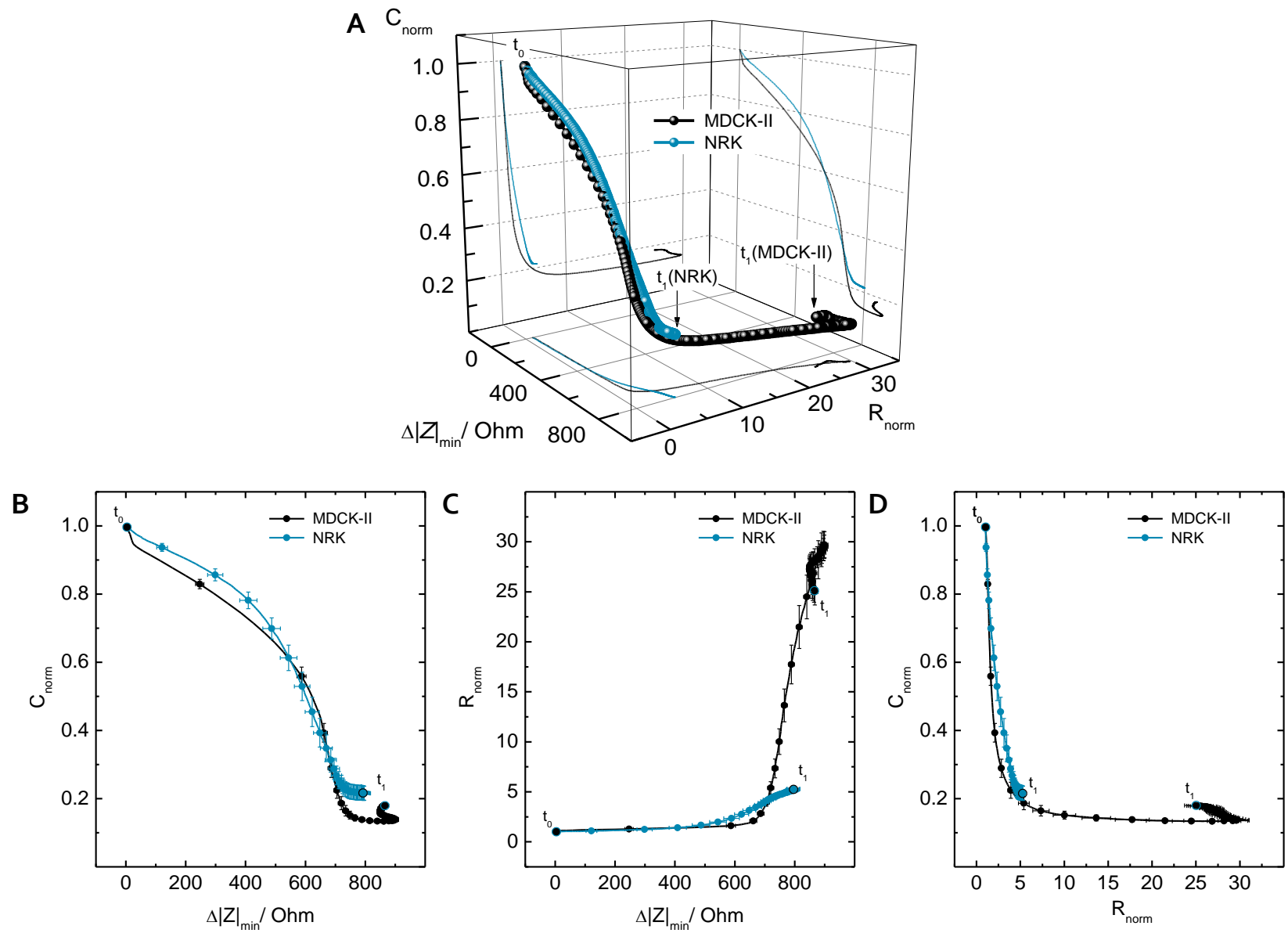

Fig. 5-31 (A) 3-D QCM-ECIS impedance plot $\left(\Delta|Z|_{\min }\right.$ vs. $R_{\text {norm }}$ vs. $\left.C_{\text {norm }}\right)$ for MDCK-II ( $\left.\rightarrow-\right)$ and NRK $(-)$ ) cell attachment and spreading on $3^{\text {rd }}$ gen. QCM sensor surfaces between $t_{0}$ and $t_{1}=20 \mathrm{~h}$. (B), (C), (D) Projections on the planes of the 3-D curve in A. $R_{\text {norm }}$ and $C_{\text {norm }}$ are displayed at the most sensitive frequency of each cell line, wich are $316 \mathrm{~Hz}$ and $40 \mathrm{kHz}$ for MDCK-II cells, and $5 \mathrm{kHz}$ and $63 \mathrm{kHz}$ for NRK cells, respectively. Data points were reduced for the sake of clarity. The time resolution between two points in $B, C$, $D$ is 28 min. (mean $\pm S E M ; N \geq 14$ ). 
One can clearly distinguish between separate phases (physiological processes) during the MDCK-II and NRK cell layer formation, based on the three QCM-ECIS sensing parameters. By plotting the parameter changes against each other one can identify four phases for MDCK-II cells (cf. Fig. 5-13 D, E, F) and three phases for NRK cells (cf. Fig. 5-14 D, E, F). Though MDCK-II and NRK cells show different kinetics in the single OCM and ECIS parameter changes (cf. Fig. 5-30), the correlation plots disclose that the changing rates of the parameters relative to each other are similar for both cell types in the first two phases of cell adhesion (Fig. 5-31). This applies for the mutual dependencies of all three parameters. Hence, similar processes take place for NRK and MDCK-II cells, which only differ in the kinetics. They can be likely assigned to cell type-independent physical cell-substrate interactions, i.e. the mechanical interaction of the viscoelastic cell bodies with the microbalance surface $\left(\uparrow \Delta|Z|_{\min }\right)$ and the membrane coverage of the working electrode due to cell settlement $\left(\downarrow C_{\text {norm }}\right)$.

In the third and last phase of NRK cell adhesion there is a slight and simultaneous increase of $\Delta|Z|_{\text {min }}$ and $R_{\text {norm }}$ (Fig. 5-31 C), while $C_{\text {norm }}$ has already reached constant values. This is likely related to the formation of cell-cell contacts (adherens junctions, desmosomes) after cell spreading. These intercellular connections join together the actin microfilaments and keratin intermediate filaments of neighboring cells ${ }^{[386-388]}$ to a mechanically stiffened supracellular cytoskeletal network ${ }^{[479]}$, which is likely associated by a little reorganization of cytoskeletal structures $\left(\uparrow \Delta|Z|_{\text {min }}\right)$, and which narrows the gap between the cells $\left(\uparrow R_{\text {norm }}\right)$. The processes of cell attachment and the formation of cell-cell junctions within the sensor area, however, overlap in time, resulting in mixed signals in this transitional period. For instance, cells attached to the surface next to each other begin to intercommunicate and to form adherens junctions, while the sensor surface is not yet completely covered with cells.

The interpretation of initial processes of NRK cell-substrate and cell-cell interaction after seeding (phases I-III) also account for MDCK-II cells. However, the 3-D curve and the 2-D projections of MDCK-II and NRK cell layer formation start to diverge from the point when MDCK-II cells cause an enormous increase in $R_{\text {norm }}$ (cf. - - in Fig. 5-30), which is most evident in the diagrams Fig. 5-31 A, C, and D. This marks the beginning of the third phase in OCM and ECIS parameter changes during MDCK-II monolayer establishment. The resistance increase observed for MDCK-II cells, thus, has to be considered as a separate process, which cannot be assigned to the parameter changes and their mutual dependencies that are related to the attachment and spreading process of cells. The drastic resistance increase is assigned to the formation of a much closer paracellular (electrical) barrier for the MDCK-II compared to the NRK cell layer, arising from either a narrower subcellular gap or tighter cell junctions of neighboring cells, or contributions of both. From the overlay of $\Delta|Z|_{\text {min }}$ and $R_{\text {norm }}$ time courses of MDCK-Il cell layer formation ( $-\Delta-$ and $-\square-$ in Fig. 5-30) one can identify that the strong response from TJ formation ( $\uparrow R_{\text {norm }}$ ) takes place in the time frame when $\Delta|Z|_{\text {min }}$ starts the second increase from the first plateau to the transient maximum around $t \approx 3-4 \mathrm{~h}$. Moreover, maximal barrier resistance and maximal oscillation damping coincide at $\sim 490 \mathrm{~min}$ after seeding (cf. $t^{\prime \prime}$ in Tab. 5-4 and $t^{\prime}$ in Tab. 5-5). Subsequent slight recoveries of $\Delta|Z|_{\min }$ and $R_{\text {norm }}$ signals to final values exhibit simultaneous time courses, too (cf. Fig. 530). Since for NRK cell attachment and spreading neither a second maximum in $\Delta|Z|_{\text {min }}$ nor a strong resistance increase can be observed like for MDCK-II, this is already a first hint for a correlation of cytoskeleton rearrangement and barrier formation in MDCK-Il cell layers.

The simultaneous, time-resolved QCM-ECIS monitorings could confirm this synchronicity of intercellular gap closure by barrier-forming tight junctions $\left(\uparrow R_{\text {norm }}\right)$ and major changes in the cytomechanics $\left(\uparrow \Delta|Z|_{\text {min }}\right)$. This becomes evident in the 2-D correlation-plot of the parameters in Fig. 5-31 C. The trace of MDCK-II cells ( $\rightarrow$ ) shows a synchronous increase (linear slope) in $R_{\text {norm }}$ and $|Z|_{\text {min }}$ to transient maximum values $\left(\Delta|Z|_{\min }: \sim 700 \rightarrow 900 \mathrm{Ohm}, R_{\text {norm }}: \sim 3 \rightarrow 30\right.$ ). Afterwards, there is a likewise 
synchronous decrease to final values that runs exactly backwards along the path of the parameter dependencies in the previous increasing phase. Hence, there is evidence of a direct correlation of mechanical alterations within the cell layer and changes in the paracellular tightness. These individually measured cell layer properties, thus, can very likely be assigned to the same or correlated physiological processes. It is well known that the TJs are linked via ZO proteins to cytoskeletal actin structures of the connected cells. Changes in either of both structures are assumed to affect the respective other. It could be demonstrated by the combined QCM-ECIS measurements that there is not only a structural but a direct functional correlation of the actin cytoskeleton and the TJs. This is corroborated by the results of cell-based assays, in which the formation or functionality of either structure was specifically perturbed by the absence of divalent cations or by treating cells with cD (cf. discussions below). The results so far lead to the conclusion that the formation of tight junctions requires for a reorganization of the actin cytoskeleton, which causes a transient stiffening of cells. It is assumed that the cell boarders of neighboring cells are laterally pressed together by the cytoskeleton stiffening in order to increase the intercellular contact area and, thus, to enable the formation of adherens junctions directly and of tight junctions indirectly. This is supported by microscopic studies of Heitmann et al. (2007) ${ }^{[22]}$, which demonstrated for MDCK-II cells at the point of maximal $\Delta|Z|_{\text {min }}$ a maximal thickening of actin belt structures, following the cell periphery close to the apical pole (zonula adherens), as well as the presence of pronounced stress fibers that interconnect individual focal adhesion sites. After the actin belt has formed and AJs (and TJs) have established, $|Z|_{\min }$ values recover again from maximal levels. It is assumed that this mirrors the reduction of the force against the substrate (surface tension), which was necessary for cell spreading and the lateral pressure on cell-cell contacts for junction establishment. Once intercellular junctions are matured and spreading pressure is reduced, tension exerted by the cytoskeleton causes a slight contraction of cells again. This could also explain the accompanied slight reduction of the maximal barrier resistance, as the contractile forces may cause an expansion of the intercellular cleft on the apical and basolateral side of the ZO to some extent again, which gives rise to current pathways and, thus, reduces the paracellular impedance. Such structural processes fail to appear in NRK cell layers. NRK cells show no distinct reorganization of the actin cytoskeleton and formation of an actin belt structure at AJs, respectively, as seen from the $\Delta|Z|_{\min }$ time course. In addition to significantly weaker adherens junctions also no barrier forming thight junctions are formed between individual cells of this type.

\section{Modeling of ECIS Data}

For a more detailed analysis of ECIS parameter changes due to the attachment and spreading of MDCK-II and NRK cells, the impedance data was fitted by the model presented in 3.4.3 Modeling the Impedance of the Cell-Electrode Interface (p. 55 ff.). The resulting values of the fitting parameters $R_{b}, \alpha$, and $C_{m}$ are compared in Fig. 5-32 for MDCK-II ( $\square$ ) and NRK ( $\square$ ) cell layers. One can identify very pronounced differences in the paracellular barrier resistance $R_{b}$ between both cell-lines. It is about 15 times higher for $\operatorname{MDCK}-I I\left(R_{b} \approx 70 \Omega \cdot \mathrm{cm}^{2}\right)$ with respect to NRK cells $\left(R_{b} \approx 5 \Omega \cdot \mathrm{cm}^{2}\right)$. This confirms the assumption of much tighter intercellular junctions in a MDCK-II cells, which was already made from the impedance real part (equivalent resistance) at low frequencies and which is also described in literature. The cell adhesion parameter $\alpha$, which represents the impedance of current flow in the subcellular cleft, as well shows distinctively $\left(\sim 5\right.$-fold) higher values for the MDCK-II $\left(\alpha \approx 23 \Omega^{1 / 2} \cdot \mathrm{cm}\right)$ compared to the NRK cell layer $\left(\alpha \approx 5 \Omega^{1 / 2} \cdot \mathrm{cm}\right)$. The $\alpha$ value is a function of the mean cell diameter, the average cell-substrate distance, and the specific resistivity of the subcellular electrolyte (cf. Eq. (49). The discrepancy of values, therefore, reports on either a bigger mean radius, or a smaller subcellular cleft, or a higher resistivity of the medium 
beneath the cells, or any combination of these of MDCK-II compared to NRK cells. Values of the third modeling parameter, the membrane capacitance $C_{m}$, differ between the two cell lines as well. MDCK-II cells $\left(C_{m} \approx 5 \mu \mathrm{F} \cdot \mathrm{cm}^{-2}\right)$ exhibit approximately twice the membrane capacitance of NRK cells ( $C_{m} \approx$ $\left.2 \mu \mathrm{F} \cdot \mathrm{cm}^{-2}\right)$. The values of the model parameters obtained for MDCK-II and NRK cell layers are in good agreement with the literature, reporting for MDCK-II and NRK cells on $R_{b}$ values of $40-150 \Omega \cdot \mathrm{cm}^{2}$ $[108,427,473-475,480-485]$ and $\sim 6 \Omega \cdot \mathrm{cm}^{2}{ }^{[7]}, \alpha$ values of $\sim 18 \Omega^{1 / 2} \cdot \mathrm{cm}^{[480,481]}$ and $\sim 4 \Omega^{1 / 2} \cdot \mathrm{cm}{ }^{[7]}$, and $C_{m}$ values of $\sim 4 \mu \mathrm{F} \cdot \mathrm{cm}^{-2}[480,481]$ and $\sim 2 \mu \mathrm{F} \cdot \mathrm{cm}^{-2}[7]$, respectively.

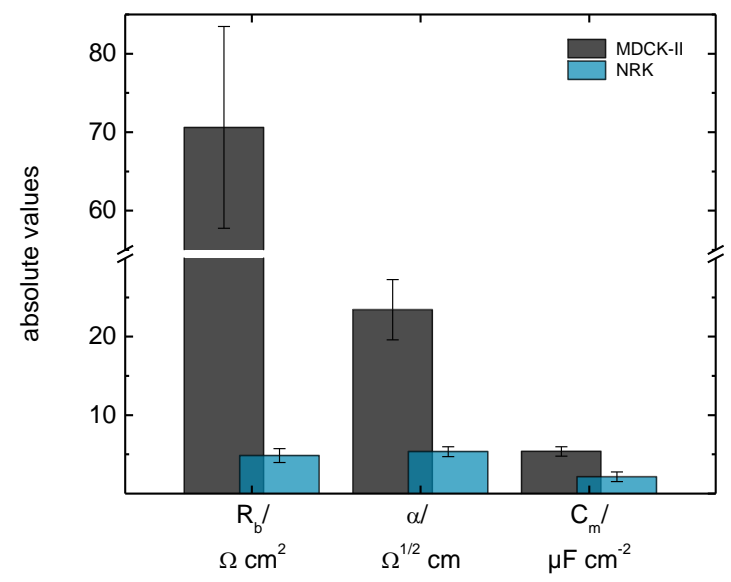

Fig. 5-32 Modeling the impedance data of cell-covered electrodes, according to the model presented in subsection 3.4.3, provided the characteristic frequency-independent parameters $R_{b}$, $\alpha$, and $C_{m}$ for MDCK-II ( $\square$ ) and NRK ( $\square$ ) cell layers (mean \pm SEM; N $\geq 2$ ). The bulk resistance $R_{\text {bulk }}$ and the CPE parameters $n$ and $A$ (cf. Eq. (18)) were determined by linearly fitting the impedance spectra at high and low frequencies, respectively. Mean parameter values are $R_{\text {bulk }}=442( \pm 19) \Omega, n=0.971( \pm 0.004), A=1.64( \pm 0.04) \cdot 10^{-5} \mathrm{~F} \cdot \mathrm{s}^{n-1} \cdot \mathrm{cm}^{-2}($ mean $\pm \mathrm{SEM} ; \mathrm{N}=10)$ for an electrode surface area of $2.05 \cdot 10^{-3} \mathrm{~cm}^{2}$.

In summary, the distinct time courses of all OCM-ECIS sensor parameters $\left(\Delta|Z|_{\text {min }}, C_{\text {norm }}\right.$ and $\left.R_{\text {norm }}\right)$ after seeding a suspension of adherent cells demonstrate the capability of the dual sensor for the timeresolved, multi-parametric, label-free, and sensitive analysis of cell-substrate and cell-cell interactions during cell layer formation. Moreover, the analysis of the absolute values and time courses of the characteristic OCM- and ECIS-mode sensor parameters uncovers significant differences in the attachment and spreading between NRK and MDCK-II cells. This demonstrates the great performance of the individual sensor technologies, and the potentiated performance and high-content information of combined measurements, which are accessible by the new OCM-ECIS double sensor, presented in this work. The fitting of the ECIS impedance data by a biophysical model provides deeper insights into the physiological origin of the measured signals. Moreover, the comparison of OCM and ECIS parameter changes in 3-D and 2-D plots enables the time-resolved illustration of parameter dependencies. This way of representing mutual dependencies of OCM and ECIS parameters in one diagram may be confusing at a first glance, and it may be hard to extract structural and functional conclusions from that. However, these diagrams comprise a lot of information and allow to make visible correlations between viscoelastic and dielectrical alterations in a cell layer, like for instance the mutual dependency of TJ formation and actin cytoskeleton rearrangement in MDCK-II cell layers. This has not been presented to date on the basis of label-free technologies. 


\subsubsection{Cell Attachment and Spreading in Absence of $\mathrm{Ca}^{2+}$ and $\mathrm{Mg}^{2+}$}

Combined OCM-ECIS measurements provided significant differences in the curve shapes of attachment and spreading, when seeding MDCK-II cells in a medium either lacking $\mathrm{Ca}^{2+}$ or $\mathrm{Mg}^{2+}$ on fibronectin-coated QCM-ECIS sensors, compared to time courses obtained in presence of physiological concentrations of $\mathrm{Ca}^{2+}(1.8 \mathrm{mM})$ and $\mathrm{Mg}^{2+}(0.81 \mathrm{mM})$ (cf. Fig. 5-15, p. 141). This is also reflected in characteristic values that were extracted from $\Delta|Z|_{\text {min }}, C$, and $R$ time courses, listed in $S / 17$ (p. 271).

\section{Impact of Divalent Cations on OCM and ECIS Cell Adhesion Profiles}

In cases when $\mathrm{Mg}^{2+}$ was present in the cell suspension, either alone or in co-presence of $\mathrm{Ca}^{2+}(-\square-$ and $\rightarrow-$ in Fig. 5-15), cells show faster attachment kinetics $\left(m, t_{m}, t_{1 / 2}\right)$ in $\Delta|Z|_{\min }$, compared to seeding conditions where $\mathrm{Mg}^{2+}$ was omitted in the extracellular medium (- - in Fig. ${ }^{-15}$ ). This becomes just as apparent in the passive electrical properties obtained from ECIS. Though the spreading rate of cells (maximal slope in capacitance) is not lowered without $\mathrm{Mg}^{2+}$, the points in time of maximal capacitance decrease $\left(t_{m}\right)$ and of $50 \%$ values $\left(t_{1 / 2}\right)$ are delayed. Hence, in absence of $\mathrm{Mg}^{2+}$, the mechanical cellsubstrate interaction is retarded and the adhesion energy is reduced (cf. Eq. (66)). These results agree with ECIS investigations made by Wegener et al. (2000) ${ }^{[89]}$, confirming that the MDCK-II cell-surface receptors for $\mathrm{FN}$ exhibit a higher binding affinity in the presence of $\mathrm{Mg}^{2+}$. The integrin $\alpha_{v} \beta_{3}$ was identified ${ }^{[486]}$ as one possible FN receptor expressed on the MDCK-II cell surface, to which a $\mathrm{Ca}^{2+}$-dependent enhancement of binding function was attributed in the presence of $\mathrm{Mg}^{2+}{ }^{[385]}$. However, there is no evidence from presented MDCK-II attachment studies for a significantly faster and/ or stronger binding of cells in the co-presence of $\mathrm{Mg}^{2+}$ and $\mathrm{Ca}^{2+}$ compared to the presence of $\mathrm{Mg}^{2+}$ alone. From $\Delta|Z|_{\text {min }}$ and $C$ time courses, one may even identify a slightly slower and little delayed MDCK-II cell attachment and spreading, if $\mathrm{Ca}^{2+}$ is co-present to $\mathrm{Mg}^{2+}$ in the extracellular medium, as if it is not. This could indicate a contrary effect of a $\mathrm{Ca}^{2+}$-dependent inhibition of integrin-mediated cell adhesion, as it has been reported for $\alpha_{v} \beta_{1}$ receptors ${ }^{[385]}$. The kinetic differences of parameter time courses, however, are little so that further time-resolved measurements are required in order to uncover statistically significant effects of both $\mathrm{Mg}^{2+}$ and $\mathrm{Ca}^{2+}$ in the early stages of cell adhesion on the basis of the QCM-ECIS sensors.

After the initial attachment process of cells on the sensor electrodes, the traces of $\Delta|Z|_{\min }, C$, and $R$ significantly differ, dependent on whether the cells were seeded in medium containing physiological concentrations of $\mathrm{Ca}^{2+}$ or not. However, no significant $\mathrm{Mg}^{2+}$-dependent differences can be seen anymore. Capacitance values (at $100 \mathrm{kHz}$ ) drop to lower values in presence of $\mathrm{Ca}^{2+}$ in buffer within the first four hours after cell inoculation as if $\mathrm{Ca}^{2+}$ was absent (- - vs. - and $-\bullet-$ in Fig. ${ }^{-15} \mathrm{C}$ ). In absence of $\mathrm{Ca}^{2+}$ capacitance values even increase slowly towards cell-free values for the rest of the investigation period, afterwards. This indicates morphological alterations within the cell layer after the initial cell anchorage and spreading on the substrate. Since the capacitance at high frequencies predominantly reports on the electrode surface coverage with cell membrane, the recovery in capacitance values indicates a partial detachment of cells or at least the formation of gaps in the cell layer by rounding up of the cells. Moreover, there is no biphasic shape in the time course of the OCM analysis parameter $\Delta|Z|_{\text {min }}$ in absence of $\mathrm{Ca}^{2+}$ (- - in Fig. 5-15 A) as it is observed in presence of $\mathrm{Ca}^{2+}(-$ and $-\mathrm{a}$ in Fig. 5-15 A) and when MDCK-II cells are seeded in complete medium on uncoated sensors (cf. Fig. 5-13 A, p. 132). As this transient maximum of cell stiffness is assigned to a reorganization of the actin cytoskeleton, the $\mathrm{QCM}$ data indicate that low calcium levels in any way inhibit such a rearrangement process of cytoskeletal structures. This cytoskeleton remodeling is associated with the formation of actin stress fiber bundles linking FAs and with the formation of an peripheral actin belt and intercellular AJs. Calcium-dependent 
integrins and the cadherins of these junctional structures are the key for the effect of $\mathrm{Ca}^{2+}$ on cell stiffness and the observations in OCM-mode measurements. It is interesting that after seeding cells without $\mathrm{Ca}^{2+}$ the level of minimal impedance is almost exactly the level of the transient maximum which is attained when cells were seeded in presence of $\mathrm{Ca}^{2+}$. Moreover, $\Delta|Z|_{\text {min }}$ values remain constant for the rest of the investigation period after the initial strong increase within two hours. This clearly reports on consistent mechanical properties of the cell bodies that remain firmly attached to the sensor surface. No significant cell detachment is detectable from this data, which would be reflected in a drop of $|Z|_{\min }$ towards cellfree values, like for instance observable in Fig. 5-17 A after treatment of the cell-layer with Saponin.

Electrochemical resistance data clearly show a $\mathrm{Ca}^{2+}$-related strong increase in the low-frequency resistance (at $630 \mathrm{~Hz}$ ) and, thus, the establishment of a transepithelial permeability barrier $(-$ and $-\bullet$ in Fig. 5-15 B, D). This indicates predominantely the formation of tight intercellular connections. This can not be observed for cells that were cultured on the OCM-ECIS sensors in medium lacking $\mathrm{Ca}^{2+}$ ( $-\square-$ in Fig. 5-15 B, D). Hence, the impedance data provide evidence that the presence of $\mathrm{Ca}^{2+}$ is essential for the establishment of cadherin-based desmosomes and AJs and, thus, indirectly also of TJs. In absence of $\mathrm{Ca}^{2+}$ this cannot be compensated for by $\mathrm{Mg}^{2+}$ as divalent cation. However, there is also a little increase in the low-frequency resistance. This may be assigned to narrowing of either the subcellular gap or of the intercellular space or to contributions of both, due to the progressing assembly of cell-substrate contact sites and any cell-cell junctions except for those requiring $\mathrm{Ca}^{2+}$. There is also evidence that $\mathrm{TJ}$ formation does not depend on $\mathrm{Mg}^{2+}$, at least not to an appreciable extent, since both the kinetics and the absolute final values of resistance increase are similar for MDCK-II cell attachment and spreading in EBSS ${ }^{--}$ containing $\mathrm{Ca}^{2+}$ only or $\mathrm{Ca}^{2+}$ and $\mathrm{Mg}^{2+}$. Besides these different time courses and degrees of $R$ and $C$ signal changes, the sensitive frequencies of both quantities for the MDCK-II cell attachment also depend on whether extracellular calcium is present or not, and are shifted to higher values in the latter case (cf. $\mathrm{SI}$ 18). Hence, the most sensitive frequencies of ECIS parameters themselves can serve as indicators for different morphological properties of cell layers of the same type. The prerequisite of calcium for TJ establishment as well as for maintaining both the structural and functional integrity of the junctional

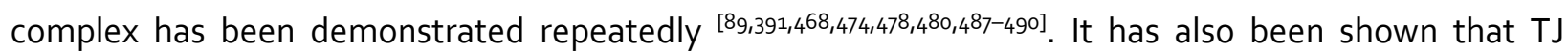
opening, by withdrawing calcium, can be reversed upon restoring $\mathrm{Ca}^{2+}[398,474,488]$, with a complete resealing achieved within $2-3 \mathrm{~h}$. There is evidence that the TJs do not depend directly on $\mathrm{Ca}^{2+}{ }^{[346,491]}$, but that the effect of low calcium on other junctional elements like ZA $[345,388,492-495]$ or desmosomes

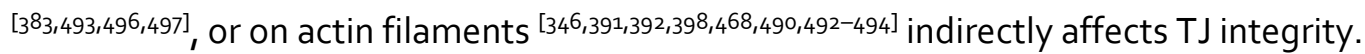

\section{Correlation of QCM and ECIS Data}

In Fig. 5-33 A, the time-resolved changes of the characteristic microbalance and electrochemical sensor parameters are merged in a single 3-D OCM-ECIS impedance diagram. The projections of the spatial curves to the planes are represented in 2-D plots in Fig. 5-33 B, C, and D. This way of representation reveals mutual parameter dependencies. From these plots, a simultaneous change of the capacitance and the minimal impedance (Fig. 5-33 B) can be observed in the first stages of MDCK-II cell attachment and spreading in EBSS ${ }^{--}$buffer containing $\mathrm{Ca}^{2+}\left(\mathrm{EBSS}^{+-}:-{ }^{-}, \mathrm{EBSS}^{++}:-{ }_{-}\right)$, just like in cell culture medium (cf. Fig. 5-31 B). The absence of $\mathrm{Ca}^{2+}\left(\right.$ EBSS $^{-+}:-{ }^{-}$) causes a somewhat smaller capacitance decrease with increasing $\Delta|Z|_{\text {min }}$. This indicates that the cells anchor to the surface and change the viscoelastic properties on the microbalance surface, however they fail to form a dense two-dimensional membrane layer on the ECIS working electrode. The curve further shows a partial recovery of the capacitance that is not accompanied by a similar recovery in $\Delta|Z|_{\text {min }}$. Hence, the $C_{\text {norm }}$ vs. $\Delta|Z|_{\min }$ plot illustrates that this is not just the reverse mechanical and electrical process of cell attachment, that is cell detachment, but 
a reduction of cell membrane while maintaining the mechanical properties close to the substrate. The $C_{\text {norm }}$ vs. $R_{\text {norm }}$ plot (Fig. 5-33 D) illustrates simultaneous changes of both parameters (a steep decrease in $C_{\text {norm }}$ and a marginal increase in $R_{\text {norm }}$ ) within the first two hours after cell inoculation. This course is independent on the presence or absence of physiological amounts of $\mathrm{Ca}^{2+}$ and $\mathrm{Mg}^{2+}$, due to the initially equal, passive processes of cell sedimentation and gravity-driven spreading on the FN-coated electrodes. These cell-substrate interactions cause the capacitance to decreases and the resistance to increase, due to the lipid bilayer coverage on the WE and as low-frequency current is forced to flow beneath and around the cell bodies, respectively. While the resistance increases strongly later in the time course in presence of $\mathrm{Ca}^{2+}(-\rightarrow,-$ in Fig. 5-33 D) due to cell-cell junction (TJ) formation, there is a reversal point in absence of $\mathrm{Ca}^{2+}$ (- - - in Fig. 5-33 D). The rearward course of parameters in the latter case, thereby, runs exactly along the same path as before the turning point, however in the opposite direction. This is assigned to exactly the converse process of cell spreading, which is the contraction and rounding up of the cells again. This morphologic alteration is what opens the way again for both low- as well as high-frequency current. From that it is concluded that in the absence of $\mathrm{Ca}^{2+}$ ions, after cell settlement and initial spreading, cells fail to establish both integrin-based adhesion contacts (FAs) to their growth substrate and cadherinbased anchoring contacts (AJs) to adjacent cells, whereas the latter probably depends on the former.

A
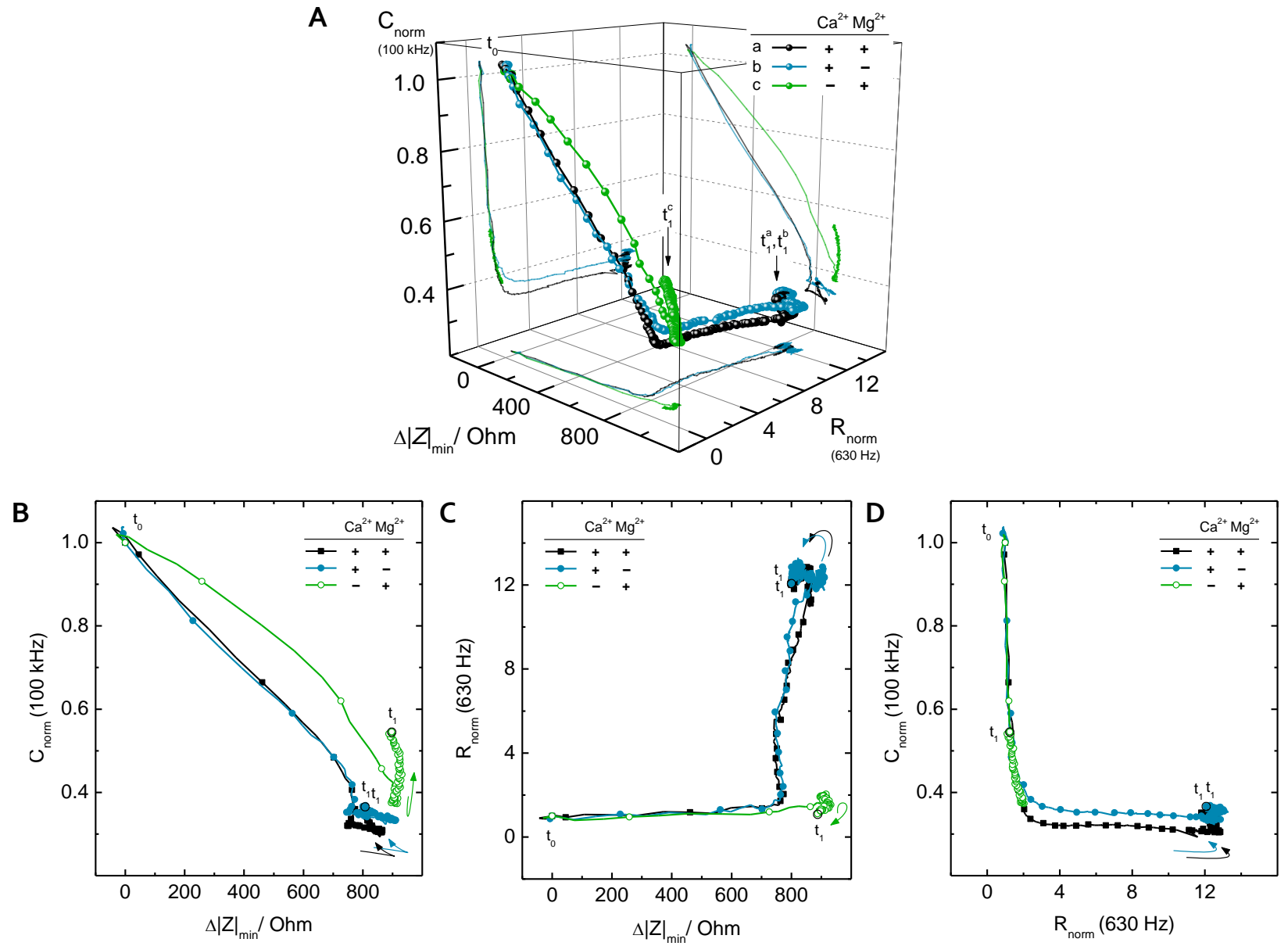

Fig. 5-33 (A) 3-D QCM-ECIS impedance plot ( $\Delta|Z|_{\min }$ vs. $R_{\text {norm }}$ vs. $C_{\text {norm }}$ ) of MDCK-II cell attachment and spreading on FN-coated sensors in EBSS $^{--}$buffer containing physiological amounts of either $\mathrm{Ca}^{2+}$ and $\mathrm{Mg}^{2+}(-)$ ), or $\mathrm{Ca}^{2+}$ only $(--)$, or $\mathrm{Mg}^{2+}$ only (- $\left.{ }^{-}\right)$. (B), (C), (D) Projections on the planes of the 3-D curve in A. $R_{\text {norm }}$ and $C_{\text {norm }}$ are displayed at the most sensitive frequencies in presence of $\mathrm{Ca}^{2+}$, which are $630 \mathrm{~Hz}$ and $100 \mathrm{kHz}$. Data points were reduced for the sake of clarity. The time resolution between two points in $B, C, D$ is 20 min. 


\section{Modeling of ECIS Data}

The fitting of typical impedance data from ECIS measurements by the model mentioned above 3.4 .3 Modeling the Impedance of the Cell-Electrode Interface) allows for a more detailed analysis of the physiological origin of the observed effects. The extracted model parameters are summarized in Fig. 534. The analysis reveals, firstly, that lacking $\mathrm{Mg}^{2+}$ in the seeding buffer inhibits the establishment of close cell-substrate contacts, as the cell-substrate adhesion parameter $\alpha$ is significantly reduced without $\mathrm{Mg}^{2+}$ $\left(\sim 1 \Omega^{1 / 2} \cdot \mathrm{cm} ; \square\right)$ compared to control conditions with both $\mathrm{Mg}^{2+}$ and $\mathrm{Ca}^{2+}\left(\sim 15 \Omega^{1 / 2} \cdot \mathrm{cm} ; \square\right)$. The absence of $\mathrm{Mg}^{2+}$, though, does not affect the establishment of an intercellular diffusion barrier within the confluent MDCK-II cell layer. The barrier resistance $R_{b}$ is $\operatorname{similar}\left(\sim 80 \Omega \cdot \mathrm{cm}^{-2}\right)$ at the end $(t=20 \mathrm{~h})$ of MDCK-II attachment and spreading investigations, whether in presence of $\mathrm{Ca}^{2+}$ alone or in co-presence of $\mathrm{Mg}^{2+}$. The membrane capacitance $C_{m}$ is moderately affected by low magnesium medium, exhibiting values of $\sim 4 \mu \mathrm{F} \cdot \mathrm{cm}^{-2}$ as long as sufficient $\mathrm{Ca}^{2+}$ is available. Depletion of extracellular $\mathrm{Ca}^{2+}$, however, causes a $50 \%$ reduction of $C_{m}$ to $\sim 2 \mu \mathrm{F} \cdot \mathrm{cm}^{-2}$ ( $\square$ in Fig. 5-34). Even more striking are a barrier resistance close to zero and the low $\alpha$ value of $\sim 3 \Omega^{1 / 2} \cdot \mathrm{cm}$ of the cell layer, $20 \mathrm{~h}$ after seeding in low calcium medium. This low resistance of paracellular current pathways, through cellular junctions and around the cell bodies, is what the real part data from impedance recordings (cf. Fig. 5-15 D) have already indicated. However, the fitting parameters now disclose contributions from both, not closing TJs between adjacent cells $\left(R_{b}\right)$ and a lower impedance of the subcellular cleft $(\alpha)$. The latter again reports either on a reduced mean radius of cells, or an increased volume of the subcellular cleft, or a lowered specific resistivity of the subcellular electrolyte, or any combination of these. Similar $R_{b}$ and $\alpha$ values have been reported by Lo et al. ${ }^{[480]}$ for confluent MDCK-II cell layers exposed to EBSS ${ }^{++}$and EBSS ${ }^{-+}$, and by Wegener et al. ${ }^{[89]}$ for MDCK-II cell attachment on typical ECIS substrates $\left(A_{\mathrm{WE}}=5 \cdot 10^{-4} \mathrm{~cm}^{2}\right)$ with and without physiological concentrations of calcium.

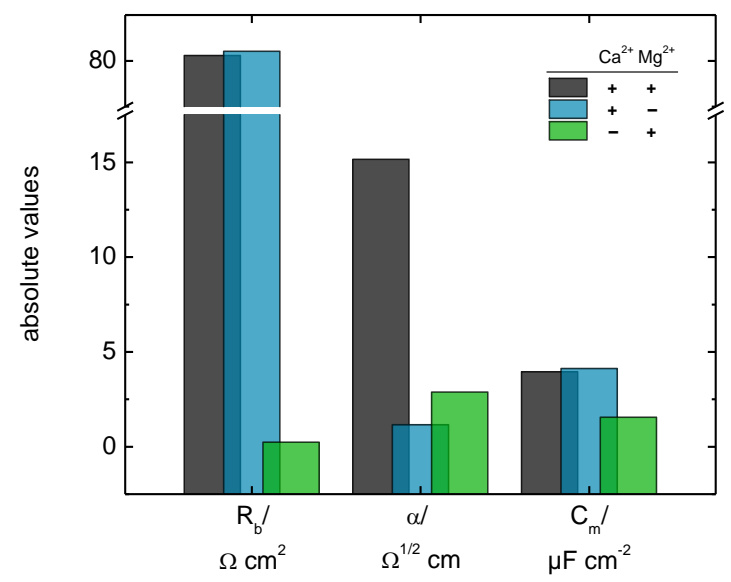

Fig. 5-34 Modeling the impedance data of cell-covered, according to the model presented in subsection 3.4.3, provided the characteristic frequency-independent parameters $R_{b}, \alpha$, and $C_{m}$ for MDCK-II cell layers $20 \mathrm{~h}$ after seeding cells on FN pre-coated 2 ElQs in EBSS-- buffer containing either $0.81 \mathrm{mM} \mathrm{Mg}^{2+}(\square), 1.8 \mathrm{mM} \mathrm{Ca}^{2+}(\square)$, or both ( $\square$ ). The bulk resistance $R_{\text {bulk }}$ and the CPE parameters $n$ and $A$ (cf. Eq. (18)) were determined by linearly fitting impedance spectra at high and low frequencies, respectively, providing mean parameter values of $R_{\text {bulk }}=560( \pm 30) \Omega$, $n=0.959( \pm 0.005), A=4.41( \pm 0.16) \cdot 10^{-6} \mathrm{~F} \cdot \mathrm{s}^{n-1} \cdot \mathrm{cm}^{-2}($ mean $\pm \mathrm{SEM} ; \mathrm{N}=6)$ for an electrode surface area of $2.05 \cdot 10^{-3} \mathrm{~cm}^{2}$. 


\section{Microscopic Evidence for QCM and ECIS Data Interpretation}

Investigations of the cell morphology by fluorescence staining and subsequent microscopic analysis of specific cellular structures support the conclusions drawn from the measured QCM-ECIS sensor data. In presence of physiological calcium concentrations there is a distinct localization of ZO-1 along the cell boundaries (Fig. 5-16 A4, B4, p. 142), illustrating a two-dimensional undisturbed tight junction network between neighboring cells. However, if cells are seeded in low-calcium medium, immunostained ZO-1 proteins of cells are not localized at the regions of cell-cell contacts, but are rather uniformly distributed in point-shaped clusters throughout the entire cytoplasm (Fig. 5-16 $\mathrm{C}_{4}$ ). Hence, TJ formation requires $\mathrm{Ca}^{2+}$ and is inhibited if it is omitted from the medium. This is what was already concluded previously from the electrochemical resistance data, where an increase in the paracellular resistance is assigned to the establishment of TJs and the sealing of the intercellular space, respectively, which fails to appear if cells are seeded in $\mathrm{Ca}^{2+}$-free buffer. $\mathrm{Mg}^{2+}$ could not significantly compensate for missing $\mathrm{Ca}^{2+}$ neither in the formation of TJs, as evident from micrographs, nor in the formation of an electrical barrier within the MDCK-II cell layer.

The micrographs also confirm the interpretation of the slight increases in the capacitance values at $100 \mathrm{kHz}$, occurring approximately three hours after seeding cells in calcium-free buffer (Fig. 5-15 C), as they reveal clearly round-shaped cells and intercellular gaps. Moreover, phase-contrast micrographs and micrographs of live-stained cells (Fig. $5-16 C_{1}, C_{2}$ ) indicate a scattered detachment of cells if $\mathrm{Ca}^{2+}$ is lacking ( $)$. This gives rise to an increased cell-free electrode area on the sensor surface, which in electrochemical terms means a decisive contribution to an increase in the capacitance. Such a partial detachment of cells may be due to weak cell-substrate adhesions (FAs) as a consequence of missing $\mathrm{Ca}^{2+}$.

Actin staining of cells unveils the disruption of filamentous actin structures and, in return, a punctual actin aggregation that is randomly distributed in the cytoplasm, if cells have been cultured in medium without calcium (Fig. 5-16 C5). These micrographs further reveal that inter-cellular spaces are left open within the confluent cell layer (indicated by $\boldsymbol{D}$ ) and that cells are mainly round-shaped in the absence of extracellular $\mathrm{Ca}^{2+}$, supporting the interpretation of ECIS results (capacitance time course) above. From actin staining also a pronounced localization of actin in the cell periphery close to the plasma membrane is evident if calcium is lacking. This is assumedly directly related to an increased stiffness of cells or, to be more accurately, a significantly increased viscoelasticity in the cell-substrate region of the microbalance. According to the $\Delta|Z|_{\text {min }}$ data in Fig. 5-15 A, the apparent stiffness is exactly at levels cell layers transiently reach during cytoskeleton rearrangement and $A J$ formation when they are seeded in presence of calcium. Microscopic investigations by Heitmann et al. (2007) ${ }^{[22]}$ at the point of the transient $\Delta|Z|_{\min }$ maximum showed a pronounced localization of actin in the cell periphery (actin belt). Moreover, in SFM studies the cortical actin network is considered to predominantly determine the mechanical properties of the cell membrane. ${ }^{[469]}$ These findings provide evidence that the actin cytoskeleton and especially the cortical actin network and the actin belt decisively determine the viscoelasticity of the cells and that these are what the $|Z|_{\text {min }}$ signal of QCM monitoring is sensitive for in cell-based assays. In this context it has to be noted that the viscoelastic properties, thereby, not only include the stiffness of cell bodies attached to the microbalance surface but also any other mechanical property within the decay length of the acoustic wave, such as liquid density and viscosity, and contributions by the ECM. Hence, a direct and exclusive allocation of $\Delta|Z|_{\min }$ values to cell stiffness has to be done with caution. For instance, it is critical to compare $\Delta|Z|_{\text {min }}$-derived stiffnesses of cell layers seeded one time with and the other time without $\mathrm{Ca}^{2+}$ in the culture medium, because of morphological differences in cell shape and different cellsubstrate contact areas. 
Similar results of morphological and structural changes of epithelial cells in $\mathrm{Ca}^{2+}$-free medium as presented in Fig. $5-16$ have been published previously ${ }^{[391,398,468,489,490]}$. On the basis of these microscopic findings together with the results from OCM-ECIS measurments, one could speculate about two things regarding the direct and indirect effect of missing $\mathrm{Ca}^{2+}$. Firstly, after sedimentation of cells, the actin cytoskeleton keeps the tension against the substrate high in order to press adjacent cells together and, thereby, to facilitate the formation of adherens junctions and tight junctions. As this fails due to the lack of crucial $\mathrm{Ca}^{2+}$ ions, the cytoskeleton remains in this tense state of spreading pressure without significant reduction sometime during the experiment. This may just as well apply for the formation of cell-substrate contacts by focal adhesions. Secondly, missing $\mathrm{Ca}^{2+}$ might not only directly affect the formation of AJs and FAs via cadherins and calcium-dependent integrins, but it also might have a direct inhibitory impact on structural alterations in the actin cytoskeleton which, however, are crucial for the establishment of cell-substrate and cell-cell adhesions. Calcium missing in the culture medium would in this case have also an indirect effect on the non-appearance of intercellular junctions. The correlation of cytoskeleton integrity and AJ/ TJ assembly and maintenance also becomes obvious in the results of cell-based QCMECIS assays with the cytoskeleton disrupting cytochalasin $D$, which are described and discussed in subsections 5.3 .1 and 5.4 .7 , respectively.

In summary, the cell attachment and spreading assays on the OCM-ECIS sensors revealed significant differences in the dielectric and micromechanical alterations close to the substrate, in both kinetics and absolute values, depending on whether cells are seeded in culture medium with or without $\mathrm{Ca}^{2+}$. Additional microscopic observations could consistently confirm the conclusions that were drawn from the obtained sensor data. The OCM-ECIS sensor approach demonstrates its big advantage over classical cellular end-point measurements in the attachment and spreading assays. As both technologies are label-free and non-invasive, the cell behavior can be monitored over time during the entire attachment and spreading process. Thereby, one obtains independent information on the quality of cell-substrate and cell-cell contacts as well as on the viscoelastic properties of the substrate-electrolyte interface. This multi-parametric readout provides high-content information and enables precise characterization of the cellular state after seeding. As the dual approach simultaneously provides information from multiple physical perspectives in a time-resolved manner, the measurements also allow to reveal correlations of morphological and machanical alterations during cell layer formation in time. Such correlations can be obtained by plotting sensor parameter changes against each other in 2-D and 3-D OCM-ECIS plots and analyzing their mutual dependencies. For instance, not only a $\mathrm{Ca}^{2+}$-dependent formation of TJs was uncovered, but also a simultaneous effect on the micromechanical properties of the cell layer, which could be assigned to a different distribution of filamentous and cortical actin. Moreover, information were obtained about the kinetics of the initial cell spreading and the subsequent rounding up of cells again in the absence of $\mathrm{Ca}^{2+}$, together with the information that the cell layer mechanics do not change during these processes.

Hence the presented OCM-ECIS sensor provides a powerful tool for testing the attachment and spreading behavior of cells upon various impacts, like for example various coatings and materials as growth substrate or any kind of modification and supplements in the seeding medium. Furthermore, the sensor can be applied as an on-line quality control for cell layer properties. Deviations from defined specifications, like for example slow spreading kinetics, non-appearance of cytoskeleton rearrangement, or the establishment of no tight barrier, can be non-invasively detected and evaluated with respect to the usability of the cell population for further studies. 


\subsubsection{Degradation of the Actin Cytoskeleton}

\section{Correlation of QCM, ECIS and Microscopic Data}

We showed that exposure of a confluent layer of MDCK-Il cells on the dual sensor platform to cytochalasin D (CD) causes siginificant responses in QCM- and ECIS-mode recordings (Fig. 5-17, p. 145). The lowest concentration of $C D, 0.1 \mu \mathrm{M}$, applied to MDCK-II cells leads to a transient drop of $|Z|_{\min }$ values ( $\rightarrow / Z / \triangle$ in Fig. 5-18 A, B, p. 147). The same applies for the low-frequency resistance (Fig. 5-18 C, $D)$. These signal changes report on an initial $C D$-induced reduction of the cytomechanical stiffness of the cell bodies, accompanied by a simultaneous opening of the paracellular barrier and of TJs, respectively. Afterwards, cytomechanics almost totally recover to pre-stimulation values and values of unstimulated (negative) control cells, respectively ( vs. in Fig. 5-18 B). Moreover, cells at least partially compensate the initial TJ disintegration within $12 \mathrm{~h}$ after adding $0.1 \mu \mathrm{M} \mathrm{CD}$. Cytochemical staining of actin demonstrates a nearly complete intactness of actin filaments in cell layers treated with $0.1 \mu \mathrm{M} \mathrm{cD}$ for $1 \mathrm{~h}$ and $25 \mathrm{~h}$ (Fig. 5-19 B1, B2, p. 149). Though these micrographs hardly differ from those of unstimulated cells (Fig. 5-19 $A_{1}, A_{2}$ ), this $C D$ concentration causes to some extent the formation of monomeric actin aggregates ( - in Fig. 5-19 B). At this low amount of $c D$ electrochemical capacitance values remain at levels of negative control throughout the whole experiment (Fig. 5-18 E, F), which reports on a confluent layer of spread and stably attached cells on the sensor surface. The situation changes, if cells are exposed to elevated concentrations of CD. Exposing MDCK-II cells to 1.0 and 5.0 $\mu \mathrm{M}$

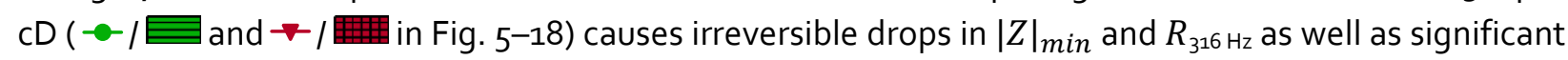
increases in $C_{40} \mathrm{kHz}$, with stationary levels reached within less than $4 \mathrm{~h}$ after stimulation for all parameters. These effects are reflected in the OCM and ECIS spectra shown in $S / 20$ (p. 273). Here, the reduction in OCM energy dissipation is evident in a decrease of the impedance magnitude near serial resonance (A) as well as by an increasing phase angle and a sharpening of the phase peak (B). The exemplary ECIS spectra clearly show gradual and drastic changes in impedance magnitude $(C)$ and impedance phase angle (D), as well as in equivalent resistance and capacitance spectra (E) upon $C D$ treatement of a confluent MDCK-II cell layer. Higher concentrations of CD thus cause faster and stronger irreversible reductions of both the cytomechanical stiffness and the functional integrity of barrier-forming TJs (Fig. 5-18). The differences of effects between 1.0 and $5.0 \mu \mathrm{M} \mathrm{CD}$ are significant only in the kinetics, however not in the final values of parameters (after $4 \mathrm{~h}$ ). Resistance values reach almost levels of the cellfree electrode within $1-2 \mathrm{~h}$ after adding $C D$ (Fig. 5-18 C, D). This reports on a complete loss of the sealing function in the epithelial cell layer and an opening of the intercellular space. A similar CD dose-dependent breakdown of the barrier resistance has been presented previously for different cell lines, such as MDCK-II cells ${ }^{[267]}$, HUVECS ${ }^{[16]}$, and fibroblasts ${ }^{[87,97,498]}$, investigated by ECIS. In contrast to these findings, Stevenson \& Begg (1994) ${ }^{[397]}$ observed less decreas in the transepithelial electrical resistance (TER) of MDCK-II cells (roughly from $-60 \%$ to $-20 \%$ ) with increasing the applied cD concentration (from $2 \mu \mathrm{g} \cdot \mathrm{mL}^{-1}$ to $20 \mu \mathrm{g} \cdot \mathrm{mL}^{-1}(\sim 4 \mu \mathrm{M}$ to $40 \mu \mathrm{M})$ ), supported by immunostained actin structures that were less disrupted at higher $\mathrm{CD}$ levels. The decrease in the motional impedance in this thesis reflects a $c D$ dosedependent loss in the micromechanical stiffness of cells (Fig. 5-18 A, B). This is assigned to the disruption of an increased amount of actin cytoskeleton structures, which is confirmed by microscopic investigations (cf. Fig. 5-19). In contrast to the barrier resistance, the load impedances of the OCM sensors are not completely reduced to cell-free values by treating cells with either 1.0 or $5.0 \mu \mathrm{M} \mathrm{CD}$. A maximal reduction of $|Z|_{\text {min }}$ to $\sim 70 \%$ of initial values $(5 \mu \mathrm{M} c D, 4 \mathrm{~h})$ is observed. These findings are consistent with previous QCM studies of CD treated MDCK-II cells [216,251,267,268], fibroblasts [222,242], lymphoblasts ${ }^{[499]}$, and FaDu cells ${ }^{[251]}$. Hence, cells do not, or at least not completely, loose cell-substrate 
contacts and largely remain firmly attached to the sensor surface at the highest CD concentration applied, despite disassembly of $\mathrm{f}$-actin. This is confirmed by micrographs of equally treated cell layers (Fig. 5-19 D). Though no discernible gaps can be noticed in the cell layers within $1-4$ hours after adding

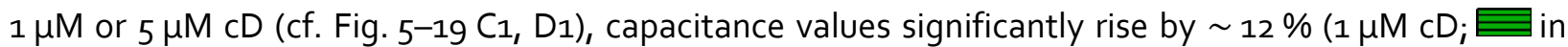
Fig. 5-18 F) and $\sim 35 \%(5 \mu \mathrm{M} \mathrm{cD}$; in Fig. 5-18 F) with respect to values of the cell-covered electrode. It is tempting to assigning this for the most part to the detachment of cells. However, this is not (yet) the case within $4 \mathrm{~h}$ after adding $\mathrm{CD}$, as micrographs and $\mathrm{OCM}$ data clearly confirm. Capacitance increases within the first $1-2$ hours after cytochalasin stimulation are more likely due to uncovered electrode areas from TJ disintegration and an accompanied contraction of cells ${ }^{[401,402]}$, which give rise to high frequency current pathways. After an incubation period of $25 \mathrm{~h}$, higher $\mathrm{CD}$ concentrations cause the formation of larger intercellular spaces and even the scattered detachment of single cells from the cellular network. This is evident from micrographs of actin-stained cell layers, where black areas indicate cell-free regions on the substrate (cf. $>$ in Fig. 5-19D2). This is explained by the cD-induced disassembly of actin filaments, which are well-known to play an essential role in cell-matrix and cell-cell adhesion, as they are structurally linked to FAs, AJs, and TJs via integrins ${ }^{[379-384]}$, cadherins ${ }^{[386-388]}$, and ZO proteins ${ }^{[345,346,391]}$, respectively. The addition of Saponin after $C D$ treatment aimed to systematically disintegrate the cell membrane and to induce a complete loss of the dielectrical properties of the cell bodys and the cell layer, respectively. However, normalized capacitance values slightly below that of an uncovered working electrode indicate remaining rests of cell membrane structures on the sensor electrodes (Fig. 5-18 E). Saponin treatment neither led to a complete removal of the acoustic load from the OCM (Fig. 5-18 B). The remaining impedance of roughly $250 \mathrm{Ohm}$ above cell-free values reports on the remaining cytoskeleton framework that stays attached to the microbalance surface and, thus, cause increased dissipation of oscillation energy. An active generation of convection in the measurement vessel would wash away such residues so that cellular contributions to both the high-frequency capacitance and load impedance are expected to completely disappear.

\section{Correlation of QCM and ECIS Data}

In Fig. 5-35 A, the $Q C M$ and ECIS sensor data from a typical time-resolved $C D$ assay are merged in a 3-D QCM-ECIS impedance diagram. Correlations of two sensor parameters, i.e. the projections of the 3-D curves in Fig. 5-35 A to the planes, are represented in Fig. 5-35 B, C, and D. Firstly, the plots uncover that the monitored parameters change in similar mutual dependency, independent of which concentration of $C D$ is applied. This can be seen best in the correlation plots in Fig. $5-35 B, C$, and $D$, where parameter changes from $t_{0}$ to $t_{1}=3 \mathrm{~h}$ follow the same path until the respective dose-dependent final value is reached. Hence, $C D$ causes the same concerted structural dielectrical and mechanical responses of the cells independent of its concentration. In detail, there is an almost linear dependency between $\Delta|Z|_{\min }$ and $R_{\text {norm }}$ after exposing cells to $\mathrm{CD}$, except the first data point after adding $5 \mu \mathrm{M} \mathrm{CD}$ (indicated with *). Thus, the breakdown of cell stiffnes and opening of intercellular junctions (TJs) are strongly related processes, which confirms a structural and functional linkage of the actin fibres and the zonula occludens. Although TJ disintegration is assumed as a consequence of actin disrupture and accompanied cell contraction, the data cannot resolve a temporal succession of cell softening and opening of junctions. Secondly, from these plots one can clearly distinguish between the effects of different amounts of $C D$ on the mechanical and dielectrical properties of a confluent MDCK-II cell layer, as the final values of parameter changes after $t_{1}=3 \mathrm{~h}$ of $\mathrm{cD}$ stimulation are localized at significantely different regions in the 3-D and 2-D diagrams, respectively. Although the presented 2-D and 3-D correlation plots of $Q C M$ and ECIS parameters may be very complex, these diagrams comprise condensed information on the 
mechanical and dielectric correlations of cell layer responses to cD-mediated actin cytoskeleton disintegration.
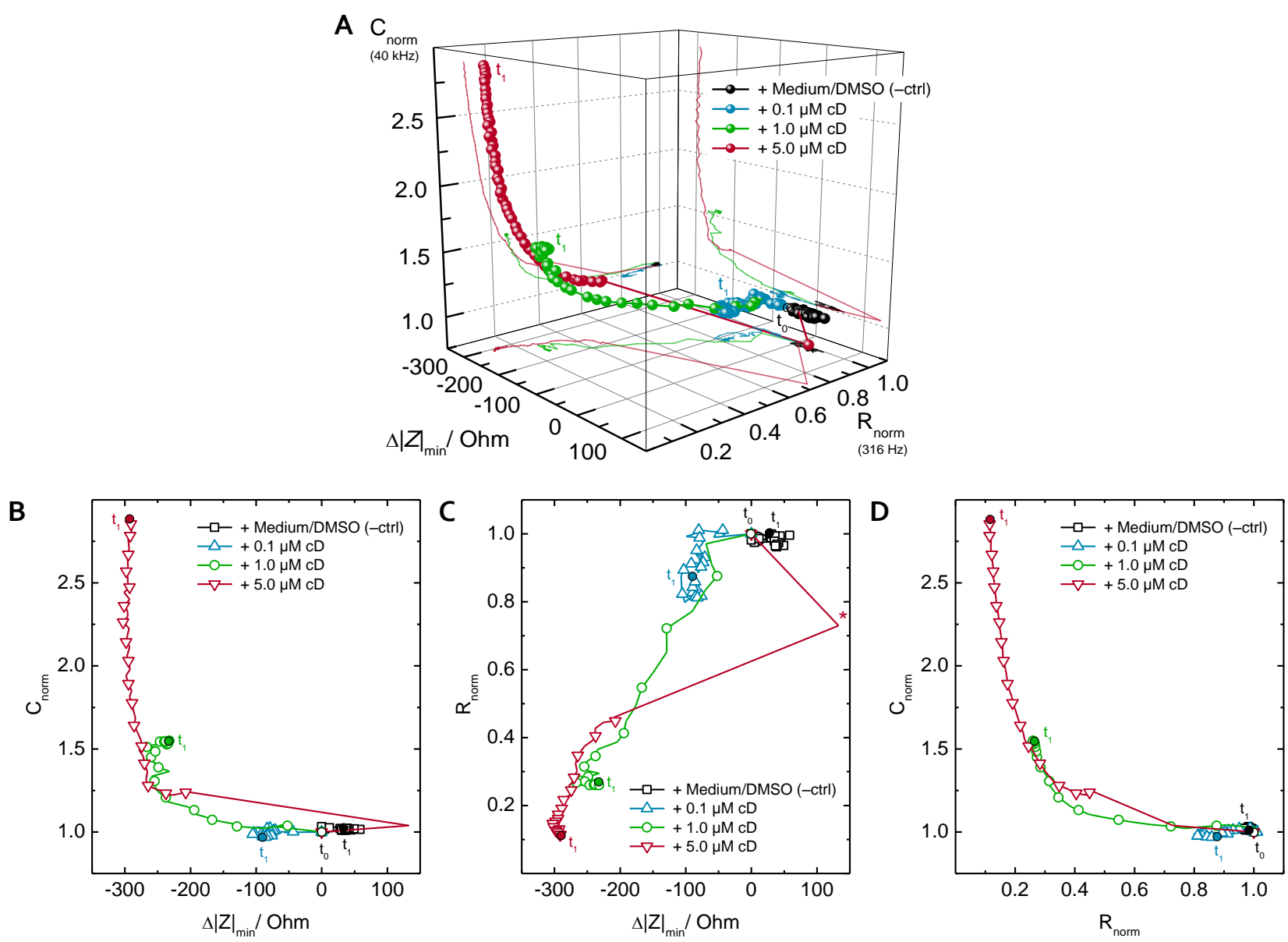

Fig. 5-35 (A) 3-D QCM-ECIS impedance plot ( $\Delta|Z|_{\min }$ vs. $R_{\text {norm }}$ vs. $C_{\text {norm }}$ ) of typical, timeresolved $\mathrm{CD}$ assays. Parameter changes are shown between the point $\left(t_{0}\right)$ of adding $0.1 \mu \mathrm{M}$, $1.0 \mu \mathrm{M}$, and $5.0 \mu \mathrm{M} \mathrm{CD}$ to a confluent MDCK-II cell layer and $t_{1}=3 \mathrm{~h}$. The time resolution between two points is 4 min. (B), (C), (D) Projections on the planes of the 3-D curves in A. $R_{\text {norm }}$ and $C_{\text {norm }}$ are displayed at the respective most sensitive frequencies, which are $316 \mathrm{~Hz}$ and $40 \mathrm{kHz}$. Data points in $B, C$, and $D$ were reduced for the sake of clarity, resulting in a time resolution of $12 \mathrm{~min}$.

\section{Modeling of ECIS Data}

A closer view on the structural alterations in the cell layer upon $C D$ treatment is accessible by modeling the ECIS data. Fig. 5-36 shows the characteristic fitting parameters, $R_{b}, \alpha$, and $C_{m}$, obtained from adjusting Eq. (50) to the experimental impedance spectra. The fitting data show a strong decrease in the barrier resistance $R_{b}$, by $\sim 80 \%$ to $\sim 8 \Omega \cdot \mathrm{cm}^{-2}$, within 30 min after adding $5 \mu \mathrm{M} \mathrm{cD}$ to the MDCK-II cell layer. In the same period the value of the cell-substrate parameter $\alpha$ drops to $\sim 13 \Omega^{1 / 2} \cdot \mathrm{cm}(\sim 50 \%$ with respect to starting values). The membrane capacitance $C_{m}$ is not affected and remains nearly constant. Two hours after $C D$ treatment the barrier resistance has completely decreased to values of the cell-free electrode and does not change anymore in the further course of the experiment. Meza \& Ibarra (1980) reported on a decrease of the TER by $75 \%$ upon treatment of MDCK-II cell layers with $5 \mu \mathrm{g} \cdot \mathrm{mL}^{-1}$ cytochalasin for two hours ${ }^{[398]}$, which is similar to findings by Stevenson \& Begg (1994) ${ }^{[397]}$ and which agrees well with the findings on the basis of the QCM-ECIS sensor approach. 
The analysis of the data from OCM-ECIS experiments further shows that the $\alpha$ value also descreases in the course of $C D$ treatment, though in contrast to $R_{b}$ slower and gradually (Fig. 5-36). It is reduced to $\sim 8 \Omega^{1 / 2} \cdot \mathrm{cm}$ after $2 \mathrm{~h}$ and to $\sim 6 \Omega^{1 / 2} \cdot \mathrm{cm}$ after $4 \mathrm{~h}$, which is still significantly above cell-free values. These data indicate a kinetic discrepancy in the structural alterations of cell-cell and cell-substrate contacts. Exposure of a cell layer to $C D$ causes a very fast loss in the barrier resistance of the cell layer, while electrical properties that are attributed to the subcellular cleft are affected delayed and less pronounced. This supports the assumption that actin cytoskeleton disruption is directly linked with the disassembly of intercellular sealing junctions and probably also of adherens junctions. The cell-substrate properties are more or less affected subsequently, as a consequence of lost intra- and extracellular mechanical stability from the actin cytoskeleton and junctions to adjacent cells. If assuming no alteration in the resistivity of the subcellular electrolyte, the gradual decrease in $\alpha$ can be assigned to either a reduction of the average cell diameter, or an increase in the mean cell-substrate distance, according to Eq. (49). It is likely a combination of both, since (i) the opening of intercellular junctions and an ongoing cell contraction goes along with a reduction in the average cell diameter, and (ii) the strength and number of actin-linked focal adhesions for cell-substrate anchorage is reduced due to $C D^{[384,500,501]}$, which is probably accompanied by an increasing average cell-substrate distance. For a more detailed analysis of structural processes during $C D$ challenges, the cell-substrate distance and its alteration have to be further investigated. Fitting of the ECIS data shows a significant increase in the membrane capacitance $C_{m}, 4 \mathrm{~h}$ after $C D$ inoculation. This could indicate an increase of membrane folding. However, such membrane foldings are typically stabilized by $\mathrm{f}$-actin, which is actually depolymerized due to $\mathrm{CD}$. This value might be explained as an artifact just as well, since $C_{m}$ values are increasingly hard to determine when $R_{b} \approx 0$ and $\alpha$ values are low. In order to confirm this suggestion, further studies focusing on this are needed, too.

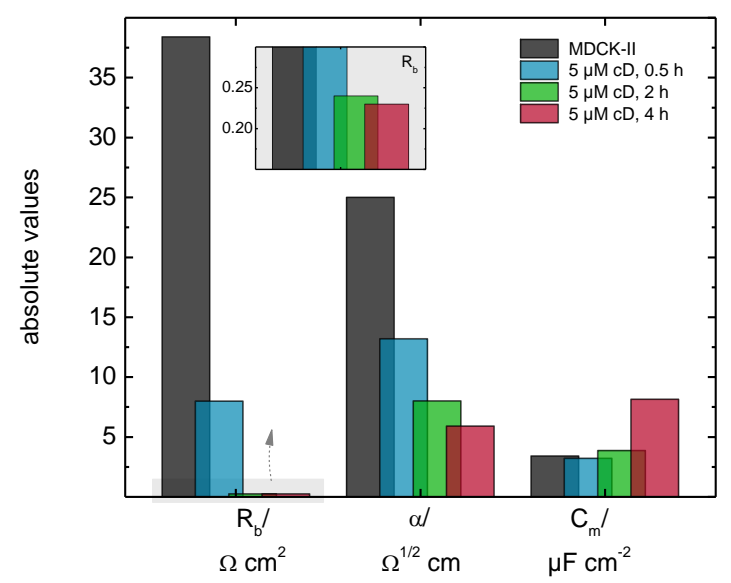

Fig. 5-36 Modeling the impedance data of cell-covered electrodes, according to the model presented in subsection 3.4.3, provided the characteristic frequency-independent parameters $R_{b}$, $\alpha$, and $C_{m}$ for MDCK-II cell layers exposed to $5.0 \mu \mathrm{M}$ Cytochalasin D (cD) for $0.5 \mathrm{~h}(\square), 2 \mathrm{~h}$ ( $\square$ ), and $4 \mathrm{~h}$ ( $\square$ ). Untreated cells serve as reference level ( $\square$ ). The bulk resistance $R_{\text {bulk }}$ and the CPE parameters $n$ and $A$ (cf. Eq. (18)) were determined by linearly fitting impedance spectra at high and low frequencies, respectively, providing values of $R_{\text {bulk }}=430 \Omega, n=0.97$, and $A=1.78 \cdot 10^{-5} \mathrm{~F} \cdot \mathrm{s}^{n-1} \cdot \mathrm{cm}^{-2}$ for an electrode surface area of $2.05 \cdot 10^{-3} \mathrm{~cm}^{2}$. 


\section{Analysis of QCM Data}

Changes in the $\mathrm{OCM}$ sensor signal, i.e. in the minimal impedance $|Z|_{\min }$, upon cell treatment with $c D$ are assigned to changes in the cytomechanics. The underlying correlation is an increase/ decrease in $|Z|_{\text {min }}$ with increasing/ decreasing stiffness of a viscoelastic microbalance loading. This dependency could be demonstrated in an empirical approach where it was investigated the change in the minimal impedance of an oscillating quartz disc upon loading it with gelatins of various stiffness (data not shown). Hence, a decrease in $|Z|_{\text {min }}$ of a microbalance loaded with a cell layer can be assigned primarily to losses in the cellular stiffness, whereas an increase of $|Z|_{\text {min }}$ l like it is for example observed in cell fixation experiments with GA and PFA (cf. 5.3.2, p. 149 ff.; discussed below in 5.4.5) reports on increasing stiffness of the soft matter on the $\mathrm{QCM}$.

However, the electrochemical data from cD assays report on a contraction and partial detachment of cells, if applying sufficiently high drug concentrations for a long period. Cell detachment is induced by an initial disassembly and reduction of FA contacts and a rounding up of cells. ${ }^{[500,501]}$ The acoustic waves of the microbalance in the region of a detaching cell thus progressively penetrate less into the viscoelastic cell body, replaced by an increasing amount of viscous culture medium. This also applies for cells that remain attached to the surface in spread shape, however increase the cell-substrate distance due to actin disintegration. These processes change the mechanical properties (the cell-to-medium ratio) near the OCM surface and, more precisely, within the decay length of the acoustic wave. The mentioned cytomechanical and cell morphological alterations due to CD treatment consequently would shift the average impedance signal towards values of the cell-free resonator immersed in medium only.

Assigning the OCM signal changes to micromechanical alterations within the cell layer only and not taking into account potential processes of cell detachment or axial cell movement may lead to a misinterpretation of data. This again illustrates the big advantage of combined measurements, which provide multi-perspective views on the cell layer properties. ECIS in addition to OCM monitoring is complementary and covers the above-mentioned uncertainties in QCM data interpretation. Capacitance data from ECIS recordings is capable of reporting sensitively on gaps in the cell layer due to cell detachment and the electrochemical resistance can provide information on height changes of the subcellular cleft. Results of $\mathrm{cD}$ studies show that capacitances increase only to a small extent within $4 \mathrm{~h}$ after stimulation. Moreover, fitting a theoretical model to the experimental ECIS data shows that resistance decreases within the first hour are predominantly due to opening of the intercellular sealing and comparatively less due to an increasing distance between the cells and the working electrode of the sensor. Hence, the pronounced $|Z|_{\text {min }}$ decreases within this period are not due to to cell detachment but can be very likely assigned to losses in the micromechanical stiffness of cells. This is consistent with several published AFM studies on cellular elasticity. In these, decreases of the Young's modulus by factors of $2-3$ are reported upon cytochalasin treatment of cell layers ${ }^{[394-396,403,404,502]}$. In cases of single cells, an even up to 7 -fold decrease of the cellular stiffness is observed ${ }^{[396]}$, which, however, is due to a higher initial stiffness of single cells compared to cells that are part of a mechanically cross-linked monolayer unit. Moreover, from AFM indentation experiments the actin cytoskeleton has been made responsible for half of the overall cellular stiffness in cell layers that form close intercellular junctions. ${ }^{[479]}$

A time-dependent effect of different cytochalasin concentrations on the cell layer elasticity was also assumed from AFM force mapping. ${ }^{[395]}$ The respective AFM studies, however, were disadvantageous for kinetic analysis of drug effects due to a poor temporal resolution of data acquisition. For instance, the cellular stiffness did already change significantly within 20 min upon drug treatment, which was roughly 
the time required for recording one elasticity map of $64 \times 64$ force curves. This again nicely points out one big advantage of real-time QCM cell sensing over AFM elastography.

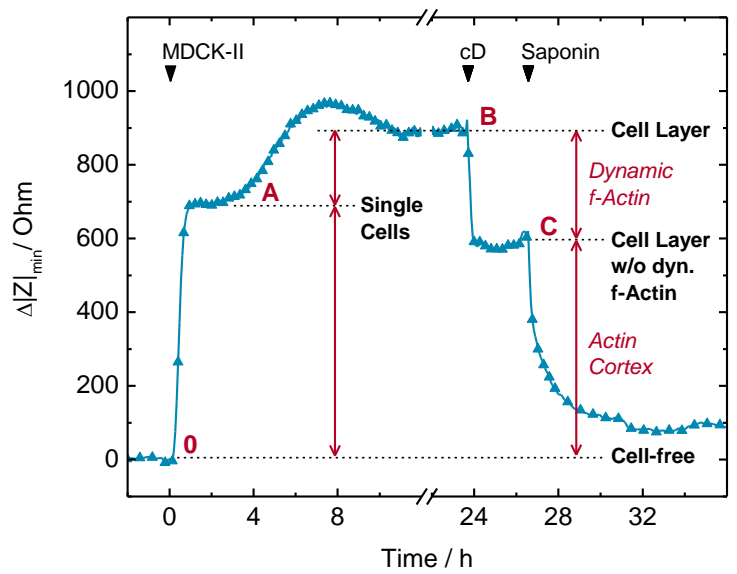

Fig. 5-37 $|Z|_{\min }$ changes during MDCK-II cell attachment/ spreading and treatment with cytochalasin $D(C D)$ reveal different mechanical contributions of different cell constituents to the measured signal. Different "mechanical levels" can be determined for (o) the cell-free sensor, (A) single cells attached to the surface, (B) a confluent monolayer of spread and interconnected cells, and $(C)$ a confluent cell layer, however without dynamic $f$-actin structures due to $C D$ treatment.

One can observe different stationary $|Z|_{\text {min }}$ values in the course of cell layer formation and cD treatment, as it can be nicely seen in the time course of a typical experiment below in Fig. 5-37. These levels and the $\Delta|Z|_{\text {min }}$ signal changes inbetween can be split into separate mechanical contributions to the load of the resonator, originating from different structural properties of individual cells and the cell layer as a whole. The default starting point of experiments was the cell-free sensor immersed in cell culture medium only ( $\triangleq$ reference level of $|Z|_{\text {min }}, \Delta|Z|_{\text {min }}=0$ ). By seeding 450 o0o MDCK-II cells per square centimeter on the quartz resonator, the minimal oscillation impedance increases in a biphasic shape to a constant level during the attachment and spreading phase ( $O \rightarrow B$ in Fig. 5-37). The endpoint reflects the mechanically equilibrated state of an established confluent cell monolayer. As discussed already above $5.4 .5 \mathrm{Cell}$ Attachment and Spreading), this signal time course can be basically separated into the following two mechanical processes taking place on the OCM surface: Firstly, the settlement of suspended, spherical cells onto the resonator and into the field of view of the acoustic wave, followed by initial cell-substrate anchoring processes ( $0 \rightarrow A$ in Fig. 5-37). These include the formation of FAs and HDs of the (still) separate cells to the underlying growth substrate and the ECM. Secondly, cells begin to spread on the surface. They thereby come in close contact to attached neighboring cells and begin with the formation of intercellular anchoring junctions (AJs, desmosomes), communicating junctions (GJs), and sealing junctions (TJs). These structural processes during cell layer formation take place under remodeling of the intracellular actin cytoskeleton. This causes a short-time mechanical stiffening of the cells, which is reflected in the observed transient maximum of $|Z|_{\text {min }}$. After functional and structural connection, the initially single cells finally form a dense and mechanically stable cell layer network ( $\rightarrow B$ in Fig. 5-37). The stiffness of the cell layer is primarily determined by the actin filament network and the resulting lateral tension in the plasma membrane. ${ }^{[396,403,404,470,502]}$

By adding the actin disrupting drug cytochalasin $D$ to the confluent cell layer, the $|Z|_{\text {min }}$ value rapidly drops within few minutes to a substantially lower level ( $B \rightarrow C$ in Fig. 5-37). The final situation on the 
microbalance is a still confluent layer of cell bodies, however, whose dynamic intracellular actin cytoskeleton network is disassembled. From experiments of cell fixation and subsequent membrane disintegration, discussed below in subsection 5.4 .8 , it is evident that the OCM load impedance and cellular stiffness are determined by the protein network and that the contribution of the plasma membrane is neglectable. It is assumed that the CD-mediated disassembly of the actin cytoskeleton mainly affects the very dynamic f-actin structures whereas the cortical actin attached to the plasma membrane largely remains intact. Therefore, the difference in the $|Z|_{\min }$ levels of " $\mathrm{B}$ " and " $\mathrm{C}$ " in Fig. 537 is assigned to the mechanical contribution of the dynamic actin cytoskeleton in the cell layer, which is about $1 / 3$ of the total $\Delta|Z|_{\text {min }}$ increase by a confluent cell layer. The remaining damping of the microbalance oscillation, between the cell-free sensor and level " $C$ ", consequently arises from the mechanical properties of the attached cell bodies lacking the very dynamic parts of $f$-actin. Regarding the cell mechanics, AFM studies in combination with fluorescence microscopy and immunostaining showed that, besides the actin microfilaments, intermediate filaments contribute decisively to the overall cell elasticity, whereas microtubules play only a minor role. ${ }^{[395,503-508]}$ The cell body mechanics are further determined by the elasticity of vinculin ${ }^{[509]}$, the viscoelasticity of the phospholipid bilayer membrane-enclosed cytoplasm, and by the mechanical interactions of all remaining cellular structures just like in a tensegrity model $[419,508,510,511]$. In this state, cytomechanics measured by OCM resonators however may not be approximated by the mechanical model of vesicles, comprising the elasticity of the plasma membrane and the viscosity of the enclosed liquid. This correlation did not apply previously for MDCK-II cells with intact actin network, since their viscous energy dissipation was found to be significantly higher. ${ }^{[227]}$ The experiments further show that disassembly of dynamic $f$-actin causes loss of only $\sim 1 / 3$ of the total energy dissipation of MDCK-II cell layers on resonators. The remaining oscillation damping is still significantly higher compared to liposomes attached to the QCM ${ }^{[227]}$, making them also a not suitable mechanical model for $\mathrm{CD}$ treated cell layers. As evident from Fig. 5-37, Saponin addition subsequent to $\mathrm{CD}$ treatment leads to an almost complete loss of the cell-caused motional impedance $\Delta|Z|_{\min }$ to cell-free levels $(C \rightarrow \sim 0$ ). The detergent causes perforation of the apical and basolateral cell membrane and, thus, disintegration of the cellular unit and of cell body micromechanics. It is assumed that upon membrane disintegration the actin cortex loses its anchorage structure and disintegrates together with it. This would consequently mean that the contribution of the cortex to the mechanical stiffness of the cells disappears. And this again would explain the observed almost complete decline of the remaining 2/3 of the $\mathrm{OCM}$ load impedance of the cell layer (level " $\mathrm{C}$ " in Fig. 5-37). The slow decrease in $|Z|_{\text {min }}$ thereby assumedly reflects the diffusion-determined detachment of cellular fragments from the microbalance surface. Moreover, the dissolution of cells without externally applied convection does not lead to a complete removal of the acoustic load from the OCM. The remaining energy dissipation of nearly $100 \mathrm{Ohm}$ is attributed to cellular fragments remaining stuck to the sensor surface.

\subsubsection{Cell Stiffening using Chemical Fixatives}

The OCM-ECIS data clearly report on different mechanical as well as dielectrical cell responses upon protein cross-linking, dependent on the kind and concentration of the fixing agent (Fig. 5-21, p. 153).

\section{Correlation of QCM and ECIS Data}

Fig. 5-38 summarizes the OCM and ECIS data, which have been obtained from time-resolved GA and PFA cell fixation assays on the dual sensor. In the 3-D plot (Fig. 5-38 A), with the three sensor parameters $\left(\Delta|Z|_{\min }, R_{\text {norm }}\right.$ and $\left.C_{\text {norm }}\right)$ as perpendicular axes, one can see the significantly different spatial curves of 
the different fixation experiments. Correlations of two sensor parameters, i.e. the projections of the 3-D curves in Fig. 5-38 A to the planes, are represented in Fig. 5-38 B-D. These diagrams enable new insights in the temporal correlation of mechanical and dielectrical changes in cell layers and, thereby, provide information on possibly associated structural alterations upon fixation.

The $C_{\text {norm }}-\Delta|Z|_{\min }$ plot (Fig. 5-38 B) shows that there is no noticeable correlation between cell stiffening and the degree of cell-coverage on the ECIS WE for the fixation of cells with any GA concentration. For $0.025 \% \mathrm{GA}\left(--^{-}\right)$there is a comparatively fast initial increase in the capacitance while $\Delta|Z|_{\text {min }}$ increases slowly in this period. For $0.1 \% \mathrm{GA}\left({ }^{-}-\right)$) the period of the fast initial rising in $C_{\text {norm }}$ is accompanied by a likewise fast increase in $\Delta|Z|_{\text {min }}$. And for $0.25 \% \mathrm{GA}(-\nabla)$ the fast and efficient stiffening of the cell layer (strong $\Delta|Z|_{\min }$ increase) likely prohibits any cellular reaction and a potential cell detachment (no change in $C_{\text {norm }}$ ). The $R_{\text {norm }}-\Delta|Z|_{\min }$ plots (Fig. 5-38 C) indicate that cellular stiffening and the opening of tight junctions take place in a similar dependency for one sort of fixative. In the first stages after adding GA to the cells, an increase in $\Delta|Z|_{\min }$ is accompanied by a decrease in $R_{\text {norm }}$. As mentioned before, the decrease in $R_{\text {norm }}$ is less pronounced with increasing GA concentrations. This is reflected in a flattening of the slopes of curves in Fig. $5-38 C(-\Delta->->-\nabla-)$. On closer examination a three-phase course of the GA-curves is identified. After the initial simultaneous increase of $\Delta|Z|_{\min }$ and decrease of $R_{\text {norm }}$ there is a horizontal phase in which $\Delta|Z|_{\text {min }}$ increases only without an accompanied change in the resistance. In the final phase, $R_{\text {norm }}$ is slightly reduced again during an ongoing increase of $\Delta|Z|_{\text {min }}$. A translation of these phases, of mechanical and dielectrical parameter changes, into separate and successive structural processes occuring in the cell layer during fixation, however, requires detailed further investigations. Especially early processes have to be investigated with a high time-resolution, as the described parameter changes in the denoted first phase take place within the first 5-10 min after addition and are based on only few data points. Effects due to compound addition itself, like changes in temperature, convection, and osmotic stress, have to be taken into account in this context, too.

The fixation of cellular proteins with $1 \%$ PFA shows a completely different correlation of mechanical and dielectrical sensor parameters. The strong decrease in $R_{\text {norm }}$, the moderate increase in $\Delta|Z|_{\text {min }}$ and the negligible change in $C_{\text {norm }}$ cause that the PFA-curves significantly differ from the GA-curves in the 3-D and 2-D correlation plots ( $\diamond-$ in Fig. $5-38$ ). This is most evident in the $R_{\text {norm }}-\Delta|Z|_{\text {min }}$ diagram. After adding PFA as fixing agent to the cells, there is a $\Delta|Z|_{\text {min }}$ increase and a $R_{\text {norm }}$ decrease, similar to the initial processes upon adding GA. After approximately 5 min almost $50 \%$ of the overall $\Delta|Z|_{\min }$ increase are reached. In contrast to the curves of GA-mediated cell fixation, $\Delta|Z|_{\min }$ increases by $\sim 100 \mathrm{Ohm}$ only slightly during the following $\sim 50 \mathrm{~min}$, whereas in this period $R_{\text {norm }}$ drastically drops by $\sim 45 \%$ with respect to values before fixation. Afterwards, $R_{\text {norm }}$ and $\Delta|Z|_{\min }$ slowly and simultaneously approach to final values. Hence, there seems to be a secondary effect of PFA on the cells, which is absent when cells are exposed to GA instead and thus is assumed to be independent of the pure protein cross-linking and cell stiffening process.

\section{Analysis of QCM Data}

The minimum impedance of the microbalance oscillation, $|Z|_{m i n}$ increases in a dose-dependent manner for the cross-linking of cellular protein with GA. Thus, this data show exactly the opposite OCM wholecell sensor response compared to the just previously discussed observations for cell layers stimulated with CD. The higher the concentration of the fixative the faster is the increase of $\Delta|Z|_{\min }$ and the earlier stationary end values are reached (Fig. 5-21 A, B). The higher the temporal and local availability of GA molecules the more cross-linking reactions take place simultaneously, which results in a faster stiffening of the cells and an earlier abreaction of any available amino group of the cells and the ECM. 
A

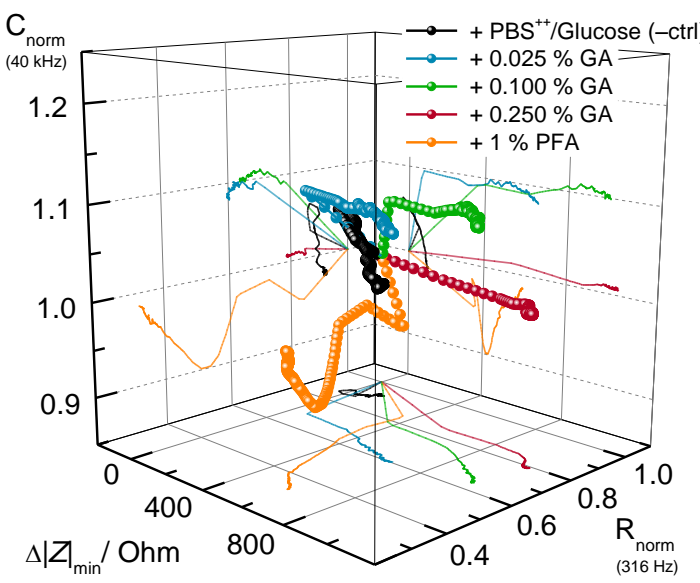

B

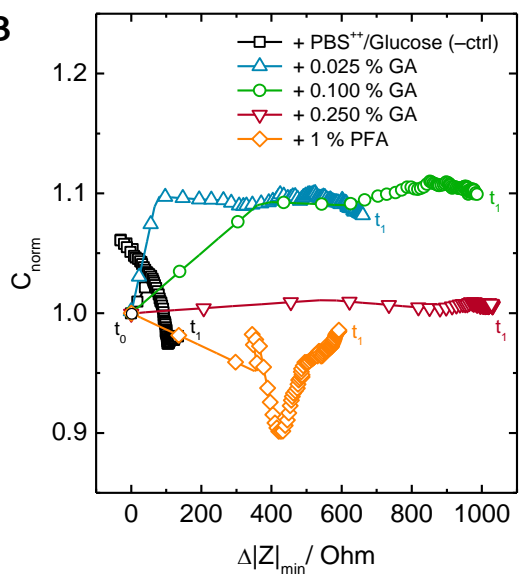

C

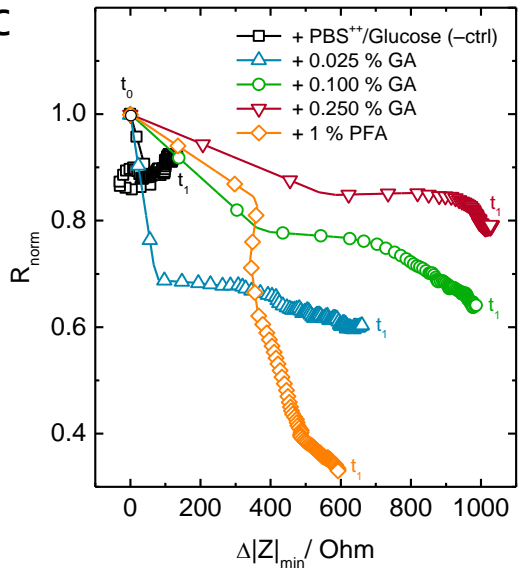

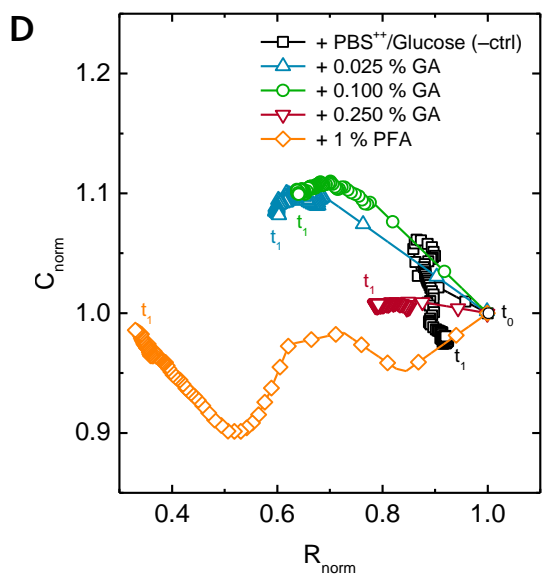

Fig. 5-38 (A) 3-D OCM-ECIS impedance plot ( $\Delta|Z|_{\min }$ vs. $R_{\text {norm }}$ vs. $\left.C_{\text {norm }}\right)$ of averaged, timeresolved cell fixation assays. Parameter changes are shown between the point $\left(t_{0}\right)$ of adding

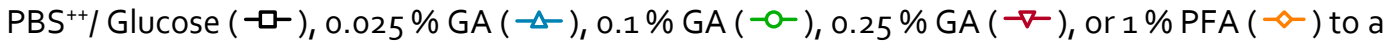
confluent MDCK-Il cell layer and $t_{1}=3 \mathrm{~h}$. (B), (C), (D) Projections on the planes of the 3 -D curve in A. $R_{\text {norm }}$ and $C_{\text {norm }}$ are displayed at the respective most sensitive frequencies, which are $316 \mathrm{~Hz}$ and $40 \mathrm{kHz}$. The time resolution between two points in $A$ is 0.6 min. Data points in $B, C$, and D were reduced for the sake of clarity, resulting in a time resolution of $1.8 \mathrm{~min}$.

Moreover, there is a significant difference between GA and PFA cross-linking. This becomes obvious from the curve shapes in the $\Delta|Z|_{\text {min }}$ time course, but also from the effects of the fixatives on the dielectrical properties of cells, which are discussed below. After adding $1 \%$ PFA to the cells, there is a steep and stepwise initial increase of $\Delta|Z|_{\min }$, followed by a slow and steady approximation to final values within $18 \mathrm{~h}$. The lowest GA concentration, for instance, causes a steady and continuous increase in $\Delta|Z|_{\min }$ from the point of addition. For all applied GA concentrations, the overall $\Delta|Z|_{\text {min }}$ increase is significantly higher compared to the cross-linking of cellular protein with $1 \%$ PFA. The main reason for these observations may arise from the different molecular structures and the related different mechanisms and properties of tissue penetration and cross-linking of the fixatives. Firstly, formaldehyde diffuses faster into the cells, causing a quick initial cross-linking of protein structures not only at the apical but also at the basal side of the cells. This leads to immediate micromechanical changes within the decay length of the acoustic wave and, thus, to an instantaneous OCM response. Secondly, the glutaraldehyde molecules perform a more efficient cross-linking once they have diffused into the cells and to the basal cell regions, due to two aldehyde groups per molecule and a longer and flexible hydrocarbon chain. The 
gradual and very steep increase of energy dissipation due to cell fixation is very clear in the OCM spectra, shown in $S I 21$ (p. 274). The resonance curve of the impedance magnitude $(S I 21 A)$ is strongly damped and flattened with progressive duration of GA-action on the cell layer. This is similarly reflected in the phase spectra $(S / 21 B)$, where a drastic decrease of the maximal phase angel and a distinct broadening of the phase peak report on increased losses of oscillation energy. The phase angle maximum even drops to values well below zero. These studies in passive-mode OCM (impedance analysis), therefore, could not be performed with any active-mode OCM, like for example the commercial Q-Sense QCM-D

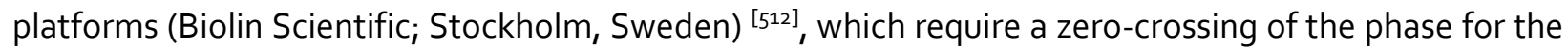
electrical compensation of oscillation damping.

Permeabilization of the plasma membrane with Saponin subsequent to cross-linking of all cellular protein, either with GA or PFA, does not alter the increased QCM damping resistance at all. This is evident in the typical $\Delta|Z|_{\text {min }}$ time course of an entire cell attachment/ fixation/ permeabilization assay in Fig. 5$20 \mathrm{~A}$ (p. 151). Equal results were reported previously for cell fixation with $2.5 \% \mathrm{GA}$ and $4 \%$ PFA, and subsequent membrane perforation with Triton-X-100. ${ }^{[268]}$ The results presented above confirm this and demonstrate that this applies even at 100-fold lower GA concentrations. In AFM studies, a removal of the plasma membrane of GA fixed cells did not change the stiffness of the cell layer. ${ }^{[408]}$ The acoustic load impedance and stiffness of fixed cells thus are solely determined by the protein framework of the cells, whereas the lipid bilayer and the cytoplasm do not contribute in a detectable extent. Wegener et al. (2000) ${ }^{[216]}$ demonstrated that the extracellular matrix (ECM) contributes to the load impedance of quartz resonators mainly in the inductance, whereas resistance contributions by the ECM are negligible compared to the values arising from attached MDCK-II cell layers. Moreover, it was shown that crosslinking of a gelatin layer, which was deposited on a quartz resonator and should imitate the ECM, does not cause an increase in the damping resistance. ${ }^{[268]}$ Hence, the observed $\Delta|Z|_{\min }$ increase $\left(|Z|_{\text {min }} \sim R_{q}\right.$, according to Eq. (44)) is also no effect which is due to the cross-linking of proteins in the ECM between the cell layer and the microbalance surface. Moreover, FLIC microscopy studies could demonstrate ${ }^{\text {[268] }}$ that the increase in the damping resistance upon fixation with PFA is not related to changes in the cellsubstrate distance. Taken together, these results provide strong evidence that the measured $\Delta|Z|_{\text {min }}$ increase and, thus, the quartz oscillation damping solely arise due to the crosslinking and stiffening of the intracellular protein.

\section{Modeling of QCM Data}

From the OCM impedance spectra, the damping resistance could be extracted by BVD modeling of the data (3.3.2 Electro-acoustic Modeling and Impedance Analysis of TSM Resonators, p. 37 ff.). In Fig. 5-39, the resistance change of the quartz oscillation is exemplarily shown for the cross-linking of cellular protein with $0.25 \% \mathrm{GA}(\rightarrow)$ and $1 \%$ PFA $(\rightarrow)$ as well as for negative control $(\rightarrow)$ ). The damping resistance of the quartz oscillation $R$ increases on average by $930( \pm 60) \mathrm{Ohm}$ (mean $\pm \mathrm{SEM} ; \mathrm{N}=12$ ), i.e. $190( \pm 6) \%$, upon coverage of a medium-loaded resonator surface with a confluent layer of MDCK-II cells. Cell fixation causes a further increase of $R$ by $2700( \pm 300) \mathrm{Ohm}(\mathrm{N}=6)$ and $910( \pm 90) \mathrm{Ohm}(\mathrm{N}=6)$ after $3 \mathrm{~h}$ of applying $0.25 \% \mathrm{GA}$ and $1 \%$ PFA, respectively (Fig. 5-39). These effects of protein cross-linking are equivalent to $350( \pm 20) \%$ (for $0.25 \% \mathrm{GA}$ ) and $220( \pm 20) \%$ (for $1 \%$ PFA) of the damping resistance caused by the cell layer. The analysis of $\Delta R$ thus provides an even more pronounced $Q C M$ response upon cell fixation as the course of the $\Delta|Z|_{\min }$ raw data already indicated with increases of $208( \pm 6) \%$ for $0.25 \%$ GA and 180 ( \pm 10$) \%$ for $1 \%$ PFA relative to the damping caused by the cell layer. 


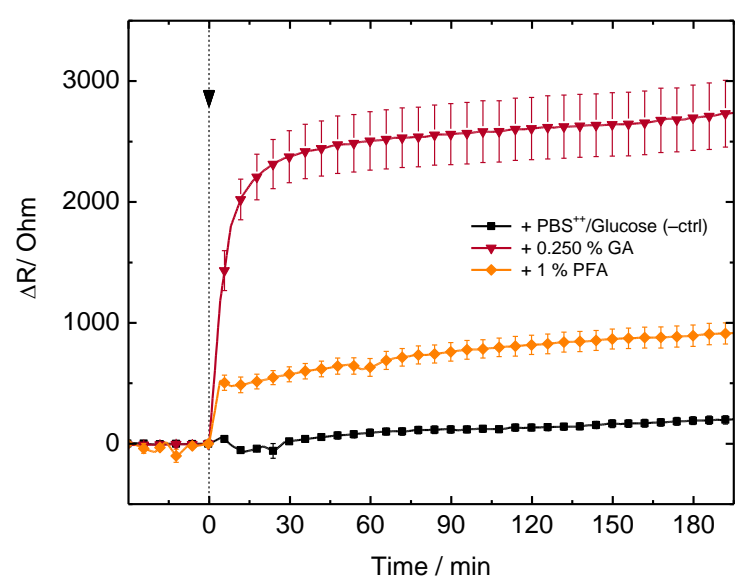

Fig. 5-39 Changes of the QCM damping resistance $\Delta R$ upon cell-layer fixation on QCM-ECIS sensors at time zero with either $0.25 \% \mathrm{GA}(\rightarrow)$ or $1 \%$ PFA $(--)$, extracted from modeling the measured impedance data with the BVD equivalent circuit (cf. 3.3.2). Cells of negative control were exposed to $\mathrm{PBS}^{++}$/ Glucose $(-)$). Significant differences in both the kinetics and the degree of resistance increase are observed. Data points were reduced for sake of clarity.

Similar results were reported previously by Reiß ${ }^{[268]}$ and Heitmann et al. ${ }^{[22]}$. Upon fixation of confluent MDCK-II cell layers with $2.5 \%$ GA they determined an increase in the damping resistance $\Delta R$ by $540( \pm 90) \%$ (in the load impedance $\Delta\left|Z_{L}\right|$ by $500( \pm 90) \%$ ) relative to the native cell layer on classical 1 ElOs. The time of half-maximal signal change was determined to $170( \pm 10) \mathrm{s}$. Nearly the same end value was obtained with a tenfold lower GA concentration, however, the time for reaching half of maximal levels then was significantly later at $690( \pm 40) \mathrm{s}$. The influence of PFA-mediated protein cross-linking on the damping resistance (load impedance) of cell-covered quartz disks was also reported. Even though cells there were exposed to a fourfold higher PFA concentration than in the studies presented here, $\Delta R$ and $\Delta\left|Z_{L}\right|$ increased by $195( \pm 8) \%$ and $190( \pm 10) \%$, respectively, only to slightly lower values compared to the results presented above. However, the differences between the 1 ElOs ( $2^{\text {nd }}$ generation $O C M$ ) used by Reiß and the $2 \mathrm{ElOs}\left(3^{\text {rd }} \mathrm{gen}\right.$. OCM) used in this thesis (the oscillation amplitudes and the Q-factor of 2ElOs are significantly lower; see discussion under 4.3.1, p. $97 \mathrm{ff}$.) anyway does not allow a direct comparison of parameter changes. As discussed above (5.4.2, p. $167 \mathrm{ff}$.), the smaller electrode size of the double-spot quartz disk causes already a significant intrinsic damping of the quartz oscillations in air (cf. Fig. 5-27, p. 168), which is $\sim 1$ kOhm higher than that of the single-spot $Q C M$, when expressed as values of the minimum impedance. If cells are grown on both types of resonators, the values of minimal impedance and phase maximum, which both are parameters for oscillation damping, amount to approximately $2800 \mathrm{Ohm}$ and $-9^{\circ}$ for $3^{\text {rd }}$ generation OCM sensors and to $900 \mathrm{Ohm}$ and $42^{\circ}$ for sensors of the $2^{\text {nd }} / 1^{\text {st }}$ generation OCM. It is assumed that the already strong damping of a cell-covered 2 ElO oscillator limits its sensitivity for detecting further big contributions to energy dissipation, like for example when cellular protein is cross-linked. By contrast, the much less intrinsically damped 1 EIO exhibits the capacity for detecting additional big dissipative contributions from the quartz surface load. It is interesting that one can observe a bigger signal change and, thus, a higher sensitivity of $3^{\text {rd }}$ compared to $2^{\text {nd }} / 1^{\text {st }}$ generation microbalances upon cell attachment and spreading (Fig. $5^{-27}$ ), whereas the $2^{\text {nd }} / 1^{\text {st }}$ generation OCM seems to report more sensitively on further, additional damping effects. Hence, there seems to be a threshold of the damping resistance above which the sensitivity of $2 \mathrm{ElO}$ s is reduced in comparison to $1 \mathrm{ElOs}$. Though absolute values and parameter changes can not be directly compared for the mentioned reasons, more than just basic trends and similarities in the OCM time courses can be 
recognized. Due to additional AFM investigations of native and fixed cell layers, the changes in the QCM damping (load impedance) could be strongly related to changes in the micromechanical stiffness (Young's modulus) of cells. ${ }^{[22,268]}$ However, AFM and QCM data of cells should be correlated with caution, since the AFM is rather sensitive to structural and mechanical alterations at the apical side of the cells, whereas the detection volume by the $\mathrm{OCM}$ is restricted to micromechanical changes close to the substratum, like for example of the basal cytoskeleton and focal adhesions.

\section{Analysis of ECIS Data}

In the course of fixation the dielectrical properties of the cell layers are significantly less affected than the mechanical readout-parameters of the dual sensor. However, interesting fixative- and dose-dependent differences are observed, especially regarding the time courses of the paracellular resistance. Crosslinking of cellular protein causes little reduction of the electrical resistance at $316 \mathrm{~Hz}$ when applying GA and a comparatively strong decrease when applying PFA (Fig. 5-21 C, D). This is noteworthy, because PFA treatment caused the smallest effect in the changes of cellular mechanics compared to GAmediated fixation, as discussed above. Hence, the dielectric structure of the cell layer is largely preserved during fixation with $G A$, whereas the paracellular current pathways are distinctively opened in the course of fixation with PFA. This reports on the enlargement of the subcellular and, more likely, intercellular cleft due to a weakening of cell-substrate and cell-cell contacts. The cross-linking mechanism and the target structures of PFA thus seem to affect the functional integrity of barrier forming tight junctions.

In dye uptake studies, where membrane impermeable FITC-labeled dextran (250 kDa) was provided extracellularly to fixated NRK cell layers, distinct differences in the intracellular fluorescence could be observed, depending on the applied fixation agent (data not shown). After the fixation of cells with either $0.5 \%$ or $1 \%$ GA, no fluorescence of FITC-dextran could be seen inside the cells, just as in negative control cells where FITC-dextran was provided extracellulary to not fixated cells. However, a clear intracellular fluorescence could be seen in cells that have been fixated with either $2 \%$ or $4 \%$ PFA directly after adding FITC-dextran. The dye was taken up by more than $50 \%$ of the cells that have been fixated with $2 \%$ PFA, and intracellular FITC-dextran could be detected across the entire cell layer that has been cross-linked with $4 \%$ PFA. This independent, microscopic investigations provide evidence that (i) GA and PFA act in different ways, and (ii) the cross-linking mechanism of PFA apparently causes an either direct or indirect perforation of the plasma membrane. This membrane permeabilization may be the reason for the electrical impedance decreases observed in the presented OCM-ECIS studies.

However, there is no detachment of cells from the cell layer, as evident from capacitance values which remain constant near starting values throughout the entire investigation period (Fig. 5-21 E, F). By contrast, the capacitance values of PFA-fixated cells on the ECIS working electrode lie even slightly below values of negative control (cf. zoom-in in Fig. 5-21E). Cell layers that were exposed to GA show concentration dependent changes of their dielectrical properties. Lower concentrations of GA thereby cause stronger reductions in the electrical resistance than higher concentrations. This means that a reduction of the GA concentration causes an increasingly strong opening of intercellular junctions during the fixation process. This is exactly the converse dose-dependent effect of GA compared to the previously discussed increases in $\Delta|Z|_{\min }$ and the Young's modulus, respectively. Hence, the bigger the amount of fixative that is available in the cells the faster cellular protein is cross-linked, the faster cells are stiffened, and the smaller is the invasiveness of the cross-linking on the functional integrity of tight junctions. Moreover, the high-frequency capacitances at $40 \mathrm{kHz}$ are also slightly increased by $\sim 10 \%$ for the two lowest GA concentrations applied ( 0.025 and $0.1 \%$ ), whereas $0.25 \%$ GA do not cause any change in the capacitance at all. Even though these changes are only marginal, too mild and supposedly gentle 
cross-linking with low amounts of fixative therefore may even cause a partial detachment of cells. It is supposed that a too slow and less invasive cross-linking action enables a physiological reaction of the cells. In sum, the OCM-ECIS data consistently show that the fixation of cells with high amounts of GA is strongly recommended, for applications where a structural and functional conservation of the cellular state and of the tight junctions between adjacent cells are desired. Too low concentrations of $G A$ and the usage of PFA for fixation, however, would per se lead to alterations within the cell layer during the fixation process. This means that in cell based assays, where cells are fixated in the mentioned manner after a stimulation for the purpose of microscopic analysis, the effects due to fixation could not be separated from the cellular responses to the stimulus, which actually are to be investigated.

Membrane permeabilization with Saponin after protein cross-linking causes a rapid drop of the barrier resistance to values slightly above that of sensors without cells and immersed in cell culture medium only (曲 in Fig. 5-21 D). This causes also an instantaneous increase in the capacitance to almost values of the cell-free level (Fig. 5-21 E). The rapid kinetics of electrochemical signal changes upon Saponin treatment can be nicely seen in the typical $R_{\text {norm }}$ and $C_{\text {norm }}$ time courses of an entire cell attachment/ fixation/ permeabilization assay in Fig. 5-20C (p.151). The corresponding spectra of impedance magnitude, phase angle, resistance and capacitance are shown in SI 21 (p. 274). For the GA- and PFAmediated cross-linking and subsequent membrane perforation by Saponin typical $R_{\text {norm }}$ and $C_{\text {norm }}$ curves with high temporal resolution are depicted in SI 24 (p. 277). The ECIS data demonstrate that perforation of the apical and basolateral plasma membrane causes an instantaneous loss of the dielectrical structure of a cell layer. The cell bodies lose their impeding character on the current flow between the working and counter electrode and the impedance of the entire system is henceforth determined only by the electrodes and the electrolyte in between. This allows us to conclude that, firstly, cross-linking of cellular protein in the forerun of membrane perforation has no reduced or delayed effect on the generation of para- and transcellular current pathways. Secondly, the remaining highly crosslinked protein framework of the cells and the ECM on the sensor surface does not impede current flow across the entire frequency window between $1 \mathrm{~Hz}$ and $1 \mathrm{MHz}$ (cf. SI $21 \mathrm{C}-\mathrm{E}, \mathrm{p} .274$ ), though it exhibits an unchanged high Young's modulus and OCM damping resistance, respectively. Thus, the cell layer dielectrics at high frequencies are exclusively determined by the capacitance of the basolateral and apical plasma membrane, and resistance contributions by the cytoplasm and intracellular structures in series to that can be fairly neglected.

\section{Modeling of ECIS Data}

In order to get a more detailed view on the structural changes that may take place within the cell layer during fixation and which cause the discussed dielectric changes, the impedance data was fitted with a transfer function (Eq. (50)) according to the model mentioned above (3.4.3). Fig. 5-40 shows the fitting parameters, $R_{b}, \alpha$, and $C_{m}$, extracted from data sets of typical cross-linking assays with $0.25 \% \mathrm{GA}$ (Fig. 5-40 A) and $1 \%$ PFA (Fig. 5-40 B). The native MDCK-Il cell layers show significantly high $R_{b}$ and $\alpha$ values $(\square)$, reflecting a moderate barrier resistance and an impedance of the subcellular cleft as typically found for MDCK-II layers. ${ }^{[473-475,480-485]}$ The reduction of the low-frequency resistance in ECIS measurements during GA fixation of cells (cf. Fig. 5-21 C, D) are mainly attributed to losses in the barrier resistance. Impedance changes in the subcellular cleft, however, seem to be negligible as evident from an almost unchanged cell-substrate parameter $\alpha$ ( $\square$ in Fig. 5-40 A). The situation is different for PFA cross-linking of cellular protein. $R_{b}$ values are almost completely reduced $24 \mathrm{~h}$ after adding $1 \%$ PFA to the cells. Final values are less than $5 \%$ compared to the barrier resistance of the native cell layer before fixation. Initially this is a strong indication that PFA causes a substantial and almost complete loss in the 
barrier function of tight junctions. However, since $\alpha$ is also significantly reduced by $\sim 60 \%$ in the course of PFA fixation ( $\square$ in Fig. 5-40 B) and considering the results of the dye uptake studies mentioned above, there is a strong hint that a PFA-mediated permeabilization of the cell membrane is the reason for the significant decrease of both $\alpha$ and $R_{b}$ values. If so, effects due to membrane permeabilization and due to TJ opening can not be clearly distinguished from each other in the decrease of $R_{b}$. The same applies for $\alpha$ and structural changes except membrane permeabilization that are reflected in changes of the parameter value. If the cell membrane is assumed to be intact and if a not changing conductivity of the subcellular medium is assumed, too, a decrease in $\alpha$ reports on a weakening of cell-substrate contacts. This is structurally related to either a decrease in the average cell diameter or an increase in the cell-substrate distance (cf. Eq. (49)). A weakening in the substrate adhesion of cells means a reduction of focal contacts. On the one hand, this leads to an increased distance between the cell bodies and the working electrode. On the other hand, this causes a less spread and more rounded cell shape at the basolateral side, which is equivalent to a reduction of the actual cell radius. Further investiagtions are required to clarify the situation and the origin of the effects observed in $\alpha$ and $R_{b}$. Irrespective of that, neither GA- nor PFA-mediated cross-linking discernibly affected the membrane capacitance $C_{m}$.

A

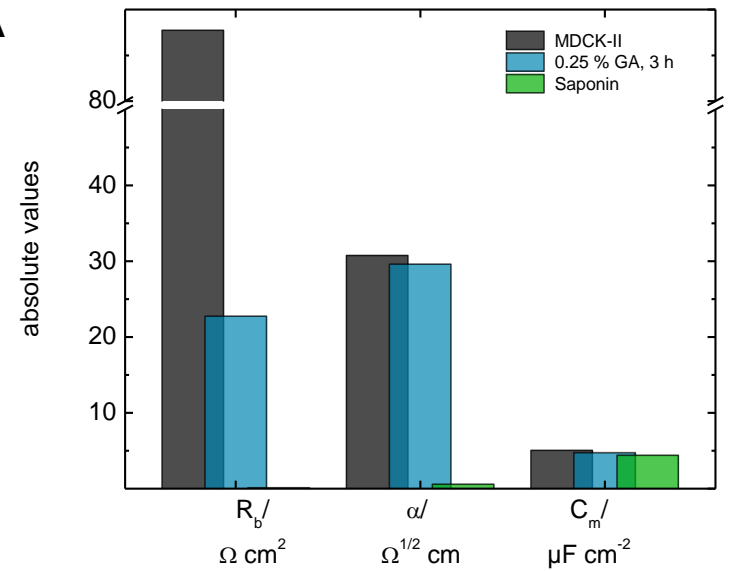

B

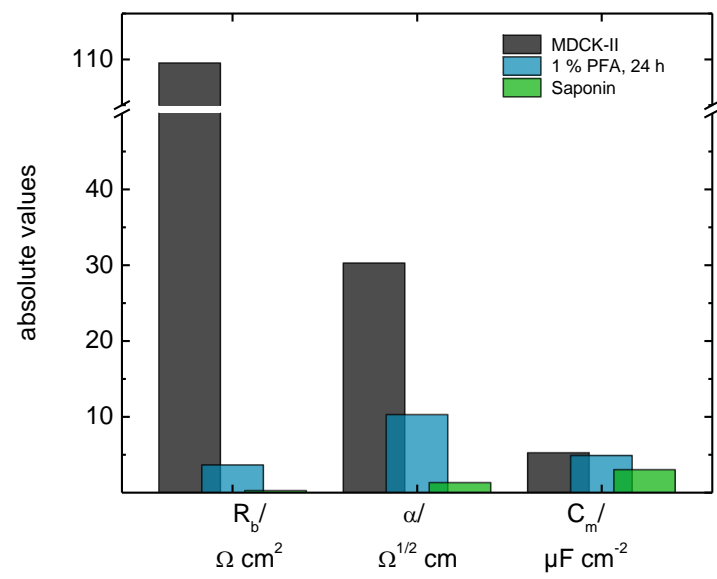

Fig. 5-40 Modeling the impedance data of cell-covered electrodes (according to the model presented in subsection 3.4.3) provided the characteristic frequency-independent parameters $R_{b}$ $\alpha$, and $C_{m}$. Data are shown for native MDCK-II cell layers ( $\square$ ), for cells exposed to (A) $0.25 \%$ GA and (B) $1 \%$ PFA ( $\square$ ), and for fixated cells successively treated wit Saponin ( $\square$ ). The bulk resistance $R_{\text {bulk }}$ and the CPE parameters $n$ and $A$ (cf. Eq. (18)) were determined by linearly fitting impedance spectra at high and low frequencies, respectively, providing values of $R_{\text {bulk }}=450 \Omega$, $n=0.98$, and $A=1.71 \cdot 10^{-5} \mathrm{~F} \cdot \mathrm{s}^{n-1} \cdot \mathrm{cm}^{-2}$ for an electrode surface area of $2.05 \cdot 10^{-3} \mathrm{~cm}^{2}$.

Membrane perforation by Saponin subsequent to fixation ( $\square$ ) causes in all cases a drastic drop in the impedance contributions of both the cell-cell contacts $\left(R_{b}\right)$ and the cell-substrate contacts $(\alpha)$. However, it should be noted that the applied model for fitting the impedance data is actually only applicable in the presence of a confluent cell layer on the working electrode, so that the fitting values after membrane permeabilization have to be considered with caution. Though basic trends and fixative-related differences are clearly evident in the fitting parameters, the presented data in Fig. 5-40 are results of only one typical measurement. To be able to extract statistically reliable statements from changes in the fitting parameters thus a larger population of data has to be modeled and analyzed. Nevertheless, the obtained parameter changes fit well with previous observations by Reiß (2004) ${ }^{[268]}$, who found distinct drops in the barrier resistances to approximately $20 \%$ and $10 \%$ with respect to the native cell layers after 
30 min of cell fixation with 2.5\% GA and $4 \%$ PFA, respectively. However, reductions in the $\alpha$ value during fixation were observed neither for GA nor for PFA. These findings have been confirmed by analysis of the cell-substrate distance in FLIC microscopy studies.

In sum, the fixation and permeabilization assays showed significantly different sensor responses in OCMmode and ECIS-mode. This results demonstrate at the same time the striking benefit of the dual OCMECIS monitoring in cell-based assays, compared to the respective separate sensor techniques. For instance, protein cross-linking with $0.25 \%$ GA induces only slight changes in the dielectrical cell layer properties, even though the fixation means a drastic impact on the cellular structure and function. This becomes evident only if one looks at the impedance data from microbalance recordings. These are capable of detecting the enormous stiffness increase of the cells on the sensor surface due to that fixation. The situation is exactly the other way round, when permeabilizing the cells after protein crosslinking. Then, the cell layer dielectrics significantly change, whereas no change in the micromechanical properties can be discovered. The presented whole-cell QCM-ECIS biosensor approach therefore has the decisive advantage of providing the complementary information on cell shape and cytomechanical changes from one and the same cell population, simultaneously, and in real-time. Together with the possibility of detecting and analyzing parameter correlations, this makes the OCM-ECIS sensor combination a useful tool for the cell-based high-content screening of substances, like for example in drug discovery.

\subsubsection{Stimulating Intracellular Signaling Cascades}

In time-resolved, combined QCM-ECIS sensing distinct cellular responses upon addition of $100 \mu \mathrm{M}$ of the membrane-permeable cyclic AMP-analogue 8-CPT-cAMP were measured. The time-courses of parameter values $\Delta|Z|_{\text {min }}, R_{\text {norm }}$ and $C_{\text {norm }}$ show significant differences in the shape as well as in the kinetics of the signal responses. After a short time delay of roughly $10 \mathrm{~min}$, there is a steep increase in $\Delta|Z|_{\min }$. Hence, the stimulation of the cells causes an increased energy dissipation by the microbalance load (Fig. 5-23 A, B, p. 158). In the electrical resistance, there is an initial but pronounced drop (-15\%). Afterwards, $R_{\text {norm }}$ considerably increases from 10 min after extracellularly providing 8-CPT-cAMP within $4 \mathrm{~h}$ to $125 \%$ of starting values (Fig. $5-23 \mathrm{C}, \mathrm{D}$ ). Hence, there is an enhancement of the cell layer barrier for electrical current at low frequencies. The capacitance values immediately respond to 8-CPT-CAMP addition by a $15 \%$ increase as well. However, the signal recovers again after $10 \mathrm{~min}$ and the overall capacitance change after $4 \mathrm{~h}$ is only $\sim 5 \%$ below reference values of the unstimulated cell layer (Fig. $5-$ $23 \mathrm{E}, \mathrm{F}$ ). These cellular responses detected by combined microbalance sensing and ECIS are reversible, when exchanging the culture medium containing $100 \mu \mathrm{M} 8$-CPT-CAMP against medium lacking the second messenger analogue. Doing so, the signals of both sensors immediately drop back to values before stimulation (Fig. 5-23 A, C, E). However, the instantaneous and strong mechanical and dielectrical sensor responses upon withdrawal of elevated CAMP concentrations seem to be followed by a kind of readjustment phase of the cells for about $4 \mathrm{~h}$. These subsequent signal oscillations can be observed for all three sensor parameters. Stable values of almost pre-stimulation levels are achieved about $10-12 \mathrm{~h}$ after CPT-cAMP removal (Fig. 5-23 B, D, F).

\section{Correlation of QCM and ECIS Data}

Fig. 5-41 displays in a 3-D and in 2-D plots the correlation of the OCM and ECIS responses of a typical CPT-cAMP assay (blue), compared to the signal change of not stimulated control cells (black). The 
dielectrical and micromechanical cell layer responses upon stimulation can be divided into three major linear phases in the $C_{\text {norm }}-\Delta|Z|_{\text {min }}$ diagram (Fig. 5-41 B). The slight $\Delta|Z|_{\min }$ decrease and $C_{\text {norm }}$ increase during the first $8 \mathrm{~min}$ after adding the membrane permeable cAMP analogue to the cells (I. phase) is followed by a totally opposite signal course. During this II. phase of approximately $60 \mathrm{~min}, C_{\text {norm }}$ decreases back to initial values and $\Delta|Z|_{\text {min }}$ simultaneously increases to a stationary level significantly above starting values, however both rates are slower compared to the signal changes in the first phase. In a last and III. phase, from hour one to four after stimulation, the coherence of both signal changes is stopped. $\Delta|Z|_{\min }$ does not significantely change anymore, whereas $C_{\text {norm }}$ exhibits an ongoing slight decrease until the end of the investigation period $\left(t_{1}=4.5 \mathrm{~h}\right)$. This plot thus uncovers no linear correlation of alterations in the cytomechanics and the transepithelial capacitance. A similar observation is made for the transepithelial resistance (at low frequencies) correlated with $\Delta|Z|_{\min }$ (Fig. 5-41 C). The resistance thereby shows almost exactly the same tri-phase correlation with $\Delta|Z|_{\text {min }}$ like the capacitance, shown in Fig. 5-41 B, but with opposite sign. Hence, this plot also indicates that $\Delta|Z|_{\min }$ and $R_{\text {norm }}$ signals report on different, non-linearly dependent structural responses of the cells as a consequence of elevated intracellular cAMP concentrations. By contrast, when plotting $C_{\text {norm }}$ vs. $R_{\text {norm }}$ changes (Fig. 5-41 D), one can identify a high correlation of parameters. This is evident from the biphasic curve, where the linear dependency of $C_{\text {norm }}$ vs. $R_{\text {norm }}$ is equal before and after the turning point, however, only with a different sign. The abrupt $R_{\text {norm }}$ decrease and $C_{\text {norm }}$ increase in the early stages mark the I. phase of parameter changes in the experiment $\left(t_{0} \rightarrow 8 \mathrm{~min}\right)$. Both values recover again in the following II. phase of the experiment, in the opposite direction of the I. phase. The initial sensor responses thereby are compensated within 45 min after stimulation and are further overcompensated with a comparatively lower slope until the end of the experiment $\left(t_{1}=4.5 \mathrm{~h}\right)$.

The mutual dependency of electrochemical capacitance and resistance changes from ECIS-mode recordings also becomes obvious, when withdrawing the second messenger analogue from the cell culture medium again. The respective 3-D and 2-D correlation plots of parameters are shown in S/ 25 (p. 277). These indicate only for $C_{\text {norm }}$ vs. $R_{\text {norm }}$ a linear dependency, since their changes after 8-CPTcAMP removal again follow almost exactly the same paths as during stimulation. This correlation can be observed for neither of the electrochemical parameters in diagrams where they are plotted vs. $\Delta|Z|_{\text {min }}$.

\section{Modeling of ECIS Data}

Impedance analysis of ECIS-mode data enables to distinguish between resistance contributions arising from the tightness of intercellular junctions (cell-cell contacts) and the resistance that arises from the properties of the subcellular cleft (cell-substrate contacts) ${ }^{[96]}$ (cf. 3.4.3). Fig. 5-42 shows the modeling parameters, $R_{b}, \alpha$, and $C_{m}$ obtained from fitting the impedance data of an exemplary assay according to the transfer function given in Eq. (50). The bar plot shows increases of both paracellular resistance parameters, $R_{b}$ and $\alpha$, upon increasing the cAMP level inside the cells. The barrier resistance $R_{b}$ thereby shows a stronger initial decrease $(t=10 \mathrm{~min}, \square)$, a slower compensation of this fast response ( $t=30 \mathrm{~min}, \square)$, and a more pronounced final increase $(t=4 \mathrm{~h}, \square)$, compared to the cell-adhesion parameter $\alpha$. As the capacitance values already indicated, the membrane capacitance $C_{m}$ is altered only to a negligible extent during the CAMP stimulation of cells. Very similar results were presented by Wegener et al. ${ }^{\left[{ }^{114}\right]}$ for porcine choroid plexus epithelial cells and by Riethmüller et al. ${ }^{[443]}$ for human umbilical vein endothelial cells (HUVECs). They could also confirm their findings on the electrical resistance change by microscopic permeability studies with FITC-labeled dextran. 
A

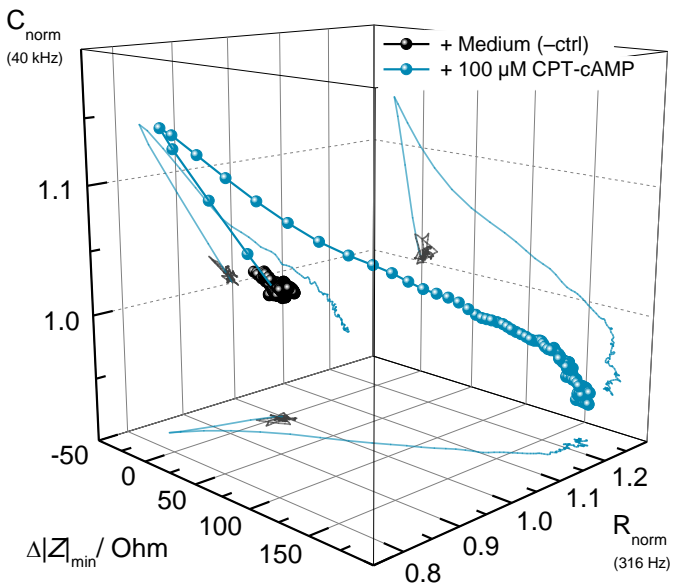

\section{B}

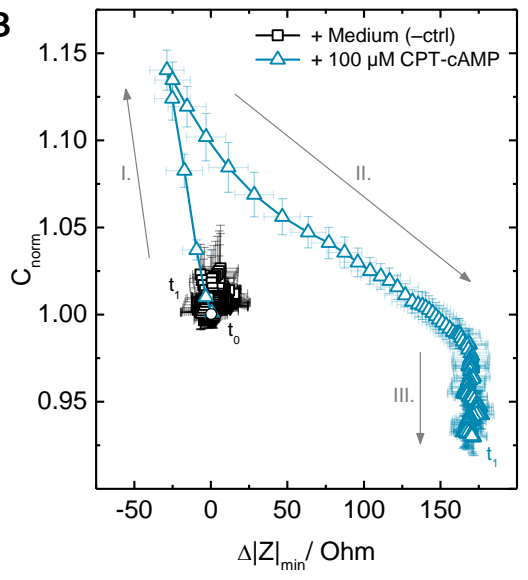

C

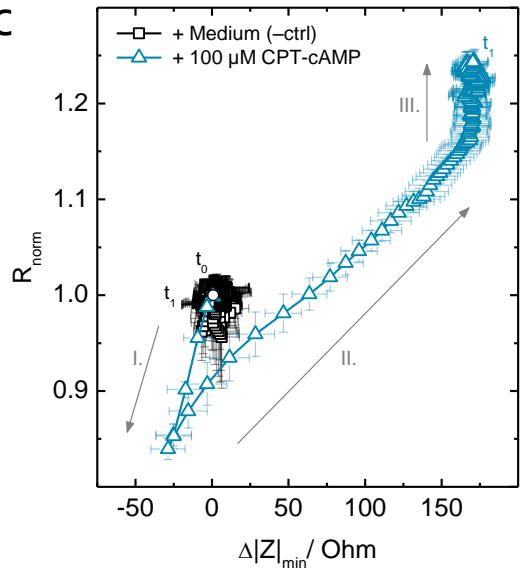

D

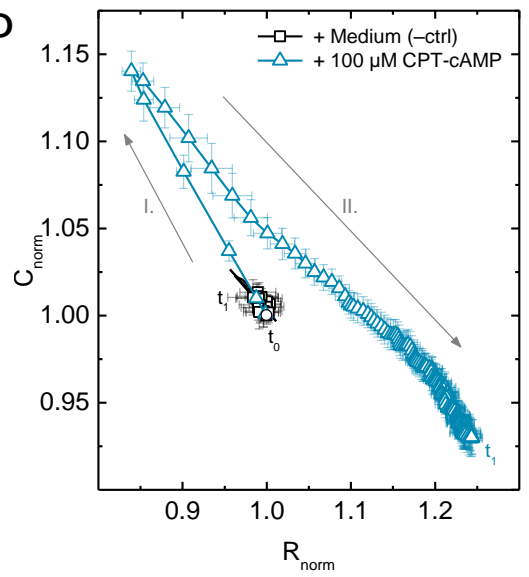

Fig. 5-41 (A) 3-D OCM-ECIS impedance plot ( $\Delta|Z|_{\min }$ vs. $R_{\text {norm }}$ vs. $\left.C_{\text {norm }}\right)$ of averaged, timeresolved cAMP assays. Parameter changes are shown between the point $\left(t_{0}\right)$ of adding $100 \mu \mathrm{M}$ of the membrane permeable CAMP-analogue 8-CPT-cAMP $(-\Delta)$ or medium only $(-\square-)$ to a confluent NRK cell layer and $t_{1}=4.5 \mathrm{~h}$. (B), (C), (D) Projections on the planes of the $3-\mathrm{D}$ curve in A. Different phases of parameter correlations are indicated. $R_{\text {norm }}$ and $C_{\text {norm }}$ are displayed at the respective most sensitive frequencies, which are $5 \mathrm{kHz}$ and $50 \mathrm{kHz}$. The time resolution between two points is $100 \mathrm{~S}$.

Similar results of an increased paracellular electrical barrier and a reduced transepithelial permeability for macromolecules, respectively, as a consequence of elevated intracellular CAMP have been reported previously for various cell types, such as Necturus gallbladder ECs ${ }^{[445]}$, BAECs ${ }^{[447]}$, HUVECs ${ }^{[443]}$, HUAECs/ HAECs ${ }^{[444]}$, BPAECs ${ }^{[446]}$, porcine choroid plexus ECs ${ }^{\left[{ }^{[14}\right]}$, and BCECs ${ }^{[448]}$. The current in transepithelial electrical resistance (TER) or ECIS studies, just as the macromolecular probes in permeability studies, have to get through the same impeding intercellular structures. These are, starting from the bulk medium above the apical cell side, first the tight intercellular junctions of zonula occludens, secondly the narrow gap between the lateral plasma membranes of adjacent cells, and thirdly the small cleft between the cells and the substrate surface. As this pathway can be electrically described as several resistors connected in series, changes in any of these structural units affects the entire paracellular impedance and the transepithelial permeability, respectively. 


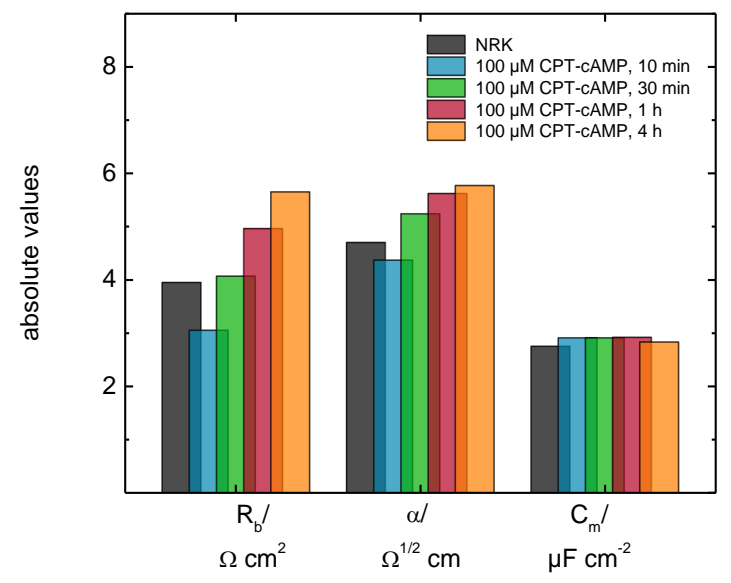

Fig. 5-42 Modeling the impedance data of cell-covered electrodes, according to the model presented in subsection 3.4.3, provided the characteristic frequency-independent parameters $R_{b}$, $\alpha$, and $C_{m}$ for NRK cell layers, exposed to $100 \mu \mathrm{M}$ 8-CPT-cAMP for $10 \mathrm{~min}(\square)$, $30 \mathrm{~min}(\square)$, $1 \mathrm{~h}(\square)$, and $4 \mathrm{~h}(\square)$. Not treated cells serve as negative control ( $\square$ ). The bulk resistance $R_{\text {bulk }}$ and the CPE parameters $n$ and $A$ (cf. Eq. (18)) were determined by linearly fitting impedance spectra at high and low frequencies, respectively, providing values of $R_{\text {bulk }}=350 \Omega$, $n=0.97, A=1.61 \cdot 10^{-5} \mathrm{~F} \cdot \mathrm{s}^{n-1} \cdot \mathrm{cm}^{-2}$ for an electrode surface area of $2.05 \cdot 10^{-3} \mathrm{~cm}^{2}$.

\section{QCM and ECIS Data Interpretation}

It has been previously reported by Petersen and Reuss ${ }^{[513]}$, Copello et al. ${ }^{[144]}$, Kottra ${ }^{[515]}$, and Vank et al. ${ }^{[516]}$ that elevation of CAMP inside Necturus gallbladder epithelium cells stimulates $\mathrm{Cl}^{-}$conductance in their apical membrane. $\mathrm{Cl}^{-}$channel activation has as well been reported for various other cell types, like for example airway and intestinal epithelial cells ${ }^{[517]}$. It is argued that an opening of $\mathrm{Cl}^{-}$channels generates an osmotic pressure and causes a water influx into the cells, which consequently leads to a certain swelling of cells. Such swelling processes could be the reasons for the observed changes in the dielectric structure of the cell layer, since a vertical and lateral expansion of the cell bodies leads to a compression of the lateral gap between neighboring cells and probably also of the cell-substrate distance. Both effects directly affect the paracellular permeability and electrical resistance and might explain the increases of $R_{b}$ and $\alpha$ values in the course of cAMP stimulation. Even though this has not yet been reported in literature for the normal rat kidney cell line which was used in the studies presented above, it has to be considered that such a mechanism is also what is monitored by the OCM-ECIS measurements. This explanation would nicely agree with the obtained ECIS data, however if there were not the OCM data which report on something completely different than cell swelling. As already known from a variety of cell-based OCM and AFM studies, like for example protein cross-linking assays (cf. subsections 5.3.2/ 5.4.8, ref. OCM ${ }^{[22,246,268]}$, and ref. AFM ${ }^{[404,408,433-439]}$ ), the increase in the quartz oscillation damping is connected with a stiffness increase in the cell-substrate region. Wegener et al. (2000) ${ }^{[216]}$ reported on a pronounced increase in the oscillation damping and, thus, also a stiffening in the micromechanics close to the resonator surface, if confluent cell layers on OCM plates are exposed to hyperosmotic culture medium. Similar results were obtained by own studies of hyperosmotic stimulation ( $395 \mathrm{mOsmol} / \mathrm{kg}$ ) of MDCK-II cells on the OCM (data not shown). Such a cell layer stiffening could be explained by an osmotically induced water efflux and the accompanied shrinking of the cells, which is well-known for a variety of epithelial cells ${ }^{[518]}$. This is confirmed by AFM studies of Steltenkamp et al. (2006) ${ }^{[439]}$ and Pietuch (2012 ${ }^{[403]}$, which report on a reversible, linear cell volume increase (decrease) and an accompanied cell softening (stiffening) under hypoosmotic (hyperosmotic) challenges of cell layers. 
Hence, the observed increase in $\Delta|Z|_{\min }$ due to increased cAMP levels has to be rather assigned to cell stiffening by shrinking and can not be coherently explained by a swelling of the cells. The approach of explaining the ECIS data by an activation of $\mathrm{Cl}^{-}$channels and an induced cell swelling, therefore, can not be confirmed by the OCM data which have been obtained simultaneously from the same cell population by the dual sensors used in these studies. Assuming a CPT-CAMP-mediated cell shrinkage on the basis of the presented OCM data, however, can in return also not explain the dielectric changes of the cell layer (increase of $R_{b}$ and $\alpha$ ), since a contraction of the cell bodies presumably causes the opposite effect by an increase of the intercellular gap or even an opening of cell-cell junctions. From the OCM and ECIS data following conclusions are draw. The induced NRK cell layer response towards increased intracellular CAMP levels can not or , at least, only to a minor degree be assigned to cell volume changes. Moreover, the correlation plots of OCM and ECIS data (Fig. 5-41) suggest that there is not a direct (linear) dependence of micromechanical and dielectrical changes within the cell layer. Hence, a complex and diverse structural responses induced by CPT-CAMP in NRK cells is assumed. Increased levels of intracellular CAMP are correlated with an activation of the PKA, which is involved in the regulatory process of cytoskeletal structures, either directly or via subsequent signal transduction cascades $[445,446,455,456]$.

Fitting to the presented OCM data, it is therefore assumed that CPT-CAMP causes a reversible stiffening of cellular structures in the cell-substrate region. Since the stiffness of the cell layer is predominantly determined by the actin cytoskeleton, in particular a reorganization or stabilization of $f$-actin structures that are located at the basal cell side and a redistribution of actin filaments from the cytoplasm into the cortex are considered. This is consistent with previous observations of a cyclic AMP-mediated thickening and aggregation of microfilaments in the submembraneous regions [445], a quantitative increase of polymerized actin ${ }^{[446]}$, and the formation of highly organized filamentous cytoskeleton structures of cells exposed to agents known to increase intracellular cAMP levels ${ }^{[454]}$. The increased actin assembly due to elevated intracellular CAMP has been also found to coincide with a barrier strengthening of the epithelial cell layer ${ }^{[445,446]}$. This was also reported by various groups independent on the cytoskeleton properties $[314,429,443,444,447-450]$. Similar findings of an enhancement of the transepithelial barrier have also been observed for agents that specifically polymerize and stabilize microfilaments ${ }^{[519]}$. The stimulation of cells with cyclic AMP thus demonstrates the same relationship of actin disassembly and increased permeability (decreased barrier resistance) as it has been observed in cytochalasin D studies (cf. subsections 5.3.1/ 5.4.7 and ref. ${ }^{[97,116,397,398,427-432]}$ ), however in the opposite direction of effects. These findings support the hypothesis of a structural correlation or even a regulatory connection between the state of polymerization of the actin cytoskeleton and the tightness of barrier forming tight junctions (cellcell contacts) and, very likely, also the quality of cell-substrate adhesions (FAs) of ECs. The regulatory hypothesis is further confirmed by the reversibility of effects, which have been observed within $\sim 4 \mathrm{~h}$ after withdrawal of CPT-CAMP from the culture medium. This theory is also supported by the signal overshoots and post-oscillations of dielectric and micromechanical sensor data, which are assigned to subsequent readjustment processes by the cells, as all ambient parameters were kept constant during this time. The exact short-term and long-term structural alterations of cells that are indicated by the OCM and ECIS signal changes after CPT-CAMP removal, however, have to be further investigated.

The OCM and ECIS results of the presented CPT-CAMP assays show a striking similarity to the cellular reactions that have been reported for endothelial cells to hydrocortisone $(\mathrm{HC})$ treatment. $\mathrm{HC}$ causes a significant improvement of the barrier properties and a reduction of paracellular permeability, respectively. ${ }^{[268,305,520,521]}$ These originate from both a strengthening of the barrier resistance $\left(R_{b}\right)$ and a reinforcement of the cell adhesion to the substrate $(\alpha)$. The membrane capacitance of the cells $\left(C_{m}\right)$ and, 
thus, the morphology of the plasma membrane however is not significantly affected by HC stimulation [268,305,521], just like in CAMP assays presented above. Additional to the mentioned TEER and ECIS investigations, fluorescence microscopic studies ${ }^{[521,522]}$ and SFM ${ }^{\left[5^{22]}\right.}$ and OCM ${ }^{[268]}$ measurements indicate a reorganization of the actin cortex as well as an accompanied significant stiffening of the cell layer due to the addition of HC. This temporal co-occurrence of effects led to the conclusion that the enhancement of the barrier properties is correlated with the reorganization and stiffening of the actin cytoskeleton via direct or indirect effects on the cell-cell and cell-substrate contacts. The effects of HC on the micromechanical and dielectrical properties of confluent MDCK-II cell layers thus show similarities to that induced by elevated intracellular cAMP, however, effects due to $\mathrm{HC}$ appear on a much longer timescale $(>24 \mathrm{~h})$ compared to that caused by cAMP $(<4 \mathrm{~h})$.

In sum, the cellular responses upon CPT-cAMP stimulation were monitored in time-resolved manner and with high reproducibility in both OCM-mode and ECIS-mode measurements of the dual sensors. The CPT-CAMP assays clearly demonstrate the applicability of the QCM-ECIS sensors for monitoring not only comparatively radical, external interventions into the cellular system, like for example by cytoskeleton disruption with CD (cf. 5.3.1/5.4.7) or protein cross-linking with GA (cf. 5.3.2/ 5.4.8), but also nondestructive, intracellular stimulations under physiological conditions. Moreover, the dual sensors provide a very sensitive and non-invasive opportunity to monitor the relaxation of the cells after a stimulation or regulatory mechanisms at the level of mechanical and dielectrical cell layer properties. The possibility of a temporal correlation of these characteristics opens even deeper insights in structural links. The combined OCM-ECIS measurements thus allow, on the basis of two independent viewpoints, to draw more comprehensive and more accurate conclusions about structural processes in a cell layer under study. This demonstrates the big benefit of the dual sensing approach.

\subsubsection{Electric Manipulation and Double-mode Impedance Analysis (ELPO-ECIS-QCM)}

In proof-of-concept studies it has been demonstrated the applicability of the newly developed electrode layout on piezoelectric quartz crystals for both, the electric manipulation of cells and the ECIS- and OCMmode measurements of cell layer responses directly thereafter in a time-resolved and non-invasive manner. Moreover, the dual sensor allowed to monitor the quality and vitality of the cell layer in the forerun of the electric field application, by tracking the characteristic OCM and ECIS parameters over time. By default, the whole attachment and spreading process of the cells on the sensor surface has been monitored by ECIS and QCM recordings, as exemplarily shown in Fig. 5-24 A, C (p. 160). The data provided meaningful information about the cell layer properties and, thus, a good basis for deciding whether a manipulation experiment makes sense or if no useful results could be expected anyway due to a problematic cell layer. This quality control prior to pulse application also enabled the detection of cell layer responses caused solely by the extracellular addition of the substance that is to be introduced into the cells by ELPO. For instance, the QCM-ECIS sensor data show no significant changes of cellular mechanics and dielectrics upon, firstly, exchanging the cell culture medium against EBSS ${ }^{++}$buffer and, secondly, adding $1 \mathrm{~g} \cdot \mathrm{L}^{-1}$ FITC-labeled dextran (250 kDa) to the cells (Fig. 5-24 A, C). Due to the molecular weight of the dextran, it is too big to freely diffuse across the cell membrane into the cells. By awaiting equilibration of parameter changes, which arise from the act of adding a substance or medium exchange, the effects of extracellular stimulation were easily distinguished from cell responses to the ELPO voltage pulse as well as any subsequent intracellular stimulation induced by this. 


\section{Electric Pulse Parameters}

In the ELPO-OCM-ECIS and microscopic studies presented values of the pulse parameters were applied as they were described in literature for the successful electroporation of FITC-dextran into NRK cells on commercial 8W1 $\mathrm{E}^{\mathrm{TM}}$ ECIS electrodes. Wegener et al. (2002) ${ }^{[120]}$, Albermann (2004) ${ }^{[523]}$, and Stolwijk (2011) $^{[7]}$ investigated the electroporation efficiency for NRK cell layers by means of dye-uptake at various pulse frequencies, amplitudes and durations. As the best ELPO parameters, for a maximum FITC-dextran loading with minimum mortality of cells, they consistently reported on a frequency of $40-100 \mathrm{kHz}$, an $\mathrm{AC}$ voltage amplitude of $3-4 \mathrm{~V}$, and a pulse duration of $200 \mathrm{~ms}$. If exposing the cells either to higher amplitudes of the electric AC field or for a longer duration to the pulse, this leads to an irreversible damage of the cell membrane which is associated with cell death. This has become a proven method in the past 10 years for the specific, electrical generation of a defined wound in the cell layer and the subsequent analysis of cell migration, cell-cell interaction, and proliferation rates by ECIS and

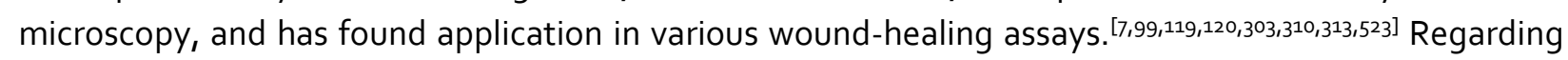
NRK cell layers cultured on $8 \mathrm{~W}_{1} \mathrm{E}^{\mathrm{TM}} \mathrm{ECIS}$ electrodes, effective and complete electrical wounding was reported for pulse durations longer than $10 \mathrm{~s}$ (at $2.5 \mathrm{~V}$ and $40 \mathrm{kHz}$ ) ${ }^{[99]}$ and for amplitudes higher than $5 \mathrm{~V}$ (at $40 \mathrm{kHz}$ for $>500 \mathrm{~ms})^{[7,120]}$. In both approaches, ELPO and wounding, the electric manipulation of cells depends on the applied pulse frequency, as it determines the fraction of the total voltage that actually drops across the cell layer. Simulations by Albermann ${ }^{[523]}$ show that the biggest fraction of the applied voltage drops across the cell layer only at sufficiently high frequencies, whereas at too low $(<5 \mathrm{kHz})$ or too high ( $>100 \mathrm{kHz}$ ) AC frequencies only a minor fraction of voltage is imposed on the adherent cells by the external electric field. The frequency dependent voltage profile thereby is determined by the dielectric properties of the cell layer under study. The calculations reveal that for leaky cell layers with a low barrier resistance $\left(R_{b}\right)$, weak cell-substrate adhesion $(\alpha)$, and low membrane capacitance $\left(C_{m}\right)$ higher frequencies for efficient electroporation are required, while for cell layers with high $R_{b}$ and $\alpha$ values the applicable frequency window is extended towards smaller frequencies. The frequency at which most of the voltage drops across the cell layer can be easily determined from the normalized impedance spectrum of the cells, since it directly emphasizes the frequency at which the cell layer contributes most to the impedance of the entire system (peak of $|Z|_{\text {norm }}(f)$ ). For the presented OCM-ECIS sensors with standard electrode layout a maximal electrical impedance contribution of the NRK cell layer with respect to the cell-free working electrode was determined at 22 ( \pm 2$) \mathrm{kHz}$ (cf. S/ $15 D$ (p. 269)), which fairly agrees with the published value. For reasons of comparability, and since $40 \mathrm{kHz}$ were found to be appropriate for ELPO and electric wounding of various different cell types ${ }^{[7]}$, this frequency was applied in the ELPOOCM-ECIS assays.

\section{Microscopic Evidence of ECIS Data}

It was shown by the analysis of fluorescence micrographs that the electric manipulation of NRK cells on the OCM-ECIS sensors, by applying a $200 \mathrm{~ms} A C$ voltage pulse of $2 \mathrm{~V}$ at $40 \mathrm{kHz}$, caused a temporal and reversible opening of pores in the cell membranes, like it is schematically illustrated in Fig. 5-43. Prior to the pulse FITC-labeled, not membrane permeable dextran $(250 \mathrm{kDa})$ was added to the cell culture medium above the cells (Fig. 5-43 A). During the electric field-mediated pore formation in the plasma membrane of the cells on the working electrode, extracellular FITC-dextran could diffuse into the perforated cells, along the concentration gradient (Fig. 5-43 B). After the post-pulse resealing of the plasma membranes, the fluorescently labeled macromolecules remained inside the cells (Fig. 5-43 C, D), causing a green fluorescence of successfully electroporated and loaded cells. 
A

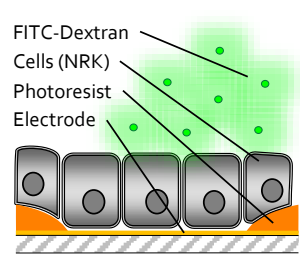

Extracellular FITC-

dextran $(250 \mathrm{kDa})$
B

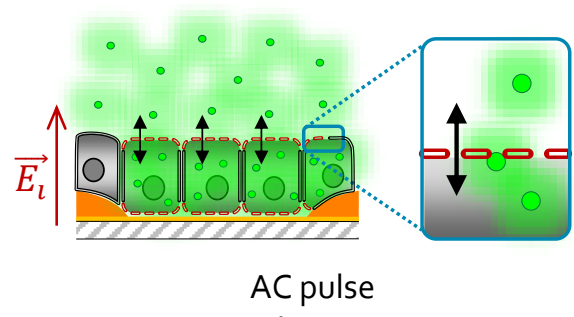

$(2 \mathrm{~V}, 40 \mathrm{kHz}, 200 \mathrm{~ms})$
C

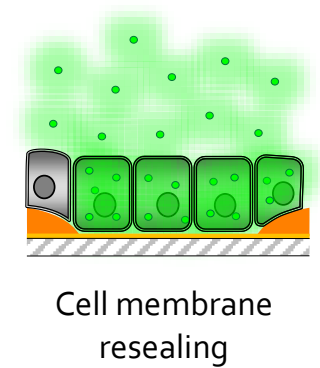

D

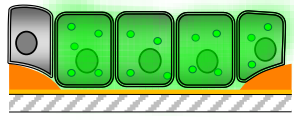

Washing

Fig. 5-43 Schematic illustration of the in situ ELPO process of adherent cells cultured on OCMECIS sensors. The active electrode area is defined by a circular hole in a photoresist coating layer, which insulates the rest of the gold electrode from the bulk electrolyte. (A) Extracellular addition of FITC-labeled dextran (250 kDa). (B) AC voltage pulse application (2 V, $40 \mathrm{kHz}, 200 \mathrm{~ms})$ and induced transient permeabilization of the basal and apical plasma membrane of the cells, which enables FITC-dextran molecules to enter the cells via pores in the apical membrane (cf. zoom-in). (C) Resealing of the cell membrane after pulsing. (D) Washing removes extracellular FITC-dextran.

The micrographs in Fig. 5-25 (p. 162) clearly indicate that only cells that adhered directly to the working electrode show a bright green fluorescence, whereas cells attached to the insulating photoresist next to the uncovered electrode show no fluorescence (Fig. 5-25 $F_{1}, F_{2}$ ). Hence, only cells on the WE were perforated by the AC pulse and show an ELPO-mediated FITC-dextran loading. Cells in the periphery of the WE were not affected at all by the applied pulse and, therefore, the dextran macromolecules could not enter the cells. Negative control cells, exposed to FITC-dextran and not electroporated, confirm that the observed dextran uptake was not an effect of passive diffusion (Fig. 5-25 E1, E2). From the results it is concluded that the AC voltage amplitude of $2 \mathrm{~V}$ is high enough to electroporate cells and to cause a significant dye-uptake on the one hand, and that it is low enough to cause no irreversible cell damage on the other hand. A doubling of the voltage amplitude for ELPO (4 V, $40 \mathrm{kHz}, 200 \mathrm{~ms})$, however illustrates the thin line between effective but non-destructive electroporation and irreversible and damaging electric wounding of the cells on the WE of the QCM-ECIS setup. Although applying of $4 \mathrm{~V}$ pulses also mediates loading of the cells with FITC-dextran just like $2 \mathrm{~V}$ pulses, the impact of membrane perforation crosses a threshold value where a complete recovery of the plasma membrane is not possible anymore and cells are irreversibly damaged. This consequently leads to a widespread cell death and scattered cell detachment from the sensor surface across the entire working electrode ( - in Fig. $5-25 G_{1}, G_{2}$ ). This can also be seen in the loading of cell nuclei with FITC-dextran $(>)$, which is not observable for milder, non-destructive ELPO conditions (cf. dark circular area of labeled cells in Fig. 5-25 $F_{1}, F_{2}$ ). In single experiments, ELPO with $4 \mathrm{~V}$ even led to an almost complete detachment of cells, as documented by the fluorescence and phase-contrast micrographs in Fig. 5-26. Thus, electroporation of NRK cells at $4 \mathrm{~V}$ ( $40 \mathrm{kHz}, 200 \mathrm{~ms}$ ) with the OCM-ECIS setup rather induces cell death and an electrical wound in the cell layer. This invasiveness differs from the literature, where a pulse amplitude of $4 \mathrm{~V}$ (at $40 \mathrm{kHz}$ for $200 \mathrm{~ms}$ ) was reported as the optimal voltage for a maximal dye-loading while maintaining a maximal survival rate of cells ${ }^{[7,120,523]}$. For the application of the ELPO-OCM-ECIS setup in wound-healing assays, however, a longer pulse duration of $>10 \mathrm{~s}$ is recommended in order to completely kill the cells on the WE and to generate a defined, cell-free wound. 


\section{Analysis of post-Pulse QCM and ECIS Signals}

The OCM and ECIS sensor data, which have been recorded immediately after electric pulsing, report on no detectable changes in the micromechanical properties close to the microbalance surface (Fig. 5-25 A), however on a significant decrease in the low-frequency resistance (Fig. 5-25 B) and an increase in the high-frequency capacitance (Fig. 5-25 C). In detail, the $2 \mathrm{~V} \mathrm{AC}$ pulse causes a short transient drop in the resistance by $20 \%$ within $10 \mathrm{~min}$, followed by a comparatively slow recovery to pre-pulse values within $\sim 2 \mathrm{~h}$. In almost similar kinetics, the capacitance value is slightly increased by $20 \%$ and recovers afterwards. The degree of the resistance drop is slightly lower as described in literature. Wegener et al. (2002) ${ }^{[120]}$ and Stolwijk (2011) ${ }^{[7]}$ reported on a fast resistance/ impedance drop to $\sim 0.7$ at $4 \mathrm{kHz}$ with respect to values before pulsing, upon the electroporation of confluent NRK cell layers on $8 \mathrm{~W}_{1} \mathrm{E}^{\mathrm{TM}} \mathrm{ECIS}$ electrodes with non-damaging $A C$ pulses $(2-4 \mathrm{~V}, 100-500 \mathrm{~ms}, 40 \mathrm{kHz})$. Values of electrical impedance and resistance of NRK cells at $4 \mathrm{kHz}$ are similar. This can be nicely seen in the normalized spectra of impedance, resistance, and capacitance in $S / 15$ (p. 269). A slow recovery of the electrical resistance/ impedance values back to pre-pulse values was consistently obtained within $30-60$ min after electric field application. The recovery time thereby is increasingly retarded when applying higher ELPO voltage amplitudes. ${ }^{[7,120,524]}$

The electroporation of NRK cells on the QCM-ECIS sensor with $4 \mathrm{VAC}$ pulses $(40 \mathrm{kHz}, 200 \mathrm{~ms})$ caused a resistance drop almost down to values of the cell-free electrode within 5-10 min (Fig. 5-25 B). Afterwards, the resistance even slightly decreased further. Roughly one hour after pulse application, resistance values start to recover again and slowly increase towards pre-pulse levels. A similar observation can be made for the capacitance values at $50 \mathrm{kHz}$ (Fig. 5-25 C). These time courses agree well with the time-delayed recovery of impedance/ resistance values as described in literature for NRK cells after the application of AC voltage pulses above certain threshold values in amplitude and duration [7,99], which caused irreversible membrane permeabilization and wounding of the cell layer. The slow recovery of values visualize the repopulation of the, at least partly, wounded cell-free area due to proliferation of cells and cell migration from the electrode periphery. By the linear extrapolation of the $R_{\text {norm }}$ and $C_{\text {norm }}$ data in Fig. 5-25 B, C in the phase of re-rise let us roughly estimate the duration the cells require for closing the electrically generated wound. This is illustrated in Fig. 5-44. The extrapolation of the capacitance data (Fig. 5-44 B) indicates a complete recovery of the cells within approximately $4 \mathrm{~h}$, and the extrapolation of the resistance recovery (Fig. 5-44 A) lets assume a full establishment of the intercellular barrier within about $5 \mathrm{~h}$ after electrical manipulation.

Though this is only an estimation and does not replace the need for complete recordings and analyses of the repopulation process, the recovery periods obtained from extrapolations agree well with the time scales reported in literature by Stolwijk ${ }^{[7]}$ and Keese et al. ${ }^{[99]}$. However, it has to be noted that the area of the working electrodes used in the cited studies is only about a quarter of that of the 2 EIO WEs used in this study. Hence, the estimated periods for re-establishment of the cell layer rather report on the recovery of a partly damaged cell layer than on a complete repopulation of a cell-free wound. In this context, however two points have to be noted. Firstly, the presented assays with $4 \mathrm{~V}(40 \mathrm{kHz}, 200 \mathrm{~ms})$ ELPO pulses actually were not aimed to wound the cell layer and, as micrographs indicate, there is not a complete removal of cells from the electrode. An undefined, partial wound and the impedance contribution of the dead or live cells and of cellular fragments remaining on the electrode probably influence the recovery of impedance values. Secondly, the working electrode diameter of the OCM-ECIS sensors, which defines the area of electrically manipulated cells, is twice the diameter of the $8 \mathrm{~W}_{1} \mathrm{E}^{\mathrm{TM}} \mathrm{ECIS}$ electrodes used in the published ELPO studies. Hence, the time for cell migration and complete 
repopulation of the QCM-ECIS working electrode after wounding probably takes longer and thus also the impedance recovery in ECIS-mode recordings.

A

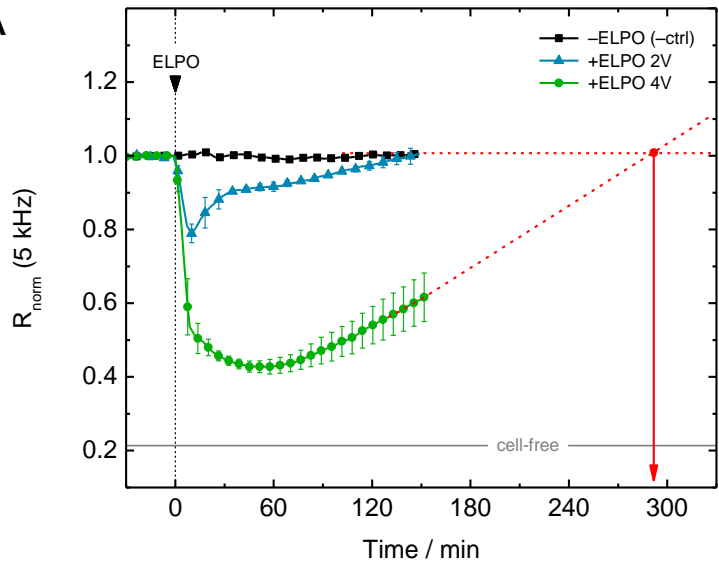

B

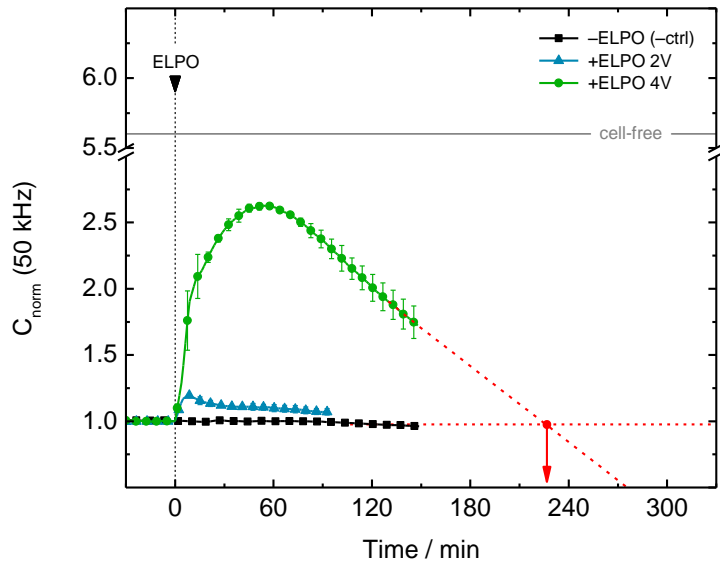

Fig. 5-44 Linear extrapolation of (A) resistance and (B) capacitance values in the phase of recovery after ELPO of NRK cell layers on the WE of OCM-ECIS sensors. The crossing of the extrapolated time course with the pre-pulse level (both indicated by dashed red lines) delivers the assumed duration that is required for the recovery of parameter values (red arrows).

In sum, at both electric pulse conditions applied in the presented studies, the electrical current pathways through the cell layer are opened at low as well as at high frequencies. The degree of the impact of the AC field on the electrical properties of the treated cells thereby strongly depends on the magnitude of the applied voltage pulse. This also determines the time the cells require for rehabilitation of the electrical properties on the working electrode. This time includes the duration for reconditioning of the porous plasma membrane, for rebuilding of maybe affected cell-substrate and cell-cell junctions, as well as for the proliferation and migration of cells into cell-free areas on the electrode, that are generated by potential electric field-mediated killing and detachment of cells. Electric manipulation and ECIS recordings with the $2 \mathrm{EIO}$ sensors, thus, demonstrate their applicability and enable access to any in situ electroporation and electrical wounding assays described for classical ECIS electrodes. In order to find out the best electroporation and electric wounding parameters for NRK cells and others on the presented system, however, extensive studies have to be carried out. Besides the amplitude, also the duration of pulsing has to be addressed.

By fitting the ECIS data that have been directly recorded after the pulse, according to the model presented in subsection 3.4.3, Albermann ${ }^{[523]}$ and Stolwijk ${ }^{[7]}$ could show that the barrier resistance $R_{b}$ and the cell-adhesion parameter $\alpha$ are strongly affected by ELPO while the membrane capacitance $C_{m}$ is almost not influenced. The observed influences of ELPO on the ECIS parameters are assumed to arise mainly from cell morphological alterations due to electroporation, as well as from cell shape recovery after the electric field has been switched off again. Impedance changes due the formation and resealing of pores in the cell membranes, however, can be neglected, since these processes occur in the range of few seconds ${ }^{[118]}$ and thereby can be temporally not resolved by our spectroscopic impedance recordings. The effect of ELPO on the cell morphology and, thus, the impedance properties of a cell layer is schematically illustrated in Fig. 5-45. 

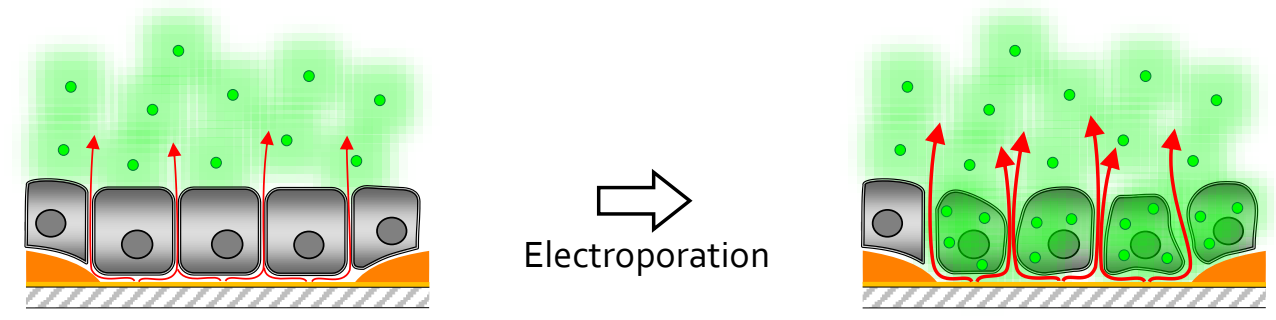

Fig. 5-45 Shematic illustration of assumed cell morphological alterations due to the exposure of the cells to an externally applied electrical field. Such cell shape changes give rise to an increase of paracellular current pathways (indicated by red arrows, quantified by number and thickness) and, thus, cause a reduction of the impedance across the cell layer.

The morphological alterations measured may arise from a weakening of cell-cell or/ and cell-substrate contacts, as a consequence of ELPO-mediated swelling/ shrinking of the cells or changes in cytoskeletal structures. Cell volume changes could be induced by the diffusion of extracellular molecules into the cells or of intracellular molecules out of the cells through the pores in the membrane that are generated by the electric field. Imbalances and disturbances in the concentration gradients of molecules caused by this lead to an osmotically-driven water efflux or influx. The extent of the described effects thereby depend on the degree of membrane permeabilization (number and size) and the time required for resealing the plasma membranes, which both are a function of pulse amplitude and pulse duration. ${ }^{\left[{ }^{225}, 526\right]}$ Hence, it seems to be evident that after ELPO the impedance values of cells recover in a duration and amplitude dependent manner. A more comprehensive discussion on the origin of dielectric alterations of cell layers due to ELPO is given by Stolwijk ${ }^{[7]}$.

\section{Sensing the Impact of ELPO on Cytomechanics by $3^{\text {rd }}$ Generation QCM}

The absence of any QCM response to ELPO of cells, neither for $2 \mathrm{~V}$ nor for $4 \mathrm{~V}$ pulses, was not expected in the first instance. The formation of pores in the cell membranes by the nondestructive $2 \mathrm{~V}$ pulses lets molecules smaller than the pore size freely diffuse into or out of the cells. This causes a temporal breakdown of chemical gradients between the cytoplasm and the bulk medium and leads to an osmotic imbalance. This again causes osmotic water influx or efflux and, thus, swelling or shrinking of cells. For osmotically stimulated cells on quartz resonators, distinct alterations in the oscillation damping $\left(\Delta|Z|_{\text {min }}\right)$ are reported ${ }^{[216]}$. Significant impedance changes of $\sim 200 \mathrm{Ohm}$ upon the hyperosmotic stimulation of cells ( $395 \mathrm{mOsmol} / \mathrm{kg}$ ) on quartz crystals have also been observed in previous experiments with the 2 ElO setup (data not shown). Hence, it was expected to sensitively detect such assumed ELPOinduced cell volume changes (change in the viscoelasticity of the load) by means of OCM-mode measurements. However, this was not the case. Even the application of $4 \mathrm{~V}$ pulses and the thereby induced - at least partial - electrical wounding of the cell layer did not cause any significant drop in the OCM signal, either. This was surprising, as the (partial) wound is generated on the ECIS working electrode exactly in the center of the microbalance electrode, where the oscillation amplitude is maximal and, thus, the QCM sensor is most sensitive for mechanical changes of the loading. The microbalance, however, is not only sensitive in the center, where the small working electrode $\left(A_{\mathrm{WE}}=2.05( \pm 0.3) \cdot 10^{-3} \mathrm{~cm}^{2}\right)$ for ECIS and ELPO is uncovered in the photoresist coating. The measured electrical oscillation impedance mirrors the average acoustic impedance of the entire, piezoelectric quartz layer that is sandwiched between the two surface electrodes $\left(A_{\mathrm{OCM}, 2 \mathrm{EIO}}=96 \cdot 10^{-3} \mathrm{~cm}^{2}\right)$ and even a little beyond. On closer inspection, it is therefore not surprising that a detachment of $\sim 2 \%$ of the cells on the microbalance, i.e. the area fraction 
of the $\mathrm{WE}$, due to electric wounding does not appreciably affect the mean oscillation properties of the quartz crystal.

Regarding the sensor layout, one could consider two strategies in order to increase the sensitivity of QCM measurements to the electrically manipulated fraction of cells on the sensor plate. This would be, firstly, a reduction in the QCM electrode size and, secondly, an increase of the WE size, or maybe a combination of both. Regarding the first approach, however, one has to consider further adverse effects on the quartz oscillation performance in terms of the damping impedance. As discussed in the previous chapter (4.3.2, p. $102 \mathrm{ff}$.), the quality factor of the microbalance $\left(Q=1 / D=X_{\text {tot }} / R_{\text {tot }}\right)$ strongly depends on the electrode area and decreases with its radius ${ }^{[288]}$. For instance, the $Q$-factor of the developed $2 \mathrm{ElO}$ sensors $\left(A_{\mathrm{OCM}, 2 \mathrm{ElO}}=9.6 \mathrm{~mm}^{2}\right)$ is $\sim 4.5$-fold lower in air compared to the classical $1 \mathrm{EIO}\left(A_{\mathrm{OCM}, 1 \mathrm{EIO}}=28.3 \mathrm{~mm}^{2}\right)$. A higher energy dissipation (lower $Q$-factor) is likely accompanied by a reduced sensitivity to viscoelastic changes of the microbalance load and a reduction of the oscillation amplitude, respectively (cf. Eq. (62)). This again is linked to a reduced penetration depth of the acoustic wave and, thus, of the sensed volume above the sensor surface. The second approach, to leave the electrode size of the resonator unchanged and to increase the working electrode area instead, however, introduces other difficulties that need to be addressed. An increase of the WE area on the $2 \mathrm{ElO}$ sensors would mean an increase in the circular hole in the photoresist of the coated resonator spot. This results in a reduction of the microbalance load and, as the experimental investigations in subsection 5.1.6 demonstrate, in an approximation of the resonance conditions (resonance frequency) of the neighboring resonators. From WE sizes of $2 \mathrm{~mm}$ in diameter an interference of oscillations of both microbalances is expected which probably negatively affects the series resonance and the readout parameters. There are several ways to increase the area of the WE (on $\mathrm{OCM}_{1}$ ) but at the same time to prevent an interference of the adjacent $\mathrm{OCM}$ spots. One approach would be to load $\mathrm{OCM}_{2}$ with a bigger rigid mass than $\mathrm{OCM}_{1}$, by e.g. a thick polymer layer on the side facing away from the cells. Secondly, one could increase the mass on $\mathrm{OCM}_{1}$ by additionally coating the side facing away from the cells. Other possibilities without additional coating processes require a change in the basic sensor layout. These comprise an increase in the electrode spacing ${ }^{[362,363]}$, a different electrode thickness of the two resonators ${ }^{[362,364]}$, variations in the quartz thickness by mesashape and convex-shape designs, or the use of quartz plates with x-axis inversion ${ }^{[352,360]}$. Regarding the ECIS-mode properties, the size of the WE area determines the sensitivity of impedance recordings for cell morphology changes. Moreover, if one wants to measure the micromotion of a cell layer, i.e. the impedance fluctuation in ECIS-mode, as an indicator for cell viability ${ }^{[96]}$, this is recommended with small electrode sizes. The sensitivity for micromotion is higher with smaller WEs, since impedance fluctuations cancel each other when larger electrodes are used. At least, the parameters used for electric manipulation of the bigger cell area have to adapted, too. With an increasing size of the $W E$, the impedance of the system is more and more determined by the bulk resistance, while the impedance contributions arising from the electrode and the cell layer in series to it are becoming increasingly smaller. If the WE is small, the total bulk resistance is dominated by the so-called constriction resistance. It is inversely proportional to the electrode size with $\rho_{b} / r_{\mathrm{WE}}$, where $r_{\mathrm{WE}}$ is the radius of the WE and $\rho_{b}$ is the specific resistance of the bulk medium, and the impedance of the electrode is inversely proportional to the square of the radius of the WE. ${ }^{\left[{ }^{[6]}\right]}$ The influence of the WE radius on the entire impedance spectrum of cell-free and cell-covered electrodes can be nicely seen by the simulated curves in Fig. 5-46. An increase in the diameter of the WE from $250 \mu \mathrm{m}$ ( $8 \mathrm{~W}_{1} \mathrm{E}^{\mathrm{TM}} \mathrm{ECIS}$ electrode) to $6.5 \mathrm{~mm}$ (1ElO electrode) causes a significant reduction of the CPE impedance of the electrode at low frequencies and of the bulk resistance at high frequencies. As a consequence, a cell layer grown on these electrodes exhibits an increasingly smaller impact on the impedance in the relevant frequency window of $10^{3}-10^{5} \mathrm{~Hz}$. In the 
spectra of impedance magnitude (Fig. 5-46 A), this becomes visible in a decrease of the area that is enclosed between the cell-free (open symbols) and the cell-covered (filled symbols) spectra. This becomes even clearer in the normalized impedance spectra in Fig.5-46 B. The ECIS sensitivity, expressed as the maximum value of the normalized impedance spectrum, for a given cell layer (values of a typical NRK cell layer are used for simulation) is reduced from almost six to less than 1.5 for a working electrode increased from $250 \mu \mathrm{m}$ in diameter $(-)$ to $6.5 \mathrm{~mm}$ in diameter $(\rightarrow)$. This means for an electric manipulation of cells that at a certain voltage amplitude less of the voltage drops across the cell layer on a bigger working electrode. ${ }^{[118]}$ Hence, in order to achieve the same impact of ELPO on the cell layer, i.e. pore formation or electrical wounding, on big as on small WEs one has to apply a much higher amplitudes of the electrical AC potential for this. However, this also increases the voltage drop across the electrode and might cause electrochemical reactions. The theoretical, fractional voltage drop $F$ across the cells can be approximated from the impedance magnitudes of the cell-free $\left(|Z|_{w / o c e l l s}\right)$ and the cell-covered electrode $\left(|\mathrm{Z}|_{\mathrm{w} / \text { cells }}\right)$ at the frequency of the electric pulse according to:

$$
F=\left(1-\frac{|\mathrm{Z}|_{\mathrm{w} / \mathrm{o} \mathrm{cells}}}{|\mathrm{Z}|_{\mathrm{w} / \text { cells }}}\right) \cdot 100[\%]
$$

A

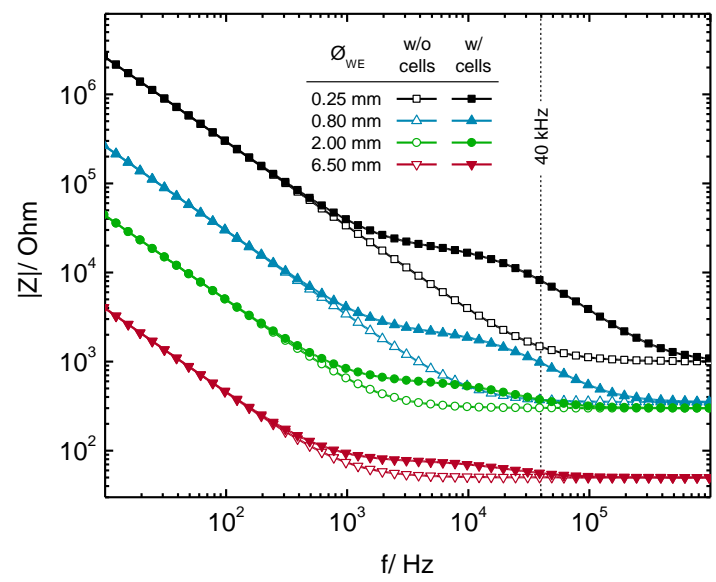

B

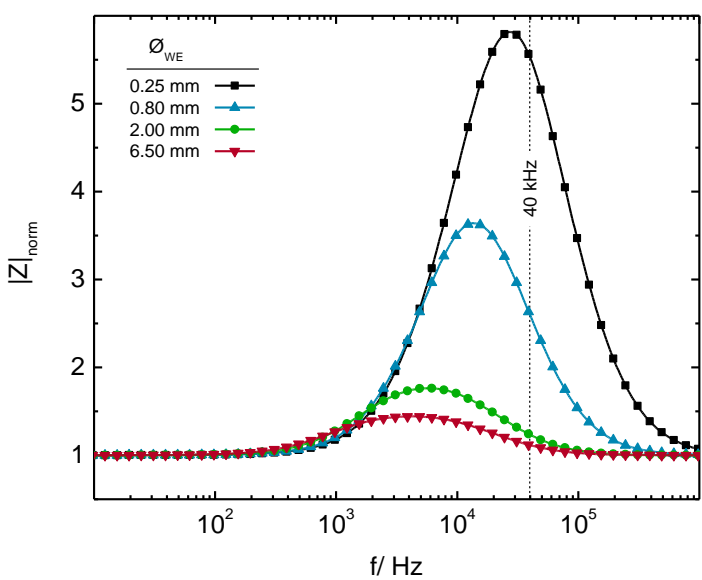

Fig. 5-46 (A) Simulated impedance magnitude spectra of cell-free (open symbols) and cellcovered (filled symbols) circular gold film electrodes, with diameters $\varnothing_{\mathrm{WE}}$ (areas $A_{\mathrm{WE}}$ ) of $0.25 \mathrm{~mm}$ $\left(5 \cdot 10^{-4} \mathrm{~cm}^{2} ;-\square,--\right), 0.80 \mathrm{~mm}\left(5 \cdot 10^{-3} \mathrm{~cm}^{2} ;-\triangle,-\right), 1.95 \mathrm{~mm}\left(3 \cdot 10^{-2} \mathrm{~cm}^{2} ;-0-,-\right)$, and $6.5 \mathrm{~mm}\left(3.3 \cdot 10^{-1} \mathrm{~cm}^{2} ;-\nabla, \rightarrow\right)$. The impedance of the CPE depends on $1 / r_{\mathrm{WE}}{ }^{2}$ and the constriction resistance of the bulk electrolyte depends on $1 / r_{\mathrm{WE}}$. Simulation parameters: $R_{b}=6 \Omega \cdot \mathrm{cm}^{2}, C_{m}=2 \mu \mathrm{F} \cdot \mathrm{cm}^{-2}, \alpha=4 \Omega^{1 / 2} \cdot \mathrm{cm}, n_{\mathrm{CPE}}=0.95, A_{\mathrm{CPE}}=1.5 \cdot 10^{-5} \mathrm{~F} \cdot \mathrm{s}^{n-1} \cdot \mathrm{cm}^{-2}, R_{\text {bulk }}$ $\left(\varnothing_{\mathrm{WE}}=0.25 \mathrm{~mm}\right)=1000 \Omega, R_{\text {bulk }}\left(\varnothing_{\mathrm{WE}}=0.80 \mathrm{~mm}\right)=350 \Omega, R_{\text {bulk }}\left(\varnothing_{\mathrm{WE}}=2.00 \mathrm{~mm}\right)=300 \Omega$, and $R_{\text {bulk }}\left(\varnothing_{\mathrm{WE}}=6.5 \mathrm{~mm}\right)=50 \Omega$. The parameter values were chosen according to experimentally found values for NRK cell layers (cf. references ${ }^{[7,523]}$ ). (B) Normalized spectra of impedance magnitude $\left(|Z|_{\text {norm }}=|Z|_{\text {w/ cells }} /|Z|_{\text {w/o cells }}\right)$ for various sizes of the working electrode, calculated from the respective spectra shown in $A$.

Applying Eq. (67) to the simulated ECIS impedance spectra shown in Fig. $5-46$ provides for the various WE sizes fractional voltage drops across the cell layer of $82 \%\left(\varnothing_{\mathrm{WE}}=0.25 \mathrm{~mm}\right), 62 \%\left(\varnothing_{\mathrm{WE}}=0.80 \mathrm{~mm}\right)$, $19 \%\left(\varnothing_{\mathrm{WE}}=2.00 \mathrm{~mm}\right)$, and $10 \%\left(\varnothing_{\mathrm{WE}}=6.5 \mathrm{~mm}\right)$, at a pulse frequency of $40 \mathrm{kHz}$. Similar theoretical results were presented by Stolwijk (2011) ${ }^{[7]}$. However, it has to be noted that Stolwijk surprisingly found that on the basis of experimental data the fractional voltage that drops across the cells actually is lower 
for smaller compared to bigger electrodes. For instance, at an electroporation pulse of $4 \mathrm{~V}, 79 \%$ of the voltage drops across a NRK cell layer grown on $8 \mathrm{~W}_{1} \mathrm{E}^{\mathrm{TM}} \mathrm{ECIS}$ electrodes $\left(\varnothing_{\mathrm{WE}}=0.25 \mathrm{~mm}\right.$ ), whereas only $70 \%$ of the voltage drops across a NRK cell layer grown on microelectrodes $\left(\varnothing_{\mathrm{WE}}=0.18 \mathrm{~mm}\right.$ ). For this reason the best electroporation amplitudes for confluent NRK cells were $4 \mathrm{~V}(40 \mathrm{kHz}, 200 \mathrm{~ms})$ on $8 \mathrm{~W}_{1} \mathrm{E}^{\mathrm{TM}}$ ECIS electrodes and $5 \mathrm{~V}(40 \mathrm{kHz}, 200 \mathrm{~ms})$ on the smaller microelectrodes. ${ }^{[7]}$ However, it has to be noted that the geometrical proportionalities of electrodes were different for $8 \mathrm{~W}_{1} \mathrm{E}^{\mathrm{TM}}$ and microelectrodes, which is considered as reason for the observations. Independent of this, the best ELPO parameters anyway have to be adapted for each electrode layout, cell line, and kind of assay, by applying empiric ECIS, dye-uptake, and live/ dead staining studies.

\subsubsection{Summary}

The results presented in this chapter convincingly demonstrate the benefits of the dual sensor approach, of simultaneous and substrate-integrated OCM and ECIS measurements, in a variety of cell-based assays. The parameter time-courses that are obtained from the two sensor modes sensitively report on mechanical alterations close to the microbalance surface and changes in the dielectric properties at the electrode-electrolyte interface, providing complementary information. Cell attachment and spreading assays on the OCM-ECIS sensors, for instance, provide considerable differences in the extent and kinetics of the readout parameters between MDCK-II and NRK cells. Hence, characteristic two- and threedimensional signal patterns can be derived, which allow to distinguish between various cell lines. Moreover, significant changes in the mechanical and dielectrical cell attachment properties can be monitored when cells are seeded in modified cell culture media. The time-resolved recordings allow to uncover not only differences in the final state of cell layer formation, as e.g. $24 \mathrm{~h}$ after cell seeding, due to a deficiency or a supplement in the culture medium, but also any kinetic delay or acceleration of parameter changes within the observation time. Once a confluent cell layer is established on the QCMECIS substrate, it can be used as a whole-cell biosensor to follow changes in the cellular micromechanics and dielectrics upon any kind of stimulus, non-invasively and in time-resolved manner. By this, doseresponse relationships could be determined for various agents. The non-invasive sensor approach enables not only to determine the effects of a drug at a certain time after stimulation, as in classical cellbased endpoint assays. It additionally allows to investigate the time-dependent mechanism of action of substances on cells, as well as their response to it, on the basis of time-resolved micromechanical and dielectrical changes of a cell layer. This could be delayed effects, regulatory overshoots, or the recovery of signals within a certain period. Another benefit of QCM measurements in combination with ECIS is the possibility to correlate the parameters from both independent sensor principles in a time-resolved manner, since the information is obtained from one and the same cell population at the same time. This provides new insights into mutual dependencies or time-independent processes in cell layers. Fig. 5-47 shows a compilation of correlation plots of OCM- and ECIS-mode parameters of the various whole-cell biosensor assays presented in this thesis. The diagrams nicely show that there are significantly different mutual dependencies of mechanical and dielectrical parameter changes, depending on the kind of cell layer stimulation. Traces are shown for cell layer treatments with 8-CPT-CAMP ( $-\square-$ ), cytochalasin D (CD; $-\Delta_{-}$), glutardialdehyde $\left(\mathrm{GA}_{;}{ }^{-}-\right)^{-}$), and paraformaldehyde (PFA; $\left.-{ }^{-}\right)$. Hence, single measurements with the combined OCM-ECIS setup provide even more high-content information from one cell layer (cytomechanics, cell-shape, barrier resistance) than the sum of separate studies on different cell layers with different experimental approaches. In a proof-of-concept study, the principle of electroporation (ELPO) was also successfully integrated in the dual sensor system. By the application of invasive but nondestructive electric fields, cells on the ECIS working electrode could be loaded in situ with 
extracellularly provided substances, while their viability was maintained. Furthermore, the generation of a defined electrical wound in cell layers on OCM-ECIS sensor surfaces by ELPO with elevated voltage amplitudes was demonstrated, too. In both approaches the cell layer properties have been monitored before ELPO and instantaneously afterwards by means of OCM-ECIS. This allows to follow over time the impact of ELPO and of introduced substances on the cell morphology, as well as the recovery of the cell layer after the electric manipulation. The ELPO-OCM-ECIS actuator-sensor approach opens the door to a wide field of possible applications in biomedical research.
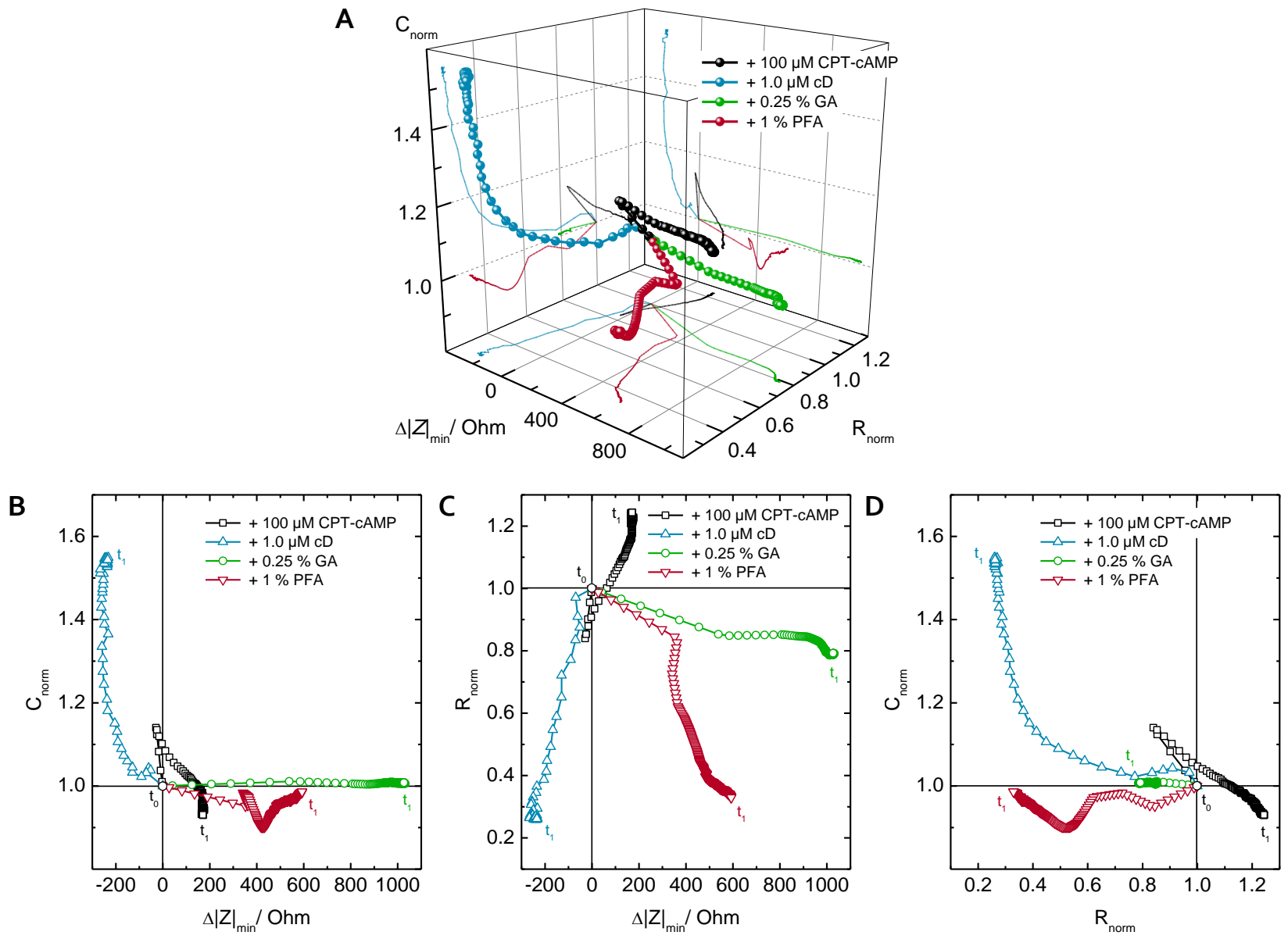

Fig. 5-47 (A) 3-D QCM-ECIS impedance plots $\left(\Delta|Z|_{\min }\right.$ vs. $R_{\text {norm }}$ vs. $\left.C_{\text {norm }}\right)$ of time-resolved cellbased assays. Parameter changes are shown for the stimulation of a confluent NRK cell layer with $100 \mu \mathrm{M} 8$ 8-CPT-cAMP ( $-\square ; t_{1}=4.5 \mathrm{~h}$; time resolution $\Delta t=0.7 \mathrm{~min}$ ) and of confluent MDCK-II cell layers with $1.0 \mu \mathrm{M}$ cytochalasin $\mathrm{D}\left(\mathrm{CD}_{;}-\Delta_{-} ; t_{1}=3 \mathrm{~h} ; \Delta t=4 \mathrm{~min}\right), 0.25 \%$ glutaraldehyde $\left(\mathrm{GA}_{;}{ }^{-}{ }^{-}\right.$; $t_{1}=3 \mathrm{~h} ; \Delta t=0.6 \mathrm{~min}$ ), and $1 \%$ paraformaldehyde (PFA; $\left.-\nabla ; t_{1}=3 \mathrm{~h} ; \Delta t=0.6 \mathrm{~min}\right) .(\mathrm{B}),(\mathrm{C}),(\mathrm{D})$ Projections on the planes of the 3-D curve in $\mathrm{A} . R_{\text {norm }}$ and $C_{\text {norm }}$ are displayed at the respective most sensitive frequencies, which are $5 \mathrm{kHz}$ and $50 \mathrm{kHz}$ for NRK, and $316 \mathrm{~Hz}$ and $40 \mathrm{kHz}$ for MDCK-Il cell layers.

Besides a reduction of the experimental effort, the dual sensor also minimizes uncertainties and problems that arise in separate studies from unavoidable divergences in general experimental conditions. These are in particular naturally different properties of cell layers from different passages, or of cell layers that were, although of the same passage, seeded on different substrates. In contrast to AFM and fluorescence microscopic end-point studies, or time-resolved AFM studies with either poor lateral or poor temporal resolution, the continuous $\mathrm{OCM}$ sensing and ECIS provide information on structural 
alterations of thousands of cells in real-time. AFM is commonly used as the default method for cell elastography measurements. ${ }^{[527-533]}$ However, cell elasticity probing by AFM either requires a large number of nanoindentation measurements on various points and cells for obtaining a representative mean stiffness value of a cell population, or force-deflection measurements are performed only at a single point on one cell for obtaining time-resolved stiffness data. None of both approaches is satisfactory for capturing fast and dynamic cytomechanical changes, for reasons of too slow data acquisition time or too low statistical validity. Cells possess a highly heterogenic nanomechanical surface structure so that the local elasticity obtained from few points of a cell can not at all represent the mechanical properties of the entire cell and even less of a whole cell layer. ${ }^{[528,529]}$ The analysis of quartz resonator damping has proven to be a feasible method to detect stiffness/ viscoelasticity changes of a cell layer cultured on the microbalance surface. . $22,205,206,210,216,223,242,245,247,252,534] \mathrm{OCM}$ measurements can achieve a time resolution of few seconds and the extracted stiffness parameter is an average value over $\sim 55000$ cells for quartzes of the $3^{\text {rd }}$ generation QCM $\left(\varnothing_{\text {Electrode }}=3.5 \mathrm{~mm}\right)$. Hence, cell viscoelasticity testing by means of OCM is capable of providing information on cytomechanics in a high temporal resolution and at the same time averaged over a whole population of cells, something which AFM-based approaches are not able to perform in reasonable time and effort. Another benefit of QCM over AFM for quantifying cell stiffness is its non-invasiveness. Whereas an indentation of cells with an AFM cantilever

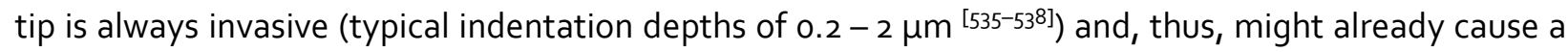
cellular response solely by the act of measurement ${ }^{[539,540]}$, cells cultured on OCM resonators don't notice anything of the measurement, since the amplitude of the quartz oscillation is in the range of a few nanometers.

In summary, various types of cell-based assays demonstrate the capability of the developed QCM-ECIS sensor platform for time-resolved, multi-parametric, label-free, and sensitive analysis of cell-substrate and cell-cell adhesion properties. The hyphenated approach of simultaneous OCM and ECIS readouts of cells, technologically integrated on one substrate surface, represents a powerful tool for a multiperspective and thus detailed analysis of cell-physiological processes in various fields of application. The implementation of ELPO on QCM-ECIS sensors also offers additional interesting application possibilities of in situ actuation and subsequent dual sensing of a cell layer.

\subsubsection{Outlook}

In this thesis a dual sensor platform on the basis of the independent transducer principles of OCM and ECIS has been developed, implemented, and established. The working QCM-ECIS sensor system now offers a wide range of possible applications. The multi-parametric readout of the whole-cell biosensor could serve as a means to get a more comprehensive insight into the reaction of cells to any kind of (bio)chemical, biological, or physical stimulus. These include amongst others environmental pollutants, toxins of biological origin, any kind of radiation, and pharmaceutical drugs. It is believed that label-free and non-invasive sensor approaches for real-time monitoring of cell behavior, such as QCM, ECIS, SPR, LAPS, or ISFET, are indispensable in future for the diagnosis and therapy of cancer. These methods allow for a better understanding of cancer cell behavior as well as of the effectiveness and mode of action of anti-cancer drugs. A combination of independent sensor technologies like ECIS-SPR ${ }^{[274]}$ or OCM-ECIS (this thesis) enable here even deeper insights. The OCM-ECIS sensor can be applied in a multitude of cellbased assays, such as cell attachment and spreading, proliferation, cell barrier function, wound healing, migration and invasion, cytotoxicity, micromotion, cell receptor responses, cell signaling, cytocompatibility testing, cell-to-cell communication, electroporation, or drug assessment. Any cellular 
assay that has been previously performed with a TSM resonator can be transferred to the dual sensor without restrictions, since the ECIS principle is directly integrated into the quartz surface so that liquid handling and the experimental setup remain unchanged. The only difference to the classical measurement chamber relates to the electronics, since now four instead of two electrodes have to be contacted (cf. Fig. 3-13 vs. Fig. 3-18).

On the basis of the QCM-ECIS sensor layout, one could think of completely new cell-based assay formats. For instance, the arrangement of two resonator spots on one quartz disc (2EIO) enables something like a QCM migration assay. If cells are seeded at a small spot at one edge of the quartz crystal, which is in a line with the two electrodes, one could monitor the time-dependent growth of the cells across the first and the second OCM spot by analyzing their oscillation impedances. This might provide access to alterations in the cellular mechanics during migration and proliferation. Moreover, one could also apply the two-electrode quartz layout in chemotaxis and haptotaxis assays. By seeding cells only on one electrode/ one half of the sensor and providing a chemical attractant at the other side of the quartz disk, or an immobilized concentration gradient of chemokines in the direction of the second OCM spot, changes in the mechanical properties of the cell-substrate contacts upon induced migration can be detected. This is considered as a very interesting and promising approach, since cellular motion depends on a rearrangement of the cytoskeleton, and the actin cytoskeleton is the determining factor for the stiffness of adherently grown cells to which the damping of the quartz oscillation is sensitive. Further assays can make use of the in situ electroporation of cells in combination with OCM-ECIS sensing. Introducing a substance into the cells on the working electrode by ELPO and monitoring the cytomechanics by the second microbalance some distance away could be applied in cell-cell communication assays for studying e.g. gap junction coupling and multiplier effects in cell layers.

Further technological developments of the presented dual sensor are imaginable. In order to meet the requirements of an efficient drug discovery, the sensor sizes have to be reduced and multiplied for highthroughput capabilities. Besides the standard 8-well ECIS arrays and their parallelization, 16-well arrays ${ }^{[107,541-543]}$ (RT-CES ${ }^{\circledR}$ system from ACEA Biosciences; San Diego, CA, USA), 96-well plates ${ }^{[12,544,545]}$, and

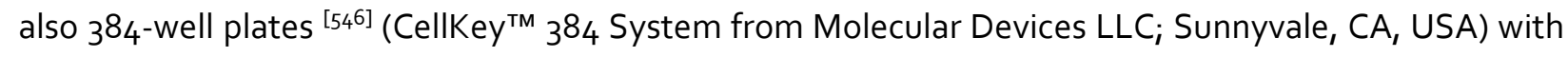
integrated electrodes for impedance readings are already in use. $\mathrm{OCM}$ arrays on monolithic quartz plates were presented, too ${ }^{[351-358,360]}$, even though a reduction in the $\mathrm{OCM}$ spot size is limited. However, new data analyzing systems and chemometric aproaches will be necessary in order to automatically analyze the huge amount of data obtained from high-throughput high-content screenings. The integration of microfluidic systems on the sensor chips by nanotechnological processes is another challenge, which however offers great possibilities. By this, cell-based systems could be established on the sensor, which mimic the real situation in tissue much better and, for example, enable the study of cells under a continuous medium flow. Instead of parallelization, an advancement of the sensors for even more comprehensive high-content screening of cells and the cellular environment are as well thinkable. In addition to the above mentioned label-free sensor technologies, basically each nondestructive surface analysis technique, such as for example also AFM and ellipsometry, can be taken into account. A promising approach is also the integration of fluorescence-optical sensor spots (optodes) onto the quartz substrate. This is already addressed in the outlook of Chapter 4 (4.3.3, p. 109 f.) and is the basis for the projects of substrate-embedded temperature and oxygen sensing presented in Chapter 6 . Further technical advancements would be the establishment of transparent metal electrodes like indium tin oxide (ITO) or the use of conductive polymers like Poly(3,4-ethylenedioxythiophene) poly(styrenesulfonate) (PEDOT:PSS) for the contacting of the quartz faces. Transparent quartz plates would have the big advantage that the sensed fraction of cells then is accessible for additional optical investigation 
methods from both sides of the quartz. However, this would also require the construction of new measurement chambers that enable the positioning of an microscope objective with a satisfactory working distance, preferably on the side facing away from the culture medium and the cells. The microscopic analysis of the cells serves by default as an optical reference method for supporting or supplementing the electrochemical or acoustic sensor data. An unobstructed view on the cell layer thus would make a troublesome and difficult removing of the quartz from the measurement chamber unnecessary. Moreover, the area above the quartz would be free for light sources, e.g. for phase-contrast microscopy or for pulsed LEDs as the excitation light for the fluorescence lifetime imaging of quartzembedded optodes. Equipped with a small incubator around the measurement chamber, this setup would enable the non-invasive, simultaneous monitoring of a cell layer and its environment by acoustic, electrochemical, and optical sensors in addition with phase-contrast and fluorescence microscopy.

Aside from cell-based applications, the ECIS-OCM sensors can also serve as a novel, substrate-integrated platform for the multi-parametric analysis and characterization of any other (bio)material on their surface. This includes, amongst others, studies of coated polymer layers, of lipid bilayer formation and alteration, of polyelectrolyte multilayer build-up and functionalization, and of (bio)molecular binding processes on particularly coated or pretreated sensor surfaces, or to pre-adsorbed receptor molecules or target structures. ${ }^{[373-377]}$ The latter includes applications in the fields of biofilm formation, protein-protein interaction, protein-DNA interaction, protein-lipid interaction, immunological binding, and any kind of molecular recognition. The sensor can provide a multitude of information on the binding characteristics, such as quality, quantity, adsorption kinetics, desorption kinetics, and binding constants of interactions. Furthermore, information on the mass, thickness, elastic modulus, and viscosity of adsorbed soft films are accessible by applying simulations with appropriate models. Additionally, it delivers complementary information on alterations in the dielectrical properties of the surface load. The two coplanar electrodes on the sensor surface can also be used for e.g. in situ electropolymerization or for any other application of the electrochemical quartz crystal microbalance (EOCM). For instance, metal films have been electrodeposited on classical 1-Electrode quartz disks and the adhesion of proteins to that have been subsequently analyzed by OCM recordings. ${ }^{[210,378,547]}$. Recently, also the adhesion and spreading properties of adherent cells on electropolymerized films have been investigated by means of OCM measurements. ${ }^{[232,548]}$ For the described electrosyntheses of polymer films on quartz disks, and for the subsequent protein adsorption/ cell attachment studies, however, the instrumentations had to be changed. Thinking of the substrate-embedded, multi-electrode layout of the $2 \mathrm{EIO}$ disks presented in this thesis, there would be no need for external electrodes and the setups could be combined, if any electrode that is required for electrochemical deposition and microbalance measurements is already integrated in the quartz surface. 


\section{Chapter 6}

\section{OCM-OCS: Optochemical Sensing of}

Temperature and $p \mathrm{O}_{2}$ in the Cell

\section{Surface Junction}

6.1 Imaging the Frictional Heat Induced by High-Amplitude Quartz Oscillations

6.2 Imaging the Oxygen Consumption Rate (OCR) beneath Adherent Cells

6.3 Discussion 
Optical chemical sensors/ sensing (OCS) have/ has been successfully used in the past two to three decades for the online analysis of various chemical and physical parameters. This is reviewed in ${ }^{[169-173]}$. One can classify OCS devices, also referred to as optodes or optrodes as the optical analogue to the electrodes, into direct (spectroscopic) and indirect, indicator-mediated sensors. Direct optodes utilize the intrinsic optical properties of an analyte for its detection, such as by IR/ FTIR/ NDIR/ UV-vis absorption spectroscopy, luminescence (autofluorescence, chemo-/ bioluminescence) measurements, and Raman/ SERS analysis. ${ }^{[169]}$ They could be realized in planar as well as in fiber optical readout configurations. In indirect OCS approaches, one makes use of indicator molecules (probes). These are immobilized in a polymer matrix or hydrogel and report indirectly on the presence and usually also on the concentration of the target analyte, by the modulation of their absorbance and/ or luminescence properties. A variety of configurations are thinkable by the coating of surfaces with such analyte-sensitive paints. The sensor "cocktail", consisting of an indicator dye and a polymer or hydrogel dissolved in a solvent, can be immobilized either at the end of an optical waveguide for fiber optical sensor platforms or on a substrate surface as a planar, large area sensor film or as sensor array. Aside from spot- and layer-based solid-state sensor platforms, optical sensors are also realized simply as soluble probes or as dyes encapsulated in soluble and inert nanoparticles, sometimes also referred to as PEBBLEs (probes encapsulated by biologically localized embedding), which especially find application in live cell and in vivo imaging of analytes ${ }^{[549-553]}$.

Due to its high sensitivity, fluorescence-based OCS is the most widely used method. Luminescent probes are reported for various analytes, such as $\mathrm{pH}^{[173,181,190,554-564]}, \mathrm{CO}_{2}{ }^{[565-574]}$ and ammonia ${ }^{[575-579]}$ (both via $\mathrm{pH} / \mathrm{H}^{+}$indicators), $\mathrm{O}_{2}{ }^{[175,178,180,182,323,580-583]}$ (reviewed in detail in $\left.{ }^{[175,323,584]}\right), \mathrm{Na}^{+[585-587]}, \mathrm{K}^{+[585,588-591]}, \mathrm{Ca}^{2+}$ ${ }^{[592-598]}$, halides ${ }^{[599-602]}$, (transition) metal ions ${ }^{[593,603-611]}$, peroxide ${ }^{[612,613]}$, glucose ${ }^{\left[614^{6617]}\right.}$ (via oxygen indicators; oxygen is consumed during glucose oxidation catalyzed by immobilized GOx), and a variety of other analytes ${ }^{[170,171]}$. There are also a large variety of luminescent probes and sensors for temperature $\left(T\right.$; reviewed in detail in $\left.{ }^{[618]}\right)$, since the lifetime of almost any luminescent dye depends on the temperature. Thus, often multi-sensors are used with one indicator for the analyte of interest and with a second indicator for the temperature ${ }^{[176-182]}$, which allows to compensate for temperature-related luminescence quenching effects $[176,177,319,320,325,326]$. Besides the conventional intensity-based measurements, other luminescence parameters such as decay time/ lifetime, anisotropy, quenching efficiency, FRET, ratiometric intensity, or dual-lifetime referencing (DLR) data are used for sensing, too. ${ }^{[170]}$

Fluorescence lifetime-based sensing and imaging approaches gained increasing importance in the past five to ten years, since they are much less susceptible to interferences and promise a better temporal and lateral resolution of the analyte sensed. The combination of long-living phosphorescent probes, like porphyrine-based luminescent transition metal or lanthanide complexes, in analyte-sensitive paints with lifetime-based imaging (FLIM) provides a powerful tool for the time-resolved 2-D mapping of an analyte. The following sections demonstrate the application of a temperature-sensitive paint (TSP) for 6. I Imaging the Frictional Heat Induced by High-Amplitude Quartz Oscillations and of a pressure-sensitive paint (PSP), which in fact is sensitive for the oxygen partial pressure, for 6.2 Imaging the Oxygen Consumption Rate (OCR) beneath Adherent Cells. 


\subsection{Imaging the Frictional Heat Induced by High-Amplitude Quartz Oscillations}

The quartz crystal microbalance is commonly known as a sensor for the sensitive quantification of deposited rigid masses (microweighing), as viscosity sensor for liquids, and as viscoelasticity sensor for not rigid loadings with both elastic and viscous properties, such as vesicles, bacteria and mammalian cells. $^{[22,197,208,211,212]}$ However, besides this wide field of micromechanical sensor applications, recently the QCM has also been applied as an actuator. A new invasive method of measuring bond rupture forces has been developed on the basis of QCM oscillations, which is referred to as rupture event scanning, or short REVS $^{\text {TM }}{ }^{[619-622]}$ Invasiveness of the OCM oscillations is generated by increasing the amplitude of the applied driving voltage. This directly correlates with an increase in the oscillation amplitude of the quartz surfaces, and the oscillation energy is also transferred to any mechanically coupled molecule or particle. "Hearing" bond breakage by OCM actuating and (bio)sensing has been applied for physisorbed microand nanospheres ${ }^{[619,623]}$, streptavidin-biotin bound latex/ polystyrene microspheres ${ }^{[619,624-626]}$, chemically bound (amide bond) latex spheres ${ }^{[619]}$, adsorbed bacteriophages ${ }^{[627]}$, complementary DNA sequences ${ }^{[628]}$, viruses/ bacteria bound to surface-attached antibodies ${ }^{[620,629-632]}$, and for established cell layers as well ${ }^{[226]}$.

High-amplitude OCM has not only been used to analyze the rupture event of established bonds but also to investigate the suppressed adsorption and binding process of particles onto the oscillating quartz surface, like for example supported lipid bilayer formation ${ }^{[633]}$, binding of streptavidin to biotin-modified supported lipid bilayers ${ }^{\left[{ }^{633}\right]}$, binding of NeutrAvidin-modified polystyrene spheres to planar biotinmodified supported lipid bilayers on $\mathrm{SiO}_{2}{ }^{[633,634]}$, DNA hybridization ${ }^{[634]}$, and cell adhesion ${ }^{[226]}$. An analytical model was presented by Zhdanov et al. ${ }^{[635]}$, describing what may happen in such experiments of binding suppression via external perturbation.

Dultsev et al. ${ }^{[619,636]}$ reported on a generated heat on the $\mathrm{OCM}$ surface due to elevated shear oscillation amplitudes of $1{ }^{\circ} \mathrm{C}$ at $1 \mathrm{~V}, 2-5^{\circ} \mathrm{C}$ at $5 \mathrm{~V}$, and of $30^{\circ} \mathrm{C}$ at $10 \mathrm{~V}$ in air, and of $7^{\circ} \mathrm{C}$ at $10 \mathrm{~V}$ in water. However, these temperatures are considered to be too small to cause the observed breakage of chemical bonds. They also argue that the temperature effect on the resonance frequency of AT-cut QCM is unimportant, as it is only weakly dependent on temperature (cf. S/ 1). Such a small shift in the frequency would lead to an insignificantly increased voltage at which particles get detached from the surface. Edvardsson et al. ${ }^{[633]}$ studying the kinetics of supported lipid bilayer formation observed similar effects at $10 \mathrm{~V}$ OCM driving voltages as at an increased ambient temperature by $\sim 10^{\circ} \mathrm{C}$. Since they calculated a theoretical heating of only $2^{\circ} \mathrm{C}$ from changes of the oscillation energy dissipation in liquid, the detected effects at $10 \mathrm{~V}$ are not assigned to a temperature rise. Heitmann et al. ${ }^{[226]}$ indirectly measured the generated surface heat via the temperature-dependent viscosity changes of glycerol on the quartz crystal, a physical parameter to which the OCM is sensitive. Based on the discovered correlation between load viscosity/ oscillation resistance and temperature, they determined a temperature increase of less than $1^{\circ} \mathrm{C}$, when operating the $\mathrm{OCM}$ at $10 \mathrm{~V}$ with glycerol as surface load. Cooper et al. ${ }^{\left[{ }^{31]}\right.}$ determined with a thermal imager a similar, not significant temperature rise $\left(<0.7^{\circ} \mathrm{C}\right)$ for $\mathrm{OCM}$ oscillations in air, when increasing the driving voltage from $0 \mathrm{~V}$ to $10 \mathrm{~V}$.

If at all, the possibly generated frictional heat of high-amplitude QCM and the related effects are usually mentioned only as a side note in literature and are considered as not important for the reported applications. Only the direct effect of heat on the bond rupture of attached particles or on the frequency 
stability of the OCM device are discussed. However, the temperature effect is considered as crucial point in many assay formats, which is confirmed by the results presented below. Especially, the effect of local heat on the convection of liquid in the measurement vessel is not at all taken into account in the literature of this research field. Instead of only estimating the temperature increase due to high-amplitude oscillations by calculations or indirect measurements, this problem is faced in this thesis by means of temperature-sensitive paints and FLIM of the temperature directly on the quartz surface. The simulation of convection profiles on the basis of this data indicate significant effects of operating the OCM at elevated driving voltages.

\subsubsection{Quartz Coating and Characterization}

The sensor cocktail, containing the temperature-sensitive indicator dye $\mathrm{Eu}(\mathrm{dnm})_{3}(\text { topo })_{2}$ (Fig. 3-23 A) and the polymer support, was immobilized on commercial $5 \mathrm{MHz}$ quartz disks (KVG Quartz Crystal

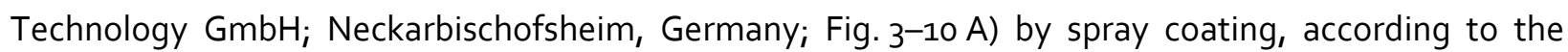
protocol in the experimental section (cf. 3.5.3, p. 67). The oscillation characteristics of the quartz disks before and after TSP-coating were verified by means of impedance analysis in the resonance region. This served as quality control of the oscillation properties on the one hand, and for the determination of the resonance frequency of the coated resonator on the other hand. In order to attain a maximal shear displacement of the quartz material for high-amplitude OCM applications, the microbalance has to be operated at its resonance frequency. This varies between different quartz disks, since there are differences in the electrode thickness and predominantly in the thickness of the coated TSP layers.
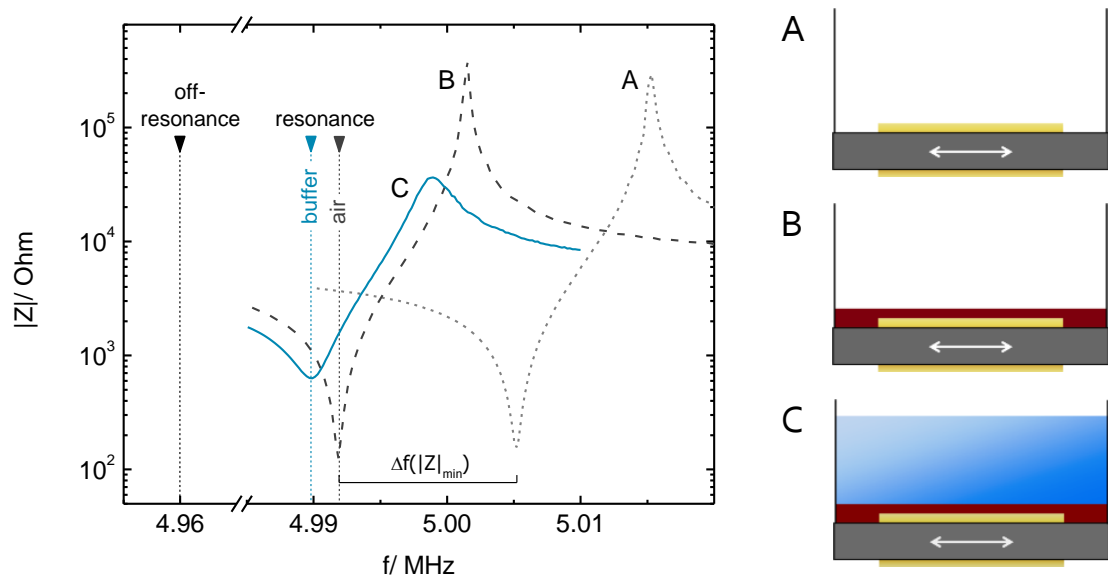

B

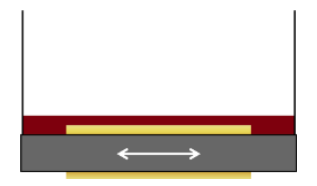

C

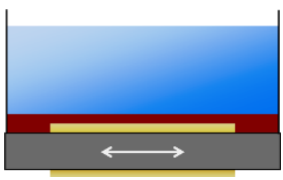

Fig. 6-1 Impedance spectra near resonance of $5 \mathrm{MHz}$ AT-cut quartz crystals: (A) blank quartz disk in air, (B) TSP-coated quartz disk in air, and (C) TSP-coated quartz disk covered with $550 \mu \mathrm{L}$ PBS $^{--}$buffer. The frequencies of resonance and off-resonance are indicated.

A set of exemplary impedance spectra is shown in Fig. 6-1. The resonance curve of the resonator is shifted to lower frequencies by the coating with a TSP layer (A $\rightarrow B$ in Fig. 6-1). However, no increase in the damping of the oscillation (increased $|Z|_{\min }$ value) due to the loading is observable. Hence, the coating layer contributes only to the storage of oscillation energy, but not to its dissipation. The thickness of the rigid sensor film could be estimated from the resonance shift $\Delta f_{|Z|_{\text {min' }}{ }^{\prime}}$ according to the Sauerbrey relation (Eq. (23)). For instance, the spectra of the blank and coated microbalance in Fig. 6-1 exhibit a resonance difference of $13300 \mathrm{~Hz}$. This is related with a film thickness of approximately $2.5 \mu \mathrm{m}$, using an 
integral mass sensitivity of $34 \mathrm{~Hz} \mathrm{~cm}^{2} \mu \mathrm{g}^{-1} .{ }^{[637]}$ The thicknesses of the used TSP films were all in the singledigit micrometer range. Loading of the TSP-coated resonators with buffer solution goes along with a further slight decrease in the resonance frequency ( $B \rightarrow C$ in Fig. $6-1$ ). The damping contribution by the viscosity and density of the liquid loading becomes apparent, too, in the increase of the minimal impedance value (cf. Impact of Different Loadings on the Resonator Oscillation in subsection 3.3.2). As mentioned previously, imaging of the temperature on the crystal surface was performed when the microbalance oscillated with high amplitudes at its resonance frequency. Reference measurements were

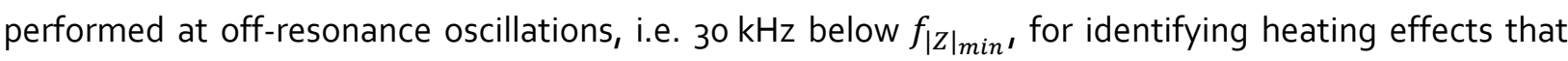
potentially do not arise from the frictional heat of resonant shear oscillations.

\subsubsection{Sensor Calibration}

Since the TSP sensor films on the quartz crystal surfaces could be reused several times, the phosphorescence lifetime properties of the sensors were always re-calibrated, before each temperatureimaging of high-amplitude quartz oscillations. The sensor responses were measured by means of FLIM in RLD method, (see subsection 3.5.2; p.65 f.). For detailed information, regarding the experimental setup and the custom-made, miniaturized OCM chamber, which has been used for the measurements, is referred to Fig. 3-24 in subsection 3.5.3 Experimental of FLIM-RLD > Hardware - Experimental Setup (p. 67) and to the photographs in S/ 6. The spectral characteristics of the TSP absorbance and emission, of the light source, and of the excitation and emission filters are illustrated in SI 26.

A

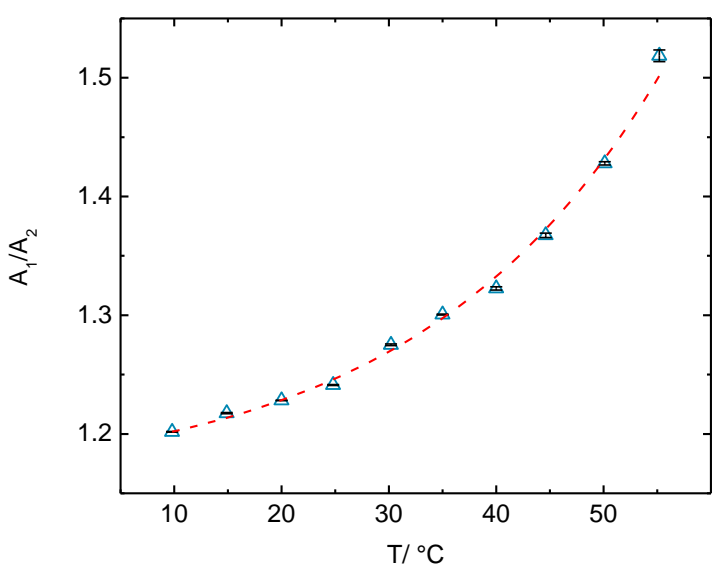

B

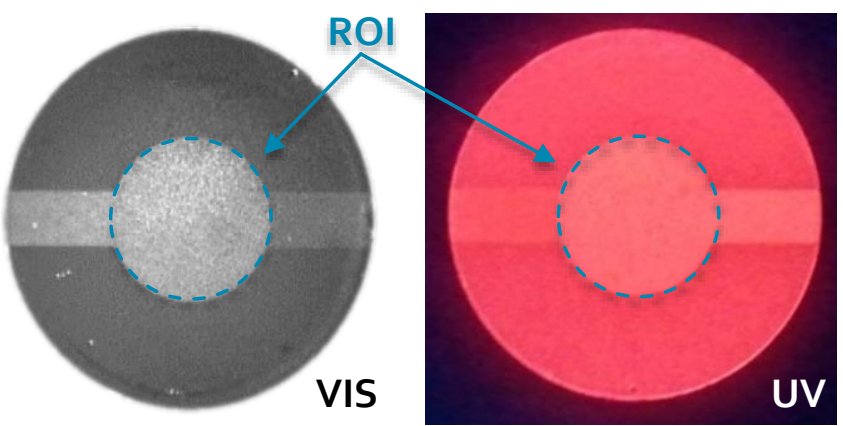

C

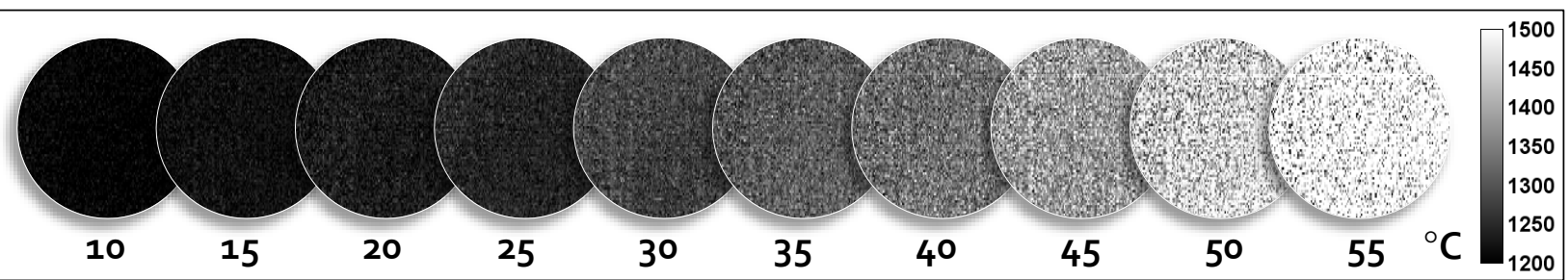

Fig. 6-2 (A) Calibration plot of a TSP-coated quartz disk in air. $A_{1} / A_{2}$ is the averaged pixel value of $A_{1} / A_{2}$-images in the $\mathrm{ROI}$ (mean $\left.\pm \mathrm{SEM} ; \mathrm{N}=7\right)$. The best fitting function $\left(A_{1} / A_{2}=\right.$ $\left.0.031( \pm 0.006) \cdot \exp \left(T / 22.8( \pm 1.8){ }^{\circ} \mathrm{C}\right)+1.154( \pm 0.008) ; R^{2}=0.9941\right)$ is indicated (dashed red line). (B) The ROI was defined as the area of the gold electrode on the OCM disk (dashed blue circles). Pictures show a TSP-coated quartz plate illuminated with visible (VIS) and UV light (red emission of the phosphorescent dye). (C) Grayscale $A_{1} / A_{2}$-images of the ROI at the various calibration temperatures. The calibration bar depicts values of $A_{1} / A_{2} \cdot 10^{3}$. 
Fig. 6-2 A shows a typical calibration plot of a TSP-coated quartz disk in air. $A_{1} / A_{2}$ is the averaged ratio of two intensity images taken successively of a defined region of interest (ROI) on the surface in the decay phase of the indicator phosphorescence (cf. Fig. 3-22; mean $\pm S E M ; N=7$ ). The ratio is related to the decay time according to Eq. (55). The area of the gold electrode is defned as the ROI, since in first approximation the quartz material oscillates only within the borders of the electrode. This is indicated by dashed circles in Fig. 6-2 B, which shows the pictures of a TSP-coated quartz disk taken in visible (VIS) and UV light. The data points of the calibration in Fig. 6-2 A could be fitted best with an exponential function of the form $y=a \cdot e^{x / b}+y_{0}$ (indicated by a dashed red line; parameter values see caption). Fig. 6-2 Cillustrates grayscale $A_{1} / A_{2}$-images of the ROI at the various calibration temperatures between $10^{\circ} \mathrm{C}$ and $55^{\circ} \mathrm{C}$ (the calibration bar depicts values of $A_{1} / A_{2} \cdot 10^{3}$ ).

\subsubsection{Imaging the Temperature of High-Amplitude OCM in Air}

The temperature imaging measurements of the quartz surface heating due to high-amplitude oscillations were performed directly in the calibration chamber, in order to ensure constant experimental conditions. The measurement temperature was set to $20^{\circ} \mathrm{C}$ by default and has been continuously monitored with a temperature sensor inside the chamber (cf. S/6). The surface electrodes of the quartz disk were connected via copper wires outside the calibration chamber with an oscilloscope (cf. Fig. 3-24, p. 68) for confirming of the resonance frequency. Afterwards, the contacts were switched to an arbitrary waveform generator. Different amplitudes of a sinusoidal AC voltage at the frequency of resonance were used for the driving of quartz oscillations. Besides $0.15 \mathrm{~V}$ as the default amplitude for OCM sensing applications, following elevated amplitudes have been applied: $0.5,1,2,3,5,7.5$, and $10 \mathrm{~V}$.

We calculated the lateral shear oscillation amplitudes $x_{r}$ of the quartz material in air, for the various driving voltages $U$ and as a function of the distance $r$ from the center of the microblanace, according to:

$$
x_{r}=1.4( \pm 0.1) \cdot 10^{-12} \mathrm{~m} / \mathrm{V} \cdot Q \cdot U_{0} \cdot e^{\left(-w r^{2} / r_{e l}{ }^{2}\right)}
$$

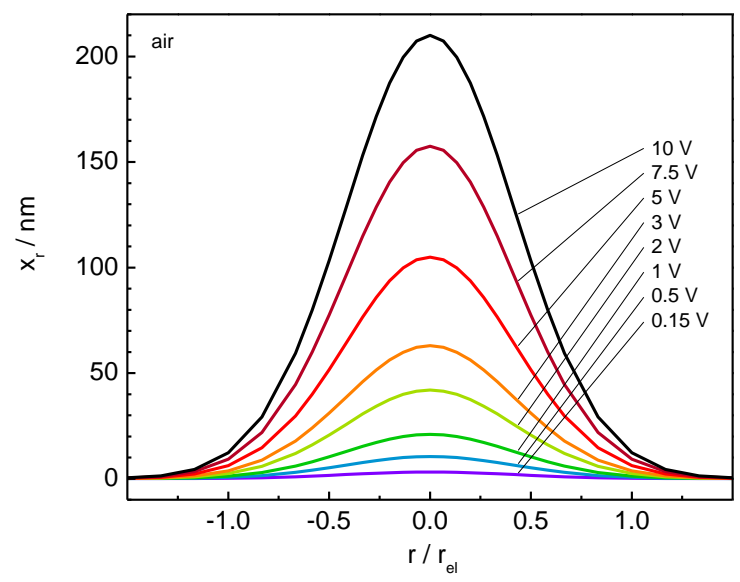

Fig. 6-3 Lateral distribution of the amplitudes of QCM shear displacement $x_{r}$ for quartz oscillations in air at the driving voltages $0.15,0.5,1,2,3,5,7.5$, and $10 \mathrm{~V}$, calculated according to Eq. (68). $x_{r}$ values are plotted as a function of the distance $r$ from the OCM disk (electrode) center, normalized to the electrode radius $r_{e l}=3 \mathrm{~mm}$. The maximal oscillation amplitudes $x_{0}$ in the center of the resonator $\left(r / r_{e l}=0\right)$ are listed in Tab. 6-1. $Q=15000, w^{\text {air }}=2.84^{[365]}$. 
This is a combination of Eq. (59), providing the maximal lateral shear amplitude $x_{0}$ in the center of the OCM electrode as a function of the $Q$-factor of the oscillation and the driving voltage $U_{0}$ (empirically determined by Borovsky et al. ${ }^{[366]}$ ), and of Eq. (62), which describes the Gaussian decay of the maximal amplitude by increasing the distance $r$ from the center of the electrode with radius $r_{e l}$ (based on a theoretical model by Martin et al. ${ }^{\left[{ }^{65}\right]}$ ). For this calculations it was used a $Q$-factor of 15000 , based on experimentally determined values of OCM oscillations in air, the electrode radius $r_{e l}$ of the $5 \mathrm{MHz}$ AT-cut quartz resonators used $\left(r_{e l}=3 \mathrm{~mm}\right)$, and a Gaussian distribution coefficient of $w^{\text {air }}=2.84^{[365]}$. The lateral oscillation amplitude distributions obtained of the various OCM operation voltages are illustrated in Fig. 6-3 as a function of the distance $r$ from the microbalance center, normalized to the electrode radius $r_{e l}$. The calculated values of the maximal shear amplitude $x_{0}$ at the resonators' center lie between $3.15 \mathrm{~nm}$ for $0.15 \mathrm{~V}$ and $210 \mathrm{~nm}$ for $10 \mathrm{~V}$ driving voltage; all values are listed in Tab. 6-1.

The quartz disk was forced to a continuous oscillation at the various amplitudes for 15 to 20 min, which turned out to be sufficiently long for the equilibration of the generated heat on the quartz surface. After this period of oscillation at each driving voltage, the phosphorescence lifetime property of the TSP was measured during the oscillation by FLIM (in RLD mode). The average brightness values of $A_{1} / A_{2}$ images within the ROI were then converted into mean temperature values, based on the previous calibration and the respective best fitting function. These are finally referenced to the starting temperature, measured of the non-oscillating quartz disk at o $\mathrm{V}$ driving voltage. Thus, values of temperature increases on the quartz crystal surface that are caused by high-amplitude shear oscillations are obtained.

The temperature course of a typical experiment is shown in Fig. $6-4 \mathrm{~A}$, where amplitudes of $0,0.15,0.5$, $1,2,3,5,7.5$, and $10 \mathrm{~V}$ were successively applied. The corresponding underlying $A_{1} / A_{2}$-images of the ROI (electrode area) are illustrated in grayscale in Fig. $6-4$ C. From these data, a gradual increase of frictional heat on the oscillating quartz surface by increasing the amplitude of shear displacement is evident. A significant increase of temperature can be noticed from roughly $2 \mathrm{~V}$ of the applied voltage. The absolute temperature increases to about $35^{\circ} \mathrm{C}$ on the faces of the quartz disk, or more precisely in the TSP layer of $<2.5 \mu \mathrm{m}$ thickness on top of the crystal, at $10 \mathrm{~V} \mathrm{OCM}$ driving voltage and $20^{\circ} \mathrm{C}$ ambient temperature. After turning off the driving voltage, the temperature on the quartz surface slowly approximates values of the calibration chamber $\left(20^{\circ} \mathrm{C}\right)$ again. Reducing the frequency of the $\mathrm{AC}$ driving voltage by $30 \mathrm{kHz}$ with respect to the resonance frequency of the resonator (off-resonance; cf. Fig. 6-1) and applying an amplitude of $10 \mathrm{~V}$ again, however, does not cause a significant change in the quartz surface temperature at all (red shaded area in Fig. 6-4 A). It remains at the same value, i.e. at the ambient temperature inside the calibration chamber, independently of whether a voltage amplitude of $10 \mathrm{~V}$ or $\circ \mathrm{V}$ is applied. This is evident from the underlying $A_{1} / A_{2}$-images as well (Fig. 6-4 $C_{i}$ off-resonance images are indicated with *). The distribution of brightness values (coloring) across the entire ROI and the average brightness value of pixels within the ROI are similar for the operation of the $\mathrm{QCM}$ at $10 \mathrm{~V}$ off resonance and at $\mathrm{OV}$. By turning the frequency back again to resonance conditions and applying a $10 \mathrm{~V}$ OCM driving amplitude, the temperature on the quartz surface again rises reproducibly to similarly high values as in the first run at $10 \mathrm{~V}$ (Fig. 6-4 A). This can be clearly seen also in Fig. 6-4 C, where both $A_{1} / A_{2}$-images obtained at $10 \mathrm{~V}$ at resonance show a similar coloring, i.e. similarly high $A_{1} / A_{2}$ pixel values.

Fig. 6-4 B shows the sensor response, converted into a temperature change, in a high temporal resolution, when the driving voltage is switched between $\mathrm{V}$ and $10 \mathrm{~V}$ at the resonance frequency. There is a steep increase in temperature to $90 \%$ of final values within $5 \mathrm{~min}$ after switching on of the voltage. Stationary values are reached at least after $20 \mathrm{~min}$ of oscillation. There is no further increase in the 
temperature anymore during further $40 \mathrm{~min}$ of quartz vibrations at $10 \mathrm{~V}$. Turning off of OCM oscillations by reducing the amplitude of the driving voltage to o $\mathrm{V}$ causes a likewise steep decrease in the surface temperature. About $90 \%$ of the total decrease are reached within $6 \mathrm{~min}$ and the equilibrium temperature is reached after roughly $30 \mathrm{~min}$.

A

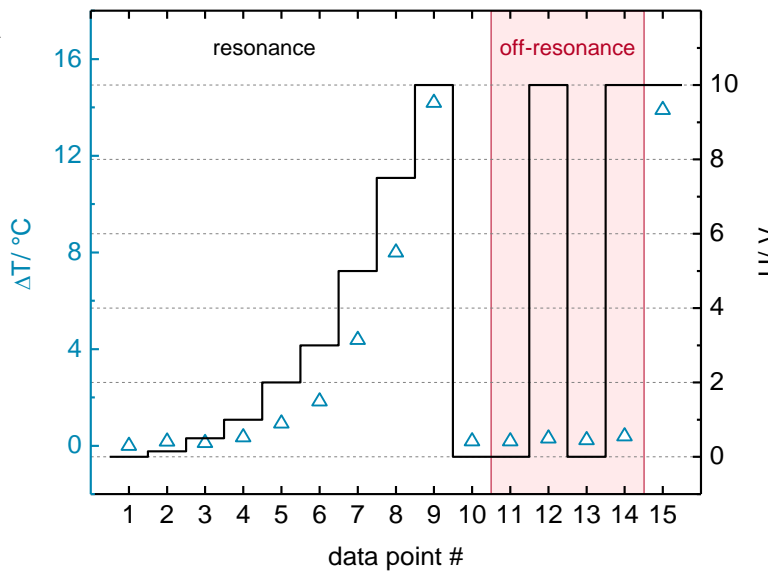

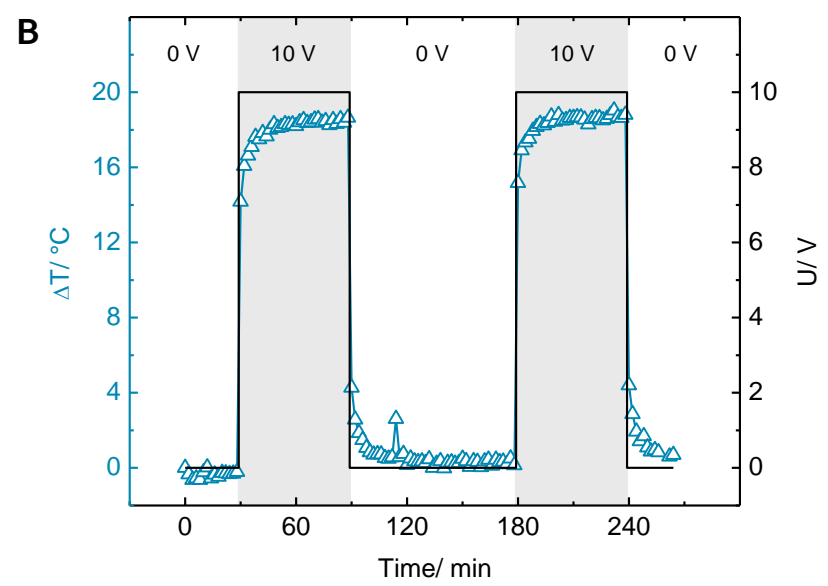

C

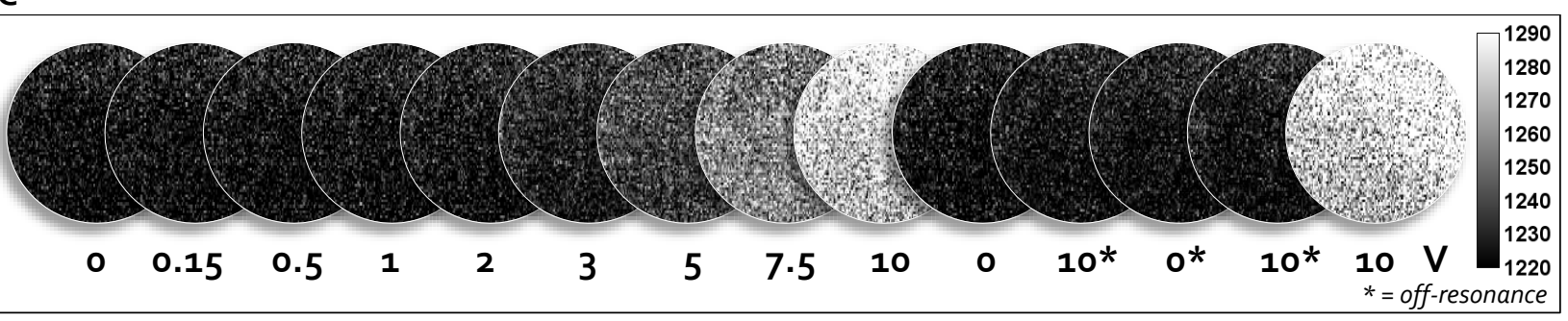

Fig. 6-4 (A) Data of a typical experiment of high-amplitude OCM with FLIM of the temperature on the quartz crystal surface by means of a TSP coating. The temperature change $\Delta T$ (blue triangles) is shown on the primary axis and the corresponding driving voltage $U$ (solid line) is scaled on the secondary axis. Absolute temperature values are obtained from transforming averaged pixel values of $A_{1} / A_{2}$-images into mean temperature values by the fitting function of a previous calibration of the sensor (cf. 6.1.2). These values are referenced to the temperature at $\circ \mathrm{V}$ driving voltage. The quartz was operated at its resonance frequency except for data points that are marked with "off-resonance" (red shaded area). Here, a frequency of $30 \mathrm{kHz}$ below the resonance was used. (B) TSP sensor response with high temporal resolution, converted into temperature changes, upon switching the OCM driving voltage at resonance between $\mathrm{o}$ and $10 \mathrm{~V}$. (C) Grayscale $A_{1} / A_{2}$-images of the ROI of the quartz crystal surface on which the data in the graph in $\mathrm{A}$ are based. Numbers below the images are the voltage amplitudes of $\mathrm{OCM}$ oscillation during imaging. Images that were taken at off-resonance $(-30 \mathrm{kHz}) \mathrm{OCM}$ driving voltages are indicated with *. The calibration bar depicts values of $A_{1} / A_{2} \cdot 10^{3}$.

Fig. 6-5 shows the average temperature change within the ROI of the quartz surface of repeated measurements, as a function of the applied driving voltage at the resonance frequency and with respect to the temperature at o $\mathrm{V} O C M$ driving voltage (mean $\pm S E M ; N \geq 11$ ). The mean temperature change due to oscillations at $10 \mathrm{~V}$ off-resonance $(-30 \mathrm{kHz})$ are illustrated as well (indicated with $* ;=6$ ). The corresponding values are listed in Tab. 6-1, together with the calculated values of the maximal shear oscillation amplitude at the respective driving voltages. While the frictional heat generated in the TSP layer is by $0.00( \pm 0.09)^{\circ} \mathrm{C}$ negligible at $0.15 \mathrm{~V}$ driving voltage and $3.15 \mathrm{~nm}$ amplitude of maximal lateral displacement, respectively, a small but already significant temperature increase by $0.9( \pm 0.2)^{\circ} \mathrm{C}$ can be detected at $1 \mathrm{~V}$ driving voltage and $21 \mathrm{~nm}$ shear oscillation amplitude in the center of the ROI, 
respectively (Tab. $6-1$ ). From $3 \mathrm{~V}$ to $10 \mathrm{~V}$ the temperature increases more than linear. The maximal operation voltage of $10 \mathrm{~V}$ and the corresponding shear oscillation amplitudes of maximal $210 \mathrm{~nm}$ in the center of the resonator cause a mean temperature increase of $17.0( \pm 0.9)^{\circ} \mathrm{C}$ on the quartz crystal surface. When operating the $\mathrm{OCM}$ at $10 \mathrm{~V}$ off-resonance, however, a not significant mean temperature change of $0.5( \pm 0.3)^{\circ} \mathrm{C}$ is measured (indicated with * in Fig. 6-5). The non-linear relationship between the applied driving voltage and the generated temperature on the QCM surface could be described best by a parabolic fitting function of the form $y=a x^{2}+b x+c$ (indicated by a dashed red line; parameter values see caption).

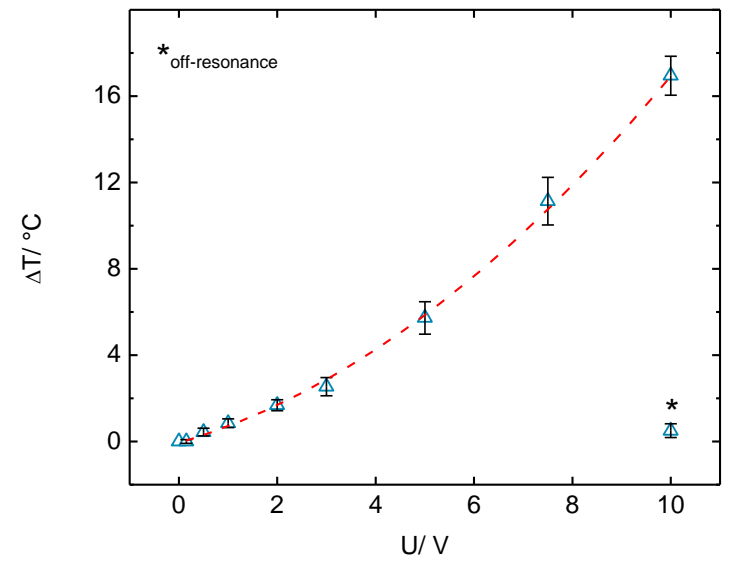

Fig. 6-5 Average temperature increase $\Delta T$ on the quartz crystal surface (in air) as a function of the applied $\mathrm{OCM}$ driving voltage $U$ at resonance frequencies (mean $\pm \mathrm{SEM} ; \mathrm{N} \geq 11$ ). The mean temperature rise of high-amplitude $\mathrm{OCM}(10 \mathrm{~V})$ at off-resonance frequency $(-30 \mathrm{kHz})$ is indicated with * $(\mathrm{N}=6)$. The underlying values are listed in Tab. 6-1. In consideration of standard errors (direct weighting), the correlation of $\Delta T$ and $U$ could be fitted best by a parabolic function of the form $\Delta T=0.103( \pm 0.011){ }^{\circ} \mathrm{C}^{2} / \mathrm{V}^{2} \cdot U^{2}+0.67( \pm 0.09){ }^{\circ} \mathrm{C} / \mathrm{V} \cdot U-0.06( \pm 0.06){ }^{\circ} \mathrm{C} \quad\left(R^{2}=0.9947\right)$, shown as dashed red line.

Tab. 6-1 Values of the maximal lateral shear oscillation amplitude $x_{0}$, calculated according to Eq. (68) $(Q=15000)$, and of the average temperature increase $\Delta T$ on the quartz disk surface in air upon high-amplitude QCM at various amplitudes $U$ and frequencies $f$ of the driving voltage (mean $\pm \mathrm{SEM})$.

\begin{tabular}{rlrrr}
\hline$U / V$ & $f$ & $x_{0} / \mathrm{nm}$ & $\Delta T /{ }^{\circ} \mathrm{C}$ & $\mathrm{N}$ \\
\hline 0.15 & resonance & 3.15 & $0.00( \pm 0.09)$ & 12 \\
0.5 & resonance & 10.5 & $0.44( \pm 0.18)$ & 11 \\
1 & resonance & 21 & $0.9( \pm 0.2)$ & 12 \\
2 & resonance & 42 & $1.7( \pm 0.3)$ & 11 \\
3 & resonance & 63 & $2.5( \pm 0.4)$ & 12 \\
5 & resonance & 105 & $5.7( \pm 0.8)$ & 12 \\
7.5 & resonance & 157.5 & $11.1 \quad( \pm 1.1)$ & 12 \\
10 & resonance & 210 & $17.0( \pm 0.9)$ & 14 \\
10 & off-resonance $(-30 \mathrm{kHz})$ & -- & $0.5 \quad( \pm 0.3)$ & 6 \\
\hline
\end{tabular}




\subsubsection{Imaging the Temperature of High-Amplitude QCM in Buffer}

Besides temperature imaging of the quartz surface during high-amplitude oscillations in air (6.1.3), measurements were also performed for high-amplitude QCM oscillations with one quartz face immersed in aqueous solution. For this purpose, the headspace above the quartz disk in the home-made OCM chamber was completely filled with PBS $^{--}$buffer $(550 \mu \mathrm{L})$ and covered with a transparent glass slide without inclusions of air bubbles (Fig. 3-24, p. 68). The procedures of sensor calibration, of resonance frequency testing by an oscilloscope, and of high-amplitude OCM operations with a power supply were the same as described previously for the measurements in air. The TSP sensor film immersed in buffer has been calibrated at fewer temperature values than in air since, firstly, the temperature increase due to high-amplitude oscillations was expected to be considerably lower in the aqueous environment due to the heat dissipation in water and, secondly, the equilibration of calibration temperatures has taken significantly longer (about $30 \mathrm{~min}$ ) under liquid than in air. Various amplitudes of a sinusoidal AC voltage at the frequency of quartz resonance were successively applied. The applied QCM driving voltages $U$ in high-amplitude measurements were $0.15,1,3,5,7.5$, and $10 \mathrm{~V}$.

It was again calculated the distribution of lateral shear oscillation amplitudes $x_{r}$ in dependence of the distance $r$ from the microbalance center, normalized to the electrode radius $r_{e l}$, and in dependence of the voltage amplitude, according to Eq. (68). A $Q$-factor value of 2500 was used for calculations, based on experimentally derived values of $5 \mathrm{MHz}$ AT-cut quartz oscillations with one face exposed to buffer. Furthermore, an electrode radius $r_{e l}$ of $3 \mathrm{~mm}$ and a Gaussian distribution coefficient of $w^{\text {water }}=2.03$, according to ${ }^{[365]}$, were inserted. In Fig. 6-6, the results of calculations are illustrated. The maximal shear oscillation amplitudes $x_{0}$ in the center of the resonator $(r / R=0)$ are listed in Tab. 6-2 for the applied driving voltages. The $x_{0}$ values are in the range between 0.5 and $35 \mathrm{~nm}$ between the lowest ( $150 \mathrm{mV}$ ) and the highest $(10 \mathrm{~V})$ voltage amplitude. These are by roughly a factor of six lower compared to the calculated oscillation amplitudes in air (cf. Tab. 6-1). This discrepancy is directly correlated with the 6fold lower $Q$-factor of the quartz oscillation in liquid, which is due to the viscosity of the loading and the accompanied increased dissipation of vibrational energy (cf. Tab. 3-6 (B) Newtonian liquid and the associated text passages, p. 42 ff.).

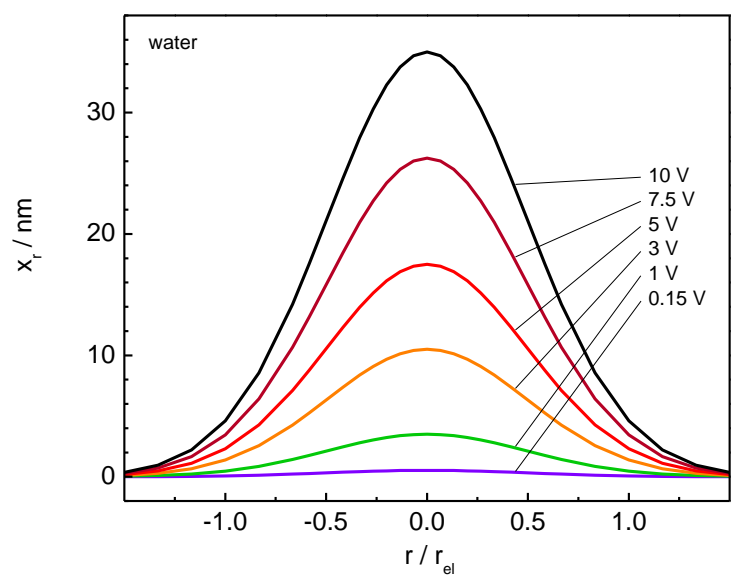

Fig. 6-6 Lateral distribution of the amplitudes of OCM shear displacement $x_{r}$ for quartz oscillations in water at the driving voltages $0.15,1,3,5,7.5$, and $10 \mathrm{~V}$, calculated according to Eq. (68). $x_{r}$ values are plotted as a function of the distance $r$ from the disk (electrode) center, normalized to the electrode radius $r_{e l}=3 \mathrm{~mm}$. The maximal oscillation amplitudes $x_{0}$ in the center of the resonator $\left(r / r_{e l}=0\right)$ are listed in Tab. 6-2. $Q=2500, w^{\text {water }}=2.03^{[365]}$. 
Fig. 6-7 shows the average temperature increase on the quartz crystal surface due to high-amplitude oscillations in PBS-- buffer from a number of separate measurements at an ambient temperature of $20^{\circ} \mathrm{C}$ (mean $\pm S E M ; N \geq 5$ ). There is also an increase of the temperature on the quartz surface in buffer with increasing the driving voltage at the resonance frequency of the resonator, just as previously observed for oscillations in air (Fig. 6-5, Tab. 6-1). The heating effect, however, is considerably lower for quartz oscillations in aqueous solution than in air. The corresponding values are listed in Tab. 6-2. A significant temperature increase of $0.68( \pm 0.05)^{\circ} \mathrm{C}$ is noticeable at a resonant $\mathrm{OCM}$ driving voltage of $5 \mathrm{~V}$ and the maximal increase in temperature is observed by $2.42( \pm 0.14)^{\circ} \mathrm{C}$ at the highest driving voltage $(10 \mathrm{~V})$. By contrast, operating the resonator at $10 \mathrm{~V}$ driving voltage off-resonance $(-30 \mathrm{kHz})$ causes no significant increase in temperature (indicated with * in Fig. 6-7). The temperature change arising from $\mathrm{OCM}$ oscillations in buffer as a function of the applied driving voltage could be fitted best with a parabolic function of the form $y=a x^{2}+b x+c$ (indicated by a dashed red line; parameter values see caption).

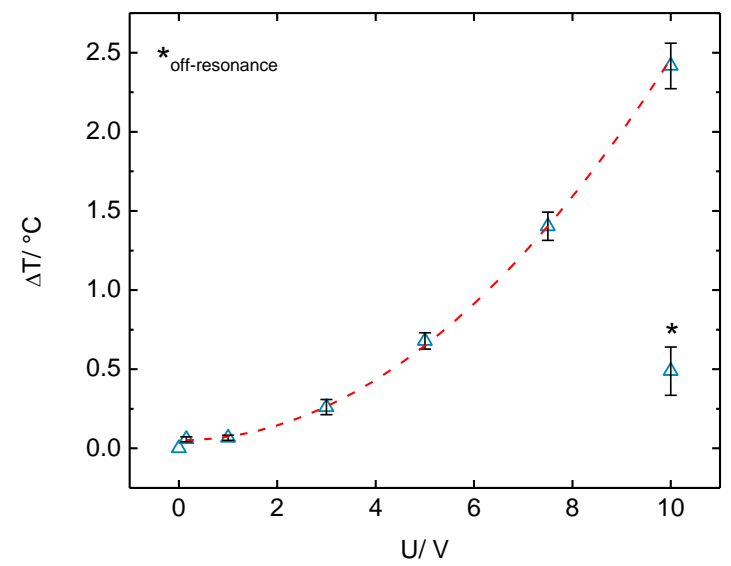

Fig. 6-7 Average temperature increase $\Delta T$ on the quartz crystal surface (in buffer) as a function of the applied QCM driving voltage $U$ at resonance frequencies (mean $\pm S E M ; N \geq 5$ ). The mean temperature rise of high-amplitude $\mathrm{OCM}(10 \mathrm{~V})$ at off-resonance frequency $(-30 \mathrm{kHz})$ is indicated with *. The underlying values are listed in Tab. 6-2. In consideration of standard errors (direct weighting), the correlation of $\Delta T$ and $U$ could be fitted best by a parabolic function of the form $\Delta T=0.0243( \pm 0.0013){ }^{\circ} \mathrm{C}^{2} / \mathrm{V}^{2} \cdot U^{2}-0.002( \pm 0.009){ }^{\circ} \mathrm{C} / \mathrm{V} \cdot U+0.052( \pm 0.008){ }^{\circ} \mathrm{C}$, shown as dashed red line $\left(R^{2}=0.9979\right)$.

Tab. 6-2 Values of the maximal lateral shear oscillation amplitude $x_{0}$, calculated according to Eq. (68) $(Q=2500)$, and of the average temperature increase $\Delta T$ on the quartz disk surface in buffer upon high-amplitude QCM at various amplitudes $U$ and frequencies $f$ of the driving voltage (mean $\pm \mathrm{SEM})$.

\begin{tabular}{rlrcc}
\hline$U / V$ & $f$ & $x_{0} / \mathrm{nm}$ & $\Delta T /{ }^{\circ} \mathrm{C}$ & $\mathrm{N}$ \\
\hline 0.15 & resonance & 0.5 & $0.058( \pm 0.016)$ & 5 \\
1 & resonance & 3.5 & $0.067( \pm 0.016)$ & 6 \\
3 & resonance & 10.5 & $0.26( \pm 0.05)$ & 6 \\
5 & resonance & 17.5 & $0.68( \pm 0.05)$ & 6 \\
7.5 & resonance & 26.3 & $1.40( \pm 0.09)$ & 7 \\
10 & resonance & 35 & $2.42( \pm 0.14)$ & 7 \\
10 & off-resonance $(-30 \mathrm{kHz})$ & -- & $0.36( \pm 0.10)$ & 5 \\
\hline
\end{tabular}


FLIM of temperature of the quartz crystal surface was also performed for high-amplitude oscillations in PBS $^{--}$buffer at an ambient temperature of $37^{\circ} \mathrm{C}$. The increases in the average temperature, thereby, are slightly lower than that observed at $20^{\circ} \mathrm{C}$. At $5 \mathrm{~V}$ and $10 \mathrm{~V}$ of resonant $\mathrm{QCM}$ driving voltages, increases by $0.39( \pm 0.08){ }^{\circ} \mathrm{C}$ and $1.9( \pm 0.3)^{\circ} \mathrm{C}$, respectively, were determined (mean $\pm \mathrm{SEM} ; \mathrm{N}=3$ ).

\subsection{Imaging the Oxygen Consumption Rate (OCR) beneath Adherent Cells}

Over the past five to ten years a steady increase in the number of publications about the use of oxygensensitive fluorescent indicators (phosphorescence quenching) in cell-based biosensing and imaging applications is observed ${ }^{[175,638,639]}$. Most of them focus on micro- and nanoparticle-based luminescent oxygen probes, where the indicator dye is encapsulated in polymeric particles and whose luminescence is quenched in the presence of oxygen. These kind of optical nanosensors are predominantly utilized for the monitoring of analyte concentrations and distributions in live cells and in vivo and are also referred

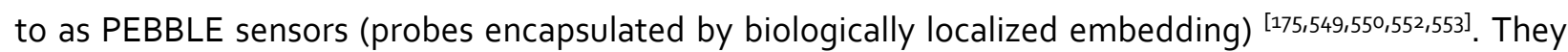
exist in variable dye-polymer combinations for the optical detection of dissolved oxygen (DO) in biological samples ${ }^{[551,581-583,640-645]}$. Recently, dual nanosensors for the simultaneous sensing and imaging of intracellular oxygen and $\mathrm{pH}$ have been described ${ }^{[646]}$. Particle-based oxygen sensors have also been prepared by embedding the luminescent indicator dyes in polyelectrolyte multilayers on luminescent nanoparticles, which serve as internal intensity reference ${ }^{[647]}$, or in polymerized phospholipid vesicles (liposomes) ${ }^{\left[{ }^{648}\right]}$. Oxygen probes on the basis of cell-penetrating $\mathrm{Pt}(\mathrm{II})$-coproporphyrin conjugates have also been successfully applied for monitoring the extracellular, intracellular and pericellular $\mathrm{O}_{2}$ concentration of respiring mammalian cells and their changes upon metabolic stimulations ${ }^{[649-652]}$.

PEBBLEs have been used for the real-time sensing of the metabolic oxygen consumption inside live cells $[581,640,641,645,646,648,653-655]$ as well as for the quantitative imaging of the oxygen distribution in vitro and in vivo $^{[189,582,645,656-659]}$ via ratiometric fluorescence intensity measurements. Besides fluorescence intensitybased nanosensors for monitoring oxygen in living cells, also fluorescence anisotropy-based ${ }^{[660]}$ and lifetime-based approaches are reported. Non-imaging fluorescence lifetime sensing (time-resolved fluorometry) of intracellular $\mathrm{O}_{2}$ has been successfully applied in plant cells, non-mammalian and mammalian cells ${ }^{[551,642,643,661-664]}$, as well as in vivo ${ }^{[582]}$. Fluorescence lifetime imaging (FLIM; also referred to as phosphorescence quenching microscopy - POM ${ }^{[665]}$ ) enables the qualitative visualization and mapping of the oxygen distribution, and the spatial and temporal quantification of $\mathrm{O}_{2}$ concentrations in complex biological samples. ${ }^{[644,658]}$ This has been presented for various cell types, such as macrophages $[666,667]$, plant cells ${ }^{[661,668]}, 2-\mathrm{D}$ mammalian cell cultures ${ }^{[649,658,667,669-674]}, 3$-D tissue culture models/ spheroids (2-D and 3-D O ${ }_{2}$ mapping) ${ }^{[673-678]}$, as well as for measurements in vivo ${ }^{[561,639,679-681]}$.

Besides soluble probes, solid-state luminescent sensors (dyes embedded in an immobilized polymer matrix) like sensor spots and planar optodes have also been applied for the oxygen sensing in biological environment, integrated in cell culture vessels or coated on growth substrates. These immobilized sensors are used for point measurements (monitoring) of the concentration of DO in the cell culture medium, like e.g. for the process control in bioreactors or in multi-well plates for the monitoring of the oxygen consumption rate (OCR) of cells. The sensor films described in literature ${ }^{[682-688]}$ and of commercially available platforms [689-693], however, have the common disadvantage of lacking biocompatibility, as usually hydrophobic polymer matrices are used for dissolving of the indicator dye. 
Such hydrophobic surfaces impede the processes of cell attachment and spreading so that adherent cells usually grow around such sensor spots but not on them. This disables the 2-D mapping of subcellular $\mathrm{O}_{2}$ distributions and one can "only" sense the average bulk oxygen concentration with such setups. Moreover, single sensor spots allow only point measurements of an analyte, which might not always be representative in a biological sample. The primary approach to facilitate cell attachment on the sensor films was hitherto their pre-coating, such as with fibronectin ${ }^{[694]}$, collagen ${ }^{[683]}$, or PDMS [694].

A new solid-state sensor platform is presented for monitoring and imaging the oxygen distribution directly beneath adherently grown cell cultures by means of a planar sensor film, i.e. an oxygen-sensitive phosphorescent dye embedded in a polymer matrix, which serves as the growth substrate. For this system, no additional hydrophilic layer or protein pre-coating is required on top of the sensor for promoting cell attachment and spreading. The biocompatibility of the sensor itself is attained by a smooth surface, generated by spin coating of the sensor cocktail, and by the hydrophilization of the cured sensor film prior to the seeding of cells. Thus, the cells can be cultured directly on the surface of the sensor layer, which means that sensing and imaging is possible in immediate contact to the cells. This enables a more sensitive and a laterally more accurate measurement of the subcellular oxygen concentration and distribution than with systems that utilize an adhesion-facilitating layer and where there is an increased diffusion distance for the dissolved oxygen between the cells and the sensor. The presented setup enables the 2-D mapping of oxygen concentrations and the spatially and temporally resolved sensing of the OCR of cells.

\subsubsection{Cytocompatibility Testing of the Sensor Film}

Circular AT-cut quartz crystal sensors were coated with a pressure-sensitive paint (PSP) by either spray coating or spin coating the sensor cocktail, according to the PSP Sensor Preparation protocol (p. 67). The cocktail consisted of the phosphorescent Pt(II)-porphyrine complex PtTFPP (Fig. 3-23 C; the oxygensensitive indicator dye) and polystyrene (polymer support), dissolved in a suitable solvent. The quartz disks were coated in order to (i) determine the average thickness of the sensor film and (ii) investigate cell attachment, cell growth and cell viability on the PSP sensor films by means of OCM recordings.

Fig. 6-8 shows the QCM impedance spectra of the blank quartz disk (black squares) and of quartz disks that were coated with the PSP (blue circles) either by spray coating (Fig. 6-8 A) or by spin coating at 4000 rpm (Fig. 6-8 B). The OCM spectra were recorded after curing of the coating layer in air. A shift in

the resonance curve $\left(\Delta f_{|Z|_{\text {min }}}\right)$ to lower frequencies upon PSP deposition is evident at either coating method. Moreover, one can recognize in the impedance spectra the rigidity of the sensor film from the absence of any damping effects on the microbalance oscillation. This allows to apply the Saverbrey relation, which links the frequency shift with an additional mass on the microbalance ( $-\Delta f \propto \Delta m$; cf. Eq. (23)). The resonance shifts report on the deposition of much ( 50-fold) more mass on the resonator by spray coating $\left(\Delta f_{|Z|_{\text {min }}} \approx-15 \mathrm{kHz}\right)$ than by spin coating at $4000 \mathrm{rpm}\left(\Delta f_{|Z|_{\text {min }}} \approx-0.3 \mathrm{kHz}\right)$. As the same sensor cocktail ( $\rightarrow$ equal density) has been used in both coating methods and since the sensors were of similar type ( $\rightarrow$ equal size and mass sensitivity), the differences in the resonance shift/ deposited mass can be directly correlated with different thicknesses of the deposited polymer layers. Average heights of the PSP layers on the microbalances of $\sim 4.2 \mu \mathrm{m}$ and $\sim 70 \mathrm{~nm}$ by spray coating and spin coating (at 4000 rpm), respectively, were calculated from the impedance properties of the QCM oscillations.

For testing the cytocompatibility of the planar sensors, MDCK-II cells were seeded on resonators with spin coated and spray coated PSPs, after sterilization and hydrophilization of the polymer surface. The cell attachment and spreading processes on the different substrates have been monitored by time- 
resolved, spectroscopic OCM sensing. It has been demonstrated repeatedly in literature $[22,197,206,219,228,240,267]$ as well as in this work that this technique (including the active-mode OCM with dissipation monitoring, QCM-D) is a valuable, label-free, and non-invasive means for the real-time monitoring of the attachment, spreading and proliferation properties of mammalian cells on their growth substrate. OCM measurements have also been applied previously for the cell-based biocompatibility testing of various materials ${ }^{[217,236]}$. The $\Delta|Z|_{\text {min }}$ time courses that were obtained from OCM recordings during cell attachment on the quartz disks with differently coated PSP layers are illustrated in Fig. 6-8 C, normalized to the point of cell inoculation. Both curves show an increase in $\Delta|Z|_{\text {min }}$. This reflects an increase in the viscoelasticity of the OCM load close to the surface, which can be assigned to the sedimentation of the viscoelastic cell bodies into the sensing volume above the crystal surface. After roughly $4 \mathrm{~h}$, the minimum impedance of the spray coated resonator does not change anymore (dashed line). In contrast to that, $|Z|_{\min }$ of the spin coated resonator (solid line) increases by the threefold within this period and even increases continuously further to approximately 5 -fold higher values after $24 \mathrm{~h}$ of seeding the cells. This change in $|Z|_{\text {min }}$ reports on the attachment and spreading of cells on the microbalance surface as well as on cell proliferation, as it is known from blank, not coated resonators (cf. Fig. 5-27D). The comparatively small $|Z|_{\text {min }}$ increase of the spray coated resonator indicates the failure of cell spreading after the initial sedimentation and anchorage of the cells to the substrate, preventing proliferation and cell growth across the entire sensor.

A
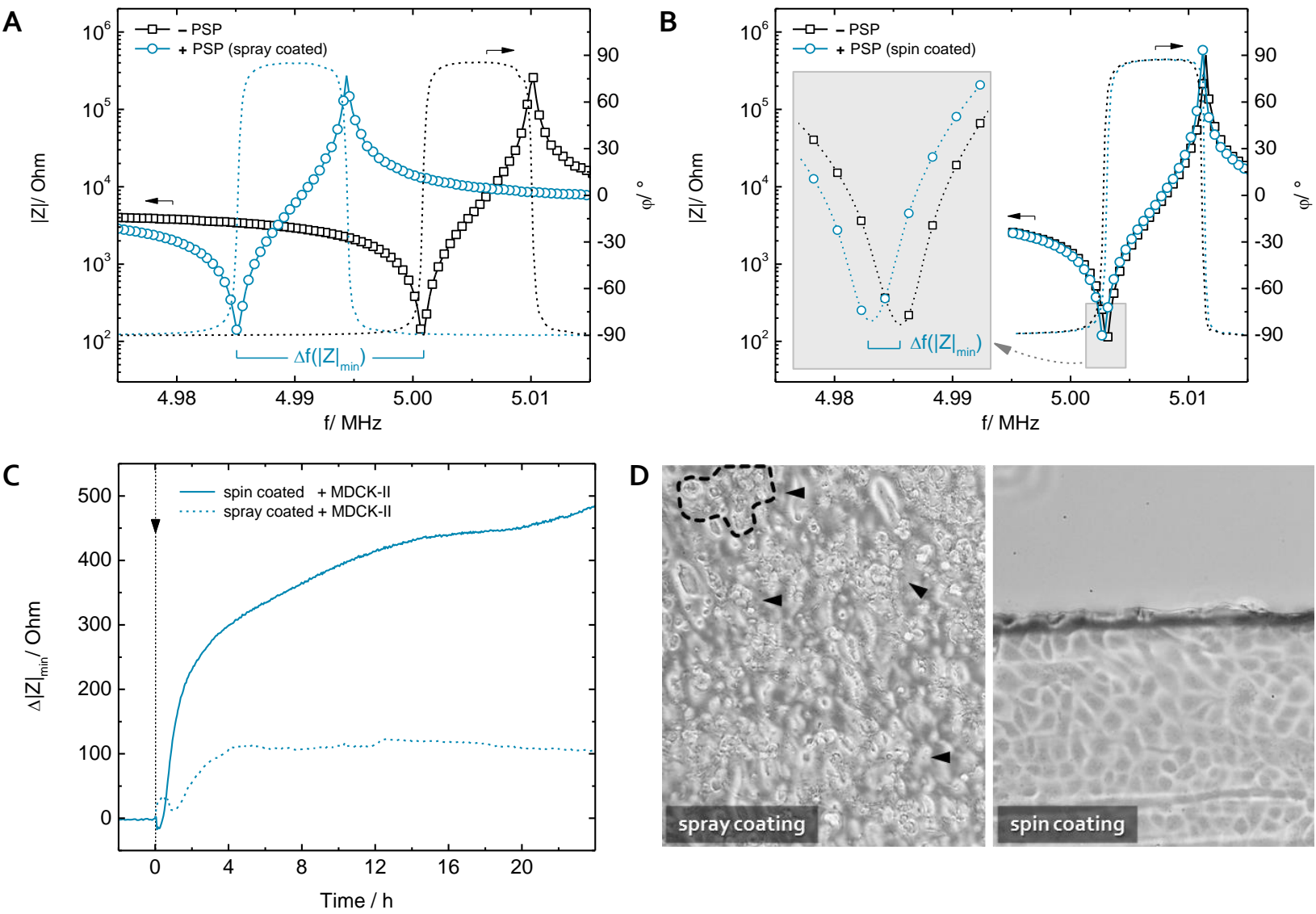

D
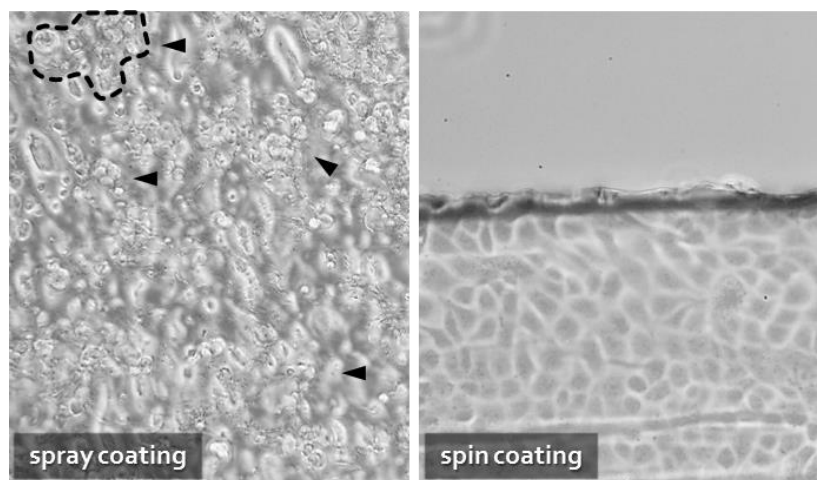

Fig. 6-8 (A), (B) Shift of the resonance curve (of the frequency of minimal impedance, $\Delta f_{|Z|_{\min }}$ ) of a $5 \mathrm{MHz}$ AT-cut quartz resonator upon (A) spray coating and (B) spin coating with a PSP. (C) Change of the magnitude of minimal impedance $\Delta|Z|_{\text {min }}$, after seeding MDCK-II cells on quartz resonators with a spray coated (dashed line) and spin coated (solid line) PSP layer. (D) Micrographs of a spray coated (left) and a spin coated (right) PSP film $24 \mathrm{~h}$ after seeding cells. 
This could be confirmed by stereomicroscopy and phase-contrast microscopy of the substrate surfaces $24 \mathrm{~h}$ after seeding the cells. The exemplary micrographs in Fig. 6-8 D show clusters of rounded cells (surrounded by a dashed line and $\longrightarrow$ ) on top of the very inhomogeneous and rough, spray coated PSP (left), and a confluent layer of MDCK II cells, which has grown up to the edge on the homogeneous, smooth and optical transparent, spin coated PSP film (right). Hence, spray coating of the PSP prevents cell spreading and their growth on the polymer surface. This is very likely due to the surface roughness that makes it impossible for the cells to form a sufficient number of strong cell-substrate contacts. For this reason, the cells remain attached in rounded shape on the substrate. By contrast, the spin coated PSP film demonstrates cytocompatibility and, thus, enables the growth of a confluent cell layer.

\subsubsection{Sensor Calibration}

The very thin sensor films ( $\sim 100 \mathrm{~nm}$ ) that were deposited on circular cover glasses by spin coating have been investigated with respect to their oxygen sensing capabilities. The sensor response (fluorescence lifetime) was calibrated in air, in dependence of the ambient temperature, and under physiological buffer at various preset oxygen partial pressures $\mathrm{pO}_{2}$ with either $\operatorname{Ar}$ or $\mathrm{N}_{2}$ as complementary gas (total pressure $p_{\text {tot }}=1$ bar). A photograph of the calibration chamber and the imaging setup with integrated microscope, temperature control, and gas mixing device is shown in $S I 7$ (p. 264). The single components of the experimental setup are specified and described in detail in 3.5.3 (p. 67 ff.; Fig. 3-25), together with the software and gate settings (p. $70 \mathrm{f}$.), and the data processing of fluorescence lifetime images (p. $72 \mathrm{f}$.) recorded by RLD (cf. 3.5.2, p. 65 ff.).

\section{Calibration in Air}

Fig. 6-9 depicts the fluorescence lifetime data of a PSP film that has been calibrated in dry air at a constant ambient temperature of $24^{\circ} \mathrm{C}$. Fig. 6-9 A shows the change of the average $A_{1} / A_{2}$ ratio (cf. Eq. (55), p. 66), calculated from phosphorescence intensity pictures which have been taken in the decay phase of the phosphorescence of the oxygen-sensitive platinum porphyrine dye. Oxygen partial pressures of $0,5,10,30,50,70,100,150,200,300,500,700,900$, and $1000 \mathrm{mbar}$ ( $p_{\text {tot }}=1000 \mathrm{mbar}$ ) were consecutively set by gas blender in the headspace of the sensor film (arrows in Fig. 6-9 A indicate the time at which the specified pressure was adjusted).

The $A_{1} / A_{2}$ value sensitively increases with increasing oxygen amount in the calibration gas mixture, due to an increasing amount of oxygen molecules that participate in the dynamic quenching of the dye luminescence. This causes a decrease in the fluorescence lifetime $\tau$ and an increase in the $A_{1} / A_{2}$ ratio, respectively. The high sensitivity of the sensor film at low oxygen concentrations is evident in the significant $A_{1} / A_{2}$ changes for rather little changes in the oxygen partial pressure $p \mathrm{O}_{2}$ (see zoom-in insets in Fig. 6-9 A). With increasing the $\mathrm{pO}_{2}$ to $500 \mathrm{mbar}$ and more, a clearly noticeable and increasing noise in the $A_{1} / A_{2}$ pixel values occurs. The time course in Fig. 6-9 $\mathrm{A}$ also indicates a rapid sensor response upon changing the oxygen concentration above the sensor. Equilibration of the $\mathrm{pO}_{2}$ above the sensor and in the polymer matrix of the embedded dye is attained within $60-90 \mathrm{~s}$, which is the data acquisition time for recording one data set of seven pairs of $A_{1}-A_{2}$ images and one blank image. The reversibility of the sensor is also evident from the exemplary calibration time course in oFig. 6-9 A, where there is an equally fast response time when switching from $100 \%$ back to $0 \%$ oxygen again at the end of the calibration.

Fig. 6-9 $\mathrm{B}$ shows the mean $A_{1} / A_{2}$ values in the range of $0-300 \mathrm{mbar} p \mathrm{O}_{2}$ (mean $\pm \mathrm{SEM} ; \mathrm{N}=7$ ). The standard errors were calculated using ImageJ software [330,331]. The underlying $A_{1} / A_{2}$ images are compiled in Fig. 6-9 $\mathrm{C}$ in pseudo colors of $A_{1} / A_{2}$ pixel values between 1.45 and 2.70. The linear regression 
of the data points at low oxygen concentrations ( $0-10 \mathrm{mbar}$ ) delivers a slope and oxygen-sensitivity of $0.068 A_{1} / A_{2}$ per $10 \mathrm{mbar} p \mathrm{O}_{2}$ (air). The $A_{1} / A_{2}$ calibration data points were fitted best by an exponential fitting function of the form $y=a \cdot e^{b \cdot x}+y_{0}$ (indicated by a dashed red line in Fig. 6-9 B; parameter values see caption). The Stern-Volmer plot, $\tau_{0} / \tau$ as a function of $p \mathrm{O}_{2}$, belonging to this calibration is shown in $S / 27 A$, together with a fit of the data according to the Stern-Volmer two-site quenching model (Eq. (53)).

A

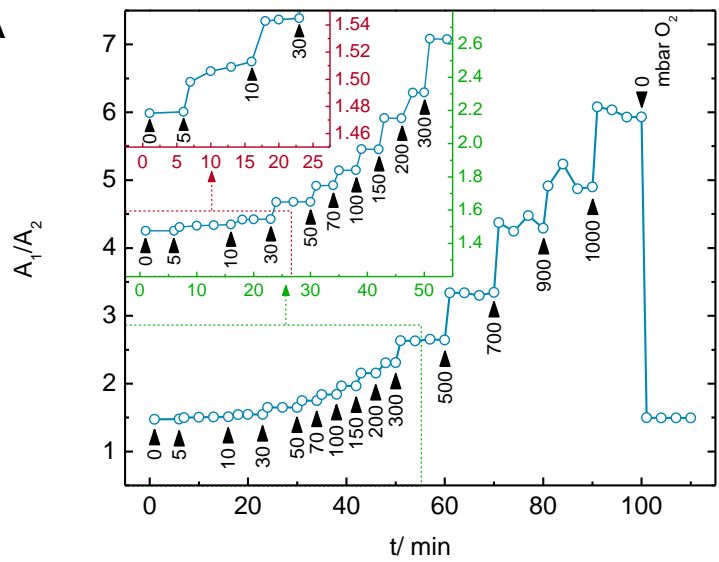

B

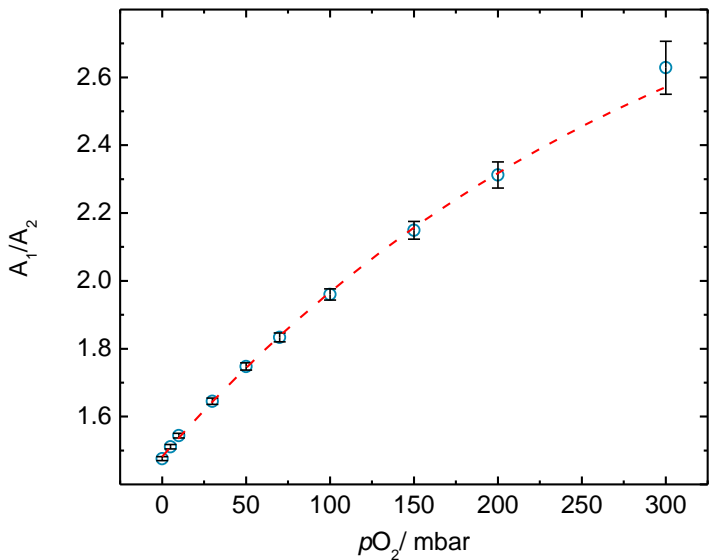

C

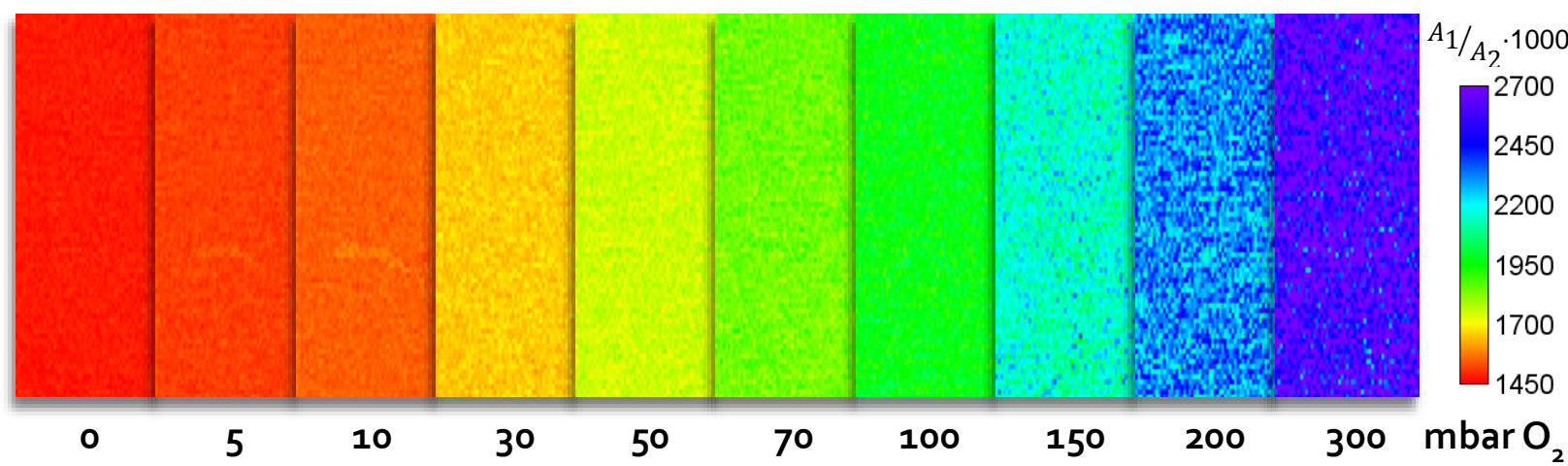

Fig. 6-9 Calibration of a PSP film in air. (A) Time course of $A_{1} / A_{2}$ values while different $p \mathrm{O}_{2}$ values were applied to the headspace above the planar sensor $\left(p_{\text {tot }}=1000 \mathrm{mbar}\right)$. Black arrows indicate the time of setting of the specified $p \mathrm{O}_{2}$. (B) Average $A_{1} / A_{2}$ values (mean $\pm \mathrm{SEM} ; \mathrm{N}=7$ ) as a function of the oxygen concentration in the physiologically relevant range of $0-300 \mathrm{mbar}$. Taking the standard errors into account (instrumental weighting), the $A_{1} / A_{2}$ calibration data were fitted best by an exponential function of the form $A_{1} / A_{2}=-1.75( \pm 0.17) \cdot \exp (-0.0032$ $\left.( \pm 0.0004) \mathrm{mbar}^{-1} \cdot p \mathrm{O}_{2}\right)+3.23( \pm 0.17)\left(R^{2}=0.9986\right)$, shown as dashed red line. (C) Pseudo-color $A_{1} / A_{2}$ images of the sensor film from which the calibration data shown in $\mathrm{B}$ are extracted. The color code covers $A_{1} / A_{2}$ values of $1.45-2.70$.

\section{Temperature Dependence}

It is well known that the intensity and lifetime of luminescence is also a function of the temperature. An increase in the temperature promotes the non-radiative relaxation of excited electrical states, which then consequently are no longer populated and available for events of radiative relaxation, i.e. fluorescence and phosphorescence (cf. 3.5.1 > Temperature Sensitivity of Luminescence, p. 64). The dependence of the fluorescence intensity and lifetime on the temperature is widely utilized for its sensing and imaging by the use of luminescent probes, like for example in the form of planar films as demonstrated above in 
section 6.1. Hence, temperature variations also affect the luminescence properties of oxygen-sensitive dyes. Without an accurate temperature control, oxygen readings are prone to error.

The planar PSP sensor was tested with respect to its cross-sensitivity to temperature changes. For this, one and the same sensor film was calibrated at $24^{\circ} \mathrm{C}$ and at physiological $37^{\circ} \mathrm{C}$ in air as described above. For temperature control a heatable metal block, surrounding the measurement chamber, and an additional thermo sensor to monitor its temperature was integrated in the experimental setup ( $\mathrm{cf} . \mathrm{SI} 7$ ). The calibration data, the average pixel values of $A_{1} / A_{2}$ images (mean $\pm \mathrm{SEM} ; \mathrm{N}=7$ ), are shown in Fig. 6 10 for both temperatures in the range of $0-300 \mathrm{mbar}$ of applied oxygen partial pressures ( $p_{\text {tot }}=1000$ mbar). The $A_{1} / A_{2}$ values are significantly higher at $37^{\circ} \mathrm{C}(\square)$ compared to $24^{\circ} \mathrm{C}(O)$ across all $p \mathrm{O}_{2}$ calibration values. The absolute fluorescence lifetime, thereby, is by $10 \%$ lower at $37^{\circ} \mathrm{C}$ than at $24^{\circ} \mathrm{C}$. The Stern-Volmer plots $\left(\tau_{0} / \tau\right.$ vs. $\left.p \mathrm{O}_{2}\right)$ corresponding to this data and the respective fitting of the lifetime data according to the two-site quenching model are depicted in $S / 27 \mathrm{~B}$. One can also observe from the calibration data in Fig. 6-10 that the noise of the $A_{1} / A_{2}$ images and the error bars, respectively, are bigger at $37^{\circ} \mathrm{C}$ than at $24^{\circ} \mathrm{C}$ at each oxygen partial pressure. Irrespective of the increased fluorescence quenching and the bigger standard errors of averaged pixel values at increased ambient temperatures, the calibration data still prove the predominant sensitivity of the PSP sensor film towards changes in the oxygen concentration in the physiologically relevant range.

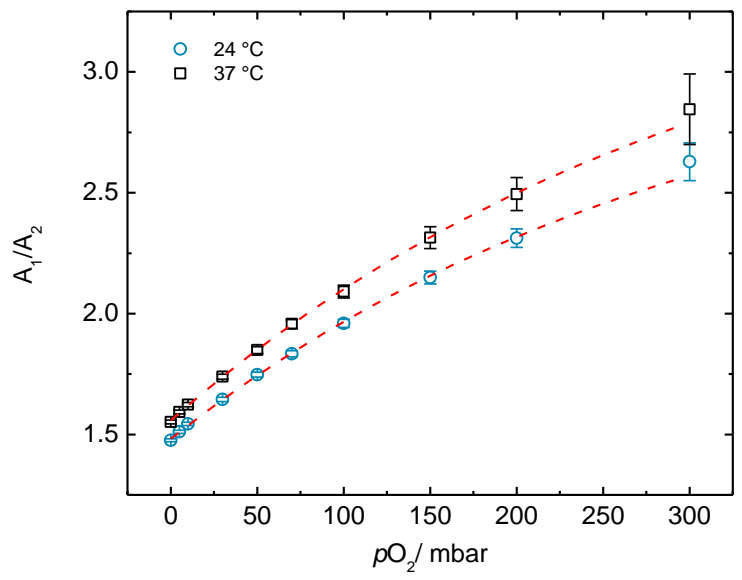

Fig. 6-10 Calibration plots of average phosphorescence lifetime images $A_{1} / A_{2}$ (mean $\pm \mathrm{SEM}$; $\mathrm{N}=7$ ) as a function of $p \mathrm{O}_{2}$ at the two temperatures $24^{\circ} \mathrm{C}(\mathrm{O})$ and $37^{\circ} \mathrm{C}(\square)$. The fits of the data points, according to functions of the form $y=a \cdot e^{b \cdot x}+y_{0}$, are indicated (dashed red lines).

\section{Calibration under Liquid}

With the aim of monitoring and imaging the concentration of DO in a buffer solution and beneath mammalian cells cultured on the PSP, the sensing performance of the spin coated PSP film was tested by its calibration under liquid. For this purpose, the PSP-coated glass disks were covered with $4 \mathrm{~mL}$ of $\mathrm{PBS}^{++}$buffer solution and gas mixtures of various ratios of $\mathrm{O}_{2}$ and $\mathrm{N}_{2}$ partial pressures were streamed through the headspace above the liquid in the calibration chamber $\left(\mathrm{pO}_{2}\right.$ (air) $=0,10,50,100,150,300$, and $500 \mathrm{mbar} ; p_{\text {tot }}($ air $\left.)=1000 \mathrm{mbar}\right)$. Fluorescence lifetime images were recorded after equilibration of the gas phase with the buffer solution and the polymer matrix of the sensor. These equilibration phases can be clearly seen in the exemplary time course of $A_{1} / A_{2}$ values in Fig. 6-11 $\mathrm{A}$, by the time that values require for reaching stationary levels upon the stepwise increases of $\mathrm{pO}_{2}$ (air). An uniform and constant 
distribution of oxygen molecules in the liquid and in the sensor matrix is attained at the earliest 15-20 min after the setting of the oxygen partial pressure in air, as evident from the inset graph in Fig. 6$11 \mathrm{~A}$, which shows in magnification the $A_{1} / A_{2}$ time course after the change from o to $10 \mathrm{mbar} p \mathrm{O}_{2}$.

A

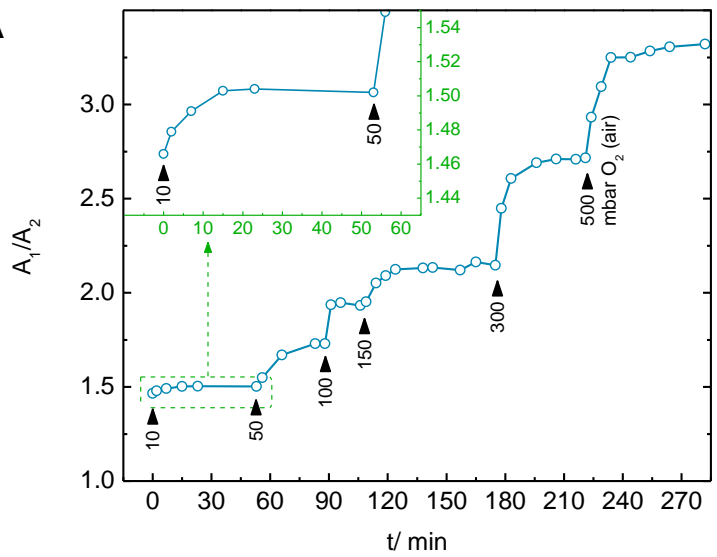

C

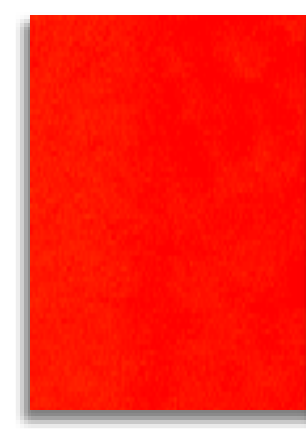

o

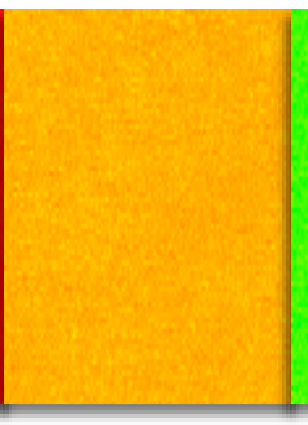

50

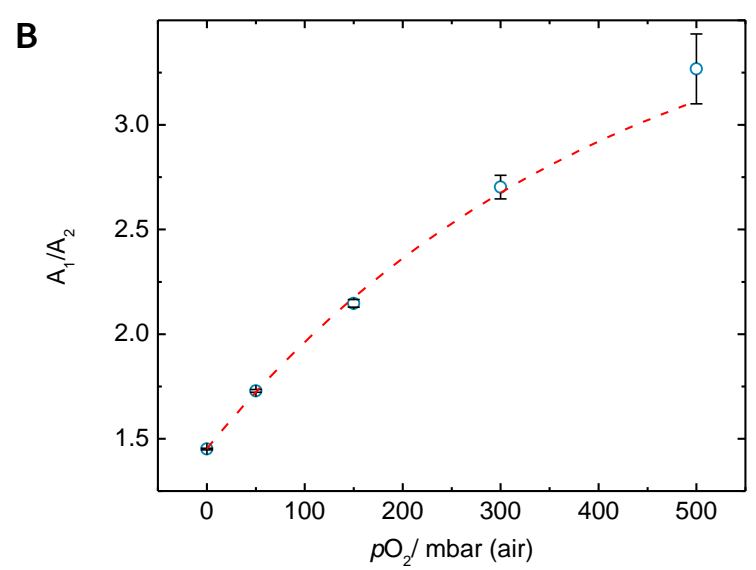

Fig. 6-11 Calibration of a PSP film in PBS ${ }^{++}$buffer. (A) Time course of $A_{1} / A_{2}$ values while different $\mathrm{pO}_{2}$ values were applied to the headspace above the liquid on the planar sensor ( $p_{\text {tot }}($ air $)=1000 \mathrm{mbar}$ ). Black arrows indicate the time of application of the specified $p \mathrm{O}_{2}$ (air). (B) Average $A_{1} / A_{2}$ values (mean $\pm \mathrm{SEM} ; \mathrm{N}=7$ ) as a function of the $\mathrm{O}_{2}$ concentration in the range of $0-500$ mbar in air. Taking the standard errors into account (instrumental weighting), the calibration data of $A_{1} / A_{2}$ were fitted best by an exponential function of the form $A_{1} / A_{2}=$ $-2.4( \pm 0.5) \cdot \exp \left(-0.0024( \pm 0.0006) \mathrm{mbar}^{-1} \cdot p \mathrm{O}_{2}\right)+3.8( \pm 0.5)\left(R^{2}=0.9986\right)$, shown as dashed red line. (C) Pseudo-color $A_{1} / A_{2}$ images of the sensor film from which the calibration data in $\mathrm{B}$ are extracted. The color code covers $A_{1} / A_{2}$ pixel values of $1.45-3.45$.

Fig. 6-11 B shows the gradual increase of the average $A_{1} / A_{2}$ values with increasing oxygen partial pressures (in air) from o mbar to 500 mbar (mean $\pm \mathrm{SEM} ; \mathrm{N}=7$ ). The standard errors of pixel values were calculated using ImageJ software ${ }^{[330,331]}$. From the linear regression of the calibration data points at low oxygen concentrations (o- $50 \mathrm{mbar}$ ) a slope of $0.056 A_{1} / A_{2}$ per $10 \mathrm{mbar} p \mathrm{O}_{2}$ (air) is obtained, reflecting the oxygen-sensitivity of the PSP sensor film in aqueous liquid. The $A_{1} / A_{2}$ images of the calibration in Fig. 6-11 A, B are illustrated in Fig. 6-11 C in pseudo colors of $A_{1} / A_{2}$ pixel values between 1.45 and 3.45 . The $A_{1} / A_{2}$ calibration data points were fitted best by an exponential function of the form $y=a \cdot e^{b \cdot x}+$ $y_{0}$ (indicated by a dashed red line in Fig. $6-11$ B; parameter values see caption). The Stern-Volmer plot, $\tau_{0} / \tau$ as a function of $p_{2}$ (air), belonging to this calibration is shown in $S / 27 C$, together with a fit of the data according to the Stern-Volmer two-site quenching model (Eq. (53)). 
The calibration data in liquid prove a high reproducibility and sensitivity for sensing and imaging small alterations in the amount of DO in the physiological range at the sensor/ liquid interface. This provides the basis for utilizing the sensor layout on the basis of PSP films presented as the growth substrate for mammalian cells and for the substrate-integrated, label-free monitoring of the OCR of cells in temporal as well as in spatial resolution.

\subsubsection{Imaging the OCR of Cells}

Fluorescence lifetime imaging and monitoring of the oxygen consumption rate of epithelial cells was performed by applying growth substrate-integrated PSP sensor films. Studies on the cytocompatibility (6.2.1) and the oxygen-sensing capabilities and sensitivities, in both air and under liquid (6.2.2), of these were presented above. After hydrophilization and pre-incubation of the PSP surfaces with serum-free medium over night, MDCK-Il cells were grown to confluence on these substrates in cell culture medium. For fluorescence imaging measurements, the culture medium was exchanged against $\mathrm{PBS}^{++}$buffer, supplemented with $1 \mathrm{~g} / \mathrm{L}$ glucose. The quality of the cell layer on the PSP, i.e. the confluence and cell morphology, was routinely checked microscopically before FLIM (cf. experimental implementation section on $\mathrm{p}$. 70).

The change of the oxygen concentration beneath the epithelial cell layer was investigated over time, after adding the respiratory chain decoupler 2,4-Dinitrophenol (2,4-DNP) to the cells. 2,4-DNP is known to act as ionophore for protons and, thus, counteracts the establishment and maintenance of the proton gradient across the mitochondria membrane. However, this is the energy source for the cells, as it is used for the production of adenosine triphosphate (ATP), which is the intracellular molecular carrier of chemical energy. Since 2,4-DNP inhibits the molecular fixation of energy in the form of ATP molecules, cells attempt to compensate for this by an increased metabolism, which is correlated with an increase in the OCR of the cells.

Typical time courses of the average $A_{1} / A_{2}$ ratio that are obtained from FLIM of oxygen-sensitive films beneath cells are shown in Fig. 6-12 A, for a cell layer treated with $100 \mu \mathrm{M}$ 2,4-DNP (-o-) and for a control cell layer, where the $\mathrm{PBS}^{++}$buffer was exchanged only (ctrl; - ). The point of addition is indicated with an arrow. A strong decrease of the $A_{1} / A_{2}$ values is observable after adding 2,4-DNP. The values approximate a stationary level of roughly 1.45 after $5.5 \mathrm{~h}$. Since the phosphorescence intensity of $A_{1}$ pictures ran into saturation in this time course, the data points marked with $\star$ were recorded with a shorter exposure time. The oxygen concentrations (with respect to air) were calculated from the $A_{1} / A_{2}$ data in Fig. 6-12 A, according to the fitting functions of the associated calibration measurements and taking account of the error propagation of measurement and fit uncertainties. Fig. 6-12 B shows the $A_{1} / A_{2}$ time courses translated into $\mathrm{pO}_{2}$ (air) values for the control cell layer (ctrl; ${ }^{-}$) ) and cells treated with 2,4-DNP (-o-). The associated calibration data of the "2,4-DNP" sensor film at various exposure times are shown in $S / 28 A, B$, together with the respective best fit. The calibration data of the PSP serving as control can be found in $S / 28 \mathrm{C}$. 
A

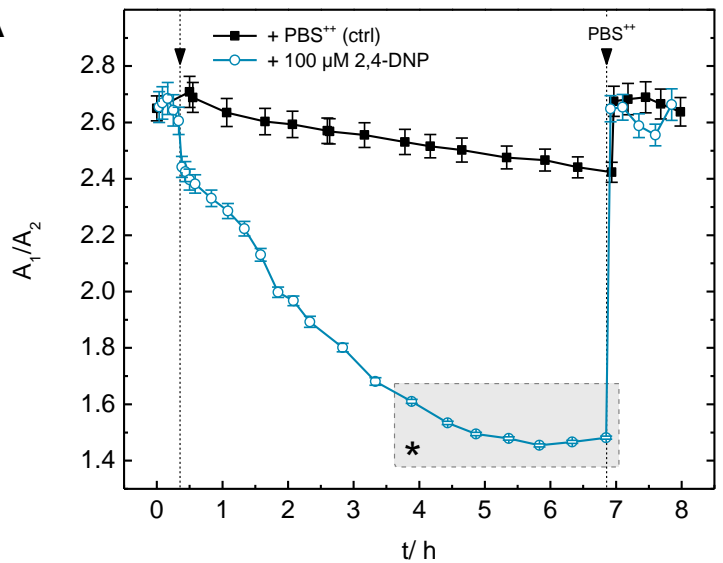

C
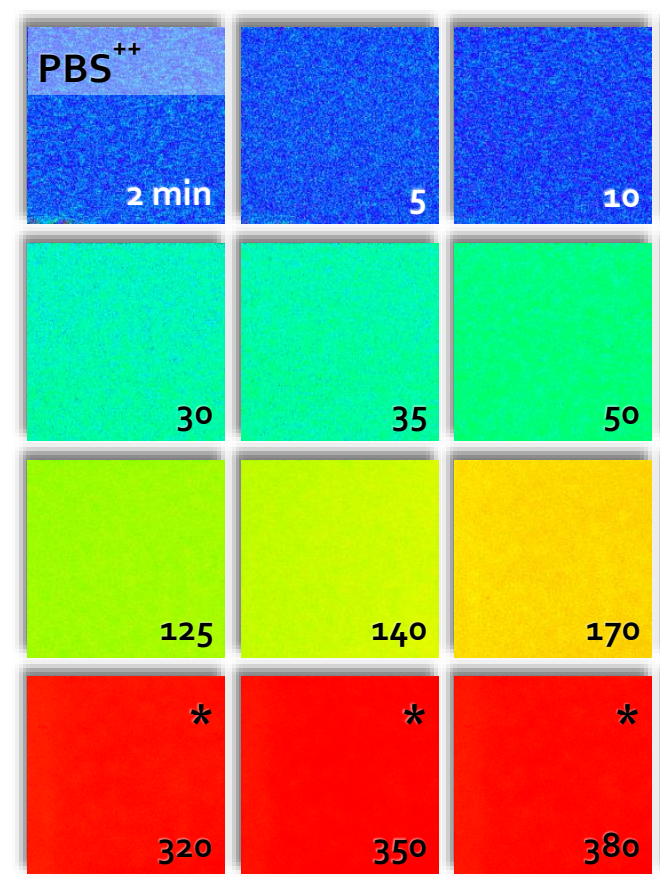

B

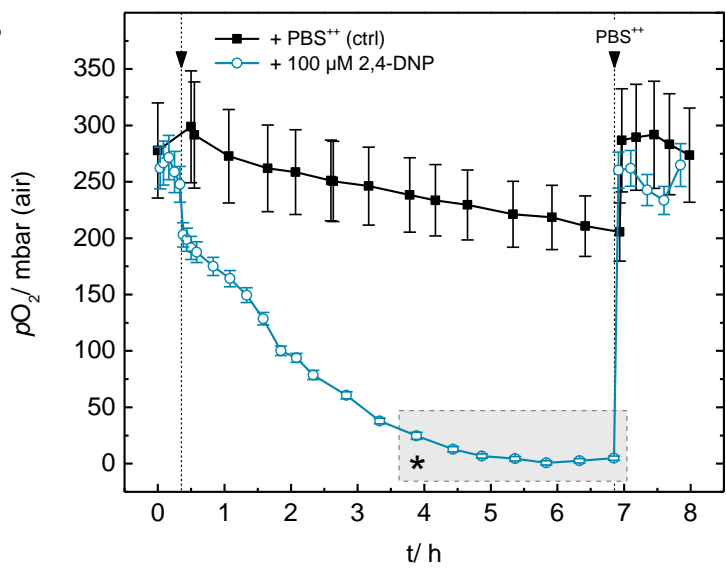

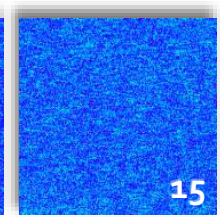
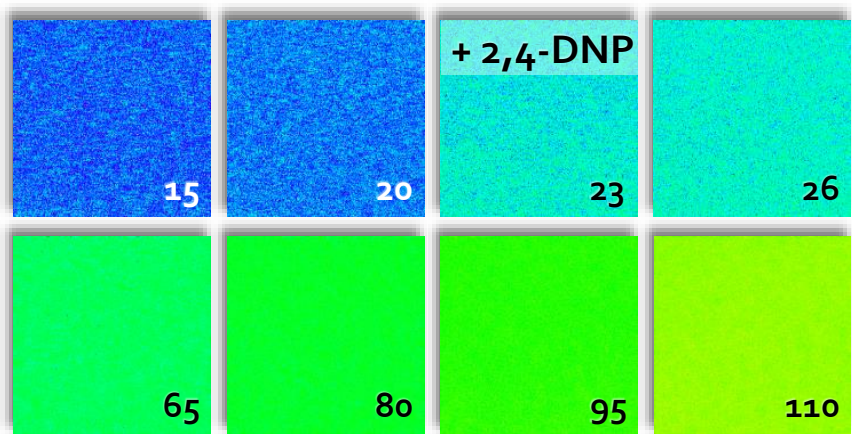

$\mathrm{pO}_{2}$ (air)
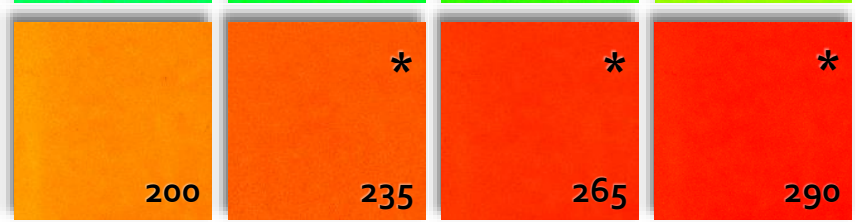

mbar
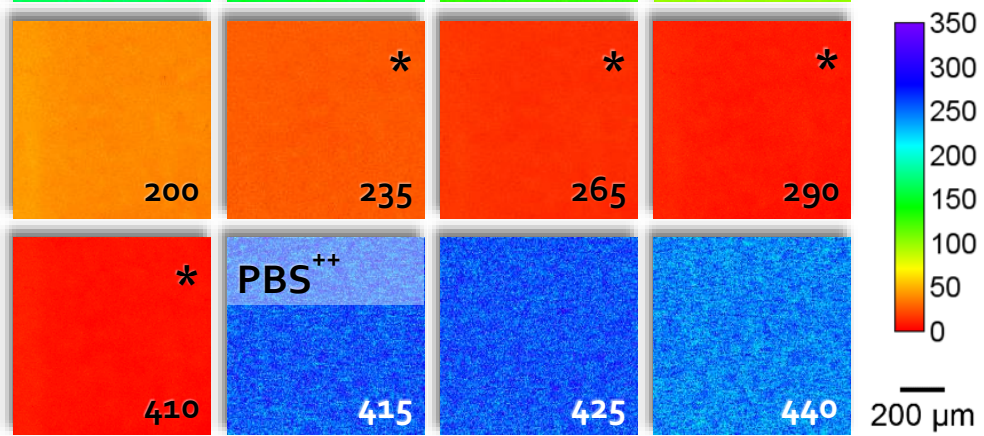

Fig. 6-12 FLIM of the OCR of cells grown on a PSP sensor film upon stimulation with the respiratory chain decoupler 2,4-DNP. (A) $A_{1} / A_{2}$ time course and (B) calculated $p \mathrm{O}_{2}$ (air) time course upon addition of either $100 \mu \mathrm{M} 2,4-\mathrm{DNP}(-\mathrm{O}-)$ or $\mathrm{PBS}^{++}\left(\mathrm{ctrl}^{-}-\right.$) to the cell layer at the indicated point of time. The corresponding calibration plots of the sensor films are summarized in $S / 28$. Data points marked with $\star$ in $A$ were recorded with a shorter exposure time and, thus, were transferred into $\mathrm{pO}_{2}$ (air) values on the basis of a separate calibration for low oxygen concentrations. After consumption of all dissolved oxygen, the medium was exchanged against air-saturated $\mathrm{PBS}^{++}$buffer, whereupon $A_{1} / A_{2}$ and $p \mathrm{O}_{2}$ (air) values show starting levels again. (C) Pseudo-color $\mathrm{PO}_{2}$ (air) images that correspond to the data points of the 2,4-DNP-stimulated cells on the PSP film in B. Numbers depict the time of recording a fluorescence lifetime image. The calibration bar displays the color code of the $\mathrm{pO}_{2}$ (air) values of the pixels. Images of the control are shown in $\mathrm{SI} 29$.

The concentration of DO strongly decreases to 0.7 ( \pm 1.8 ) mbar (air) within $330 \mathrm{~min}$ after the addition of the respiratory chain decoupler 2,4-DNP, which is a decrease to $0.3( \pm 0.7) \%$ with respect to the dissolved oxygen concentration at the beginning. The linear regression of the time course in the region of the initial decreasing phase $(t<1.5 \mathrm{~h})$ provides a negative slope and OCR of approximately $1.25( \pm 0.10) \mathrm{mbar} \cdot \mathrm{min}^{-1}$ $\left(=0.52( \pm 0.04) \%(\mathrm{v} / \mathrm{v}) \cdot \mathrm{min}^{-1}=31( \pm 2) \%(\mathrm{v} / \mathrm{v}) \cdot \mathrm{h}^{-1}\right) \mathrm{pO}_{2}($ air $)$ for the 2,4-DNP stimulated cell layer. One can 
also observe a slight and continuous reduction of dissolved oxygen beneath the control cell layer, with an OCR of $\sim 0.20( \pm 0.01) \mathrm{mbar} \cdot \mathrm{min}^{-1}\left(=0.069( \pm 0.003) \%(\mathrm{v} / \mathrm{v}) \cdot \mathrm{min}^{-1}=4.14( \pm 0.18) \%(\mathrm{v} / \mathrm{v}) \cdot \mathrm{h}^{-1}\right) p \mathrm{O}_{2}$ (air). The concentration of the DO decreased to $74( \pm 15) \% p \mathrm{O}_{2}$ (air) within seven hours with respect to the beginning of the experiment. However, the OCR is about $85 \%$ less and the total decrease of the DO is only $\sim 26 \%$ compared to the stimulated cell layer. An exchange of the measurement solution against air-saturated $\mathrm{PBS}^{++}$buffer after an incubation time of roughly seven hours results for both 2,4-DNP- and $\mathrm{PBS}^{++}$-treated cell layers in a recovery of the DO to starting values again (Fig. 6-12 A, B). Fig. 6-12 C illustrates sections of the underlying $\mathrm{pO}_{2}$ (air) images, obtained from FLIM of the PSP beneath the 2,4DNP-treated cell layer and subsequent conversion of $A_{1} / A_{2}$ images. Each micrograph corresponds to one data point in Fig. 6-12 A and B, respectively. The respective points in time (in min) are indicated in the images as well as the times of substance additions. The calculated oxygen concentration of each pixel is illustrated in pseudo-colors between o and $350 \mathrm{mbar} \mathrm{O}_{2}$ (air) (cf. calibration bar in Fig. 6-12 C). Comparative images showing the $\mathrm{pO}_{2}$ (air) distribution beneath the control cell layer during the time of investigation are depicted in $\mathrm{SI} 29$.

Stereomicroscopic and phase-contrast microscopic investigations of the cell layers after the experiment show over a wide range a clearly visible destruction of the cell layer integrity and striking changes in the morphology of individual cells, independently on whether the cells have been treated with 2,4-DNP or as control. This is evident in the micrographs of a confluent cell layer before FLIM measurements in Fig. $6-$ $13 \mathrm{~A}$, and of the same cell layer afterwards in Fig. 6-13 B. Fig. 6-13 A shows that cells grow similarly on the PSP film (+PSP; area outside the grey line) and on areas where the PSP film was removed (-PSP; area inside the grey line). After FLIM (Fig. 6-13 B), cells that were attached to the PSP film obviously were irreversibly damaged, whereas cells with no PSP beneath retained their morphology and very likely also remained vital.

A

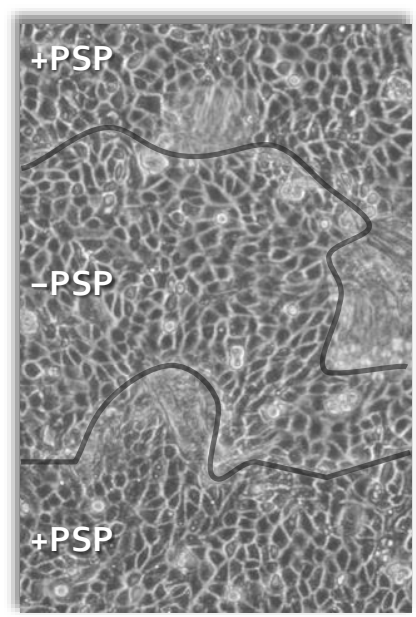

B

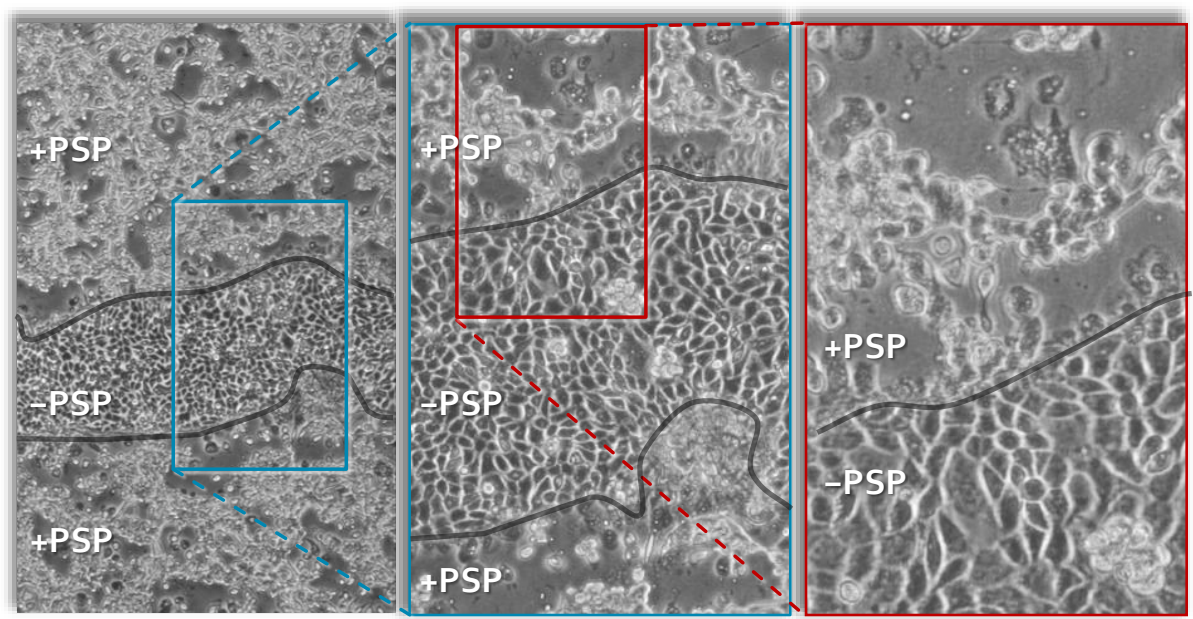

Fig. 6-13 Micrographs of MDCK-Il cells that were grown on a cover glass with a PSP-coated (+PSP) and non-coated (-PSP) areas. Pictures of the same section of cells were taken (A) before and (B) after FLIM measurements. 


\subsection{Discussion}

\subsubsection{Imaging the Frictional Heat Induced by High-Amplitude Quartz Oscillations}

We provided experimental data about the temperature on the surface of $5 \mathrm{MHz}$ AT-cut quartz crystal resonators operated at various elevated driving voltages and shear oscillations, respectively. The resonators were forced to oscillation at voltages between 0 and $10 \mathrm{~V}$, which is associated with lateral shear amplitudes of up to $210 \mathrm{~nm}$ and $35 \mathrm{~nm}$ in the center of the electrode when the quartz is operated in air (cf. Fig. 6-3) or with one face exposed to water (cf. Fig. 6-6), respectively. Temperature sensing and imaging was performed by time-resolved FLIM (in RLD mode) of TSP films coated on OCM disks. The experimental data demonstrated an increase in the surface temperature with increasing shear amplitudes. The maximal driving amplitude of $10 \mathrm{~V}$ caused mean temperature increases of $17.0( \pm 0.9)^{\circ} \mathrm{C}$ in air and $2.42( \pm 0.14)^{\circ} \mathrm{C}$ with one side of the resonator immersed in water across the electrode area of the quartz crystal (cf. Tab. 6-1 and Tab. 6-2). These experimental values are roughly in the middle of the temperature increases reported in the literature by Dultsev et al. ${ }^{[619,636]}$, Edvardsson et al. ${ }^{[633]}$, Heitmann et al. ${ }^{[226]}$, and Cooper et al. ${ }^{[631]}$ (cf. introductory paragraph of chapter 6.1, p. 223 f.).

\section{FLIM Properties of TSP on Quartz Resonators}

As the illumination of a TSP-coated quartz disk with UV light in Fig. 6-2 B already indicates, the phosphorescence intensity of the sensor film is slightly brighter at regions where the gold film of the electrodes and their supply lines are below. Moreover, one can observe slightly higher average $A_{1} / A_{2}$ values and quenching of luminescence, respectively, above the gold compared to the surrounding quartz material (data not shown). There are two possible reasons for this. Firstly, the increased phosphorescence intensity might be an optical effect. The indicator molecules embedded in the polymer matrix emit luminescence in all spatial directions when excited by light of appropriate energy. Hence, the emitted light also radiates in the direction of the gold beneath the TSP film. Here, a certain degree of the emitted light is reflected back from the polished gold surface in a way that they reach the detector (CCD), in addition to the photons that reach the CCD the most direct route. Thus, the detector collects more photons from the dye molecules above the gold than from those dye molecules which are located "only" above the bare quartz material, despite an actually equal luminescence intensity. Hence, the gold electrode actually acts as an amplifier of the emitted luminescence. Secondly, the increased values of $A_{1} / A_{2}$ images, related with a decreased fluorescence lifetime, at the regions of the OCM electrodes might arise from an additional quenching effect. It is known that metal surfaces like gold are efficient quenchers of luminescence via nonradiative energy transfer or charge transfer from the excited states of luminescent molecules, whereas quartz is not. ${ }^{[695-701]}$ However, for this hypothesis one has to assume that a high fraction of dye molecules is located in the bottom region of the polymer matrix close to the gold surface, since the rate of electronic coupling and energy transfer between the excited europium(III) complexes and gold becomes slower and less efficient with increasing distance in between. ${ }^{[699,702,703]}$ This would mean that most dye molecules subside in the polystyrene film, resulting in an uneven spatial distribution of the dye molecules. To address this observation, the average distance of dye molecules to the gold surface and their spatial distribution in the polymer matrix have to be investigated. The issue of photon reflection and luminescence amplification due to the gold electrode could be addressed by the 
use of electrodes that are transparent for the wavelength of the luminescence light, such as for example ITO electrodes or electrodes made of transparent conductive polymers.

\section{The Origin of Heating and the Effect of Water}

The operation of the resonator at $-30 \mathrm{kHz}$ off-resonance led to no significant increase in the surface temperature even though the amplitude of the driving voltage was set to the maximum of $10 \mathrm{~V}$ (cf. Fig. $6-$ ${ }_{4} \mathrm{~A}$ ). Switching the frequency to the fundamental resonance of the resonator, however, caused an instantaneous and significant increase of the temperature on the resonator surface (cf. Fig. $6-5$ and Fig. 6-7). This provides evidence that the dissipated heat arises solely from the oscillation of the quartz material and is not due to the pure energy input by applying a high voltage on the faces of the quartz plate. The AC potential is converted into mechanical oscillation energy due to the electromechanical coupling of the piezoelectric material when meeting the resonance conditions. The shear displacement of atoms in the $\mathrm{SiO}_{2}$ lattice increases in amplitude with increasing $\mathrm{AC}$ voltage amplitudes. This in turn causes an increase in the frictional heat within the resonating crystal and finally results in a heating of the entire quartz plate. At off-resonance frequencies, however, the transversal displacement of charges within the quartz crystal is intrinsically damped so that there is no or only a small amplitude of shear displacement and oscillation of atoms, respectively. With the absence of the oscillation there are consequently no frictional losses and, thereby, no heating of the quartz crystal and the TSP film on its surface.

Besides the frequency and the amplitude of the OCM driving voltage, the temperature on the quartz crystal surface also strongly depends on the heat dissipation into the surrounding media, i.e. its heat capacity. The specific heat capacities $c_{p}$ of water and air at $300 \mathrm{~K}$ are $4.18 \mathrm{~kJ} /(\mathrm{kg} \mathrm{K}){ }^{[704]}$ and $1.005 \mathrm{~kJ} /(\mathrm{kg} \mathrm{K})^{[705]}$, respectively. The bigger heat capacity of water with respect to air (factor of four) in addition to the smaller oscillation amplitudes of a quartz disk immersed in water (Fig. 6-6) compared to air (Fig. 6-3) (factor of six) both explain the much lower surface temperature that is imaged for quartz oscillations in water than in air at equal amplitudes of the driving voltage (cf. Fig. 6-7 vs. Fig. 6-5 and Tab. 6-2 vs. Tab. 6-1). The heating of the quartz surface in contact with water during high-amplitude OCM operation is nevertheless significant. The generated heat gradient in the liquid has a serious influence on (bio)sensor applications and studies of molecular interactions on the microbalance surface, as will be discussed below.

\section{Consequences for (Bio)sensor Applications using High-Amplitude QCM}

Heitmann ${ }^{\left[{ }^{006]}\right.}$ presented a 2-D temperature profile that was simulated by means of the finite element method for a $5 \mathrm{MHz}$ quartz disk with one side exposed to water and operated at $10 \mathrm{~V}$, taking the heat capacities and heat conductivities of the relevant materials and media into account. This is illustrated in Fig. 6-14 A. The temperature simulation resulted in a heating of the water in close proximity to the quartz surface by a maximum of $4.5^{\circ} \mathrm{C}$ in the center of the electrode. Though it is slightly higher, this theoretical value is approximately in the order of the experimentally determined temperature increase presented above.

On the basis of the axial and lateral temperature gradient of the simulated profile in Fig. $6-14 A$, the thermally-driven convection (thermophoresis) and velocity profile within the measurement vessel was simulated. The calculations were performed by Prof. Dieter Braun (Systems Biophysics, LMU Munich). The results are visualized in Fig. 6-14 B. There is a distinct convection current above a quartz crystal that resonates at $10 \mathrm{~V}$ and thereby generates local heat on its surface. In the center of the quartz, where the 
frictional heat of the oscillation and warming of the water is maximal, an upward liquid flow arises that is perpendicular to the quartz surface. This induces two oppositely directed lateral liquid flows. One occurs close to the quartz surface directed from the edges to the center, symmetrically from all sides. The second stream is generated at a distance of about $3 \mathrm{~mm}$ above the surface directed from the upflowing liquid in the center to the periphery of the vessel (ambient temperature is assumed at a distance of $4 \mathrm{~mm}$ above the crystal). Both streams in parallel to the surface possess a maximum velocity of approximately $8 \cdot 10^{-4} \mathrm{~m} \cdot \mathrm{s}^{-1}$. The liquid sinks onto the quartz surface at the edge of the measurement chamber, where the thermally-driven liquid circulation begins again. This thermophoretic fluid circulation and buoyancy force above the quartz center have to be taken into account when the interaction of suspended small molecules, particles, or cells with the surface of the microbalance are studied, as it considerably counteracts the rate of sedimentation.

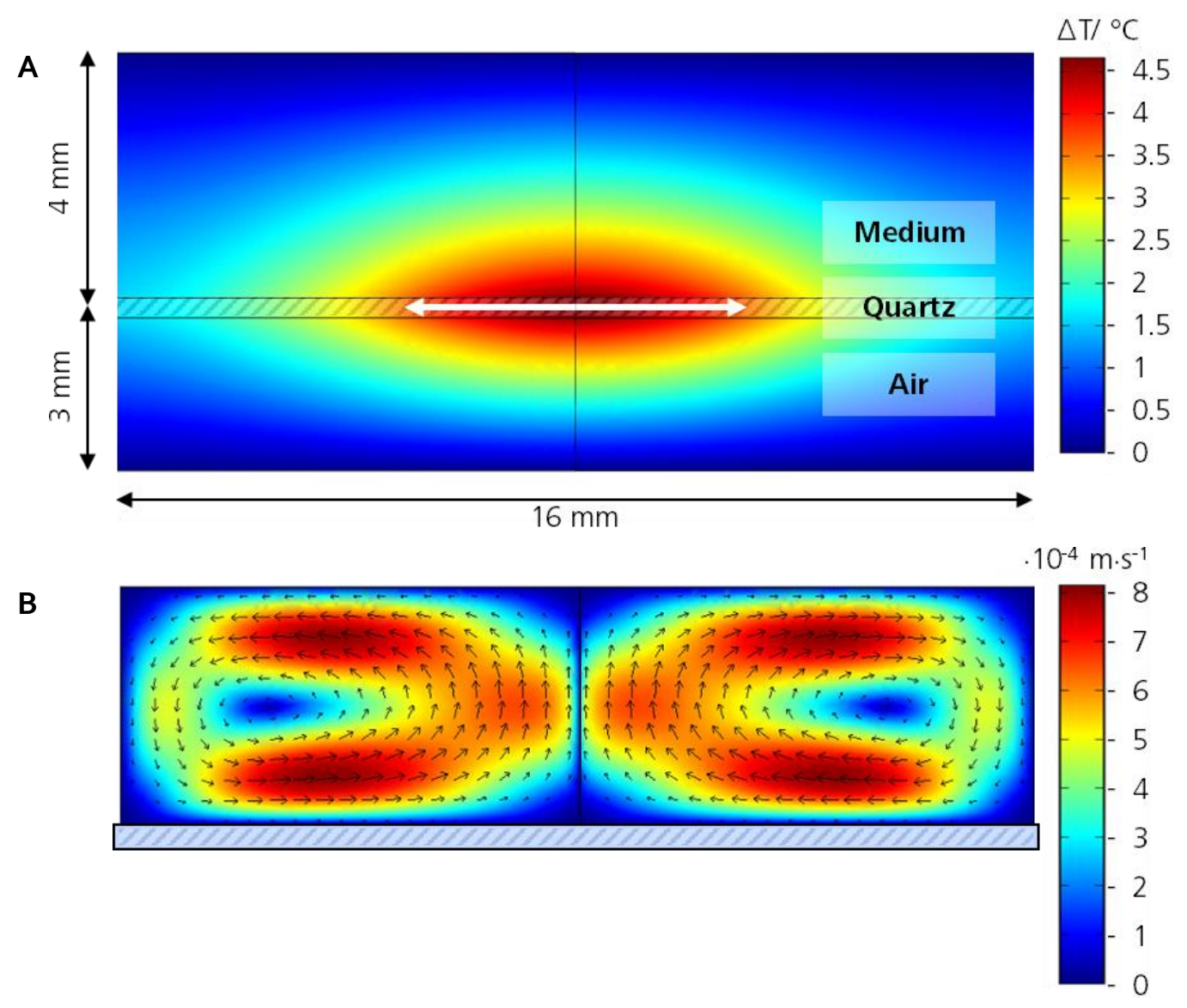

Fig. 6-14 Simulated profiles of (A) surface temperature and (B) induced convection (thermophoresis) in the liquid of an AT-cut $5 \mathrm{MHz}$ quartz crystal, whose top side is immersed in water and which is forced to oscillation at resonance with a $10 \mathrm{~V}$ driving voltage. Calculations were performed by Prof. Dieter Braun (Systems Biophysics, LMU Munich), A is adapted from [706].

In the following the convection velocity above the resonator and the rate of sedimentation of mammalian cells that might be injected into such a OCM chamber are opposed, and the consequences of the balance of forces for monitoring and analyzing the properties of cell attachment and spreading on the microbalance surface are discussed. In general, the sedimentation rate of a particle in a viscous fluid is determined by the gravitation force and the opposing viscosity force and buoyancy force. The effective gravitational force $\overrightarrow{F_{G}}$, considering the buoyancy force, is given by: 


$$
\overrightarrow{F_{G}}=\left(\rho_{p}-\rho_{f}\right) \cdot V_{p} \cdot \vec{g}
$$

where $\rho_{p}$ and $\rho_{f}$ are the densities of the particle and the fluid, respectively, $V_{p}$ is the volume of the particle, and $\vec{g}$ is the gravitational acceleration $\left(g=9.81 \mathrm{~m} \cdot \mathrm{s}^{-2}\right)$. The counteracting force of viscosity (frictional force) $\overrightarrow{F_{f}}$ of a small spherical particle in a viscous fluid is described by the Stokes' law:

$$
\overrightarrow{F_{f}}=6 \pi \cdot r_{p} \cdot \eta_{f} \cdot \overrightarrow{v_{p}}
$$

where $r_{p}$ is the radius of the particle, $\eta_{f}$ is the viscosity of the fluid, and $\overrightarrow{v_{p}}$ is the velocity of the particle. In the case of floating, the force of viscosity is equal to the gravitational force $\left(\overrightarrow{F_{G}}=\overrightarrow{F_{f}}\right)$. The combination of Eq. (69) and Eq. (70) provides for spherical particles $\left(V_{p}=4 / 3 \cdot r_{p}^{3} \pi\right)$ the magnitude $v$ of the sedimentation velocity by:

$$
v_{p}=\frac{2}{9} \cdot \frac{\left(\rho_{p}-\rho_{f}\right)}{\eta_{f}} \cdot r_{p}^{2} \cdot g
$$

Using Eq. (71), we can appraise the sedimentation velocity of suspended (spherical) mammalian cells in the gravity field of the earth through the liquid onto the resonator surface. The densities of mammalian cells at physiological conditions are in the range of 1.05 and $1.10 \mathrm{~g} \cdot \mathrm{cm}^{-3}$, and their radii are between 2.5 and $12 \mu \mathrm{m} \cdot{ }^{[707]}$ The density of water at $37^{\circ} \mathrm{C}$ is $993.3287 \mathrm{~kg} \cdot \mathrm{m}^{-3}{ }^{[708]}$ and the viscosity of water was calculated to $6.917 \cdot 10^{-4} \mathrm{~kg} \cdot \mathrm{m}^{-1} \cdot \mathrm{s}^{-1}\left(T=37^{\circ} \mathrm{C}\right){ }^{[708,709]}$. By choosing a cell density of $1.05 \mathrm{~g} \cdot \mathrm{cm}^{-3}$ and by taking an average cell radius of $10 \mu \mathrm{m}$, one obtains a cell sedimentation rate $v_{p}$ of approximately $1.8 \cdot 10^{-5} \mathrm{~m} \cdot \mathrm{s}^{-1}\left(=1.1 \mathrm{~mm} \cdot \mathrm{min}^{-1}=6.4 \mathrm{~cm} \cdot \mathrm{h}^{-1}\right)$, according to Eq. (71). Hence, cell sedimentation competes against a $10-40$ times higher vertical convection above the center of the resonator, which is according to Fig. $6-14 \mathrm{~B}$ in the range of $2-7 \cdot 10^{-4} \mathrm{~m} \cdot \mathrm{s}^{-1}$. On the basis of these calculations it is straightforward to conclude that cell sedimentation and, consequently, the attachment and spreading of cells on the resonator surface is significantly impaired at elevated oscillation amplitudes. For QCM driving voltages of $10 \mathrm{~V}$ and shear amplitudes of $35 \mathrm{~nm}$, respectively, a complete prevention of cell attachment is assumed. With decreasing voltage amplitudes less surface heat due to internal friction of the quartz is generated, resulting in a lower local heating of water, a lower temperature gradient inside the liquid reservoir, less convection, an increasing dominance of the gravitational force with respect to the buoyancy force (including vertical convection) and, thus, an increasing probability for a successful attachment of suspended mammalian cells on the surface.

These findings agree with the experimental data reported by Heitmann et al. [226], who "observed a significant retardation of cell adhesion when the driving voltage was raised from non-invasive 0.15 to $5 \mathrm{~V}$ or higher". Based on the above results and remarks, the impeded cell adhesion, however, is likely not (only) a direct effect of the elevated lateral oscillation amplitude of the growth surface that prohibits cellsubstrate interactions. It is more likely an indirect effect of the elevated oscillation amplitudes, arising from an increased frictional heat inside the quartz material and causing thermophoresis in the measurement vessel that opposes the cell sedimentation process. Thus, the cells are not able to establish focal adhesions to the growth surface, since they can hardly elude the flow and are permanently flushed away from the surface. However, cell attachment seems to be slightly favored away from the main flow at the edges and in the direct center of the quartz, where the convection velocity is significantly reduced (dark blue areas in Fig. 6-14 B). Moreover, one can see from Eq. (71) that the sedimentation rate $v_{p}$ of a particle increases with increasing density $\rho_{p}$ and predominantly with increasing size, as $v_{p}$ increases with 
the square of the particle radius $r_{p}$. This might explain the observations made by Heitmann et al. ${ }^{[226]}$ who detected to a certain degree the attachment of $3^{T} 3$ cells on a quartz surface, despite high-amplitude oscillations and the associated frictional heat and fluid convection, in contrast to the $\sim 40 \%$ smaller MDCK-II and NRK cells which seem to attach not at all. Making the local heat and the convection responsible for the experimentally observed phenomena is supported by the statement of Edvardsson et al. ${ }^{6344}$, who argue for the size of a particle being the determining factor rather than the nature and strength of specific biorecognition reactions for surface binding at a certain amplitude of oscillation.

Based on the size-dependence of the sedimentation rate of a particle (Eq. (71)), the effect of the counteracting convection becomes more important in surface-interaction studies and (bio)sensing of particles that are smaller in size than mammalian cells, such as bacteria, vesicles, nanobeads, DNA sequences, antibodies, and enzymes. This counts for studies where the interaction is suppressed by highamplitude $\mathrm{OCM}{ }^{[633,634]}$ as well as for studies of REVS ${ }^{\mathrm{TM}}{ }^{\left[619-63^{22}\right]}$. Concerning the latter, the effect of a bulk temperature increase due to the frictional heat of high-amplitude OCM was already considered as too low to cause bond breakage on the sensor surface. ${ }^{[619,636]}$ However, the event of bond breakage occurring at elevated shear amplitudes might be promoted by the local heat and the induced convection across the surface (lateral force acts on binding). This has not been taken into account in the literature to date.

\subsubsection{Imaging the Oxygen Consumption Rate (OCR) beneath Adherent Cells}

\section{General Remarks}

We demonstrated a new sensor layout that enables the time-resolved monitoring and two-dimensional imaging of DO in a cell culture vessel and in immediate proximity to cultured adherent cells, as the indicator dye is directly integrated into the growth substrate. The sensing principle is based on oxygensensitive phosphorescent dye molecules and the technology of fluorescence (phosphorescence) lifetime imaging (RLD), which features several benefits with respect to luminescence intensity-based measurements. Lifetime-based sensing is much less susceptible to interference as for instance background fluorescence, inhomogeneity of the excitation light, and an inhomogeneous distribution of dye molecules in the sensor matrix. They therefore promise a better lateral resolution of the sensed analyte. The indicator dyes embedded in a polymer matrix were immobilized as a planar sensor film, also referred to as pressure-sensitive paint (PSP), on cover glasses. Cells were successfully cultivated on these films, since simple and effective procedures for coating and hydrophilization of the PSP films were found, making them highly cytocompatible just like standard tissue culture substrates. The biocompatibility of the PSP substrates were checked microscopically as well as by means of time-resolved OCM measurements of cell spreading kinetics (cf. Fig. 6-8). OCM sensing enables to monitor sensitively and without any labelling the entire process of cell attachment and spreading. It provides meaningful data about both the kinetics and the quality of cell-substrate interactions and, thus, is a valuable tool for testing the cytocompatibility of materials.

There is a large variety of fluorescent dyes that change luminescence properties in dependence of the oxygen concentration ${ }^{[175]}$. In this thesis the platinum porphyrin complex PtTFPP (cf. Fig. 3-23 C) was applied for the phosphorescence-based sensing of oxygen, since it has a sufficiently long lifetime for the FLIM method and since it is well-known for its good response to oxygen, high photostability, and high quantum yield of the triplet energy transfer to oxygen. The PSP show a sensitive and reversible response to changes in the oxygen partial pressure $\left(\mathrm{pO}_{2}\right)$ in the headspace of the sensor in air (Fig. 6-9) as well as 
of a buffer-covered sensor film (Fig. 6-11). In the latter case, the concentration of DO differs from the concentration in air. The solubility of $\mathrm{O}_{2}$ in water depends on the salinity of the liquid, the temperature, and the overall pressure according to Henry's law. The oxygen values presented in this thesis do not quantify the oxygen concentration in the liquid or in the sensor matrix but are expressed relative to the partial pressure (saturation) in the gas phase (in mbar, relative to a total pressure of 1 bar).

The sensor readout shows a significantly higher noise in the images at higher oxygen concentrations, as evident in the calibration plots in Fig. 6-9-Fig. 6-11. This trend becomes even clearer in the calibration plot in Fig. 6-15, which covers oxygen partial pressures from zero up to $1000 \mathrm{mbar}(0-100 \%(\mathrm{v} / \mathrm{v})$ ). However, this observation is hardly surprising, when one considers that the luminescence intensity is more and more quenched with increasing oxygen concentrations. This results in a lowering of the quantum yield of phosphorescence emission and convergence to values of the background fluorescence (cf. $A_{2}\left({ }^{\circ}\right)$ in Fig. 6-15 for $p \mathrm{O}_{2}>500$ mbar). Big standard deviations in $A_{1} / A_{2}$ values (cf. $\mathrm{O}$ in Fig. 6-15 for $p \mathrm{O}_{2}>500 \mathrm{mbar}$ ) and thus in calculated $p \mathrm{O}_{2}$ values arise from individual pixels in $A_{2}$ images that report on completely quenched luminescence and which then exhibit irrational values after background correction and calculation of the $A_{1} / A_{2}$ ratio. This might become a problem for applications with high oxygen partial pressures (levels of approximately $400 \mathrm{mbar}$ and higher), which could be due to either a higher oxygen fraction $x \mathrm{O}_{2}$ or an elevated total pressure $p_{\text {tot }}\left(\mathrm{O}_{2}=x \mathrm{O}_{2} \cdot p_{\text {tot }}\right)$. However, these oxygen partial pressures are more than twice as high as in atmospheric air $\left(\mathrm{O}_{2}=210 \mathrm{mbar}\right.$ at $1013.25 \mathrm{mbar}$ air pressure) and lie far above physiologically relevant concentrations. The measurements presented here were performed at atmospheric pressure in the range between o mbar and $300 \mathrm{mbar} p \mathrm{O}_{2}$, where the sensor film provides almost noise-free images. Moreover, monitoring of the metabolism of microbes and living cells is always related with a decrease of oxygen with respect to the oxygen saturation in liquid, as oxygen is consumed and not produced. The problem of too big quenching rates by oxygen thus does not arise in cell-based applications. The oxygen sensors on the basis of luminescence quenching feature a special property in this context: they become increasingly accurate and more sensitive with a decreasing concentration of the analyte, i.e. an increasing metabolic rate and oxygen consumption by the living organisms.

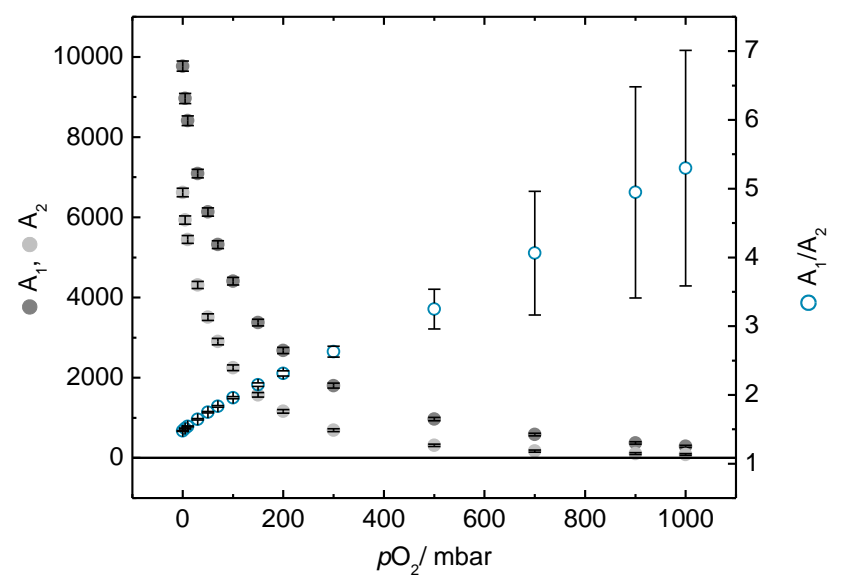

Fig. 6-15 Average $A_{1}(\odot), A_{2}(\odot)$, and $A_{1} / A_{2}(\bigcirc)$ values (mean $\pm \mathrm{SEM} ; \mathrm{N}=7$ ) as a function of the oxygen partial pressure, in the range from zero to 1 bar $\mathrm{pO}_{2}$ (total pressure 1 bar).

In an exemplary measurement an increase in the oxygen consumption rate (OCR) of mammalian cells was stimulated by the addition of the respiratory chain decoupler 2,4-DNP. With the cells grown across 
the entire PSP sensor surface, the time course of the changing DO concentration beneath the cell layer was sensitively monitored and imaged (cf. Fig. 6-12). A strong decrease of the DO amount in $\mathrm{PBS}^{++}$buffer (equilibrated at $37^{\circ} \mathrm{C}$ with atmospheric air) was measured after airtight sealing of the measurement chamber and adding $100 \mu \mathrm{M}$ of the decoupler to the confluent cell layer. A supply of oxygen from the air outside the measurement chamber was excluded, since diffusion of oxygen through the polystyrene material was negligible (diffusion rate of $\mathrm{O}_{2}$ in $\mathrm{PS} \approx 10^{-7} \mathrm{~cm}^{2} \cdot \mathrm{s}^{-1}$ ) ${ }^{[710]}$. The decrease of $\mathrm{O}_{2}$ detected can be directly assigned to the OCR of the cell layer, due to the closed system.

\section{Effect of Temperature on FLIM of $p \mathrm{O}_{2}$}

A very critical issue in luminescence-based oxygen sensing is the control of temperature. There is a distinct dependence of the sensor response on the ambient temperature, with increased $A_{1} / A_{2}$ values with increasing temperature (cf. Fig. 6-10). As shown in section 6.1, the temperature-dependence of luminescence can be utilized for the sensitive measurement of temperature changes (reviewed in ${ }^{[618]}$ ). The increased $A_{1} / A_{2}$ values at higher temperatures but similar $p \mathrm{O}_{2}$ are due to a more effective quenching of the indicator phosphorescence, since, besides the triplet-triplet annihilation of excited dye molecules by molecular oxygen, excited electrical states are additionally depopulated by a thermally-driven radiationless deactivation ( $\rightarrow$ decrease in the average lifetime). Consequently, less excited dye molecules exist so that the quantum yield for the emission of energy in the form of phosphorescence radiation is reduced ( $\rightarrow$ decrease in the average emission intensity; cf. subsection 3.5.1, p. 62 ff.). If one has this temperature effect not in mind and if the PSP is exposed to temperature fluctuations during the measurement because of no temperature control, this could lead to an under- or overestimation of oxygen. For instance, a decrease of the ambient temperature from $37^{\circ} \mathrm{C}$ to $24^{\circ} \mathrm{C}$ of one of the sensor films at a constant $\mathrm{PO}_{2}$ of 200 mbar causes a reduction of the $A_{1} / A_{2}$ ratio from 2.49 to 2.31 (cf. Fig. $6-$ 10). Regardless of the temperature, this reduction would report on a decrease of the oxygen partial pressure to about $150 \mathrm{mbar}$, although this has not changed at all.

Besides the higher $A_{1} / A_{2}$ values, one can also observe from the data in Fig. 6-10 an increase in the noise (SEM) of the average $A_{1} / A_{2}$ pixel values at a defined $p \mathrm{O}_{2}$ with increasing the temperature. This is due to the additional thermal quenching of the luminescence. This has the same effect on the deviation of pixel brightness values like an increase in the oxygen concentration, which was addressed in the previous subsection. The higher the concentration of oxygen molecules is and the more the phosphorescence is already quenched through this, the bigger is the effect of the additional thermal quenching on both the mean value and the variance of pixel values of $A_{1} / A_{2}$ images.

Hence, there is an indispensable necessity for exact temperature control, when applying luminescent probes for sensing other analytes than temperature, such as oxygen for example. It is the same the other way round. For instance, if one wants to analyze a system with respect to temperature while there is a changing total pressure or a varying percentage of oxygen (change in $p \mathrm{O}_{2}$ ), one has to assure that the temperature-sensitive luminescence is not cross-sensitive to triplet-triplet annihilation by oxygen. In such applications, this is solved by default by the encapsulation of the temperature indicator dye into an oxygen-blocking polymer, either in the form of a particle or in a planar sensor film. ${ }^{[176-181,320,326]}$ In addition to that, the introduction of an additional oxygen sensor would be helpful as a reference and control. This underlines that all possibilities of luminescence quenching have to be considered, since otherwise this may lead to a misinterpretation of the measured data. This criteria are met in the presented OCS measurements due the use of a pressure- and temperature-controlled calibration chamber. 


\section{Generation of Reactive Singlet Oxygen}

The microscopic evaluation of the cell layers after oxygen imaging experiments disclose that the cells grown on the PSP film apparently were damaged in the course of FLIM, while cells grown directly on cover glasses exhibited an undamaged cell morphology at the end of experiments (cf. Fig. 6-13). Since the cells initially have grown equally well on the entire sensor surface, independently on whether there was PSP pre-coated or not (Fig. 6-13 A), and since these cells have been equally treated throughout the experiment, damaging of the cells could only result from an energy conversion process in the PSP, i.e. dye excitation (absorbance of light energy), phosphorescence emission (emission of energy in the form of radiation), non-radiative emission (emission of energy in the form of heat), or dynamic quenching by oxygen (energy transfer to oxygen molecules). Except for the last-mentioned process, the photophysical processes taking place do not release energy that is harmful to the cells. However, the energy transferred from excited platinum(II)-porphyrine molecules to ${ }^{3} \mathrm{O}_{2}$ molecules by triplet-triplet annihilation causes the formation of singlet oxygen species ${ }^{1} \mathrm{O}_{2}$ (cf. Jablonski diagram in Fig. $3-21$, p. 62). ${ }^{[71-713]}$ The cytotoxic effects do not arise from energy dissipation of singlet oxygen in the form of radiation or radiationless relaxation but it is rather the high chemical reactivity of singlet oxygen which makes this species cytotoxic. These photosensitized singlet oxygen molecules can react with nearby organic molecules of biological samples, such as proteins, lipids, and nucleic acids, and thereby irreversibly damage essential cellular structures, resulting in cell death (phototoxicity). ${ }^{[14-716]}$ In this context, one also has to think of a possible polymer degradation and affected physical properties (mechanical stability, solubility, hydrophobicity, etc.) of the polymer matrix by the reaction with singlet oxygen. ${ }^{[17-720]}$ Changes in the chemical structure of the polymer due to oxidation by singlet oxygen may alter the polymers' quenching abilities, resulting in a drift in the determination of oxygen. The oxidation of nearby (bio)molecules by singlet oxygen additionally means that the oxygen molecules are consumed during this reaction. The consumption of the analyte triggered by the readout of the sensor, however, is a non-negligible disadvantage of the measurement system, because results are prone to error, i.e. an underestimation of oxygen. ${ }^{[721]}$

Despite the phototoxic effect of the applied phosphorescent dye (via singlet oxygen), a steady decrease in the concentration of dissolved oxygen still could be measured in the experiments presented in subsection 6.2.3. On the one hand this is assigned to the oxygen consumption of the cells directly attached to the PSP sensor film $\left(A=1.13 \mathrm{~cm}^{2}\right)$. Even though phototoxicity causes rounding up of the cells and irreversible cell damage during the assay, the cells do not instantaneously stop metabolism and dye due to the phototoxic stress. It may be assumed that the cells might rather boost metabolism as a counter-reaction to the stress. On the other hand, the respiration of the cells that are attached to the PSP-free areas of the measurement chamber $\left(A=7.91 \mathrm{~cm}^{2}\right)$ also has to be taken into account. These cells are not affected by the cytotoxic effect of singlet oxygen and consume the dissolved oxygen in the bulk solution throughout the assay. Though this respiration of the cells surrounding the sensor surface contributes to the total consumption of DO during the assay, this "bulk effect" is considered as not significant for the OCR monitored at the early stages of the assay. This is almost exclusively assigned to the respiration rate of the cells on the sensor surface, due to the small medium volume between the cells and the sensor and the very short distance for diffusion of oxygen molecules. This allows a very sensitive and fast detection of the OCR of animal cells and surpasses all approaches presented in literature that are based on the detection of oxygen respiration of adherent cells via the concentration of DO in the bulk medium. 
One can think of various approaches to address the problem of reactive and cytotoxic singlet oxygen species, which are generated by photodynamic quenching. Firstly, one could increase the distance between the indicator dyes and cellular structures. This increases the diffusion distance for singlet oxygen molecules and, thus, also the probability of abreaction until they come into the immediate vicinity of the biological sample. For instance, this could be realized by the deposition of an additional polymer layer (without the indicator dye) on top of the PSP film. However, this is accompanied by the disadvantage that the diffusion distance is also increased for triplet oxygen, resulting in a probably slower response time of the sensor and a lower lateral resolution. Ceroni et al. ${ }^{[716]}$ reported on a significantly reduced phototoxicity of "dendritically-protected" PEGylated (polyethylenglycol-coated) porphyrinbased phosphorescent oxygen probes. Enko et al. ${ }^{\left[{ }^{[21]}\right.}$ demonstrated the introduction of stabilizers such as DABCO (1,4-Diazabicyclo $[2,2,2]$ octane) into the polymers, which can act as effective quenchers of singlet oxygen. These enable the deactivation of the reactive species to its triplet ground state via charge transfer or electronic energy transfer processes. ${ }^{[711,722,723]}$ This means that, on the one hand, the phototoxicity for biological samples is reduced and the integrity of the polymer is largely maintained due to fast abreaction of ${ }^{1} \mathrm{O}_{2}$, and, on the other hand, the analyte is also not consumed by a chemical reaction as a consequence of the sensor readout. Besides DABCO ${ }^{[721-725]}$, there is a number of other reported compounds which are able to effectively quench ${ }^{1} \mathrm{O}_{2}$. These include tertiary amines ${ }^{[723,726,727]}$, sulfur compounds ${ }^{[728]}$, nickel(II) complexes ${ }^{[725]}$, azide ${ }^{[729]}$, and typical natural quenchers like vitamin $\mathrm{E}$

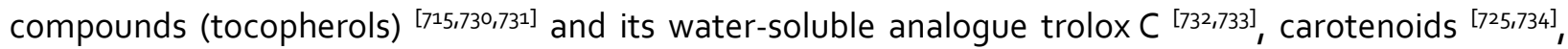
melatonin ${ }^{[715]}$, aloesaponarin I ${ }^{[735]}$, flavonoids ${ }^{[736,737]}$ and tea catechins ${ }^{[738]}$, and others ${ }^{[739]}$. By adding such antioxidants and scavenger of free radicals to the culture medium, these ingredients could intercept reactive species like singlet oxygen before they come into contact with cells and can cause irreversible damage and cell death.

In sum, the presented approach of a cytocompatible planar sensor for the optochemical monitoring of DO and the OCR in cell-based assays significantly differs from any other electrochemical and optical approach presented in literature to date. Firstly, oxygen is not consumed during the measurement. This key feature of the luminescence-based approach makes it superior with respect to electrochemical techniques, where oxygen is consumed in an electrochemical reaction (cf. 1.1.1 Electrochemical Transducers for $L a b e l$-free $C B B$, p. 4 f.). Secondly, the presented method clearly differs from the wide field of particle-based optochemical oxygen sensing in CBAs. There, luminescent (nano)particles are either dissolved in the cell culture medium or introduced into the cells for monitoring the extracellular, intracellular, and/ or pericellular $\mathrm{O}_{2}$ concentration of respiring mammalian cells (cf. introduction of section 6.2, p. 232 f.). These particle-based approaches thus entail the inherent disadvantage that the system for readout directly interfers with the biological system under study. Hence, the cells do not remain in their natural physiological state on the basis of establishing the measuring system alone. This is not the case in the presented planar sensor system, where cells are cultured on a cytocompatible sensor surface and are not challenged with any additives for readout. Since the luminescent probe here is immobilized in a planar sensor matrix and thus is not in direct contact with the cells, this approach is referred to as label-free. Additionally, the generation of reactice singlet oxygen is much more critical directly inside the cells (particle-based sensor) than somewhat farther away in the growth substrate (planar sensor), considering the lifetime of singlet oxygen and the much shorter diffusion distance to the biological system. Finally, the presented sensor films also possess a significant advantage compared to planar optochemical oxygen sensors already presented: they are cytocompatible. Thus, cells directly grow on the sensor surface. Instad of measuring the concentration of DO in the bulk "only", this allows a 
very sensitive and fast monitoring of the OCR of adherent living cells. Moreover, the presented setup also enables the 2-D imaging of local oxygen concentrations in the small volume beneath the cell layer.

\subsubsection{Outlook}

As already mentioned in the previous discussion, temperature control is a critical issue in luminescence quenching-based sensors. Since temperature itself is also an important parameter in cellular assays, the combined integration of TSP and PSP readout spots in the growth substrate for cells would be of great advantage. In this way, there is a thermometer in the immediate vicinity of the cells as temperature control for cell culture conditions on the one hand and for the temperature-correction of oxygen measurements on the other hand. Such dual OCS films for temperature and $p \mathrm{O}_{2}$ have already been described multiple times in the literature ${ }^{[176,177,179,180,740-748]}$, even though not for their application in cellbased assays. The integration of further optodes into the growth substrate of cells for planar multiple OCS is possible as well. These could be implemented either in the form of a sensor array/ multispot sensor

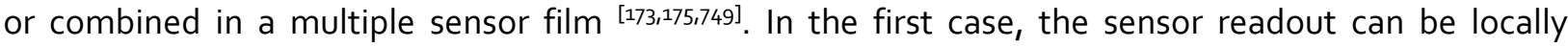
separated, while the latter case requires an optical separation of indicator dye properties (excitation/ emission wavelength, lifetime) in order to distinguishing the sensor responses towards different target analytes. For imaging purposes, like of the temporal and spatial change of analyte concentrations beneath adherent cells, the multiple sensor is the approach of choice. Besides the $\mathrm{O}_{2} / \mathrm{T}$ sensors, layerbased dual OCS were also published for the analyte combinations $\mathrm{O}_{2} / \mathrm{CO}_{2}{ }^{[184,185]}, \mathrm{O}_{2} / \mathrm{pH}^{[186-192]}$, and $\mathrm{O}_{2} /$ glucose ${ }^{[193]}$. A planar triple sensor for the analytes $\mathrm{O}_{2} / \mathrm{T} / \mathrm{pH}^{[194]}$ and the first quadruple OCS film for the simultaneous monitoring of $\mathrm{O}_{2} / \mathrm{T} / \mathrm{pH} / \mathrm{CO}_{2}{ }^{[181]}$ were presented recently, too. However, the readout of the latter sensor was not carried out by an imaging method but by means of two separate fiber-optical detector channels, equipped with different light sources and filters. Imaging of this sensor film would either require two independent imaging systems (i.e. two light sources, two cameras, and different combinations of excitation and emission filters), or, if using only one camera for imaging, one has to switch between two optical readout sets (light source + excitation filter + emission filter) for sensing different analytes, what would be manually quite laborious.

Instead or, even better, in addition to the multiplication of OCS for multi-analyte sensing and imaging, the planar phosphorescent sensor film could be coated on the surface of a piezoelectric quartz crystal. This would combine the optical sensors with the independent sensor principle of acoustic resonators and of the quartz crystal microbalance (OCM; cf. section 3.3), respectively. This multi-sensoric platform then would enable the label-free monitoring of the mechanical cell-substrate interactions and of viscoelastic changes at the sensor surface in the course of cellular assays, in addition to the simultaneous imaging of parameters like $\mathrm{O}_{2}, \mathrm{pH}$ and temperature. This approach of high-content screening of cell-based assays could even be further advanced by the replacement of classical OCM sensors with the double-spot QCM sensors or the OCM-ECIS sensors that are introduced in this thesis in Chapter 4 and Chapter 5 , respectively. By this, the readout spots of OCM measurements were doubled and, in addition to the optical and acoustic sensor principles, an electrochemical transducer platform was integrated into the growth substrate for the complementary electric cell-substrate impedance sensing (ECIS; cf. section 3.4 and Chapter 5). Thus, time-resolved information on cell adhesion, cell morphology changes, epithelial barrier function, and cytotoxicity of substances or other stimuli are accessible. The additional OCM and ECIS sensors allow to monitor the cell vitality and eventual rounding up and detachment of cells during FLIM measurements and, thereby, could replace the microscopic control of the cell layer quality. The integration of ECIS also opens the door to all ECIS-related sensor and actuator approaches, like the 
monitoring of micromotion and cell migration or the introduction of xenobiotics into cells by electroporation (ELPO; cf. subsections 3.4 .5 and 5.3.4). Potential applications and further technologic developments regarding the double-spot QCM sensors and the OCM-ECIS sensors, including sensor parallelization and the use of transparent electrodes, it is referred to the respective outlook sections in Chapter 4 (4.3.3, p. 109 ff.) and Chapter 5 (5.4.12, p. 218 ff.).

Regarding the experimental setup of FLIM of substrate-embedded sensor films, one could think of an inversion. In the presented setup (Fig. 3-25 and SI 7), an upright microscope is used for FLIM microscopy of the PSP, equipped with a 5 -fold objective (working distance $=14.5 \mathrm{~mm}$ ) for magnification. However, objectives with higher magnification for a better lateral resolution could not be used, as the working distances were too small for focusing the PSP film on the bottom of the petri dish. To overcome this limitation, one could either build a shallower chamber, or use objectives with an extra-long working distance, or exchange the upright microscope against an inverse one. Using an inverse microscope comprises the big advantage to get quite close to the cells and their growth substrate, which is the sensor film in our case. Another improvement would be the coupling of the excitation light directly through the objective. This would make the light source (LED) opposite of the objective redundant and would increase the working space on top of the measurement chamber. Moreover, optical effects like scattering, absorption, and reflection of the excitation light by the lid of the chamber, the culture medium above the cells, and by the cell layer itself could be avoided in this way. Such effects have to be considered especially when using an excitation light of small wavelength in the UV region. 


\section{Chapter 7 \\ Summary/ Zusammenfassung}

7.1 Summary

254

7.2 Zusammenfassung

257 


\subsection{Summary}

Various approaches have been pursued on the basis of electrochemical or piezoelectric transducers, particularly of the quartz crystal microbalance (OCM), to monitor non-invasively and in real-time cellular states and reactions with substrate-embedded sensors. On the one hand, these comprised the technical development of piezoelectric sensors with multiple read-out spots and the integration of additional noninvasive (electro- and optochemical) sensor technologies on the OCM surface. On the other hand, a variety of studies and cell-based assays (CBAs) have been performed in order to test the sensor performances and to gain a deeper understanding of the sensors' readout parameters with respect to their information content about the biophysical properties and the metabolic behavior of cells. Fig. 7-1 presents an overview of the different projects on the basis of OCM sensor disks presented in this thesis.

In the first project (Fig. 7-1 A) a novel electrode layout was designed on the basis of commercial $5 \mathrm{MHz}$ AT-cut quartz disks to implement two independent readout spots on the QCM surface. This also comprised the construction of new measurement chambers for the electrical actuation and sensing of quartz oscillations. These two-electrode $\mathrm{OCM}$ sensors (2EIO) are also referred to as multichannel $\mathrm{OCM}$ (MOCM).

The developed MOCM sensor spots on one quartz disk exhibited a strong interference, even though they were operated sequentially, which is in contrast to the results of previous theoretical calculations. The resonances could be successfully decoupled by coating half of the quartz surface and one sensor spot, respectively, with a thin and rigid film of photoresist. This quartz loading with mass caused a shift in the resonance spectra of the coated resonator to lower frequencies and efficient decoupling. The operation of the decoupled MOCM sensors demonstrated both, a sensitive and equal change in the oscillation characteristics of the two resonators upon loading of the quartz with medium. The $Q$-factor was not significantly different for the two resonators, qualifying the MOCM for its application in CBAs.

Building on the preceding development of the double-electrode quartz disks, a novel electrode layout has been realized at the sensor surface, which enables the complementary electrochemical (impedance spectroscopical) characterization of the substrate-liquid interface in addition to its mechanical characterization by the piezoelectric transducers (Fig. 7-1 B). This layout was achieved by removing a small area of the insulating photoresist on the coated electrode in the photolithographic process. By this, a coplanar electrode arrangement of a small working electrode and a bigger counter electrode was created. This sensor combination on the basis of the MOCM is an improvement of the so-called $2^{\text {nd }}$ generation $Q C M$ to what we call the $3^{\text {rd }}$ generation $Q C M$, and which is also referred to as OCM-ECIS.

Various electrode layouts, varying in size and number of the working electrode(s), were fundamentally characterized microscopically and by profilometry regarding the geometrical properties and by means of impedance spectroscopy with respect to the sensing performances in OCM- and ECIS-mode. An optimal electrode layout was identified and defined as standard for subsequent applications in CBAs. In both OCM- and ECIS studies of cell-covered sensor surfaces significant changes in the characteristic sensing parameters with respect to the cell-free electrodes are measurable. In addition to the measurement of absolute signal changes, the transducer technologies of OCM and ECIS also enable to monitor the kinetic changes of the readout parameters with high temporal resolution. This allows to use 
the dual sensors for monitoring and analyzing the states of adherent cell cultures in any kind of assay, label-free, non-invasively, and in real-time.

Mechanical (OCM-mode) and the dielectric (ECIS-mode) characteristics of cell adhesion were simultaneously measured for two different cell lines (MDCK-II and NRK), with high reproducibility for each. The total and kinetic parameter changes in both sensing modes distinguished clearly and were specific for the cell lines under test. The signals from both OCM-mode and ECIS-mode recordings also reported on significant impacts of the presence/ absence of bivalent cations $\left(\mathrm{Ca}^{2+}, \mathrm{Mg}^{2+}\right)$ on the attachment and spreading kinetics and behavior of MDCK-II cells. Aside from cell adhesion studies, the cytomechanical and cell morphological reactions towards various stimuli were monitored and analyzed by QCM-ECIS in a multitude of cellular assays: systematic softening and stiffening of cells (using agents for disassembling the actin cytoskeleton and cross-linking protein structures, respectively), intracellular stimulation (using a second messenger analogue), as well as electrical manipulation (electroporation (ELPO) and wounding) of cell layers (applying invasive voltage pulses). The applicability of electrical actuation and the subsequent non-invasive, time-resolved, and dual sensing with the electrodes of the OCM-ECIS substrates has been successfully demonstrated.

The monitoring of CBAs with the dual QCM-ECIS sensor chips developed in this thesis provides not only a multiplication of the information gain due to the complementarity of OCM and ECIS readout parameters. The simultaneous, time-resolved measurements also enable the kinetic correlation of the sensor signals in novel 2-D and 3-D diagrams, which offers the hitherto unprecedented opportunity for a more detailed view and analysis of the coherence or consecutiveness of mechanical and morphological/ dielectric changes of a cell layer under study.

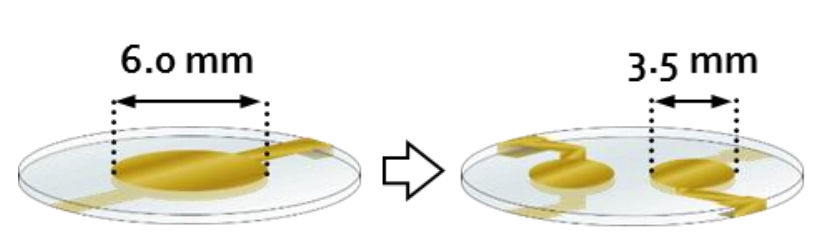

MOCM

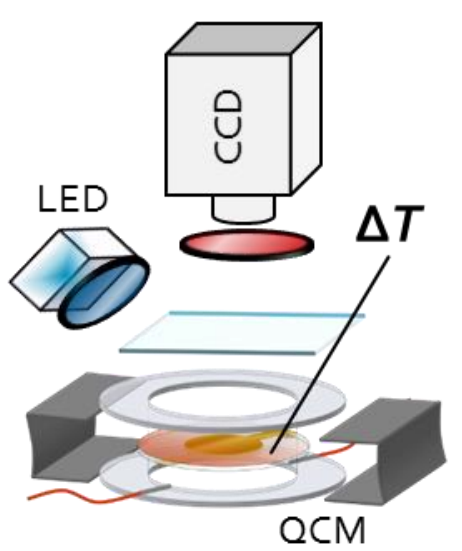

A

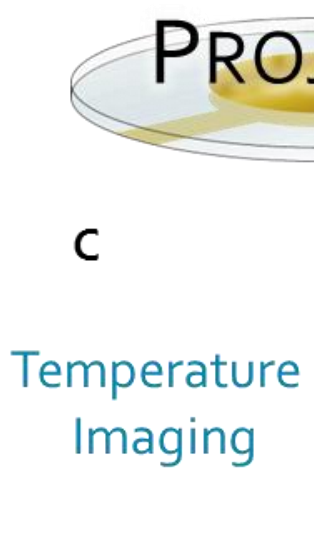

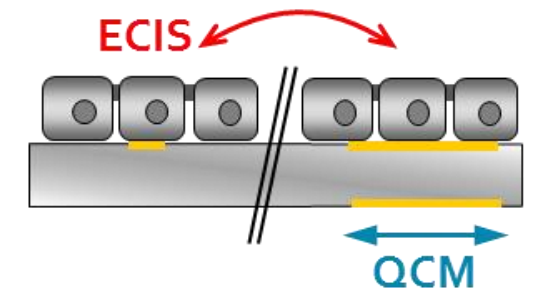

B

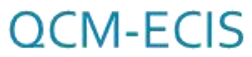

Fig. 7-1 Projects on the basis of QCM resonators, presented in this thesis. 
A third research project focused on the combination of optical-chemical sensors (OCS) with the piezoelectric (OCM) sensor technology. For this purpose, the quartz crystal surface was coated with a polymer film with embedded phosphorescent indicator dye for the target analyte. The luminescence properties were measured by means of fluorescence (phosphorescence) lifetime imaging (FLIM).

By using a temperature-sensitive paint (TSP), an increase in temperature on the sensor surface upon high-amplitude oscillations was monitored and imaged this way in one project (Fig. 7-1 C). Based on this experimentally determined local heating on the OCM surface and the thereby generated temperature gradient in the liquid above the resonator, a thermophoretic convection in the fluid has been simulated. Theoretical considerations showed that the convection profile in the measurement vessel counteracts and even largely prevents the sedimentation of cells onto the sensor surface. It is suggested that the effect of thermophoresis is crucial especially in studies of biomolecular interactions on QCM surfaces at elevated shear amplitudes and driving voltages, respectively, which however has not been considered in literature to date.

The phosphorescence quenching capability of oxygen was utilized in a second imaging project to monitor and image the local concentration and distribution of oxygen on the growth substrate of cells by means of a so-called pressure(/oxygen)-sensitive paint (PSP) (Fig. 7-1 D). A home-made experimental setup was constructed for sensor calibration and the imaging of subcellular oxygen, consisting of a FLIM setup coupled to an upright microscope and a temperature- and oxygen-controlled calibration and measurement chamber suitable for cellular applications. The cytocompatible sensor films have been characterized under various test conditions (in air, under medium, at different temperatures) regarding their sensitivity and response characteristics to different oxygen partial pressures. The oxygen consumption of cells adherently grown on the sensor film was successfully monitored and imaged by this setup. The time-resolved measurements demonstrated a significantly faster consumption of oxygen of a cell layer stimulated with a respiration chain decoupler compared to an unstimulated control cell layer.

Taken together, various technical improvements of piezoelectric sensors (OCM) have been realized (MOCM, OCM-ECIS, ELPO-OCM-ECIS, OCM-OCS), which provide a significant information gain in cellbased applications. The sensors developed enable the high-content screening (HCS) of adherent cell lines in a wide range of assay formats and provide complementary physico-chemical information for obtaining a more complete picture of the state of cells and their reactions in contact to diverse stimuli. All sensor techniques share the characteristics of time-resolved, label-free, and non-invasive monitoring. This allows to disclose and analyze even the kinetics, delayed effects, recoveries, and fluctuations of physicochemical alterations of a studied cell layer, in addition to the absolute parameter changes, which is a valuable improvement compared to classical endpoint assays. The approach of combined, independent sensor systems also provides the novel possibility to bring parameters obtained by the different readout technologies from one cell layer in a temporal correlation, by which new insights into physiological relationships are possible. 


\subsection{Zusammenfassung}

Auf Basis von piezoelektrischen und elektrochemischen Signalwandlerprinzipien, im Speziellen der Quarzmikrowaagen-Technik (OCM), wurden verschiedene Ansätze verfolgt, um mittels in das Wachstumssubstrat integrierter Sensoren zelluläre Zustände und Reaktionen zeitaufgelöst und nichtinvasiv zu verfolgen. Diese umfassten zum Einen die technische Weiterentwicklung der bestehenden piezoelektrischen Sensorsysteme hinsichtlich Multiplizierung und Integration weiterer nicht-invasiver (elektrochemischer und optisch-chemischer) Sensortechnologien in die OCM-Oberfläche. Zum Anderen wurden zahlreiche Studien und zell-basierte Assays mit den entwickelten Systemen durchgeführt, um sowohl die Sensorperformance zu testen als auch ein tieferes Verständnis über die ausgelesenen Sensorparameter hinsichtlich deren Informationsgehalt über die biophysikalischen Eigenschaften und das metabolische Verhalten von Zellschichten zu erhalten. Fig. 7-1 gibt einen Überblick über die in dieser Arbeit vorgestellten Projekte.

In einem ersten Entwicklungsschritt wurde auf der Basis von kommerziell erhältlichen $5 \mathrm{MHz}$ AT-cut Quarzplättchen ein neues Elektroden-Layout entworfen um zwei unabhängige Auslesespots auf der Quarzoberfläche zu generieren (Fig. 7-1 A). Dies beinhaltete auch die Konstruktion neuer Messkammern für die elektrische Kontaktierung der entsprechenden Elektrodenpaare auf dem Kristall. Diese DoppelElektroden OCM Sensoren (2EIO) werden auch als "Multichannel OCM" (MOCM) bezeichnet.

Entgegen vorheriger theoretischer Berechnungen wiesen die entwickelten MOCM-Sensoren selbst bei sequenziellem Betrieb eine Interferenz auf. Durch Belackung einer Hälfte der Quarzoberfläche bzw. einer der beiden OCM-Spots mit einem dünnen und rigiden Polymerfilm gelang es, die Resonanzen zu entkoppeln. Diese Massenbeladung resultierte in einer Resonanzverschiebung des entsprechenden Oszillators hin zu kleineren Frequenzen und damit in einer effizienten Entkopplung. Das Betreiben der entkoppelten MOCM Sensoren zeigte, dass bei Beladung des Quarzes mit Medium beide Sensorspots sensitiv und in gleichem Ausmaß ihre Schwingungscharakteristik verändern. Der Qualitätsfaktor der beiden Oszillatoren kann als gleich angesehen werden, wodurch sich die MOCM-Sensoren für Anwendungen in zellbasierten Assays qualifizieren.

Aufbauend auf den entwickelten Doppel-Elektroden Quarzen wurde auf der Sensoroberfläche ein Elektrodenlayout verwirklicht, das neben der mechanischen Charakterisierung der Substrat-Medium Grenzfläche durch die piezoelektrischen Signalwandler eine zusätzliche, komplementäre elektrochemische (impedanzspektroskopische) Charakterisierung ermöglicht (Fig. 7-1 B). Dieses Layout wurde durch das photolithographische Freilegen eines kleinen Bereiches der mit isolierendem Photopolymer beschichteten Elektrode erreicht. Hierdurch entsteht eine koplanare Anordnung aus einer kleinen Arbeitselektrode und einer größeren Gegenelektrode. Dieser kombinierte Ansatz auf Basis der MOCM ist eine Weiterentwicklung der 2. OCM-Generation zur 3. OCM-Generation und wird auch als "OCMECIS" bezeichnet.

Verschiedene Elektrodenlayouts, variierend in der Größe und Anzahl der Arbeitselektroden, wurden grundlegend charakterisiert, sowohl mikroskopisch und profilometrisch in der Abmessung der integrierten Arbeitselektrode, als auch impedanzspektroskopisch in deren sensorischen Leistungsfähigkeiten im OCM- und ECIS-Modus. Basierend auf diesen Messungen wurde ein optimales 
Elektrodenlayout ermittelt und als Standard für nachfolgende Anwendungen in zellbasierten Assays definiert. Sowohl in den OCM- als auch in den ECIS-Messungen von mit tierischen Zellen bewachsenen Sensoroberflächen sind signifikante Messsignale gegenüber dem zellfreien Zustand detektierbar. Neben dem Messen absoluter Signale lassen sich mit beiden Technologien auch die kinetischen Änderungen charakteristischer Sensorparameter in hoher zeitlicher Auflösung verfolgen. Dies ermöglicht den Einsatz dieser dualen OCM-ECIS Sensoren für die markierungsfreie, nicht-invasive und zeitaufgelöste Überwachung und Zustandsanalyse von Zellschichten in allen erdenklichen Assays mit adhärenten Zellkulturen.

Es wurden simultan die mechanischen (OCM-Modus) als auch die dielektrischen (ECIS-Modus) Adhäsionscharakteristiken zweier unterschiedlicher Zelllinien (MDCK-II und NRK) jeweils mit hoher Reproduzierbarkeit gemessen. Die dabei erhaltenen Absolutwerte und Kinetiken der Sensorparameter unterschieden sich signifikant und waren zelltyp-spezifisch. Die Signale aus Messungen im OCM- und ECIS-Modus zeigten auch deutliche Einflüsse der An-/ Abwesenheit bivalenter Kationen $\left(\mathrm{Ca}^{2+}, \mathrm{Mg}^{2+}\right)$ auf die Kinetik und das Verhalten von Adhäsion und Spreitung von MDCK-II Zellen auf. Neben diesen Zelladhäsions-Untersuchungen wurden in einer Vielzahl zellulärer Assays die zytomechanischen und zellmorphologischen Reaktionen auf unterschiedliche Stimuli untersucht: systematisches Erweichen und Versteifen von Zellen (durch Agenzien, die das Aktin-Zytoskellet abbauen bzw. Proteinstrukturen quervernetzen), intrazelluläre Stimulation (mittels eines sekundären Botenstoff-Analogons), sowie gezielte elektrische Manipulation (Elektroporation (ELPO) und Verwundung) von Zellschichten (durch Applizieren invasiver Spannungspulse). Die Anwendbarkeit der elektrischen Aktorik und anschließender nicht-invasiver, zeitaufgelöster und dualer Sensorik mit den Elektroden der OCM-ECIS Substrate wurde erfolgreich demonstriert.

Das Überwachen zellbasierter Assays mit den in dieser Arbeit entwickelten dualen OCM-ECIS Sensorchips führt nicht nur zu einer Multiplikation des Informationsgewinnes durch die Komplementarität der OCM- und ECIS- Ausleseparameter. Die simultanen, zeitaufgelösten Messungen ermöglichen darüber hinaus auch die kinetische Korrelation der Sensorsignale in neuartigen 2D- und 3D-Diagrammen, was bis dato noch nicht dagewesene Möglichkeiten zur Analyse kohärenter oder sequentieller mechanischer und morphologischer/ dielektrischer Änderungen der untersuchten Zellschichten bietet.

In einem dritten Forschungsschwerpunkt wurde die Kombination von optisch-chemischen Sensoren (OCS) mit den piezoelektrischen OCM Sensoren näher untersucht. Hierzu wurde die Quarzoberfläche mit einem Polymerfilm beschichtet, in dem ein phosphoreszierender Indikatorfarbstoff für den Zielanalyten eingebettet ist. Die Lumineszenzeigenschaft wurde mittels "fluorescence (phosphorescence) lifetime imaging" (FLIM) ausgelesen.

Mit Hilfe eines temperatursensitiven Anstriches (TSP) konnte in einem Projekt auf diese Weise die Erhöhung der Oberflächentemperatur auf dem Sensor, erzeugt durch Quarzoszillationen bei hoher Amplitude, überwacht und abgebildet werden (Fig. 7-1 C). Auf Basis dieser experimentell bestimmten lokalen Erwärmung auf der OCM Oberfläche und des daraus resultierenden Temperaturgradienten in der Flüssigkeit über dem Quarz wurde eine thermophoretische Konvektion in der Flüssigkeit simuliert. Theoretische Abschätzungen ergaben, dass dieses Konvektionsprofil der Sedimentation von Zellen auf die Sensoroberfläche entgegenwirkt und diese sogar weitgehend verhindert. Es wird angenommen, dass diese Thermophorese vor allem auch bei biomolekularen Interaktionsstudien auf OCM-Substraten bei erhöhten Scheramplituden einen maßgeblichen Einfluss hat, der jedoch bisher nicht in Betracht gezogen wurde. 
In einem zweiten Imaging-Projekt (Fig.7-1D) wurde die Phosphoreszenzlöschungseigenschaft von Sauerstoff ausgenutzt, um mittels eines sauerstoffsensitiven Anstriches (PSP) auf dem Wachstumssubstrat von Zellen die lokale Sauerstoffkonzentration und -verteilung bildgebend zu messen. Für die Kalibration der Sensorfilme und das subzelluläre Sauerstoff-Imaging wurde eigens ein experimenteller Aufbau, bestehend aus einer an ein Epifluoreszenzmikroskop gekoppelten Imaging-Apparatur und einer für Zellanwendungen geeigneten thermostatisierbaren und Sauerstoff-kontrollierten Kalibrations- und Messkammer, entwickelt. Die zytokompatiblen Polymerfilme wurden unter verschiedenen Testbedingungen (an Luft, unter Medium, bei unterschiedlichen Temperaturen) hinsichtlich ihrer Sensitivität und dem Ansprechverhalten auf sich ändernde Sauerstoffpartialdrücke charakterisiert. Mit diesem Setup wurde erfolgreich der Sauerstoffverbrauch von auf dem Sensorfilm kultivierten adhärenten Zellen verfolgt und bildgebend gemessen. Die zeitaufgelösten Messungen zeigten einen signifikant schnelleren Sauerstoffverbrauch einer mit einem Atmungskettenentkoppler stimulierten Zellschicht im Vergleich zu einer nicht stimulierten Kontroll-Zellpopulation.

Zusammengefasst wurden verschiedene messtechnische Erweiterungen von piezoelektrischen Sensoren (OCM) erreicht (MOCM, OCM-ECIS, ELPO-OCM-ECIS, OCM-OCS), die erhebliche Zugewinne an Information in zellbasierten Anwendungen ermöglichen. Die entwickelten Sensoren machen ein multiparametrisches Screenen von adhärenten Zelllinien in vielzähligen Assayformaten möglich und liefern komplementäre physikochemische Informationen, um ein umfassenderes Bild über den Zustand von Zellen und deren Reaktionen auf unterschiedlichste Stimuli zu erhalten. Alle Sensorprinzipien teilen die Charakteristiken der zeitaufgelösten, label-freien und nicht-invasiven Messung. Dies erlaubt, im Gegensatz zu den klassischen Endpunktassays, neben absoluten Änderungen von Parametern auch die Kinetiken, verzögerte Effekte, Erholungen und Schwankungen in den Änderungen physikochemischer Eigenschaften der untersuchten Zellschichten aufzulösen und zu analysieren. Der Ansatz der SensorKombination bietet zudem die neuartige Möglichkeit, die durch die verschiedenen Auslesetechniken von einer Zellpopulation erhaltenen Parameter in zeitliche Korrelation zu bringen, wodurch neue Einblicke in physiologische Zusammenhänge möglich sind. 



\section{Appendix}

$\begin{array}{lll}\text { A } & \text { Supplementary Information (SI) } & 262 \\ \text { B } & \text { Abbreviations, Acronyms, and Symbols } & 281 \\ \text { C } & \text { List of Special (Bio)Chemical Reagents } & 284\end{array}$ 


\section{A Supplementary Information (SI)}

SI 1 Frequency dependence of AT-cut $\left(35^{\circ} 10^{\prime}\right)$ quartz disks on temperature an changes in the cutting angle ${ }^{[282]}$

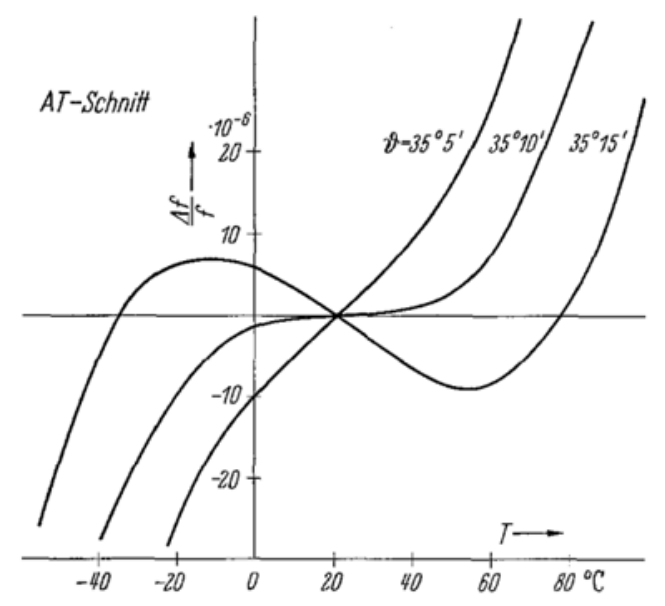

SI 2 Longitudinal piezoelectric effect

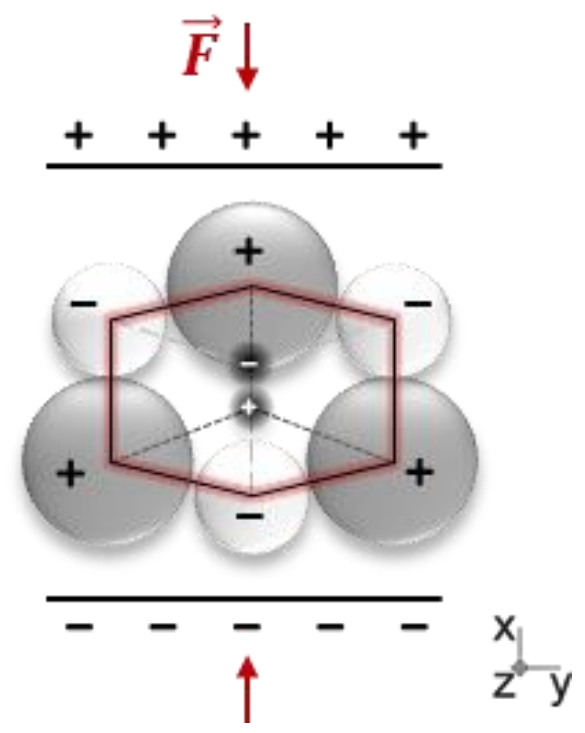

SI 3 Sauerbrey relationship between adsorbed rigid mass layer and resonance frequency shift

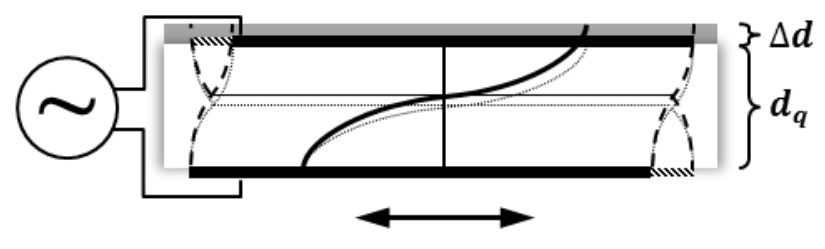


SI 4 Exponential damping of the shear wave in a viscous liquid

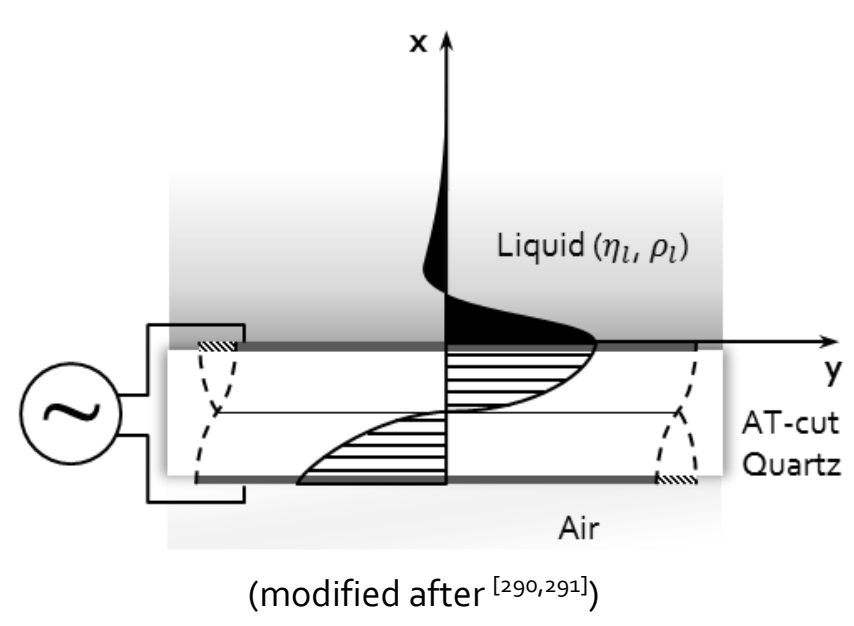

SI 5 The complex impedance of a BVD circuit in 3-D plots. (A), (C) 3-D Bode plots and (B), (D) 3-D Nyquist plots of an (A), (B) undamped and a (C), (D) damped BVD oscillator; $L_{q}=0.0467 \mathrm{H}$, $C_{q}=2.1655 \cdot 10^{-14} \mathrm{~F}, C_{0}=7 \cdot 10^{-12} \mathrm{~F}, R_{q}=10 \mathrm{Ohm}$ in $\mathrm{A}$ and $\mathrm{B}, R_{q}=1 \mathrm{kOhm}$ in $\mathrm{C}$ and $\mathrm{D}$.

A

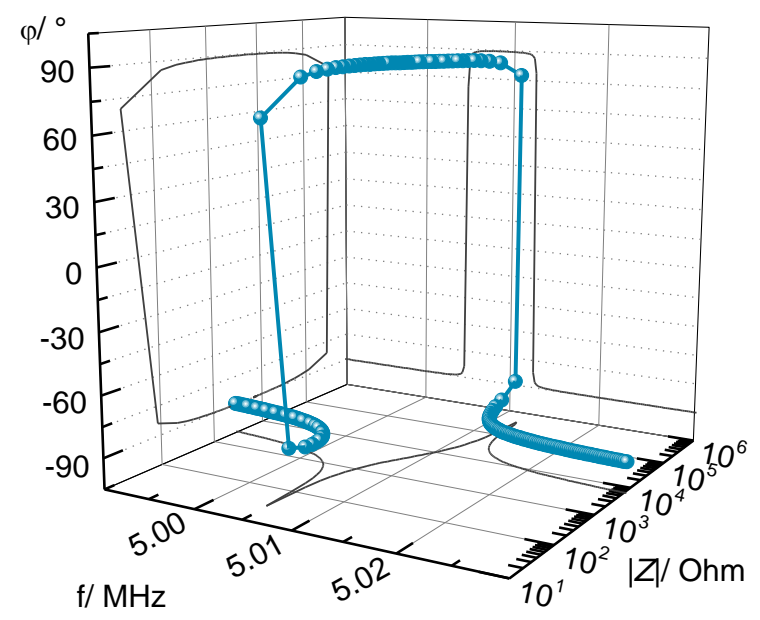

C

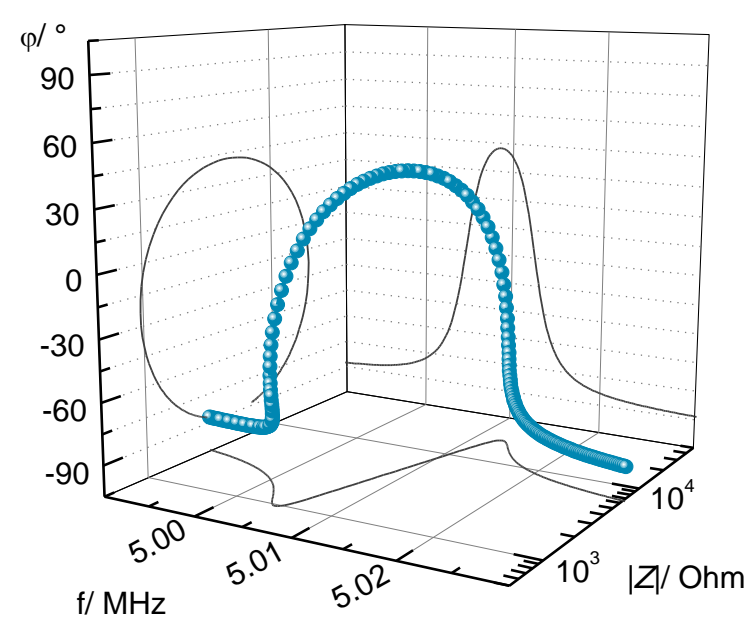

B

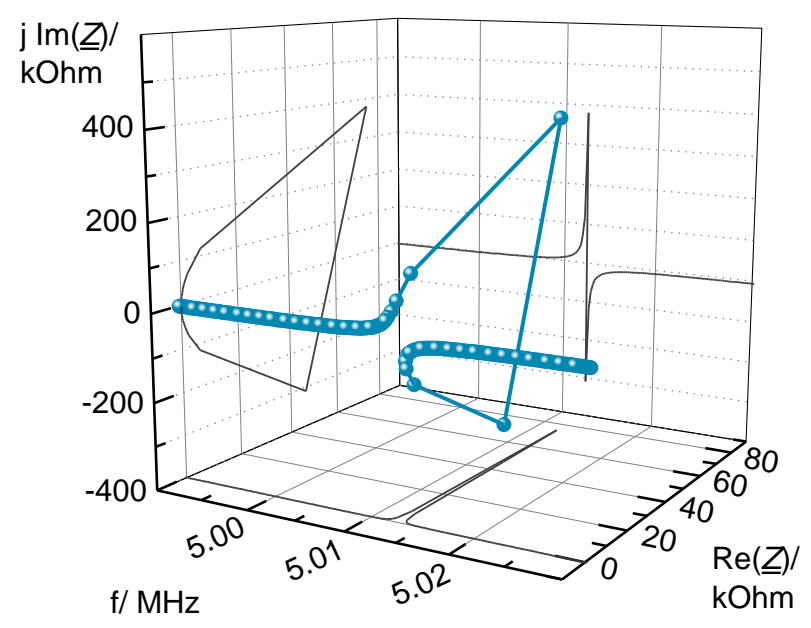

D

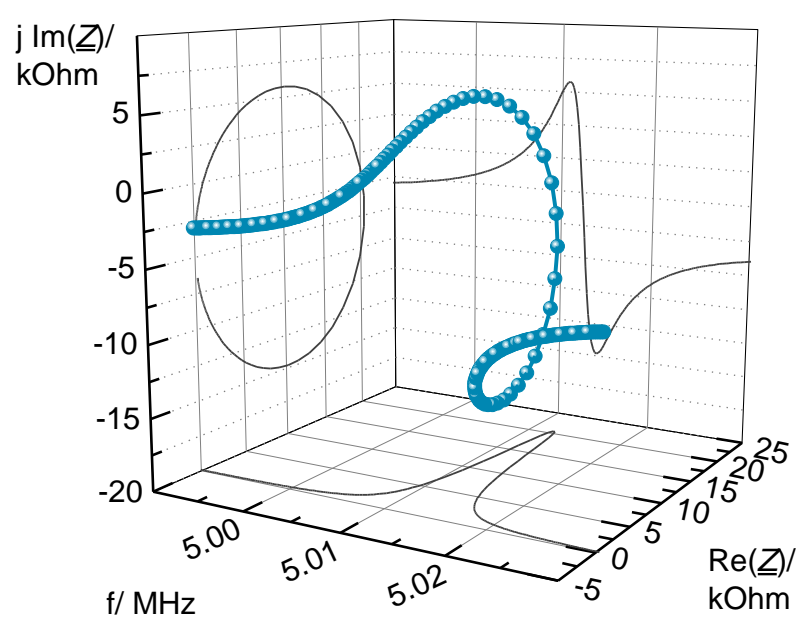




\section{SI 6 TSP imaging setup}

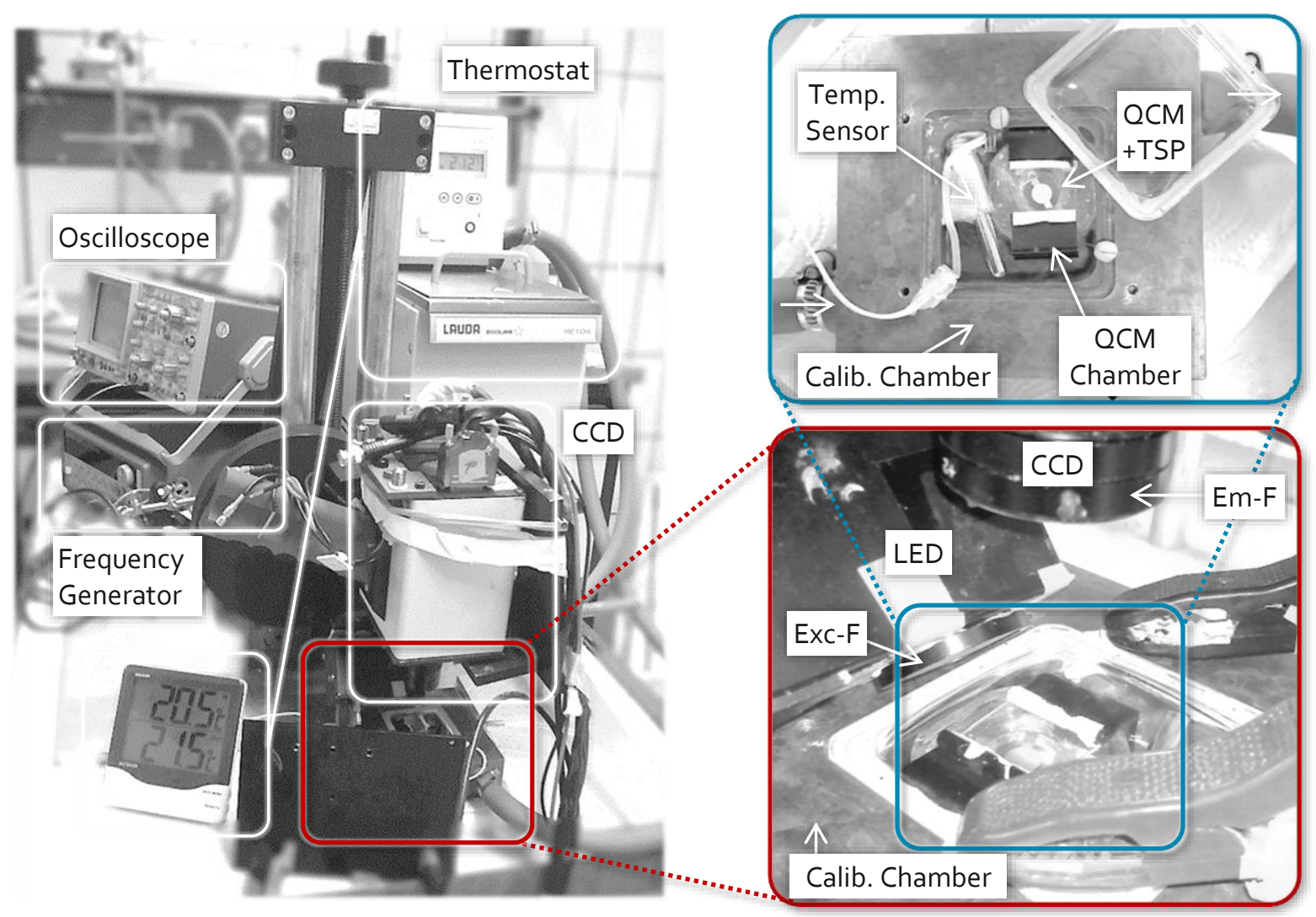

SI 7 PSP imaging setup with microscope

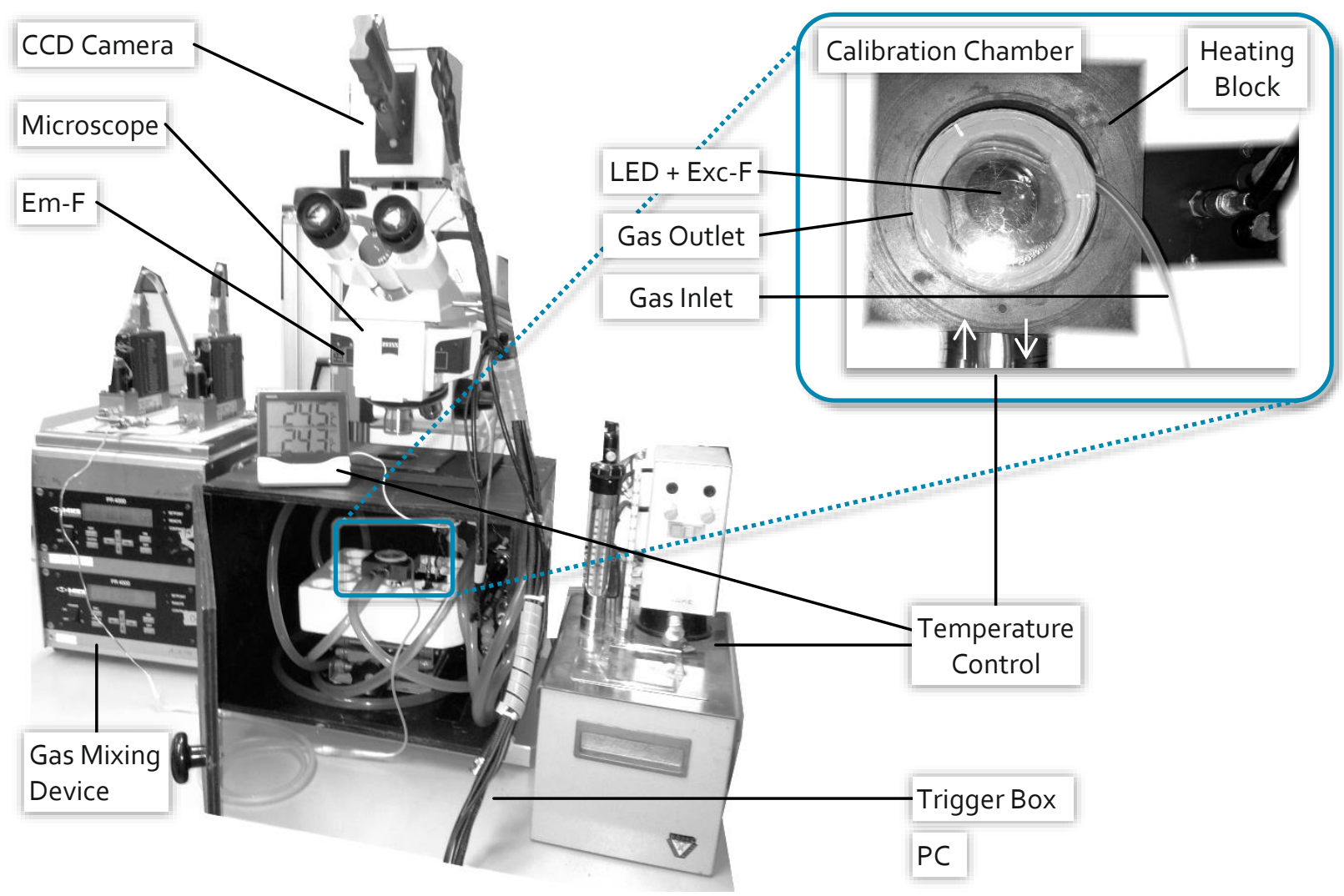


SI 8 Maths script for image processing in ImagexTGi software

\begin{tabular}{|c|c|c|c|}
\hline Maths Script \#1 & \multicolumn{3}{|c|}{ Maths Script \#2 } \\
\hline $1=1-7 *(1)$ & $1=1-7^{*}(1)$ & $1=1 *(.14286)$ & $3=1+3 *(1)$ \\
\hline $2=2-7^{*}(1)$ & $2=2-7 *(1)$ & $2=2 *(.14286)$ & $5=3+5 *(1)$ \\
\hline $3=3-7 *(1)$ & $3=3-7 *(1)$ & $3=3 *(.14286)$ & $8=5+8 *(1)$ \\
\hline $4=4-7 *(1)$ & $4=4-7 *(1)$ & $4=4^{*}(.14286)$ & $10=8+10 *(1)$ \\
\hline $5=5-7 *(1)$ & $5=5-7 *(1)$ & $5=5^{*}(.14286)$ & $12=10+12 *(1)$ \\
\hline $6=6-7^{*}(1)$ & $6=6-7 *(1)$ & $6=6 *(.14286)$ & $14=12+14 *(1)$ \\
\hline $8=8-7^{*}(1)$ & $8=8-7^{*}(1)$ & $8=8 *(.14286)$ & $4=2+4 *(1)$ \\
\hline $9=9-7^{*}(1)$ & $9=9-7 *(1)$ & $9=9 *(.14286)$ & $6=4+6 *(1)$ \\
\hline $10=10-7^{*}(1)$ & $10=10-7^{*}(1)$ & $10=10 *(.14286)$ & $9=6+9 *(1)$ \\
\hline $11=11-7^{*}(1)$ & $11=11-7 *(1)$ & $11=11 *(.14286)$ & $11=9+11 *(1)$ \\
\hline $12=12-7^{*}(1)$ & $12=12-7^{*}(1)$ & $12=12 *(.14286)$ & $13=11+13 *(1)$ \\
\hline $13=13-7^{*}(1)$ & $13=13-7 *(1)$ & $13=13 *(.14286)$ & $15=13+15^{*}(1)$ \\
\hline $14=14-7 *(1)$ & $14=14-7^{*}(1)$ & $14=14 *(.14286)$ & $16=14 / 15 *(1000)$ \\
\hline $\begin{array}{l}15=15-7^{*}(1) \\
\text { End }\end{array}$ & $15=15-7 *(1)$ & $15=15 *(.14286)$ & End \\
\hline
\end{tabular}

SI 9 (A) 3-D Bode and (B) 3-D Nyquist plot of $\mathrm{OCM} 2$ on a not decoupled $2 \mathrm{ElO}$ (dark grey) and of $\mathrm{QCM} 2$ on a $2 \mathrm{ElO}$ that is decoupled by the deposition of a rigid polymer film on $\mathrm{QCM}_{1}$ (blue)

A

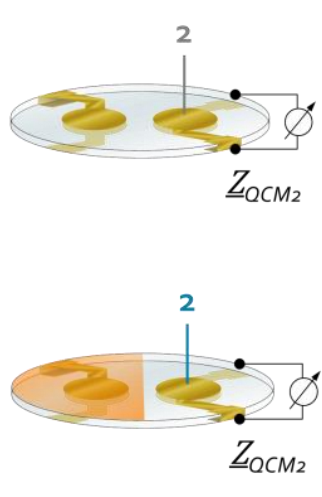

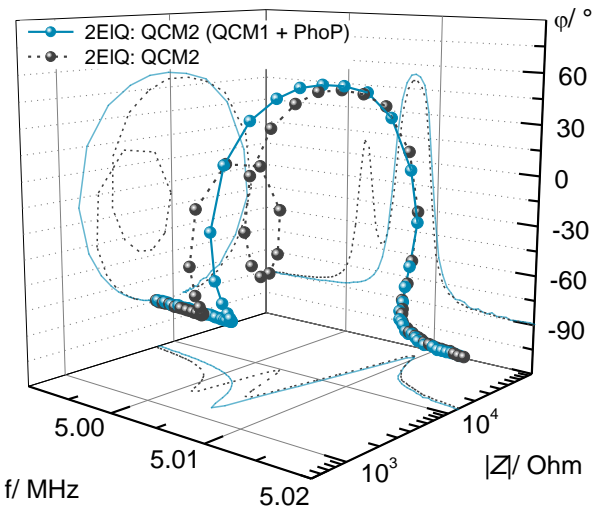

B

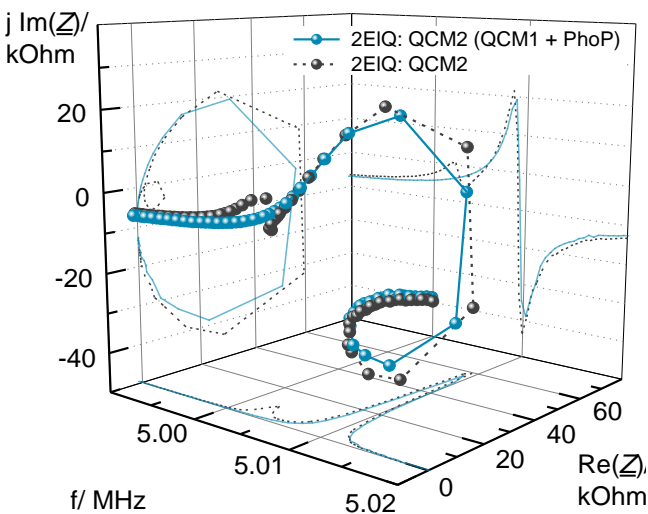


SI 10 (A) 3-D Bode plot and (B) three-dimensional $R-C$ - $f$ plot of a QCM-ECIS sensor in ECIS-mode, under medium (black) and covered with a MDCK-Il cell layer (blue)
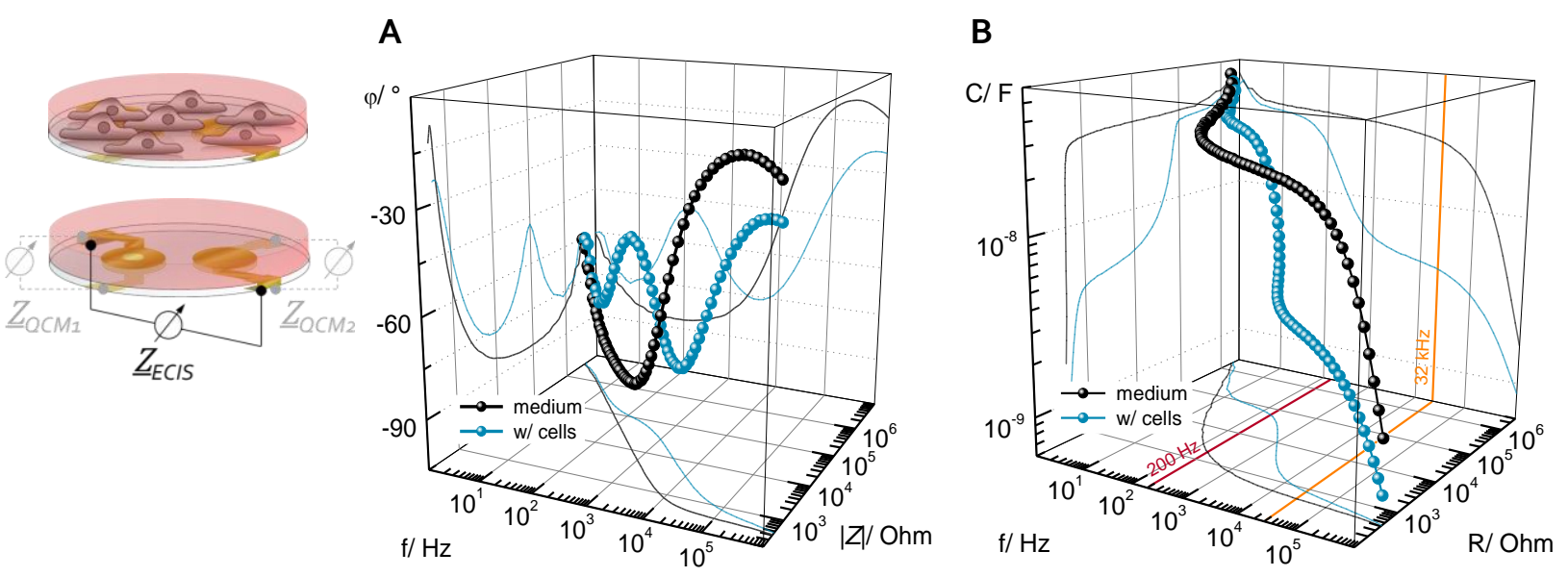

SI 11 OCM-mode properties of OCM-ECIS designs with ECIS WE diameters of (A) $250 \mu \mathrm{m}$, (B) $1000 \mu \mathrm{m}$, and (C) $2000 \mu \mathrm{m}$ in air. (1) Phase shift spectra, (2) 3-D Bode plots for PhoP-coated $\mathrm{OCM}_{1}$ (w/ PhoP; black) and PhoP-uncovered $\mathrm{QCM}_{2}$ (w/o PhoP; blue), and (3) exemplary Nyquist plots of $\mathrm{QCM} 1$ oscillations.

A1

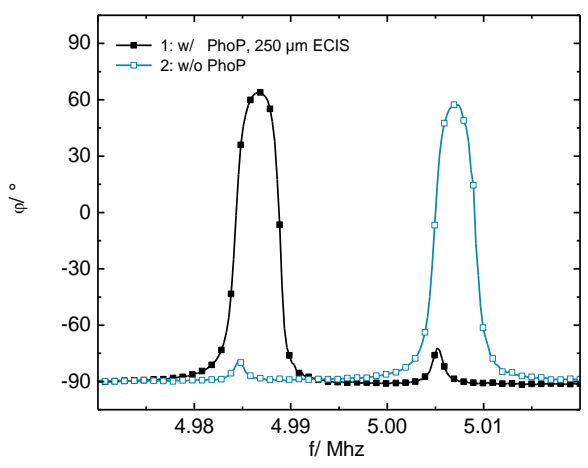

B1

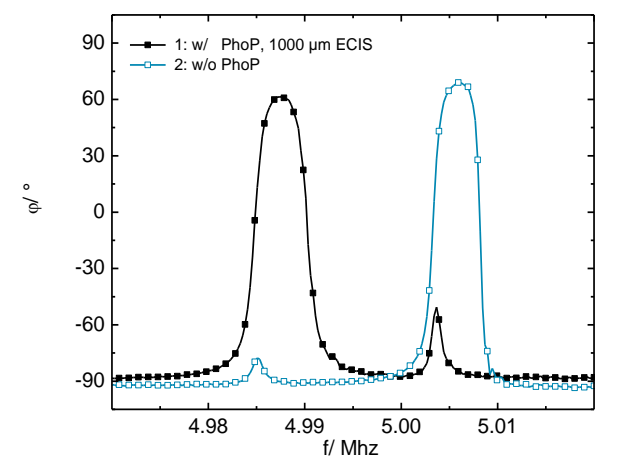

A2

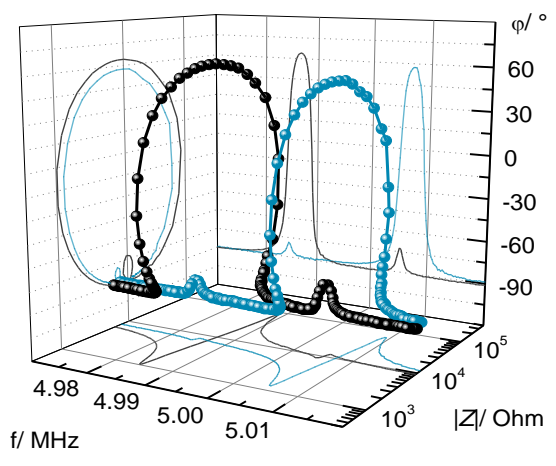

B2

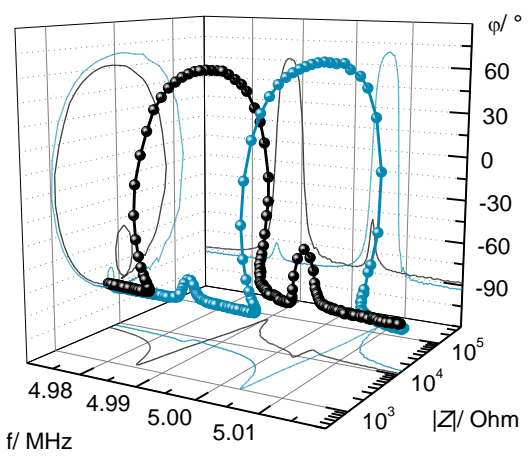

A3

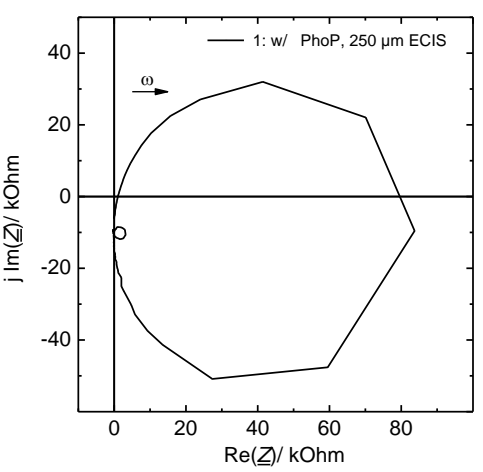

B3

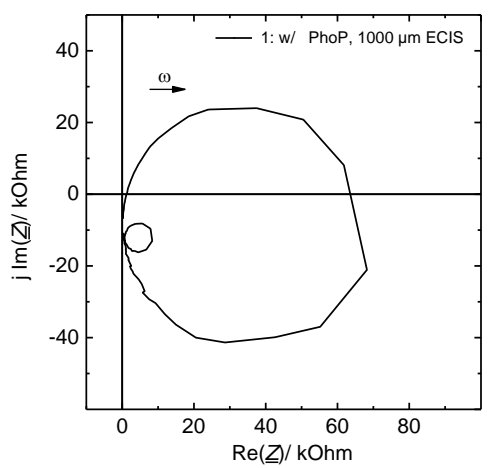


C1

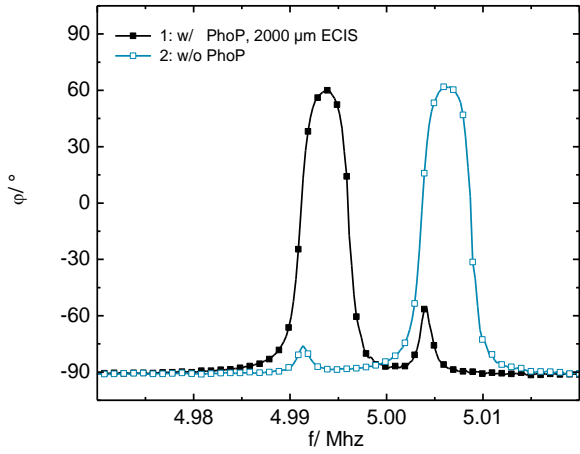

$\mathrm{C}_{2}$

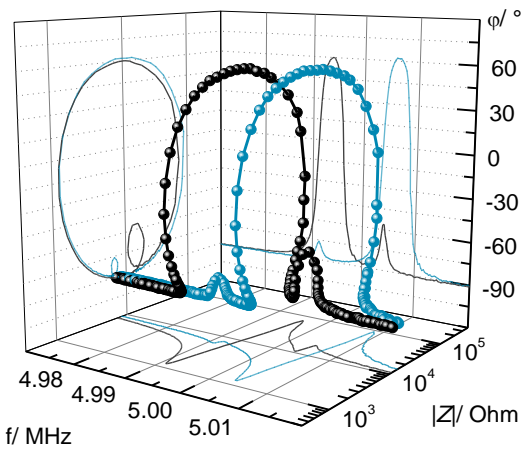

$\mathrm{C}_{3}$

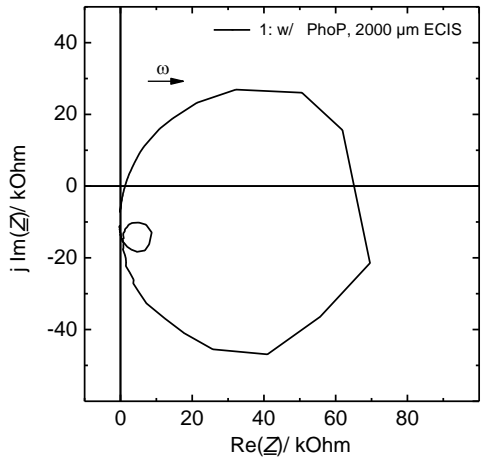

SI 12 ECIS-mode properties of QCM-ECIS prototypes comprising ten WEs with a diameter of each electrode of either $\varnothing_{\text {mask }}=250 \mu \mathrm{m}$ (black squares) or $\varnothing_{\text {mask }}=500 \mu \mathrm{m}$ (blue triangles). Spectra of absolute (A) resistance and (C) capacitance of the sensors exposed to medium (open symbols) and covered with cells (filled symbols). Normalized spectra of (B) resistance and (D) capacitance. Data points were reduced for sake of clarity.

A

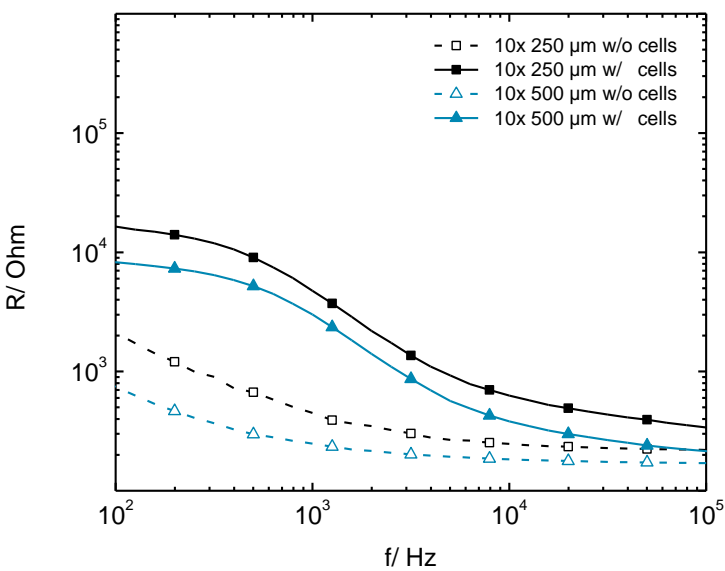

C

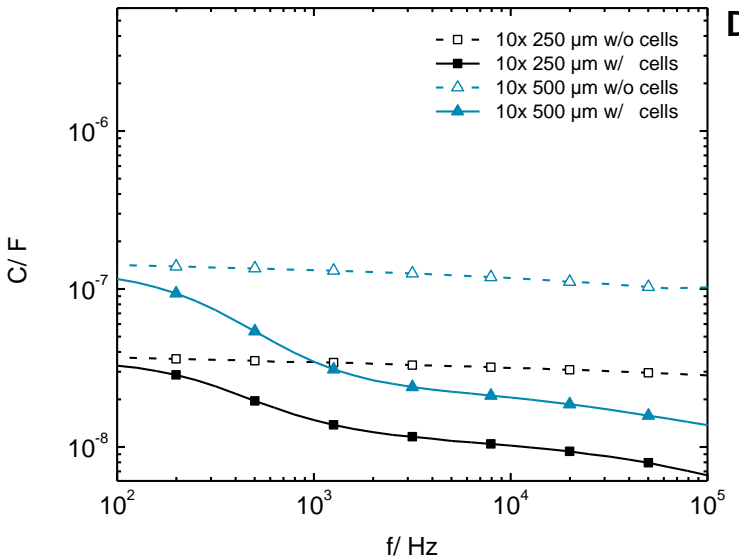

B

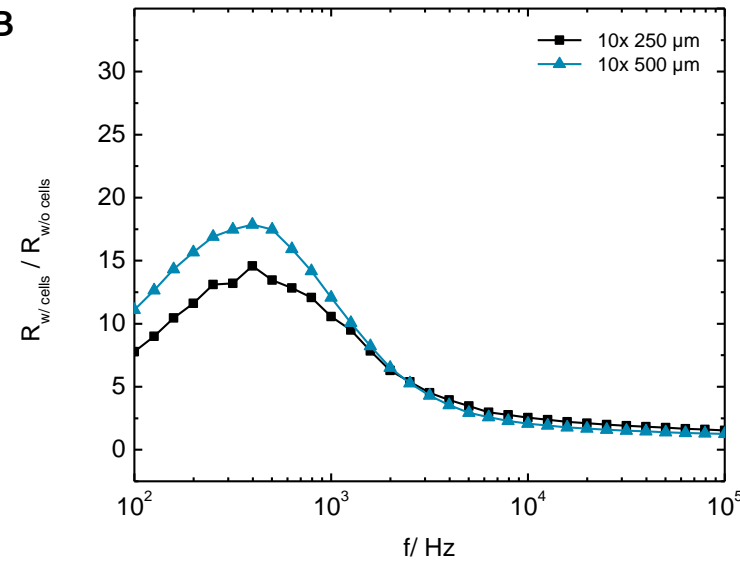

D

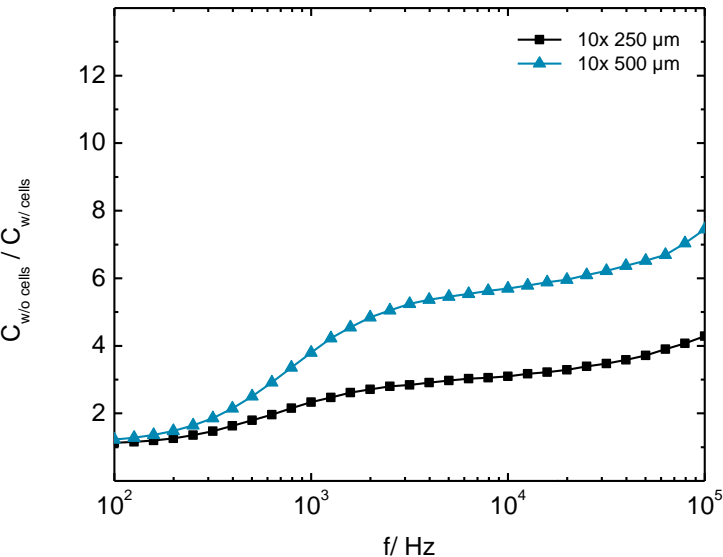


SI 13 (A) 2-D correlation of ECIS parameters $\left(C_{\text {norm }}\right.$ vs. $\left.R_{\text {norm }}\right)$ and (B), (C), (D) 3-D time courses of QCM- and ECIS-mode impedance parameters during MDCK-II cell attachment and spreading on newly developed 2 ElOs ((B) $C_{\text {norm }}$ vs. $R_{\text {norm }}$ vs. $t$, (C) $C_{\text {norm }}$ vs. $\Delta|Z|_{\min }$ vs. $t$, and (D) $R_{\text {norm }}$ vs. $\Delta|Z|_{\min }$ vs. $t$ ).

A

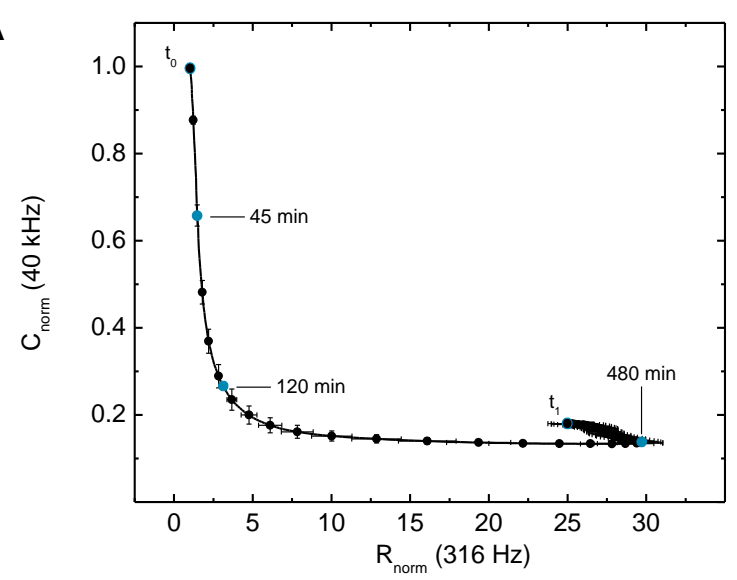

C

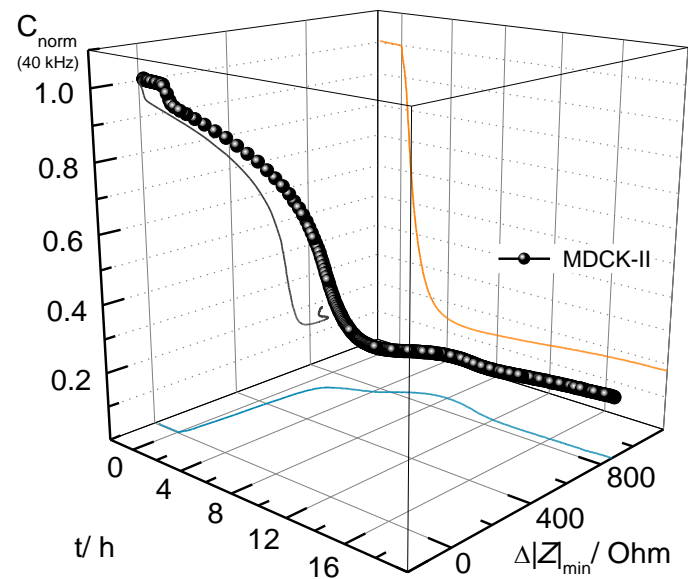

B $\mathrm{C}$

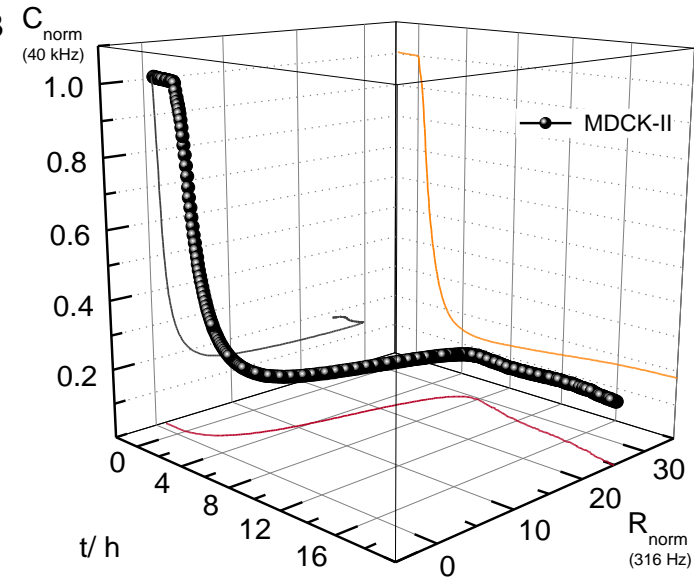

D R

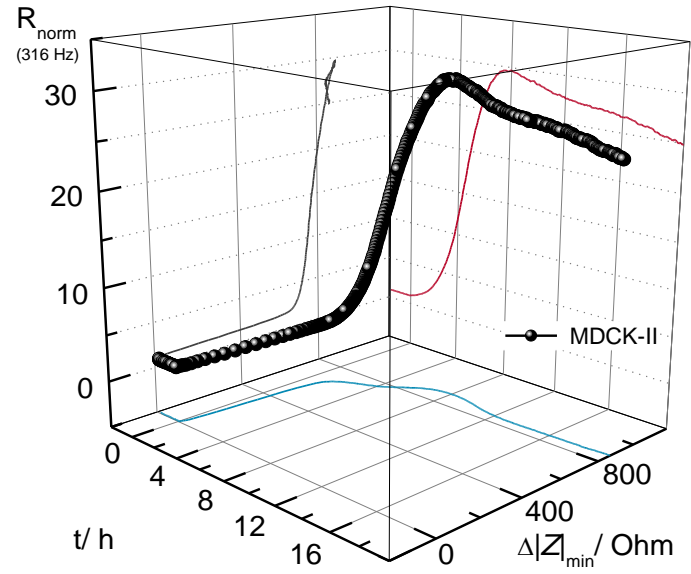

SI 14 OCM-mode (A) impedance spectra and (B) phase shift spectra of resonators OCM1 (filled symbols) and QCM2 (open symbols) on a OCM-ECIS sensor, performing oscillations in air (grey squares), loaded with medium (black triangles) and covered with a confluent layer of NRK cells (blue circles). (C) 3-D Bode and (D) 3-D Nyquist plot exemplarily shown for QCM1.

A

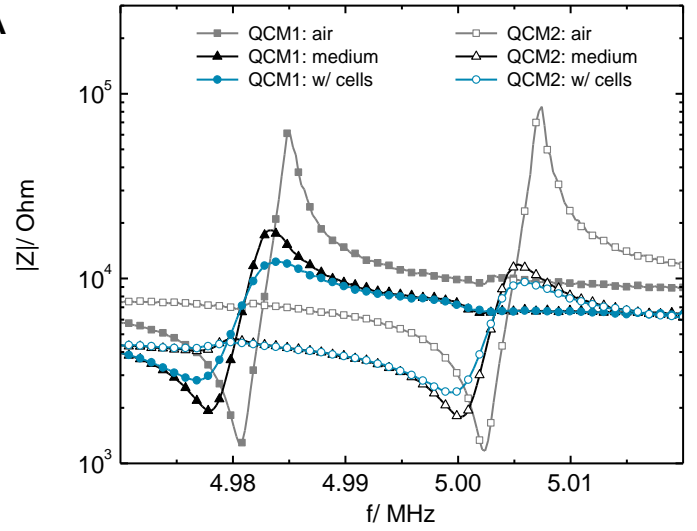

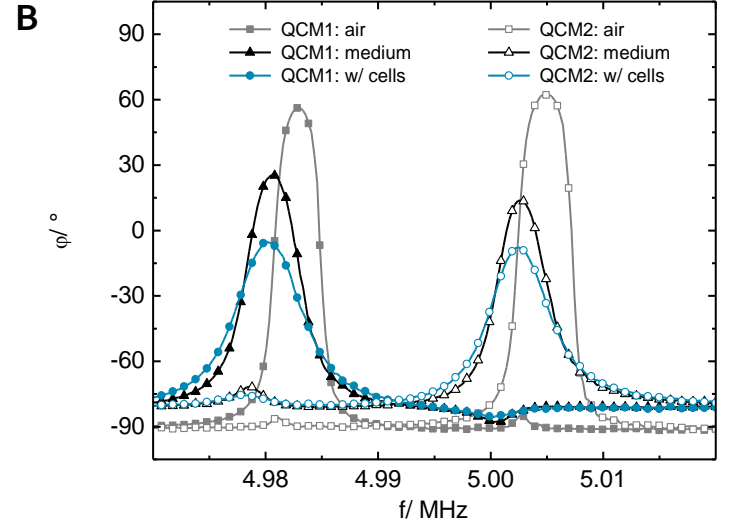


C

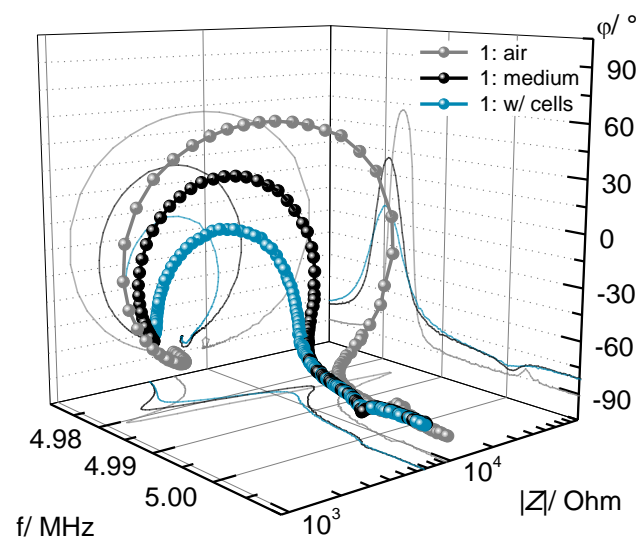

D

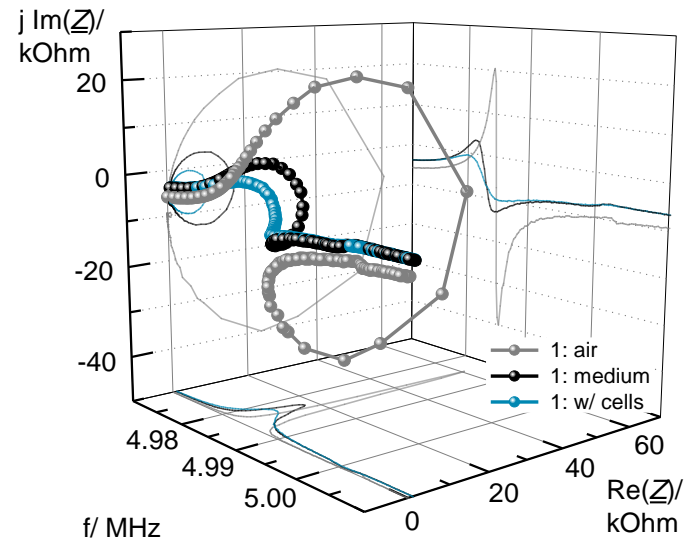

SI 15 ECIS-mode spectra of (A) impedance, (B) phase shift, (C) resistance (red squares) and (C) capacitance (orange circles) of a QCM-ECIS sensor loaded with medium (open symbols) and with a confluent layer of NRK cells (filled symbols). (D) Normalized spectra of impedance (black triangles), resistance (red squares) and capacitance (orange circles) with indicated mostsensitive frequencies for NRK cells. (E) 3-D Bode plot, (F) 3-D R-C-f plot, (G) 2-D and (H) 3-D Nyquist plot of the sensor under medium (black) and covered with NRK cells (blue).

A

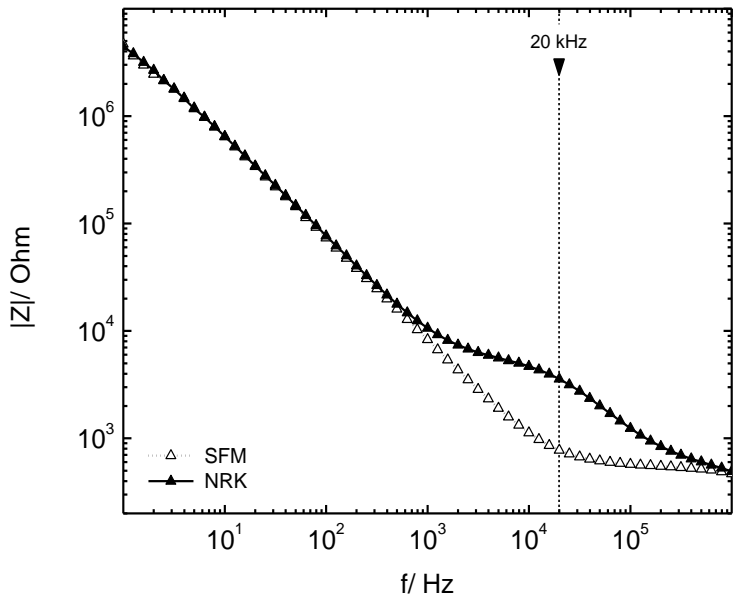

C

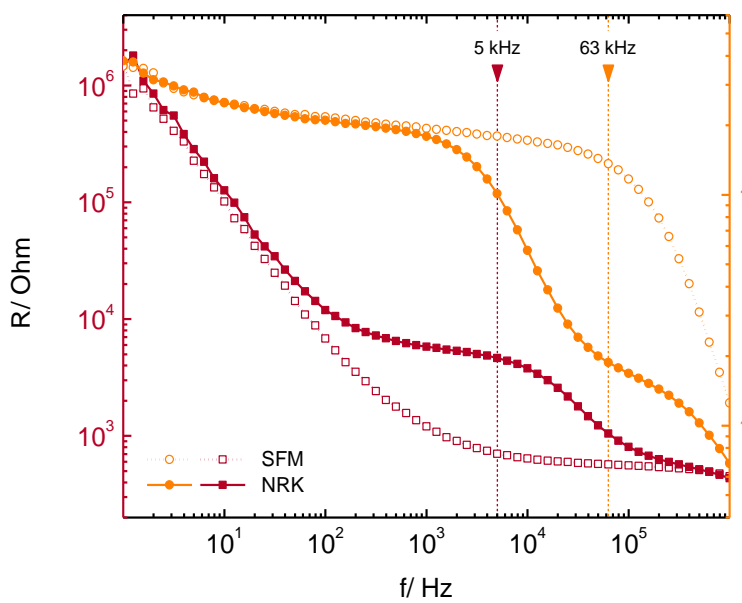

B
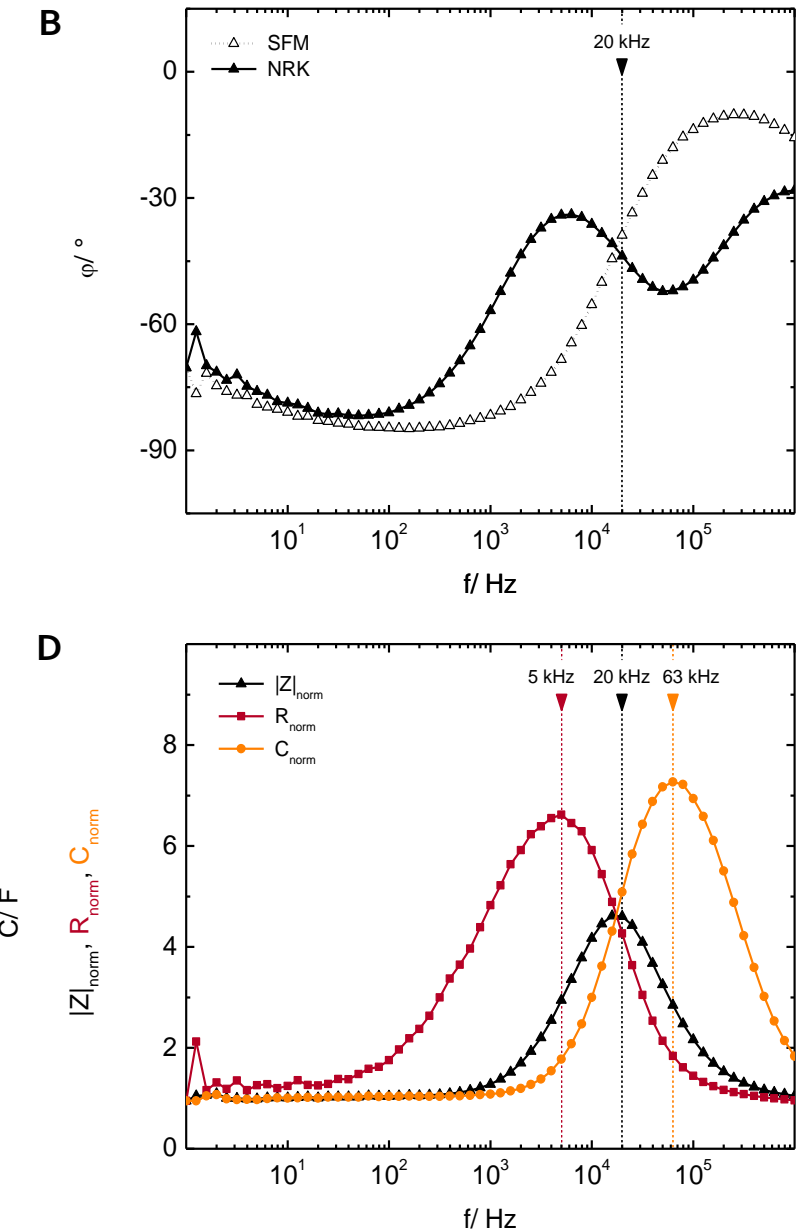
E

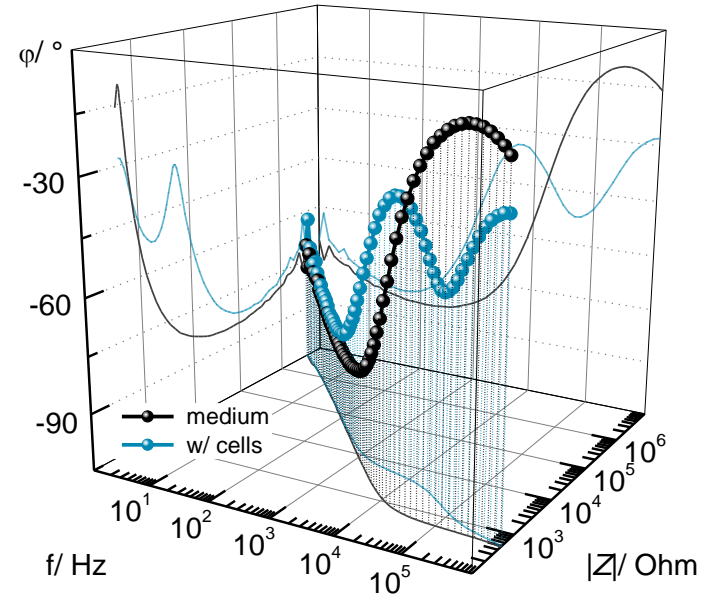

G

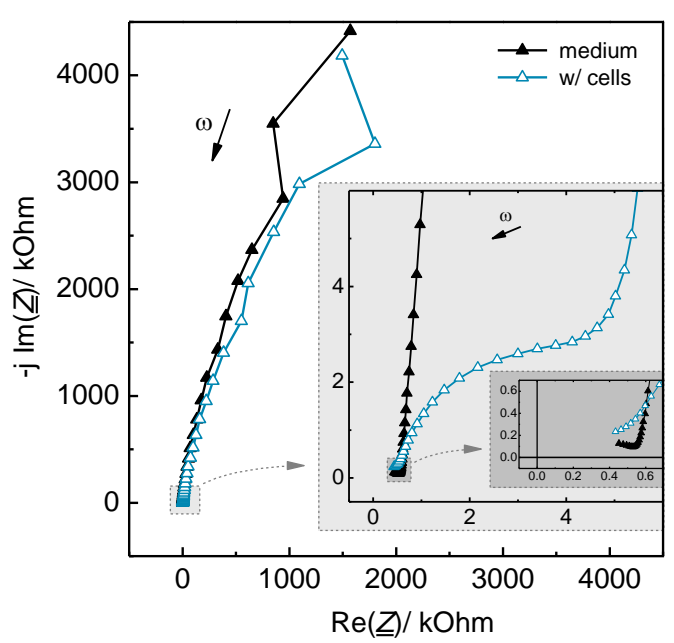

$\mathbf{F}$

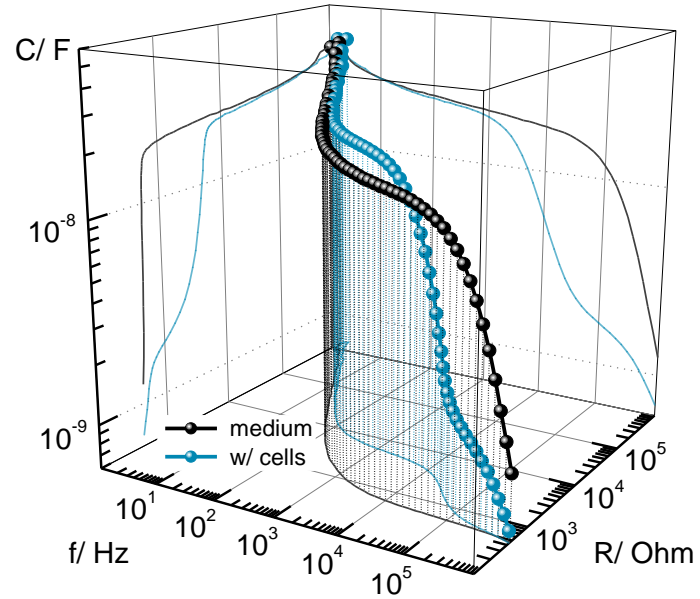

$\mathrm{H}$

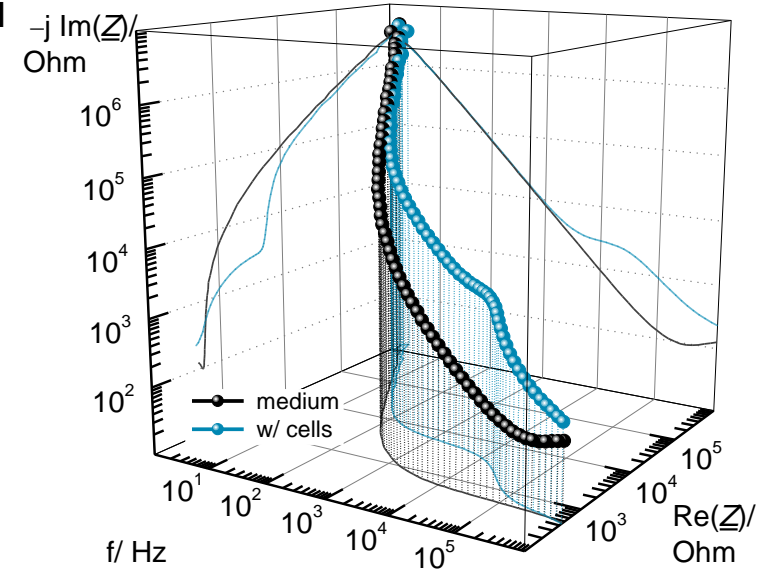

SI 16 (A) 2-D correlation of ECIS parameters ( $C_{\text {norm }}$ vs. $\left.R_{\text {norm }}\right)$ and (B), (C), (D) 3-D time courses of OCM- and ECIS-mode impedance parameters during NRK cell attachment and spreading on newly developed 2 ElOs (B) $C_{\text {norm }}$ vs. $R_{\text {norm }}$ vs. $t$, (C) $C_{\text {norm }}$ vs. $\Delta|Z|_{\min }$ vs. $t$, and (D) $R_{\text {norm }}$ vs. $\Delta|Z|_{\min }$ vs. $t$ ).

A

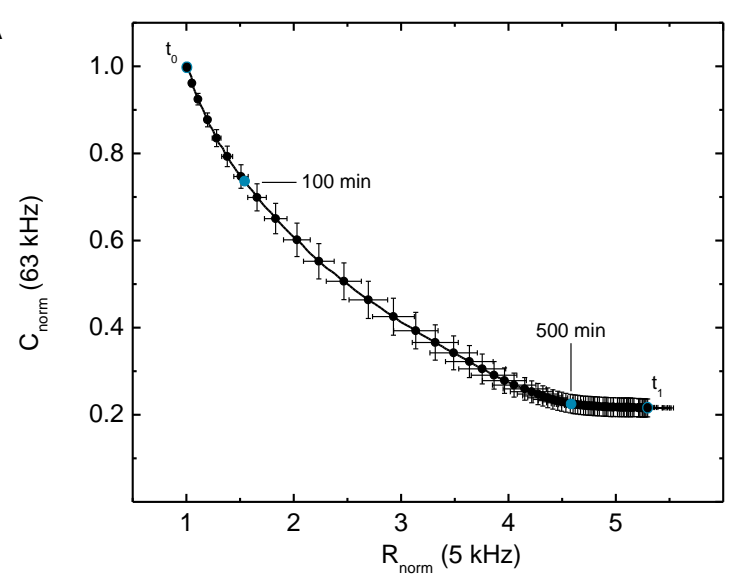

B $\mathrm{C}$

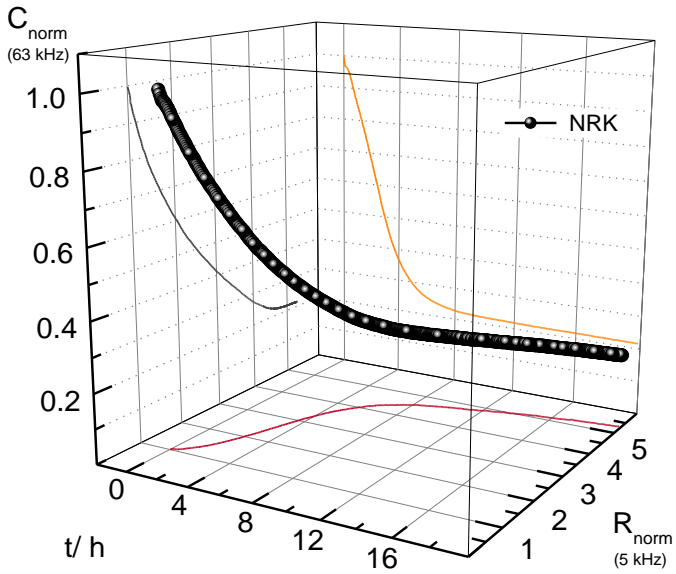


C

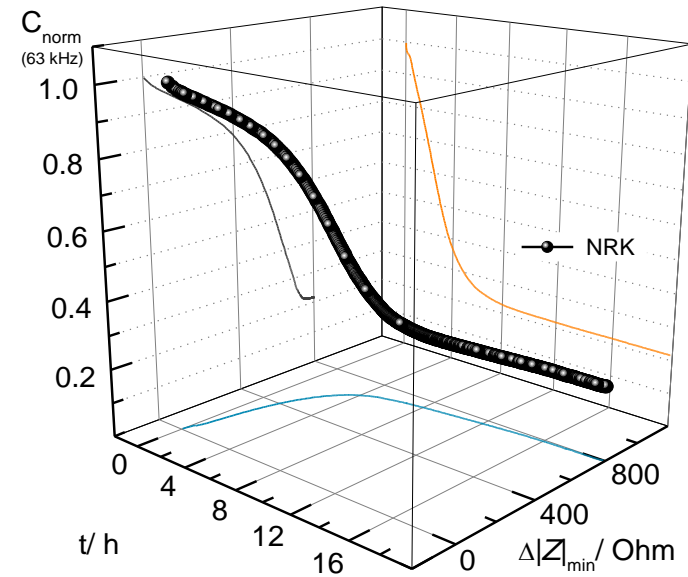

D $R$

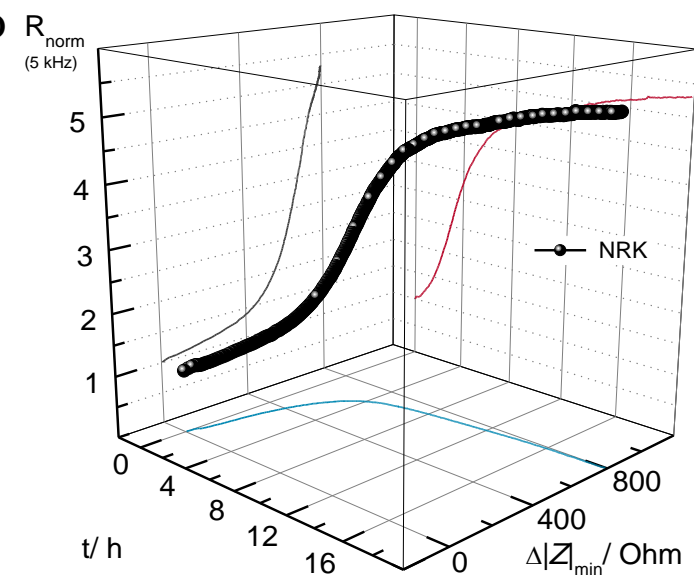

SI 17 Characteristic shear oscillation parameters (OCM-mode) and electrochemical impedance parameters (ECIS-mode) in the time course of MDCK-II cell attachment and spreading on FNcoated QCM-ECIS sensor surfaces in EBSS ${ }^{--}$buffer containing both $\mathrm{Ca}^{2+}(1.8 \mathrm{mM})$ and $\mathrm{Mg}^{2+}$ $(0.81 \mathrm{mM})$, or $\mathrm{Ca}^{2+}$ only or $\mathrm{Mg}^{2+}$ only. Calculated averaged sensitive frequencies $\left(f_{\text {sens }}^{\text {calc }}\right)$ and sampling frequencies used for time-dependent analysis $\left(f_{\text {sens }}^{\text {used }}\right)$ of resistance $(R)$ and capacitance $(C)$ are listed. Absolute and normalized mean values of $R$ and $C$ are obtained from averaged curves at points of cell inoculation $\left(t_{0}\right)$ and at the end of experiments $\left(t_{1}=20 \mathrm{~h}\right)$. Maximal slopes $m=\Delta Y / \Delta t\left(Y=\Delta|Z|_{\text {min }}, R, C\right)$ were calculated by linear regression around $t_{m}$ ( \pm 5 data points) considering standard errors (instrumental weighting). $t_{1 / 2}(Y)$ marks the time when half of the final signals are attained. (mean $\pm S E M ; N \geq 2$ ).

\begin{tabular}{|c|c|c|c|c|c|c|c|c|}
\hline $\begin{array}{l}|Z|_{\min }: \\
\text { EBSS }^{--}\end{array}$ & & & $\begin{array}{l}|Z|_{\min }\left(t_{0}\right) / \\
\mathrm{Ohm}\end{array}$ & $\begin{array}{l}t_{m} / \\
\min \end{array}$ & $\begin{array}{c}m=\Delta|Z|_{\min } / \Delta t / \\
\mathrm{Ohm} \cdot \mathrm{min}^{-1}\end{array}$ & $\begin{array}{l}t_{1 / 2} l \\
\min \end{array}$ & $\begin{array}{l}t_{1} / \\
\min \end{array}$ & $\begin{array}{c}\Delta|Z|_{\min }\left(t_{1}\right) / \\
\text { Ohm }\end{array}$ \\
\hline$+\mathrm{Ca}^{2+}+\mathrm{Mg}^{2+}$ & & & $1870( \pm 40)$ & 35 & $19.6( \pm 0.2)$ & 35 & 1200 & $810( \pm 40)$ \\
\hline$+\mathrm{Ca}^{2+}-\mathrm{Mg}^{2+}$ & & & $1700( \pm 70)$ & 40 & $19.2( \pm 0.1)$ & 45 & 1200 & $780( \pm 50)$ \\
\hline$-\mathrm{Ca}^{2+}+\mathrm{Mg}^{2+}$ & & & $1910( \pm 90)$ & 35 & $23.9( \pm 0.2)$ & 35 & 1200 & $830( \pm 20)$ \\
\hline $\begin{array}{l}R: \\
\text { EBSS }^{--}\end{array}$ & $\begin{array}{l}f_{\text {sens }}^{\text {calc }} / \\
\mathrm{kHz}\end{array}$ & $\begin{array}{c}f_{\text {sens }}^{\text {usd }} \text { I } \\
\mathrm{kHz}\end{array}$ & $\begin{array}{l}R\left(t_{0}\right) / \\
\text { Ohm }\end{array}$ & $\begin{array}{l}t_{m} / \\
\min \end{array}$ & $\begin{array}{c}m=\Delta R / \Delta t \\
\mathrm{kOhm} \cdot \mathrm{h}^{-1}\end{array}$ & $\begin{array}{l}t_{1 / 2} l \\
\min \end{array}$ & $\begin{array}{l}t_{1} / \\
\min \end{array}$ & $\begin{array}{c}R_{\text {norm }}\left(t_{1}\right) / \\
\%\end{array}$ \\
\hline$+\mathrm{Ca}^{2+}+\mathrm{Mg}^{2+}$ & $0.57( \pm 0.03)$ & 0.63 & $2550( \pm 8)$ & 245 & $5.0( \pm 0.2)$ & 240 & 1200 & $1150( \pm 70)$ \\
\hline$+\mathrm{Ca}^{2+}-\mathrm{Mg}^{2+}$ & & 0.63 & $2200( \pm 300)$ & 225 & $6.8( \pm 0.2)$ & 240 & 1200 & $1400( \pm 200)$ \\
\hline$-\mathrm{Ca}^{2+}+\mathrm{Mg}^{2+}$ & $10.2( \pm 0.5)$ & 0.63 & $2900( \pm 400)$ & 55 & $2.0( \pm 0.1)$ & 40 & 1200 & $110( \pm 10)$ \\
\hline$-\mathrm{Ca}^{2+}+\mathrm{Mg}^{2+}$ & & 10 & $729( \pm 7)$ & 50 & $1.71( \pm 0.02)$ & 40 & 1200 & $192( \pm 4)$ \\
\hline $\begin{array}{l}\boldsymbol{C}: \\
\text { EBSS }^{--}\end{array}$ & $\begin{array}{c}f_{\text {sens }}^{\text {calc }} / \\
\mathrm{kHz}\end{array}$ & $\begin{array}{c}f_{\text {sens }}^{\text {used }} / \\
\mathrm{kHz}\end{array}$ & $\begin{array}{c}C\left(t_{0}\right) / \\
\mathrm{nF}\end{array}$ & $\begin{array}{l}t_{m} / \\
\min \end{array}$ & $\begin{array}{c}m=\Delta C / \Delta t \\
\mathrm{nF} \cdot \mathrm{h}^{-1}\end{array}$ & $\begin{array}{l}t_{1 / 2} l \\
\min \end{array}$ & $\begin{array}{c}t_{1} / \\
\min \end{array}$ & $\begin{array}{c}C_{\text {norm }}\left(t_{1}\right) / \\
\%\end{array}$ \\
\hline$+\mathrm{Ca}^{2+}+\mathrm{Mg}^{2+}$ & $100( \pm 10)$ & 100 & $5.160( \pm 0.006)$ & 40 & $-5.10( \pm 0.06)$ & 45 & 1200 & $38( \pm 2)$ \\
\hline$+\mathrm{Ca}^{2+}-\mathrm{Mg}^{2+}$ & & 100 & $6( \pm 1)$ & 50 & $-5.76( \pm 0.12)$ & 50 & 1200 & $31( \pm 5)$ \\
\hline$-\mathrm{Ca}^{2+}+\mathrm{Mg}^{2+}$ & $290( \pm 40)$ & 100 & $5.4( \pm 0.3)$ & 40 & $-5.03( \pm 0.05)$ & 35 & 1200 & $54.9( \pm 0.5)$ \\
\hline$-\mathrm{Ca}^{2+}+\mathrm{Mg}^{2+}$ & & 316 & $3.3( \pm 0.3)$ & 40 & $-2.69( \pm 0.02)$ & 35 & 1200 & $49( \pm 2)$ \\
\hline
\end{tabular}


SI 18 Normalized spectra of impedance (black triangles), resistance (red squares) and capacitance (orange circles) calculated from spectra of standard QCM-ECIS sensors coated with FN and $20 \mathrm{~h}$ after sensor inoculation with MDCK-II cells in (A) EBSS ${ }^{--}$buffer containing $\mathrm{Ca}^{2+}$ and (B) $\mathrm{Ca}^{2+}$-free EBSS $^{--}$buffer. Note the different scales of the $y$-axis in A and B.

A

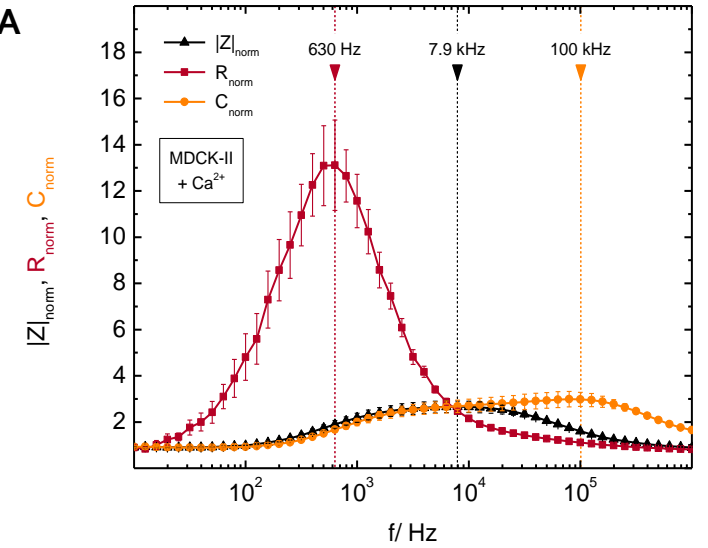

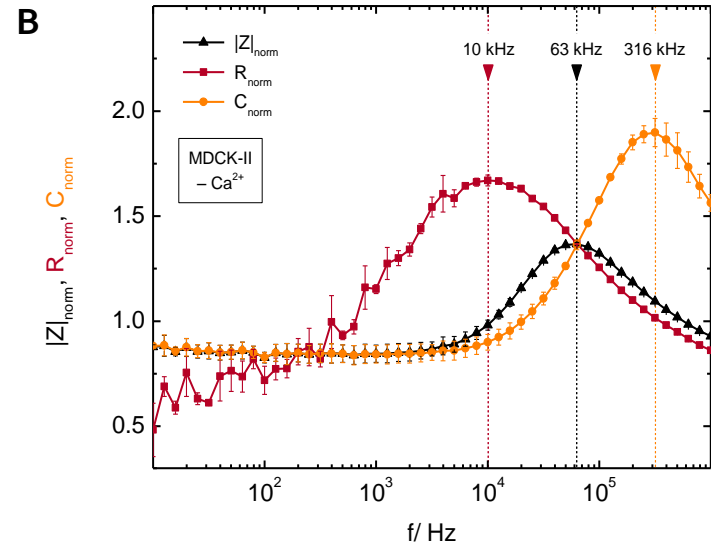

SI 19 Micrographs of MDCK-II cells seeded to confluence on glass plates in (A) EBSS ${ }^{++}$, (B) EBSS ${ }^{+-}$, and (C) EBSS $^{-+}$. Cells were triple stained for DNA (blue), ZO-1 (red), and f-actin (green) the day after seeding.
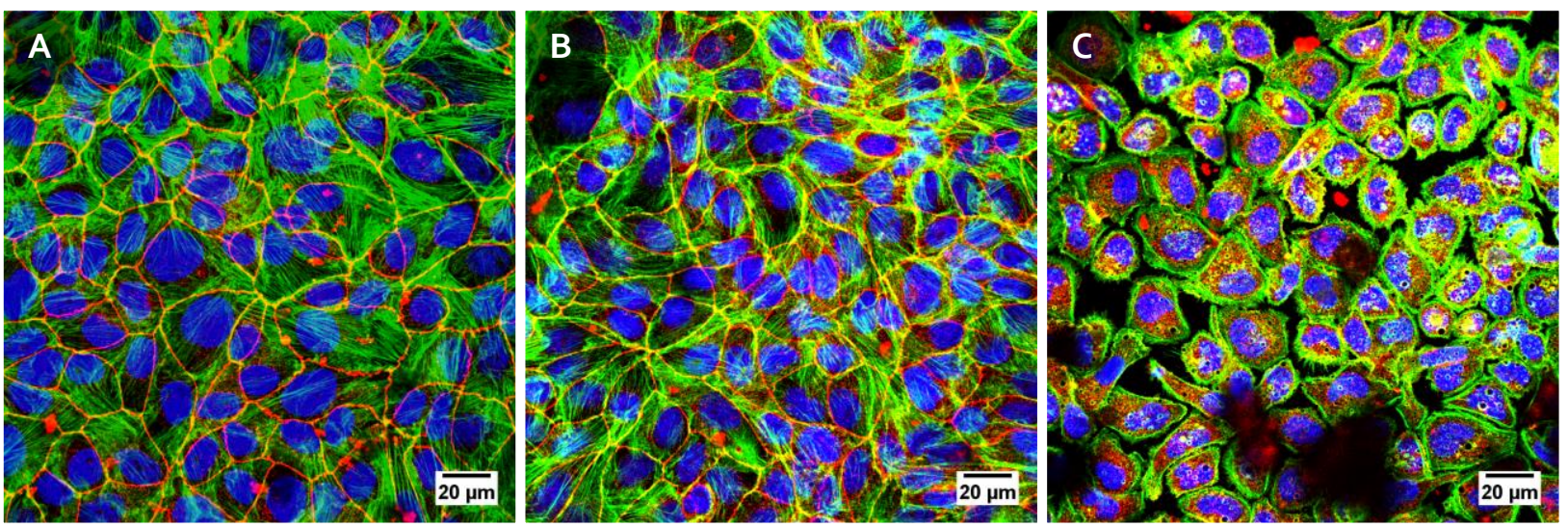
SI 20 OCM impedance spectra (A: $|Z|(f), \mathbf{B}: \varphi(f))$, ECIS impedance spectra (C: $|Z|(f)$, D: $\varphi(f))$, and ECIS resistance and capacitance spectra (E) of a OCM-ECIS sensor: in air, covered with a confluent layer of MDCK-II cells, with cells that have been treated with $5 \mu \mathrm{M}$ Cytochalasin $D(C D)$ for $0.5 h, 2 h$, and $4 h$, and with cells treated with Saponin.

A

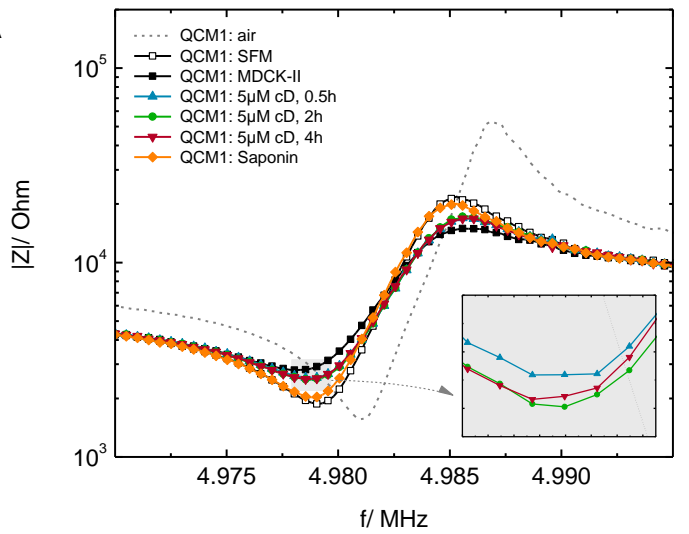

C

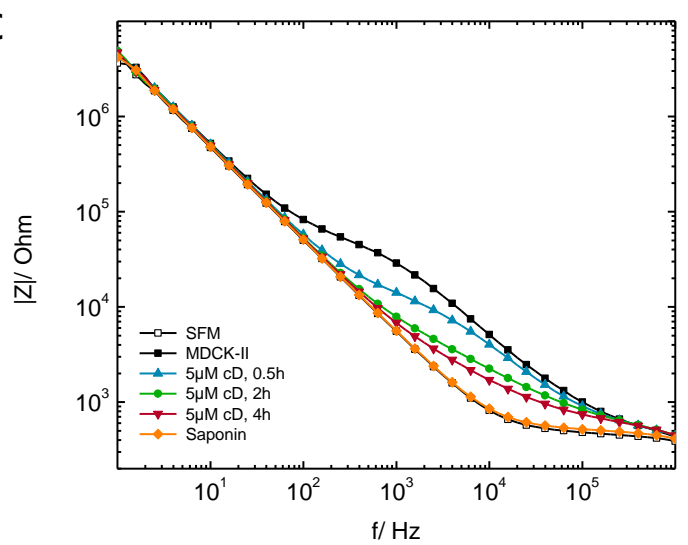

E

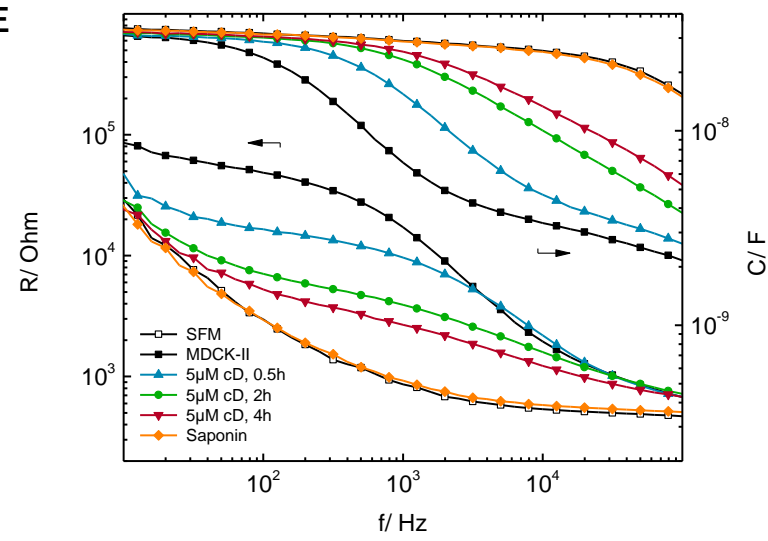

B

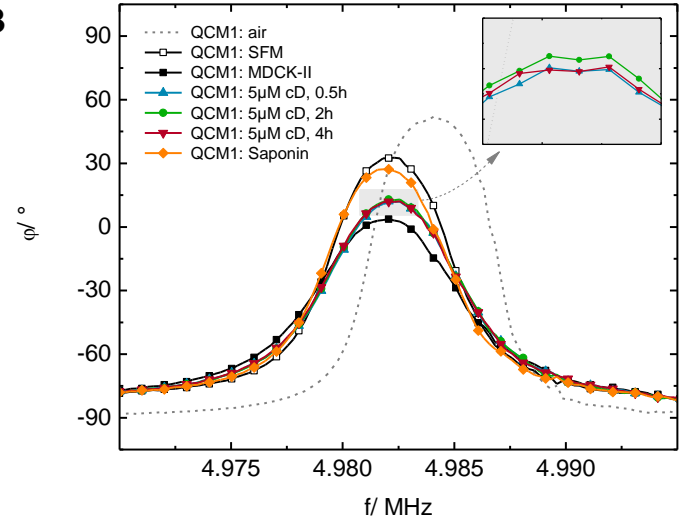

D

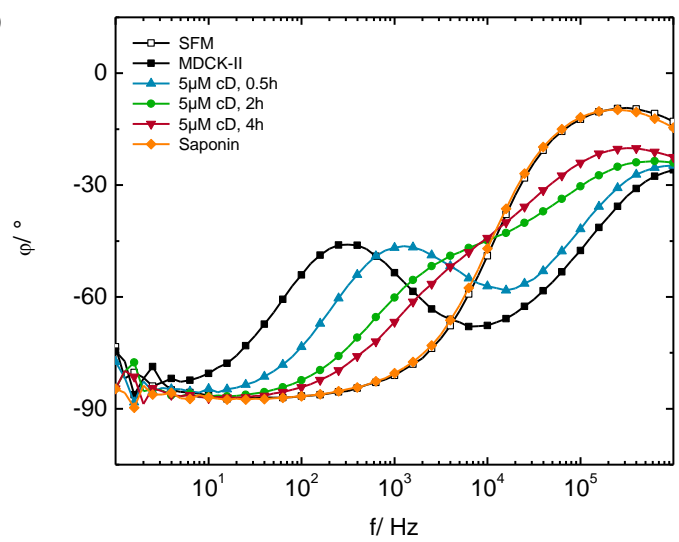


SI 21 OCM impedance spectra (A: $|Z|(f)$, B: $\varphi(f))$, ECIS impedance spectra (C: $|Z|(f), \mathbf{D}: \varphi(f))$, and ECIS resistance and capacitance spectra $(E)$ of a QCM-ECIS sensor: in air, covered with a confluent layer of MDCK-II cells, with cells that have been treated with $0.25 \%$ glutaraldehyde (GA) for $5 \mathrm{~min}, 10 \mathrm{~min}$, and $3 \mathrm{~h}$, and with cells treated with Saponin after GA incubation.

A

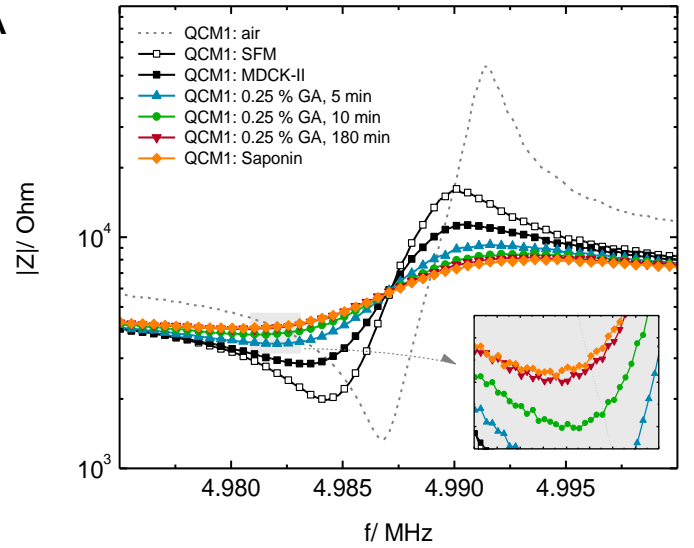

C

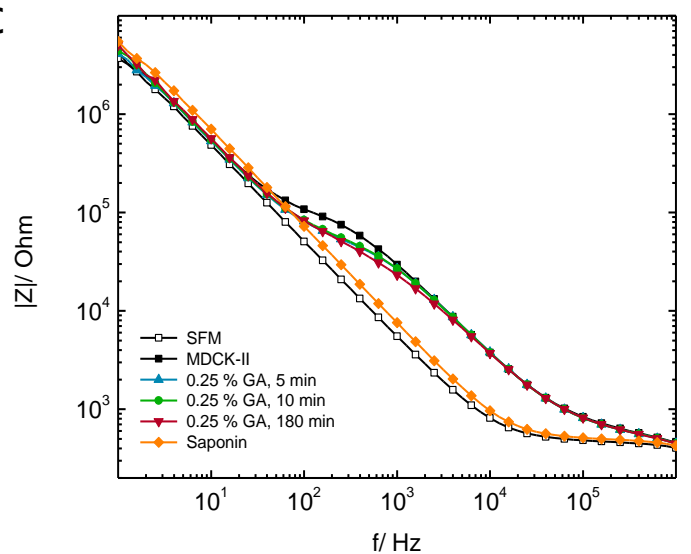

B

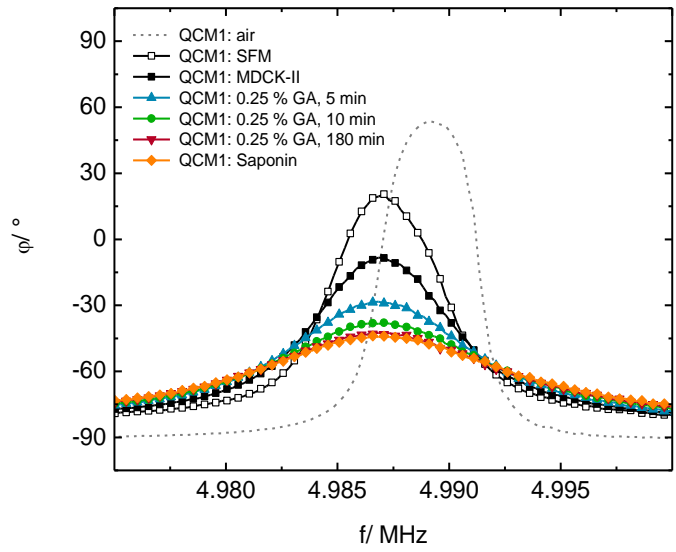

D

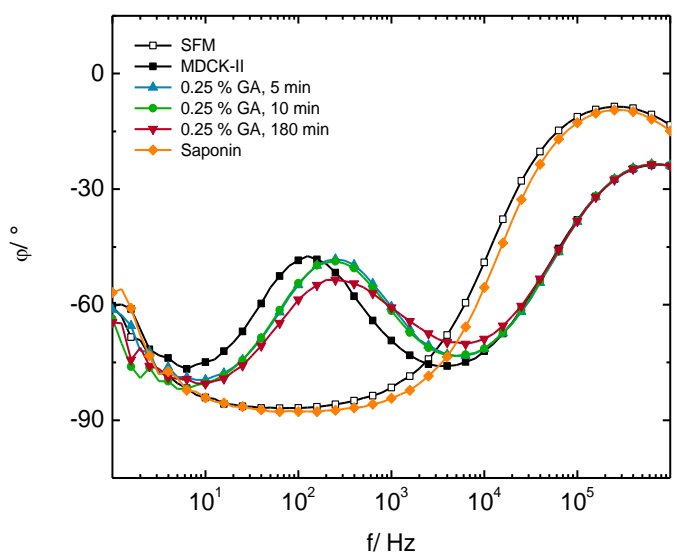

E

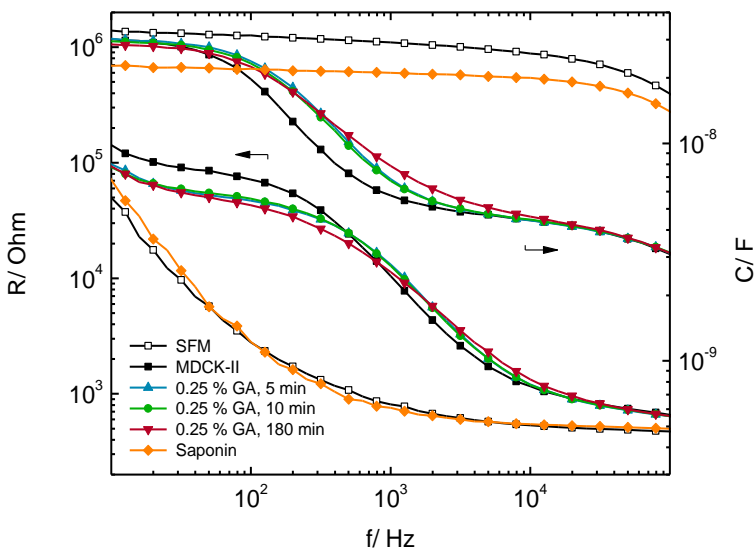


SI 22 OCM impedance spectra (A: $|Z|(f), \mathbf{B}: \varphi(f))$, ECIS impedance spectra (C: $|Z|(f)$, D: $\varphi(f))$, and ECIS resistance and capacitance spectra (E) of a OCM-ECIS sensor: in air, covered with a confluent layer of NRK cells, and with cells that have been treated with $100 \mu \mathrm{M}$ CPT-CAMP for $10 \mathrm{~min}, 30 \mathrm{~min}, 1 \mathrm{~h}$, and $4 \mathrm{~h}$.

A

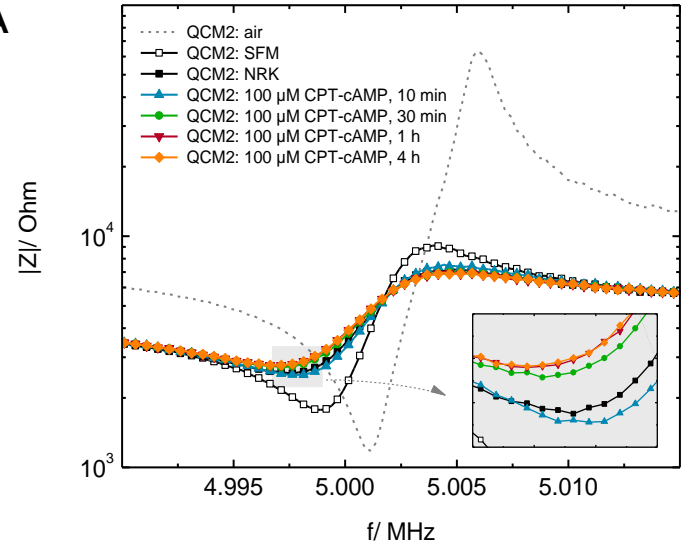

C

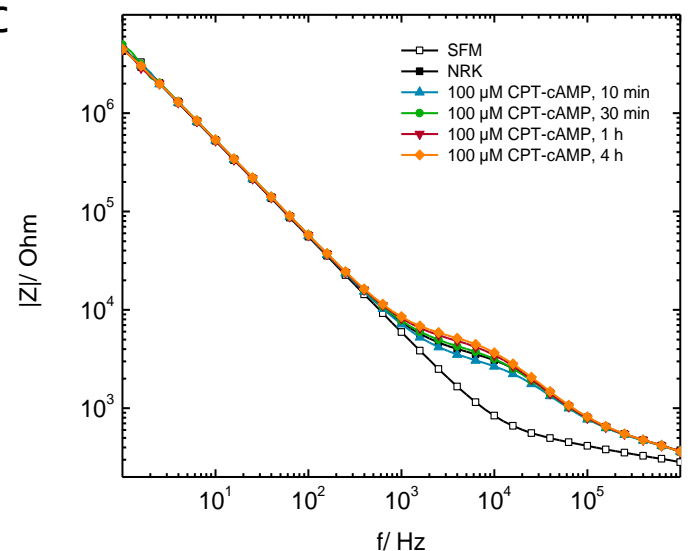

E

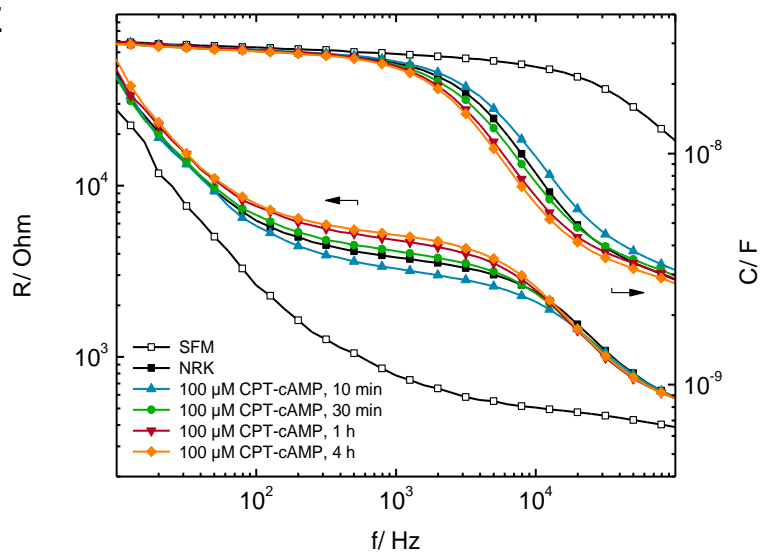

(1)
B

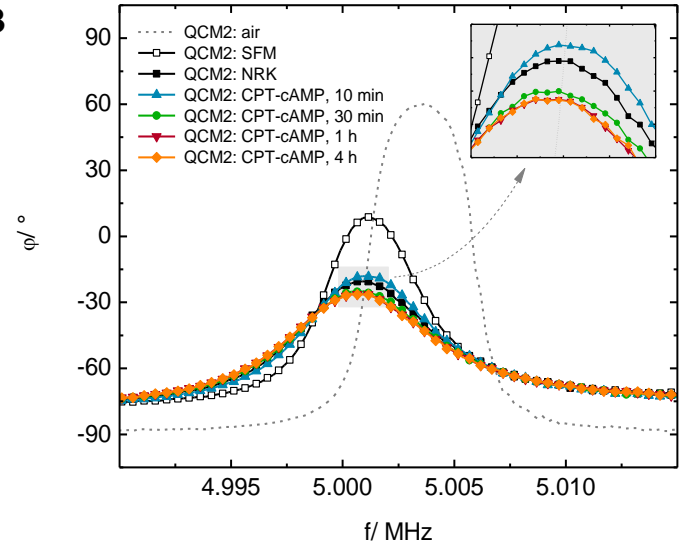

D

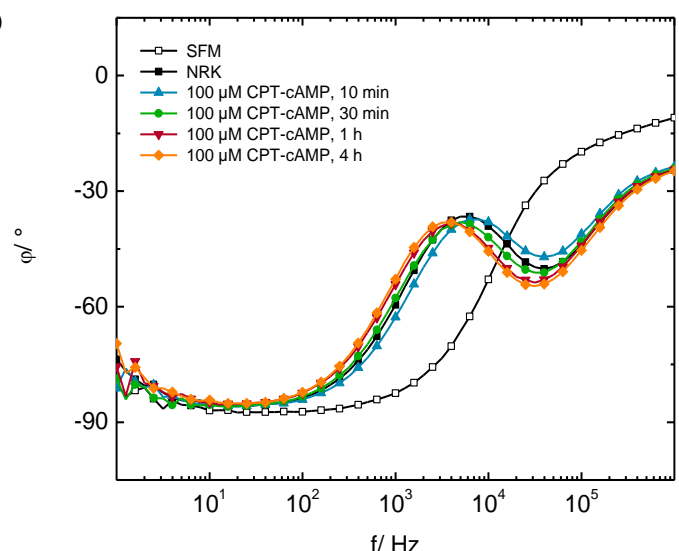


SI 23 OCM impedance spectra (A: $|Z|(f), \mathbf{B}: \varphi(f))$, ECIS impedance spectra (C: $|Z|(f), \mathbf{D}: \varphi(f))$, and ECIS resistance and capacitance spectra (E) of a QCM-ECIS sensor: in air, covered with a confluent layer of NRK cells, after medium exchange against $\mathrm{EBSS}^{++}$, after exposing cells extracellularly to membrane-impermeable FITC-dextran $(250 \mathrm{kDa})$, and at extreme values of parameter changes $\sim 1 / 2-1 \mathrm{~h}$ and at final recording values $\sim 2 \frac{1}{2} \mathrm{~h}$ after ELPO $(4 \mathrm{~V}, 200 \mathrm{~ms}$, $40 \mathrm{kHz}$ ).

A

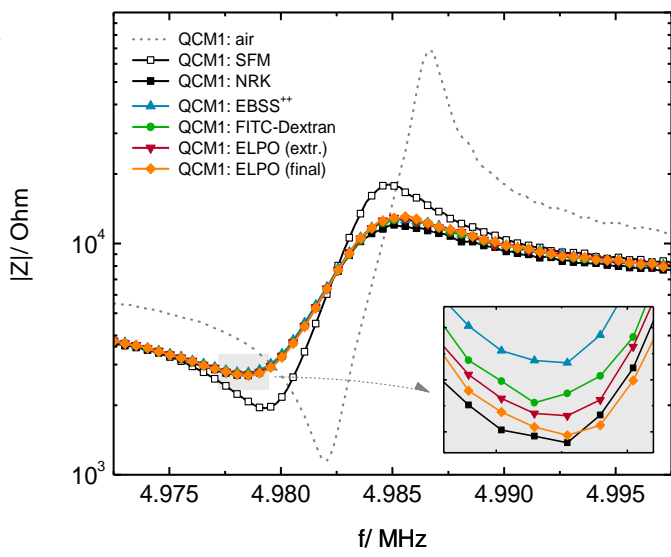

C

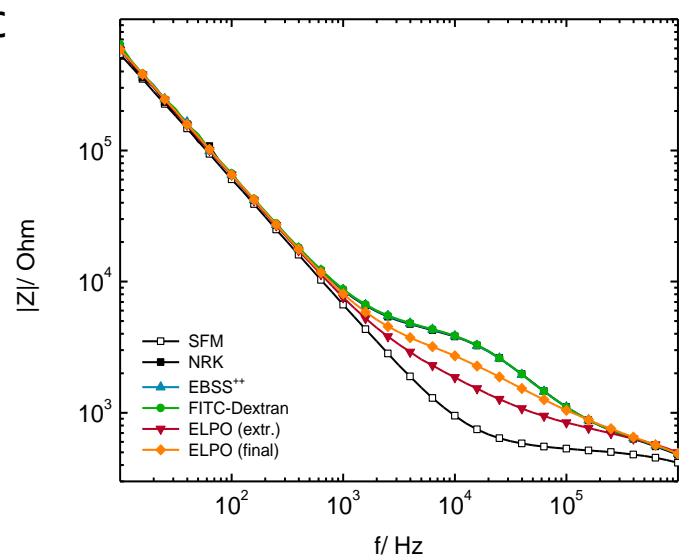

$E$

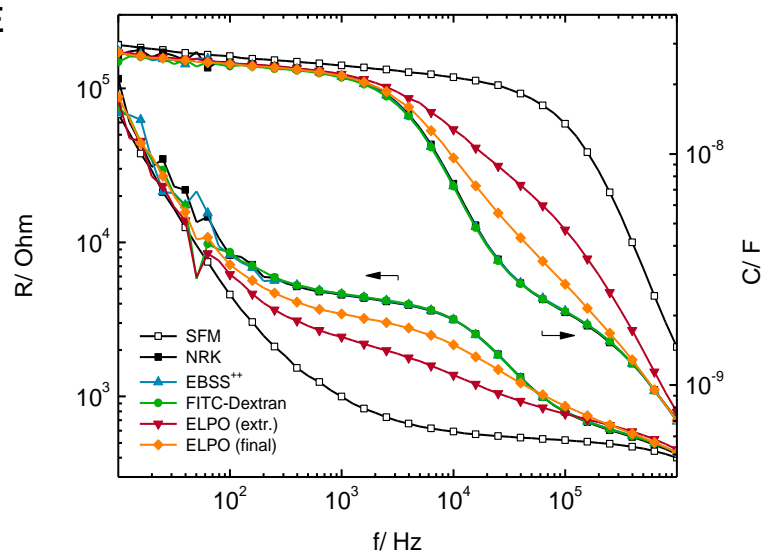

B

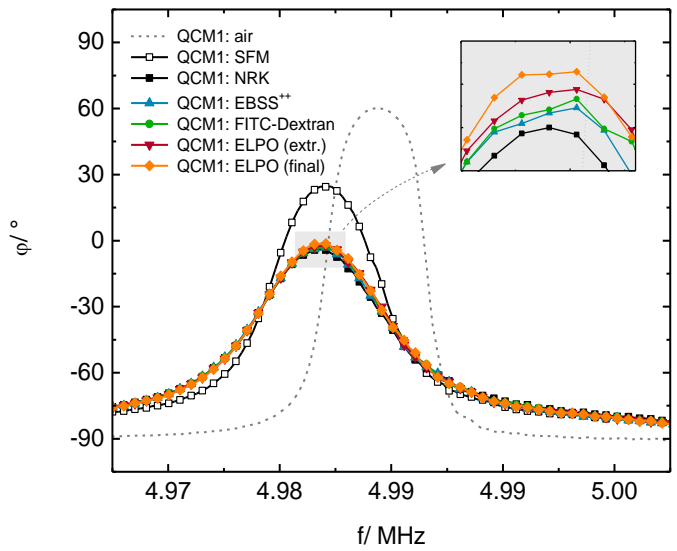

D

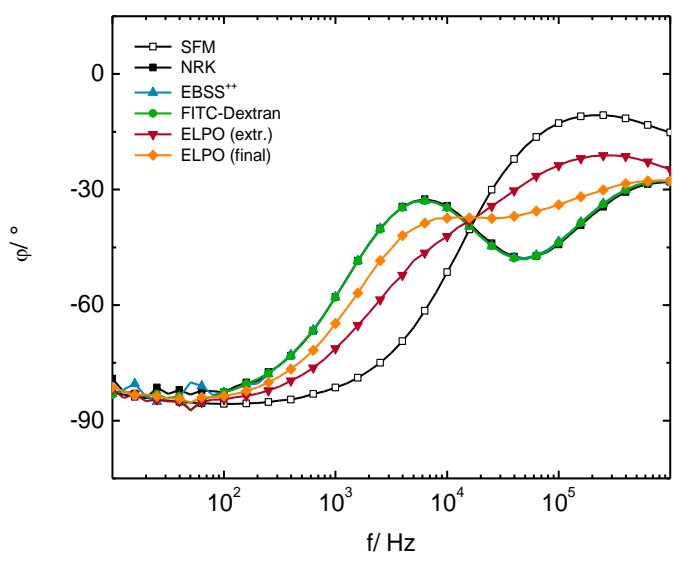


SI 24 Exemplary $R_{\text {norm }}\left(-{ }^{-}\right)$and $C_{\text {norm }}\left({ }^{-}-\right)$time curves at sensitive frequencies of MDCK-II cells fixated at time zero with either (A) $0.1 \%$ GA or (B) 1 \% PFA. Perforation of the plasma membrane with Saponin subsequent to fixation is indicated. Cell-free values of $R_{\text {norm }}$ and $C_{\text {norm }}$ are indicated by horizontal dashed lines.

A

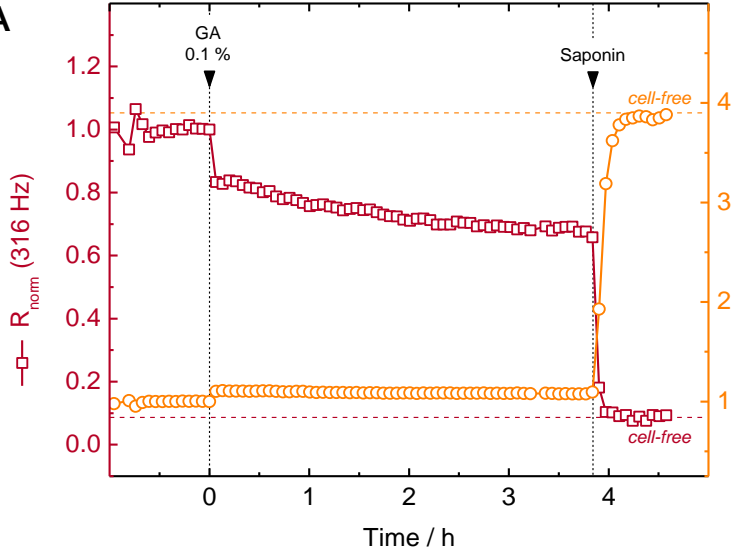

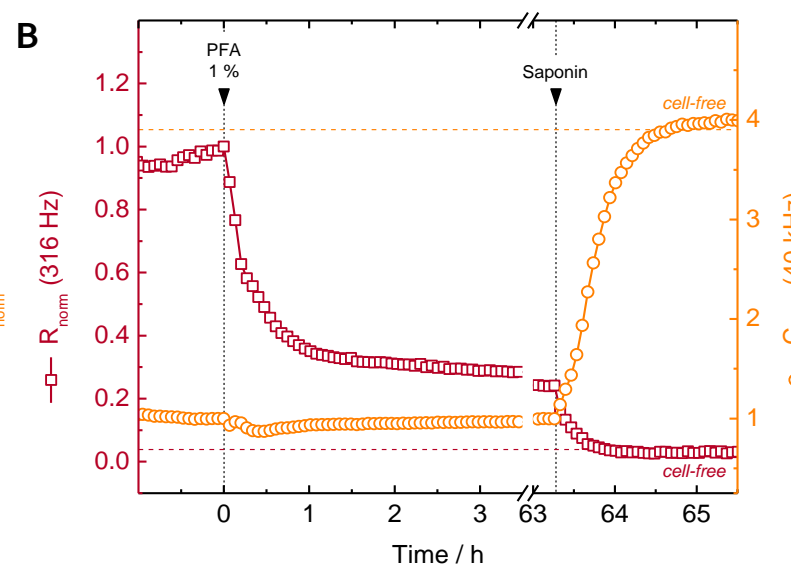

SI 25 (A) 3-D OCM-ECIS impedance plot ( $\Delta|Z|_{\min }$ vs. $R_{\text {norm }}$ vs. $\left.C_{\text {norm }}\right)$ of averaged, time-resolved cAMP assays. Parameter changes are shown between the point $\left(t_{0}\right)$ of adding $100 \mu \mathrm{M}$ of the membrane permeable CAMP-analogue 8-CPT-cAMP $(--)$ or medium only $(-\square)$ to a confluent NRK cell layer and $t_{1}=4.5 \mathrm{~h}$, as well as after withdrawing 8-CPT-cAMP from the cells again $\left(t_{1}\right.$ $\left.\rightarrow t_{2}=8 \mathrm{~h} ;-\Delta\right)$. (B), (C), (D) Projections on the planes of the curves in A. $R_{\text {norm }}$ and $C_{\text {norm }}$ are displayed at the most sensitive frequencies, which are $316 \mathrm{~Hz}$ and $40 \mathrm{kHz}$. Time resolution: $100 \mathrm{~s}$.
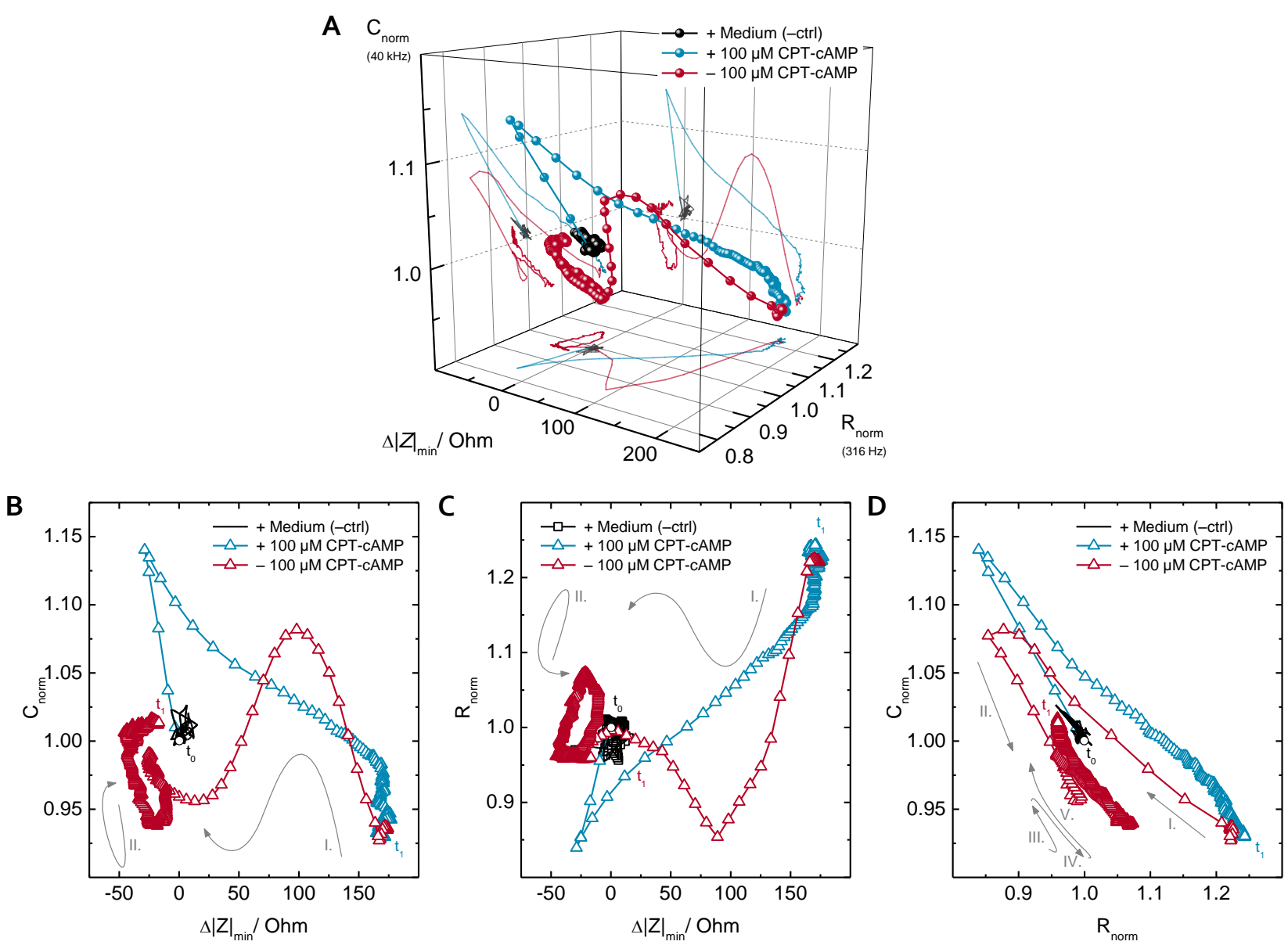
SI 26 Normalized spectra of absorbance "A" and emission "E" of (1) Eu(dnm) $)_{3}(\text { topo })_{2}$ and (2) $\mathrm{Eu}(\text { benzac) })_{3}$ (phen), emission of the excitation LED ( $405 \mathrm{~nm}$ with BG 12 filter), and transmission spectra "T" of the emission filter (EmF) D610/6oM.

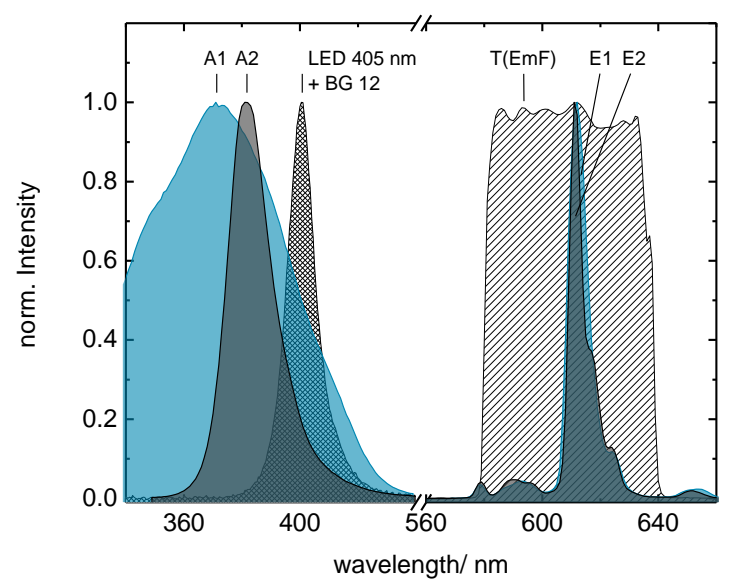

SI 27 Stern-Volmer plots obtained from FLIM of PSP sensor films calibrated at various $p \mathrm{O}_{2}$ values (total pressure 1 bar) (A) in dry air, (B) in air at $24^{\circ} \mathrm{C}$ and $37^{\circ} \mathrm{C}$, and $(\mathrm{C})$ under $\mathrm{PBS}^{++}$buffer. The best fits of the data points, taking the standard errors into account, according to the two-site quenching model are indicated by dashed red lines. The respective values of the fitting parameters are depicted in the associated tables.

A

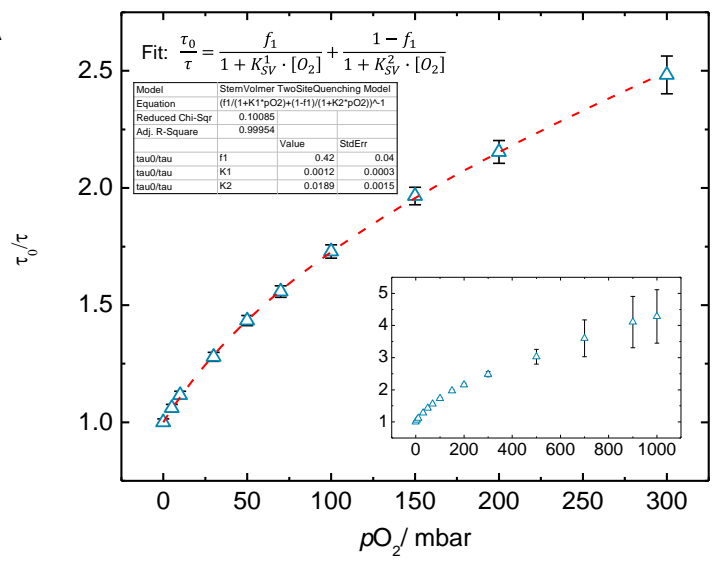

C

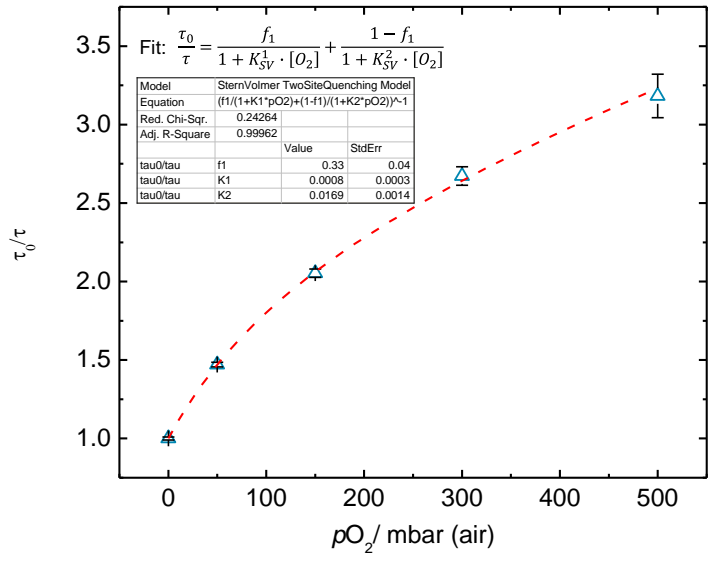

B

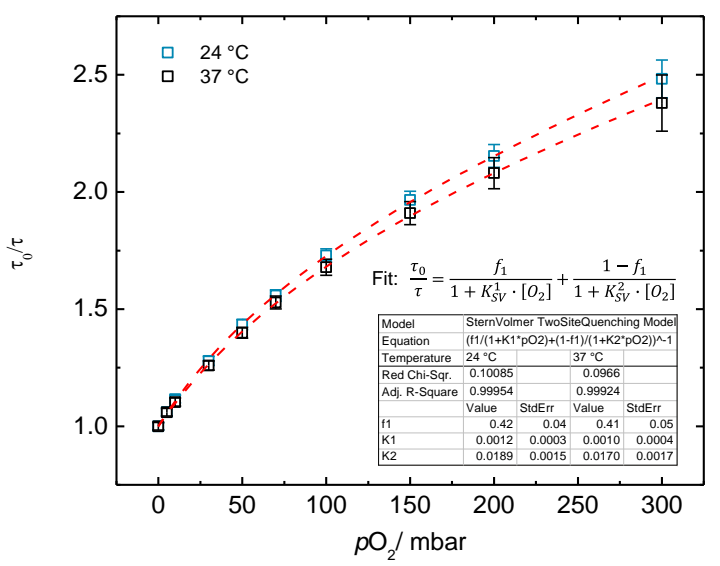


SI 28 Calibration plots, of average $A_{1} / A_{2}$ pixel values (mean $\pm \mathrm{SEM} ; \mathrm{N}=7$ ) as a function of $p \mathrm{O}_{2}$ (total pressure 1 bar), corresponding to the data shown in Fig. 6-12. Calibration points were measured by FLIM (in RLD mode) of the PSPs under PBS ${ }^{++}$buffer at $37^{\circ} \mathrm{C}$. (A), (B) Calibration of the PSP beneath the 2,4-DNP-treated MDCK-II cell layer, measured at an exposure time of (A) $2000 \mathrm{~ms}$ for $A_{1} / A_{2}$ values above 1.65 , and (B) $1500 \mathrm{~ms}$ for values below 1.65 (indicated with $\star$ in Fig. $6-$ 12). (C) Calibration of the PSP beneath the control cell layer. The best fits of the data points, taking the standard errors into account, are indicated by dashed red lines. The respective values of the fitting parameters are depicted in the associated tables.

A
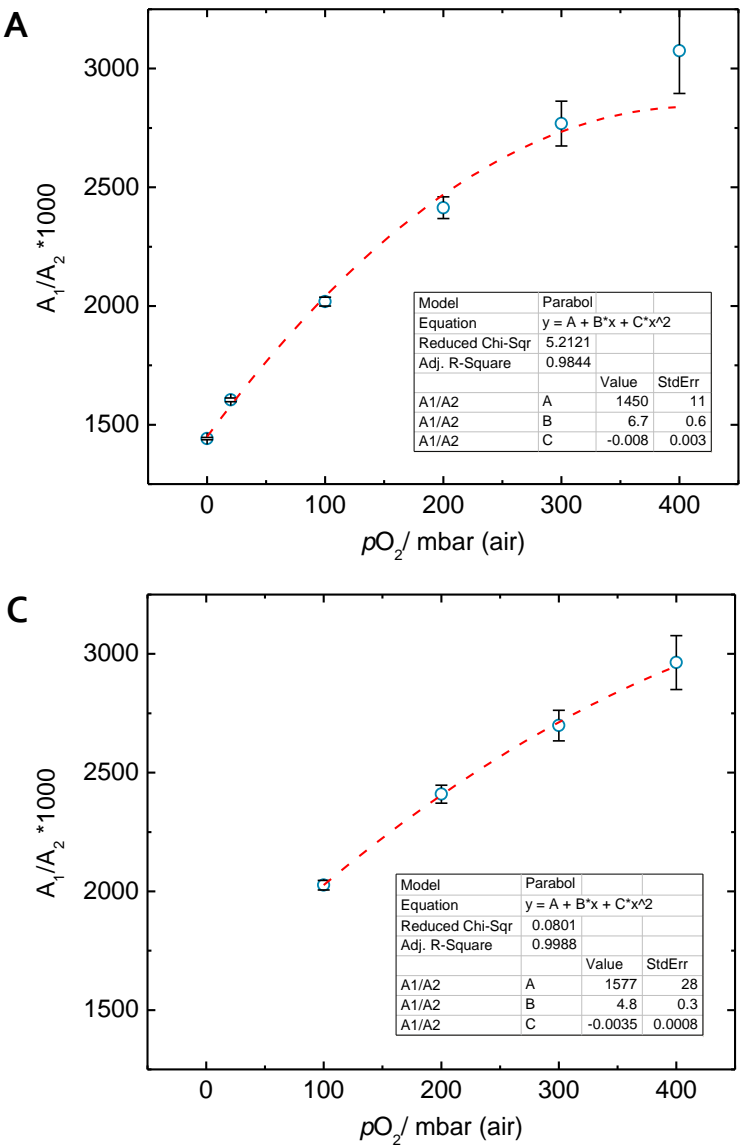

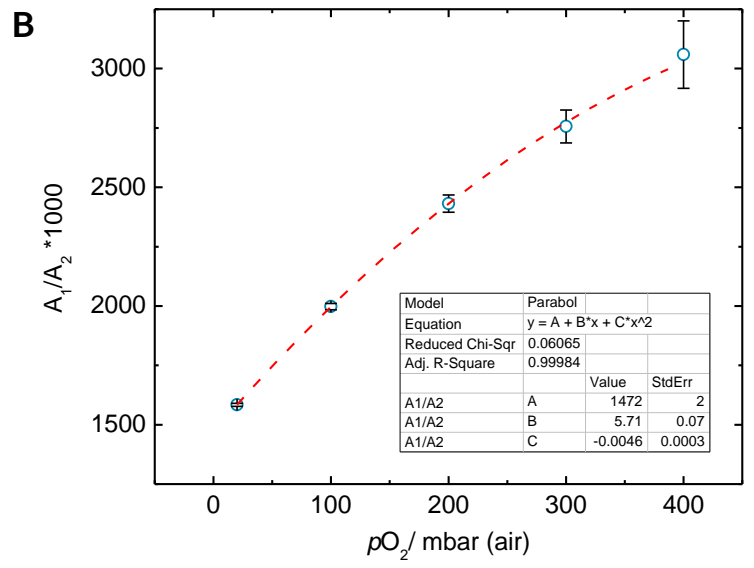


SI $29 \mathrm{pO}_{2}$ (air) images in pseudo-colors corresponding to the data points of the control cell layer in Fig. 6-12 B. Numbers depict the time of recording a fluorescence lifetime image. Points of $\mathrm{PBS}^{++}$ exchange are indicated as well. The calibration bar displays the color code of the $p \mathrm{O}_{2}$ (air) values of the pixels. The underlying calibration used for the conversion of $A_{1} / A_{2}$ to $p \mathrm{O}_{2}$ (air) images is shown in SI $28 \mathrm{C}$.

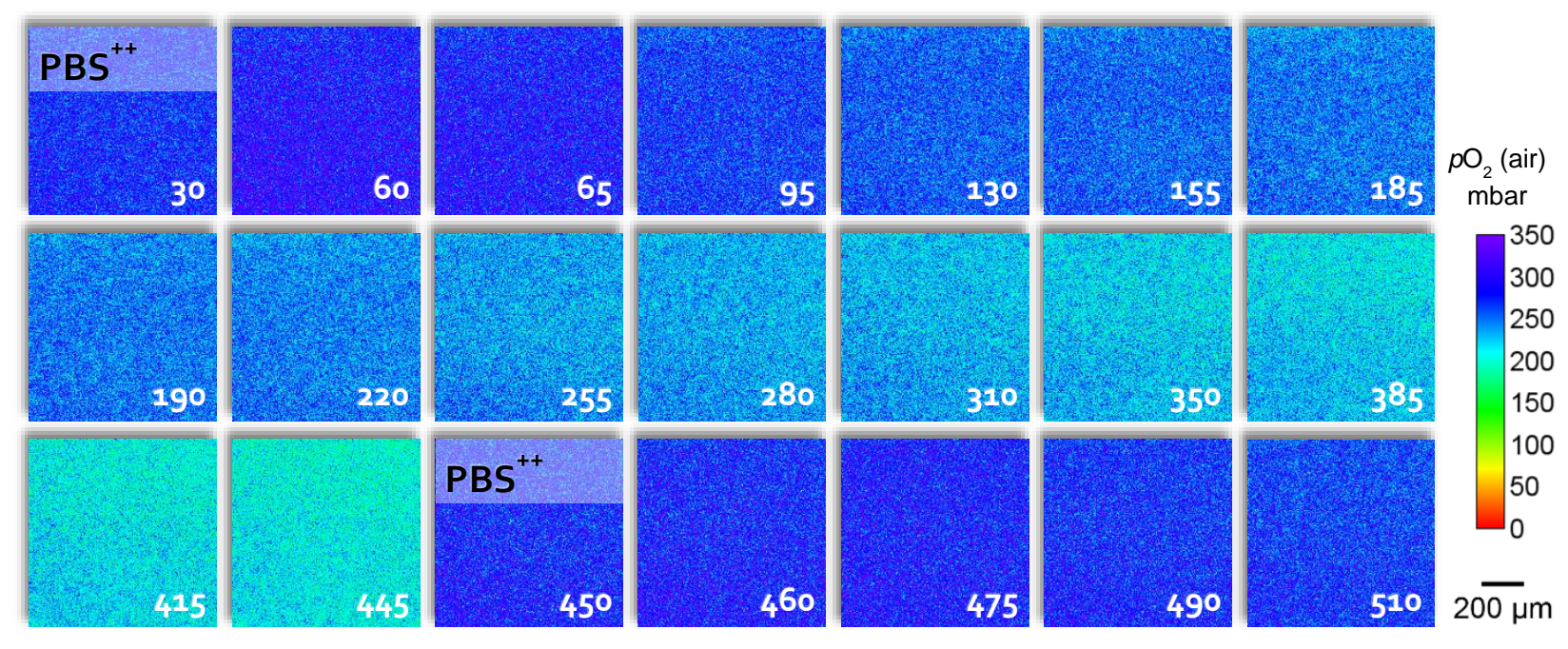




\section{B Abbreviations, Acronyms, and Symbols}

\section{List of Abbreviations and Acronyms}

+ ctrl

IEIO

2,4-DNP
$2-D$
2 EIO

3-D
$8-C P T-C A M P$
$8 \mathrm{~W}_{1} \mathrm{E}^{\mathrm{TM}}$

AC

AFM

AJ

ATP

BAEC

$B C E C$

$B O D$

BPAEC

CAMP

CBB

$C D$

CE

$\mathrm{CMS}^{\circledR}$

CPT-CAMP

ctrl

$-c t r l$

DAPI

DC

DM

DMEM

DMSO

DO

DSMZ
Positive Control

1-Electrode Quartz; $5 \mathrm{MHz}$ AT-cut quartz disk $\left(\varnothing_{q}=14 \mathrm{~mm}\right)$ with one electrode on either side

$\left(\varnothing_{\mathrm{E}}=6 \mathrm{~mm}\right)$

2,4-Dinitrophenol

Two-dimensional

2-Electrode Quartz; $5 \mathrm{MHz}$ AT-cut quartz disk $\left(\varnothing_{\mathrm{q}}=14 \mathrm{~mm}\right)$ with two electrodes on either side

$\left(\emptyset_{\mathrm{E}}=3.5 \mathrm{~mm}\right)$

Three-dimensional

8-(4-Chlorophenylthio)adenosine $3^{\prime}, 5^{\prime}$-cyclic monophosphate

ECIS array comprising 8 wells with 1 working electrode in each; trademark of Applied Biophysics, Troy, NY, USA

Alternating Current

Atomic Force Microscopy

Adherens Junction

Adenosine Triphosphate

Bovine Aortic Endothelial Cells

Bovine Corneal Endothelial Cells

Biological Oxygen Demand

Bovine Pulmonary Artery

Endothelial Cells

Adenosine $3^{\prime}, 5^{\prime}$-cyclic

monophosphate

Cell-Based Biosensor/ Biosensing

Cytochalasin D

Counter Electrode

Cell Montitoring System ${ }^{[64]}$

see 8-CPT-cAMP

Control

Negative Control

4',6-diamidin-2-phenylindol

Direct Current

Dichroitic Mirror

Dulbecco's Modified Eagle's

Medium

Dimethylsulfoxide

Dissolved Oxygen

German Collection of

Microorganisms and Cell Cultures
EBSS $^{--}$

Earles' Balanced Salt Solution, w/ o $\mathrm{Ca}^{2+}$ and $\mathrm{Mg}^{2+}$

EBSS $^{+-} \quad$ Earles' Balanced Salt Solution, w/ $\mathrm{Ca}^{2+}$ and $\mathrm{w} / \mathrm{o} \mathrm{Mg}^{2+}$

EBSS $^{-+} \quad$ Earles' Balanced Salt Solution, w/ o $\mathrm{Ca}^{2+}$ and $\mathrm{w} / \mathrm{Mg}^{2+}$

EBSS $^{++} \quad$ Earles' Balanced Salt Solution, w/ $\mathrm{Ca}^{2+}$ and $\mathrm{Mg}^{2+}$

EC Epithelial Cells

ECAR Extracellular Acidification Rate

ECIS ${ }^{\circledR} \quad$ Electric Cell-Substrate Impedance Sensing; registered trademark of Applied Biophysics, Troy, NY, USA

ECM Extracellular Matrix

EDTA Ethylenediaminetetraacetic acid

ELPO Electroporation

EnFET Enzyme Field-Effect Transistor

Eu(benzac) $)_{3}$ (phen) Tris(benzoylacetonato)mono(phenanthroline)europium(III)

$\mathrm{Eu}(\mathrm{dnm})_{3}(\mathrm{topo})_{2} \quad$ Tris(dinaphthoylmethane)bis(trioctylphosphine oxide)europium(III)

FA

Focal Adhesion

FCS Fetal Calf Serum

FED Field-Effect Device

FITC Fluorescein Isothiocyanate

FLIM Fluorescence (Phosphorescence) Lifetime Imaging

FN Fibronectin

GA Glutaraldehyde

GJ Gap Junction

GOx Glucose Oxidase Enzyme

HAEC Human Aortic Endothelial Cells

HC Hydrocortisone

HCS High-Content Screening

HD Hemidesmosome

HTS High-Throughput Screening

HUAEC Human Umbilical Artery Endothelial Cells

HUVEC Human Umbilical Vein Endothelial Cells

IA Impedance Analyzer

IDEs Interdigital Electrodes

IF Intermediate Filaments 
IS

ISFET

ITO

IUPAC

LAPS

LED

MDCK-II

ME

MEM

MISFET

MLAPS

MLC

MOSFET

NRK

OCR

OCS

$\mathrm{PBS}^{--}$

$\mathrm{PBS}^{++}$

PEBBLE

PEDOT

PFA

PhoP

PKA

PMT
Infrared Radiation

Impedance Spectroscopy

Ion-Selective Field-Effect

Transistor

Indium Tin Oxide

International Union of Pure and Applied Chemistry

Light-Addressable Potentiometric Sensor

Light-Emitting Diode

Madin Darby Canine Kidney cell line, strain II

Microelectrode

Minimum Essential Medium Eagle

Metal-Insulation-Semiconductor

Field-Effect Transistor

Multiple Light-Addressable

Potentiometric Sensor

Metal-Ligand Complex

Metal-Oxide-Semiconductor Field-

Effect Transistor

Normal Rat Kidney cell line, strain $52 \mathrm{E}$

Oxygen Consumption Rate

Optical Chemical Sensor/ Sensing

Phosphate Buffered Saline, w/ o

$\mathrm{Ca}^{2+}$ and $\mathrm{Mg}^{2+}$

Phosphate Buffered Saline, $\mathrm{w} / \mathrm{Ca}^{2+}$ and $\mathrm{Mg}^{2+}$

Probes Encapsulated By Biologically Localized Embedding

Poly(3,4-ethylenedioxythiophene)

Paraformaldehyde

Photopolymer

Protein kinase A

Photomultiplier Tube
PSP

PSS

PtTFPP

QCM

Q-factor

REVS

RI

RIfS

RLD

$\mathrm{ROI}$

rpm

RT

RWG

SDM

SEM

SFM

$\mathrm{SI}$

SPR

TER, TEER

TIR

TJ

TSM

TSP

UV

VIS

$V_{\text {rms }}$

w/

$w / o$

WE

ZO
Pressure-Sensitive Paint

Poly(styrenesulfonate)

$5,10,15,20$-Tetrakis- $(2,3,4,5,6$ pentafluorophenyl)-porphyrin-

platinum(II)

Quartz Crystal Microbalance

Quality Factor; cf. Eq. (63)

Rupture Event Scanning

Refractive Index

Reflectometric Interference

Spectroscopy

Rapid Lifetime Determination

Region Of Interest

Rounds Per Minute

Room Temperature

Resonant Waveguide Grating

Standard Deviation of Mean

Standard Error of Mean

Serum-Free Medium

Supplementary Information

Surface Plasmon Resonance

Transepithelial/Transendothelial

Electrical Resistance

Total Internal Reflection

Tight Junction

Thickness Shear Mode

Temperature-Sensitive Paint

Ultraviolet Radiation

Visible Radiation

Root-Mean-Square Voltage

with

without

Working Electrode

Zonula Occludens 


\section{List of Symbols}

$\varnothing$

$\alpha$

$\varepsilon$

$\eta$

\section{Diameter}

Model Parameter, $\alpha=r_{C} \cdot \sqrt{\rho_{\text {sub }} / d}\left[\Omega^{1 / 2} \cdot \mathrm{cm}\right]$

Decay Length

Electric Permittivity

Viscosity

Angle of Incident Light

Wavelength

Wave Frequency

Density;

Specific Resistance

Luminescence Lifetime

Phase Angle, Phase Shift

Radial Frequency, $\omega=2 \pi f$

Electric Potential [V];

Quantum Yield

Electrical Flux, $\Psi=\iint_{A} \vec{D} \cdot d \vec{A}[\mathrm{~A} \cdot \mathrm{s}]$

Area

\section{Susceptance}

Piezoelectrically Stiffened Quartz Elastic

Constant, $\overline{c_{66}}=c_{66}+e_{26}{ }^{2} / \epsilon_{22}$, for AT-cut Quartz:

$\overline{c_{66}}=2.947 \cdot 10^{10} \mathrm{~kg} \cdot \mathrm{m}^{-1} \cdot \mathrm{s}^{-2}$

Specific Heat Capacity

Capacitance;

Circumference

Distance

Quartz Thickness,

$d_{q}=330 \mu \mathrm{m}$ for $f_{s}=5 \mathrm{MHz}$

Electric Displacement Field,

$\vec{D}=\varepsilon_{0} \varepsilon_{r} \vec{E}\left[\mathrm{~A} \cdot \mathrm{s} \cdot \mathrm{m}^{-2}\right]$

Euler's Number, Base of the Natural

Logarithm,

$e \approx 2.71828$

Piezoelectric Stress Constant;

for AT-cut Quartz:

$e_{26}=9.54 \cdot 10^{-2} \mathrm{~A} \cdot \mathrm{s} \cdot \mathrm{m}^{-2}$

$\vec{E} \quad$ Electrical Field;

$\vec{E}(\vec{r})=-\nabla \Phi(\vec{r})$

Young's Modulus

Frequency

Fractional voltage drop across a cell layer

[\%], cf. Eq. (67)

Force

Gravitational Acceleration
Conductance

Complex Shear Modulus

Height;

Plank Constant

Complex Current

Current Amplitude

Electrical Current;

Luminescence Intensity

$\operatorname{Im}(Z) \quad$ Imaginary Part of the Complex

Impedance

Imaginary Unit

Spring Constant;

Rate Constant

$K_{S V} \quad$ Stern-Volmer Quenching Constant

$L \quad$ Inductance

m Mass

M Molar Mass

$n_{i} \quad$ Refractive Index of Medium $i$

$\mathrm{N} \quad$ Number of Values Used for Averaging

$\mathrm{pO}_{2} \quad$ Oxygen Partial Pressure

$p_{\text {tot }} \quad$ Total Preesure

$q \quad$ Charge

$Q \quad$ Quality Factor, $Q$-factor,

$Q=1 / D=X_{\text {tot }} / R_{\text {toti }}$

Quencher

$r \quad$ Radius

Damping Constant $\left(r=\eta_{q} \pi^{2} / d_{q}^{2}\right)$

$R$

Resistance

$\operatorname{Re}(Z) \quad$ Real Part of the Complex Impedance

$t \quad$ Time

$T \quad$ Temperature

$\underline{u} \quad$ Complex Voltage

$\hat{u} \quad$ Voltage Amplitude

U Voltage

$v \quad$ Velocity

V Volume

w Gaussian Distribution Coefficient $w^{\text {air }}=2.84, w^{\text {water }}=2.03$

$x \quad$ Shear Amplitude;

Displacement

Reactance

$\underline{Z} \quad$ Complex Impedance

$|Z| \quad$ Impedance Magnitude

Z Impedance 


\section{List of Special (Bio)Chemical Reagents}

2,4-Dinitrophenol (2,4-DNP)

4',6-diamidin-2-phenylindol (DAPI)

5,10,15,20-Tetrakis-(2,3,4,5,6-pentafluorophenyl)porphyrin-platinum(II) (PtTFPP)

8-(4-Chlorophenylthio)adenosine $3^{\prime}, 5^{\prime}$-cyclic monophosphate sodium salt (8-CPT-CAMP)

Alexa Fluor ${ }^{\circledR} 488$ phalloidin

Alexa Fluor ${ }^{\circledR} 546$ rabbit anti-mouse lgG $(\mathrm{H}+\mathrm{L})$

Cytochalasin D (CD)

Dimethylsulfoxide (DMSO)

FITC-Dextran $250 \mathrm{kDa}$

Glutaraldehyde (GA)

LIVE/DEAD ${ }^{\circledR}$ viability/ cytotoxicity kit

Paraformaldehyde (PFA)

Photoresist $A Z{ }^{\circledR} \mathrm{ECl}_{3027}$

Poly(vinylidene-chloride/ acrylonitrile) (80:20)

Sylgard ${ }^{\circledR} 182$ silicone elastomer kit

THF

TI Prime

Tolvene

Tris(benzoylacetonato)-mono(phenanthroline)europium(III) (Eu(benzac) ${ }_{3}$ (phen))

Tris(dinaphthoylmethane)-bis(trioctylphosphine oxide)-europium(III) (Eu(dnm) ${ }_{3}$ (topo $)_{2}$ )

TRITC-phalloidin

Triton-X-100

ZO-1 mouse monoclonal antibody
Sigma-Aldrich; St. Louis, MO, USA

Sigma-Aldrich; St. Louis, MO, USA

Porphyrine Systems GbR; Appen, Germany

Sigma-Aldrich; St. Louis, MO, USA

Life Technologies; Carlsbad, CA, USA

Life Technologies; Carlsbad, CA, USA

Sigma-Aldrich; St. Louis, MO, USA

Sigma-Aldrich; St. Louis, MO, USA

Sigma-Aldrich; St. Louis, MO, USA

Merck KGaA; Darmstadt, Germany

Molecular Probes, Life Technologies; Carlsbad, CA, USA

Merck Schuchardt OHG; Hohenbrunn

Microchemicals; Ulm, Germany

Polysciences, Inc.; Warrington, PA, USA

Dow Corning; Midland, MI, USA

Sigma-Aldrich; St. Louis, MO, USA

Microchemicals; Ulm, Germany

Merck; Darmstadt, Germany

Sigma-Aldrich; St. Louis, MO, USA

synthesized at the institute, according to Peng's procedure [329]

Sigma-Aldrich; St. Louis, MO, USA

Sigma-Aldrich; St. Louis, MO, USA

Life Technologies; Carlsbad, CA, USA 
References 
[1] D. R. Thévenot, K. Toth, R. A. Durst, G. S. Wilson, "Electrochemical Biosensors: Recommended Definitions and Classification", Pure and Applied Chemistry 1999, 71, 2333-2348.

[2] Y. Fang, "Label-Free Cell-Based Assays with Optical Biosensors in Drug Discovery", ASSAY and Drug Development Technologies 2006, 4, 583-595.

[3] B. Xi, N. Yu, X. Wang, X. Xu, Y. A. Abassi, "The Application of Cell-Based Label-Free Technology in Drug Discovery.", Biotechnology Journal 2008, 3, 484495 .

[4] T. S. Hug, "Biophysical Methods for Monitoring CellSubstrate Interactions in Drug Discovery.", ASSAY and Drug Development Technologies 2003, 1, 479-488.

[5] P. Banerjee, A. K. Bhunia, "Mammalian Cell-Based Biosensors for Pathogens and Toxins", Trends in biotechnology 2009, 27, 179-188.

[6] J. J. Pancrazio, J. P. Whelan, D. A. Borkholder, W. Ma, D. A. Stenger, "Development and Application of CellBased Biosensors", Annals of Biomedical Engineering 1999, 27, 697-711.

[7] J. A. Stolwijk, "Electric Manipulation and Impedance Analysis of Adherent Cells on Gold-Film Electrodes", Thesis, University of Regensburg, 2011.

[8] F.-G. Banica, Chemical Sensors and Biosensors: Fundamentals and Applications (Google eBook), John Wiley \& Sons, 2012.

[9] X. Fan, I. M. White, S. I. Shopova, H. Zhu, J. D. Suter et al., "Sensitive Optical Biosensors for Unlabeled Targets: A Review.", Analytica Chimica Acta 2008, 620, 8-26.

[10] O. Liu, C. Wu, H. Cai, N. Hu, J. Zhou et al., "Cell-Based Biosensors and Their Application in Biomedicine", Chemical Reviews 2014, 114, 6423-6461.

[11] S. Michaelis, R. Robelek, J. Wegener, "Studying CellSurface Interactions In Vitro: A Survey of Experimental Approaches and Techniques", in Tissue Engineering III: Cell - Surface Interactions for Tissue Culture (Eds.: C. Kasper, F. Witte, R. Pörtner), Springer Berlin Heidelberg, Berlin - Heidelberg, 2012, 33-66.

[12] Y. Fang, "Label-Free Biosensors for Cell Biology", International Journal of Electrochemistry 2011, 2011, 116.

[13] J. W. Chan, D. K. Lieu, "Label-Free Biochemical Characterization of Stem Cells Using Vibrational Spectroscopy", Journal of Biophotonics 2009, 2, 656668.

[14] I. Notingher, "Raman Spectroscopy Cell-Based Biosensors", Sensors 2007, 7, 1343-1358.

[15] S. M. Shamah, B. T. Cunningham, "Label-Free CellBased Assays Using Photonic Crystal Optical Biosensors", The Analyst 2011, 136, 1090.

[16] Y. Fang, "Label-Free and Non-Invasive Biosensor Cellular Assays for Cell Adhesion", Journal of Adhesion Science and Technology 2010, 24, 1011-1021.

[17] T. Ona, J. Shibata, "Advanced Dynamic Monitoring of Cellular Status Using Label-Free and Non-Invasive CellBased Sensing Technology for the Prediction of Anticancer Drug Efficacy", Analytical and Bioanalytical Chemistry 2010, 398, 2505-2533.
[18] R. Halai, M. A. Cooper, "Using Label-Free Screening Technology to Improve Efficiency in Drug Discovery.", Expert Opinion on Drug Discovery 2012, 7, 123-131.

[19] M. A. Cooper, "Current Biosensor Technologies in Drug Discovery", Drug Discovery 2006.

[20] K. Bizet, C. Gabrielli, H. Perrot, "Biosensors Based on Piezoelectric Transducers", Analusis 1999, 7, 609-616.

[21] M. Saitakis, E. Gizeli, "Acoustic Sensors as a Biophysical Tool for Probing Cell Attachment and Cell/surface Interactions.", Cellular and Molecular Life Sciences 2012, 69, 357-371.

[22] V. Heitmann, B. Reiß, J. Wegener, "The Quartz Crystal Microbalance in Cell Biology: Basics and Applications", in Piezoelectric Sensors (Eds.: C. Steinem, A. Janshoff), Springer, Berlin - Heidelberg, 2007, 303-338.

[23] A. Errachid, N. Zine, J. Samitier, J. Bausells, "FETBased Chemical Sensor Systems Fabricated with Standard Technologies", Electroanalysis 2004, 16, 1843-1851.

[24] M. Brittinger, P. Fromherz, "Field-Effect Transistor with Recombinant Potassium Channels: Fast and Slow Response by Electrical and Chemical Interactions", Applied Physics A: Materials Science and Processing 2005, 81, 439-447.

[25] M. Pabst, G. Wrobel, S. Ingebrandt, F. Sommerhage, A. Offenhäusser, "Solution of the Poisson-Nernst-Planck Equations in the Cell-Substrate Interface", European Physical Journal E 2007, 24, 1-8.

[26] M. Schmidtner, P. Fromherz, "Functional Na+ Channels in Cell Adhesion Probed by Transistor Recording.", Biophysical journal 2006, 90, 183-189.

[27] P. Wang, G. Xu, L. Qin, Y. Xu, Y. Li et al., "Cell-Based Biosensors and Its Application in Biomedicine", Sensors and Actuators B: Chemical 2005, 108, 576-584.

[28] Y. Mourzina, T. Mai, A. Poghossian, Y. Ermolenko, T. Yoshinobu et al., "K+ -Selective Field-Effect Sensors as Transducers for Bioelectronic Applications", Electrochimica Acta 2003, 48, 3333-3339.

[29] K. M. L. May, Y. Wang, L. G. Bachas, K. W. Anderson, "Development of a Whole-Cell-Based Biosensor for Detecting Histamine as a Model Toxin", Analytical Chemistry 2004, 76, 4156-4161.

[30] D. Braeken, D. R. Rand, a Andrei, R. Huys, M. E. Spira et al., "Glutamate Sensing with Enzyme-Modified Floating-Gate Field Effect Transistors.", Biosensors \& Bioelectronics 2009, 24, 2384-9.

[31] L. Yin, J. Chou, W. Chung, T. Sun, "Glucose ENFET Doped with $\mathrm{MnO}_{2}$ Powder", Sensors and Actuators B: Chemical 2001, 76, 187-192.

[32] K. Y. Park, S. B. Choi, M. Lee, B. K. Sohn, S. Y. Choi, "ISFET Glucose Sensor System with Fast Recovery Characteristics by Employing Electrolysis", Sensors and Actuators B: Chemical 2002, 83, 90-97.

[33] X. L. Luo, J. J. Xu, W. Zhao, H. Y. Chen, "A Novel Glucose ENFET Based on the Special Reactivity of $\mathrm{MnO}_{2}$ Nanoparticles", Biosensors \& Bioelectronics 2004, 19, 1295-1300.

[34] X. L. Luo, J. J. Xu, W. Zhao, H. Y. Chen, "Glucose Biosensor Based on ENFET Doped with $\mathrm{SiO}_{2}$ 
Nanoparticles", Sensors and Actuators B: Chemical 2004, 97, 249-255.

[35] J. C. Chen, J. C. Chou, T. P. Sun, S. K. Hsiung, "Portable Urea Biosensor Based on the Extended-Gate Field Effect Transistor", Sensors and Actuators B: Chemical 2003, 91, 180-186.

[36] A. P. Soldatkin, J. Montoriol, W. Sant, C. Martelet, N. Jaffrezic-Renault, "A Novel Urea Sensitive Biosensor with Extended Dynamic Range Based on Recombinant Urease and ISFETs", Biosensors \& Bioelectronics 2003, 19, 131-135.

[37] W. Sant, M. L. Pourciel, J. Launay, T. Do Conto, A. Martinez et al., "Development of Chemical Field Effect Transistors for the Detection of Urea", Sensors and Actuators B: Chemical 2003, 95, 309-314.

[38] F. Aouni, R. Mlika, C. Martelet, H. Ben Ouada, N. Jaffrezic-Renault et al., "Modelling of the Potentiometric Response of ENFETs Based on Enzymatic Multilayer Membranes", Electroanalysis 2004, 16, 1907-1911.

[39] A. V Rebriiev, N. F. Starodub, "Enzymatic Biosensor Based on the ISFET and Photopolymeric Membrane for the Determination of Urea", Electroanalysis 2004, 16, 1891-1895.

[40] D. Niwa, K. Omichi, N. Motohashi, T. Homma, T. Osaka, "Organosilane Self-Assembled MonolayerModified Field Effect Transistors for on-Chip Ion and Biomolecule Sensing", Sensors and Actuators B: Chemical 2005, 108, 721-726.

[41] J.-J. Xu, W. Zhao, X.-L. Luo, H.-Y. Chen, "A Sensitive Biosensor for Lactate Based on Layer-by-Layer Assembling $\mathrm{MnO}_{2}$ Nanoparticles and Lactate Oxidase on Ion-Sensitive Field-Effect Transistors.", Chemical Communications 2005, 792-794.

[42] A. Poghossian, M. J. Schöning, P. Schroth, A. Simonis, H. Lüth, "An ISFET-Based Penicillin Sensor with High Sensitivity, Low Detection Limit and Long Lifetime", Sensors and Actuators B: Chemical 2001, 76, 519-526.

[43] A. Poghossian, T. Yoshinobu, A. Simonis, H. Ecken, H. Lüth et al., "Penicillin Detection by Means of FieldEffect Based Sensors: EnFET, Capacitive EIS Sensor or LAPS?", Sensors and Actuators B: Chemical 2001, 78, 237-242.

[44] A. P. Soldatkin, V. N. Arkhypova, S. V Dzyadevych, A. $\checkmark$ El'skaya, J. M. Gravoueille et al., "Analysis of the Potato Glycoalkaloids by Using of Enzyme Biosensor Based on pH-ISFETs", Talanta 2005, 66, 28-33.

[45] M. Lehmann, W. Baumann, M. Brischwein, H. J. Gahle, I. Freund et al., "Simultaneous Measurement of Cellular Respiration and Acidification with a Single CMOS ISFET", Biosensors \& Bioelectronics 2001, 16, 195-203.

[46] B. Wolf, M. Brischwein, V. Lob, J. Ressler, J. Wiest, "Cellular Signaling: Aspects for Tumor Diagnosis and Therapy.", Biomedizinische Technik. Biomedical engineering 2007, 52, 164-8.

[47] J. Wiest, T. Stadthagen, M. Schmidhuber, M. Brischwein, J. Ressler et al., "Intelligent Mobile Lab for Metabolics in Environmental Monitoring", Analytical Letters 2006, 39, 1759-1771.
[48] J. Wiest, M. Brischwein, "Cellular Assays with Multiparametric Bioelectronic Sensor Chips", CHIMIA 2005, 59, 243-246.

[49] E. Thedinga, A. Kob, H. Holst, A. Kever, S. Drechsler et al., "Online Monitoring of Cell Metabolism for Studying Pharmacodynamic Effects", Toxicology and Applied Pharmacology 2007, 220, 33-44.

[50] S. Seeland, M. Török, H. Kettiger, A. Treiber, M. Hafner et al., "A Cell-Based, Multiparametric Sensor Approach Characterises Drug-Induced Cytotoxicity in Human Liver HepG2 Cells", Toxicology in Vitro 2013, 27, 11091120.

[51] T. Schwarzenberger, P. Wolf, M. Brischwein, R. Kleinhans, F. Demmel et al., "Impedance Sensor Technology for Cell-Based Assays in the Framework of a High-Content Screening System", Physiological Measurement 2011, 32, 977-993.

[52] J. Ressler, H. Grothe, E. Motrescu, B. Wolf, "New Concepts for Chip-Supported Multi-Well-Plates: Realization of a 24-Well-Plate with Integrated Impedance-Sensors for Functional Cellular Screening Applications and Automated Microscope Aided CellBased Assays", in Conference Proceedings of the 26th Annual International Conference of the IEEE Engineering in Medicine and Biology Society, 2004, 2074-2077.

[53] A. M. Otto, M. Brischwein, A. Niendorf, T. Henning, E. Motrescu et al., "Microphysiological Testing for Chemosensitivity of Living Tumor Cells with Multiparametric Microsensor Chips", Cancer Detection and Prevention 2003, 27, 291-296.

[54] A. M. Otto, M. Brischwein, E. Motrescu, E. Cabala, H. Grothe et al., "Chips instead of Mice: Cells on Bioelectronic Sensor-Chips as an Alternative to Animal Experiments", ALTEX: Alternatives to Animal Experimentation 2004, Supp. Linz, 70-76.

[55] V. Lob, T. Geisler, M. Brischwein, R. Uhl, B. Wolf, "Automated Live Cell Screening System Based on a 24Well-Microplate with Integrated Micro Fluidics.", Medical \& Biological Engineering \& Computing 2007, 45, 1023-1028.

[56] S. Kustermann, F. Boess, A. Buness, M. Schmitz, M. Watzele et al., "A Label-Free, Impedance-Based Real Time Assay to Identify Drug-Induced Toxicities and Differentiate Cytostatic from Cytotoxic Effects.", Toxicology in Vitro 2013, 27, 1589-1595.

[57] R. Kubisch, U. Bohrn, M. Fleischer, E. Stütz, "CellBased Sensor System Using L6 Cells for Broad Band Continuous Pollutant Monitoring in Aquatic Environments", Sensors 2012, 12, 3370-3393.

[58] A. S. Kocincová, S. Nagl, S. Arain, C. Krause, S. M. Borisov et al., "Multiplex Bacterial Growth Monitoring in 24-Well Microplates Using a Dual Optical Sensor for Dissolved Oxygen and $\mathrm{pH}^{\mathrm{N}}$, Biotechnology and Bioengineering 2008, 100, 430-438.

[59] R. Kleinhans, M. Brischwein, P. Wang, B. Becker, F. Demmel et al., "Sensor-Based Cell and Tissue Screening for Personalized Cancer Chemotherapy.", Medical \& Biological Engineering \& Computing 2012, 50, 117-26.

[6o] R. Ehret, W. Baumann, M. Brischwein, M. Lehmann, T. Henning et al., "Multiparametric Microsensor Chips for 
Screening Applications.", Fresenius' Journal of Analytical Chemistry 2001, 369, 30-35.

[61] L. Ceriotti, A. Kob, S. Drechsler, J. Ponti, E. Thedinga et al., "Online Monitoring of BALB/3 $3 \mathrm{~T}_{3}$ Metabolism and Adhesion with Multiparametric Chip-Based System", Analytical Biochemistry 2007, 371, 92-104.

[62] M. Brischwein, E. R. Motrescu, E. Cabala, a M. Otto, H. Grothe et al., "Functional Cellular Assays with Multiparametric Silicon Sensor Chips.", Lab on a Chip 2003, 3, 234-240.

[63] W. Baumann, E. Schreiber, G. Krause, S. Stüwe, A. Podssun et al., "Multiparametric Neurosensor Microchip", Proc. Eurosensors XVI 2002, 1169-1172.

[64] W. H. Baumann, M. Lehmann, A. Schwinde, R. Ehret, M. Brischwein et al., "Microelectronic Sensor System for Microphysiological Application on Living Cells", Sensors and Actuators B 1999, 55, 77-89.

[65] M. Wu, A. Neilson, A. L. Swift, R. Moran, J. Tamagnine et al., "Multiparameter Metabolic Analysis Reveals a Close Link between Attenuated Mitochondrial Bioenergetic Function and Enhanced Glycolysis Dependency in Human Tumor Cells", American Journal of Physiology. Cell Physiology 2007, 01862, C125-C136.

[66] M. J. Schöning, A. Poghossian, "Bio FEDs (Field-Effect Devices): State-of-the-Art and New Directions", Electroanalysis 2006, 18, 1893-1900.

[67] F. Hafner, "Cytosensor Microphysiometer: Technology and Recent Applications", Biosensors \& Bioelectronics 2000, 15, 149-158.

[68] A. Poghossian, S. Ingebrandt, A. Offenhäusser, M. J. Schöning, "Field-Effect Devices for Detecting Cellular Signals.", Seminars in Cell \& Developmental Biology 2009, 20, 41-48.

[69] H. M. McConnell, J. C. Owicki, J. W. Parce, D. L. Miller, G. T. Baxter et al., "The Cytosensor Microphysiometer: Biological Applications of Silicon Technology.", Science 1992, 257, 1906-1912.

[70] J. C. Owicki, L. J. Bousse, D. G. Hafeman, G. L. Kirk, J. D. Olson et al., "The Light-Addressable Potentiometric Sensor: Principles and Biological Applications.", Annual Review of Biophysics and Biomolecular Structure 1994, 23, 87-113.

[71] M. J. Schöning, T. Wagner, C. Wang, R. Otto, T. Yoshinobu, "Development of a Handheld 16 Channel Pen-Type LAPS for Electrochemical Sensing", Sensors and Actuators B: Chemical 2005, 108, 808-814.

[72] W. Yicong, W. Ping, Y. Xuesong, Z. Gaoyan, H. Huiqi et al., "Drug Evaluations Using a Novel Microphysiometer Based on Cell-Based Biosensors", Sensors and Actuators B: Chemical 2001, 80, 215-221.

[73] W. Yicong, W. Ping, Y. Xuesong, Z. Qingtao, L. Rong et al., "A Novel Microphysiometer Based on MLAPS for Drugs Screening", Biosensors \& Bioelectronics 2001, 16, 277-286.

[74] Z. Qintao, W. Ping, W. J. Parak, M. George, G. Zhang, "Novel Design of Multi-Light LAPS Based on Digital Compensation of Frequency Domain", Sensors and Actuators B: Chemical 2001, 73, 152-156.

[75] T. Wagner, T. Yoshinobu, C. Rao, R. Otto, M. J. Schöning, "'All-in-One" Solid-State Device Based on a
Light-Addressable Potentiometric Sensor Platform", Sensors and Actuators B: Chemical 2006, 117, 472-479.

[76] T. Wagner, C. Rao, J. P. Kloock, T. Yoshinobu, R. Otto et al., "'LAPS Card"-A Novel Chip Card-Based LightAddressable Potentiometric Sensor (LAPS)", Sensors and Actuators B: Chemical 2006, 118, 33-40.

[77] T. Yoshinobu, M. J. Schöning, R. Otto, K. Furuichi, Y. Mourzine et al., "Portable Light-Addressable Potentiometric Sensor (LAPS) for Multisensor Applications", Sensors and Actuators, B: Chemical 2003, 95, 352-356.

[78] S. E. Eklund, R. G. Thompson, R. M. Snider, C. K. Carney, D. W. Wright et al., "Metabolic Discrimination of Select List Agents by Monitoring Cellular Responses in a Multianalyte Microphysiometer", Sensors 2009, 9, 2117-2133.

[79] S. E. Eklund, R. M. Snider, J. Wikswo, F. Baudenbacher, A. Prokop et al., "Multianalyte Microphysiometry as a Tool in Metabolomics and Systems Biology", Journal of Electroanalytical Chemistry 2006, 587, 333-339.

[80] A. Das, Y.-H. Lin, C.-S. Lai, "Miniaturized AmorphousSilicon Based Chemical Imaging Sensor System Using a Mini-Projector as a Simplified Light-Addressable Scanning Source", Sensors and Actuators B: Chemical 2014, 190, 664-672.

[81] Y.-H. Lin, A. Das, C.-S. Lai, "A Simple and Convenient Set-up of Light Addressable Potentiometric Sensors (LAPS) for Chemical Imaging Using a Commercially Available Projector as a Light Source", International Journal of Electrochemical Science 2013, 8, 7062-7074.

[82] C. F. Werner, T. Wagner, K. Miyamoto, T. Yoshinobu, M. J. Schöning, "High Speed and High Resolution Chemical Imaging Based on a New Type of OLEDLAPS Set-Up", Sensors and Actuators B: Chemical 2012, 175, 118-122.

[83] T. Yoshinobu, H. Ecken, A. B. Md. Ismail, H. Iwasaki, H. Lüth et al., "Chemical Imaging Sensor and Its Application to Biological Systems", Electrochimica Acta 2001, 47, 259-263.

[84] L. C. Clark Jr, "Monitor and Control of Blood and Tissue Oxygen Tensions", ASAIO Journal 1956, 2, 41-48.

[85] L. C. Clark Jr, C. Lyons, "Electrode Systems for Continuous Monitoring in Cardiovascular Surgery", Annals of the New York Academy of Sciences 1962, 102, 29-45.

[86] M. I. Prodromidis, M. I. Karayannis, "Enzyme Based Amperometric Biosensors for Food Analysis", Electroanalysis 2002, 14, 241-261.

[87] I. Giaever, C. R. Keese, "Monitoring Fibroblast Behavior in Tissue Culture with an Applied Electric Field.", Proceedings of the National Academy of Sciences of the United States of America 1984, 81, 3761-3764.

[88] I. Giaever, C. R. Keese, "A Morphological Biosensor for Mammalian Cells.", Nature 1993, 366, 591-592.

[89] J. Wegener, C. R. Keese, I. Giaever, "Electric CellSubstrate Impedance Sensing (ECIS) as a Noninvasive Means to Monitor the Kinetics of Cell Spreading to Artificial Surfaces", Experimental Cell Research 2000, 259, 158-166. 
[90] A. Janshoff, A. Kunze, S. Michaelis, V. Heitmann, B. Reiss et al., "Cell Adhesion Monitoring Using Substrate-Integrated Sensors", Journal of Adhesion Science and Technology 2010, 24, 2079-2104.

[91] C. Tiruppathi, A. B. Malik, P. J. Del Vecchio, C. R. Keese, I. Giaever, "Electrical Method for Detection of Endothelial Cell Shape Change in Real Time: Assessment of Endothelial Barrier Function.", Proceedings of the National Academy of Sciences of the United States of America 1992, 89, 7919-7923.

[92] L. Reddy, H.-S. Wang, C. R. Keese, I. Giaever, T. J. Smith, "Assessment of Rapid Morphological Changes Associated with Elevated CAMP Levels in Human Orbital Fibroblasts.", Experimental Cell Research 1998, $245,360-367$.

[93] T. Martin, W. Jiang, "Tight Junctions in Cancer Metastasis and Their Investigation Using ECIS (Electric Cell-Substrate Impedance Sensing)", in Electric CellSubstrate Impedance Sensing and Cancer Metastasis (Ed.: W.G. Jiang), Springer Netherlands, 2012, 119130.

[94] T. J. Smith, H.-S. Wang, M. G. Hogg, R. C. Henrikson, C. R. Keese et al., "Prostaglandin E2 Elicits a Morphological Change in Cultured Orbital Fibroblasts from Patients with Graves Ophthalmopathy.", Proceedings of the National Academy of Sciences of the United States of America 1994, 91, 5094-5098.

[95] C.-M. Lo, C. R. Keese, I. Giaever, "pH Changes in Pulsed $\mathrm{CO}_{2}$ Incubators Cause Periodic Changes in Cell Morphology", Experimental Cell Research 1994, 213, 391-397.

[96] I. Giaever, C. R. Keese, "Micromotion of Mammalian Cells Measured Electrically.", Proceedings of the National Academy of Sciences of the United States of America 1991, 88, 7896-7900.

[97] C.-M. Lo, C. R. Keese, I. Giaever, "Monitoring Motion of Confluent Cells in Tissue Culture", Experimental Cell Research 1993, 204, 102-109.

[98] D. Lovelady, T. Richmond, A. Maggi, C.-M. Lo, D. Rabson, "Distinguishing Cancerous from Noncancerous Cells through Analysis of Electrical Noise", Physical Review E 2007, 76, 1-10.

[99] C. R. Keese, J. Wegener, S. R. Walker, I. Giaever, "Electrical Wound-Healing Assay for Cells in Vitro.", Proceedings of the National Academy of Sciences of the United States of America 2004, 101, 1554-1559.

[100] B. Plunger, C. Choi, T. Sparer, "Electrical Cell-Substrate Impedance Sensing for Measuring Cellular Transformation, Migration, Invasion, and Anticancer Compound Screening", in Electric Cell-Substrate Impedance Sensing and Cancer Metastasis (Ed.: W.G. Jiang), Springer Netherlands, 2012, 55-69.

[101] K. Szaszi, M. Vandermeer, Y. Amoozadeh, "Epithelial Wound Healing and the Effects of Cytokines Investigated by ECIS", in Electric Cell-Substrate Impedance Sensing and Cancer Metastasis (Ed.: W.G. Jiang), Springer Netherlands, 2012, 131-175.

[102] C. Liu, J. Tam, A. Sanders, D. Jiang, C. Ko et al., "Electric Cell-Substrate Impedance Sensing as a Screening Tool for Wound Healing Agents", in Electric Cell-Substrate
Impedance Sensing and Cancer Metastasis (Ed.: W.G. Jiang), Springer Netherlands, 2012, 203-216.

[103] D. Bosanquet, K. Harding, W. Jiang, "ECIS, Cellular Adhesion and Migration in Keratinocytes", in Electric Cell-Substrate Impedance Sensing and Cancer Metastasis (Ed.: W.G. Jiang), Springer Netherlands, 2012, 217-237.

[104] C. R. Keese, K. Bhawe, J. Wegener, I. Giaever, "RealTime Impedance Assay to Follow the Invasive Activities of Metastatic Cells in Culture.", BioTechniques 2002, 33, 842-850.

[105] A. Sanders, V. Saravolac, M. Mason, W. Jiang, "ECIS as a Tool in the Study of Metastasis Suppressor Genes: Epithelial Protein Lost In Neoplasm (EPLIN)", in Electric Cell-Substrate Impedance Sensing and Cancer Metastasis (Ed.: W.G. Jiang), Springer Netherlands, 2012, 41-54

[106] W. Jiang, L. Ye, H. Ren, A. Kift-Morgan, N. Topley et al., "Tumour-Endothelial and Tumour-Mesothelial Interactions Investigated by Impedance Sensing Based Cell Analyses", in Electric Cell-Substrate Impedance Sensing and Cancer Metastasis (Ed.: W.G. Jiang), Springer Netherlands, 2012, 177-193.

[107] S. Rahim, A. Üren, "A Real-Time Electrical Impedance Based Technique to Measure Invasion of Endothelial Cell Monolayer by Cancer Cells", Journal of Visualized Experiments 2011, e2792.

[108] M. S. Balda, J. A. Whitney, C. Flores, S. González, M. Cereijido et al., "Functional Dissociation of Paracellular Permeability and Transepithelial Electrical Resistance and Disruption of the Apical-Basolateral Intramembrane Diffusion Barrier by Expression of a Mutant Tight Junction Membrane Protein", The Journal of Cell Biology 1996, 134, 1031-1049.

[109] C. E. Campbell, M. M. Laane, E. Haugarvoll, I. Giaever, "Monitoring Viral-Induced Cell Death Using Electric Cell-Substrate Impedance Sensing.", Biosensors \& Bioelectronics 2007, 23, 536-542.

[110] J. A. Stolwijk, S. Michaelis, J. Wegener, "Cell Growth and Cell Death Studied by Electric Cell-Substrate Impedance Sensing", in Electric Cell-Substrate Impedance Sensing and Cancer Metastasis (Ed.: W.G. Jiang), Springer Netherlands, 2012, 85-117.

[111] C. Xiao, J. H. T. Luong, "On-Line Monitoring of Cell Growth and Cytotoxicity Using Electric Cell-Substrate Impedance Sensing (ECIS).", Biotechnology Progress 2003, 19, 1000-1005.

[112] L. Ceriotti, J. Ponti, F. Broggi, A. Kob, S. Drechsler et al., "Real-Time Assessment of Cytotoxicity by Impedance Measurement on a 96-Well Plate", Sensors and Actuators B: Chemical 2007, 123, 769-778.

[113] K. B. Male, B. Lachance, S. Hrapovic, G. Sunahara, J. H. T. Luong, "Assessment of Cytotoxicity of Quantum Dots and Gold Nanoparticles Using Cell-Based Impedance Spectroscopy.", Analytical chemistry 2008, 80, 5487-5493.

[114] T. M. Curtis, M. W. Widder, L. M. Brennan, S. J. Schwager, W. H. van der Schalie et al., "A Portable CellBased Impedance Sensor for Toxicity Testing of Drinking Water.", Lab on a Chip 2009, 9, 2176-2183. 
[115] M. Tarantola, D. Schneider, E. Sunnick, H. Adam, S. Pierrat et al., "Cytotoxicity of Metal and Semiconductor Nanoparticles Indicated by Cellular Micromotility.", ACS nano 2009, 3, 213-222.

[116] D. Opp, B. Wafula, J. Lim, E. Huang, J.-C. Lo et al., "Use of Electric Cell-Substrate Impedance Sensing to Assess in Vitro Cytotoxicity.", Biosensors \& Bioelectronics 2009, 24, 2625-2629.

[117] F. Asphahani, M. Zhang, "Cellular Impedance Biosensors for Drug Screening and Toxin Detection.", The Analyst 2007, 132, 835-841.

[118] P. M. Ghosh, C. R. Keese, I. Giaever, "Monitoring Electropermeabilization in the Plasma Membrane of Adherent Mammalian Cells.", Biophysical Journal 1993, $64,1602-1609$.

[119] J. A. Stolwijk, C. Hartmann, P. Balani, S. Albermann, C. R. Keese et al., "Impedance Analysis of Adherent Cells after in Situ Electroporation: Non-Invasive Monitoring during Intracellular Manipulations.", Biosensors \& Bioelectronics 2011, 26, 4720-4727.

[120] J. Wegener, C. R. Keese, I. Giaever, "Recovery of Adherent Cells after in Situ Electroporation Monitored Electrically", Biotechniques 2002, 33, 348-357.

[121] F. Alexander, D. T. Price, S. Bhansali, "Optimization of Interdigitated Electrode (IDE) Arrays for Impedance Based Evaluation of Hs 578T Cancer Cells", Journal of Physics: Conference Series 2010, 224, 012134.

[122] J. Mamouni, L. Yang, "Interdigitated MicroelectrodeBased Microchip for Electrical Impedance Spectroscopic Study of Oral Cancer Cells", Biomedical Microdevices 2011, 13, 1075-1088.

[123] C. Caviglia, A. Heiskanen, T. L. Andresen, J. Emnéus, "Comparison of Microelectrode Sensing Configurations for Impedimetric Cell Monitoring", in Proceedings of the International Workshop on Impedance Spectroscopy, 2012.

[124] B. Rappaz, B. Breton, E. Shaffer, G. Turcatti, "Digital Holographic Microscopy: A Quantitative Label-Free Microscopy Technique for Phenotypic Screening", Combinatorial Chemistry \& High Throughput Screening 2014, 17, 80-88.

[125] J. Kühn, E. Shaffer, J. Mena, B. Breton, J. Parent et al., "Label-Free Cytotoxicity Screening Assay by Digital Holographic Microscopy", ASSAY and Drug Development Technologies 2013, 11, 101-107.

[126] D. Bettenworth, P. Lenz, P. Krausewitz, M. Brückner, S. Ketelhut et al., "Quantitative Stain-Free and Continuous Multimodal Monitoring of Wound Healing In Vitro with Digital Holographic Microscopy", PLOS ONE 2014, 9, e107317.

[127] R. Robelek, "Surface Plasmon Resonance Sensors in Cell Biology: Basics and Application", Bioanalytical Reviews 2009, 1, 57-72.

[128] H. N. Daghestani, B. W. Day, "Theory and Applications of Surface Plasmon Resonance, Resonant Mirror, Resonant Waveguide Grating, and Dual Polarization Interferometry Biosensors.", Sensors 2010, 10, 96309646.

[129] G. Proll, L. Steinle, F. Pröll, M. Kumpf, B. Moehrle et al., "Potential of Label-Free Detection in High-Content-
Screening Applications.", Journal of Chromatography. A 2007, 1161, 2-8.

[130] M. N. Velasco-Garcia, "Optical Biosensors for Probing at the Cellular Level: A Review of Recent Progress and Future Prospects.", Seminars in Cell \& Developmental Biology 2009, 20, 27-33.

[131] X. D. Hoa, a. G. Kirk, M. Tabrizian, "Towards Integrated and Sensitive Surface Plasmon Resonance Biosensors: A Review of Recent Progress", Biosensors \& Bioelectronics 2007, 23, 151-160.

[132] J. Homola, "Present and Future of Surface Plasmon Resonance Biosensors", Analytical and Bioanalytical Chemistry 2003, 377, 528-539.

[133] Y. Yanase, T. Hiragun, K. Ishii, T. Kawaguchi, T. Yanase et al., "Surface Plasmon Resonance for Cell-Based Clinical Diagnosis.", Sensors 2014, 14, 4948-4959.

[134] R. H. Ritchie, "Plasma Losses by Fast Electrons in Thin Metal Films", Physical Review 1957, 106, 874-881.

[135] E. Kretschmann, "Die Bestimmungen Optischer Konstanten von Metallen Durch Anregung van Oberflächen Plasma-Schwingungen", Zeitschrift für Physik 1971, 241, 313-324.

[136] B. Liedberg, I. Lundström, E. Stenberg, "Principles of Biosensing with an Extended Coupling Matrix and Surface Plasmon Resonance", Sensors and Actuators B: Chemical 1993, 11, 63-72.

[137] B. Liedberg, C. Nylander, I. Lundström, "Biosensing with Surface Plasmon Resonance--How It All Started.", Biosensors \& Bioelectronics 1995, 10, i-ix.

[138] M. Golosovsky, V. Lirtsman, V. Yashunsky, D. Davidov, B. Aroeti, "Midinfrared Surface-Plasmon Resonance: A Novel Biophysical Tool for Studying Living Cells", Journal of Applied Physics 2009, 105, DOI 10.1063/1.3116143.

[139] R. L. Rich, D. G. Myszka, "Advances in Surface Plasmon Resonance Biosensor Analysis.", Current Opinion in Biotechnology 2000, 11, 54-61.

[140] F. Yu, S. Tian, D. Yao, W. Knoll, "Surface Plasmon Enhanced Diffraction for Label-Free Biosensing", Analytical Chemistry 2004, 76, 3530-3535.

[141] Y. Fang, A. M. Ferrie, N. H. Fontaine, P. K. Yuen, "Optical Biosensors for Monitoring Dynamic Mass Redistribution in Living Cells Mediated by Epidermal Growth Factor Receptor Activation", Analytical Chemistry 2005, 77, 5720-5725.

[142] Y. Fang, A. M. Ferrie, N. H. Fontaine, J. Mauro, J. Balakrishnan, "Resonant Waveguide Grating Biosensor for Living Cell Sensing.", Biophysical Journal 2006, 91, 1925-1940.

[143] Y. Yanase, H. Suzuki, T. Tsutsui, T. Hiragun, Y. Kameyoshi et al., "The SPR Signal in Living Cells Reflects Changes Other than the Area of Adhesion and the Formation of Cell Constructions", Biosensors \& Bioelectronics 2007, 22, 1081-1086.

[144] M. Hide, T. Tsutsui, H. Sato, T. Nishimura, K. Morimoto et al., "Real-Time Analysis of Ligand-Induced Cell Surface and Intracellular Reactions of Living Mast Cells Using a Surface Plasmon Resonance-Based Biosensor.", Analytical Biochemistry 2002, 302, 28-37. 
[145] V. Yashunsky, V. Lirtsman, M. Golosovsky, D. Davidov, B. Aroeti, "Real-Time Monitoring of Epithelial Cell-Cell and Cell-Substrate Interactions by Infrared Surface Plasmon Spectroscopy", Biophysical Journal 2010, 99, 4028-4036.

[146] Y. Yanase, A. Araki, H. Suzuki, T. Tsutsui, T. Kimura et al., "Development of an Optical Fiber SPR Sensor for Living Cell Activation", Biosensors \& Bioelectronics 2010, 25, 1244-1247.

[147] V. Chabot, C. M. Cuerrier, E. Escher, V. Aimez, M. Grandbois et al., "Biosensing Based on Surface Plasmon Resonance and Living Cells.", Biosensors \& bioelectronics 2009, 24, 1667-73.

[148] M. Vala, R. Robelek, M. Bocková, J. Wegener, J. Homola, "Real-Time Label-Free Monitoring of the Cellular Response to Osmotic Stress Using Conventional and Long-Range Surface Plasmons.", Biosensors \& bioelectronics 2013, 40, 417-421.

[149] M. Tanaka, T. Hiragun, T. Tsutsui, Y. Yanase, H. Suzuki et al., "Surface Plasmon Resonance Biosensor Detects the Downstream Events of Active PKC $\beta$ in AntigenStimulated Mast Cells", Biosensors \& Bioelectronics 2008, 23, 1652-1658.

[150] J. G. Quinn, S. O'Neill, A. Doyle, C. McAtamney, D. Diamond et al., "Development and Application of Surface Plasmon Resonance-Based Biosensors for the Detection of Cell-Ligand Interactions.", Analytical Biochemistry 2000, 281, 135-143.

[151] K. Giebel, C. Bechinger, S. Herminghaus, M. Riedel, P. Leiderer et al., "Imaging of Cell/substrate Contacts of Living Cells with Surface Plasmon Resonance Microscopy.", Biophysical Journal 1999, 76, 509-516.

[152] M. G. Somekh, S. Liu, T. S. Velinov, C. W. See, "HighResolution Scanning Surface-Plasmon Microscopy", Applied Optics 2000, 39, 6279-6287.

[153] K. Watanabe, K. Matsuura, F. Kawata, K. Nagata, J. Ning et al., "Scanning and Non-Scanning Surface Plasmon Microscopy to Observe Cell Adhesion Sites", Biomedical Optics Express 2012, 3, 354.

[154] A. W. Peterson, M. Halter, A. Tona, K. Bhadriraju, A. L. Plant, "Surface Plasmon Resonance Imaging of Cells and Surface-Associated Fibronectin.", BMC cell biology 2009, 10, 16 .

[155] A. W. Peterson, M. Halter, A. Tona, K. Bhadriraju, A. L. Plant, "Using Surface Plasmon Resonance Imaging to Probe Dynamic Interactions between Cells and Extracellular Matrix.", Cytometry Part A 2010, 77A, 895-903.

[156] K. Tiefenthaler, W. Lukosz, "Sensitivity of Grating Couplers as Integrated-Optical Chemical Sensors", Journal of the Optical Society of America B 1989, 6, 209.

[157] J. J. Ramsden, S.-Y. Li, E. Heinzle, J. E. Prenosil, "Optical Method for Measurment of Nuber and Shape of Attached Cells in Real Time", Cytometry 1995, 102, 97-102.

[158] T. Nuutinen, P. Karvinen, J. Rahomäki, P. Vahimaa, "Resonant Waveguide Grating (RWG): Overcoming the Problem of Angular Sensitivity by Conical, Broad-Band Illumination for Fluorescence Measurements", Analytical Methods 2012, 281-284.
[159] N. Zaytseva, W. Miller, V. Goral, J. Hepburn, Y. Fang, "Microfluidic Resonant Waveguide Grating Biosensor System for Whole Cell Sensing", Applied Physics Letters 2011, 98, DOI 10.1063/1.3582611.

[160] R. Horváth, H. C. Pedersen, N. Skivesen, D. Selmeczi, N. B. Larsen, "Optical Waveguide Sensor for on-Line Monitoring of Bacteria.", Optics letters 2003, 28, 12331235

[161] B. Cunningham, P. Li, B. Lin, J. Pepper, "Colorimetric Resonant Reflection as a Direct Biochemical Assay Technique", Sensors and Actuators B: Chemical 2002, $81,316-328$.

[162] S.-Y. Li, J. J. Ramsden, J. E. Prenosil, E. Heinzle, "Measurement of Adhesion and Spreading Kinetics of Baby Hamster Kidney and Hybridoma Cells Using an Integrated Optical Method.", Biotechnology Progress 1994, 10, 520-524.

[163] Y. Fang, "Resonant Waveguide Grating Biosensor for Microarrays", in Optical Guided-Wave Chemical and Biosensors II (Eds.: M. Zourob, A. Lakhtakia), SpringerVerlag, Berlin Heidelberg, 2010, 27-42.

[164] J. J. Ramsden, S.-Y. Li, J. E. Prenosil, E. Heinzle, "Kinetics of Adhesion and Spreading of Animal Cells", Biotechnology and Bioengineering 1994, 43, 939-945.

[165] Y. Fang, G. G. Li, J. Peng, "Optical Biosensor Provides Insights for Bradykinin B(2) Receptor Signaling in $A_{431}$ Cells.", FEBS letters 2005, 579, 6365-6374.

[166] Y. Fang, "Probing Cancer Signaling with Resonant Waveguide Grating Biosensors", Expert Opinion on Drug Discovery 2010, 5, 1237-1248.

[167] Y. Fang, A. M. Ferrie, G. Li, "Probing Cytoskeleton Modulation by Optical Biosensors", FEBS Letters 2005, 579, 4175-4180.

[168] R. Schröder, J. Schmidt, S. Blättermann, L. Peters, N. Janssen et al., "Applying Label-Free Dynamic Mass Redistribution Technology to Frame Signaling of $G$ Protein-coupled Receptors Noninvasively in Living Cells", Nature Protocols 2011, 6, 1748-176o.

[169] C. McDonagh, C. S. Burke, B. D. MacCraith, "Optical Chemical Sensors", Chemical Reviews 2008, 108, 400422.

[170] S. M. Borisov, O. S. Wolfbeis, "Optical Biosensors.", Chemical Reviews 2008, 108, 423-461.

[171] O. S. Wolfbeis, "Fiber-Optic Chemical Sensors and Biosensors.", Analytical Chemistry 2008, 80, 42694283.

[172] O. S. Wolfbeis, "Fiber-Optic Chemical Sensors and Biosensors.", Analytical Chemistry 2006, 78, 38593874 .

[173] M. I. J. Stich, L. H. Fischer, O. S. Wolfbeis, "Multiple Fluorescent Chemical Sensing and Imaging", Chemical Society Reviews 2010, 39, 3102-3114.

[174] J. R. Lakowicz, Principles of Fluorescence Spectroscopy, Springer, New York, 2006.

[175] X. Wang, O. S. Wolfbeis, "Optical Methods for Sensing and Imaging Oxygen: Materials, Spectroscopies and Applications.", Chemical Society Reviews 2014, 43, 3666-3761. 
[176] L. M. Coyle, M. Gouterman, "Correcting Lifetime Measurements for Temperature", Sensors and Actuators B 1999, 61, 92-99.

[177] J. Hradil, C. Davis, K. Mongey, C. Mcdonagh, B. D. Maccraith, "Temperature-Corrected PressureSensitive Paint Measurements Using a Single Camera and a Dual-Lifetime Approach", Measurement Science and Technology 2002, 13, 1552-1557.

[178] M. I. J. Stich, O. S. Wolfbeis, "Fluorescence Sensing and Imaging Using Pressure-Sensitive Paints and Temperature-Sensitive Paints", in Standardization and Quality Assurance in Fluorescence Measurements I (Ed.: U. Resch-Genger), Springer, Berlin - Heidelberg, 2008, 429-461.

[179] M. I. J. Stich, S. Nagl, O. S. Wolfbeis, U. Henne, M. Schaeferling, "A Dual Luminescent Sensor Material for Simultaneous Imaging of Pressure and Temperature on Surfaces", Advanced Functional Materials 2008, 18, 1399-1406.

[180] L. H. Fischer, S. M. Borisov, M. Schaeferling, I. Klimant, O.S. Wolfbeis, "Dual Sensing of $\mathrm{pO}_{2}$ and Temperature Using a Water-Based and Sprayable Fluorescent Paint", The Analyst 2010, 135, 1224-1229.

[181] S. M. Borisov, R. Seifner, I. Klimant, "A Novel Planar Optical Sensor for Simultaneous Monitoring of Oxygen, Carbon Dioxide, pH and Temperature.", Analytical and Bioanalytical Chemistry 2011, 400, 24632474.

[182] L. Fischer, "New Materials for Temperature and Pressure Sensitive Fluorescent Paints", Thesis, University of Regensburg, 2012.

[183] M. Naciri, D. Kuystermans, M. Al-Rubeai, "Monitoring $\mathrm{pH}$ and Dissolved Oxygen in Mammalian Cell Culture Using Optical Sensors", Cytotechnology 2008, 57, 245250.

[184] S. M. Borisov, C. Krause, S. Arain, O. S. Wolfbeis, "Composite Material for Simultaneous and Contactless Luminescent Sensing and Imaging of Oxygen and Carbon Dioxide", Advanced Materials 2006, 18, 15111516.

[185] C. R. Schroeder, G. Neurauter, I. Klimant, "Luminescent Dual Sensor for Time-Resolved Imaging of $\mathrm{pCO}_{2}$ and $\mathrm{pO}_{2}$ in Aquatic Systems", Microchimica Acta 2007, 158, 205-218.

[186] G. S. Vasylevska, S. M. Borisov, C. Krause, O. S. Wolfbeis, "Indicator-Loaded Permeation-Selective Microbeads for Use in Fiber Optic Simultaneous Sensing of $\mathrm{pH}$ and Dissolved Oxygen", Chemistry of Materials 2006, 18, 4609-4616.

[187] C. R. Schröder, L. Polerecky, I. Klimant, "TimeResolved $\mathrm{pH} / \mathrm{pO}_{2}$ Mapping with Luminescent Hybrid Sensors", Analytical Chemistry 2007, 79, 60-70.

[188] Y. Tian, B. R. Shumway, A. Cody Youngbull, Y. Li, A. K.$\mathrm{Y}$. Jen et al., "Dually Fluorescent Sensing of $\mathrm{pH}$ and Dissolved Oxygen Using a Membrane Made from Polymerizable Sensing Monomers", Sensors and Actuators B: Chemical 2010, 147, 714-722.

[189] R. J. Meier, S. Schreml, X. Wang, M. Landthaler, P. Babilas et al., "Simultaneous Photographing of Oxygen and $\mathrm{pH}$ in Vivo Using Sensor Films.", Angewandte
Chemie (International ed. in English) 2011, 50, 1089310896.

[190] R. J. Meier, "Luminescent Single and Dual Sensors for In Vivo Imaging of $\mathrm{pH}$ and $\mathrm{pO}_{2}$ ", Thesis, University of Regensburg, 2011.

[191] H. Lu, Y. Jin, Y. Tian, W. Zhang, M. R. Holl et al., "New Ratiometric Optical Oxygen and pH Dual Sensors with Three Emission Colors for Measuring Photosynthetic Activity in Cyanobacteria", Journal of Materials Chemistry 2011, 21, 19293.

[192] R. Liu, T. Xiao, W. Cui, J. Shinar, R. Shinar, "Multiple Approaches for Enhancing All-Organic Electronics Photoluminescent Sensors: Simultaneous Oxygen and pH Monitoring", Analytica Chimica Acta 2013, 778, 7078.

[193] L. Zhang, F. Su, S. Buizer, H. Lu, W. Gao et al., "A Dual Sensor for Real-Time Monitoring of Glucose and Oxygen", Biomaterials 2013, 34, 9779-9788.

[194] M. I. J. Stich, M. Schaeferling, O. S. Wolfbeis, "Multicolor Fluorescent and Permeation-Selective Microbeads Enable Simultaneous Sensing of $\mathrm{pH}$, Oxygen, and Temperature", Advanced Materials 2009, 21, 2216-2220.

[195] L. Li, D. R. Walt, "Dual-Analyte Fiber-Optic Sensor for the Simultaneous and Continuous Measurement of Glucose and Oxygen.", Analytical chemistry 1995, 67, 3746-3752.

[196] D. S. Ballantine, R. M. White, S. J. Martin, A. J. Ricco, E. T. Zellers et al., Acoustic Wave Sensors: Theory, Design, and Physico-Chemical Applications, Academic Press, San Diego, 1996.

[197] A. Janshoff, H.-J. Galla, C. Steinem, "Piezoelectric Mass-Sensing Devices as Biosensors-An Alternative to Optical Biosensors?", Angewandte Chemie (International ed. in English) 2000, 39, 4004-4032.

[198] S.-M. Chang, H. Muramatsu, C. Nakamura, J. Miyake, "The Principle and Applications of Piezoelectric Crystal Sensors", Materials Science and Engineering: C 2000, 12, 111-123.

[199] B. A. Čavić, M. Thompson, G. L. Hayward, "Acoustic Waves and the Study of Biochemical Macromolecules and Cells at the Sensor-liquid Interface", Analyst 1999, $124,1405-1420$.

[200] J. W. Grate, S. J. Martin, R. M. White, "Acoustic Wave Microsensors - Part I", Analytical Chemistry 1993, 65, 940-948.

[201] J. W. Grate, S. J. Martin, R. M. White, "Acoustic Wave Microsensors. Part II", Analytical Chemistry 1993, 65, 987-996.

[202] R. Lec, "Piezoelectric Biosensors: Recent Advances and Applications", IEEE International Frequency Control Symposium and PDA Exhibition 2001, 419-429.

[203] Y. Montagut, J. G. Narbon, Y. Jiménez, C. March, A. Montoya et al., "OCM Technology in Biosensors", in Biosensors - Emerging Materials and Applications (Ed.: P.P.A. Serra), InTech, 2011, 630.

[204] M. Thompson, A. Kipling, "Thickness-Shear-Mode Acoustic Wave Sensors in the Liquid Phase. A Review", Analyst 1991, 116, 881-890. 
[205] D. Johannsmann, "Studies of Viscoelasticity with the OCM", in Piezoelectric Sensors (Eds.: C. Steinem, A. Janshoff), Springer, Berlin - Heidelberg, 2007, 49-109.

[206] J. Wegener, A. Janshoff, C. Steinem, "The Quartz Crystal Microbalance as a Novel Means to Study CellSubstrate Interactions In Situ", Cell Biochemistry and Biophysics 2001, 34, 121-151.

[207] R. Lucklum, "Non-Gravimetric Contributions to OCR Sensor Response.", The Analyst 2005, 130, 1465-73.

[208] R. E. Speight, M. A. Cooper, "A Survey of the 2010 Quartz Crystal Microbalance Literature.", Journal of Molecular Recognition 2012, 25, 451-73.

[209] K. A. Marx, "Quartz Crystal Microbalance: A Useful Tool for Studying Thin Polymer Films and Complex Biomolecular Systems at the Solution-Surface Interface.", Biomacromolecules 2003, 4, 1099-1120.

[210] K. A. Marx, "The Quartz Crystal Microbalance and the Electrochemical OCM: Applications to Studies of Thin Polymer Films, Electron Transfer Systems, Biological Macromolecules, Biosensors, and Cells", in Piezoelectric Sensors (Eds.: C. Steinem, A. Janshoff), Springer, Berlin - Heidelberg, 2007, 371-424.

[211] M. A. Cooper, V. T. Singleton, "A Survey of the 2001 to 2005 Quartz Crystal Microbalance Biosensor Literature: Applications of Acoustic Physics to the Analysis of Biomolecular Interactions", Journal of Molecular Recognition 2007, 20, 154-184.

[212] B. Becker, M. A. Cooper, "A Survey of the 2006-2009 Quartz Crystal Microbalance Biosensor Literature.", Journal of Molecular Recognition 2011, 24, 754-787.

[213] C. I. Cheng, Y.-P. Chang, Y.-H. Chu, "Biomolecular Interactions and Tools for Their Recognition: Focus on the Quartz Crystal Microbalance and Its Diverse Surface Chemistries and Applications.", Chemical Society Reviews 2012, 41, 1947-71.

[214] J. Xi, J. Chen, M. Garcia, L. Penn, "Quartz Crystal Microbalance in Cell Biology Studies", J Biochip Tissue Chip 2013, S5, 1-9.

[215] J. Wegener, A. Janshoff, H.-J. H.-J. Galla, "Cell Adhesion Monitoring Using a Quartz Crystal Microbalance: Comparative Analysis of Different Mammalian Cell Lines.", European Biophysics Journal 1998, 28, 26-37.

[216] J. Wegener, J. Seebach, A. Janshoff, H.-J. Galla, "Analysis of the Composite Response of Shear Wave Resonators to the Attachment of Mammalian Cells", Biophysical Journal 2000, 78, 2821-2833.

[217] M. S. Lord, C. Modin, M. Foss, M. Duch, A. Simmons et al., "Monitoring Cell Adhesion on Tantalum and Oxidised Polystyrene Using a Quartz Crystal Microbalance with Dissipation.", Biomaterials 2006, 27, 4529-37.

[218] C. Modin, A.-L. Stranne, M. Foss, M. Duch, J. Justesen et al., "OCM-D Studies of Attachment and Differential Spreading of Pre-Osteoblastic Cells on $\mathrm{Ta}$ and $\mathrm{Cr}$ Surfaces.", Biomaterials 2006, 27, 1346-54.

[219] A. Janshoff, J. Wegener, M. Sieber, H.-J. Galla, "Double-Mode Impedance Analysis of Epithelial Cell Monolayers Cultured on Shear Wave Resonators.", European Biophysics Journal 1996, 25, 93-103.
[220] T. Zhou, K. A. Marx, M. Warren, H. Schulze, S. J. Braunhut, "The Quartz Crystal Microbalance as a Continuous Monitoring Tool for the Study of Endothelial Cell Surface Attachment and Growth.", Biotechnology Progress 2000, 16, 268-77.

[221] Z. Fohlerová, P. Skládal, J. Turánek, "Adhesion of Eukaryotic Cell Lines on the Gold Surface Modified with Extracellular Matrix Proteins Monitored by the Piezoelectric Sensor.", Biosensors \& Bioelectronics 2007, 22, 1896-901.

[222] F. Li, J. H.-C. Wang, O.-M. Wang, "Monitoring Cell Adhesion by Using Thickness Shear Mode Acoustic Wave Sensors.", Biosensors \& Bioelectronics 2007, 23, 42-50.

[223] F. Li, J. H.-C. Wang, Q.-M. Wang, "Thickness Shear Mode Acoustic Wave Sensors for Characterizing the Viscoelastic Properties of Cell Monolayer", Sensors and Actuators B: Chemical 2008, 128, 399-406.

[224] J. Li, C. Thielemann, U. Reuning, D. Johannsmann, "Monitoring of Integrin-Mediated Adhesion of Human Ovarian Cancer Cells to Model Protein Surfaces by Quartz Crystal Resonators: Evaluation in the Impedance Analysis Mode.", Biosensors \& Bioelectronics 2005, 20, 1333-1340.

[225] C. Galli Marxer, M. Collaud Coen, T. Greber, U. F. Greber, L. Schlapbach, "Cell Spreading on Quartz Crystal Microbalance Elicits Positive Frequency Shifts Indicative of Viscosity Changes.", Analytical and Bioanalytical Chemistry 2003, 377, 578-586.

[226] V. Heitmann, J. Wegener, "Monitoring Cell Adhesion by Piezoresonators: Impact of Increasing Oscillation Amplitudes.", Analytical Chemistry 2007, 79, 33923400.

[227] B. Reiss, A. Janshoff, C. Steinem, J. Seebach, J. Wegener, "Adhesion Kinetics of Functionalized Vesicles and Mammalian Cells: A Comparative Study", Langmuir 2003, 19, 1816-1823.

[228] C. Fredriksson, S. Khilman, B. Kasemo, D. M. Steel, "In Vitro Real-Time Characterization of Cell Attachment and Spreading.", Journal of Materials Science. Materials in Medicine 1998, 9, 785-8.

[229] L. Tan, O. Xie, X. Jia, M. Guo, Y. Zhang et al., "Dynamic Measurement of the Surface Stress Induced by the Attachment and Growth of Cells on Au Electrode with a Quartz Crystal Microbalance.", Biosensors \& Bioelectronics 2009, 24, 1603-9.

[230] H.-C. Chou, T.-R. Yan, K.-S. Chen, "Detecting Cells on the Surface of a Silver Electrode Quartz Crystal Microbalance Using Plasma Treatment and Graft Polymerization.", Colloids and Surfaces. B, Biointerfaces 2009, 73, 244-9.

[231] R. C. Ebersole, R. P. Foss, M. D. Ward, "Piezoelectric Cell Growth Sensor", Nature Biotechnology 1991, 9, 450-454

[232] M. Guo, J. Chen, Y. Zhang, K. Chen, C. Pan et al., "Enhanced Adhesion/spreading and Proliferation of Mammalian Cells on Electropolymerized Porphyrin Film for Biosensing Applications.", Biosensors \& Bioelectronics 2008, 23, 865-71.

[233] D. Le Guillou-Buffello, M. Gindre, P. Johnson, P. Laugier, V. Migonney, "An Alternative Quantitative 
Acoustical and Electrical Method for Detection of Cell Adhesion Process in Real-Time", Biotechnology and Bioengineering 2011, 108, 947-962.

[234] M. S. Lord, C. Modin, M. Foss, M. Duch, A. Simmons et al., "Extracellular Matrix Remodelling during Cell Adhesion Monitored by the Quartz Crystal Microbalance.", Biomaterials 2008, 29, 2581-7.

[235] K. A. Marx, T. Zhou, M. Warren, S. J. Braunhut, "Quartz Crystal Microbalance Study of Endothelial Cell Number Dependent Differences in Initial Adhesion and SteadyState Behavior: Evidence for Cell-Cell Cooperativity in Initial Adhesion and Spreading.", Biotechnology Progress 2003, 19, 987-99.

[236] S. Michaelis, "Non-Invasive Biosensors to Characterize the Cell-Material Interface", Thesis, Westfälische Wilhelms-University Münster, 2010.

[237] P. J. Molino, O. M. Hodson, J. F. Quinn, R. Wetherbee, "The Quartz Crystal Microbalance: A New Tool for the Investigation of the Bioadhesion of Diatoms to Surfaces of Differing Surface Energies.", Langmuir 2008, 24, 6730-7.

[238] P. J. Molino, O. M. Hodson, J. F. Quinn, R. Wetherbee, "Utilizing QCM-D to Characterize the Adhesive Mucilage Secreted by Two Marine Diatom Species inSitu and in Real-Time.", Biomacromolecules 2006, 7, 3276-82.

[239] A. L. J. Olsson, H. C. van der Mei, H. J. Busscher, P. K. Sharma, "Influence of Cell Surface Appendages on the Bacterium-Substratum Interface Measured Real-Time Using OCM-D.", Langmuir 2009, 25, 1627-32.

[240] M. Rodahl, F. Höök, C. Fredriksson, C. A. Keller, A. Krozer et al., "Simultaneous Frequency and Dissipation Factor OCM Measurements of Biomolecular Adsorption and Cell Adhesion", Faraday Discussions 1997, 107, 229-246.

[241] A. L. Schofield, T. R. Rudd, D. S. Martin, D. G. Fernig, C. Edwards, "Real-Time Monitoring of the Development and Stability of Biofilms of Streptococcus Mutans Using the Quartz Crystal Microbalance with Dissipation Monitoring.", Biosensors \& Bioelectronics 2007, 23, 407-13.

[242] N. Tymchenko, E. Nilebäck, M. V Voinova, J. Gold, B. Kasemo et al., "Reversible Changes in Cell Morphology due to Cytoskeletal Rearrangements Measured in Real-Time by QCM-D.", Biointerphases 2012, 7, 43.

[243] S. J. Braunhut, D. McIntosh, E. Vorotnikova, T. Zhou, K. A. Marx, "Detection of Apoptosis and Drug Resistance of Human Breast Cancer Cells to Taxane Treatments Using Quartz Crystal Microbalance Biosensor Technology.", Assay and Drug Development Technologies 2005, 3, 77-88.

[244] J. Elsom, M. I. Lethem, G. D. Rees, a C. Hunter, "Novel Quartz Crystal Microbalance Based Biosensor for Detection of Oral Epithelial Cell-Microparticle Interaction in Real-Time.", Biosensors \& Bioelectronics 2008, 23, 1259-65.

[245] J. Fatisson, F. Azari, N. Tufenkji, "Real-Time QCM-D Monitoring of Cellular Responses to Different Cytomorphic Agents.", Biosensors \& Bioelectronics 2011, 26, 3207-12.
[246] H.-W. Kang, H. Muramatsu, "Monitoring of Cultured Cell Activity by the Quartz Crystal and the Micro CCD Camera under Chemical Stressors.", Biosensors \& Bioelectronics 2009, 24, 1318-1323.

[247] K. A. Marx, T. Zhou, A. Montrone, D. Mclntosh, S. J. Braunhut, "Quartz Crystal Microbalance Biosensor Study of Endothelial Cells and Their Extracellular Matrix Following Cell Removal: Evidence for Transient Cellular Stress and Viscoelastic Changes during Detachment and the Elastic Behavior of the Pure Matrix.", Analytical Biochemistry 2005, 343, 23-34.

[248] L. Tan, X. Jia, X. Jiang, Y. Zhang, H. Tang et al., "In Vitro Study on the Individual and Synergistic Cytotoxicity of Adriamycin and Selenium Nanoparticles against Bel7402 Cells with a Quartz Crystal Microbalance.", Biosensors \& Bioelectronics 2009, 24, 2268-72.

[249] G. Wang, A. H. Dewilde, J. Zhang, A. Pal, M. Vashist et al., "A Living Cell Quartz Crystal Microbalance Biosensor for Continuous Monitoring of Cytotoxic Responses of Macrophages to Single-Walled Carbon Nanotubes.", Particle and Fibre Toxicology 2011, 8, 4.

[250] Y. Zhou, X. Jia, L. Tan, Q. Xie, L. Lei et al., "Magnetically Enhanced Cytotoxicity of Paramagnetic SeleniumFerroferric Oxide Nanocomposites on Human Osteoblast-like MG-63 Cells.", Biosensors \& Bioelectronics 2010, 25, 1116-21.

[251] M. Tarantola, E. Sunnick, D. Schneider, A.-K. Marel, A. Kunze et al., "Dynamic Changes of Acoustic Load and Complex Impedance as Reporters for the Cytotoxicity of Small Molecule Inhibitors.", Chemical Research in Toxicology 2011, 24, 1494-506.

[252] H.-W. Kang, H. Muramatsu, B.-J. Lee, Y.-S. Kwon, "Monitoring of Anticancer Effect of Cisplatin and 5Fluorouracil on HepG2 Cells by Quartz Crystal Microbalance and Micro CCD Camera.", Biosensors \& Bioelectronics 2010, 26, 1576-81.

[253] K. A. Marx, T. Zhou, A. Montrone, D. Mclntosh, S. J. Braunhut, "A Comparative Study of the Cytoskeleton Binding Drugs Nocodazole and Taxol with a Mammalian Cell Quartz Crystal Microbalance Biosensor: Different Dynamic Responses and Energy Dissipation Effects", Analytical Biochemistry 2007, 361, 77-92.

[254] K. A. Marx, T. Zhou, A. Montrone, H. Schulze, S. J. Braunhut, "A Quartz Crystal Microbalance Cell Biosensor: Detection of Microtubule Alterations in Living Cells at nM Nocodazole Concentrations.", Biosensors \& Bioelectronics 2001, 16, 773-82.

[255] M. Pax, J. Rieger, R. H. Eibl, C. Thielemann, D. Johannsmann, "Measurements of Fast Fluctuations of Viscoelastic Properties with the Quartz Crystal Microbalance", The Analyst 2005, 130, 1474-1477.

[256] V. M. Gun'ko, L. I. Mikhalovska, I. N. Savina, R. V Shevchenko, S. L. James et al., "Characterisation and Performance of Hydrogel Tissue Scaffolds", Soft Matter 2010, 6, 5351-5358.

[257] L. Sandrin, L. Coche-Guérente, A. Bernstein, H. Basit, P. Labbé et al., "Cell Adhesion through Clustered Ligand on Fluid Supported Lipid Bilayers.", Organic \& Biomolecular Chemistry 2010, 8, 1531-4. 
[258] R. Knerr, B. Weiser, S. Drotleff, C. Steinem, A. Göpferich, "Measuring Cell Adhesion on RGDModified, Self-Assembled PEG Monolayers Using the Quartz Crystal Microbalance Technique.", Macromolecular Bioscience 2006, 6, 827-38.

[259] M. Tagaya, T. Ikoma, T. Takemura, N. Hanagata, T. Yoshioka et al., "Effect of Interfacial Proteins on Osteoblast-like Cell Adhesion to Hydroxyapatite Nanocrystals.", Langmuir 2011, 27, 7645-53.

[260] J. Y. Chen, M. Li, L. S. Penn, J. Xi, "Real-Time and LabelFree Detection of Cellular Response to Signaling Mediated by Distinct Subclasses of Epidermal Growth Factor Receptors", Analytical Chemistry 2011, 31413146.

[261] H. J. Ko, T. H. Park, "Piezoelectric Olfactory Biosensor: Ligand Specificity and Dose-Dependence of an Olfactory Receptor Expressed in a Heterologous Cell System.", Biosensors \& Bioelectronics 2005, 20, 132732.

[262] R. Yang, J. Y. Chen, N. Xi, K. W. C. Lai, C. Qu et al., "Characterization of Mechanical Behavior of an Epithelial Monolayer in Response to Epidermal Growth Factor Stimulation.", Experimental cell research 2012, 318, 521-6.

[263] A. Sapper, J. Wegener, A. Janshoff, "Cell Motility Probed by Noise Analysis of Thickness Shear Mode Resonators.", Analytical Chemistry 2006, 78, 51845191.

[264] M. Tarantola, A. A.-K. Marel, E. Sunnick, H. Adam, J. Wegener et al., "Dynamics of Human Cancer Cell Lines Monitored by Electrical and Acoustic Fluctuation Analysis", Integrative Biology 2010, 2, 139-150.

[265] S. Mohri, J. Shimizu, N. Goda, T. Miyasaka, A. Fujita et al., "Measurements of $\mathrm{CO}_{2}$, Lactic Acid and Sodium Bicarbonate Secreted by Cultured Cells Using a Flowthrough Type $\mathrm{pH} / \mathrm{CO}_{2}$ Sensor System Based on ISFET", Sensors and Actuators B: Chemical 2006, 115, 519-525.

[266] S. Mohri, A. Yamada, N. Goda, M. Nakamura, K. Naruse et al., "Application of a Flow-through Type $\mathrm{pH} / \mathrm{CO}_{2}$ Sensor System Based on ISFET for Evaluation of the Glucose Dependency of the Metabolic Pathways in Cultured Cells", Sensors and Actuators B: Chemical 2008, 134, 447-450.

[267] C. Steinem, A. Janshoff, J. Wegener, W.-P. Ulrich, W. Willenbrink et al., "Impedance and Shear Wave Resonance Analysis of Ligand-Receptor Interactions at Functionalized Surfaces and of Cell Monolayers.", Biosensors \& Bioelectronics 1997, 12, 787-808.

[268] B. Reiß, "Mikrogravimetrische Untersuchung Des Adhäsionskontakts Tierischer Zellen: Eine Biophysikalische Studie", Thesis, Westälische Wilhelms-University Münster, 2004.

[269] IMOLA-IVD. cellasys GmbH, "Munich, Germany", http://www.cellasys.com/, accessed: 2015.02.26.

[270] Bionas Discovery 2500 system. Bionas GmbH, "Rostock, Germany", http://www.bionasdiscovery.com/, accessed: 2015.02.26.

[271] J. Wiest, "Zellbasierte Toxizitätsbestimmung Mittels Elektrochemischer Mikrosensorik", BIOspektrum 2014, $20,344-345$.
[272] J. Wiest, M. Schmidhuber, D. Grundl, M. Brischwein, H. Grothe et al., "Environmental Engineering Using Living Cells as Signal Transducers", IEEE Africon 2007, DOI 10.1109/AFRCON.2007.4401515.

[273] T. Geisler, J. Ressler, H. Harz, B. Wolf, R. Uhl, "Automated Multiparametric Platform for HighContent and High-Throughput Analytical Screening on Living Cells", IEEE Transactions on Automation Science and Engineering 2006, 3, 169-176.

[274] S. Michaelis, J. Wegener, R. Robelek, "Label-Free Monitoring of Cell-Based Assays: Combining Impedance Analysis with SPR for Multiparametric Cell Profiling", Biosensors \& Bioelectronics 2013, 49, 63-70.

[275] J. Kim, S. Kim, T. Ohashi, H. Muramatsu, S.-M. Chang et al., "Construction of Simultaneous SPR and OCM Sensing Platform.", Bioprocess and Biosystems Engineering 2010, 33, 39-45.

[276] M. A. Cooper, "Non-Optical Screening Platforms: The next Wave in Label-Free Screening?", Drug Discovery Today 2006, 11, 1068-1074.

[277] D. Ende, K.-M. Mangold, "Impedanzspektroskopie", Chemie in unserer Zeit 1993, 27, 134-140.

[278] V. F. Lvovich, Impedance Spectroscopy: Applications to Electrochemical and Dielectric Phenomena, John Wiley \& Sons Inc., Hoboken (NJ, USA), 2012.

[279] J. R. Macdonald, E. Barsoukov, Impedance Spectroscopy: Theory, Experiment, and Applications (2nd Edition), Wiley, Hoboken (NJ, USA), 2005

[280] S. J. Grimnes, O. G. Martinsen, Bioimpedance and Bioelectricity Basics, Academic Press, 2000.

[281] J. Lichter, "Crystals and Oscillators", NEL Frequency Controls Inc. Application Note JL9113 Rev. C 2006, 1-16.

[282] G. Sauerbrey, "Verwendung von Schwingquarzen Zur Wägung Dünner Schichten Und Zur Mikrowägung", Zeitschrift für Physik A Hadrons and Nuclei 1959, 155, 206-222.

[283] G. Gautschi, Piezoelectric Sensorics: Force, Strain, Pressure, Acceleration and Acoustic Emission Sensors, Materials and Amplifiers, Springer, Berlin - Heidelberg, 2002.

[284] C. Köhnlein, "Der Piezoeffekt am Beispiel des Quarzkristalls", http://www. piezoeffekt.de/.

[285] Boston Piezo-Optics Inc., "Material Properties: Crystal Quartz", http://bostonpiezooptics.com/crystal-quartz.

[286] A. Sapper, "Mechanics and Dynamics of Liposomes and Cells Studied by OCM and ECIS", Thesis, Johannes Gutenberg-University Mainz, 2006.

[287] R. F. Schmitt, J. W. Allen, J. F. Vetelino, J. Parks, C. Zhang, "Bulk Acoustic Wave Modes in Quartz for Sensing Measurand-Induced Mechanical and Electrical Property Changes", Sensors and Actuators B: Chemical 2001, 76, 95-102.

[288] R. Lucklum, F. Eichelbaum, "Interface Circuits for OCM Sensors", in Piezoelectric Sensors (Eds.: C. Steinem, A. Janshoff), Springer, Berlin - Heidelberg, 2007, 3-47.

[289] C. E. Reed, K. K. Kanazawa, J. H. Kaufman, "Physical Description of a Viscoelastically Loaded AT-Cut Quartz Resonator", Journal of Applied Physics 1990, 68, 19932001. 
[290] K. K. Kanazawa, J. G. Gordon, "The Oscillation Frequency of a Quartz Resonator in Contact with Liquid", Analytica Chimica Acta 1985, 175, 99-105.

[291] K. K. Kanazawa, J. G. Gordon, "Frequency of a Quartz Microbalance in Contact with Liquid", Analytical Chemistry 1985, 1771, 1770-1771.

[292] S. J. Martin, V. E. Granstaff, G. C. Frye, "Characterization of a Quartz Crystal Microbalance with Simultaneous Mass and Liquid Loading", Analytical Chemistry 1991, 2281, 2272-2281.

[293] D. A. Buttry, M. D. Ward, "Measurement of Interfacial Processes at Electrode Surfaces with the Electrochemical Quartz Crystal Microbalance", Chemical Reviews 1992, 92, 1355-1379.

[294] H. Muramatsu, E. Tamiya, I. Karube, "Computation of Equivalent Circuit Parameters of Quartz Crystals in Contact with Liquids and Study of Liquid Properties", Analytical Chemistry 1988, 60, 2142-2146.

[295] V. E. Bottom, Introduction to Quartz Crystal Unit Design, Van Nostrand Reinhold Company, New York, 1982.

[296] M. Yang, M. Thompson, "Multiple Chemical Information from the Thickness Shear Mode Acoustic Wave Sensor in the Liquid Phase", Analytical Chemistry 1993, 65, 1158-1168.

[297] H. L. Bandey, S. J. Martin, R. W. Cernosek, a R. Hillman, "Modeling the Responses of Thickness-Shear Mode Resonators under Various Loading Conditions.", Analytical Chemistry 1999, 71, 2205-2214.

[298] J. Li, C. Thielemann, U. Reuning, D. Johannsmann, "Monitoring of Integrin-Mediated Adhesion of Human Ovarian Cancer Cells to Model Protein Surfaces by Quartz Crystal Resonators: Evaluation in the Impedance Analysis Mode", Biosensors and Bioelectronics 2005, 20, 1333-1340.

[299] Z. Lin, M. D. Ward, "The Role of Longitudinal Waves in Quartz Crystal Microbalance Applications in Liquids", Analytical Chemistry 1995, 67, 685-693.

[300] Solartron Analytical, "1260A Impedance/ Gain-Phase Analyzer", http://www.solartronanalytical.com/ourproducts/potentiostats/Model-126oA.aspx.

[301] H. E. Park, D. Kim, H. S. Koh, S. Cho, J.-S. Sung et al., "Real-Time Monitoring of Neural Differentiation of Human Mesenchymal Stem Cells by Electric CellSubstrate Impedance Sensing.", Journal of Biomedicine \& Biotechnology 2011, 2011, 485173.

[302] M. Bellotti, W. Bast, A. Berra, F. J. Bonetto, "Effects of Osmolarity on Human Epithelial Conjunctival Cells Using an Electrical Technique.", Graefe's Archive for Clinical and Experimental Ophthalmology 2011, 249, 1875-1882.

[303] C. Wiesner, M. Pflüger, J. Kopecky, D. Stys, B. Entler et al., "Implementation of ECIS Technology for the Characterization of Potential Therapeutic Drugs That Promote Wound-Healing.", GMS Krankenhaushygiene interdisziplinär 2008, 3, 1-2.

[304] J. Müller, C. Thirion, M. W. Pfaffl, "Electric CellSubstrate Impedance Sensing (ECIS) Based Real-Time Measurement of Titer Dependent Cytotoxicity Induced by Adenoviral Vectors in an IPI-2I Cell Culture Model.", Biosensors \& Bioelectronics 2011, 26, 2000-2005.
[305] S. Arndt, J. Seebach, K. Psathaki, H.-J. Galla, J. Wegener, "Bioelectrical Impedance Assay to Monitor Changes in Cell Shape during Apoptosis", Biosensors \& Bioelectronics 2004, 19, 583-594.

[306] L. R. Arias, C. a Perry, L. Yang, "Real-Time Electrical Impedance Detection of Cellular Activities of Oral Cancer Cells.", Biosensors \& Bioelectronics 2010, 25, 2225-2231.

[307] E. Noiri, E. Lee, J. Testa, J. Quigley, D. Colflesh et al., "Podokinesis in Endothelial Cell Migration: Role of Nitric Oxide", American Journal of Physiology. Cell Physiology 1998, 274, C236-C244.

[308] J. Hong, K. Kandasamy, M. Marimuthu, C. S. Choi, S. Kim, "Electrical Cell-Substrate Impedance Sensing as a Non-Invasive Tool for Cancer Cell Study.", The Analyst 2011, 136, 237-245.

[309] I. H. Heijink, S. M. Brandenburg, J. A. Noordhoek, D. S. Postma, D.-J. Slebos et al., "Characterisation of Cell Adhesion in Airway Epithelial Cell Types Using Electric Cell-Substrate Impedance Sensing.", The European Respiratory Journal 2010, 35, 894-903.

[310] C.-C. Hsu, W.-C. Tsai, C. P.-C. Chen, Y.-M. Lu, J.-S. Wang, "Effects of Negative Pressures on Epithelial Tight Junctions and Migration in Wound Healing.", American Journal of Physiology. Cell Physiology 2010, 299, C528-C534.

[311] D. Sharma, J. Wang, P. P. Fu, S. Sharma, A. Nagalingam et al., "Adiponectin Antagonizes the Oncogenic Actions of Leptin in Hepatocellular Carcinogenesis.", Hepatology 2010, 52, 1713-22.

[312] K. M. Argraves, P. J. Gazzolo, E. M. Groh, B. A. Wilkerson, B. S. Matsuura et al., "High Density Lipoprotein-Associated Sphingosine 1-Phosphate Promotes Endothelial Barrier Function.", The Journal of Biological Chemistry 2008, 283, 25074-81.

[313] L. Charrier, Y. Yan, A. Driss, C. L. Laboisse, S. V Sitaraman et al., "ADAM-15 Inhibits Wound Healing in Human Intestinal Epithelial Cell Monolayers.", American Journal of Physiology. Gastrointestinal and Liver Physiology 2005, 288, G346-G353.

[314] J. Wegener, A. Hakvoort, H.-J. Galla, "Barrier Function of Porcine Choroid Plexus Epithelial Cells Is Modulated by CAMP-Dependent Pathways in Vitro.", Brain Research 2000, 853, 115-124.

[315] J. C. Weaver, Y. A. Chizmadzhev, "Theory of Electroporation: A Review", Bioelectrochemistry and Bioenergetics 1996, 41, 135-160.

[316] L. Raptis, K. L. Firth, "Electroporation of Adherent Cells in Situ.", DNA and Cell Biology 1990, 9, 615-621.

[317] M. N. Teruel, T. Meyer, "Electroporation-Induced Formation of Individual Calcium Entry Sites in the Cell Body and Processes of Adherent Cells.", Biophysical Journal 1997, 73, 1785-1796.

[318] L. H. Raptis, H. L. Brownell, K. L. Firth, L. W. Mackenzie, "A Novel Technique for the Study of Intercellular, Junctional Communication: Electroporation of Adherent Cells on a Partly Conductive Slide.", DNA and Cell Biology 1994, 13, 963975

[319] H.-F. Ji, Y. Shen, J. P. Hubner, B. F. Carroll, R. H. Schmehl et al., "Temperature-Independent Pressure- 
Sensitive Paint Based on a Bichromophoric Luminophore", Applied Spectroscopy 2000, 54, 856863.

[320] M. A. Woodmansee, J. C. Dutton, "Treating Temperature-Sensitivity Effects of Pressure-Sensitive Paint Measurements", Experiments in Fluids 1998, 24, 163-174.

[321] A. Jablonski, "Über Den Mechanismus Der Photolumineszenz von Farbstoffphosphoren", Zeitschrift für Physik 1935, 94, 38-46.

[322] J. H. Bell, E. T. Schairer, L. A. Hand, R. D. Mehta, "Surface Pressure Measurements Using Luminescent Coatings", Annual Review of Fluid Mechanics 2001, 33, 155-206.

[323] M. Quaranta, S. M. Borisov, I. Klimant, "Indicators for Optical Oxygen Sensors.", Bioanalytical Reviews 2012, 4, 115-157.

[324] D. Möller, Luft: Chemie, Physik, Biologie, Reinhaltung, Recht, Walter De Gruyter, 2003.

[325] K. S. Schanze, B. F. Carroll, S. Korotkevitch, M. J. Morris, "Temperature Dependence of Pressure Sensitive Paints", AIAA Journal 1997, 35, 306-310.

[326] S. Gouin, M. Gouterman, "Ideality of PressureSensitive Paint. II. Effect of Annealing on the Temperature Dependence of the Luminescence", Journal of Applied Polymer Science 2000, 77, 2805-2814.

[327] R. J. Woods, S. Scypinski, L. J. C. Love, H. A. Ashworth, "Transient Digitizer for the Determination of Microsecond Luminescence Lifetimes", Analytical Chemistry 1984, 56, 1395-1400.

[328] R. M. Ballew, J. N. Demas, "An Error Analysis of the Rapid Lifetime Determination Method for the Evaluation of Single Exponential Decays", Analytical Chemistry 1989, 61, 30-33.

[329] H. Peng, M. Stich, J. Yu, L. Sun, "Luminescent Europium (III) Nanoparticles for Sensing and Imaging of Temperature in the Physiological Range", Advanced Materials 2010, 22, 716-719.

[330] C. A. Schneider, W. S. Rasband, K. W. Eliceiri, "NIH Image to ImageJ: 25 Years of Image Analysis", Nature Methods 2012, 9, 671-675.

[331] W. S. Rasband, "ImageJ", http://imagej.nih.gov/ij/.

[332] F. Zernike, "Diffraction Theory of the Knife-Edge Test and Its Improved Form, the Phase-Contrast Method", Monthly Not. of R. A. S. 1934, 94, 377-384.

[333] D. B. Murphy, R. Oldfield, S. Schwartz, M. W. Davidson, "Introduction to Phase Contrast Microscopy", http://www.microscopyu.com/articles/phasecontrast/ph asemicroscopy.html.

[334] "Phasenkontrast", www.zeiss.de.

[335] M. Abramowitz, M. W. Davidson, "Introduction to Phase Contrast", http://www.olympusmicro.com/primer/techniques/phas econtrast/phase.html.

[336] Nikon Instruments, "Zoom Stereomicroscope SMZ 1500",

http://www.nikoninstruments.com/en_DE/Products/Mic roscope-

Systems/Stereomicroscopes/SMZ1500/(key_features).
[337] P. E. Nothnagle, W. Chambers, M. W. Davidson, "Introduction to Stereomicroscopy", http://www.microscopyu.com/articles/stereomicroscopy /stereointro.html.

[338] M. Minsky, "Microscopy Apparatus", U.S. Patent 3013467, 1961.

[339] M. Minsky, "Memoir on Inventing the Confocal Scanning Microscope", Scanning 1988, 10, 128-138.

[340] S. W. Paddock, T. J. Fellers, M. W. Davidson, "Confocal Microscopy: Basic Concepts", http://www.microscopyu.com/articles/confocal/confocal introbasics.html.

[341] T. W. James, C. Jope, "Visualization by Fluorescence of Chloroplast DNA in Higher Plants by Means of the DNA-Specific Probe 4'6-Diamidino-2-Phenylindole", Journal of Cell Biology 1978, 79, 623-630.

[342] J. Kapuscinski, "DAPI: A DNA-Specific Fluorescent Probe", Biotechnic \& Histochemistry 1979, 70, 220-233.

[343] E. Wulf, A. Deboben, F. A. Bautz, H. Faulstich, T. Wieland, "Fluorescent Phallotoxin, a Tool for the Visualization of Cellular Actin", Proceedings of the National Academy of Sciences of the United States of America 1979, 76, 4498-4502.

[344] H. Faulstich, S. Zobeley, G. Rinnerthaler, J. V Small, "Fluorescent Phallotoxins as Probes for Filamentous Actin", Journal of Muscle Research and Cell Motility 1988, 9, 370-383.

[345] B. Gumbiner, "Structure, Biochemistry, and Assembly of Epithelial Tight Junctions", American Journal of Physiology. Cell Physiology 1987, 253, C749-C758.

[346] B. R. Stevenson, J. M. Anderson, S. Bullivant, "The Epithelial Tight Junction: Structure, Function and Preliminary Biochemical Characterization.", Molecular and Cellular Biochemistry 1988, 83, 129-145.

[347] N. G. Papadopoulos, G. V Dedoussis, G. Spanakos, a D. Gritzapis, C. N. Baxevanis et al., "An Improved Fluorescence Assay for the Determination of Lymphocyte-Mediated Cytotoxicity Using Flow Cytometry.", Journal of Immunological Methods 1994, 177, 101-111.

[348] P. L. Moore, I. C. MacCoubrey, R. P. Haugland, "A Rapid, pH Insensitive, Two Color Fluorescence Viability (cytotoxicity) Assay", Journal of Cell Biology 1990, 111, $58 \mathrm{a}$.

[349] D. Johannsmann, "Viscoelastic, Mechanical, and Dielectric Measurements on Complex Samples with the Quartz Crystal Microbalance.", Physical Chemistry Chemical Physics 2008, 10, 4516-4534.

[350] R. D. Vaughan, G. G. Guilbault, "Piezoelectric Immunosensors", in Piezoelectric Sensors (Eds.: C. Steinem, A. Janshoff), Springer, Berlin - Heidelberg, 2007, 237-280.

[351] E. Zampetti, S. Pantalei, A. Macagnano, E. Proietti, C. Di Natale et al., "Use of a Multiplexed Oscillator in a Miniaturized Electronic Nose Based on a Multichannel Quartz Crystal Microbalance", Sensors and Actuators B: Chemical 2008, 131, 159-166.

[352] T. Abe, M. Esashi, "One-Chip Multichannel Quartz Crystal Microbalance (OCM) Fabricated by Deep RIE", Sensors and Actuators A: Physical 2000, 82, 139-143. 
[353] T. Tatsuma, Y. Watanabe, N. Oyama, K. Kitakizaki, M. Haba, "Multichannel Quartz Crystal Microbalance", Analytical Chemistry 1999, 71, 3632-3636.

[354] X. Jin, Y. Huang, A. Mason, X. Zeng, "Multichannel Monolithic Quartz Crystal Microbalance Gas Sensor Array", Analytical Chemistry 2009, 81, 595-603.

[355] J. Rabe, S. Büttgenbach, J. Schröder, P. Hauptmann, "Monolithic Miniaturized Quartz Microbalance Array and Its Application to Chemical Sensor Systems for Liquids", IEEE Sensors Journal 2003, 3, 361-368.

[356] H. Ogi, H. Nagai, Y. Fukunishi, T. Yanagida, M. Hirao et al., "Multichannel Wireless-Electrodeless QuartzCrystal Microbalance Immunosensor", Analytical Chemistry 2010, 82, 3957-3962.

[357] K. Jaruwongrungsee, T. Maturos, "Analysis of Quartz Crystal Microbalance Sensor Array with Circular Flow Chamber", International Journal of Applied Biomedical Engineering 2009, 2, 50-54.

[358] G. S. Huang, M.-T. Wang, M.-Y. Hong, "A Versatile QCM Matrix System for Online and High-Throughput Bio-Sensing.", The Analyst 2006, 131, 382-387.

[359] K. Seidler, M. Polreichová, P. a Lieberzeit, F. L. Dickert, "Biomimetic Yeast Cell Typing-Application of QCMs.", Sensors 2009, 9, 8146-8157.

[360] A. Tuantranont, A. Wisitsora-at, P. Sritongkham, K. Jaruwongrungsee, "A Review of Monolithic Multichannel Quartz Crystal Microbalance: A Review.", Analytica Chimica Acta 2011, 687, 114-128.

[361] AZ Electronic Materials GmbH (Germany), "AZ ${ }^{\circledR} \mathrm{ECl}$ 3000 Photoresist - Universal i-Line/Crossover Photoresist Series", http://www.microchemicals.com/micro/az_eci_3027_ph otoresist.pdf.

[362] S. Berg, D. Johannsmann, "Laterally Coupled Quartz Resonators", Analytical Chemistry 2001, 73, 1140-1145.

[363] F. Shen, K. H. Lee, S. J. O. Shea, P. Lu, T. Y. Ng, "Frequency Interference Between Two Quartz Crystal Microbalances", IEEE Sensors Journal 2003, 3, 274-281.

[364] F. Lu, H. P. Lee, P. Lu, S. P. Lim, "Finite Element Analysis of Interference for the Laterally Coupled Quartz Crystal Microbalances", Sensors and Actuators A: Physical 2005, 119, 90-99.

[365] B. A. Martin, H. E. Hager, "Velocity Profile on Quartz Crystals Oscillating in Liquids", Journal of Applied Physics 1989, 65, 2630-2635.

[366] B. Borovsky, B. Mason, J. Krim, "Scanning Tunneling Microscope Measurements of the Amplitude of Vibration of a Quartz Crystal Oscillator", Journal of Applied Physics 2000, 88, 4017-4021.

[367] D. Johannsmann, L.-O. Heim, "A Simple Equation Predicting the Amplitude of Motion of Quartz Crystal Resonators", Journal of Applied Physics 2006, 100, 094505 .

[368] K. E. Kasza, A. C. Rowat, J. Liu, T. E. Angelini, C. P. Brangwynne et al., "The Cell as a Material.", Current Opinion in Cell Biology 2007, 19, 101-107.

[369] P. J. Cumpson, "Ouartz Crystal Microbalance: A New Design Eliminates Sensitivity Outside the Electrodes, Often Wrongly Attributed to the Electric Fringing
Field", Journal of Vacuum Science \& Technology A: Vacuum, Surfaces, and Films 1997, 15, 2407-2412.

[370] S. Ghafouri, M. Thompson, "Electrode Modification and the Response of the Acoustic Shear Wave Device Operating in Liquids", The Analyst 2001, 126, 21592167.

[371] M. Rodahl, F. Höök, B. Kasemo, "OCM Operation in Liquids: An Explanation of Measured Variations in Frequency and $\mathrm{Q}$ Factor with Liquid Conductivity.", Analytical chemistry 1996, 68, 2219-2227.

[372] Q. Xie, C. Xiang, X. Yang, Y. Zhang, M. Li et al., "Simultaneous Impedance Measurements of Two OneFace Sealed Resonating Piezoelectric Quartz Crystals for in Situ Monitoring of Electrochemical Processes and Solution Properties", Analytica Chimica Acta 2005, 533, 213-224.

[373] A. Sabot, S. Krause, "Simultaneous Quartz Crystal Microbalance Impedance and Electrochemical Impedance Measurements. Investigation into the Degradation of Thin Polymer Films.", Analytical chemistry 2002, 74, 3304-3311.

[374] E. Briand, M. Zäch, S. Svedhem, B. Kasemo, S. Petronis, "Combined OCM-D and EIS Study of Supported Lipid Bilayer Formation and Interaction with Pore-Forming Peptides.", The Analyst 2010, 135, 343-50.

[375] H. He, Q. Xie, Y. Zhang, S. Yao, "A Simultaneous Electrochemical Impedance and Quartz Crystal Microbalance Study on Antihuman Immunoglobulin G Adsorption and Human Immunoglobulin G Reaction.", Journal of Biochemical and Biophysical Methods 2005, 62, 191-205.

[376] O. Xie, Y. Zhang, M. Xu, Z. Li, Y. Yuan et al., "Combined Quartz Crystal Impedance and Electrochemical Impedance Measurements during Adsorption of Bovine Serum Albumin onto Bare and Cysteine- or Thiophenol-Modified Gold Electrodes", Journal of Electroanalytical Chemistry 1999, 478, 1-8.

[377] Q. Xie, C. Xiang, Y. Yuan, Y. Zhang, L. Nie et al., "A Novel Dual-Impedance-Analysis EQCM System-Investigation of Bovine Serum Albumin Adsorption on Gold and Platinum Electrode Surfaces.", Journal of Colloid and Interface Science 2003, 262, 107-115.

[378] E. M. Pinto, D. M. Soares, C. M. a. Brett, "Interaction of BSA Protein with Copper Evaluated by Electrochemical Impedance Spectroscopy and Quartz Crystal Microbalance", Electrochimica Acta 2008, 53, 74607466.

[379] R. O. Hynes, "Integrins: A Family of Cell Surface Receptors.", Cell 1987, 48, 549-54.

[380] R. O. Hynes, "Integrins: Versatility, Modulation, and Signaling in Cell Adhesion.", Cell 1992, 69, 11-25.

[381] K. Green, J. Jones, "Desmosomes and Hemidesmosomes: Structure and Function of Molecular Components.", The FASEB Journal 1996, 10, 871-881.

[382] A. Sonnenberg, J. Calafat, H. Janssen, H. Daams, L. M. van der Raaij-Helmer et al., "Integrin Alpha 6/beta 4 Complex Is Located in Hemidesmosomes, Suggesting a Major Role in Epidermal Cell-Basement Membrane 
Adhesion.", The Journal of Cell Biology 1991, 113, 907917.

[383] D. R. Garrod, "Desmosomes and Hemidesmosomes.", Current Opinion in Cell Biologypinion in cell biology 1993, 5, 30-40.

[384] K. Burridge, K. Fath, T. Kelly, G. Nuckolls, C. Turner, "Focal Adhesions: Transmembrane Junctions between the Extracellular Matrix and the Cytoskeleton.", Annual Review of Cell Biology 1988, 4, 487-525.

[385] D. Kirchhofer, J. Grzesiak, M. D. Pierschbacher, "Calcium as a Potential Physiological Regulator of Integrin-Mediated Cell Adhesion.", The Journal of Biological ChemistryBological chemistry 1991, 266, 4471-4477.

[386] A. J. Freemont, J. A. Hoyland, "Cell Adhesion Molecules.", Clinical Molecular Pathology 1996, 49, M321-M330.

[387] H. Herrmann, H. Bär, L. Kreplak, S. V Strelkov, U. Aebi, "Intermediate Filaments: From Cell Architecture to Nanomechanics", Nature Reviews. Molecular Cell Biology 2007, 8, 562-573.

[388] M. Takeichi, "Cadherins: A Molecular Family Important in Selective Cell-Cell Adhesion", Annual Review of Biochemistry 1990, 59, 237-252.

[389] C. M. O'Connor, J. U. Adams, Essentials of Cell Biology, MA: NPG Education, Cambridge, 2010.

[390] S. Tsukita, M. Furuse, M. Itoh, "Multifunctional Strands in Tight Junctions", Nature Reviews. Molecular Cell Biology 2001, 2, 285-293.

[391] D. R. Pitelka, B. N. Taggart, S. T. Hamamoto, "Effects of Extracellular Calcium Depletion on Membrane Topography and Occluding Junctions of Mammary Epithelial Cells in Culture.", The Journal of Cell Biology 1983, 96, 613-624.

[392] L. S. Rodgers, A. S. Fanning, "Regulation of Epithelial Permeability by the Actin Cytoskeleton.", Cytoskeleton 2011, 68, 653-66o.

[393] E. L. Elson, "Cellular Mechanics as an Indicator of Cytoskeletal Structure and Function", Annual Review of Biophysics and Biophysical Chemistry 1988, 17, 397430.

[394] T. Wakatsuki, B. Schwab, N. C. Thompson, E. L. Elson, "Effects of Cytochalasin D and Latrunculin B on Mechanical Properties of Cells.", Journal of Cell Science 2001, 114, 1025-1036.

[395] C. Rotsch, M. Radmacher, "Drug-Induced Changes of Cytoskeletal Structure and Mechanics in Fibroblasts: An Atomic Force Microscopy Study", Biophysical Journal 2000, 78, 520-535.

[396] C. Rotsch, F. Braet, E. Wisse, M. Radmacher, "AFM Imaging and Elasticity Measurements on Living Rat Liver Macrophages", Cell Biology International 1997, 21, 685-696.

[397] B. R. Stevenson, D. A. Begg, "ConcentrationDependent Effects of Cytochalasin D on Tight Junctions and Actin Filaments in MDCK Epithelial Cells.", Journal of Cell Science 1994, 107, 367-375.

[398] I. Meza, G. Ibarra, "Occluding Junctions and Cytoskeletal Components in a Cultured Transporting
Epithelium", The Journal of Cell Biology 1980, 87, 746754.

[399] S. Brown, J. Spudich, "Cytochalasin Inhibits the Rate of Elongation of Actin Filament Fragments", The Journal of Cell Biology 1979, 83, 657-662.

[400] E. Urbanik, R. Ware, "Actin Filament Capping of Cytochalasins and Cleaving Activity B, D, E, and H", Archives of Biochemistry and Biophysics 1989, 269, 181187.

[401] I. Yahara, F. Harada, S. Sekita, "Correlation between Effects of 24 Different Cytochalasins on Cellular Structures and Cellular Events and Those on Actin in Vitro", The Journal of Cell Biology 1982, 92, 69-78.

[402] J. A. Cooper, "Effects of Cytochalasin and Phalloidin on Actin.", The Journal of Cell Biology 1987, 105, 14731478.

[403] A. Pietuch, "Membrane Mechanics Governs Cell Mechanics in Epithelial Cell: How Surface Area Regulation Ensures Tension Homeostasis", Thesis, Georg-August-Universität Göttingen, 2012.

[404] H. W. Wu, T. Kuhn, V. T. Moy, "Mechanical Properties of L929 Cells Measured by Atomic Force Microscopy: Effects of Anticytoskeletal Drugs and Membrane Crosslinking.", Scanning 1998, 20, 389-397.

[405] B. a Smith, B. Tolloczko, J. G. Martin, P. Grütter, "Probing the Viscoelastic Behavior of Cultured Airway Smooth Muscle Cells with Atomic Force Microscopy: Stiffening Induced by Contractile Agonist.", Biophysical Journal 2005, 88, 2994-3007.

[406] C. Roduit, S. Sekatski, G. Dietler, S. Catsicas, F. Lafont et al., "Stiffness Tomography by Atomic Force Microscopy.", Biophysical Journal 2009, 97, 674-677.

[407] S. Kasas, X. Wang, H. Hirling, R. Marsault, B. Huni et al., "Superficial and Deep Changes of Cellular Mechanical Properties Following Cytoskeleton Disassembly.", Cell Motility and the Cytoskeleton 2005, 62, 124-132.

[408] K. B. Grimm, H. Oberleithner, J. Fels, "Fixed Endothelial Cells Exhibit Physiologically Relevant Nanomechanics of the Cortical Actin Web.", Nanotechnology 2014, 25, 1-7.

[409] R. M. Hochmuth, "Measuring the Mechanical Properties of Individual Human Blood Cells", Journal of Biomechanical Engineering 1993, 115, 515-519.

[410] R. M. Hochmuth, "Micropipette Aspiration of Living Cells.", Journal of biomechanics 2000, 33, 15-22.

[411] M. Sato, D. P. Theret, L. T. Wheeler, N. Ohshima, R. M. Nerem, "Application of the Micropipette Technique to the Measurement of Cultured Porcine Aortic Endothelial Cell Viscoelastic Properties.", Journal of biomechanical engineering 1990, 112, 263-268.

[412] C. A. Erickson, "The Deformability of BHK Cells and Polyoma Virus-Transformed BHK Cells in Relation to Locomotory Behaviour.", Journal of Cell Science 1980, $44,187-200$

[413] O. Thoumine, O. Cardoso, J. J. Meister, "Changes in the Mechanical Properties of Fibroblasts during Spreading: A Micromanipulation Study.", European Biophysics Journal 1999, 28, 222-234.

[414] J. Dai, M. P. Sheetz, "Mechanical Properties of Neuronal Growth Cone Membranes Studied by Tether 
Formation with Laser Optical Tweezers.", Biophysical journal 1995, 68, 988-96.

[415] a R. Bausch, F. Ziemann, a a Boulbitch, K. Jacobson, E. Sackmann, "Local Measurements of Viscoelastic Parameters of Adherent Cell Surfaces by Magnetic Bead Microrheometry.", Biophysical Journal 1998, 75, 2038-49.

[416] a R. Bausch, W. Möller, E. Sackmann, "Measurement of Local Viscoelasticity and Forces in Living Cells by Magnetic Tweezers.", Biophysical Journal 1999, 76, 573-9.

[417] B. Fabry, G. Maksym, J. Butler, M. Glogauer, D. Navajas et al., "Scaling the Microrheology of Living Cells", Physical Review Letters 2001, 87, 1-4.

[418] S. Hu, L. Eberhard, J. Chen, J. C. Love, J. P. Butler et al., "Mechanical Anisotropy of Adherent Cells Probed by a Three-Dimensional Magnetic Twisting Device.", American Journal of Physiology. Cell Physiology 2004, 287, C1184-91.

[419] N. Wang, J. P. Butler, D. E. Ingber, "Mechanotransduction across the Cell Surface and through the Cytoskeleton.", Science 1993, 260, 11241127.

[420] N. Wang, "Mechanical Interactions Among Cytoskeletal Filaments", Hypertension 1998, 32, 162165.

[421] H. Huang, J. Sylvan, M. Jonas, R. Barresi, P. T. C. So et al., "Cell Stiffness and Receptors: Evidence for Cytoskeletal Subnetworks.", American Journal of Physiology. Cell Physiology 2005, 288, C72-80.

[422] K. Nagayama, Y. Nagano, M. Sato, T. Matsumoto, "Effect of Actin Filament Distribution on Tensile Properties of Smooth Muscle Cells Obtained from Rat Thoracic Aortas.", Journal of biomechanics 2006, 39, 293-301.

[423] J. Bereiter-Hahn, "Scanning Acoustic Microscopy Visualizes Cytomechanical Responses to Cytochalasin D.", Journal of microscopy 1987, 146, 29-39.

[424] I. Karl, J. Bereiter-Hahn, "Tension Modulates Cell Surface Motility: A Scanning Acoustic Microscopy Study.", Cell motility and the cytoskeleton 1999, 43, 349-59.

[425] N. Petersen, W. McConnaughey, "Dependence of Locally Measured Cellular Deformability on Position on the Cell, Temperature, and Cytochalasin B", Proceedings of the National Academy of Sciences of the United States of America 1982, 79, 5327-5331.

[426] J. Rheinlaender, T. E. Schäffer, "Mapping the Mechanical Stiffness of Live Cells with the Scanning lon Conductance Microscope", Soft Matter 2013, 9, 32303236.

[427] O. Kovbasnjuk, U. Szmulowicz, K. Spring, "Regulation of the MDCK Cell Tight Junction", The Journal of Membrane Biology 1998, 104, 93-104.

[428] O. Frederiksen, P. Leyssac, "Effects of Cytochalasin B and Dimethylsulphoxide on Isosmotic Fluid Transport by Rabbit Gall-Bladder in Vitro", The Journal of Physiology 1977, 265, 103-118.

[429] J. Anderson, C. Van Itallie, "Tight Junctions and the Molecular Basis for Regulation of Paracellular
Permeability", American Journal of Physiology. Gastrointestinal and Liver Physiology 1995, 269, G467G475.

[430] J. L. Madara, D. Barenberg, S. Carlson, "Effects of Cytochalasin D on Occluding Junctions of Intestinal Absorptive Cells: Further Evidence That the Cytoskeleton May Influence Paracellular Permeability and Junctional Charge Selectivity.", The Journal of Cell Biology 1986, 102, 2125-2136.

[431] J. Madara, "Intestinal Absorptive Cell Tight Junctions Are Linked to Cytoskeleton", American Journal of Physiology 1987, 253, C171-C175.

[432] J. Matthews, K. Tally, J. Smith, C. Awtrey, "F-Actin Differentially Alters Epithelial Transport and Barrier Function", Journal of Surgical Research 1994, 56, 505509.

[433] J. H. Hoh, C.-A. Schoenenberger, "Surface Morphology and Mechanical Properties of MDCK Monolayers by Atomic Force Microscopy.", Journal of Cell Science 1994, 107, 1105-1114.

[434] Y.-T. Yang, C.-C. K. Lin, J.-D. Liao, C.-W. Chang, M.-S. Ju, "Continuous Depth-Sensing Nano-Mechanical Characterization of Living, Fixed and Dehydrated Cells Attached on a Glass Substrate.", Nanotechnology 2010, 21, 1-7.

[435] G. S. Watson, J. A. Watson, S. Myhra, "Morphology, Mechanical Properties and Manipulation of Living Cells: Atomic Force Microscopy", in Surface Structure and Properties of Microbial Cells (Ed.: E.P. Ivanova), 2006, 1-29.

[436] S. Shroff, D. Saner, R. Lal, "Dynamic Micromechanical Properties of Cultured Rat Atrial Myocytes Measured by Atomic Force Microscopy", American Journal of Physiology. Cell Physiology 1995, 38, C286-C292.

[437] J. L. Hutter, J. Chen, W. K. Wan, S. Uniyal, M. Leabu et al., "Atomic Force Microscopy Investigation of the Dependence of Cellular Elastic Moduli on Glutaraldehyde Fixation", Journal of Microscopy 2005, 219, 61-68.

[438] S. B. Velegol, B. E. Logan, "Contributions of Bacterial Surface Polymers, Electrostatics, and Cell Elasticity to the Shape of AFM Force Curves", Langmuir 2002, 18, 5256-5262.

[439] S. Steltenkamp, C. Rommel, J. Wegener, A. Janshoff, "Membrane Stiffness of Animal Cells Challenged by Osmotic Stress.", Small 2006, 2, 1016-20.

[440] S. Baumgarten, R. Robelek, "Surface Plasmon Resonance (SPR) Sensors for the Rapid, Sensitive Detection of the Cellular Response to Osmotic Stress", Sensors and Actuators B: Chemical 2011, 156, 798-804.

[441] D. Voet, J. G. Voet, C. W. Pratt, Fundamentals of Biochemistry, John Wiley \& Sons Inc., Weinheim, 2002.

[442] R. Molina, D.-Y. Han, X.-F. Su, R.-Z. Zhao, M. Zhao et al., "Cpt-cAMP Activates Human Epithelial Sodium Channels via Relieving Self-Inhibition.", Biochimica et Biophysica Acta 2011, 1808, 1818-1826.

[443] C. Riethmüller, P. Jungmann, J. Wegener, H. Oberleithner, "Bradykinin Shifts Endothelial Fluid Passage from Para- to Transcellular Routes.", Pflügers Archiv: European Journal of Physiology 2006, 453, 157165 
[444] E. G. Langeler, V. W. van Hinsbergh, "Norepinephrine and lloprost Improve Barrier Function of Human Endothelial Cell Monolayers: Role of cAMP.", The American Journal of Physiology 1991, 260, C1052C1059.

[445] M. E. Duffey, B. Hainau, S. Ho, C. J. Bentzel, "Regulation of Epithelial Tight Junction Permeability by Cyclic AMP", Nature 1981, 294, 451-453.

[446] T. J. Stelzner, J. V Weil, R. F. O'Brien, "Role of Cyclic Adenosine Monophosphate in the Induction of Endothelial Barrier Properties.", Journal of Cellular Physiology 1989, 139, 157-166.

[447] J. Wegener, S. Zink, P. Rösen, H.-J. Galla, "Use of Electrochemical Impedance Measurements to Monitor Beta-Adrenergic Stimulation of Bovine Aortic Endothelial Cells.", Pflügers Archiv: European Journal of Physiology 1999, 437, 925-934.

[448] M. Shivanna, S. P. Srinivas, "Elevated cAMP Opposes (TNF-Alpha)-Induced Loss in the Barrier Integrity of Corneal Endothelium.", Molecular Vision 2010, 16, 1781-90.

[449] E. G. Langeler, I. Snelting-Havinga, V. W. van Hinsbergh, "Passage of Low Density Lipoproteins through Monolayers of Human Arterial Endothelial Cells. Effects of Vasoactive Substances in an in Vitro Model", Arteriosclerosis, Thrombosis, and Vascular Biology 1989, 9, 550-559.

[450] S. A. Casnocha, S. G. Eskin, E. R. Hall, L. V Mclntire, "Permeability of Human Endothelial Monolayers: Effect of Vasoactive Agonists and CAMP", Journal of Applied Physiology 1989, 67, 1997-2005.

[451] H. Jacobson, "Altered Permeability in the Proximal Tubule Response to Cyclic AMP", American Journal of Physiology 1979, 236, F71-F79.

[452] M. S. Balda, L. Gonzalez-Mariscal, "Assembly and Sealing of Tight Junctions: Possible Participation of GProteins, Phospholipase C, Protein Kinase $C$ and Calmodulin", The Journal of Membrane Biology 1991, 122, 193-202.

[453] C. Ramachandran, R. V Patil, N. a Sharif, S. P. Srinivas, "Effect of Elevated Intracellular CAMP Levels on Actomyosin Contraction in Bovine Trabecular Meshwork Cells.", Investigative Ophthalmology \& Visual Science 2011, 52, 1474-85.

[454] K. G. Bensch, P. M. Davison, M. A. Karasek, "Factors Controlling the in Vitro Growth Pattern of Human Microvascular Endothelial Cells", Journal of Ultrastructure Research 1983, 82, 76-89.

[455] Y. Ohta, T. Akiyama, E. Nishida, H. Sakai, "Protein Kinase $C$ and CAMP-Dependent Protein Kinase Induce Opposite Effects on Actin Polymerizability.", FEBS letters 1987, 222, 305-310.

[456] A. K. Howe, "Regulation of Actin-Based Cell Migration by cAMP/PKA.", Biochimica et Biophysica Acta 2004, 1692, 159-174.

[457] J. Voldman, "Electrical Forces for Microscale Cell Manipulation.", Annual Review of Biomedical Engineering 2006, 8, 425-454.

[458] C. D. McCaig, A. M. Rajnicek, B. Song, M. Zhao, "Controlling Cell Behavior Electrically: Current Views and Future Potential", Physiological Reviews 2005, 85, 943-978.

[459] G. Tai, B. Reid, L. Cao, M. Zhao, "Electrotaxis and Wound Healing Experimental Methods to Study Electric Fields as a Directional Signal for Cell Migration", in Chemotaxis (Eds.: T. Jin, D. Hereld), Humana Press, 2009, 77-97.

[460] I. H. Heijink, A. J. M. van Oosterhout, A. Kapus, "Epidermal Growth Factor Receptor Signalling Contributes to House Dust Mite-Induced Epithelial Barrier Dysfunction.", The European Respiratory Journal 2010, 36, 1016-26.

[461] R. J. Ablin, H. G. Kynaston, M. D. Mason, W. G. Jiang, "Prostate Transglutaminase (TGase-4) Antagonizes the Anti-Tumour Action of MDA-7/IL-24 in Prostate Cancer.", Journal of Translational Medicine 2011, 9, 49.

[462] J. M. van Gils, J. Stutterheim, T. J. van Duijn, J. J. Zwaginga, L. Porcelijn et al., "HPA-1a Alloantibodies Reduce Endothelial Cell Spreading and Monolayer Integrity.", Molecular Immunology 2009, 46, 406-15.

[463] Applied BioPhysics, "ECIS Cultureware ${ }^{\mathrm{TM}}$, http://www.biophysics.com/cultureware.php.

[464] E. N. da C. Andrade, "XLI. A Theory of the Viscosity of Liquids.-Part I", Philosophical Magazine Series 7 1934, 17, 497-511.

[465] C. V. Raman, "A Theory of the Viscosity of Liquids", Nature 1923, 111, 532-533.

[466] Sartorius AG, Handbuch Der Elektroanalytik Teil 3 - Die Elektrische Leitfähigkeit, Göttingen, n.d.

[467] M. Arnold, E. A. Cavalcanti-Adam, R. Glass, J. Blümmel, W. Eck et al., "Activation of Integrin Function by Nanopatterned Adhesive Interfaces", ChemPhysChem 2004, 5, 383-388.

[468] B. Buendia, M. H. Bré, G. Griffiths, E. Karsenti, "Cytoskeletal Control of Centrioles Movement during the Establishment of Polarity in Madin-Darby Canine Kidney Cells.", The Journal of Cell Biology 1990, 110, 1123-35.

[469] C. Rotsch, K. Jacobson, M. Radmacher, "Dimensional and Mechanical Dynamics of Active and Stable Edges in Motile Fibroblasts Investigated by Using Atomic Force Microscopy", Proceedings of the National Academy of Sciences 1999, 96, 921-926.

[470] G. Salbreux, G. Charras, E. Paluch, "Actin Cortex Mechanics and Cellular Morphogenesis.", Trends in Cell Biology 2012, 22, 536-545.

[471] N. C. Gauthier, T. A. Masters, M. P. Sheetz, "Mechanical Feedback between Membrane Tension and Dynamics.", Trends in Cell Biology 2012, 22, 527535 .

[472] T. Frisch, O. Thoumine, "Predicting the Kinetics of Cell Spreading", Journal of Biomechanics 2002, 35, 11371141.

[473] D. Misfeldt, "Transepithelial Transport in Cell Culture", Proceedings of the National Academy of Sciences 1976, 73, 1212-1216.

[474] M. Cereijido, E. Robbins, "Polarized Monolayers Formed by Epithelial Cells on a Permeable and Translucent Support", The Journal of Cell Biology 1978, $77,853-880$. 
[475] J. Richardson, V. Scalera, N. Simmons, "Identification of Two Strains of MDCK Cells Which Resemble Separate Nephron Tubule Segments", Biochimica et Biophysica Acta 1981, 673, 26-36.

[476] M. Cereijido, J. Ehrenfeld, "Structural and Functional Membrane Polarity in Cultured Monolayers of MDCK Cells", The Journal of Membrane Biology 1980, 52, 147159.

[477] N. Simmons, "lon Transport in "Tight" Epithelial Monolayers of MDCK Cells", The Journal of Membrane Biology 1981, 59, 105-114.

[478] L. Gonzalez-Mariscal, B. De Ramirez, M. Cereijido, "Tight Junction Formation in Cultured Epithelial Cells (MDCK)", The Journal of Membrane Biology 1985, 86, 113-125.

[479] A. R. Harris, L. Peter, J. Bellis, B. Baum, A. J. Kabla et al., "Characterizing the Mechanics of Cultured Cell Monolayers", Proceedings of the National Academy of Sciences 2012, 109, 16449-16454.

[480] C.-M. Lo, C. R. Keese, I. Giaever, "Impedance Analysis of MDCK Cells Measured by Electric Cell-Substrate Impedance Sensing.", Biophysical Journal 1995, 69, 2800-2807.

[481] J. Wegener, M. Sieber, H.-J. Galla, "Impedance Analysis of Epithelial and Endothelial Cell Monolayers Cultured on Gold Surfaces.", Journal of Biochemical and Biophysical Methods 1996, 32, 151-70.

[482] B. R. Stevenson, J. M. Anderson, D. A. Goodenough, M. S. Mooseker, "Tight Junction Structure and ZO-1 Content Are Identical in Two Strains of Madin-Darby Canine Kidney Cells Which Differ in Transepithelial Resistance.", The Journal of Cell Biology 1988, 107, 2401-2408.

[483] E. B. Griepp, W. J. Dolan, E. S. Robbins, D. D. Sabatini, "Participation of Plasma Membrane Proteins in the Formation of Tight Junctions by Cultured Epithelial Cells.", The Journal of Cell Biology 1983, 96, 693-702.

[484] M. Erben, S. Decker, H. Franke, H.-J. Galla, "Electrical Resistance Measurements on Cerebral Capillary Endothelial Cells--a New Technique to Study Small Surface Areas.", Journal of Biochemical and Biophysical Methods 1995, 30, 227-238.

[485] M. Hein, C. Madefessel, B. Haag, K. Teichmann, A. Post et al., "Implications of a Non-Lamellar Lipid Phase for the Tight Junction Stability. Part II: Reversible Modulation of Transepithelial Resistance in High and Low Resistance MDCK-Cells by Basic Amino Acids, Ca2+, Protamine and Protons.", Chemistry and Physics of Lipids 1992, 63, 223-233.

[486] C.-A. Schoenenberger, A. Zuk, G. M. Zinkl, D. Kendall, K. S. Matlin, "Integrin Expression and Localization in Normal MDCK Cells and Transformed MDCK Cells Lacking Apical Polarity.", Journal of Cell Science 1994, 107, 527-541.

[487] A. Sedar, J. Forte, "Effects of Calcium Depletion on the Junctional Complex between Oxyntic Cells of Gastric Glands", The Journal of cell biology 1964, 173-188.

[488] A. Martinez-Palomo, I. Meza, G. Beaty, M. Cereijido, "Experimental Modulation of Occluding Junctions in a Cultured Transporting Epithelium.", The Journal of Cell Biology 1980, 87, 736-745.
[489] J. Kartenbeck, M. Schmelz, W. W. Franke, B. Geiger, "Endocytosis of Junctional Cadherins in Bovine Kidney Epithelial (MDBK) Cells Cultured in Low $\mathrm{Ca}_{2}+$ Ion Medium.", The Journal of Cell Biology 1991, 113, 881892.

[490] S. Citi, "Protein Kinase Inhibitors Prevent Junction Dissociation Induced by Low Extracellular Calcium in MDCK Epithelial Cells.", The Journal of Cell Biology 1992, 117, 169-178.

[491] B. R. Stevenson, D. A. Goodenough, "Zonulae Occludentes in Junctional Complex-Enriched Fractions from Mouse Liver: Preliminary Morphological and Biochemical Characterization.", The Journal of Cell Biology 1984, 98, 1209-1221.

[492] T. Volberg, B. Geiger, J. Kartenbeck, W. W. Franke, "Changes in Membrane-Microfilament Interaction in Intercellular Adherens Junctions upon Removal of Extracellular Ca2+ Ions.", The Journal of Cell Biology 1986, 102, 1832-1842.

[493] J. Kartenbeck, E. Schmid, W. Franke, B. Geiger, "Different Modes of Internalization of Proteins Associated with Adhaerens Junctions and Desmosomes: Experimental Separation of Lateral Contacts Induces Endocytosis", The EMBO Journal $1982,725-732$.

[494] T. Volk, B. Geiger, "A-CAM: A 135-kD Receptor of Intercellular Adherens Junctions. I. Immunoelectron Microscopic Localization and Biochemical Studies.", The Journal of Cell Biology 1986, 103, 1441-1450.

[495] M. Takeichi, "The Cadherins: Cell-Cell Adhesion Molecules Controlling Animal Morphogenesis.", Development (Cambridge, England) 1988, 102, 639655.

[496] F. M. Watt, D. L. Mattey, D. R. Garrod, "CalciumInduced Reorganization of Desmosomal Components in Cultured Human Keratinocytes.", The Journal of Cell Biology 1984, 99, 2211-2215.

[497] D. L. Mattey, D. R. Garrod, "Splitting and Internalization of the Desmosomes of Cultured Kidney Epithelial Cells by Reduction in Calcium Concentration.", Journal of Cell Science 1986, 85, 113124 .

[498] D. C. Lovelady, J. Friedman, S. Patel, D. A. Rabson, C.M. Lo, "Detecting Effects of Low Levels of Cytochalasin $B$ in $3^{T} 3$ Fibroblast Cultures by Analysis of Electrical Noise Obtained from Cellular Micromotion.", Biosensors \& Bioelectronics 2009, 24, 2250-4.

[499] M. Saitakis, A. Tsortos, E. Gizeli, "Probing the Interaction of a Membrane Receptor with a SurfaceAttached Ligand Using Whole Cells on Acoustic Biosensors.", Biosensors \& Bioelectronics 2010, 25, 1688-93.

[500] W. M. Petroll, L. Ma, "Direct, Dynamic Assessment of Cell-Matrix Interactions Inside Fibrillar Collagen Lattices", Cell Motility and the Cytoskeleton 2003, 264, 254-264.

[501] N. O. Carragher, M. C. Frame, "Focal Adhesion and Actin Dynamics: A Place Where Kinases and Proteases Meet to Promote Invasion.", Trends in Cell Biology 2004, 14, 241-249. 
[502] U. G. Hofmann, C. Rotsch, W. J. Parak, M. Radmacher, "Investigating the Cytoskeleton of Chicken Cardiocytes with the Atomic Force Microscope.", Journal of Structural Biology 1997, 119, 84-91.

[503] D. Stamenović, Z. Liang, J. Chen, N. Wang, "Effect of the Cytoskeletal Prestress on the Mechanical Impedance of Cultured Airway Smooth Muscle Cells.", Journal of Applied Physiology 2002, 92, 1443-50.

[504] P. Kollmannsberger, B. Fabry, "Linear and Nonlinear Rheology of Living Cells", Annual Review of Materials Research 2011, 41, 75-97.

[505] H. Haga, S. Sasaki, K. Kawabata, E. Ito, T. Ushiki et al., "Elasticity Mapping of Living Fibroblasts by AFM and Immunofluorescence Observation of the Cytoskeleton", Ultramicroscopy 2000, 82, 253-258.

[506] P. A. Janmey, U. Euteneuer, P. Traub, M. Schliwa, "Viscoelastic Properties of Vimentin Compared with Other Filamentous Biopolymer Networks.", The Journal of Cell Biology 1991, 113, 155-160.

[507] W. R. Trickey, T. P. Vail, F. Guilak, "The Role of the Cytoskeleton in the Viscoelastic Properties of Human Articular Chondrocytes.", Journal of orthopaedic research: official publication of the Orthopaedic Research Society 2004, 22, 131-9.

[508] N. Wang, D. Stamenović, "Contribution of Intermediate Filaments to Cell Stiffness, Stiffening, and Growth", American Journal of Physiology. Cell Physiology 2000, 279, C188-C194.

[509] W. H. Goldmann, R. Galneder, M. Ludwig, A. Kromm, R. M. Ezzell, "Differences in F9 and 5.51 Cell Elasticity Determined by Cell Poking and Atomic Force Microscopy.", FEBS Letters 1998, 424, 139-142.

[510] D. E. Ingber, "Cellular Tensegrity: Defining New Rules of Biological Design That Govern the Cytoskeleton.", Journal of Cell Science 1993, 104, 613-627.

[511] P. Pullarkat, P. Fernandez, a Ott, "Rheological Properties of the Eukaryotic Cell Cytoskeleton", Physics Reports 2007, 449, 29-53.

[512] F. Höök, B. Kasemo, "The OCM-D Technique for Probing Biomacromolecular Recognition Reactions", in Piezoelectric Sensors (Eds.: C. Steinem, A. Janshoff), Springer, Berlin - Heidelberg, 2007, 425-447.

[513] K.-U. Petersen, L. Reuss, "Cyclic AMP-Induced Chloride Permeability in the Apical Membrane of Necturus Gallbladder Epithelium", Journal of General Physiology 1983, 81, 705-729.

[514] J. Copello, T. A. Heming, Y. Segal, L. Reuss, "cAMPActivated Apical Membrane Chloride Channels in Necturus Gallbladder Epithelium. Conductance, Selectivity, and Block.", Journal of General Physiology 1993, 102, 177-199.

[515] G. Kottra, "Calcium Is Not Involved in the cAMPMediated Stimulation of $\mathrm{Cl}$ - Conductance in the Apical Membrane of Necturus Gallbladder Epithelium.", Pflügers Archiv: European Journal of Physiology 1995, 429, 647-658.

[516] C. Vank, E. Frömter, G. Kottra, "Activation of an Apical $\mathrm{Cl}$-Conductance by Extracellular ATP in Necturus Gallbladder Is Mediated by cAMP and Not by [Ca2+]i", Pflügers Archiv: European Journal of Physiology 1999, 438, 486-496.
[517] M. P. Anderson, D. N. Sheppard, H. A. Berger, M. J. Welsh, "Chloride Channels in the Apical Membrane of Normal and Cystic Fibrosis Airway and Intestinal Epithelia", American journal of physiology. Lung Cellular and Molecular Physiology 1992, 263, L1-L14.

[518] L. Reuss, "Tight Junction Permeability to lons and Water", in Tight Junctions (Eds.: M. Cereijido, J.M. Anderson), CRC Press LLC, Boca Raton, FL, USA, 2001, 61-88.

[519] J. S. Alexander, H. B. Hechtman, D. Shepro, "Phalloidin Enhances Endothelial Barrier Function and Reduces Inflammatory Permeability in Vitro", Microvascular Research 1988, 35, 308-315.

[520] D. Hoheisel, T. Nitz, H. Franke, J. Wegener, A. Hakvoort et al., "Hydrocortisone Reinforces the BloodBrain Barrier Properties in a Serum Free Cell Culture System.", Biochemical and Biophysical Research Communications 1998, 244, 312-316.

[521] C. Weidenfeller, S. Schrot, A. Zozulya, H.-J. Galla, "Murine Brain Capillary Endothelial Cells Exhibit Improved Barrier Properties under the Influence of Hydrocortisone.", Brain Research 2005, 1053, 162-174.

[522] S. Schrot, C. Weidenfeller, T. E. Schäffer, H. Robenek, H.-J. Galla, "Influence of Hydrocortisone on the Mechanical Properties of the Cerebral Endothelium in Vitro.", Biophysical Journal 2005, 89, 3904-3910.

[523] S. Albermann, "In Situ Elektroporation Adhärenter Säugerzellen", Thesis, Westfälische WilhelmsUniversity Münster, 2004.

[524] P. M. Ghosh, C. R. Keese, I. Giaever, "Morphological Response of Mammalian Cells to Pulsed Ac Fields", Bioelectrochemistry and Bioenergetics 1994, 33, 121133.

[525] M. P. Rols, J. Teissié, "Electropermeabilization of Mammalian Cells. Quantitative Analysis of the Phenomenon.", Biophysical Journal 1990, 58, 10891098.

[526] M. P. Rols, J. Teissié, "Electropermeabilization of Mammalian Cells to Macromolecules: Control by Pulse Duration.", Biophysical Journal 1998, 75, 1415-1423.

[527] T. G. Kuznetsova, M. N. Starodubtseva, N. I. Yegorenkov, S. a Chizhik, R. I. Zhdanov, "Atomic Force Microscopy Probing of Cell Elasticity.", Micron 2007, 38, 824-833.

[528] H. K. Webb, V. K. Truong, J. Hasan, R. J. Crawford, E. P. Ivanova, "Physico-Mechanical Characterisation of Cells Using Atomic Force Microscopy - Current Research and Methodologies.", Journal of Microbiological Methods 2011, 86, 131-139.

[529] O. Guo, Y. Xia, M. Sandig, J. Yang, "Characterization of Cell Elasticity Correlated with Cell Morphology by Atomic Force Microscope.", Journal of Biomechanics 2012, 45, 304-309.

[530] H. Sato, M. Katano, T. Takigawa, T. Masuda, "Estimation for the Elasticity of Vascular Endothelial Cells on the Basis of Atomic Force Microscopy and Young's Modulus of Gelatin Gels", Polymer Bulletin 2001, 47, 375-381.

[531] E. A-Hassan, W. F. Heinz, M. D. Antonik, N. P. D'Costa, S. Nageswaran et al., "Relative Microelastic Mapping of 
Living Cells by Atomic Force Microscopy", Biophysical Journal 1998, 74, 1564-1578.

[532] W. R. Bowen, R. W. Lovitt, C. J. Wright, "Application of Atomic Force Microscopy to the Study of Micromechanical Properties of Biological Materials", Biotechnology Letters 2000, 22, 893-903.

[533] H. X. You, L. Yu, "Atomic Force Microscopy Imaging of Living Cells: Progress, Problems and Prospects.", Methods in Cell Science 1999, 21, 1-17.

[534] R. Yang, J. Y. Chen, N. Xi, K. W. C. Lai, C. Qu et al., "Characterization of Mechanical Behavior of an Epithelial Monolayer in Response to Epidermal Growth Factor Stimulation.", Experimental Cell Research 2012 318, 521-526.

[535] L. Sirghi, J. Ponti, F. Broggi, F. Rossi, "Probing Elasticity and Adhesion of Live Cells by Atomic Force Microscopy Indentation", European Biophysics Journal 2008, 37, 935-945.

[536] M. Plodinec, M. Loparic, C. a Monnier, E. C. Obermann, R. Zanetti-Dallenbach et al., "The Nanomechanical Signature of Breast Cancer.", Nature Nanotechnology 2012, 7, DOI 10.1038/nnano.2012.167.

[537] A. R. Harris, G. T. Charras, "Experimental Validation of Atomic Force Microscopy-Based Cell Elasticity Measurements.", Nanotechnology 2011, 22, 1-10.

[538] S. Leporatti, D. Vergara, A. Zacheo, V. Vergaro, G. Maruccio et al., "Cytomechanical and Topological Investigation of MCF-7 Cells by Scanning Force Microscopy.", Nanotechnology 2009, 20, 1-6.

[539] B. M. G. O’Hagan, P. Doyle, J. M. Allen, K. Sutton, G. McKerr, "The Effects of Atomic Force Microscopy upon Nominated Living Cells", Ultramicroscopy 2004, 102, 15 .

[540] H. X. You, J. M. Lau, S. Zhang, L. Yu, "Atomic Force Microscopy Imaging of Living Cells: A Preliminary Study of the Disruptive Effect of the Cantilever Tip on Cell Morphology", Ultramicroscopy 2000, 82, 297-305.

[541] J. Z. Xing, L. Zhu, J. A. Jackson, S. Gabos, X.-J. Sun et al., "Dynamic Monitoring of Cytotoxicity on Microelectronic Sensors.", Chemical Research in Toxicology 2005, 18, 154-161.

[542] K. Solly, X. Wang, X. Xu, B. Strulovici, W. Zheng, "Application of Real-Time Cell Electronic Sensing (RTCES) Technology to Cell-Based Assays.", Assay and Drug Development Technologies 2004, 2, 363-372.

[543] J. M. Atienza, J. Zhu, X. Wang, X. Xu, Y. Abassi, "Dynamic Monitoring of Cell Adhesion and Spreading on Microelectronic Sensor Arrays.", Journal of Biomolecular Screening 2005, 10, 795-805.

[544] K. Astanina, Y. Simon, C. Cavelius, S. Petry, A. Kraegeloh et al., "Superparamagnetic Iron Oxide Nanoparticles Impair Endothelial Integrity and Inhibit Nitric Oxide Production", Acta Biomaterialia 2014, 10, 4896-4911.

[545] R. R. Foster, L. Armstrong, S. Baker, D. W. L. Wong, E. C. Wylie et al., "Glycosaminoglycan Regulation by VEGFA and VEGFC of the Glomerular Microvascular Endothelial Cell Glycocalyx in Vitro.", The American Journal of Pathology 2013, 183, 604-616.
[546] J. Hartigan, C. Liu, W. Downey, "Moving Forward with Label-Free Technology", Drug Discovery World 2010, $12,41-48$.

[547] K. A. Marx, T. Zhou, D. Long, "Electropolymerized Films Formed from the Amphiphilic Decyl Esters of Dand L-Tyrosine Compared to L-Tyrosine Using the Electrochemical Quartz Crystal Microbalance.", Biomacromolecules 2005, 6, 1698-706.

[548] K. A. Marx, T. Zhou, D. McIntosh, S. J. Braunhut, "Electropolymerized Tyrosine-Based Thin Films: Selective Cell Binding via Peptide Recognition to Novel Electropolymerized Biomimetic Tyrosine RGDY Films.", Analytical Biochemistry 2009, 384, 86-95.

[549] Y.-E. Koo Lee, R. Kopelman, "Optical Nanoparticle Sensors for Quantitative Intracellular Imaging", Wiley Interdisciplinary Reviews: Nanomedicine and Nanobiotechnology 2009, 1, 98-110.

[550] Y.-E. Koo Lee, R. Kopelman, R. Smith, "Nanoparticle PEBBLE Sensors in Live Cells and in Vivo", Annual Review of Analytical Chemistry 2009, 2, 57-76.

[551] S. M. Borisov, I. Klimant, "Luminescent Nanobeads for Optical Sensing and Imaging of Dissolved Oxygen", Microchimica Acta 2009, 164, 7-15.

[552] S. M. Buck, Y.-E. Koo Lee, E. Park, H. Xu, M. A. Philbert et al., "Optochemical Nanosensor PEBBLEs: Photonic Explorers for Bioanalysis with Biologically Localized Embedding.", Current Opinion in Chemical Biology 2004, 8, 540-546.

[553] H. A. Clark, S. L. R. Barker, M. Brasuel, M. T. Miller, E. Monson et al., "Subcellular Optochemical Nanobiosensors: Probes Encapsulated by Biologically Localised Embedding (PEBBLEs)", Sensors and Actuators B: Chemical 1998, 51, 12-16.

[554] Z. XU, A. Rollins, R. Alcala, R. E. Marchant, "A Novel Fiber-Optic pH Sensor Incorporating Carboxy SNAFL2 and Fluorescent Wavelength-Ratiometric Detection", Journal of Biomedical Materials Research 1998, 39, 9-15.

[555] J. E. Whitaker, R. P. Haugland, F. G. Prendergast, "Spectral and Photophysical Studies of Benzo[c]xanthene Dyes: Dual Emission pH Sensors", Analytical Biochemistry 1991, 194, 330-344.

[556] J. Han, K. Burgess, "Fluorescent Indicators for Intracellular pH", Chemical Reviews 2010, 110, 27092728.

[557] B. M. Weidgans, C. Krause, I. Klimant, O. S. Wolfbeis, "Fluorescent $\mathrm{pH}$ Sensors with Negligible Sensitivity to Ionic Strength", Analyst 2004, 129, 645-650.

[558] B. M. Weidgans, "New Fluorescent Optical pH Sensors with Minimal Effects of lonic Strength", Thesis, University of Regensburg, 2004.

[559] A. S. Vasylevska, A. A. Karasyov, S. M. Borisov, C. Krause, "Novel Coumarin-Based Fluorescent $\mathrm{pH}$ Indicators, Probes and Membranes Covering a Broad pH Range", Analytical and Bioanalytical Chemistry 2007, 387, 2131-2141.

[560] O. S. Wolfbeis, E. Fürlinger, H. Kroneis, H. Marsoner, "Fluorimetric Analysis", Fresenius' Zeitschrift für analytische Chemie 1983, 314, 119-124. 
[561] S. Schreml, R. J. Meier, O. S. Wolfbeis, M. Landthaler, R. M. Szeimies et al., "2D Luminescence Imaging of $\mathrm{pH}$ in Vivo", Proceedings of the National Academy of Sciences of the United States of America 2011, 108, 2432-2437.

[562] H. Offenbacher, O. S. Wolfbeis, E. Fürlinger, "Fluorescence Optical Sensors for Continuous Determination of near-Neutral pH Values", Sensors and Actuators 1986, 9, 73-84.

[563] Z. Zhujun, W. R. Seitz, "A Fluorescence Sensor for Quantifying pH in the Range from 6.5 to 8.5", Analytica Chimica Acta 1984, 160, 47-55.

[564] D. Wencel, B. D. MacCraith, C. McDonagh, "High Performance Optical Ratiometric Sol-gel-Based $\mathrm{pH}$ Sensor", Sensors and Actuators B: Chemical 2009, 139, 208-213.

[565] Z. Zhujun, W. R. Seitz, "A Carbon Dioxide Sensor Based on Fluorescence", Analytica Chimica Acta 1984, 160, 305-309.

[566] A. Mills, Q. Chang, "Fluorescence Plastic Thin-Film Sensor for Carbon Dioxide", Analyst 1993, 118, 839843.

[567] A. Mills, "Optical Sensors for Carbon Dioxide and Their Applications", in Sensors for Environment, Health and Security (Ed.: M.-I. Baraton), Springer Netherlands, 2009, 347-370.

[568] C.-S. Chu, Y.-L. Lo, "Highly Sensitive and Linear Optical Fiber Carbon Dioxide Sensor Based on Sol-gel Matrix Doped with Silica Particles and HPTS", Sensors and Actuators B: Chemical 2009, 143, 205-210.

[569] C. S. Burke, A. Markey, R. I. Nooney, P. Byrne, C. McDonagh, "Development of an Optical Sensor Probe for the Detection of Dissolved Carbon Dioxide", Sensors and Actuators B: Chemical 2006, 119, 288-294.

[570] M. Cajlaković, A. Bizzarri, V. Ribitsch, "Luminescence Lifetime-Based Carbon Dioxide Optical Sensor for Clinical Applications.", Analytica Chimica Acta 2006, 573-574, 57-64

[571] R. Ali, S. M. Saleh, R. J. Meier, H. A. Azab, I. I. Abdelgawad et al., "Upconverting Nanoparticle Based Optical Sensor for Carbon Dioxide", Sensors and Actuators B: Chemical 2010, 150, 126-131.

[572] J. Sipior, S. Bambot, M. Romauld, G. M. Carter, J. R. Lakowicz et al., "A Lifetime-Based Optical $\mathrm{CO}_{2}$ Gas Sensor with Blue or Red Excitation and Stokes or AntiStokes Detection", Analytical Biochemistry 1995, 227, 309-318.

[573] G. Neurauter, I. Klimant, O. S. Wolfbeis, "Microsecond Lifetime-Based Optical Carbon Dioxide Sensor Using Luminescence Resonance Energy Transfer", Analytica Chimica Acta 1999, 382, 67-75.

[574] C. von Bültzingslöwen, A. K. McEvoy, C. McDonagh, B. D. MacCraith, "Lifetime-Based Optical Sensor for High-Level pCO2 Detection Employing Fluorescence Resonance Energy Transfer", Analytica Chimica Acta 2003, 480, 275-283.

[575] O. S. Wolfbeis, H. E. Posch, "Fibre-Optic Fluorescing Sensor for Ammonia", Analytica Chimica Acta 1986, $185,321-327$
[576] A. Lobnik, O. S. Wolfbeis, "Sol-Gel Based Optical Sensor for Dissolved Ammonia", Sensors and Actuators B: Chemical 1998, 51, 203-207.

[577] K. Waich, T. Mayr, I. Klimant, "Fluorescence Sensors for Trace Monitoring of Dissolved Ammonia", Talanta 2008, 77, 66-72.

[578] K. Waich, S. M. Borisov, T. Mayr, I. Klimant, "Dual Lifetime Referenced Trace Ammonia Sensors", Sensors and Actuators B: Chemical 2009, 139, 132-138.

[579] Q. Chang, J. Sipior, J. R. Lakowicz, G. Rao, "A LifetimeBased Fluorescence Resonance Energy Transfer Sensor for Ammonia.", Analytical Biochemistry 1995, 232, 92-97.

[580] D. B. Papkovsky, "New Oxygen Sensors and Their Application to Biosensing", Sensors and Actuators B: Chemical 1995, 29, 1-6.

[581] Y.-E. Koo Lee, E. E. Ulbrich, G. Kim, H. Hah, C. Strollo et al., "Near Infrared Luminescent Oxygen Nanosensors with Nanoparticle Matrix Tailored Sensitivity.", Analytical Chemistry 2010, 82, 84468455 .

[582] N. W. Choi, S. S. Verbridge, R. M. Williams, J. Chen, J.Y. Kim et al., "Phosphorescent Nanoparticles for Quantitative Measurements of Oxygen Profiles in Vitro and in Vivo.", Biomaterials 2012, 33, 2710-2722.

[583] R. I. Dmitriev, D. B. Papkovsky, "O2-Sensitive Probes Based on Phosphorescent Metalloporphyrins", in Phosphorescent Oxygen-Sensitive Probes, Springer Basel, Basel, 2012, 1-28.

[584] B. A. DeGraff, J. N. Demas, "Luminescence-Based Oxygen Sensors", Reviews in Fluorescence 2005 2005, 125-151.

[585] A. P. de Silva, S. A. de Silva, "Fluorescent Signalling Crown Ethers; "Switching on" of Fluorescence by Alkali Metal Ion Recognition and Binding in Situ", Chemical Communications 1986, 1709.

[586] A. Minta, R. Y. Tsien, "Fluorescent Indicators for Cytosolic Sodium", The Journal of Biological Chemistry 1989, 264, 19449-19457.

[587] H. He, M. A. Mortellaro, M. J. P. Leiner, S. T. Young, R. J. Fraatz et al., "A Fluorescent Chemosensor for Sodium Based on Photoinduced Electron Transfer", Analytical Chemistry 2003, 75, 549-555.

[588] H.-F. Ji, R. Dabestani, G. M. Brown, O. Ridge, "A Supramolecular Fluorescent Probe, Activated by Protons To Detect Cesium and Potassium lons, Mimics the Function of a Logic Gate", Journal of the American Chemical Society 2008, 122, 9306-9307.

[589] A. Thibon, V. C. Pierre, "A Highly Selective Luminescent Sensor for the Time-Gated Detection of Potassium.", Journal of the American Chemical Society 2009, 131, 434-435.

[590] H. Ueyama, M. Takagi, S. Takenaka, "A Novel Potassium Sensing in Aqueous Media with a Synthetic Oligonucleotide Derivative. Fluorescence Resonance Energy Transfer Associated with Guanine Quartet-", Journal of the American Chemical Society 2002, 124, 14286-14287.

[591] E. a Weitz, V. C. Pierre, "A Ratiometric Probe for the Selective Time-Gated Luminescence Detection of 
Potassium in Water", Chemical Communications 2011, 47, 541-543.

[592] P. H. Cobbold, T. J. Rinktt, "Fluorescence and Bioluminescence Measurement of Cytoplasmic Free Calcium", Biochemical Journal 1987, 248, 313-328.

[593] B. Valeur, I. Leray, "Design Principles of Fluorescent Molecular Sensors for Cation Recognition", Coordination Chemistry Reviews 2000, 205, 3-40.

[594] R. Y. Tsien, G. Grynkiewicz, "Stilbene-Type Fluorophore, Two Aminodiacetic Acid Moieties", U.S. Patent 4,603, 209, 1986.

[595] R. Y. Tsien, T. Pozzan, T. J. Rink, "Calcium Homeostasis in Intact Lymphocytes: Cytoplasmic Free Calcium Monitored With a New, Intracellularly Trapped Fluorescent Indicator", The Journal of Cell Biology 1982, 94, 325-334.

[596] D. A. Williams, F. S. Fay, "Intracellular Calibration of the Fluorescent Calcium Indicator Fura-2", Cell Calcium 1990, 11, 75-83.

[597] A. Miyawaki, J. Llopis, R. Heim, J. M. Mccaffery, J. A. Adams et al., "Fluorescent Indicators for Ca2+ Based on Green Fluorescent Proteins and Calmodulin", Nature 1997, 388, 882-887.

[598] X. Hun, Z. Zhang, "Preparation of a Novel Fluorescence Nanosensor Based on Calcein-Doped Silica Nanoparticles, and Its Application to the Determination of Calcium in Blood Serum", Microchimica Acta 2007, 159, 255-261.

[599] E. Urbano, H. Offenbacher, O. S. Wolfbeis, "Optical Sensor for Continuous Determination of Halides", Analytical Chemistry 1984, 56, 427-429.

[600] C. D. Geddes, "Optical Halide Sensing Using Fluorescence Quenching: Theory, Simulations and Applications - a Review", Measurement Science and Technology 2001, 12, R53-R88.

[601] F. Munkonge, E. W. F. W. Alton, C. Andersson, H. Davidson, A. Dragomir et al., "Measurement of Halide Efflux from Cultured and Primary Airway Epithelial Cells Using Fluorescence Indicators.", Journal of Cystic Fibrosis 3 2004, 171-176.

[602] C. D. Geddes, K. Apperson, J. Karolin, D. J. Birch, "Chloride-Sensitive Fluorescent Indicators.", Analytical Biochemistry 2001, 293, 60-66.

[603] S. M. Ng, R. Narayanaswamy, "Fluorescence Sensor Using a Molecularly Imprinted Polymer as a Recognition Receptor for the Detection of Aluminium Ions in Aqueous Media", Analytical and Bioanalytical Chemistry 2006, 386, 1235-1244.

[604] H. Li, Y. Zhang, X. Wang, Z. Gao, "A Luminescent Nanosensor for $\mathrm{Hg}(\mathrm{II})$ Based on Functionalized CdSe/ZnS Quantum Dots", Microchimica Acta 2007, 160, 119-123.

[605] O. Oter, K. Ertekin, C. Kirilmis, M. Koca, "Spectral Characterization of a Newly Synthesized Fluorescent Semicarbazone Derivative and Its Usage as a Selective Fiber Optic Sensor for copper(II)", Analytica Chimica Acta 2007, 584, 308-314.

[606] H. Wu, J. Liang, H. Han, "A Novel Method for the Determination of $\mathrm{Pb}_{2}+$ Based on the Quenching of the
Fluorescence of CdTe Quantum Dots", Microchimica Acta 2007, 161, 81-86.

[607] M. Shamsipur, M. Sadeghi, A. Garau, V. Lippolis, "An Efficient and Selective Flourescent Chemical Sensor Based on 5-(8-Hydroxy-2-Quinolinylmethyl)-2,8Dithia-5-Aza-2,6-Pyridinophane as a New Fluoroionophore for Determination of iron(III) lons. A Novel Probe for Iron Speciation.", Analytica Chimica Acta 2013, 761, 169-177.

[608] Y. Yan, Z. Che, X. Yu, X. Zhi, J. Wang et al., "Fluorescence "on-off-on" Chemosensor for Sequential Recognition of $\mathrm{Fe}_{3}+$ and $\mathrm{Hg}_{2}+$ in Water Based on Tetraphenylethylene Motif", Bioorganic \& Medicinal Chemistry 2013, 21, 508-513.

[609] T. Mayr, C. Igel, G. Liebsch, I. Klimant, O. S. Wolfbeis, "Cross-Reactive Metal Ion Sensor Array in a Micro Titer Plate Format", Analytical Chemistry 2003, 75, 43894396.

[610] T. Carofiglio, C. Fregonese, G. J. Mohr, F. Rastrelli, U. Tonellato, "Optical Sensor Arrays: One-Pot, Multiparallel Synthesis and Cellulose Immobilization of $\mathrm{pH}$ and Metal Ion Sensitive Azo-Dyes", Tetrahedron 2006, 62, 1502-1507.

[611] L. Prodi, F. Bolletta, M. Montalti, N. Zaccheroni, "Luminescent Chemosensors for Transition Metal Ions", Coordination Chemistry Reviews 2000, 205, 5983.

[612] B. C. Dickinson, C. J. Chang, "A Targetable Fluorescent Probe for Imaging Hydrogen Peroxide in the Mitochondria of Living Cells.", Journal of the American Chemical Society 2008, 130, 9638-9.

[613] O. S. Wolfbeis, A. Dürkop, "A Europium-lon-Based Luminescent Sensing Probe for Hydrogen Peroxide", Angewandte Chemie (International ed. in English) 2002, 41, 4495-4498.

[614] S. Cavaliere-Jaricot, M. Darbandi, E. Kuçur, T. Nann, "Silica Coated Quantum Dots: A New Tool for Electrochemical and Optical Glucose Detection", Microchimica Acta 2007, 160, 375-383.

[615] A. Duerkop, M. Schaeferling, O. S. Wolfbeis, "Glucose Sensing and Glucose Determination Using Fluorescent Probes and Molecular Receptors", in Glucose Sensing (Eds.: C.D. Geddes, J.R. Lakowicz), Springer, 2006, 351-375.

[616] A. Pasic, H. Koehler, L. Schaupp, T. R. Pieber, I. Klimant, "Fiber-Optic Flow-through Sensor for Online Monitoring of Glucose.", Analytical and Bioanalytical Chemistry 2006, 386, 1293-1302.

[617] J. C. Pickup, F. Hussain, N. D. Evans, O. J. Rolinski, D. J. S. Birch, "Fluorescence-Based Glucose Sensors.", Biosensors \& Bioelectronics 2005, 20, 2555-65.

[618] X. Wang, O. S. Wolfbeis, R. J. Meier, "Luminescent Probes and Sensors for Temperature.", Chemical Society reviews 2013, 42, 7834-7869.

[619] F. N. Dultsev, V. P. Ostanin, D. Klenerman, "'Hearing" Bond Breakage. Measurement of Bond Rupture Forces Using a Quartz Crystal Microbalance", Langmuir 2000, $16,5036-5040$.

[620] M. A. Cooper, "Biosensing Using Rupture Event Scanning (REVS) ${ }^{\mathrm{TM} "}$, Measurement Science and Technology 2003, 1888, 1888-1893. 
[621] M. A. Cooper, "Resonant Acoustic Profiling (RAP TM) and Rupture Event Scanning (REVS $\left.{ }^{\mathrm{TM}}\right)^{\prime \prime}$, in Piezoelectric Sensors (Eds.: C. Steinem, A. Janshoff), Springer, Berlin - Heidelberg, 2007, 449-479.

[622] E. R. Hirst, Y. J. Yuan, W. L. Xu, J. E. Bronlund, "BondRupture Immunosensors--a Review.", Biosensors \& Bioelectronics 2008, 23, 1759-68.

[623] F. Dultsev, E. Kolosovsky, I. Mik, "A New Procedure to Record the Rupture of Bonds between Macromolecules and the Surface of the Quartz Crystal Microbalance (QCM)", Langmuir 2012, 28, 1379313797.

[624] Y. J. Yuan, M. J. Van Der Werff, H. Chen, E. R. Hirst, W. L. Xu et al., "Bond Rupture of Biomolecular Interactions by Resonant Quartz Crystal.", Analytical Chemistry 2007, 79, 9039-44.

[625] M. J. van der Werff, Y. J. Yuan, E. R. Hirst, W. L. Xu, H. Chen et al., "Quartz Crystal Microbalance Induced Bond Rupture Sensing for Medical Diagnostics", IEEE Sensors Journal 2007, 7, 762-769.

[626] Y. J. Yuan, Y. Zhao, W. L. Xu, "Characterization of Molecular Interactions of an Immobilized Biotinylated Monolayer and Streptavidin-Coated Microspheres by Bond-Rupture Scanning.", Analytica Chimica Acta 2010, 680, 59-64.

[627] F. N. Dultsev, R. E. Speight, M. T. Fiorini, J. M. Blackburn, C. Abell et al., "Direct and Quantitative Detection of Bacteriophage by "Hearing" Surface Detachment Using a Quartz Crystal Microbalance.", Analytical Chemistry 2001, 73, 3935-9.

[628] F. N. Dultsev, E. A. Kolosovsky, I. A. Mik, A. A. Lomzov, D. $V$ Pyshnyi, "OCM-Based Measurement of Bond Rupture Forces in DNA Double Helices for Complementarity Sensing.", Langmuir 2014, 30, 37953801.

[629] Cooper, F. N. Dultsev, T. Minson, V. P. Ostanin, C. Abell et al., "Direct and Sensitive Detection of a Human Virus by Rupture Event Scanning.", Nature Biotechnology 2001, 19, 833-7.

[630] E. Saphire, P. Parren, "Listening for Viral Infection", Nature Biotechnology 2001, 19, 823-824.

[631] M. A. Cooper, F. N. Dultsev, V. P. Ostanin, D. Klenerman, "Separation and Detection of Bacteria Using Rupture Event Scanning.", Analytica Chimica Acta 2011, 702, 233-8.

[632] S. K. Ghosh, V. P. Ostanin, C. L. Johnson, C. R. Lowe, A. a Seshia, "Probing Biomolecular Interaction Forces Using an Anharmonic Acoustic Technique for Selective Detection of Bacterial Spores.", Biosensors \& Bioelectronics 2011, 29, 145-50.

[633] M. Edvardsson, M. Rodahl, B. Kasemo, F. Höök, "A Dual-Frequency OCM-D Setup Operating at Elevated Oscillation Amplitudes.", Analytical Chemistry 2005, 77, 4918-26.

[634] M. Edvardsson, M. Rodahl, F. Höök, "Investigation of Binding Event Perturbations Caused by Elevated OCMD Oscillation Amplitude.", The Analyst 2006, 131, 8228.

[635] V. P. Zhdanov, M. Edvardsson, F. Höök, B. Kasemo, "Suppression of Binding Events via External
Perturbation with Emphasis on OCM", Chemical Physics Letters 2006, 424, 214-217.

[636] F. N. Dultsev, E. a Kolosovsky, "Quartz Crystal Microbalance as a Sensing Active Element for Rupture Scanning within Frequency Band.", Analytica Chimica Acta 2011, 687, 75-81.

[637] J. Wegener, "Impedanzspektroskopische Und Mikrogravimetrische Untersuchung an Barrierebildenden Zellen Auf Planaren Goldelektroden", Thesis, Westfälische WilhelmsUniversity Münster, 1998.

[638] R. I. Dmitriev, D. B. Papkovsky, "Optical Probes and Techniques for $\mathrm{O}_{2}$ Measurement in Live Cells and Tissue", Cellular and Molecular Life Sciences 2012, 69, 2025-2039.

[639] D. B. Papkovsky, R. I. Dmitriev, "Biological Detection by Optical Oxygen Sensing.", Chemical Society Reviews 2013, 42, 8700-8732.

[640] Y.-E. Koo Lee, Y. Cao, R. Kopelman, S. M. Koo, M. Brasuel et al., "Real-Time Measurements of Dissolved Oxygen inside Live Cells by Organically Modified Silicate Fluorescent Nanosensors.", Analytical Chemistry 2004, 76, 2498-2505.

[641] Y. Cao, Y.-E. Koo Lee, R. Kopelman, "Poly(decyl Methacrylate)-Based Fluorescent PEBBLE Swarm Nanosensors for Measuring Dissolved Oxygen in Biosamples", The Analyst 2004, 129, 745-750.

[642] A. Fercher, S. M. Borisov, A. V Zhdanov, I. Klimant, D. B. Papkovsky, "Intracellular $\mathrm{O}_{2}$ Sensing Probe Based on Cell-Penetrating Phosphorescent Nanoparticles", Acs Nano 2011, 5499-5508.

[643] M. P. Coogan, J. B. Court, V. L. Gray, A. J. Hayes, S. H. Lloyd et al., "Probing Intracellular Oxygen by Quenched Phosphorescence Lifetimes of Nanoparticles Containing Polyacrylamide-Embedded $\left[\mathrm{Ru}\left(\mathrm{dpp}\left(\mathrm{SO}_{3} \mathrm{Na}\right)_{2}\right)_{3}\right] \mathrm{Cl}_{2}$.", $\quad$ Photochemical \& Photobiological Sciences 2010, 9, 103-109.

[644] X. Wang, J. A. Stolwijk, M. Sperber, R. J. Meier, J. Wegener et al., "Ultra-Small, Highly Stable, and Membrane-Impermeable Fluorescent Nanosensors for Oxygen", Methods and Applications in Fluorescence 2013, 1, 035002

[645] X. Wang, H. H. Gorris, J. A. Stolwijk, R. J. Meier, D. B. M. Groegel et al., "Self-Referenced RGB Colour Imaging of Intracellular Oxygen", Chemical Science 2011, 2, 901-906.

[646] X. Wang, J. A. Stolwijk, T. Lang, M. Sperber, R. J. Meier et al., "Ultra-Small, Highly Stable, and Sensitive Dual Nanosensors for Imaging Intracellular Oxygen and $\mathrm{pH}$ in Cytosol", Journal of the American Chemical Society 2012, 134, 17011-4.

[647] K. B. Guice, M. E. Caldorera, M. J. McShane, "Nanoscale Internally Referenced Oxygen Sensors Produced from Self-Assembled Nanofilms on Fluorescent Nanoparticles.", Journal of Biomedical Optics 2005, 10, 064031_1-10.

[648] Z. Cheng, C. A. Aspinwall, "Nanometre-Sized Molecular Oxygen Sensors Prepared from Polymer Stabilized Phospholipid Vesicles.", The Analyst 2006, 131, 236-243. 
[649] U. Neugebauer, Y. Pellegrin, M. Devocelle, R. J. Forster, W. Signac et al., "Ruthenium Polypyridyl Peptide Conjugates: Membrane Permeable Probes for Cellular Imaging", Chemical Communications 2008, 2, 5307-5309.

[650] R. I. Dmitriev, A. V Zhdanov, G. V Ponomarev, D. V Yashunski, D. B. Papkovsky, "Intracellular OxygenSensitive Phosphorescent Probes Based on CellPenetrating Peptides", Analytical Biochemistry 2010, 398, 24-33.

[651] R. I. Dmitriev, H. M. Ropiak, G. V Ponomarev, D. V Yashunsky, D. B. Papkovsky, "Cell-Penetrating Conjugates of Coproporphyrins with Oligoarginine Peptides: Rational Design and Application for Sensing Intracellular O2", Bioconjugate Chemistry 2011, 22, 2507-2518.

[652] R. I. Dmitriev, A. V Zhdanov, G. Jasionek, D. B. Papkovsky, "Assessment of Cellular Oxygen Gradients with a Panel of Phosphorescent Oxygen-Sensitive Probes.", Analytical Chemistry 2012, 84, 2930-2938.

[653] H. Xu, J. W. Aylott, R. Kopelman, T. J. Miller, M. A. Philbert, "A Real-Time Ratiometric Method for the Determination of Molecular Oxygen Inside Living Cells Using Sol - Gel-Based Spherical Optical Nanosensors with Applications to Rat C6 Glioma", Analytical Chemistry 2001, 73, 4124-4133.

[654] C. Wu, B. Bull, K. Christensen, J. McNeill, "Ratiometric Single-Nanoparticle Oxygen Sensors for Biological Imaging.", Angewandte Chemie (International ed. in English) 2009, 48, 2741-2745.

[655] X.-H. Wang, H.-S. Peng, Z. Chang, L.-L. Hou, F.-T. You et al., "Synthesis of Ratiometric Fluorescent Nanoparticles for Sensing Oxygen", Microchimica Acta 2012, 178, 147-152.

[656] J. Napp, T. Behnke, L. Fischer, C. Würth, M. Wottawa et al., "Targeted Luminescent Near-Infrared PolymerNanoprobes for In Vivo Imaging of Tumor Hypoxia.", Analytical Chemistry 2011, 83, 9039-9346.

[657] V. Tsytsarev, H. Arakawa, S. Borisov, E. Pumbo, R. S. Erzurumlu et al., "In Vivo Imaging of Brain Metabolism Activity Using a Phosphorescent Oxygen-Sensitive Probe", Journal of Neuroscience Methods 2013, 216, 146-151.

[658] A. Fercher, T. C. O'Riordan, A. V Zhdanov, R. I. Dmitriev, D. B. Papkovsky, "Imaging of Cellular Oxygen and Analysis of Metabolic Responses of Mammalian Cells", in Live Cell Imaging (Ed.: D.B. Papkovsky), Humana Press, New York, 2010, 257-273.

[659] J. Hofmann, R. J. Meier, A. Mahnke, V. Schatz, F. Brackmann et al., "Ratiometric Luminescence 2D in Vivo Imaging and Monitoring of Mouse Skin Oxygenation", Methods and Applications in Fluorescence 2013, 1, 045002.

[66o] T. Horvath, E. Monson, J. Sumner, H. Xu, R. Kopelman, "Use of Steady-State Fluorescence Anisotropy with Pebble Nanosensors for Chemical Analysis", in Biomedical Nanotechnology Architectures and Applications (Eds.: D. Bornhop, D. Dunn, R.J. Mariella, C. Murphy, D. Nicolau, S. Nie, et al.), SPIE, 2002, 486492.
[661] E. Schmälzlin, J. T. van Dongen, I. Klimant, B. Marmodée, M. Steup et al., "An Optical Multifrequency Phase-Modulation Method Using Microbeads for Measuring Intracellular Oxygen Concentrations in Plants.", Biophysical Journal 2005, 89, 1339-1345.

[662] M. C. Hogan, "Phosphorescence Quenching Method for Measurement of Intracellular in Isolated Skeletal Muscle Fibers", Journal of Applied Physiology 1999, 86, 720-724.

[663] T. C. O'Riordan, A. V Zhdanov, G. V Ponomarev, D. B. Papkovsky, "Analysis of Intracellular Oxygen and Metabolic Responses of Mammalian Cells by TimeResolved Fluorometry.", Analytical Chemistry 2007, 79, 9414-9419.

[664] H. Liu, H. Yang, X. Hao, H. Xu, Y. Lv et al., "Development of Polymeric Nanoprobes with Improved Lifetime Dynamic Range and Stability for Intracellular Oxygen Sensing", Small 2013, 9, 26392648.

[665] A. S. Golub, R. N. Pittman, "pO2 Measurements in the Microcirculation Using Phosphorescence Quenching Microscopy at High Magnification", American Journal of Physiology. Heart and Circulatory Physiology 2008, 294, $\mathrm{H}_{2905}-\mathrm{H}_{2} 916$.

[666] H. C. Gerritsen, R. Sanders, A. Draaijer, C. Ince, Y. K. Levine, "Fluorescence Lifetime Imaging of Oxygen in Living Cells", Journal of Fluorescence 1997, 7, 11-15.

[667] B. Korzeniowska, "Nanoparticle-Based Intracellular Diagnostics", Thesis, Dublin City University, 2012.

[668] C. Ast, E. Schmälzlin, H.-G. Löhmannsröben, J. T. van Dongen, "Optical Oxygen Micro- and Nanosensors for Plant Applications.", Sensors 2012, 12, 7015-7032.

[669] W. Zhong, P. Urayama, M.-A. Mycek, "Imaging Fluorescence Lifetime Modulation of a RutheniumBased Dye in Living Cells: The Potential for Oxygen Sensing", Journal of Physics D: Applied Physics 2003, 36, 1689-1695.

[670] D. Sud, W. Zhong, D. Beer, M.-A. Mycek, "Measurement of Intracellular Oxygen Levels Using Fluorescence Lifetime Imaging Microscopy (FLIM)", in Proc. SPIE 5859, Photon Migration and Diffuse-Light Imaging II (Eds.: K. Licha, R. Cubeddu), 2005, 585907_1-10.

[671] E. Schmälzlin, B. Walz, I. Klimant, B. Schewe, H.-G. Löhmannsröben, "Monitoring Hormone-Induced Oxygen Consumption in the Salivary Glands of the Blowfly, Calliphora Vicina, by Use of Luminescent Microbeads", Sensors and Actuators B: Chemical 2006, 119, 251-254.

[672] R. N. Pittman, A. S. Golub, H. Carvalho, "Measurement of Oxygen in the Microcirculation Using Phosphorescence Quenching Microscopy", in Oxygen Transport to Tissue XXXI (Eds.: E. Takahashi, D.F. Bruley), Springer, New York, 2010, 157-162.

[673] X. Wang, D. E. Achatz, C. Hupf, M. Sperber, J. Wegener et al., "Imaging of Cellular Oxygen via Two-Photon Excitation of Fluorescent Sensor Nanoparticles", Sensors and Actuators B: Chemical 2013, 188, 257-262.

[674] A. V Kondrashina, R. I. Dmitriev, S. M. Borisov, I. Klimant, I. O. Brien et al., "A Phosphorescent Nanoparticle-Based Probe for Sensing and Imaging of 
(Intra)Cellular Oxygen in Multiple Detection Modalities", Advanced Functional Materials 2012, 22, 4931-4939.

[675] R. I. Dmitriev, A. V Zhdanov, Y. M. Nolan, D. B. Papkovsky, "Imaging of Neurosphere Oxygenation with Phosphorescent Probes", Biomaterials 2013, 34, 9307-9317.

[676] R. I. Dmitriev, S. M. Borisov, A. V Kondrashina, J. M. P. Pakan, U. Anilkumar et al., "Imaging Oxygen in Neural Cell and Tissue Models by Means of Anionic CellPermeable Phosphorescent Nanoparticles.", Cellular and Molecular Life Sciences 2014, DOI 10.1007/s00018014-1673-5.

[677] R. I. Dmitriev, D. B. Papkovsky, "Multi-Parametric O2 Imaging in Three-Dimensional Neural Cell Models with the Phosphorescent Probes", in Neuronal Cell Death (Eds.: L. Lossi, A. Merighi), Springer New York, 2015, 55-71.

[678] R. I. Dmitriev, A. V Kondrashina, K. Koren, I. Klimant, A. V Zhdanov et al., "Small Molecule Phosphorescent Probes for $\mathrm{O}_{2}$ Imaging in 3D Tissue Models", Biomaterials Science 2014, 2, 853.

[679] R. D. Shonat, A. C. Kight, "Oxygen Tension Imaging in the Mouse Retina", Annals of Biomedical Engineering 2003, 31, 1084-1096.

[680] A. S. Golub, M. A. Tevald, R. N. Pittman, "Phosphorescence Quenching Microrespirometry of Skeletal Muscle in Situ", American Journal of Physiology. Heart and Circulatory Physiology 2011, 300, $\mathrm{H}_{135}-\mathrm{H}_{143}$.

[681] S. Sakadzić, E. Roussakis, M. A. Yaseen, E. T. Mandeville, V. J. Srinivasan et al., "Two-Photon HighResolution Measurement of Partial Pressure of Oxygen in Cerebral Vasculature and Tissue", Nature Methods 2010, 7, 755-759.

[682] A. K. McEvoy, C. M. McDonagh, B. D. MacCraith, "Dissolved Oxygen Sensor Based on Fluorescence Quenching of Oxygen-Sensitive Ruthenium Complexes Immobilized in Sol-Gel-Derived Porous Silica Coatings", Analyst 1996, 121, 785-788.

[683] V. Nock, R. J. Blaikie, T. David, "Oxygen Control For Bioreactors And In-Vitro Cell Assays", in 4th International Conference on Advanced Materials and Nanotechnology (AMN-4), 8-12 Feb 2009. AIP Conference Proceedings, Dunedin, New Zealand, 2009, 67-70.

[684] L. Tolosa, Y. Kostov, P. Harms, G. Rao, "Noninvasive Measurement of Dissolved Oxygen in Shake Flasks.", Biotechnology and Bioengineering 2002, 80, 594-597.

[685] C. Wittmann, H. M. Kim, G. John, E. Heinzle, "Characterization and Application of an Optical Sensor for Quantification of Dissolved O2 in Shake-Flasks", Biotechnology Letters 2003, 25, 377-380.

[686] G. T. John, I. Klimant, C. Wittmann, E. Heinzle, "Integrated Optical Sensing of Dissolved Oxygen in Microtiter Plates: A Novel Tool for Microbial Cultivation.", Biotechnology and Bioengineering 2003, $81,829-836$.

[687] R. D. Guarino, L. E. Dike, T. a Haq, J. A. Rowley, J. B. Pitner et al., "Method for Determining Oxygen Consumption Rates of Static Cultures from Microplate
Measurements of Pericellular Dissolved Oxygen Concentration.", Biotechnology and Bioengineering 2004, 86, 775-787.

[688] H.-L. Pang, N.-Y. Kwok, L. M.-C. Chow, C.-H. Yeung, K.-Y. Wong et al., "ORMOSIL Oxygen Sensors on Polystyrene Microplate for Dissolved Oxygen Measurement", Sensors and Actuators B: Chemical 2007, 123, 120-126.

[689] PreSens Precision Sensing $\mathrm{GmbH}$, "Regensburg, Germany", http://www.presens.de/.

[69o] DASGIP Information and Process Technology GmbH, "Jülich, Germany", http://www. dasgip.de/.

[691] Oxford Optronix Ltd., "Abingdon, United Kingdom", http://www.oxford-optronix.com/.

[692] Pyro Science GmbH, "Aachen, Germany", http://www.pyro-science.com/.

[693] Luxcel Biosciences Ltd., "Cork, Ireland", http://luxcel.com/.

[694] P. C. Thomas, M. Halter, A. Tona, S. R. Raghavan, A. L. Plant et al., "A Noninvasive Thin Film Sensor for Monitoring Oxygen Tension during in Vitro Cell Culture", Analytical Chemistry 2009, 81, 345-355.

[695] P. Avouris, B. N. J. Persson, "Excited States at Metal Surfaces and Their Non-Radiative Relaxation", The Journal of Physical Chemistry 1984, 571, 837-848.

[696] D. H. Waldeck, A. P. Alivisatos, C. B. Harris, "Nonradiative Damping of Molecular Electronic Excited States by Metal Surfaces", Surface Science $1985,158,103-125$

[697] X.-L. Zhou, X.-Y. Zhu, J. M. White, "Photodissociation of Intraadsorbate Bonds at Adsorbate-Metal Interfaces", Accounts of Chemical Research 1990, 23, 327-332.

[698] X.-L. Zhou, X.-Y. Zhu, J. M. White, "Photochemistry at Adsorbate/metal Interfaces", Surface Science Reports 1991, 13, 73-220.

[699] H. Imahori, H. Norieda, Y. Nishimura, I. Yamazaki, K. Higuchi et al., "Chain Length Effect on the Structure and Photoelectrochemical Properties of SelfAssembled Monolayers of Porphyrins on Gold Electrodes", The Journal of Physical Chemistry. B 2000, 104, 1253-1260.

[700] P. J. R. Roche, M. C.-K. Cheung, K. Y. Yung, A. G. Kirk, V. P. Chodavarpu et al., "Application of Gold Quenching of Luminescence to Improve Oxygen Sensing Using a Ruthenium (4,7-Diphenyl-1,10phenanthroline) ${ }_{3} \mathrm{Cl}_{2}$ :TEOS Thin Film", Sensors and Actuators B: Chemical 2010, 147, 581-586.

[701] D. Ghosh, N. Chattopadhyay, "Gold and Silver Nanoparticles Based Superquenching of Fluorescence: A Review", Journal of Luminescence 2015, 160, 223-232.

[702] H. Imahori, Y. Kashiwagi, Y. Endo, T. Hanada, Y. Nishimura et al., "Structure and Photophysical Properties of Porphyrin-Modified Metal Nanoclusters with Different Chain Lengths", Langmuir 2004, 20, 7381.

[703] G. Schneider, G. Decher, N. Nerambourg, R. Praho, M. H. V Werts et al., "Distance-Dependent Fluorescence Quenching on Gold Nanoparticles Ensheathed with 
Layer-by-Layer Assembled Polyelectrolytes", Nano Letters 2006, 6, 530-536.

[704] R. H. Perry, D. W. Green, J. O. Maloney, Perry's Chemical Engineers' Handbook, McGraw-Hill Book Company, New York, 2008.

[705] J. Hilsenrath, Tables of Thermal Properties of Gases: Comprising Tables of Thermodynamic and Transport Properties of Air, Argon, Carbon Dioxide, Carbon Monoxide, Hydrogen, Nitrogen, Oxygen, and Steam, U.S. Dept. Of Commerce, National Bureau Of Standards, 1955

[706] V. Heitmann, "Funktionelle Bedeutung Spezifischer Zell-Matrix-Interaktionen: Eine Biophysikalische Studie", Thesis, Westfälische Wilhelms-University Münster, 2008.

[707] G. Adam, P. Läuger, G. Stark, Physikalische Chemie Und Biophysik, Springer, Berlin - Heidelberg, 2009.

[708] R. C. Weast, CRC Handbook of Chemistry and Physics, CRC Press, Boca Raton, FL, USA, 1988.

[709] L. Korson, W. Drost-Hansen, F. J. Millero, "Viscosity of Water at Various Temperatures", The Journal of Physical Chemistry 1969, 73, 34-39.

[710] S. Arain, S. Weiss, E. Heinzle, G. T. John, C. Krause et al., "Gas Sensing in Microplates with Optodes: Influence of Oxygen Exchange between Sample, Air, and Plate Material", Biotechnology and Bioengineering 2005, 90, 271-280.

[711] C. Schweitzer, R. Schmidt, "Physical Mechanisms of Generation and Deactivation of Singlet Oxygen.", Chemical Reviews 2003, 103, 1685-1757.

[712] P. Lechtken, "Singulett-Sauerstoff", Chemie in unserer Zeit 1974, 8, 11-16.

[713] M. C. DeRosa, R. J. Crutchley, "Photosensitized Singlet Oxygen and Its Applications", Coordination Chemistry Reviews 2002, 234, 351-371.

[714] H. Sies, H. de Groot, "Role of Reactive Oxygen Species in Cell Toxicity", Toxicology Letters 1992, 64, 547-551.

[715] M. Valko, H. Morris, M. T. D. Cronin, "Metals, Toxicity and Oxidative Stress.", Current medicinal chemistry 2005, 12, 1161-1208.

[716] P. Ceroni, A. Y. Lebedev, E. Marchi, M. Yuan, T. V Esipova et al., "Evaluation of Phototoxicity of Dendritic Porphyrin-Based Phosphorescent Oxygen Probes: An in Vitro Study.", Photochemical \& Photobiological Sciences 2011, 10, 1056-1065.

[717] J. F. Rabek, B. Ranby, "Role of Singlet Oxygen in PhotoOxidative Degradation and Photostabilization of Polymers", Polymer Engineering and Science 1975, 15, 15-18.

[718] G. S. Egerton, A. G. Morgan, "The Photochemistry of Dyes. IV-The Role of Singlet Oxygen and Hydrogen Peroxide in Photosensitised Degradation of Polymers", Journal of the Society of Dyers and Colourists 1971, 87, 268-277.

[719] M. L. Kaplan, P. G. Kelleher, "Oxidation of a Polymer Surface with Gas-Phase Singlet $(1 \Delta \mathrm{g})$ Oxygen", Science 1970, 169, 1206-1207.

[720] A. Zweig, W. A. Henderson Jr, "Singlet Oxygen and Polymer Photooxidations. I. Sensitizers, Quenchers, and Reactants.", Journal of Polymer Science, Polymer Chemistry Edition 1975, 13, 717-736.

[721] B. Enko, S. M. Borisov, J. Regensburger, W. Bäumler, G. Gescheidt et al., "Singlet Oxygen-Induced Photodegradation of the Polymers and Dyes in Optical Sensing Materials and the Effect of Stabilizers on These Processes", Journal of Physical Chemistry A 2013, $117,8873-8882$.

[722] C. Ouannes, T. Wilson, "Quenching of Singlet Oxygen by Tertiary Amines. Effect of DABCO", Journal of the American Chemical Society 1968, 46, 6527-6528.

[723] E. A. Ogryzlo, C. W. Tang, "Quenching of Oxygen ( $1 \Delta \mathrm{g})$ by Amines", Journal of the American Chemical Society 1970, 92, 5034-5036.

[724] S. R. Ricketts, P. Douglas, "A Simple Colorimetric Luminescent Oxygen Sensor Using a Green LED with Pt Octaethylporphyrin in Ethyl Cellulose as the Oxygen-Responsive Element", Sensors and Actuators B: Chemical 2008, 135, 46-51.

[725] J. P. Guillory, C. F. Cook, "Energy Transfer Processes Involving Ultraviolet Stabilizers. Quenching of Singlet Oxygen.", Journal of Polymer Science, Polymer Chemistry Edition 1973, 11, 1927-1937.

[726] R. S. Atkinson, D. R. G. Brimage, R. S. Davidson, E. Gray, "Use of Tertiary Amino-Groups as Substituents to Stabilise Compounds towards Attack by Singlet Oxygen", Journal of the Chemical Society, Perkin Transactions 1 1973, 960-964.

[727] I. B. C. Matheson, J. Lee, "Quenching of Photophysically Formed Singlet $(1 \Delta \mathrm{g})$ Oxygen in Solution by Amines", Journal of the American Chemical Society 1972, 94, 3310-3313.

[728] R. A. Ackerman, I. Rosenthal, J. N. Pitts Jr, "Singlet Oxygen in the Environmental Sciences. $X$. Absolute Rates of Deactivation of $\mathrm{O}_{2}(1 \Delta \mathrm{g})$ in the Gas Phase by Sulfur Compounds", The Journal of Chemical Physics 1971, 54, 4960.

[729] N. Hasty, P. B. Merkel, P. Radlick, D. R. Kearns, "Role of Azide in Singlet Oxygen Reactions: Reaction of Azide with Singlet Oxygen.", Tetrahedron Letters 1972, 13, 49-52.

[730] S. Kaiser, P. Di Mascio, M. E. Murphy, H. Sies, "Physical and Chemical Scavenging of Singlet Molecular Oxygen by Tocopherols", Archives of Biochemistry and Biophysics 1990, 277, 101-108.

[731] K. Mukai, K. Daifuku, K. Okabe, T. Tanigaki, K. Inoue, "Structure-Activity Relationship in the Quenching Reaction of Singlet Oxygen by Tocopherol (vitamin E) Derivatives and Related Phenols. Finding of Linear Correlation between the Rates of Quenching of Singlet Oxygen and Scavenging of Peroxyl and Phenoxyl Ra", Journal of Organic Chemistry 1991, 56, 4188-4192.

[732] K. Ohara, K. Kikuchi, T. Origuchi, S. Nagaoka, "Singlet Oxygen Quenching by Trolox $C$ in Aqueous Micelle Solutions", Journal of Photochemistry and Photobiology B: Biology 2009, 97, 132-137.

[733] M. Y. Jung, D. B. Min, "ESR Study of the Singlet Oxygen Quenching and Protective Activity of Trolox on the Photodecomposition of Riboflavin and Lumiflavin in Aqueous Buffer Solutions", Journal of Food Science 2009, 74, 2-8. 
[734] A. Ouchi, K. Aizawa, Y. Iwasaki, T. Inakuma, J. Terao et al., "Kinetic Study of the Quenching Reaction of Singlet Oxygen by Carotenoids and Food Extracts in Solution. Development of a Singlet Oxygen Absorption Capacity (SOAC) Assay Method", Journal of Agricultural and Food Chemistry 2010, 58, 9967-9978.

[735] S. Nagaoka, A. Fujii, M. Hino, M. Takemoto, M. Yasuda et al., "UV Protection and Singlet Oxygen Quenching Activity of Aloesaponarin I", Journal of Physical Chemistry B 2007, 111, 13116-13123.

[736] S. Nagai, K. Ohara, K. Mukai, "Kinetic Study of the Quenching Reaction of Singlet Oxygen by Flavonoids in Ethanol Solution", Journal of Physical Chemistry $B$ 2005, 109, 4234-4240.

[737] C. Tournaire, S. Croux, M.-T. Maurette, I. Beck, M. Hocquaux et al., "Antioxidant Activity of Flavonoids: Efficiency of Singlet Oxygen ( $1 \Delta \mathrm{g}$ ) Quenching", Journal of Photochemistry and Photobiology B: Biology 1993, 19, 205-215.

[738] K. Mukai, S. Nagai, K. Ohara, "Kinetic Study of the Quenching Reaction of Singlet Oxygen by Tea Catechins in Ethanol Solution", Free Radical Biology and Medicine 2005, 39, 752-761.

[739] F. Wilkinson, W. P. Helman, A. B. Ross, "Rate Constants for the Decay and Reactions of the Lowest Electronically Excited Singlet State of Molecular Oxygen in Solution. An Expanded and Revised Compilation", Journal of Physical and Chemical Reference Data 1995, 24, 663-1021.

[740] M. E. Köse, B. F. Carroll, K. S. Schanze, "Preparation and Spectroscopic Properties of Multiluminophore Luminescent Oxygen and Temperature Sensor Films", Langmuir 2005, 21, 9121-9129.

[741] C. Baleiz̃a, S. Nagl, M. Schäferling, M. N. BerberanSantos, O. S. Wolfbeis, "Dual Fluorescence Sensor for Trace Oxygen and Temperature with Unmatched Range and Sensitivity", Analytical Chemistry 2008, 80, 6449-6457.
[742] S. M. Borisov, O. S. Wolfbeis, "Temperature-Sensitive Europium (III) Probes and Their Use for Simultaneous Luminescent Sensing of Temperature and Oxygen", Analytical Chemistry 2006, 78, 5094-5101.

[743] S. M. Borisov, A. S. Vasylevska, C. Krause, O. S. Wolfbeis, "Composite Luminescent Material for Dual Sensing of Oxygen and Temperature", Advanced Functional Materials 2006, 16, 1536-1542.

[744] L. H. Fischer, M. I. J. Stich, O. S. Wolfbeis, N. Tian, E. Holder et al., "Red- and Green-Emitting iridium(III) Complexes for a Dual Barometric and TemperatureSensitive Paint", Chemistry - A European Journal 2009, 15, 10857-10863.

[745] L. H. Fischer, C. Karakus, R. J. Meier, N. Risch, O. S. Wolfbeis et al., "Referenced Dual Pressure- and Temperature-Sensitive Paint for Digital Color Camera Read out", Chemistry - A European Journal 2012, 18, 15706-15713.

[746] H. Lam, G. Rao, J. Loureiro, L. Tolosa, "Dual Optical Sensor for Oxygen and Temperature Based on the Combination of Time Domain and Frequency Domain Techniques", Talanta 2011, 84, 65-70.

[747] S. Nagl, M. I. J. Stich, M. Schäferling, O. S. Wolfbeis, "Method for Simultaneous Luminescence Sensing of Two Species Using Optical Probes of Different Decay Time, and Its Application to an Enzymatic Reaction at Varying Temperature", Analytical and Bioanalytical Chemistry 2009, 393, 1199-1207.

[748] B. Zelelow, G. E. Khalil, G. Phelan, B. Carlson, M. Gouterman et al., "Dual Luminophor Pressure Sensitive Paint: II. Lifetime Based Measurement of Pressure and Temperature", Sensors and Actuators B: Chemical 2003, 96, 304-314.

[749] S. Nagl, O. S. Wolfbeis, "Optical Multiple Chemical Sensing: Status and Current Challenges.", The Analyst 2007, 132, 507-511. 



\section{Acknowledgement}

I thank my supervisor Prof. Dr. Joachim Wegener for issuing this thesis, for any support during the work and for the excellent working conditions in his group.

Moreover, I thank the board of examinars for their thesis reports and the oral examination as well as the chairman of the committee for leading the doctoral exam.

Not least, I want to thank all members of the institute, all former colleagues and especially all friends of the working group for all the help. 

$\mathrm{CV}$

The CV is omitted in this electronic version due to privacy reasons. 



\section{Eidesstattliche Erklärung}

Hiermit erkläre ich an Eides statt, dass ich die vorliegende Arbeit selbst verfasst habe und keine anderen als die angegebenen Hilfsmittel verwendet habe.

München, den 11. Dezember 2015

Maximilian Oberleitner 
